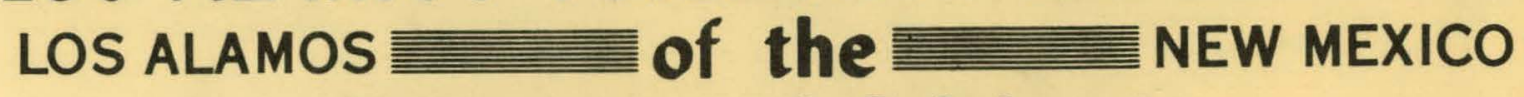
University of California

\title{
PROCEEDINGS OF AN ADVANCED NUCLEAR PROPULSION SYMPOSIUM
}

\author{
UNITED STATES \\ ATOMIC ENERGY COMMISSION \\ CONTRACT W-7405-ENG. 36
}




\section{DISCLAIMER}

This report was prepared as an account of work sponsored by an agency of the United States Government. Neither the United States Government nor any agency Thereof, nor any of their employees, makes any warranty, express or implied, or assumes any legal liability or responsibility for the accuracy, completeness, or usefulness of any information, apparatus, product, or process disclosed, or represents that its use would not infringe privately owned rights. Reference herein to any specific commercial product, process, or service by trade name, trademark, manufacturer, or otherwise does not necessarily constitute or imply its endorsement, recommendation, or favoring by the United States Government or any agency thereof. The views and opinions of authors expressed herein do not necessarily state or reflect those of the United States Government or any agency thereof. 


\section{DISCLAIMER}

Portions of this document may be illegible in electronic image products. Images are produced from the best available original document. 


\section{LEG A L NOTICE}

This report was prepared as an account of Government sponsored work. Neither the United States, nor the Commission, nor any person acting on behalf of the Commission:

A. Makes any warranty or representation, expressed or implied, with respect to the accuracy, completeness, or usefulness of the information contained in this report, or that the use of any information, apparatus, method, or process disclosed in this report may not infringe privately owned rights; or

B. Assumes any liabilities with respect to the use of, or for damages resulting from the use of any information, apparatus, method, or process disclosed in this report.

As used in the above, "person acting on behalf of the Commission" includes any employee or contractor of the Commission, or employee of such contractor, to the extent that such employee or contractor of the Commission, or employee of such contractor prepares, disseminates, or provides access to, any information pursuant to his employment or contract with the Commission, or his employment with such contractor.

All LA... MS reports are informal documents, usually prepared for a special purpose and primarily prepared for use within the Laboratory rather than for general distribution. This report has not been edited, reviewed, or verified for accuracy. All LA... MS reports express the views of the authors as of the time they were written and do not necessarily reflect the opinions of the Los Alamos Scientific Laboratory or the final opinion of the authors on the subject.

Printed in USA. Price $\$ 7.00$. Avallable from the

Clearinghouse for Federal Scientific

and Technical Information,

National Bureau of Standards,

U. S. Department of Commerce, Springfield, Virginia 


\title{
LOS ALAMOS SCIENTIFIC LABORATORY LOS ALAMOS University of California
}

Report written: January 1965

Report distributed: June 1, 1965

\section{PROCEEDINGS OF AN ADVANCED NUCLEAR PROPULSION SYMPOSIUM}

\author{
Compiled by
}

Ralph S. Cooper 
THIS PAGE

WAS INTENTIONALLY

LEFT BLANK 
FOREWORD

In April 1964, a small symposium was held at Los Alamos on the subject of advanced nuclear propulsion, by which was meant those concepts beyond the solid core heat exchanger using nuclear energy to produce a high thrust propulsion system. The attendance was limited, but an attempt was made to have at least representatives of all groups active in the field. No formal papers were required and much of the time was spent in discussion, but it was felt that a record of the proceedings would be useful. Transcriptions were made from tape recordings through the courtesy of W. E. Matheson and D. E. Knapp of the Douglas Aircraft Company who also handled the work of obtaining edited copies from the authors. Editing was generally kept to a minimum, so these proceedings retain the informal character of the meeting. Some of the material reported was work in progress and therefore preliminary, and due caution should be exercised in using or quoting results contained herein. We have tried to eliminate the repetition of material which has since been published, and in those cases usually have included only the abstract and a reference to the work.

The bulk of the material presented was unclassified (as determined by the organizations making the presentation) and is included in this volume. The classified material will be published separately in a supplement to this volume. Together they make a reasonably complete but brief survey of the field as of Apr11 1964. Althouglh the primary purpose of the mecting was an exchange of information among a few workers in the field it is hoped that the proceedings will be useful to others who were unable to attend. 
THIS PAGE

WAS INTENTIONALLY

LEFT BLANK 


\section{TABLE OF CONTENTS}

Page

FOREWORD $\quad$. . 3

INTRODUCTION

Ralph S. Cooper
Los Alamos Scientific Laboratory, Los Alamos, N. Mex.

SPACE TRANSPORTATION

M. W. Hunter, II

Bellcomm, Inc., Washington, D. C.

BNL FLUIDIZED BED STUDIES

L. P. Hatch

Brookhaven National Laboratory, Upton, L. I., N. Y.

FLASH X-RAY ANALYSIS OF FLUTIIZED BEDS

Jacob B. Romero

The Boeing Company, Seattle, Wash.

FLUIDIZED BEDS

A. C. Juveland, H. P. Deinken, and J. E. Dougherty

Los Alamos Scientific Laboratory, Los Alamos, N. Mex.

FLUIDIZED BEDS

Ralph Cooper

Lus Alamus Sulentffic Laboratory, Los Alamos, N. Mex.

THE LIQUID CORE NUCLEAR ROCKET

W. Louis Barrett, Jr.

The Boeing Company, Seattle, Wash.

THE LIQUID-CORE NUCLEAR ROCKET

Jerry Grey

Princeton University, Princeton, N. J.

EXPERIMENTAL WORK IN RELATION TO THE GLOW-PLUG CONCEPT

J. W. McKee

General Electric Company, Santa Barbara, Calif. 
TABLE OF CONTENTS (Continued)

THE TRANSPARENT PARTITION GASEOUS CORE REACTOR CONCEPT

D. E. Knapp

Douglas Aircraft Company, Inc., Santa Monica, Calif.

WORK ON NUCLEAR LIGHT BULB AT UAC RESEARCH

LABORATORIES

George H. McLafferty

United Aircraft Corporation Research Laboratories, Hartford, Conn.

A TRANSPIRATION-COOLED NOZZLE AS APPLIED TO A GAS CORE NUCLEAR PROPULSION SYSTEM

Elliot L. Kramer and Sig Gronich

(Presented by D. E. Knapp)

Douglas Aircraft Company, Inc., Santa Monica, Calif.

HIGH-TEMPERATURE GAS INTERACTIONS

Jerry Grey

Princeton University, Princeton, N. J.

COAXIAL FLOW GASEOUS NUCLEAR REACTOR CONCEPT

Frank E. Rom

NASA-Lewis Research Center, Cleveland, Ohio

COAXIAL FLOW RESEARCH STUDIES

Robert G. Ragsdale

NASA-Lewis Research Center, Cleveland, Ohio

COAXIAL FLOW RADIATION HEAT TRANSFER ANALYSIS

Albert F. Kascak

NASA-Lewis Research Center, Cleveland, Ohio

GAS CORE REACTORS

Henry J. Stumpf

Jet Propulsion Laboratory, Pasadena, Calif.

SEPARATION MEASUREMENTS IN BINARY SPECIES

GASEOUS VORTEX FLOWS AND FLOW VISUALIZATION STUDIES IN A WATER VORTEX

T. J. Pivirotto and E. J. Roschke

(Presented by Paul F. Massier)

Jet Propulsion Laboratory, Pasadena, Calif. 
TABLE OF CONTENTS (Continued)

Page

VORTICES AND VORTEX MATRICES

Martin L. Rosenzweig and Steve Lewellen

Aerospace Corporation, Los Angeles, Calif.

NUCLEAR PROPULSION SCHEMES USING MAGNETO-

HYDRODYNAMIC CONVERSION TECHNIQUES

Richard J. Rosa and Thomas R. Brogan

Avco-Everett Research Laboratory, Everett, Mass.

VORTEX GAS ACCELERATOR

Stirling A. Colgate

University of California, Livermore, Calif.

FUEL CONTAINMENT IN THE GASEOUS-CORE

NUCLEAR ROCKET BY MHD-DRIVEN VORTICES

Jacob B. Romero

The Boeing Company, Seattle, Wash.

CRITICALITY OF GAS CORE REACTORS

315

R. J. Holl

Douglas Aircraft Company, Santa Monica, Calif.

CAVITY REACTOR

George A. Jarvis

Los Alamos Scientific Laboratory, Los Alamos, N. Mex. CRITICALITY

C. B. Mills

Los Alamos Scientific Laboratory, Los Alamos, N. Mex. 
THIS PAGE WAS INTENTIONALLY LEFT BLANK 


\title{
INTRODUCTION
}

\author{
Ralph S. Cooper \\ Los Alamos Scientific Laboratory \\ Los Alamos, New Mexico
}

The field of advanced nuclear propulsion is of special interest to the Laboratory, and several of us here have been examining various aspects of it. Over the next three days we are having a meeting of those people who are actively involved in research on this topic. This is the first. such gathering to include all high thrust concepts ranging from fluidized particle beds to pulsed nuclear rockets.

In our regular sessions we shall deal only with the propulsion systems themselves rather than with the overall mission analyses. Most of the specialists realize the value of a high thrust, high performance system, but for our more general audience at this colloquium and as a keynote speaker we shall have a talk by Mr. Maxwell Hunter on the potential of these systems for manned space transportation.

Mr. Hunter has a degree in Mathematics and Physics and an advanced degree in Aeronautical Engineering. He was involved in the design of aircraft and missiles for the Douglas Aircraft Company for many years. This involved complicated, sophisticated systems such as the Nike missile which was coupled to various upper stages and a complex electronic radar and control system. Furthermore he worked on the Thor rocket which has recently scored over twenty consecutive successful launches.

The remarkable thing is that through all the hard engineering Max has done, he has maintained a very far-sighted and optimistic attitude toward propulsion systems and their use. His views are always stimulating and provoking and are worth serious consideration. 


\section{SPACE TRANSPORTATION}

M. W. Hunter, II

Flight Systems Division

Bellcomm, Inc.

Washington, D. C.

Well, that is an introduction that leaves me wondering where to go next. The business of having been the Chief Design Engineer at Douglas when the Thor was designed is a pretty good entree to practically any place in the world these days. It hadn't occurred to me that the only exception was here. I prefer to create the illusion of utter honesty at this point. I'm going to need it as I go. Honesty, therefore, compels me to bring the record of the Delta missile up-to-date. After 22 successes in a row, there have been two failures in a row. This apparently is not the year of the Delta. My friends at Douglas tell me that one of them may have been the fault of the payload, but you know the problem of trying to figure out afterwards who really did it. However, we can't be quite as bushy-tailed about the Delta this spring as we were up until January.

I think I should give you a little introduction as to where part of this briefing came from and particularly to explain the order I am going to use: There was a panel discussion at the American Nuclear Society in November. Some of you were there. This panel consisted of an unusual collection of people, namely, Stan Ulam, Wernher von Braun, myself, and Isaac Asimov. A great deal of hilarity was generated about the question of who was the science fiction man on the panel. Or, if you went down the list of names as I gave them, which was the way we were sitting at the table, which end was the rational one and which the science fiction, or were they the same?

This did put me in the position of being after von Braun and before Asimov, and it seemed right from the beginning that this was going to involve a problem in getting from the sublime to the ridiculous or vice versa. At any rate, I had to come up with something to bridge this gap with science fiction. I knew that von Braun would cover normal, mundane nuclear rockets 
without fail. So I gave a two-phase talk, one phase of which involved primarily gaseous fission engines, and the other involved a little bit of speculation on interstellar travel. Since we're going to be discussing gaseous fission and things like that for the next three days, I'm going to invert this order. I will talk about the interstellar situation first, and then we will simmer down to something nice and rational. We will be getting more rational as we go this time. With any luck, by the time we get to the end, I'll be rational enough for you to believe it.

One must carefully understand the ground rules when speculating about interstellar travel. Compared to most discussions of vehicles, systems or capabilities, the ground rules are totally different. In the latter half of this talk, I'm going to pay a great deal of attention to confining myself to such things as radiator temperatures which are reasonable, and various other practicalities. When one sits back and discusses interstellar travel, however, one talks of not just now or the next century, but of cosmic time scales. Vast advances in technology throughout the centuries are assumed, and all engineering problems are assumed solvable. One worries only about violating physical fundamentals. The more intelligent people worry about whether we even know what fundamentals to violate, but that makes the story even more complicated. In general, one talks about grand things. Are there other civilizations out there? If there are, are the fundamental barriers due to Einstein's limitations on velocity of travel so great that no. civilization imaginable could ever hope to travel such distances? Should we listen, as the radio astronomers say, and hope to learn something from these supercivilizations? The discussion is always in the context of an overall deep phllosophical sort of thing. That's the context of the first part of this talk. I will tell you when I shift gears and get rational. Unfortunately, you may have to be told this.

We can delineate these two regions by means of Figure 1 , which is a plot of specific impulse versus dilution ratio for perfect containment. Fission rockets are on the lower curve and fusion rockets on the higher. A perfect mass annihilation system is shown: at the top. I've defined several regions on Figure 1. If we were to operate a rocket with nothing but nuclear fuel (very low dilution ratio), a very high specific impulse, over a million seconds, would result. The temperatures are just tremendous, however, and no one knows how to begin to handle them. A lot of hydrogen, or some other propellant, can be put through the reactor to decrease the temperature. The solid core region down at the bottom, which we're all familiar with, is limited to a low value of specific impulse because of the temperature limitations on the solid-core materials. We can get higher performance by going to gaseous-core rockets or Orion which at least do not run headlong into the 
SPECIFIC IMPULSE FROM NUCLEAR REACTIONS

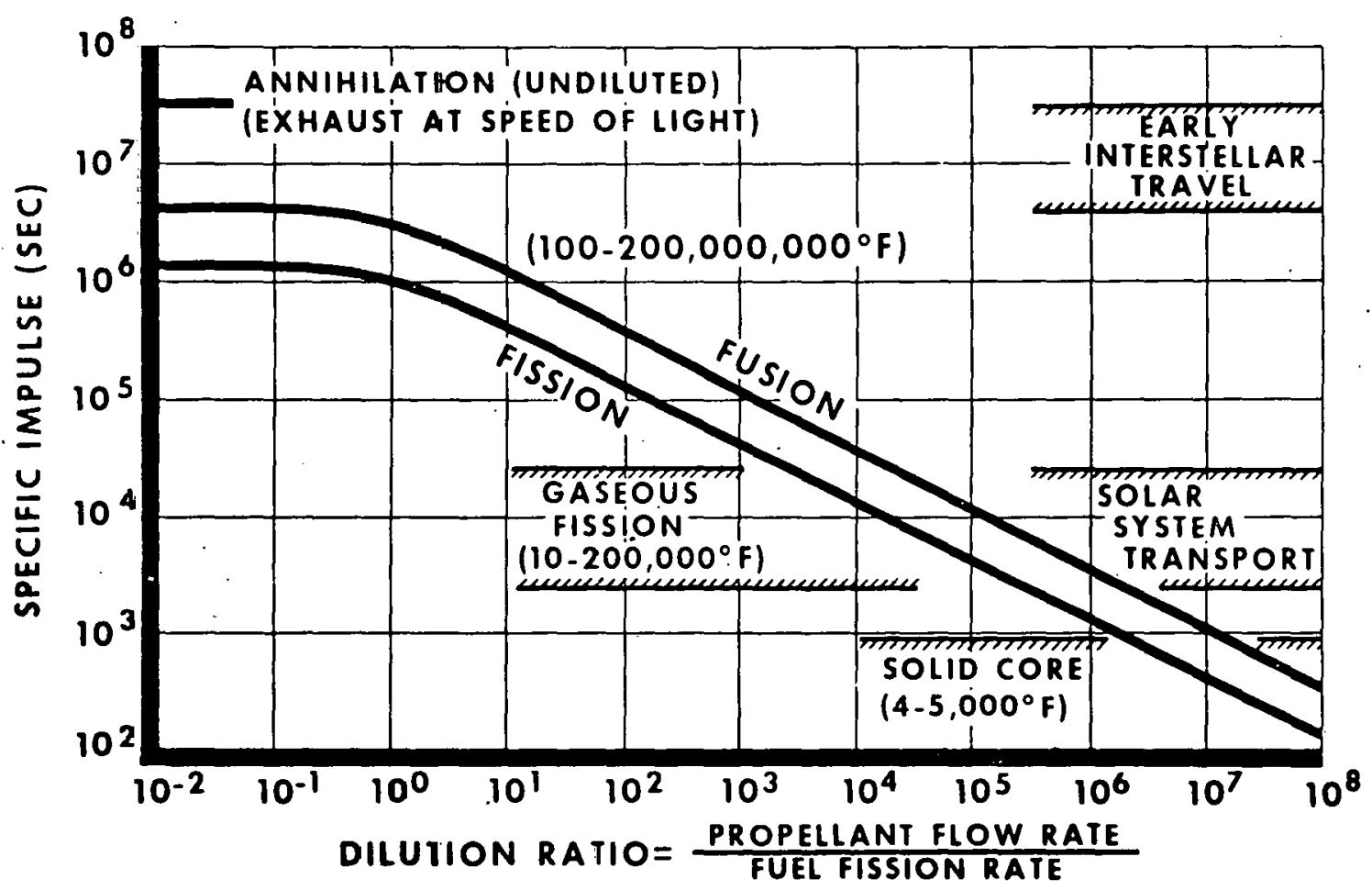

Figure 1 
materials temperature barrier. This higher region I have labeled the Solar System Transport Region, and is the region $I^{\prime} \mathrm{m}$ going to cover in the second half of the talk. The top region, labeled Early Interstellar Travel, is the region of the first part of my talk. I'm going to cover only undiluted fusion rockets. I will not bother with mass annihilation rockets, although people who discuss interstellar travel are not at all adverse to describing rocket ships operating with 100 percent efficiency on the complete annihilation of matter. It's bad enough to talk about undiluted fusion rockets, which I' $\mathrm{m}$ sure you'll recognize we do not know how to build.

Under interstellar ground rules, some very interesting things materialize. I find that I disagree with a number of basic points which some people seem to think are great. Figure 2 contains most of my complaints all in one place. It shows a curve of initial weight of rocket over final weight as a function of rocket maximum velocity divided by the velocity of light. This curve is for what I call a perfect fusion rocket. This means that not only is the fusion reaction running like mad with perfect efficiency while throwing only fusion fuel out the back, but in addition the rocket has a reasonable thrust/weight ratio like one or two. I haven't the remotest idea of how to build anything like that. Still, at least it is something that I am using a fairly legitimate fusion reaction rather than talking matter annihilation.

The weight variation of Figure 2 was calculated including relativistic effects. The interesting thing, as we all know, is due to a curve ball thrown by Einstein. In the region of one-third the speed of light, the rocket initial weight is about 100 times the final weight. In actuality, we build probes today with weight ratios in the thousands, so that fusion rockets of up to 0.4 the speed of light are imaginable. From there on, however, they start getting very, very large. To get very close to the speed of light, the weight of the rocket becomes ridiculous. Now almost everyone who studies interstellar travel assumes that it does not make sense until 99 percent of the speed of light has been attained. You can guess the kind of rocket required at that speed. I didn't even bother to plot it, and I am almost fearless as far as plotting rocket weights is concerned.

I, myself, do not understand why people seem to have this compulsion to examine casually low velocity rockets, then immediately jump to 99 percent of the speed of light. As a so-called engineer, I've made many mistakes in my life by taking only one point at each end of a curve and thinking I understood what went on in between. At one-third the speed of light, the travel duration in earth time is only three times that at the speed of light. Of course, we might decide to approach the speed of light in order to reduce ship time by means of the time dilation effect. This relativistic time dilation is also shown on Figure 2. 


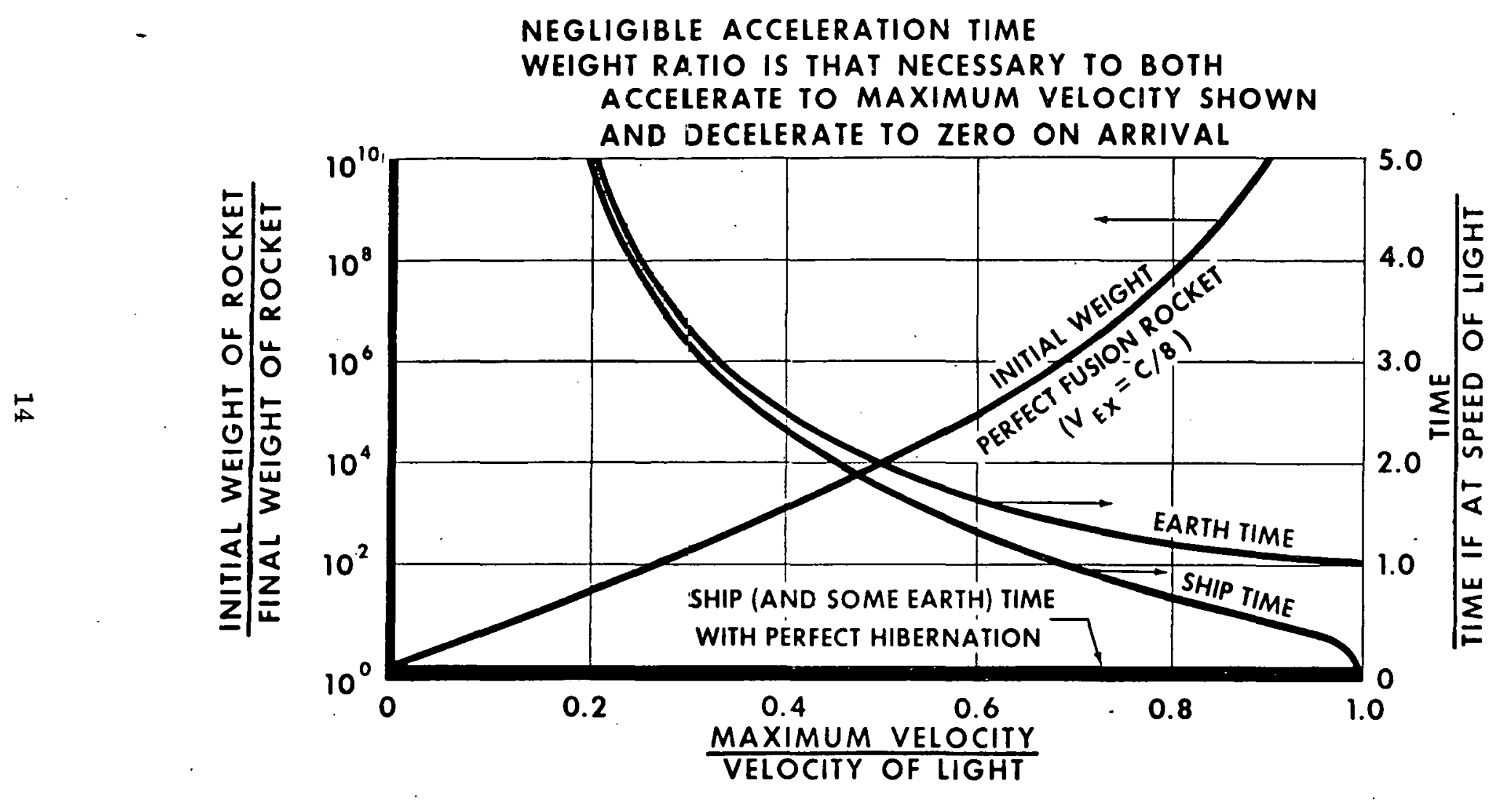

Figure 2. Interstellar Travel - Comparison of Relativistic and Biological Time Dilation Effects 
Approaching the speed of light closely is the only way open to physicists for dilating time. Presumably, there are no narrow-minded physicists here, however, and we all recognize that there are other disciplines in the world. One of them is biology. Although I am never quite sure of what is going on in the field of biology, some pretty weird things have been happening in the last few years. I get the impression that we are getting closer and closer, by deep freeze and other techniques, to learning about hibernation. Hibernation is biological time dilation. With biological time dilation, it is conceivable not only that one could come clear down to zero time, but also that this could be both for ship and for some earth time. If your wife loves you enough, she, too, can step into a deep freeze until you get back. This brings up a small question as to who has the key to the deep freeze. Regardless of such practical problems, the point is you can't dilate earth time by ship velocity, no matter how fast you drive the ship.

The question of whether one is at all interested in ships which travel at one-third the speed of light, or feel that almost the speed of light is required, therefore, has a great deal to do with a totally different discipline from physics. If the blologists do something about hibernation, they will exert a much greater leverage, both on earth and in the ability to build reasonable starships, than any possible attempt to drive ships out to the speed of light. So far as I am concerned, the people that make analyses with speeds only 1 percent lower than the speed of light, then conclude, "This is preposterous; we could never go there," are really performing a pretty naive systems analysis of interstellar travel.

Even at only one-third the speed of light, these are pretty cute ships. Other than bombs, I'm not sure that this Laboratory has done a very good job of controlling fusion reactions yet; and this rocket must be light weight, have perfect efficiency, and be safe. Furthermore, this ship, compared to one utilizing a gaseous fission engine, must control about three orders of magnitude higher thermal fluxes in order to keep from vaporizing. In addition, there is another factor of about four orders of magnitude on total power generated to obtain these speeds. Because the resulting shielding penalties are pretty horrendous, the actual payload carried will be a small fraction of the final weight.

Figure 3 is a plot of the initial power of a perfect rocket with final weight of 10,000 pounds as a function of maximum design velocity. The right-hand scale gives the power which would have to be rejected by a radiator system, assuming 10 percent of the energy soaked into the structure. Also shown is a typical number for a gaseous fission engine of about 2,500 seconds specific impulse and one million pounds of thrust, the sort of engines 
FUSION ROCKET POWER

POWER RADIATED

BY SUN

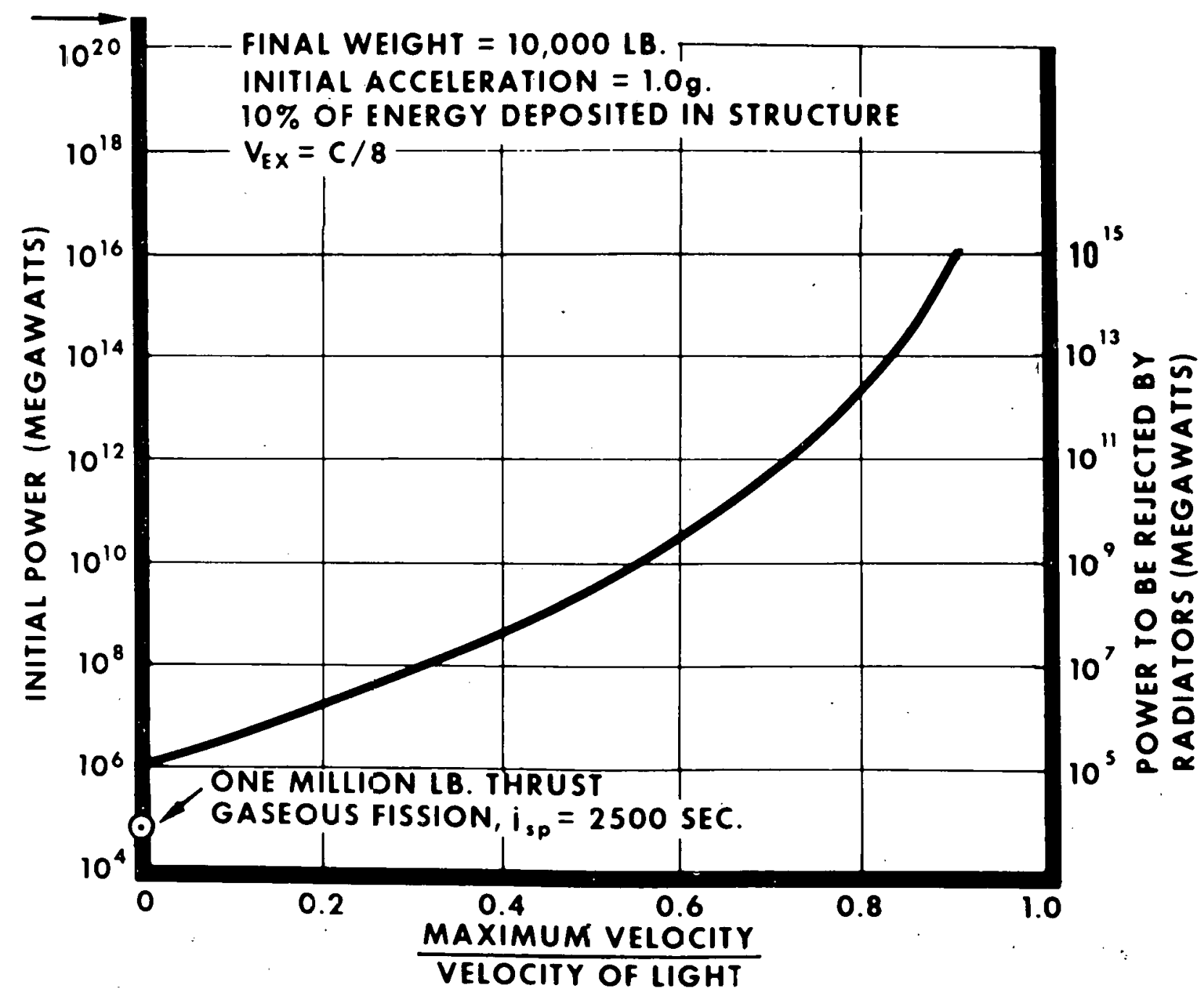

Figure 3 
we'll talk about later. For a ship to generate 0.3 the velocity of light, it must improve three orders of magnitude or so in its energy handling capability for the same thrust level. If you did get these reactions running, if you could understand how to do this at a reasonable weight, we still have three orders of magnitude of energy which somehow has to be taken in and out of the structure, or we're going to vaporize the ship right on the spot. So, even if you could turn around tomorrow and say, "Here's the engine," it's not clear at all that we could use it on these missions.

On the other hand, this is only 3 orders of magnitude, not 30 orders of magnitude. In any given year, 3 orders of magnitude sounds pretty grim to us, but that kind of number has been known to be run over in development programs in a relatively few decades. There are ways in which it might be possible to cut this number down. Ten percent of energy soaked into the structure is typical of a gaseous fission engine. An Orion system does not put as high a percentage of its energy into the structure. Any case where a fusion reaction would be different from a fission reaction and put less energy into the structure lowers the number. When the reaction is not moderated, then we might have the reaction running in a relatively transparent engine shell, so that a lot of the energy would go straight through. If the opaqueness were only 1 percent, that would be an order of magnitude. I' $m$ not saying that I know even remotely how to begin this. 'I'm simply throwing out some suggestions to indicate that from here to there just may not be centuries, it may be something like decades. Many people throw up their hands and say, "Forevermore, there will never be any interstellar travel. It doesn't make any sense." They are saying that forevermore we' re not going to improve our energy control by three orders of magnitude. I' $m$ not sure that is a suitably cosmic viewpoint.

I couldn't resist spotting the power of the sun on Figure 3 . In the region beyond 96 percent of the velocity of light, the rocket is putting out more power than the whole sun. Once again, it's easy to decide that it's a pretty preposterous idea - and it is. Although, I don't know; I don't trust you people. I think maybe a design that would do that might be appealing to some here.

Now that we have settled the fact that we can have such ships, it seemed appropriate to present a picture of the whole galaxy as seen by a starship designer. Figure 4 shows the number of stars in our galaxy versus the distance in light years away from the star we're located near now. I would prefer not to put much of my reputation behind the accuracy of these curves. The top curve shows the total number of stars. Presumably, a good astrophysicist, at least for a while, would be interested in a close look 
ASSUMPTIONS: $5 \%$ OF STARS ARE F, G, K TYPES WITH PLANETS 5. $\times 10^{\circ}$ YEARS FOR STAR TO PRODUCE A SOCIETY $5 \times 10^{8}$ YEAR SPAN FOR BIOLOOGICAL DATA 50,000 YEARS ACTIVELY COMMUNICATING CIVILIZATION

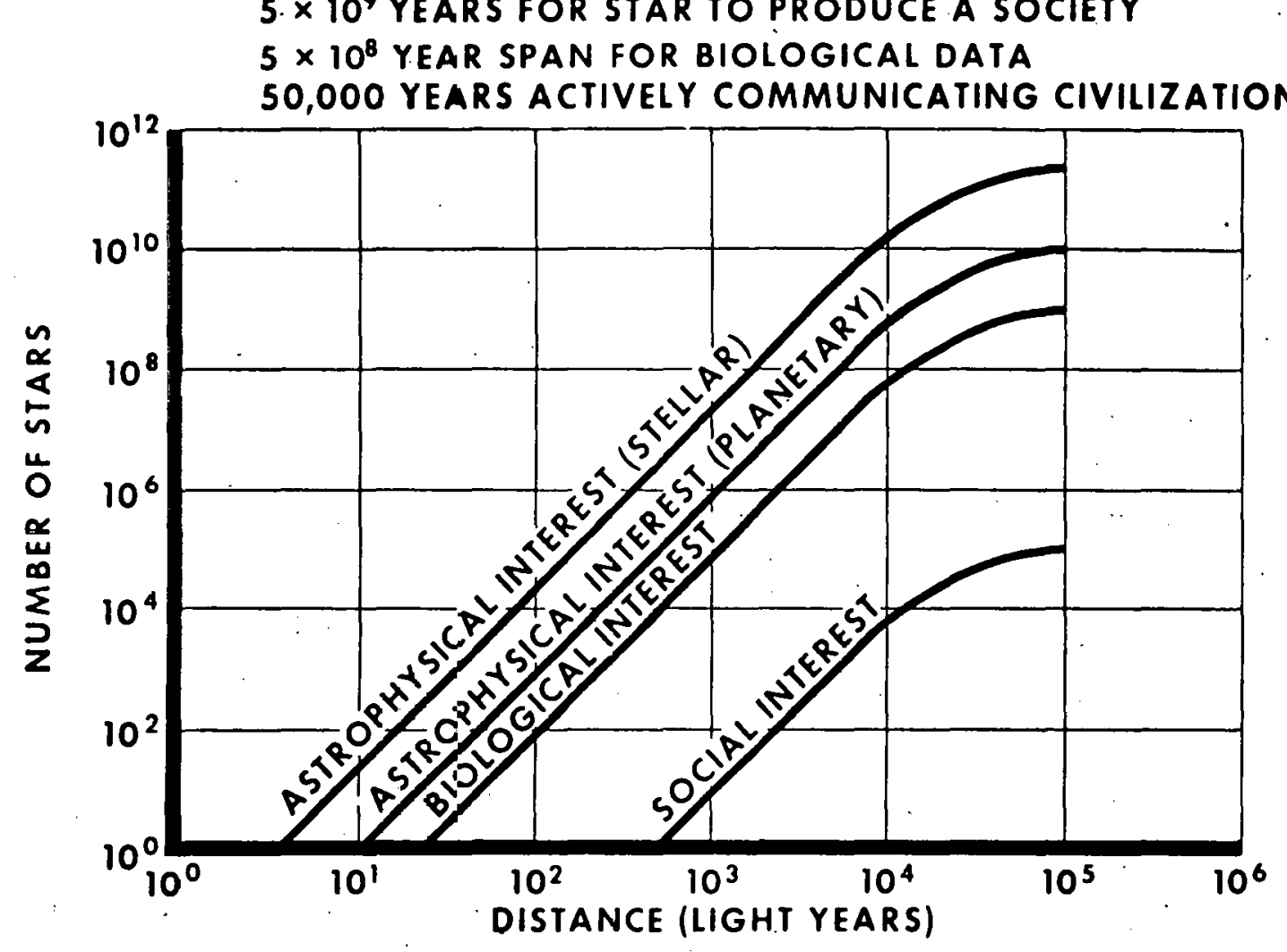

Figure 4. Galactic Community 
at most any of them. Furthermore, we have reason to suspect that $F, G$, and $\mathrm{K}$ type stars, considering their rates of rotation, have planetary systems. They constitute about 5 percent of the stars. They are likely to be of more lasting interest than stars without planets. This was the basis for drawing the curve labeled planetary astrophysical interest.

This still leaves the question of contact with an alien race. Since the radio-astronomers say we should do nothing but listen for the rest of our lives, the question of the probability of an alien transmission arises. It is, to say the least, a difficult estimate to make. We have a pretty good reason to believe that there are an awful lot of stellar systems with planets. We also have a lot of reason to believe, due to the researches on chemical evolution, that life would arise spontaneously on most of these. There still remains the question of the rise of intelligence and the rise of culture. Furthermore, if a culture reaches the point where it wants to communicate, how long will it have the urge? Our culture has not been communicating very 1ong. Over any distance, it's only a few decades and in terms of written records, only a few millenia. It could be that after another 5,000 years, the human race won't have a scientific culture. We may be living at the height of the scientific society. Maybe in another hundred years, it'11 all be philosophical and no one will develop anything - a hundred years, that is! Perhaps our descendants will not care about communicating with anyone. Even today, there are a lot of people on this planet that I couldn't care less about communicating with. I might add that this is healthily returned with respect to me by a lot of people on the same planet.

The bottom curve labeled social interest assumed that life would develop at each $F, G$, and $K$ type star, that after 5 billion years it would produce a society, and that the average society would only be actively interested in communicating with other civilizations for about 50,000 years. The 5 billion years is based on precisely one data point; namely, the time required by our star to produce a society. I've often wondered what will happen if we get two data points on that subject. The 50,000 years is based on even less data. If those assumptions are correct, however, the bottom curve results. It is not surprising that there is a tendency for the radio-astronomers to say that we should never try to go to the stars. The galaxy is a big place and there should be plenty of communicating societies, but the nearest one is a very long ways off. If only currently communicating societies interest us, perhaps all we should do is listen from here, and hope to learn something.

I think the astronomers are missing a point, not even counting the fact that I don't think they know very much about rockets. There is another 
class of stellar system which should interest us. This interest is created because a ship that goes there is, in a way, a time machine. We only possess deliberate communication records of a society on this planet for a few thousand years. We have looked hundreds of millions of years into the past, however, learning things of biological interest such as the patterns of the development of life. Therefore, if one goes to a place and explores, one can look both back and ahead in time as compared with the limited real time contact with any currently communicating society. I don't think anyone in this room has ever talked to a dinosaur, but we've learned quite a bit about the age of the dinosaurs over a hundred million years ago. You may not know whether to bring micro-biologists or archeologists, but you are able to look both back and forward in time. If you assume 500 million years as the time during which a planet has biological interest based on our own use of data from a comparable time on this planet, then the remaining curve on Figure 4 results.

The probable time of data return from the stars is shown on Figure 5 . For travel, it was assumed that the ships would travel at one-third the speed of light, then transmit data back at the speed of light after arrival. For communicating, the assumption was that a signal was received from the most probable distance tomorrow which we immediately returned to this advanced civilization which then, in turn, sent it back to earth. The travel curves show data return if you start sending ships tomorrow and the communication curve is the time for data return if you receive a signal tomorrow.

The receipt of any signal tomorrow from an alien race would be extremely stimulating, and it is obviously well worth listening. It would seem that if you stick only to listening, however, it would take 1,000 years for a reply if we heard tomorrow from the most probable distance. If one travels for purely stellar physics interests, one can get results much earlier. Even for planetary interests as well as stellar, the results are earlier. In fact, within 100 years, information should have been picked up from 15 or so stars with planets, one or two of which should have data of biological interest. If one sticks to only listening, another 900 years must pass before anything happens.

It is apparently fashionable today to say, "Only communicating is the thing to do. Travel is nonsense, and belongs back on the cereal boxes." But only the bottom curve of Figure 5 is available to the listeners and thinkers, while the other curves are available to the 'goers and doers.' I wish to make a historical point which is true, regardless of what you may think today in our current intellectual framework. All of the history of this race is squarely on the side of the 'goers and doers.' 


\section{ASSUMPTIONS:}

$5 \%$ OF STARS ARE F, G,K TYPES WITH PLANETS $5 \times 10^{\circ}$ YEARS FOR STAR TO PRODUCE A SOCIETY $5 \times 10^{8}$ YEAR SPAN FOR BIOLOGICAL DATA 50,000 YEARS ACTIVELY COMMUNICATING CIVILIZATION STARSHIP VELOCITY AVERAGES $1 / 3$ SPEED OF LIGHT

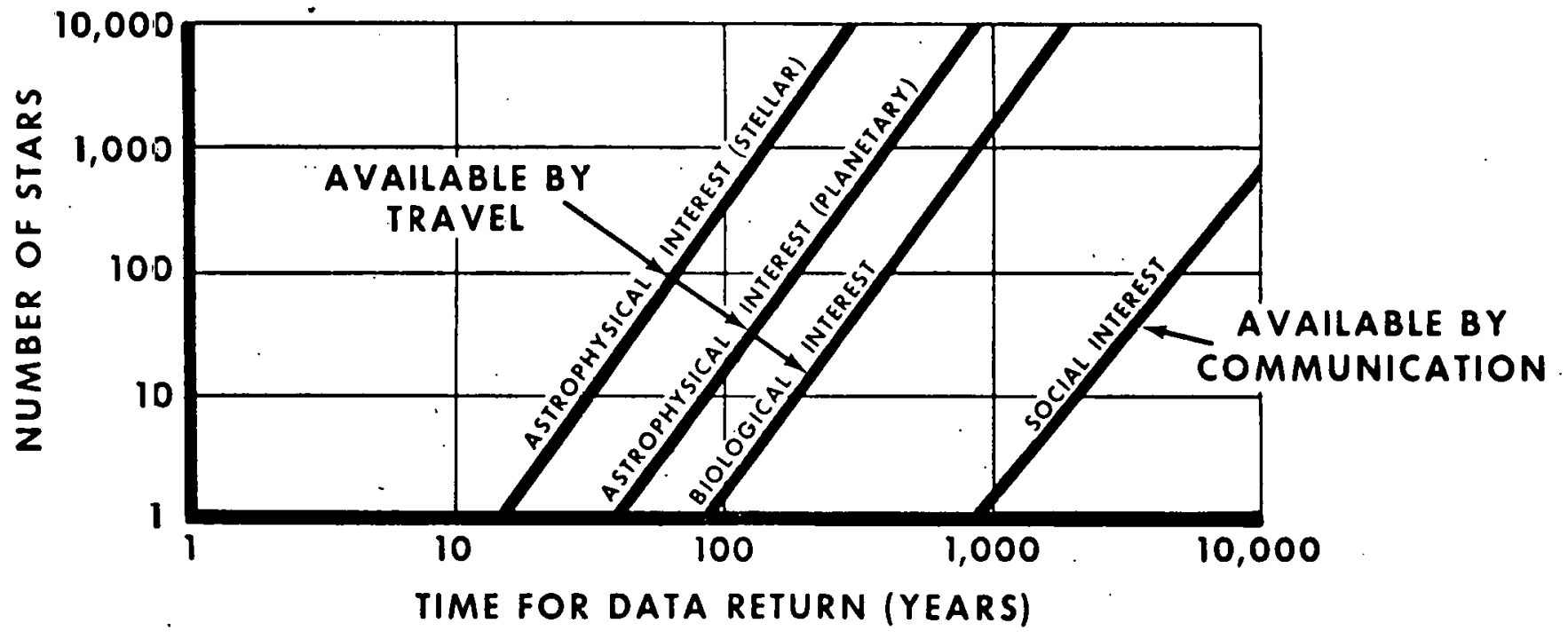

Figure 5. Galactic Community 
Now, here is where the gear shift comes in. And if any one of you have, on the back of an envelope, designed a fusion rocket for that starship while I was talking, please keep quiet until I get through with the rest of this. We'll discuss it afterward.

Now, to regress to the region of solar system transportation. I'm going to confine my discussion almost completely to gaseous fission rockets. The reason is not that I have decided that they are the things to be used rather than Orion or electrical rockets. The reason is simply that I have done more thinking about them. I think that I can see how to combine gaseous fission engines with advanced vehicles more efficiently than is possible with the other engine types. I believe that not enough thought has been given to the engine/vehicle interaction. There was a statement in the letter of invitation to this symposium to the effect that everybody knows what to do with 1800 seconds specific impulse. I happen to disagree. I don't think anybody knows what to do with 1800 seconds specific impulse, and I think we wouldn't know what to do with a good space engine if it walked up and bit us. I will try to prove those opinions as I go. I've used the "we" advisedly because I don't think I know either. Rather than getting into a big mish-mash by attempting to cover all various forms of propulsion, I will just stick with some mythical gaseous fission rockets. Presumably in the next 3 days we will discuss which engines you really should do this with, if you should do it at all.

Figure 6 is a presentation of operating cost in dollars per pound of payload versus total velocity capability for single-stage vehicles with chemical, nuclear solid-core, and two different kinds of gaseous fission rocket engines. These curves were calculated four years ago when I was at Douglas for a paper by myself, Bill Mathiesen and Bob Trapp that was given at the I. A. F. Congress in Stockholm. We thought this was pretty interesting, but other than shocking an occasional person here and there, not very much has happened as a result. The thing that has been interesting to me is the fact that here was a clear indication that one could get out to extremely high velocities for a very low transportation cost. Velocities so high that you could open up the whole solar system for exploration with reasonable costs. Yet almost everyone believes it is extremely hard to do a little bit of space flight down in the low velocity region where we talk about just barely going to the moon.

Figure 6 also is an interesting indication of the fact that you shouldn't drive a rocket faster than it wants to be driven. This is something that apparently a lot of people are forgetting. Theoretically, a rocket can go up to any velocity, not counting Einstein. But the way to make an inefficient 


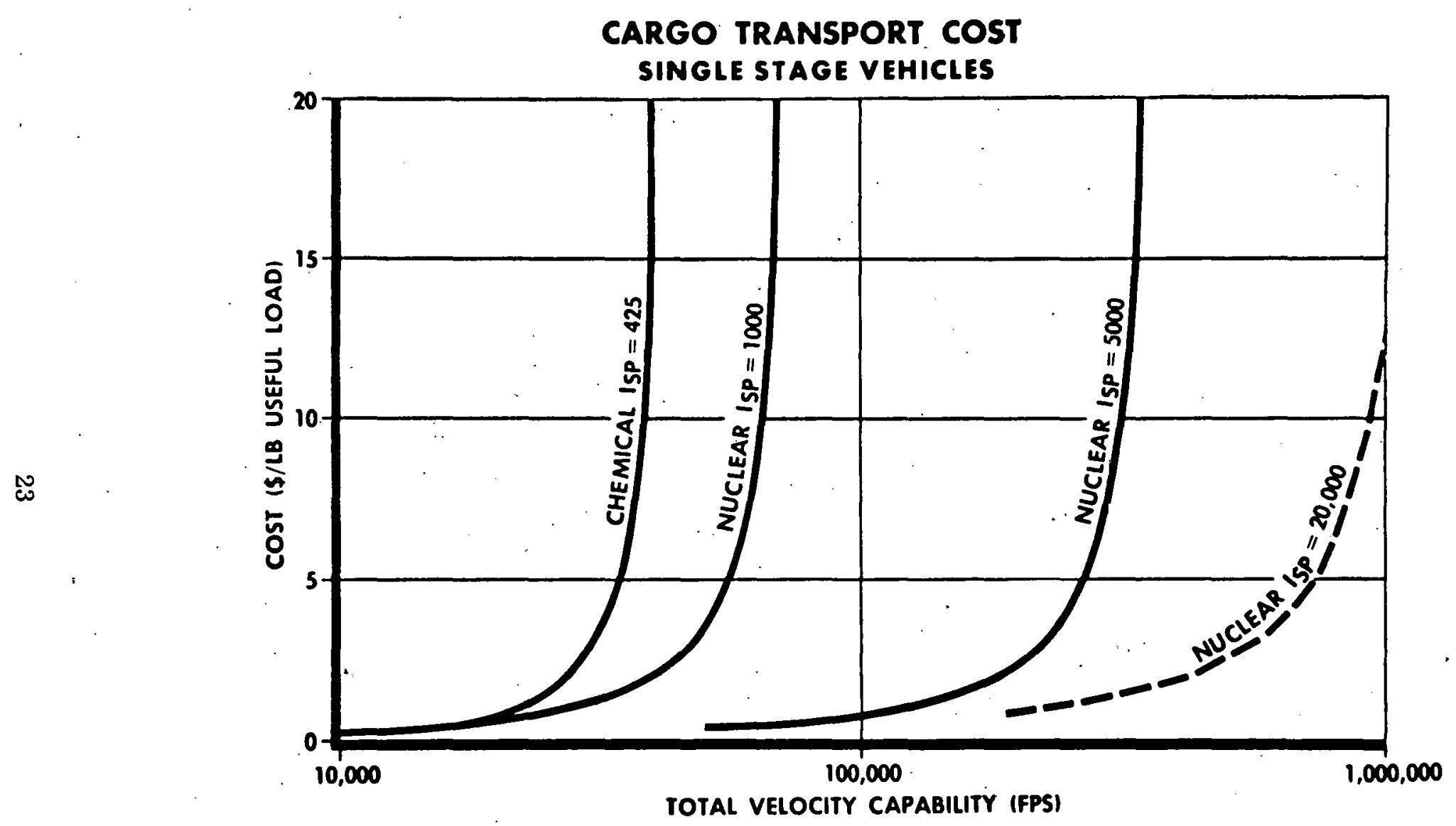

Figure 6 
rocket go to very high velocity is to stage it, and stage it, and stage it. One carries fuel to carry the fuel to carry the fuel that's going to be used later. Once the weight starts to pyramid, it's a logarithmic function and it just plain gets ridiculous in a hurry. So one has to be very careful about taking something like a solid-core nuclear rocket and deciding to perform missions at 200,000 fps. You can stack up all that equipment if you want to do it, but it's a horrendous thing.

A question that has bothered me quite a bit is why more attention was not paid to these curves. Consequently, I'm going to break down some of the assumptions used in these curves, then build new curves back up with this year's assumptions. Perhaps I can make the story more believable.

Now, there are two big ringers in the curves of Figure 6. One is the obvious one. At that stage of the game, nobody had the foggiest idea of how to build a gaseous fission engine at all, let alone one with 5,000 to 20,000 seconds specific impulse. That, right off the bat, caused everybody to throw up their hands and forget it. The second ringer is that we used transport airplane operating cost assumptions. To put it mildly, we used recovery and reuse assumptions which were not the standard thing in rocket work. I'm going to examine both of these assumptions in today's light.

Since so little was known of gaseous fission engines four years ago, the previous study assumed a thrust/weight ratio of 30 independent of specific impulse. 'This was recognized to be a very sporty assumption since, even if the containment problem could be solved, the achievement of specific impulses beyond about 3,000 seconds requires the use of a radiator to reject excess heat which cannot be handled by the thermal capacity of the propellant utilized. Although it was originally felt that such radiators would represent an intolerable decrease of thrust/weight ratio, it has since been pointed out that this is not true if high temperature radiators are used. Figure 7 shows a current estimate of the variation of the thrust/weight ratio with $i_{\text {sp }}$ achievable for a gaseous nuclear rocket system with radiator using as a basis an assumed thrust/weight ratio of 20 , at an $i_{\text {fn }}$ of 2,500 seconds. The values fall off substantially at high specific impulses compared to the assumptions of 4 years ago, but are still greater than one to beyond 10,000 seconds specific impulse.

It should be pointed out that the use of water, ammonia, methane, or other non-hydrogen working fluids should be seriously considered in gaseous fission engines from the start. Not only are better ship designs permissible due to small tankage sizes and ease of propellant storability, but the use of a higher density propellant might well ease the fuel containment problem if a vortex system is used. If so, it could result in smaller, lighter engines. 


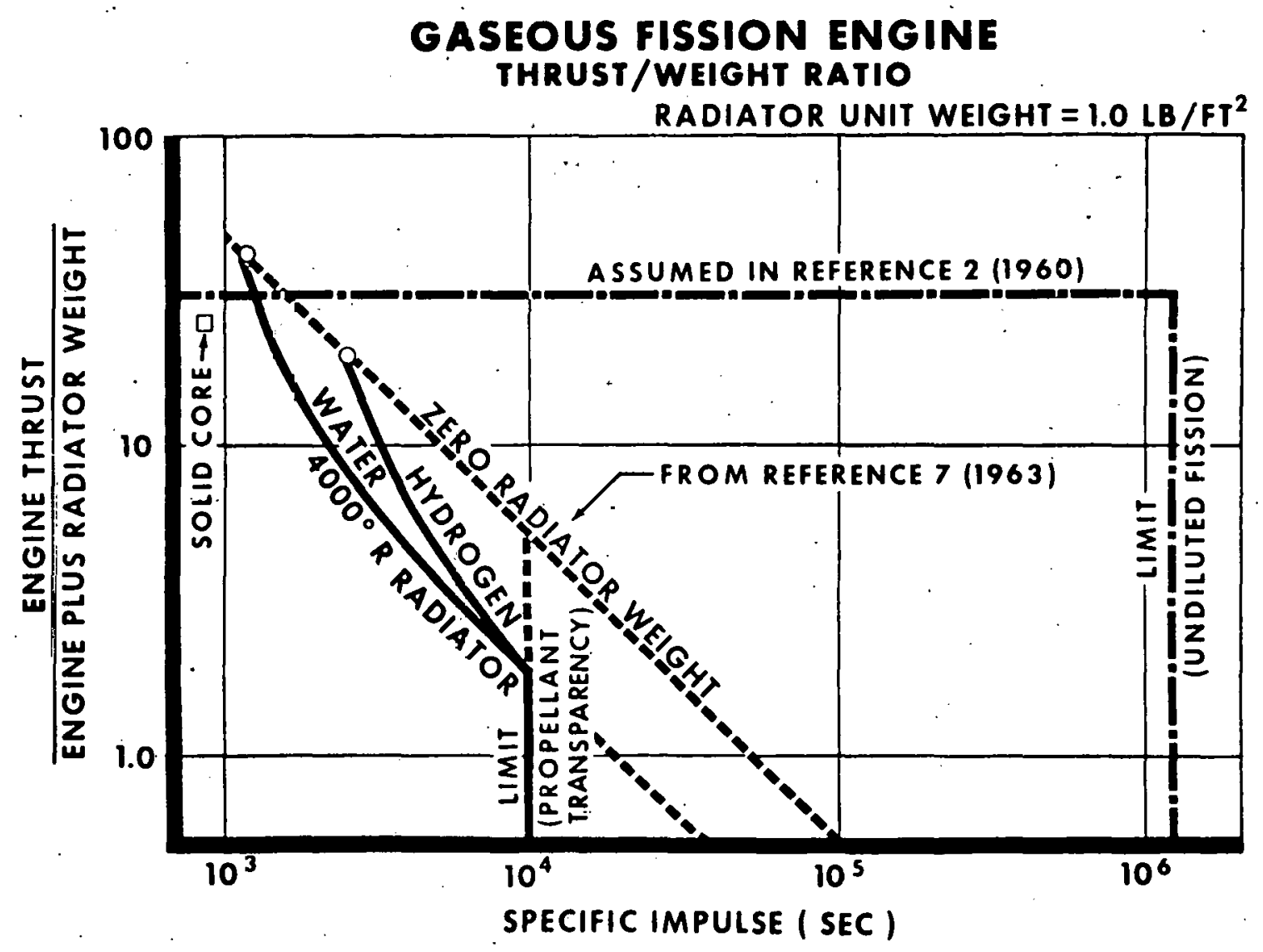

Figure 7. (References. Cited Are From ZENI, Presented by Maxwell W. Hunter, II, to The Executive Secretary, National Aeronautics and Space Council, March 1964). 
It might also result in earlier development programs if the ability to prove adequate containment occurred at an earlier time.

An interesting result in Figure 7 is that the value of thrust/weight ratio as the specific impulse approaches 10,000 seconds is independent of propellant used. This is because the reduced propellant flow at such a high specific impulse results in such small thermal capacity in the incoming fuel that the engine must be almost completely cooled by the radiator system. The propellant to fuel burned ratio required for a given specific impulse is independent of propellant used. Hence, the engine uses the same amount of energy to generate a given specific impulse, the same fraction of energy must be rejected by the radiator, and the radiator area is unaffected by the type of propellant used.

It seems clear that an engine design cooled by radiator alone should be investigated. Such an engine might be easier to develop since a major interaction between propellant and cooling system would be severed. Furthermore, such an engine could more easily use a variety of propellants. This could be very helpful in early planetary exploring.

A limitation on specific impulse of 10,000 seconds has been shown tentatively in Figure 7, assuming that the engine would be of the type which transfers heat from the fission plasma to the propellant by radiation: This is due to an unfortunate tendency of the propellants examined to date. Although adequately opaque to absorb the radiant energy at medium-high temperatures, they apparently become transparent at very high temperatures. At the moment seeding the flow, which is very effective at low temperatures, does not look promising at high temperatures.

One other point of interest in connection with the thrust/weight ratios of gaseous fission engines is the power conversion weight thus achieved. Electrical propulsion enthusiasts feel extremely optimistic when power conversion weights of the order of 10 pounds per kilowatt are mentioned. A gaseous fission engine of 2,500 seconds $i_{\mathrm{sp}}$ and $\mathrm{T} / \mathrm{W}$ of 20 achieves about one-thousandth of a pound per kilowatt. In other words, gaseous fission engines are almost certain to be 10,000 times better than electrical rockets in power conversion weights.' 'The fabulous effect of this number on spaceship design must be understood if anyone expects to make rational development decisions on future propulsion systems.

I can defend the 1963 curves of Figure 7 today. Not very well, but at least I can begin to defend them. Four years ago, the 1960 assumption was nothing that could be defended at all. However, I have always liked the calculations we made then. It influenced me in feeling strongly that, at least 
theoretically, there was a great deal more which could be done with nuclear rockets than anyone realized, far more than just trivial improvements in our current systems.

As part of the process of understanding spaceship operating costs, it is instructive to consider first only the fuel and propellant cost. This is true because this cost represents the minimum achievable. It is important to understand the mechanics of achleving a low fuel and propellant cost, particularly when truly reusable ships are used. In transport aircraft practice, the amount of reuse is so high that initial airframe costs are only a small fraction of the operating cost, and fuel costs represent about one-half the total. Thus, we shall examine fuel costs for their basic limitations on performance, and then see how closely these limits can be approached with reusable ships.

Fuel and propellant costs as a function of total velocity increment for chemical, solid core nuclear, and gaseous nuclear rockets are shown in Figure 8. Compared to the assumptions of Figure 6, the specific impulse of the high energy chemical has been increased to represent a modern, highpressure system; the solid core nuclear has been decreased in view of current development difficulties; and a number of different propellants and degrees of containment are shown for gaseous fission engines. All curves are for single-stage ships with structural assumptions more conservative than those of Figure 6 and each specifically sized for the velocity shown.

It is evident that, on this basis alone, a gaseous fission engine without radiators and with separation ratio of $10^{-3}$ is not significantly better than a solid core engine. Gaseous engines with better containment would be much better. It is also evident that gaseous engines with space radiators, but with specific impulse limited to 10,000 seconds, can drive ships up to about one-half million feet per second and still maintain reasonable fuel cost. The attainment of a fuel separation ratio of $10^{-4}$ is almost as effective as perfect fuel containment.

The optimum fuel cost curves for gaseous fission engines with radiators were obtained by determining the optimum specific impulse for each velocity and separation ratio. This is necessary since too low a specific impulge will result in excessive propellant cost while too high a specific impulse will result in excessive fuel cost. The optimum specific impulse is much higher than 10,000 seconds for all velocities beyond a few hundred thousand feet per second. Hence, these curves represent a future capability presently unattainable due to the propellant transparency problem at high temperatures previously mentioned. If it were not for this, gaseous fission ships could be driven to almost one million feet per second before fuel costs became a limitation. 


\section{SINGLE STAGE SPACESHIPS}

FUEL AND PROPELLANIT COSTS

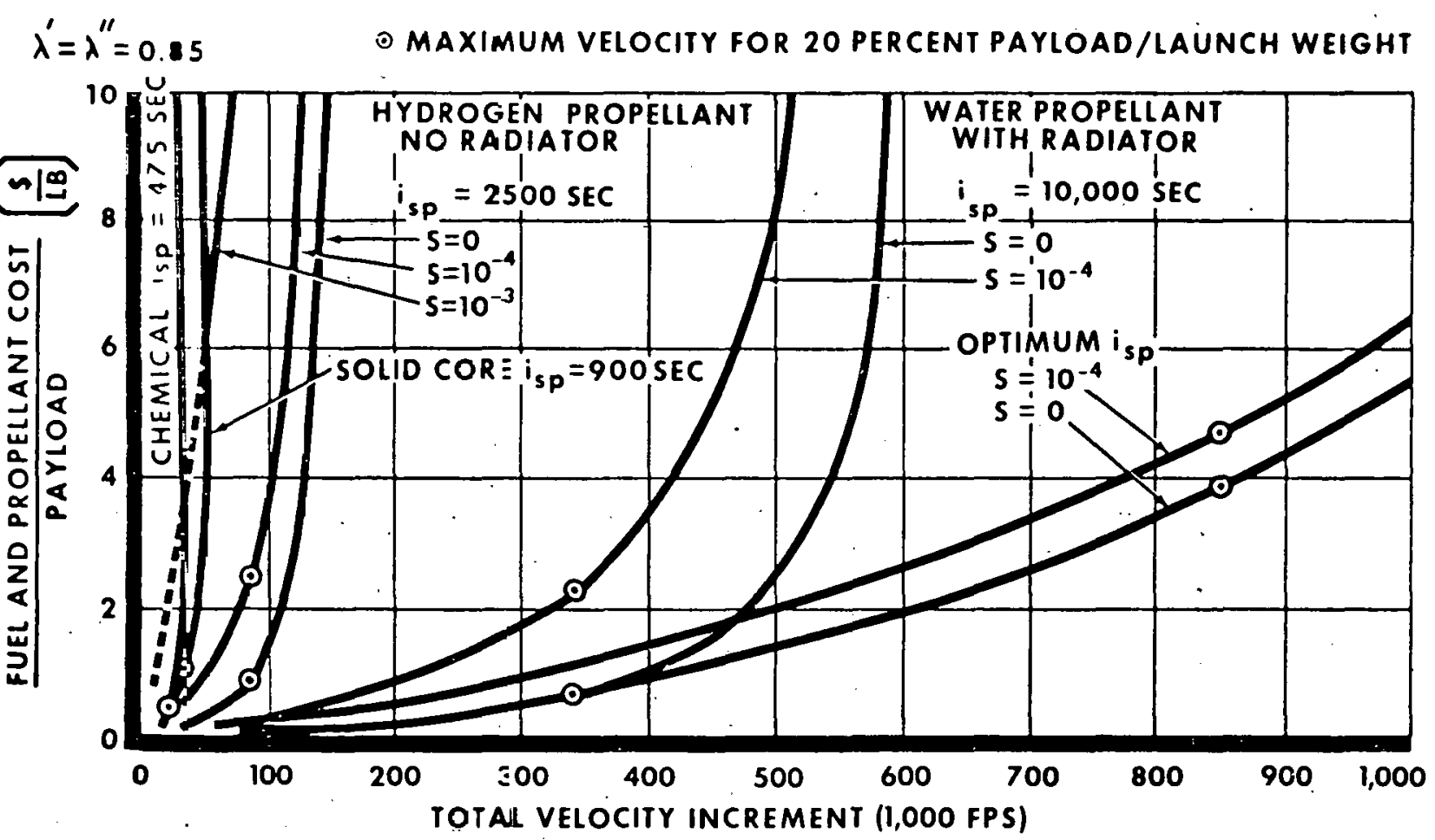

Figure 8 
Under certain circumstances, a great deal can be learned about spaceship design without a detailed knowledge of the missions to be performed. In recent years, there has been a tendency to become so detail-missionoriented that ship design is not even attempted until the exact mission is clearly understood. This may be a valid procedure when only one mission is in sight, although even then the inevitable lack of versatility usually leads to needless redesign much earlier than anticipated. In transport airplane design, the basic design procedure usually centers around the calculation of airplane operating characteristics as a function of range. The maximum range required comes from a knowledge of the total mission complex, but the airplane design is refined primarily by using general curves as a function of range, rather than by a detailed series of specific mission analyses.

When considering total solar system transportation as we are, it is. clear that we face a variety of missions. It is also very unclear as to which of these will be paramount. One way of approaching the problem is to present the characteristics of the vehicle as a function of total velocity increment which the ship can achieve. This is exactly analogous to the use of range in aircraft design practice. In this way, an understanding of the ship's basic ability to deliver payload to a certain speed economically. can be rather easily understood. The complex mission analyses, then, can be made to reflect the maximum design velocity increment required.

This approach was actually used in the 1960 study, and Figure 6 represents one of the results. Figure 6, however, contains assumptions as to degree of reuse achieved by the vehicle which, although consistent with transport aircraft practice, may not apply to space transportation. At least, if they do, their application must be better documented.

The 1960 study assumed a large number of reuses per vehicle, somewhat analogous to the number of times a transport airplane is reused. A transport aircraft is actually utilized about 50 percent of the time, and average flight durations are less than 4 hours. It is clear, therefore, that such vehicles are used over one thousand times per year. However, space travel durations are much longer, and it is obvious that the interaction between travel duration and number of reuses must be considered.

For the lunar mission, it is clear that large numbers of reuses are feasible. Typically, 100 flights per year (50. each way) can be envisioned on the basis of 2-day travel times, one day turn around time at each terminal, with Sundays and 2 weeks off for vacation. Over a 10-year ship lifetime, 1,000 uses will be achieved. 
One can get a feeling for the number of interplanetary uses by assuming a certain ship total life. Typically, transport aircraft are designed for 40,000 hours (4.6 years) total life. On the basis of slightly less than 50 percent utilization, such a vehicle would last for 10 years. They always last much longer, but the amortization time of the airframe is usually about 40,000 hours, since new equipment always becomes available in even shorter time.

Selecting a suitable lifetime for a spaceship presents a considerable technical dilemma. One viewpoint would simply take 10 years as above. An even shorter lifetime might technically: be justified due to the severe aerodynamic environments associated with atmospheric entries, and the generally unknown operational environment of space. This type of assumption has become standard in this country recently. If you don't understand the problem, assume it's horrible.

It may well be, however, that spaceships will last much longer than transport aircraft. The transport has its main propulsion system operating continually during flight, and is also continually facing the temperatures and gust loads within our atmosphere. The question is whether spaceship operating life should be determined by the total time of operation, or only by the times during which the main engines operate and/or it is within an atmosphere. In other words, is a spaceship coasting between planets actually operating in the aircraft transport sense, or is it merely parked in space, breathing quietly, waiting for its next mission.

One can make an excellent case for the latter point of view in terms of the general environment that the ship faces, either from space or its own propulsion systems, while coasting. The ship would have to be on interplanetary runs for several centuries in order to build up 40,000 hours of engine and atmospheric operation. It is, however, bound to be replaced by better equipment within a few decades. As a base for calculations, this report assumes 25 years ship useful lifetime.

The variation of various weights as a function of velocity is shown in Figure 9 for both specific impulse limited to 10,000 seconds and for the optimum specific impulse. These curves are for ships designed for 20 percent payload, then operated at lower velocities by off-loading propellant and at higher velocities by off-loading payload. Thus, these curves represent a penalty for using a single ship for multiple missions, just as in other forms of transportation.

By using. suitable planetary travel time data, which is not yet easy to come by, the weight data of Figure 9, the same fuel plus propellant cost 


\section{SPACESHIP PAYLOAD CAPABILITY}

DESIGNI POINT - 20 PERCENT PAYLOAD AT MAXIMUM WEIGHT

SPECIFIC IMPULSE $=10,000$ SEC

-OOPTIMUM SPECIFIC IMPULSE

OZERO PAYLOAD

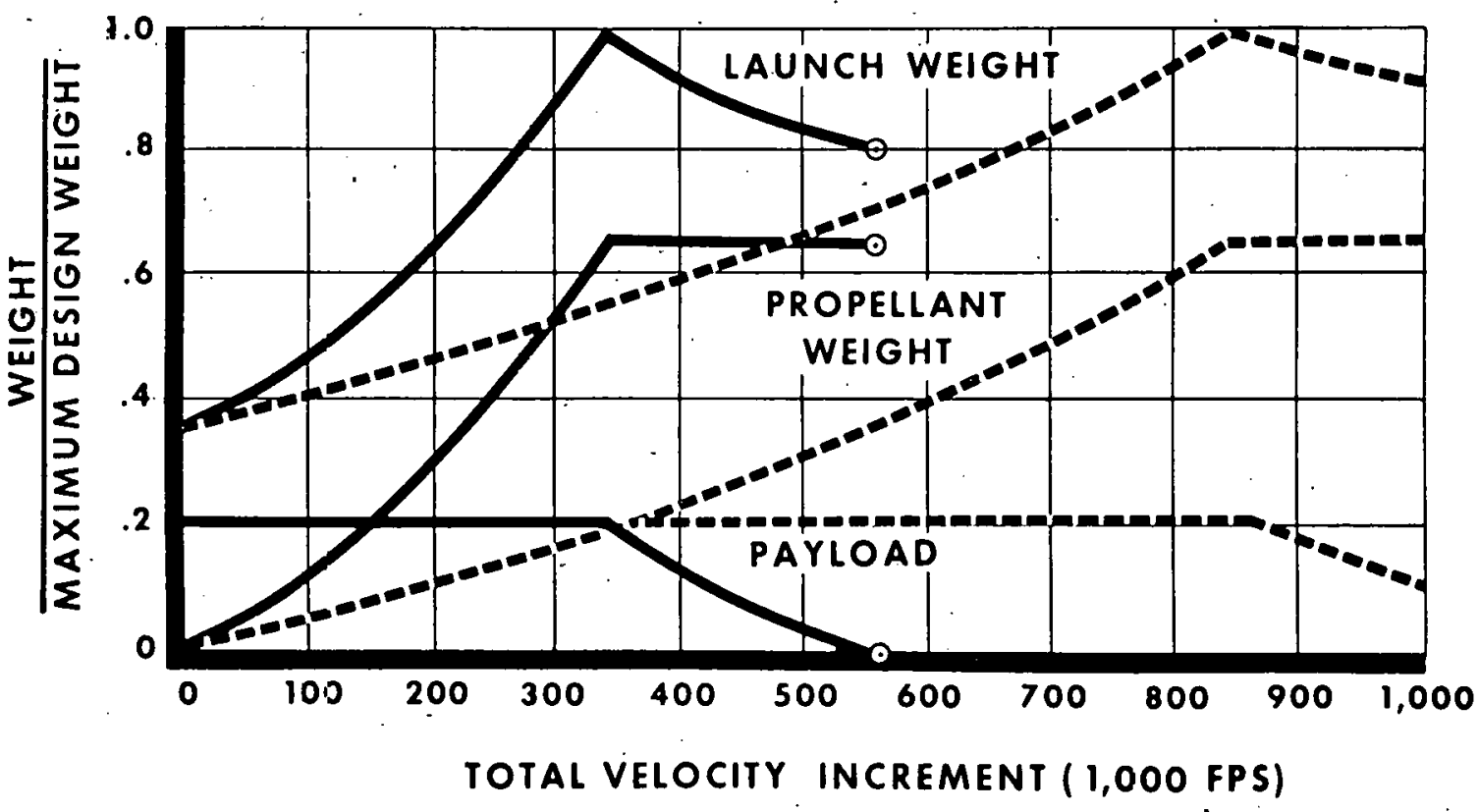

Figure 9 
assumptions as in Figure 8, and assuming a vehicle cost of $\$ 100$ per pound, the curves of Figure 10 were obtained. One hundred dollars per pound is the currently estimated cost of a supersonic transport. These curves show fuel cost plus amortized airframe cost as a function of design velocity increment for the missions selected.

The lowest curves on Figure 10 are fuel cost only. Comparing them with the other curves show that for operations as far out as the planet Saturn, the structural costs are comparable to fuel costs. Further improvements in convenience of operation can be achieved with engines not limited to 10,000 seconds specific impulse. In that case, velocity increments beyond a half-million feet per second are economically reasonable.

The average travel time between planets corresponding to the velocities of Figure 10 are shown in Figure 11. These two Figures taken together give a better feel for solar system transportation than Figure 6 alone. With specific impulse limited to 10,000 seconds, the solar system as far as Jupiter is available with travel times not exceeding 4 months. Inner solar system travel times need not exceed 2 months. The advantage of optimum specific impulse becomes more evidẹnt at Saturn and beyond.

The curves of Figures 10 and 11 apply for a given ship design velocity only if the ship can be refueled at each terminal. If it must carry its own fuel for the return journey, then it must operate at half the total velocity shown. Except for Pluto, refueling bases at the major planets are much more needed than at the minor ones, as can be seen by Figure 10. Refueling bases could be expected to be located on the surfaces of all the minor planets, although it may require some design effort in the case of Venus and Pluto.

The major planets are a different situation. Their surfaces are extremely forbidding as far as we know, to the extent that we are not even sure they have solid surfaces. It makes sense in that case to establish bases on one of the satellites of each of the four major planets. The curves are drawn with that assumption. If we do decide to penetrate to the surface of these planets, then the velocity requirements for doing this when operating from one of the satellites is a reasonable number. Thus, bases on the larger planets' satellites not only greatly facilitate the convenience of transportation, but also present a reasonable base for surface exploration, if required.

These particular curves are also calculated for the average flight times involved in year around operations between all planets. There are no launch window restrictions. If you're ever going to have a transportation system, 
SINGLE STAGE SPACESHIPS

FUEL, PROPELLANT AND STRUCTURE COSTS

SPECIFIC IMPULSE $=10,000$ SEC

WATER PROPELLANT, $s=10-4$

25 YEAR SHIP LIFETIME

SPECIFIC IMPULSE $=10,000$
$-\infty$ OPTIMUM SPECIFIC IMPULSE

REFUELING AT ALL TERMINALS

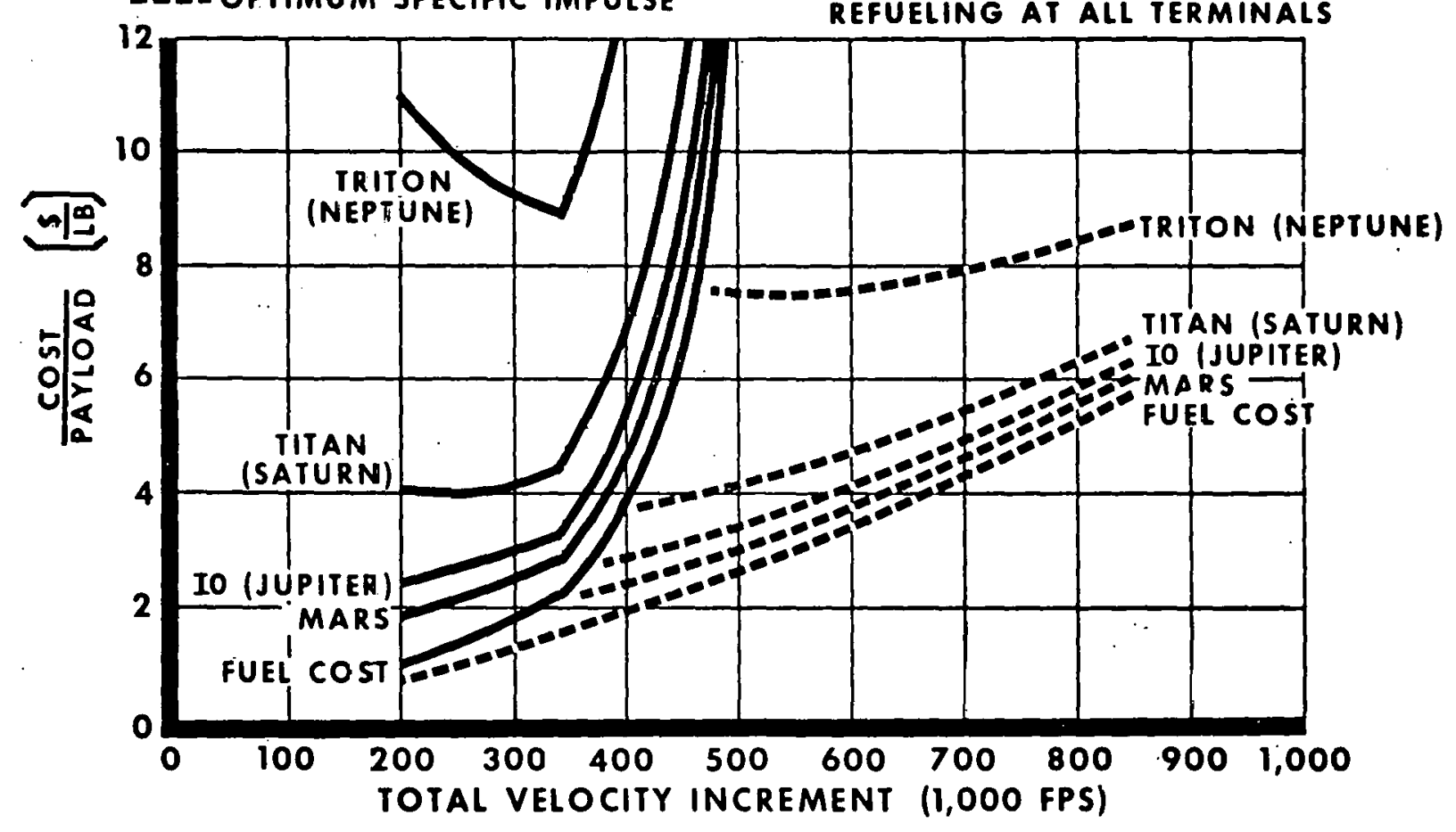

Figure 10 
SINGLE STAGE SPACESHIPS

FUEL, PROPELLANT AND STRUCTURE COSTS

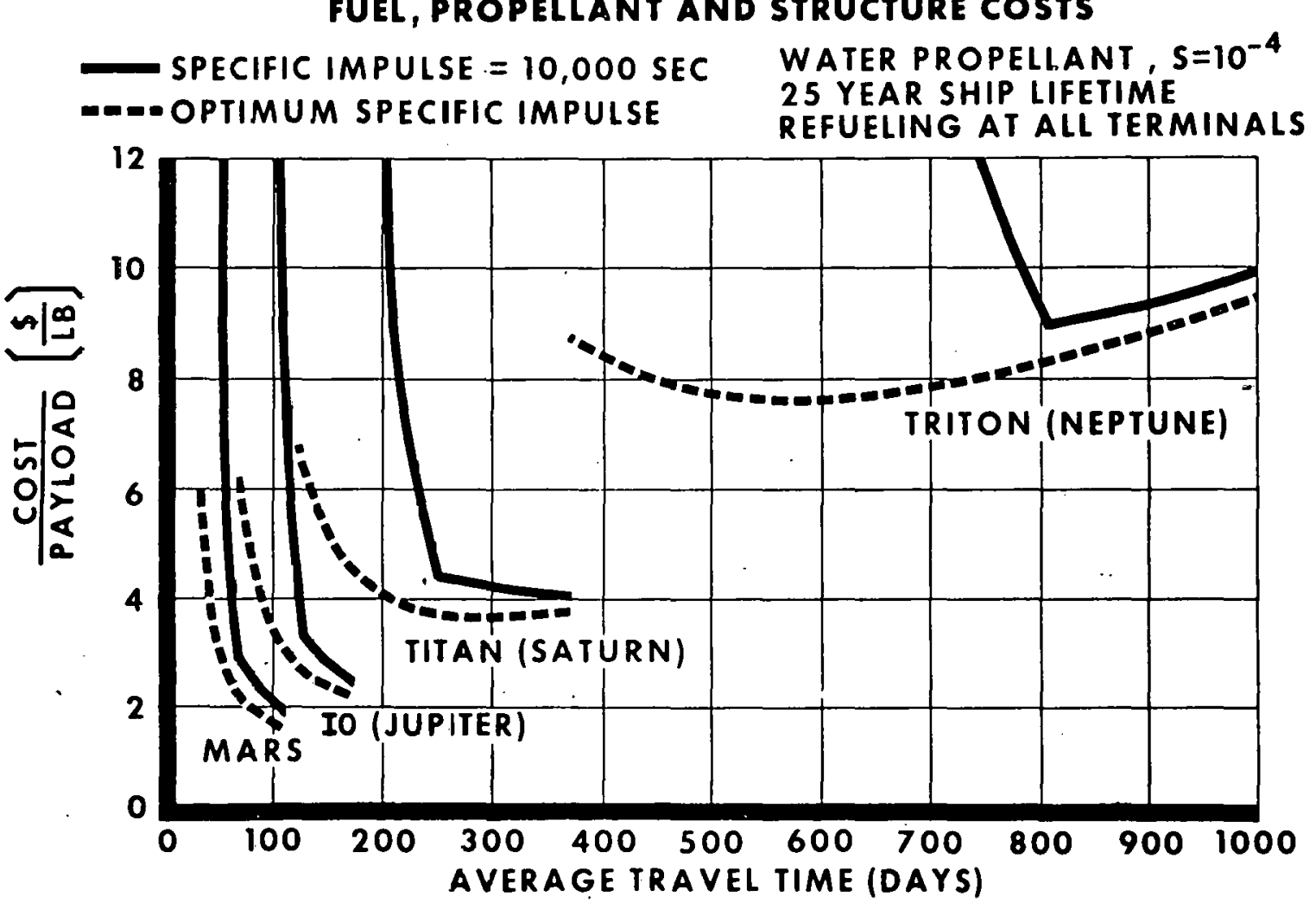

Figure 11 
you're going to have to be able to go when you want to. You cannot spend most of the time waiting. A rough averaging process between the best and worst times of the year was used in an attempt to make this a realistic transportation assumption.

A few words about perspective on these curves are in order. There is nothing magic here except a ridiculous willingness to plot curves wherever the data is leading, rather than stopping somewhere. Both better and worse situations may well occur. Even the case of specific impulse limited to 10,000 seconds requires gaseous fission engines with radiators, and most people today would rather agree to engines without radiators. In that case, the velocity increment achieved will be only about 25 percent of the curves shown. Furthermore, the economic penalty of, if necessary, ejecting a critical mass of fuel in the process of shutting down the engine has not been included. This will be on the order of $\$ 100,000$ per shutdown.

On the other hand, perfect containment might be achieved. We might design ships for each velocity increment, rather than use the single design assumed here. Furthermore, one can get a greater utilization of vehicles by the expedient of refueling the vehicles which go on deep space missions. This is preferable to multi-stage vehicles, since a fleet of ships used for refueling can also be used for other missions. No attempt will be made here to present detailed curves showing the effects of refueling. Cursory checks show that over 200,000 fps can be added for reasonable cost with only two refuelings.

The greatest conservatism of all in Figures 10 and 11 is, of course, in the magnitude of the ordinate scale. Costs beyond $\$ 12$ per pound have not been plotted so that the entire set of curves is about 100 to 1,000 times lower than virtually all space cost analyses to date. This must be clearly remembered as we discuss the performance of these ships.

I can't resist making one more solar system point here. So far, only travel between Earth and the other planets has been discussed. There is also the question of travel between planets other than Earth. The use of bases in other parts of the solar system to aid in the exploration of the even more remote portions should be considered. In fact, such considerations might well dictate the strategic location of bases.

At first thought, it would seem to be a good idea, for instance, to use a base on one of the farther planets, say Saturn, to permit further exploration of the more remote planets like Pluto. Although this is an intriguing thought, such deep bases will have only limited utility. The reason is the extremely long synodic periods which exist among the outer planets since 
they move so slowly around the Sun. In the worst case of all, the synodic period between Neptune and Pluto is slightly over 500 years. In addition to the long synodic period, the difference between travel at the optimum time of the year and the worst time of the year becomes more extreme the farther the planet is located from the Sun.

One way of illustrating this is shown in Figure 12, where the effects of basing on selected planets is shown for a constant ship velocity. It is true that a deep space base will be closer than Earth to the other deep space objects when in favorable position, but equally true that it will be much farther away during the worst conditions. Surprisingly enough, the base : wants to be reasonably close to the Sun, once again emphasizing that the Sun is the center of the solar system. Although Mercury might be the best planetary base of all, the Earth is still sufficiently close to the Sun that it represents a pretty good compromise. Thus, the major space logistics support operations could, from a celestial mechanics viewpoint, be 10cated efficiently on the Earth or its Moon. This is very convenient since the known industrial and research bases of the solar system also happen to be located in that vicinity.

The slow movement of the outer planets leads to some interesting paradoxes. One would naturally assume that a base on Triton would be an excellent place from which to explore Pluto, since Neptune is at $30.09 \mathrm{~A}$. U. from the Sun, while Pluto is 39.5 A.U. However, it turns out that Neptune at the moment is already leading Pluto around the Sun, and pulling away. In fact, in approximately 9 years, Neptune will be farther away from Pluto than Earth ever is. Furthermore, due to the long synodic period of Neptune and Pluto, that statement will be true for somewhat over the next 300 years. It would be nice to be sure that every other statement in this discussion will be true for that duration.

I threw that in as a bit of tidbit. I'm gradually working back down to our more normal systems, and there's a point that I want to harp on further - that is, this whole question of structural reuse. As I indicated before, Figures 10 and 11 show costs that are less than $\$ 10$ per pound throughout the entire solar system. Yet large and elaborate studies are made these days proving that it's going to take many hundred dollars per pound to go to the moon, no matter what we do with recovery, reuse, or anything else. It's quite clear that either I'm insane, or a lot of other people are, or we have to have an explanation. It was easier to give an explanation than to prove everyone else insane.

Figure 6 was drawn with what I like to refer to as "transportation" type assumptions for operating cost. Maintenance costs, for instance, were 


\section{SOLAR SYSTEM SYNODIC PERIODS}

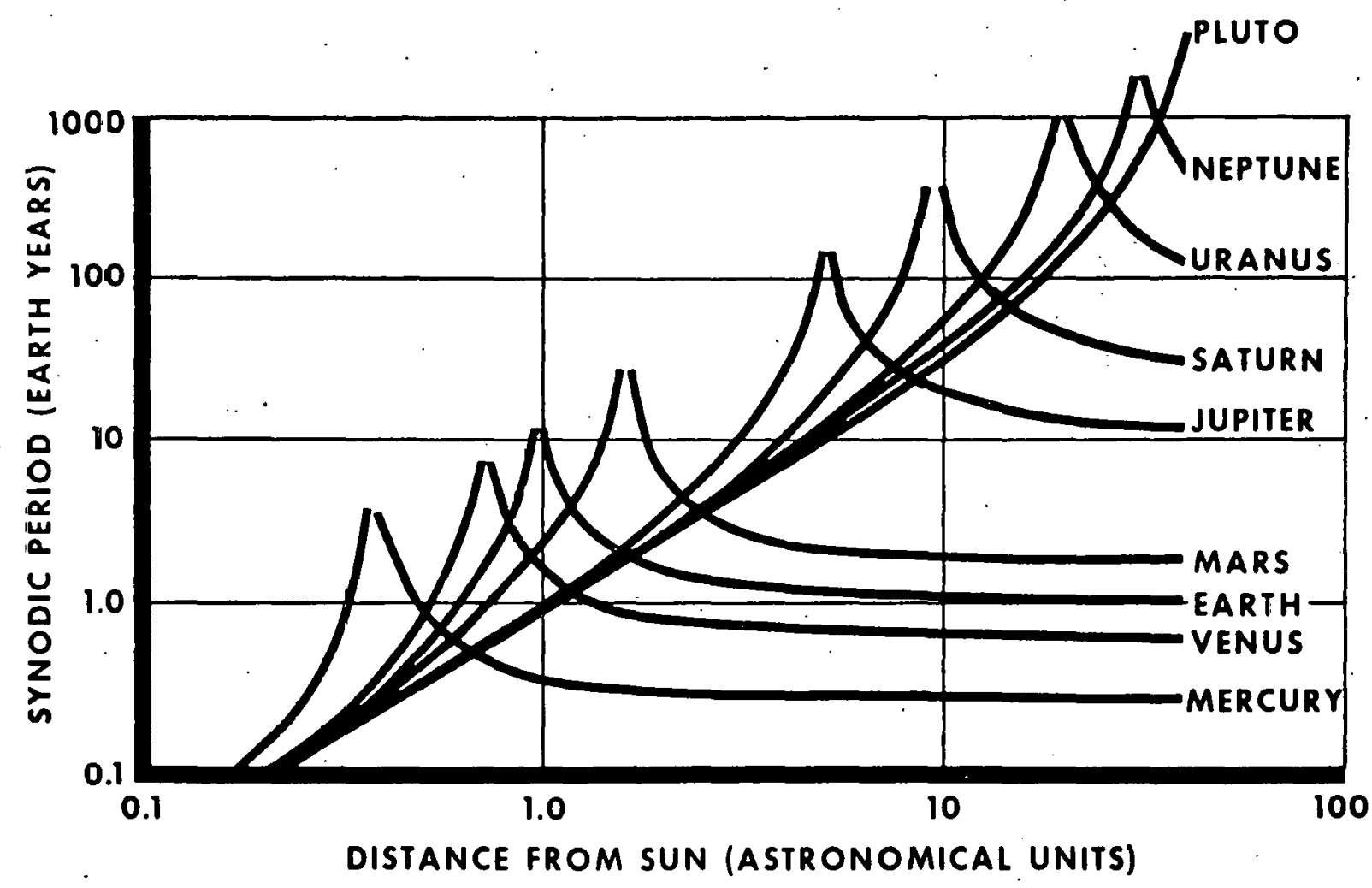

Figure 12 
taken írom normal air transport practice. The vehicles were assumed to be as reusable as transport aircraft. This is not the fashion when calculating rocket operating costs today. Almost all of our rocket builders, including myself, have been doing nothing but "ammunition" work for a large number of years. It is axiomatic that, if you want to talk about recovery and reuse, you should not talk to an ammunition builder.

To illustrate what can happen with different classes of maintenance and reuse assumption, I will shift gears drastically and discuss merely placing objects on Earth orbit with chemical propulsion. In Figure 13, I assumed, arbitrarily, about $\$ 300.00$ per pound of payload as typical of current day orbital transportation systems with no reuse at all. It so happens, however, that if you consider the actual price of high energy fuel needed for orbital velocities with advanced rockets, it is only on the order of $\$ 1.00$ per pound of payload. Figure 13 is simply a plot of operating cost as a function of recovery reliability and refurbishment cost with these assumptions.

It is quite fashionable, whenever over-all system analyses for recoverable space vehicles are performed, to assume that recovery reliabilities will be around 75 percent. After all, that is the recovery experience to date. Also, refurbishment costs around 25 percent are quite likely to be used. The shaded region brackets the se assumptions, and is typical of a good, solid, rational ammunition type analysis. It is evident that after spending that much money on refurbishment between flights with that low a recovery reliability, an improvement of at most two in over-all cost performance is the best to be expected.

Also shown on Figure 13 is what had already been achieved many decades ago in air transportation. This is what happens when you think like a transportation man. The recovery reliability is so close to 1.00 that you can't possibly see it on this scale. The same is true of the maintenance cost, which is on the order of 0.04 percent. If anyone here thinks that a DC-8 is less complicated than a Thor, just take a good look at the inside workings of a DC-8 some day. Wonder, then, at the fact that a few people turn it around, give it some fuel, pat it on the head, and it takes off again. This is what we should be trying for in future spaceships. There is an improvement of a factor of 100 over current operations to be made. I do not want you to get the impression that I am all for airplane designers. I think they, too, are irrational conservatives. But if useful design techniques have been developed, I think they should be used in space.

As a matter of fact, you can get rougher with this. You can make a calculation on what would have happened in our air transport system last year if the philosophy of our ammunition people had been used in running it. 


\section{TRANSPORTATION VS AMMUNITION COMPARISON OF RE-USE ASSUMPTIONS}

\section{ASSUMPTIONS:}

COST WITH NO RE-USE $=301 \mathrm{\$} / \mathrm{LB}$

FUEL COST $=1 \$ /$ LB

VEHICLE LIFE $=1000$ FLIGHTS

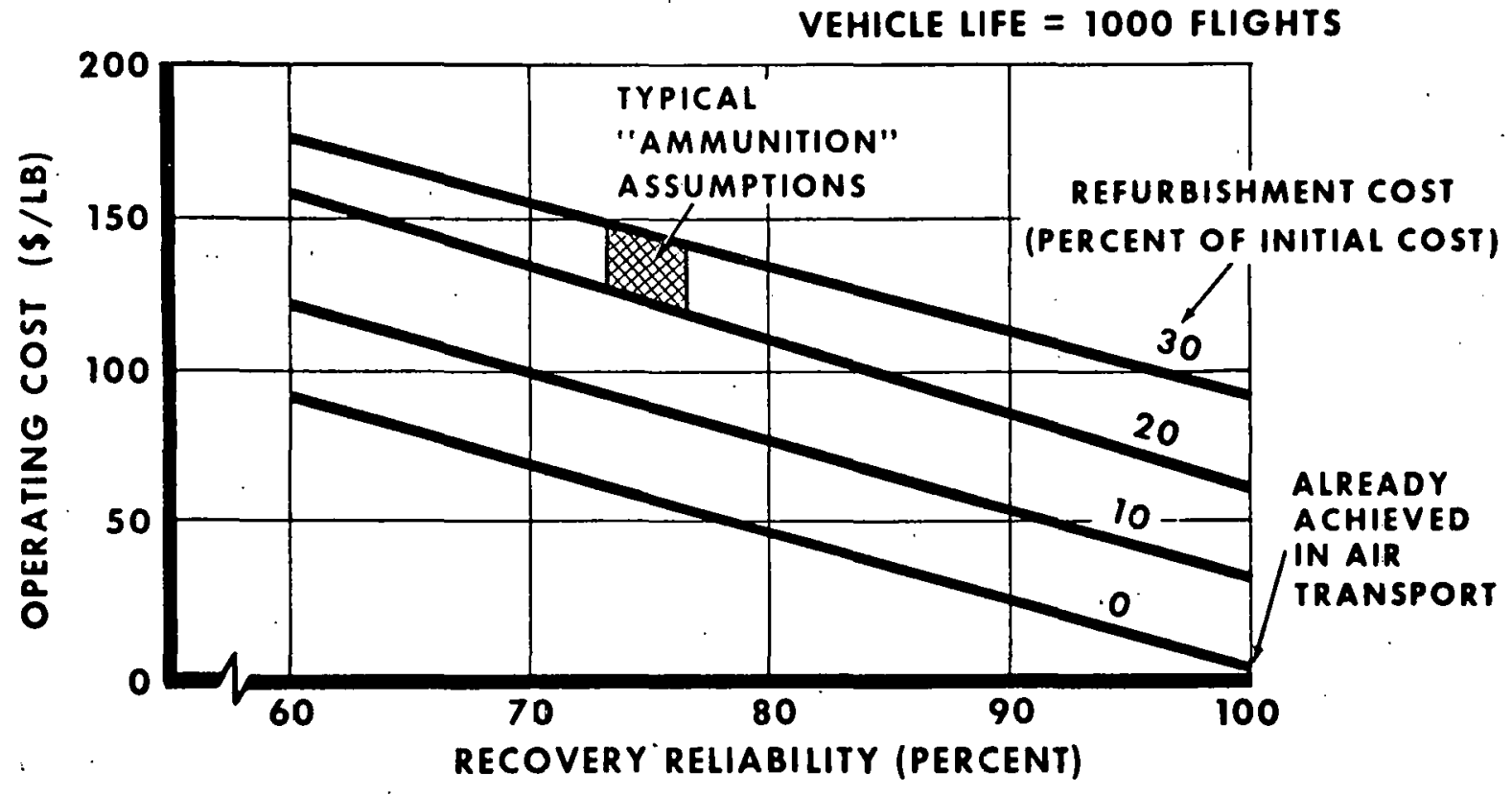


If you do that, you'd find that we would have killed 4 million people last year. You will also find that the attrition of equipment and refurbishment costs are so high that it would cost you $\$ 10,000$ for a ticket to anywhere. The only way out of the ammunition dilemma, surprisingly enough, is the kind of advance propulsion we're planning to talk about the next few days. You have to get enough margin into the propulsion so that you can have extra weight available both for use and reuse.

An interesting interaction exists between the containment capabilities of gaseous fission systems and the cost of boost to orbit. Attempts are frequently made to show that high fuel consumption gaseous fission systems (either Orion or co-axial systems) would be acceptable after all, since the extra economic penalty which they incur compared to the cost of chemical boost to orbit is relatively small. This conclusion would obviously be strongly influenced by the wide spread of orbital costs mentioned.

Figure 14 shows typical interactions between containment of fuel and economics of boost to orbit. The point is obvious. Chemical take-off is not too bad. If chemical boost to orbit is tolerated at all, however, it must be of the economical "transportation" variety, or it will completely cripple the ability of gaseous fission engines to explore economically the solar system. This to me is the real challenge of advanced propulsion. This is also why I think there's a tremendous interaction between the engine and vehicle. It is not just a matter of sitting down with a specific impulse, and making one simple performance calculation. The big gain is made by the interaction of the engine and the development of transportation techniques.

Figure 15 is a sketch of one result of using a gaseous fission engine to power a reusable spaceship. We all, by now, expect manned rockets to be hundreds of feet.long. If drawn to the scale of Figure 15, Saturn V would be two pages 1ong. It would have a little bit of payload on the front. If, however, we were to combine the kind of nuclear rocket engine we would like to have (running on water or ammonia rather than hydrogen) with a reusable structure for the entire ship, a possible result would be the ship shown. This is a typical case of about a million pounds gross weight with cargo weight on the order of 200,000 pounds.

It turns out, not surprisingly, that for reasonable economy, large payload fractions, perhaps even more than 20 percent, are required. Note that 20 percent cargo at a density of 10 pounds per cubic foot (standard transport airplane practice) when combined with the required propellant results in a rocket vehicle with 60 percent of its length devoted to cargo and crew. The engine and propellant take up only a small portion at the rear, just like "Buck Rogers" has said it should all along. 
TRANSPORTATION VS AMMUNITION EFFECT OF FUEL CONTAINMENT ON ORBITAL COST

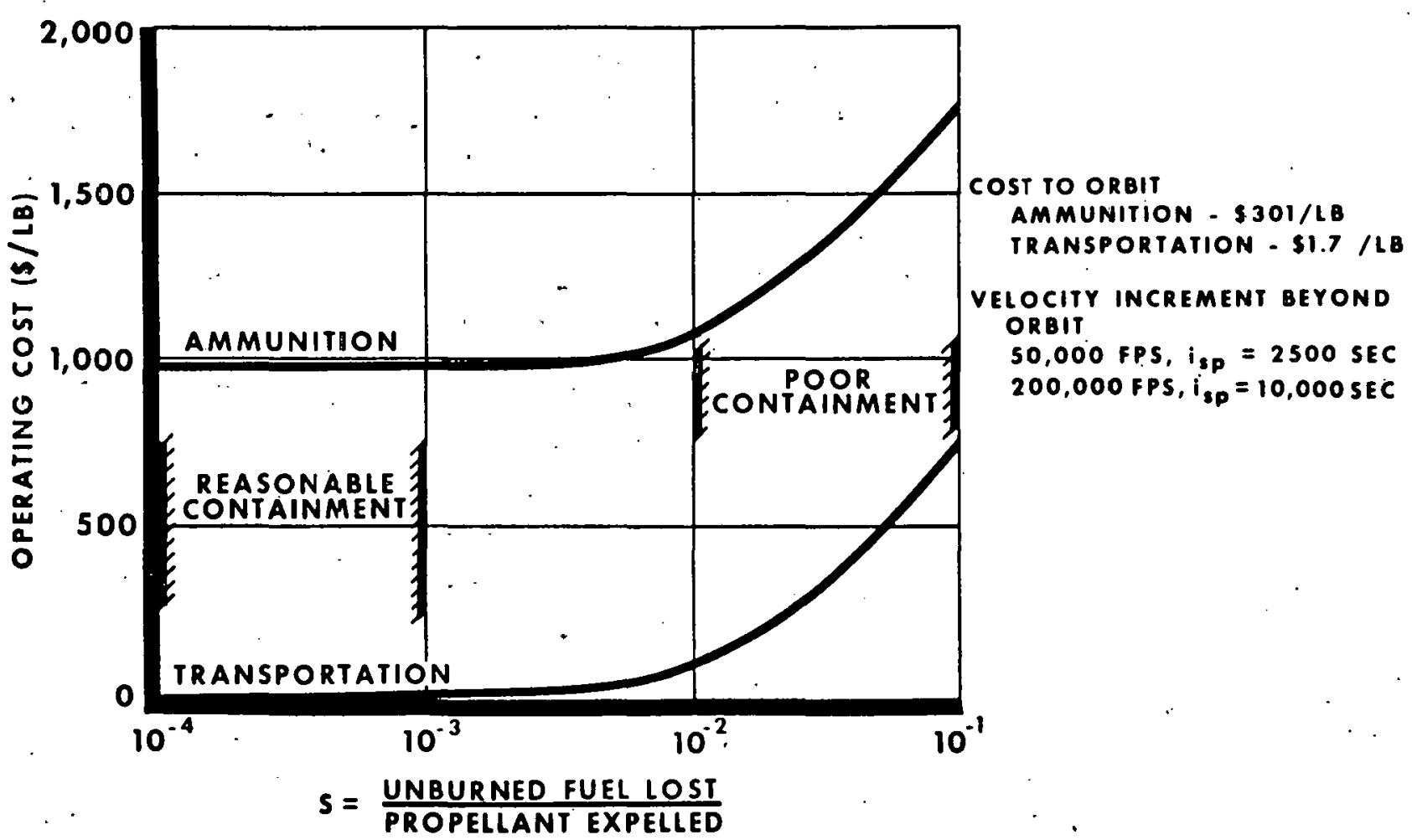



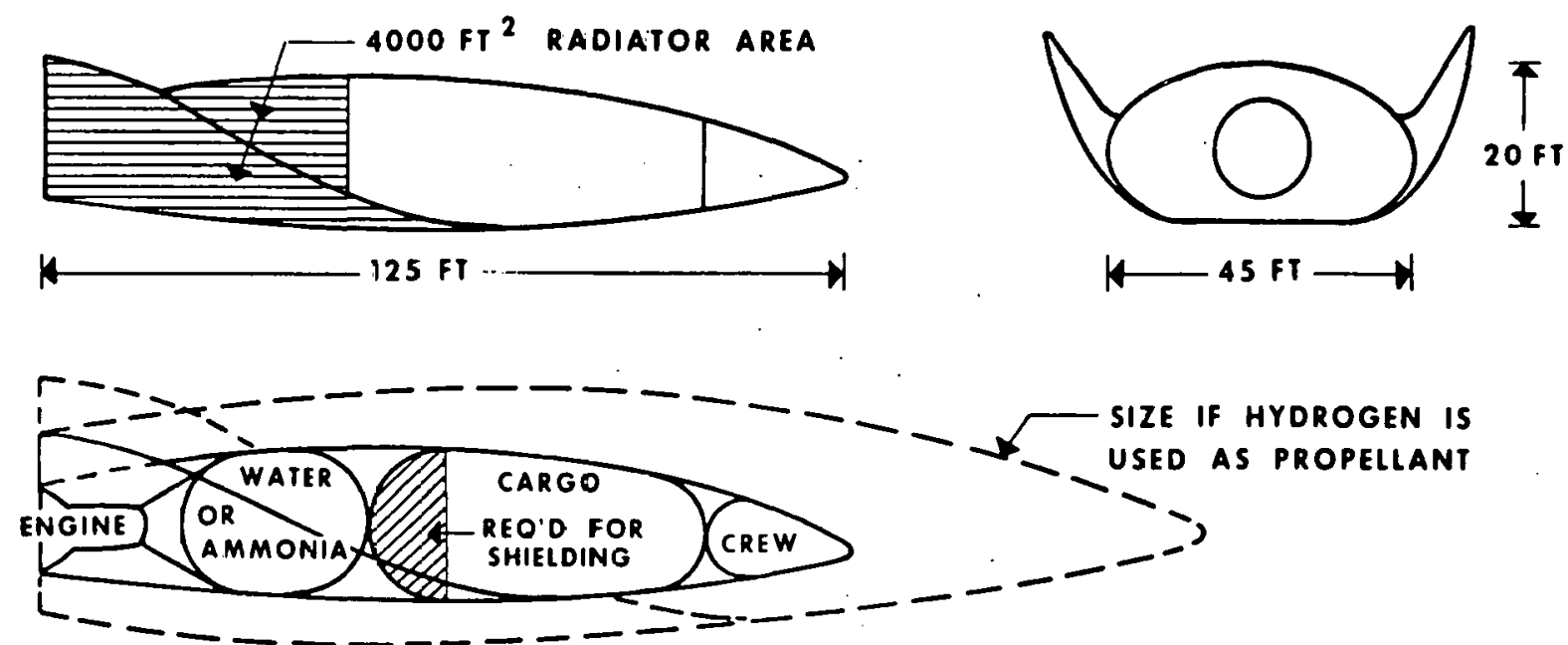

LAUNCHING WEIGHT $=1,000, C 00$ LB (500 TONS)

CARGO WEIGHT $=200,000$ LB (100 TONS)

PROPELLANT WEIGHT $=630,000$ LB (315 TONS)

VELOCITY INCREMENT $=80,000 \mathrm{FI} / \mathrm{SEC}$ (WITHOUT USE OF RADIATORS)

$\sim 500,000 \mathrm{FT} / \mathrm{SEC}\left(\begin{array}{l}\text { WITH USE OF } 5000^{\circ} \mathrm{F} \text { RADIATORS } \\ 2 \text { DAY ACCELERATION TIME TO .5 } \\ \Delta \mathrm{V}\end{array}\right)$

TO MOON (WITHOUT USE OF PADIATORS)

10,000,000 LB (5000 TONS) CARGO DELIVERED PER YEAR PER SHIP

(ONE SHIP EQUIVALENT TO 300 SATURN V LAUNCHINGS)

Figure 15. Gaseous Fission Powered Spaceship 
An interesting example of the change in design philosophy with such ships is in the matter of shielding. Indications are that about 20,000 pounds of shielding weight would be required. This is a severe penalty for most rockets. Since cargo itself is effective shielding material, however, by the simple expedient of never flying this ship with less than 10 percent cargo aboard, properly packaged, the shielding penalty is reduced effectively to zero.

If such a ship were used without radiators on the engine but with hydrogen propellant, it would be able to generate about 80,000 feet per second. This is more than needed for a lunar round trip. We can hence examine the effect of this ship on a lunar run, performing like a normal transport airplane. Logical assumptions, as previously discussed, would lead to 50 flights a year, and the ship can carry 100 tons per flight. By maneuvering a little bit with that number, I concluded that one ship like this is equivalent to 300 Saturn V launches per year.

This is the kind of thing that we're driving at. Incidentally, in our scientific operations in the Antarctic, we deliver to the Antarctic about 50,000 tons a year. Ten such ships, shuttling back and forth to the moon, could mount the same magnitude of operation on the moon as we mount in Antarctica. This is, to say the least, an interesting capability.

Some points should be made about the safety of gaseous fission spaceships. Contrary to most opinion, a gaseous fission rocket is probably a lot safer to use than a solid core rocket. Several reasons for this are listed on Figure 16. The fission products are always in vapor form, so there is never a fuel element burnup problem if emergency atmospheric entry is necessary. On return trips, one need never overfly a city with a fission product load, since the products can be ejected into space and the landing made aerodynamically. After landing, of course, the ship is radioactive only to the extent that any material has been locally activated. This will be very small with proper material selection and is certainly far lower than when the fission product load is a permanent feature of the structure. Hence, the servicing problem would be nowhere near as great as with the case of aircraft nuclear propulsion.

Furthermore, in case of an accident, the fission product load is always small. A million pounds of thrust is, after all, only $1 / 2$ kiloton of thrust, and the actual fission products created are comparable to those from kiloton, not megaton, bombs. As a matter of fact, some interesting calculations indicate that an accident as low as 5,000 feet in the air yields almost no exposure on the ground due to the effectiveness of atmospheric dispersal of the small fission product load on board. 
FISSION PRODUCTS ALWAYS IN VAPOR FORM

NO BURN-UP PROBLEM AS IN ROVER OR POWER SUPPLIES

NO FISSION PRODUCTS ABOARD UPON RETURN NEVER OVERFLY WITH FISSION PRODUCT LOAD

SERVICING PROBLEM NOT GREAT AS IN ANP APPLICATION

FISSION PRODUCT LOAD SMALL IN CASE OF ACCIDENT MEGAPOUND THRUST IS ONLY KILO (NOT MEGA) TON EQUIVALENT ONLY MILLIREM EXPOSURE IF ACCIDENT ABOVE 5000 FT.

HIGH VELOCITY EXHAUST JET

VELOCITY OF AT LEAST 100,000 FT/SEC EXCEEDS EARTH ESCAPE SPEED MOST PRODUCTS ACTUALLY EJECTED FROM SOLAR SYSTEM.

CHARACTERISTICS ALSO AFFECT DEVELOPMENT PROCEDURES

TIME BETWEEN STATIC TESTS REDUCED

ENGINE DJES NOT CONTAIN PRODUCTS AFTER TEST

FUEL ELEMENT FABRICATION DELAYS AVOIDED

MAY NOT BE A LOGICAL EXTENSION OF SOLID CORES.

PROGRESS IS RARELY LOGICAL

ICBM WAS NOT EXTRAPOLATION OF WINGED AIRCRAFT.

Figure 16. Gaseous Fission Spaceship Safety 
In addition to the fact that the fission product load is small, one can do interesting things by recognizing the fact that the exhaust jet velocity is actually higher than solar system escape speed. If trajectories are properly programmed, once out of the Earth's atmosphere, most of the fission products ejected with the exhaust will be thrown completely out of the solar system. This is the one way of not contaminating space. Space, incidentally, is a doggone big place, and the contamination of small local radiation belts, atmospheres, or planets should not be confused with all of space. Even our Sun, which is continually making a real attempt at space contamination compared to any puny spaceship, has not succeeded to any great distance.

Some of the characteristics which make a gaseous fission rocket different from solid core propulsion systems also result in development differences. There is a tendency for many people to believe that gaseous fission engines are a logical extension of Rover. At the risk of losing a number of friends, I would like to point out that they probably are not a logical extension of Rover. For instance, the time between reactor tests should be greatly reduced for gaseous engines. One need not fabricate fuel elements between tests, and does not have to live with fission products imbedded within the engine. The handling advantages in operation previously mentioned also extend to the engine development process and the development program should be a lot easier to run than that of a solid core engine. Gaseous fission engines may not be a logical extension of solid core engines at all.

A good space engine can also have a profound effect on spaceship development cost. It is not just a question of a high degree of reuse, the re is also the effect of making the ship abortable during any part of the flight.

Transportation systems not only achieve very high reuse, they contain sufficient redundancy to permit flying with partial equipment failures, and also have the ability to abort successfully from any flight condition. It is this last capability which is very important to the development program of such ships.

The savings in development cost of not losing ships continually is obvious, yet our ammunition thinkers are so used to the massive throwaway that they usually claim that recoverable equipment would be more expensive to develop since it is more complicated. This might be true of the recovery of marginal peiforming rockets, but would not be true of a properly designed reusable spaceship which would not be marginal with a gaseous fission engine.

It simply is not possible to overemphasize this difference in development philosophy. Commercial transports are extensively tested, and much of the equipment refined by flight tests. They become reliable pieces of 
equipment for expenditures very small in space budget terms, because it is possible to test the equipment over and over for a reasonable expenditure. If a high performance propulsion system can permit us for the first time to pursue a space vehicle program with the very efficient development techniques of transport aircraft systems, we will be very remiss if too blind to even consider these techniques.

The use of a reusable and abortable ship from the start of the development program can well have a profound interaction on engine development tests. This interaction will be enhanced greatly if the engine is a "tractable" engine. A "tractable" engine is one which has "benign failure modes." In other words, it does not explode catastrophically when it fails. There is an excellent chance that gaseous fission engines will tend to go out rather than explode when trouble occurs.

If the engine is tractable, and the ship abortable, then flight failures consist mostly of unscheduled landings. The ship is then capable of testing the main engine without the extreme sensitivity to component malfunction which exists in ammunition development programs. This can be a very large leverage on total development costs. Clearly, there must be extensive ground testing of the engines. However, it may be much easier to arrange partial duration ground runs in enclosed areas than it is to arrange total duration ground runs. The total duration runs could then be performed in the ship.

Many engine developments in the past have made extensive use of flying test beds when ground facilities were not adequate. The technique should not be ignored if the flight vehicles are able to reintroduce it. This is one of the examples of development techniques available with transportation devices which are not within the realm of experience of ammunition developers.

The final point I wish to make is, I'm against logical progress.

A common mistake in development thinking seems to be a tendency to relate the basic performance achieved by a device with its development difficulty. It seems so logical to assume orderly progress in development programs. Actually, many major programs are not a result of orderly progress. One of the most recent interesting examples is the development of the ICBM. These ballistic missiles penetrate to their targets at a Mach number of 25. Orderly progress would have dictated that we build first fleets of supersonic bombers, then fleets of hypersonic bombers. Only after that would we consider whether or not Mach number 25 penetrators were desirable.

The fact is that Mach number 25 ballistic missiles are considerably 
easier to build than hypersonic bombers (and evidently Mach number 3 bombers). Their performance is attained in a different manner, with different engines (not breathing air) and in a different flight region (out of the atmosphere). They are not a Mach number 25 airplane.

The gaseous fission spaceship has many analogous elements. It is easy to achieve $500,000 \mathrm{fps}$ out in space, as long as the engine is capable of it. A ship which never carries fission products aboard need never fight the safety problems of solid core nuclear rockets, or even the analogous problems of nuclear airplanes. An abortable transport rocket is a different development job than the building of larger ammunition. We must look at the gaseous fission ship in terms of its difficulty of development, not in awe of its possible accomplishments. 


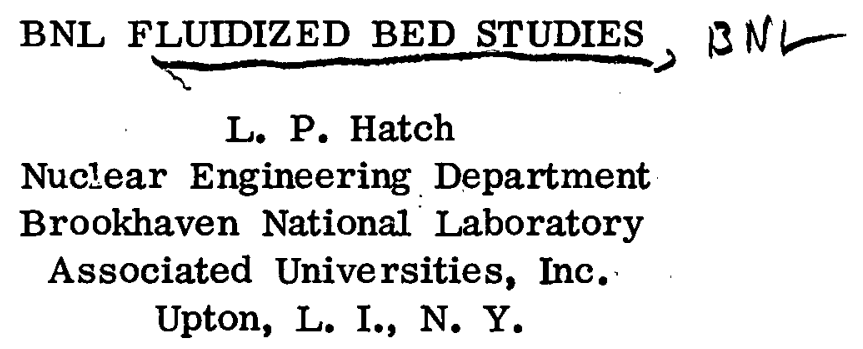

The rotating fluidized bed reactor concept offers a major advantage in the large amount of surface area presented for transfer of heat from the solid fuel to the gas coolant. So the system design features the fuel in the form of a bed of solid particles held in suspension in the flowing gas coolant, and confined to the reactor core by virtue of centrifugal force.

At the Brookhaven National Laboratory we have carried out exploratory studies on the over-all performance and mechanical aspects of rotating fluidized beds over the past 4 or 5 years. This year, however, we were able to set up a more formal program with direct support from the AEC. There have been considerable delays in getting equipment designed and built and full-time personnel assigned to the study.

The first slide (Figure 1) shows a simple representation of a fluidized bed with the granular material uniformly dispersed under a 1-g field. One view shows the bed in the settled state; the other view shows the bed with fluid passing upward and expanding it to a greater height. Fluidized bed studies of the early days were carried out with liquid flow, so that you have uniform fluidization and a uniform dispersion of the material. Moreover, unless the liquid is in highly turbulent motion, the top of the bed will be flat and will remain essentially stationary so long as the velocity and the viscosity of the fluid remain the same. In the gas fluidized system, however, there is a definite tendency for bubbles to form and for a portion of the gas to pass through the bed in the form of bubbles. Whether the original bubbles pass all the way through the bed, or the bubbles collapse and new ones form, is not too well known, but the latter is probably a safe assumption. The question that arises in nuclear reactor design considerations, however, is 

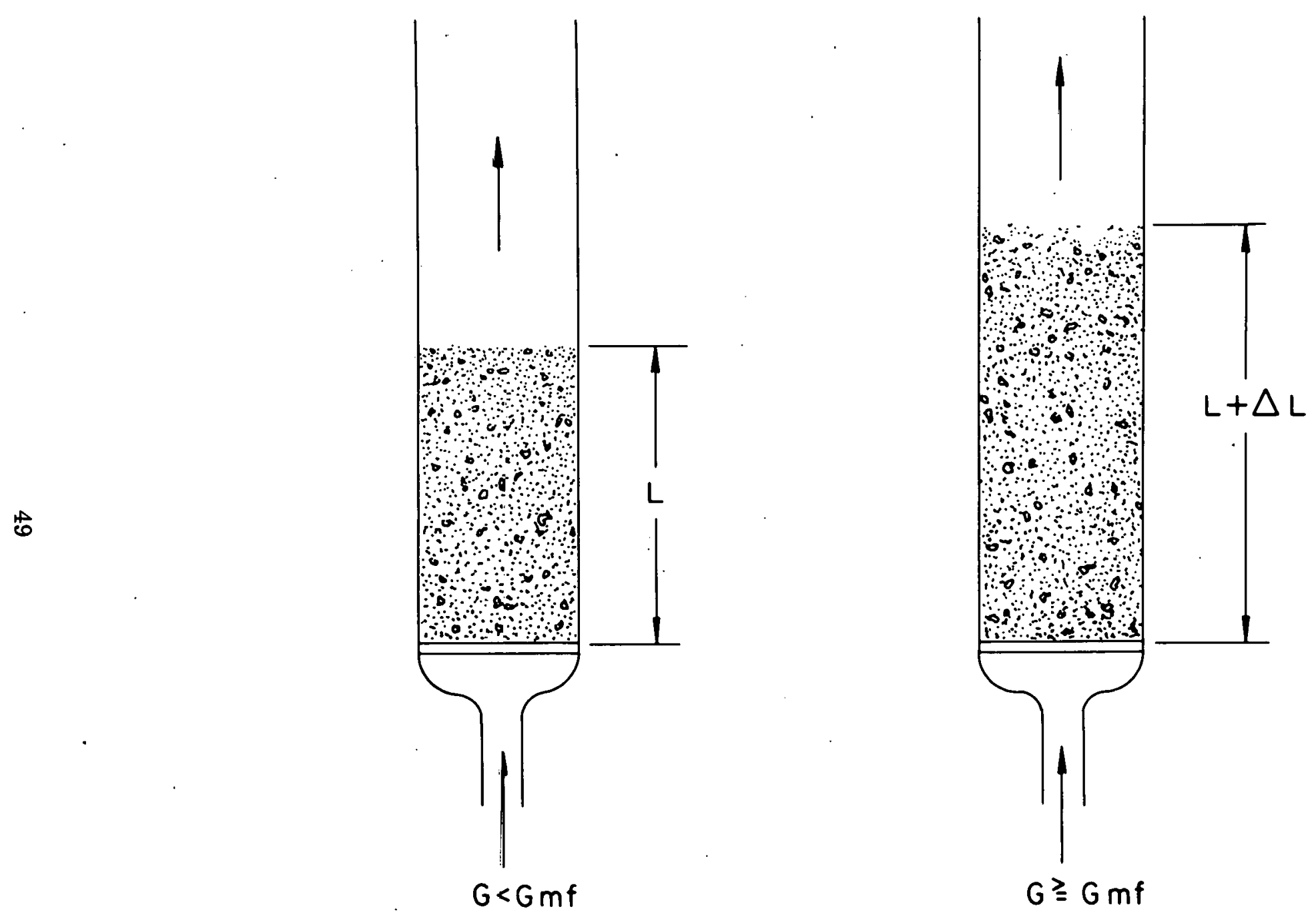

Gmf-MASS VELCCITY FOR MINIMUM FLUIDIZATION

Figure 1. Simple Fluidized Bed in 1-g Field. 
how much of the gas would pass through the bed not directly contacted with particles to maintain efficient heat transfer. To some extent heat would transfer in the form of radiation, but largely one would look to convective transfer.

Figure 2 shows a diagram of a rotating fluidized bed with the particles held in place, against the inward flow-induced drag forces, by means of centrifugal force. Both the bed and the container are rotated; the gas enters through the tubular shaft, then flows inward through a porous wall which serves to support the ked. The annular bed is about 6 inches in diameter and 6 inches long. Initially, as against the wall in the lower zone of the container, with only moderate gas flow, the material becomes fluidized and redistributes itself more or less evenly over the length of the container in the pattern of a cylindrical annulus. One would be thinking ultimately, for a full-scale propulsion system reactor, of a length-to-diameter ratio of two or three. There is a problem, of course, that as the gas flows axially down the center zone toward the discharge opening, it has a tendency to carry along some of the particles; but with sufficient $g$ forces on the system, that does not seem to be a serious problem.

Figure 3 is a photograph of a 6 -inch unit with two views from above. View (a) shows the cylindrical bed in the settled state with the gas flow cut off. View (b) shows the bed in the fluidized state with the flow on. The diffuse appearance along the inner zone is due to the formation of bubbles, presumably, and is quite different from the clear cut inner face in view (a). But, there is definitely a high degree of retention of particles; and we have seen no real evidence of escape of particles, which, in this case, were about. $120 \mu$ in diameter. Questions have been raised as to how such a system would operate in an upside down position wherein the normal forces of gravity and the discharge flow of the gas would be in the same direction. So we mounted the 6-inch unit in an upside down position, using a rubber stopper in the discharge opening to hold the bed material until we established rotation, and the material assumed its angle of repose at the wall. Then, with the stopper removed, material was fluidized with airflow, and it redistributed itself uniformily in the cylindrical pattern, as before.

Figure 4 shows a unit of more advanced design with a bed diameter of 10 inches and length of 1 inch. Gas flow, again, is up through the center tubular shaft. The unit has been operated at $2,000 \mathrm{~g}^{\prime} \mathrm{s}$, and we have obtained high-speed moving pictures of the bed.

Figure 4A shows a line-drawing design of a new unit of about the same dimensions which is intended for operation up to $10,000 \mathrm{~g}^{\prime} \mathrm{s}$. The flow and the pressures will be much higher than before and, for this reason, an 


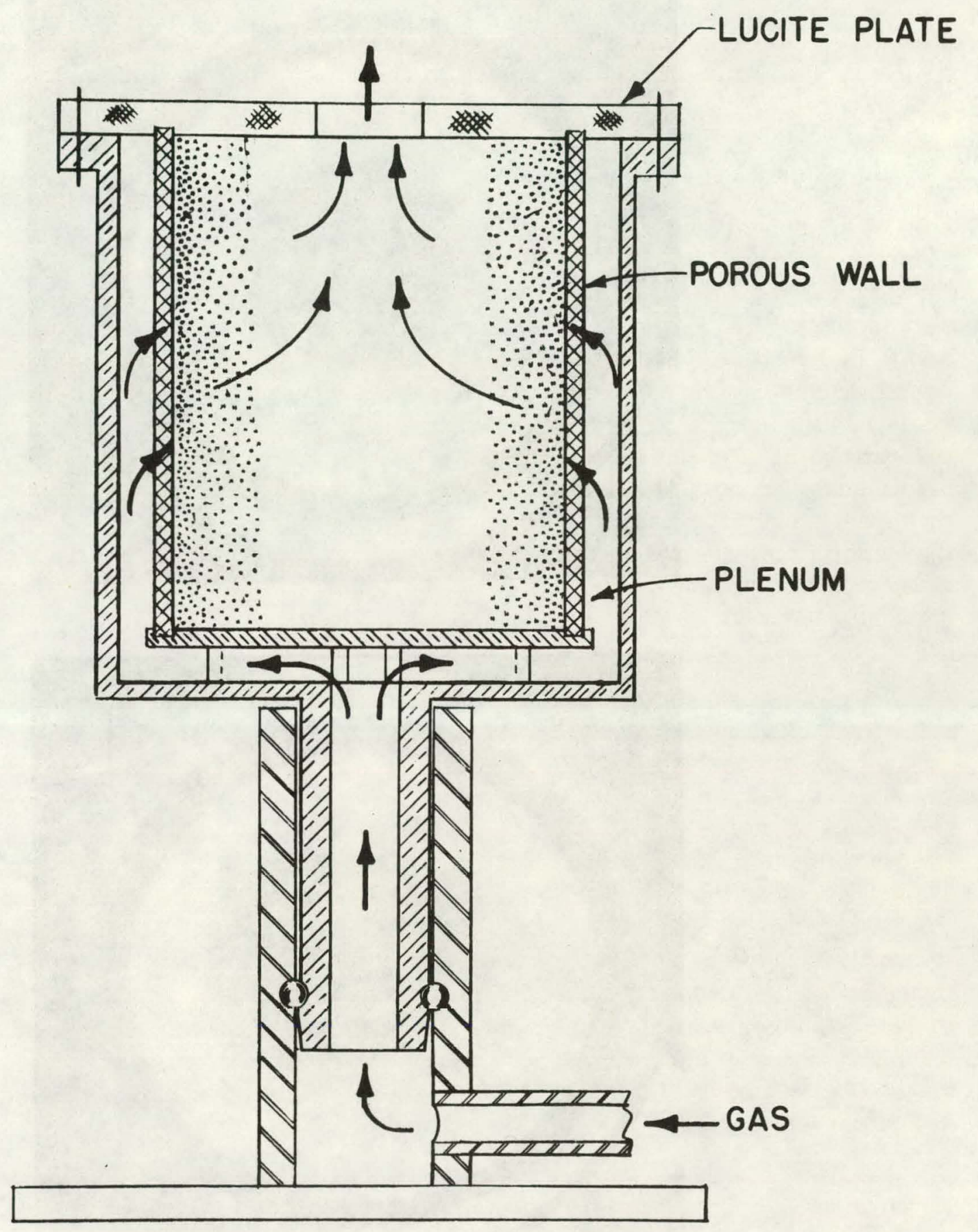

\section{ROTATING FLUIDIZED BED}

Figure 2. Rotating Fluidized Bed in Multiple-g Field 

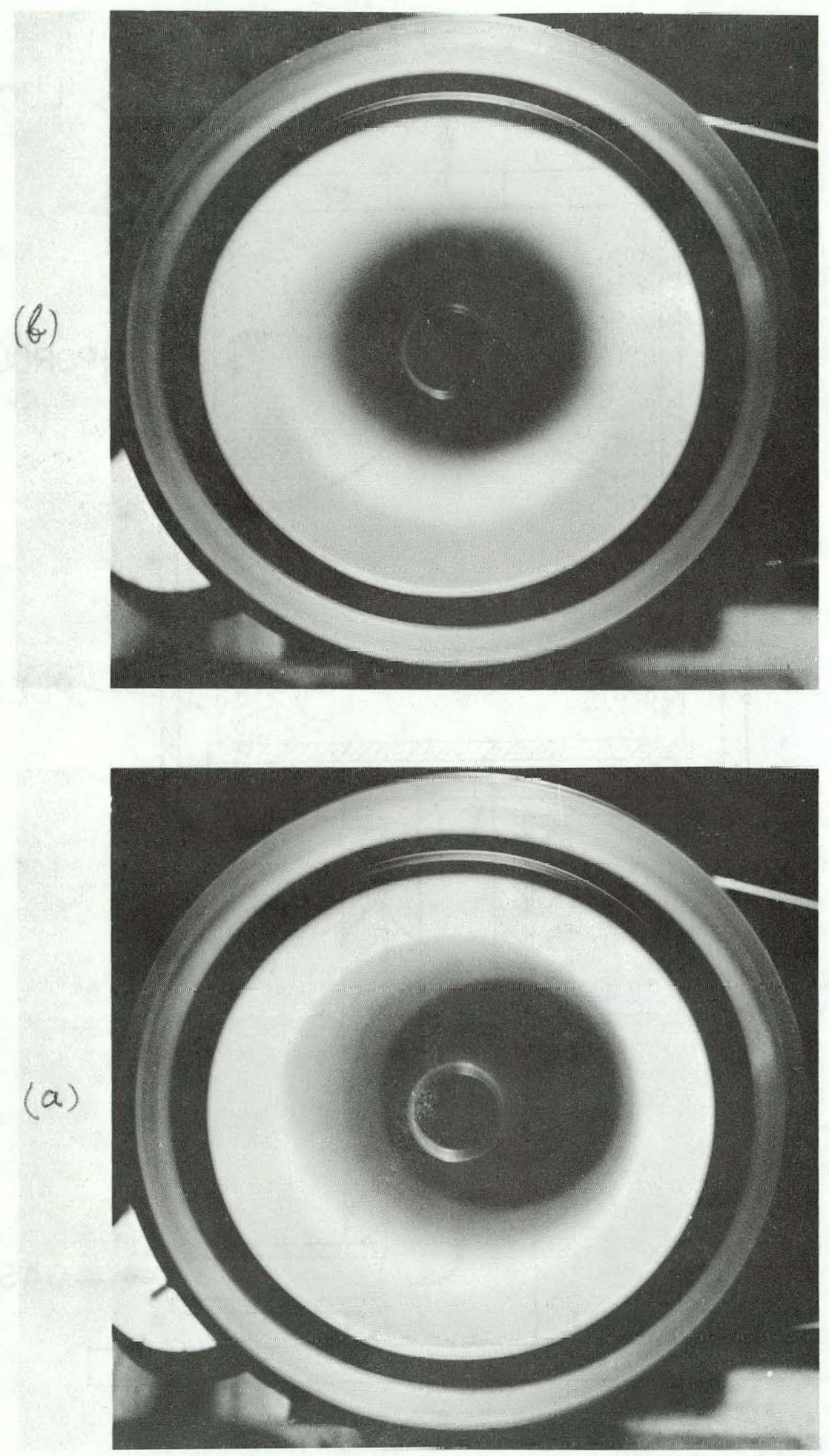

Figure 3. Photograph of Rotating Particle Bed (a) Unfluidized (b) Fluidized 

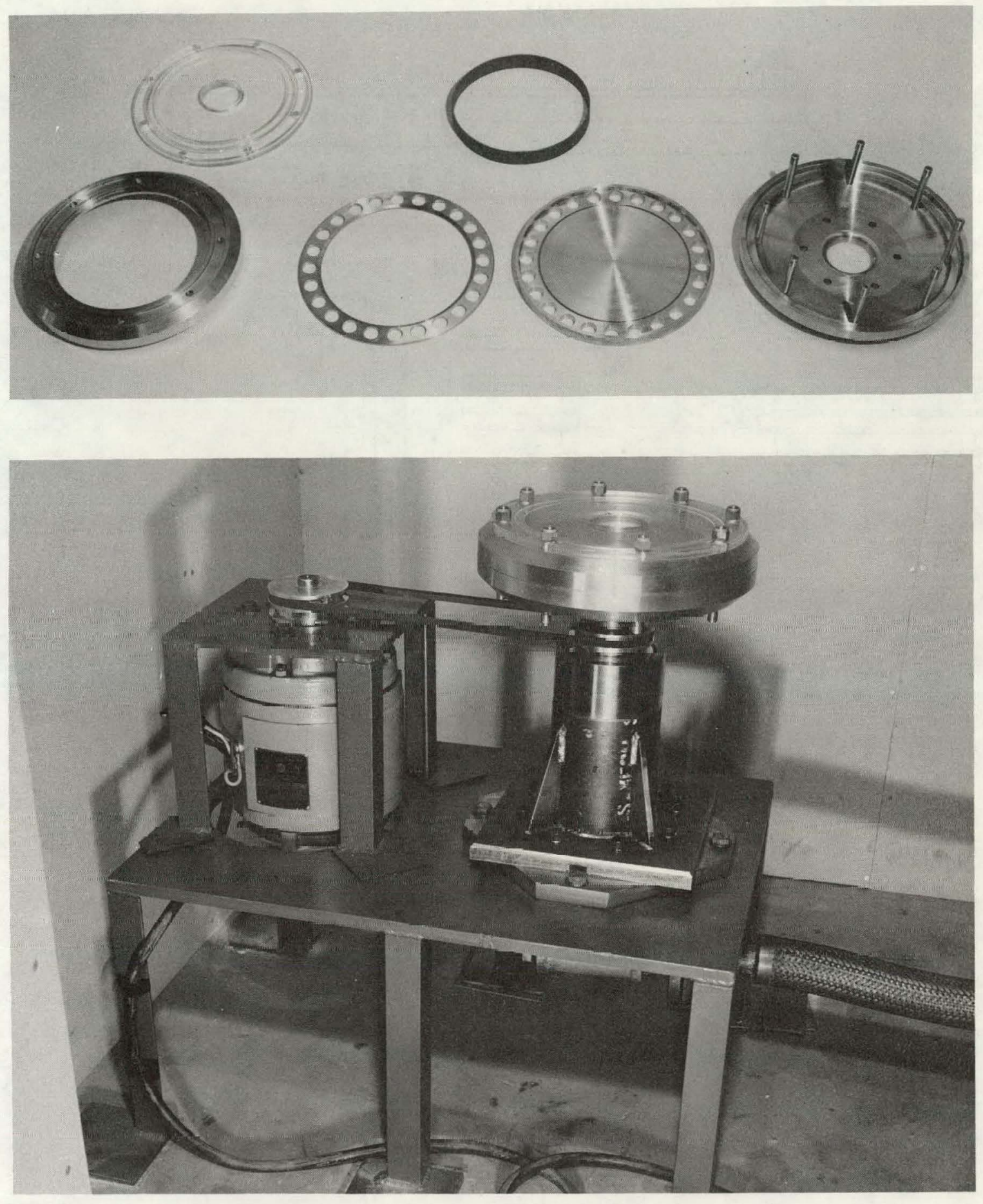

Figure 4. Apparatus for Studying Rotating Fluidized Bed Fields up to $10,000 \mathrm{~g}^{\prime} \mathrm{s}$ 


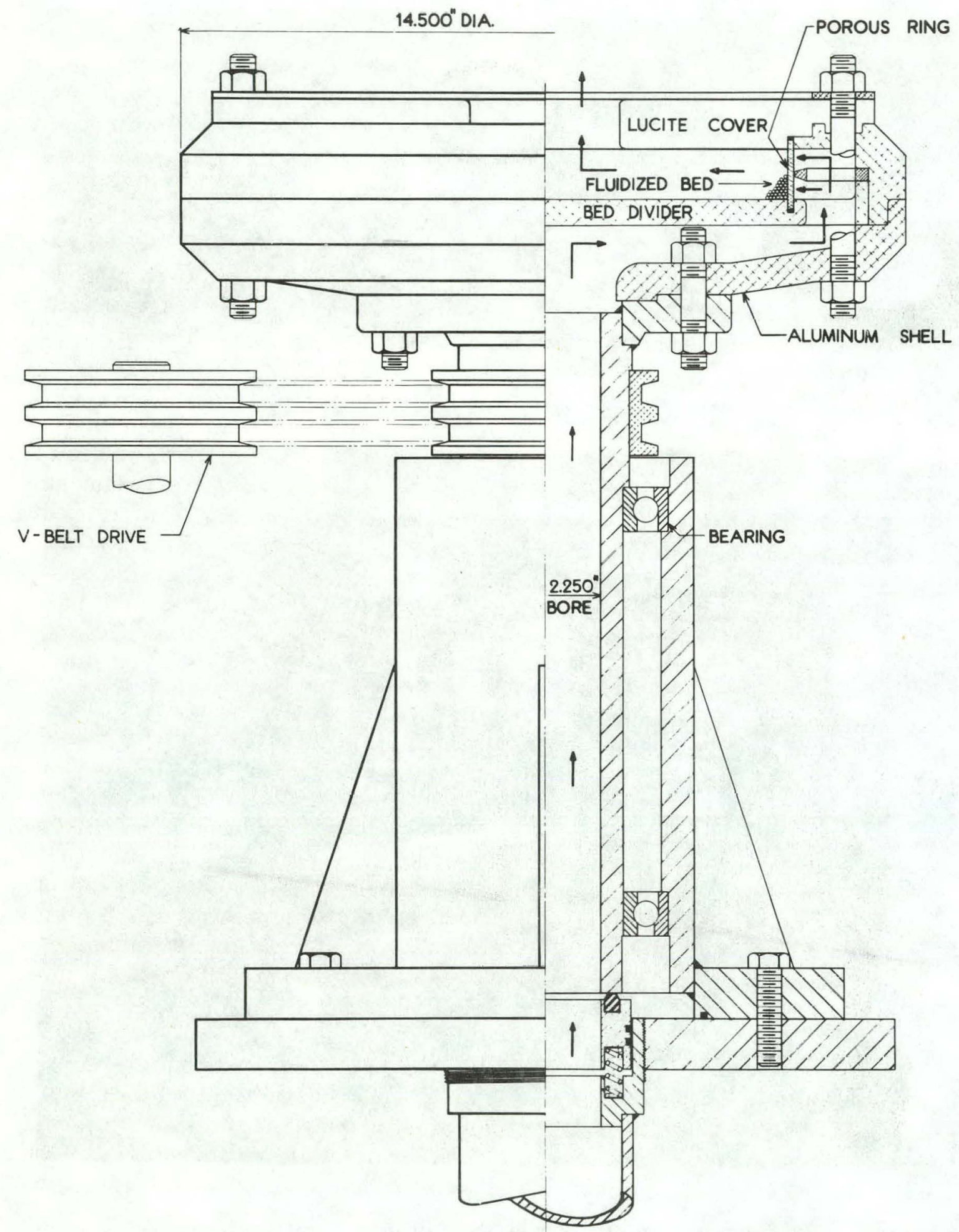

Figure 4A. Rotating Fluidized Bed Assembly 
intermediate support ring is shown midway along the 1-inch length of the porous wall. We have not operated this unit with any bed material as yet and are now doing some reworking of the inlet gas seals at the bottom. We haven't thought too much about how we would undertake to make measurements on the system beyond the point of obtaining over-all gas flows, pressures, and general performance values. Component parts and the assembled unit are shown; for maximum strength the bowl is machined from a single plate of aluminum. The unit is mounted in a room with sand bags placed along the walls for safety. Local experience, gained from mechanical failure of high-speed neutron choppers, is reflected in the design of the unit.

Question: What are the dimensions, about a foot or so?

Answer: The porous cylindrical wall is about 10 inches in diameter and 1 inch long. Because of difficulties of carrying out quantitative measurements on the rotating beds, we may want to make some initial experiments with non-rotating or $1-\mathrm{g}$ systems. This would apply especially with respect to the determination of coefficients of heat transfer between the particles and the gas. Since we are so highly concerned about heat transfer in the system and trying to foresee maximum value from it, I think that it may be necessary to look first at the simpler systems, and possibly to use analogy to mass transfer relationships.

Finally, in Figure 5 we have some of the beginning ideas, in a conceptual scheme, for a nuclear propulsion system with a one-million pound thrust and 20,000 MW capacity. The bed would be about 3-1/2 feet in diameter and 7 feet long. With $100 \mu$ fuel particles and a bed thickness of 3 inches, we have something like 150 to 200 thousand square feet of surface area for heat transfer. About $2000 \mathrm{~g}$ 's would be required to sustain the bed with a hydrogen coolant flow of one thousand pounds per second (specific impulse : of 1000 seconds). The velocity of the gas flowing through the bed would be on the order of 300 linear feet per second, and rotational speed would be $1500 \mathrm{rpm}$. So the mechanical requirements for strength and support of the rotating unit do not seem to be prohibitive.

Question: How much power is required to drive it?

Answer: This we haven't figured out. It's substantial; - something like 3 horsepower for the 10-inch unit described previously. However, it would certainly be small compared with the 20,000 megawatts produced by the reactor. For the 1 million pound thrust system, the reactor would be thermal or slightly epithermal with an 18-inch thick beryllium metal reflector around the outside. This would be in the form of plates, and the entering gas would absorb the heat released in the reflector which would be something on the order of 2000 megawatts. 


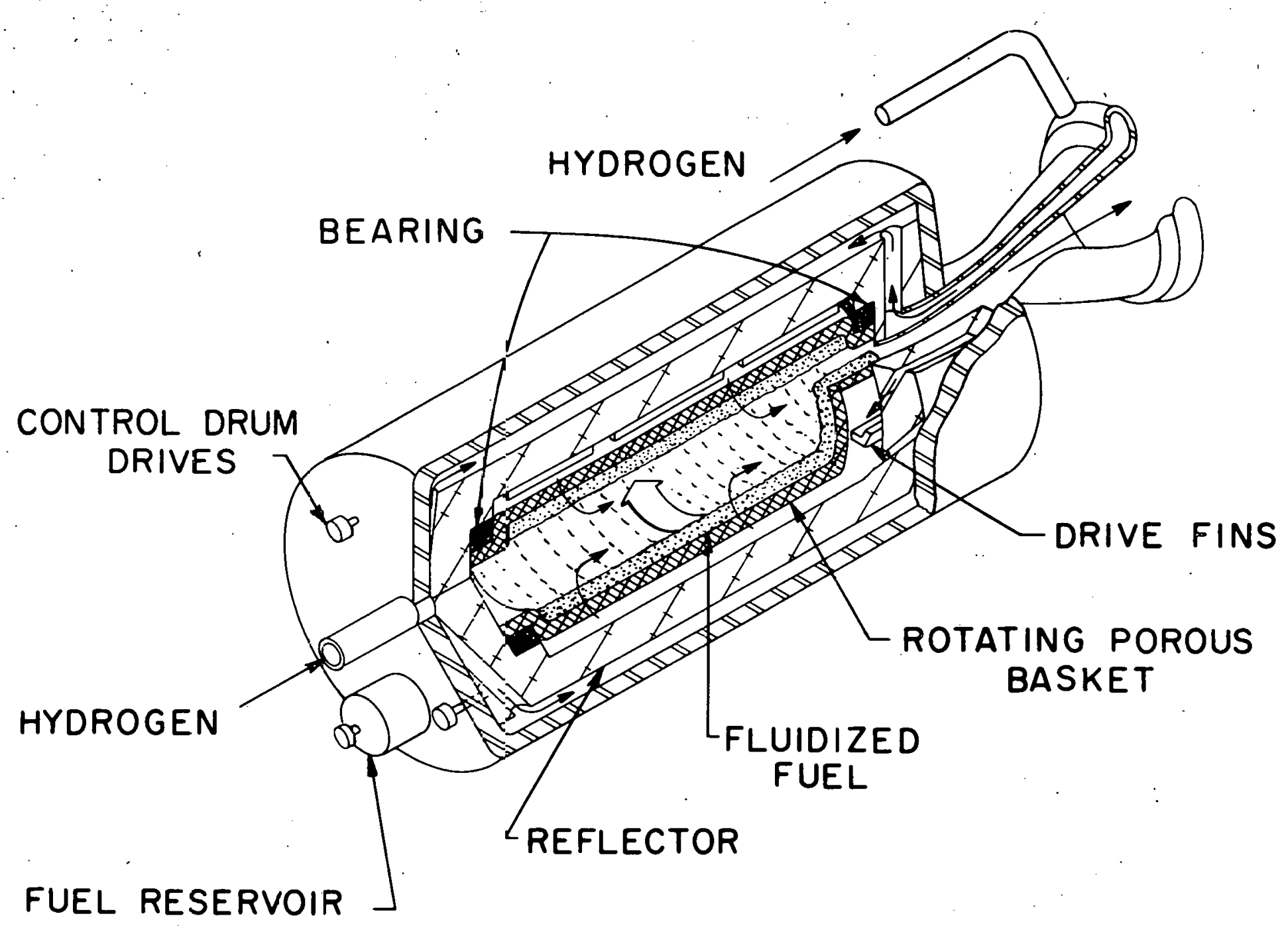

Figure 5. Conceptual Scheme for Ut:lizing Rotating Fluidized Bed Reactor for Rocket Propulsion 
The question that immediately comes to mind is how high a temperature could one expect with such a reactor. For a specific impulse of 1000 seconds with hydrogen, the temperatures would have to be in the neighborhood of $5500^{\circ} \mathrm{F}$. So the questions of thermal stability of the particles and temperature difference between particles and gas are paramount. Even without melting would the particles adhere or fuse together to any serious extent?

At this point, I want to refer to the results of an experiment carried out at Los Alamos in 1963 as part of an investigation of rotating fluidized beds for nuclear propulsion reactors. That investigation, which began about the same time as the Brookhaven study, included a very important series of experiments on $\mathrm{ZrC}$ particles fluidized under $1 \mathrm{gg}$ fields at temperatures as high as $3000^{\circ} \mathrm{C}$. It was found that the particles did not fuse at the high temperature when the bed was fluidized, but did fuse at the high temperatures when the bed was not fluidized. Now, with high gravity field systems and high gas velocities, the increased agitation among the particles should tend to further discourage fusing.

With respect to heat transfer, we have had to make the unsupported assumptions that the coefficient might be as high as 1000, in English units. If the coefficients are not that high, we have to reduce the size of the fuel particles to increase specific surface, and to correspondingly increase the g's on the system.

Question: Have you been speaking in terms of rotated porous walls rather than rotating just the bed?

Answer: Yes. We actually have been thinking both ways, but the tangential entry system does not seem to lend itself to uniform distribution of particles; whereas with the rotating porous-wall container, a particle bed of uniform thickness is readily formed. The tangential flow system might be much simpler from a mechanical standpoint, but behavior of the particles is much less predictable.

Question: What performance are you talking about in this? I mean what specific impulse would you achieve if this worked?

Answer: Well, we would hope for a specific impulse of 1000 seconds, or even 900 seconds, but the temperature is going to have to be $4500^{\circ} \mathrm{F}$ or higher.

Remark from Audience: I can't help interjecting that if you get, say a thousand seconds and if this particularly lends itself to the type of space transportation system into orbit that Max is talking about, it may just beat 
everything else. It's good enough performance for getting into orbit, as a matter of principle, but by some margin. I suppose I'm caught right in the middle between - I'm probably more prone to think in the conventional way of going up in stages from something that we? re reasonably sure of, the other systems which are far out in the operation sense, of course offer a much better promise, but when one thinks of the practical problems.

Question: This is sort of a high-speed ball-mill in a sense; how fast are you losing particles due to change in size?

Answer: Not too fast, well I have no answer for that, because I don't know, but I don't think it's a serious problem.

Question (Von Chain): What temperature would your rotating drum have?

Answer (Cooper): I've looked at this and it depends very crucially on what the thermal conductivity of this dust bed is, but the hydrogen has such a heat capacity that using a number which was one or two hundred times that of copper for dust bed conductivity the inlets were very cold, and as a matter of fact, this is a change from the ordinary dust bed or fluidized bed work that the chemical engineers do, there the conductivity is so large compared to the flows that the dust bed is assumed to be at a uniform temperature. Now with the sort of calculations I mentioned there is a tremendous temperature rise and depending upon the heat transfer it looks as if the dust bed may be one or two hundred degrees away from the gas temperature. That means you would be in the regime where you have actually very cold inlets. What the actual conductivity of the bed is, is unknown.

Answer (J. Grey): Operating the bed at a somewhat uniform temperature instead of with a high temperature difference between the inlet and outlet is just hard to conceive. The bed becomes less dense with depth with the high temperature ratio when you fluidize it.

Question: You mean sparse on the inside?

Answer (J. Grey): Yes. There is an increase in the bed expansion capability of the gas as it passes through; viscosity goes up, velocity goes up as the gas density goes down. The change in the effective drag force on individual particles is by a factor maybe of five as it reaches the inside of the bed, compared to its fluidizing capability as it enters.

Answer (Cooper): Jerry, pardon me - I have looked at this also and one may reach the situation where the lower portion of the bed did not fluidize; and actually that would be very good, because when its a fixed bed you get better heat transfer than a fluidized bed. This is because you get more gas bypassing in a gas fluidized system. 
Question: The problem of abrasion and particle loss?

Answer: I think that is not a serious problem - I can't back this statement with any numbers. For long-term operation, it could be a problem; but for high thrust propulsion, the system would not be operating too long. One indication that abrasion may not be a major problem is the absence of sintering at high temperatures. This, in turn, may suggest reduced contact between particles, and that the gas actually acts as a buffer.

Question: What is the pressure drop through the bed?

Answer: It is equal to the weight of the bed under the $\mathrm{g}$ system; so with a $2000 \mathrm{~g}$ system, you have several hundred psi pressure drop with a 3-inchthick bed.

Question: Assuming it's completely fluidized?

Answer: Yes.

Question: Is there any difference in the behavior on the high $\mathrm{g}$ bed compared to the (Romero) one $\mathrm{g}$ bed?

Answer: I' $\mathrm{m}$ sure there is. I hope it's a better behavior; in fact, we are trying to see whether if one has high enough $g^{\prime} s$ on the system the tendency to bubble formation may be reduced - possibly may be eliminated. All the forces acting on the particles are increased, but the individual behavior of single particles and not of groups of particles may be more pronounced with the high $\mathrm{g}$ system. This is one thing we want to look into carefully. I think you could say that bed performance certainly is no worse at $2000 \mathrm{~g}^{\prime} \mathrm{s}$, compared with $1 \mathrm{~g}$, and I wouldn't assume it would be the same, so it's either going to be substantially worse or better.

We have high speed moving pictures (Figures 6 and 7) of rotating fluidized beds, and these show up the bubble formation quite well. You can actually see individual particles dropping back from a surface eruption, or bubble burst. Individual particles swept into the region above the bed are not supported by sufficient drag forces which are down maybe by a factor of 10 from the drag forces obtaining in the bed.

Question: What are the particles here?

Answer: These are glass beads, spherical particles, $120 \mu$ in diameter. The pictures were taken at a rate of 8000 frames per second, and the length of time for a complete series is 1 second. We have observed partial fluidization of a bed by introducing an inner layer of glass spheres, darkened by gamma irradiation, and noting the back mixing of these spheres into the otherwise white bed. 


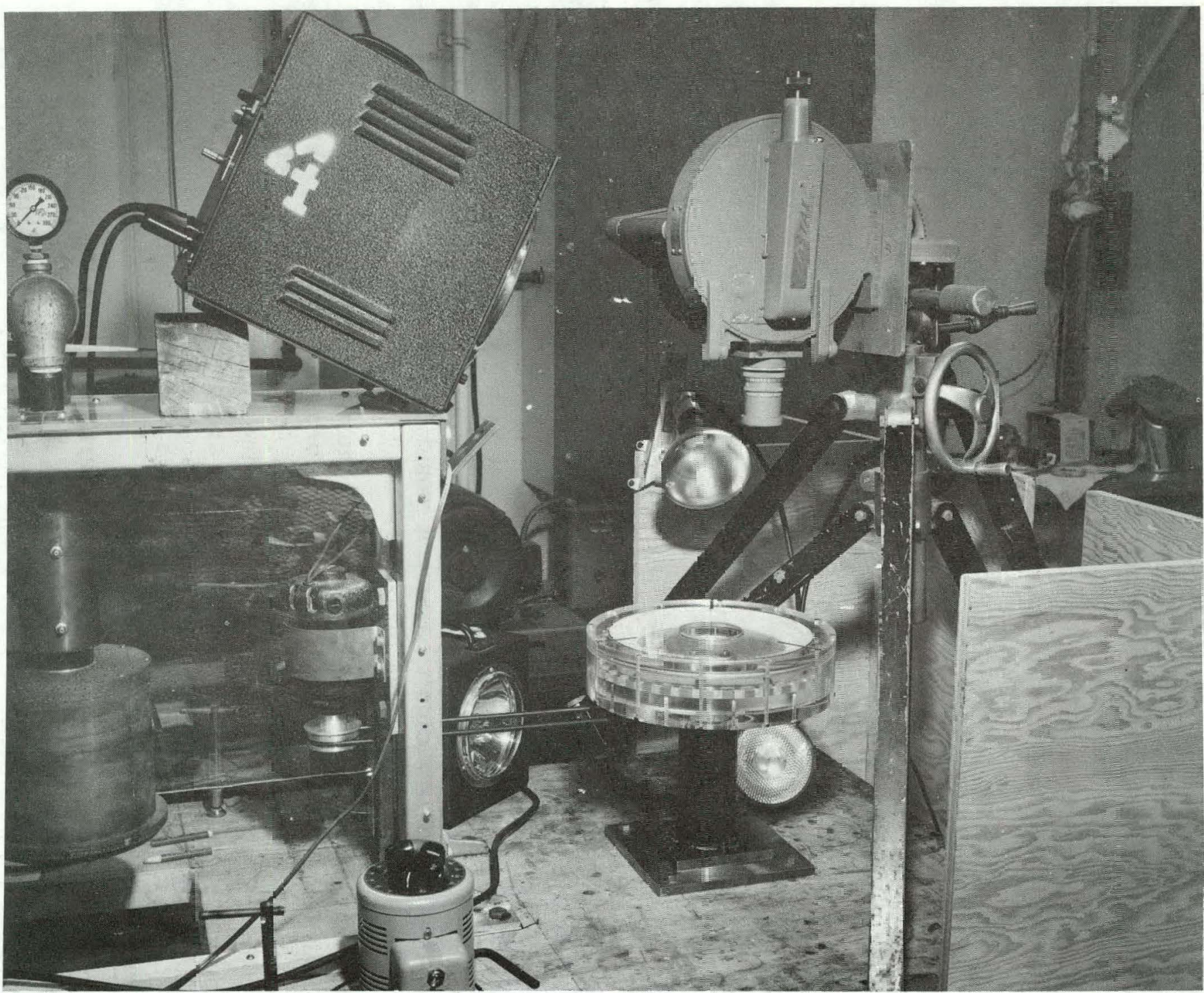

Figure 6. Apparatus Used in Photographing Rotating Fluidized Beds: 1,000 - 2,009 g's 


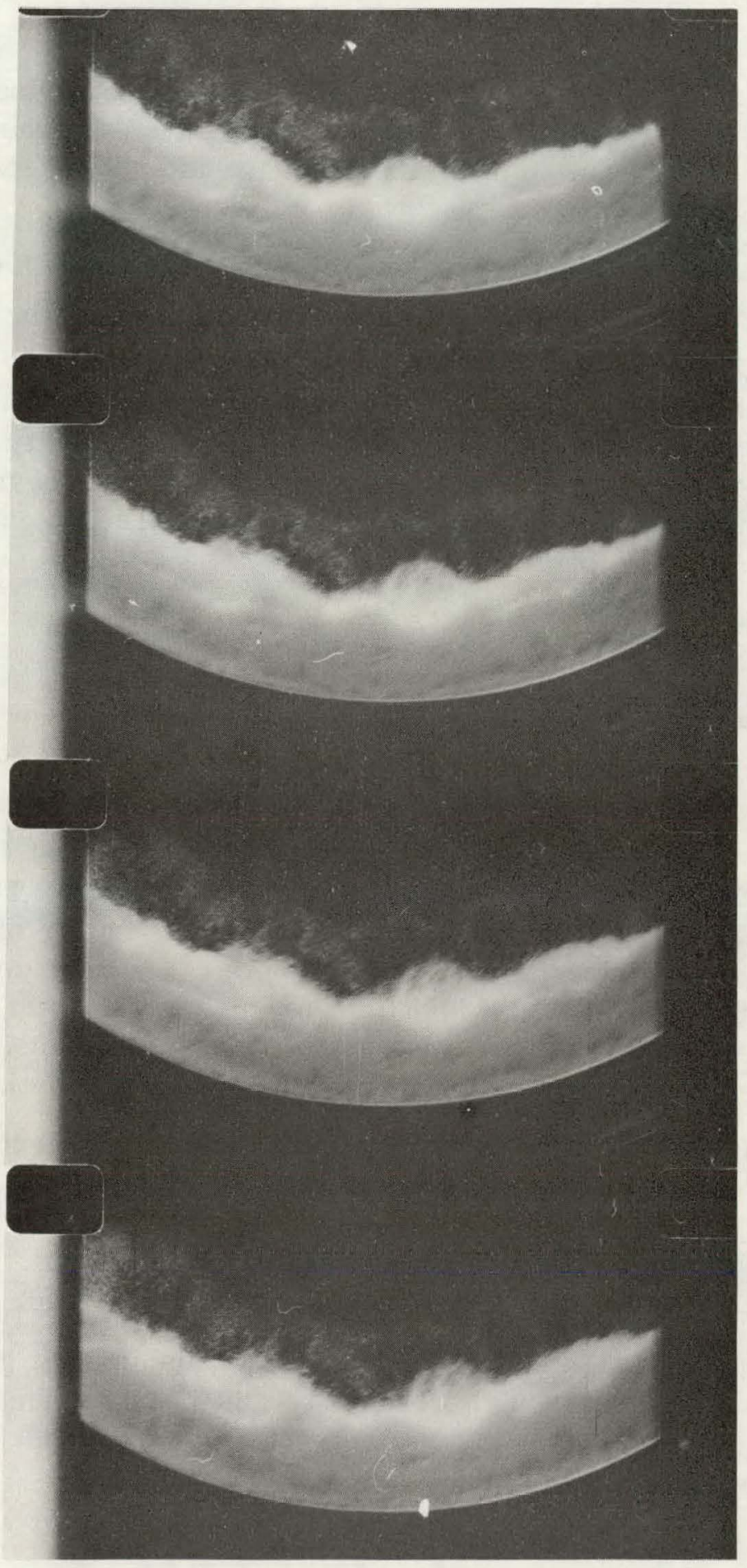

Figure 7. Sequential High-Speed Photographs of Rotating Fluidized Bed Showing Gas Bubble Formation 
Question (Taylor): Did you have a figure in mind for the typical ratio of internal energy to kinetic energy of the gas as it leaves the surface of the bed; in other words, is it the velocity it will have after it's gone clear through an outer nozzle, or is it essentially stagnant?

Answer: I don't have such figures in mind but the velocity of the gas in the bed for a propulsion system might be 300 feet per second.

Question (Von Ohain): In case the bubbles produce the heat or rather the particles produce the heat as they are so narrowly packed, isn't there the danger that you form streamers and the rest is not sufficiently cooled and melt together, in other words that you get very nonuniform heat transfer in a system where the amount of particles in the mass per cubic centimeter is so large in comparison to the gas mass?

Answer: That's a possibility. That's one reason why the gas bubble formation is always of great concern.

Question: There is a certain instability, you know the moment you cool this then, the temperature rises tremendously until certain things start to melt and

Answer: Yes, very high heat rate production, but I think that intense agitation of the system may be of considerable importance here. The experience at Los Alamos with a 1-g system, which did not show fusing of the zirconium carbide bed at a temperature of $3000^{\circ} \mathrm{C}$, offers a great deal of hope in this regard. I might say that the high temperature experiment was of major importance because it provided at least a partial answer to questions about fusing of particles at temperatures near the melting point of the material.

Question (Barrett): What is the main thing that the experiment will tell you that you don't know now?

Answer: What experiment?

Question: Well, the experiment you're doing on fluidized bed.

Answer: All we are doing now is observing the beds in high gravity fields to note their behavior, mainly with respect to gas bubble formation. But we plan to carry out quantitative measurements on fluid flow and heat removal properties of high gravity systems. 


\title{
FLASH X-RAY ANALYSIS OF FLUIDIZED BEDS
}

\author{
Jacob B. Romero \\ The Boeing Company \\ Seattle, Washington
}

First of all, I would like to clear some common misconceptions regarding fluidized beds and fluidized bed reactors, and also to define more clearly the fluidized process. I do not think there is much advantage to fluidized bed over graphite reactors for propulsion purposes, except perhaps in the event that thermal stresses become very important in the graphite core. With fluidized beds, one might be able to eliminate these stresses and thus make designs possible.

I have given talks before on this subject, and after finishing I am asked what a fluidized bed is; so it might be worthwhile here to define a fluidized bed before beginning. Actually, the fluidized process is very simple. Visualize for the moment a container filled with particles which rest on a porous support through which gas can be introduced. If gas is now forced through the particles, and the flowrate (say G in pounds per square foot per hour) versus pressure drop is measured, we will find that, up to a point, the pressure drop varies linearly with flowrate. This flow range is called the packed bed region; there is no particle movement. At a particular flowrate, the pressure drop suddenly becomes constant and very nearly equal to the weight of the bed per unit area. At this point, the particles are suspended and the bed is fluidized. The flowrate at which this occurs is called the point of minimum or incipient fluidization usually referred to as $\mathrm{G}_{\mathrm{mf}_{\mathrm{f}}}$. Further increases in flowrate result in bubble formation which by-pass the bed in much the same way that a gas bubble by-passes a liquid.

Another common misconception concerns the heat transfer between the particles and the gas in the bed. This is not necessarily lower in the fluidized bed than in the packed bed despite the bubble flow. This misconception apparently has arisen from the fact that one thinks of bubbles as 
by-passing the bed without contacting particles. But, in a fluidized bed, the gas circulates through these bubbles - a fact which has been shown through some excellent work in Great Britain. ${ }^{1}$ The gas circulation, of course, results in good contact between gas and particles. W. L. Barrett of our laboratory will show you results tomorrow on fluidized bed heat transfer experiments which yielded large values of heat transfer coefficients.

The talk today considers flash $\mathrm{x}$-ray experiments and analysis of fluidized beds. The main purpose of these experiments was to visualize the internal structure of fluidized beds. In this manner, we were able to learn more about density gradients and other properties within the bed. Do we have high density regions within which might affect heat transfer or reactor nucleonics, and what are the sizes and shapes of bubbles? These are some of the questions we were trying to answer.

The apparatus used was a flash $\mathrm{x}$-ray machine which may be familiar to some of you. It might be simply said that this machine is a high speed, $\mathrm{x}$-ray unit, which produces a tremendous dose of radiation in a matter of tenths of microseconds. Thus, it essentially stops all motion and, for this reason, is used to analyze high speed phenomena such as bullets penetrating materials or explosions. So, it is easy to see that in a fluidized bed the $\mathrm{x}$-ray unit stops all motion, and we are able to see conditions within the bed at any time.

From these visualizations, we are able to obtain useful data on the bed densities, the shape and sizes of bubbles, and bubble formation. By firing two flash $x$-ray units in sequence, we can also measure the velocity of bubbles as they rise through the bed. Data of this nature serve as a test of the Two-Phase Theory of Fluidization first proposed in $1952 .^{2}$ This theory states that all the excess flow, that is all the flow above minimum, should by-pass the bed as bubbles. Now, if this is the case, the dense phase part of the bed will maintain a constant density with flowrate. On the practical side, this is also of great importance to the nucleonics of these reactors. In addition, these data serve to support some of the newer theories on fluidized beds, in which the bed is assumed to be liquidlike.

\section{EXPERIMENTAL APPARATUS}

Slide 1 is a photograph of the apparatus used for fluidization showing the fluidizing column and flow meters. A 3-inch-square plexiglass column

1. Wace, P. F., and S. J. Burnett, Trans. Inst. Chem. Engrs., $\underline{39}, 168$ (1961).

2. Toomey, R. D., and H. F. Johnstone, Chem. Engr. Progr., 48, 220 (1952). 


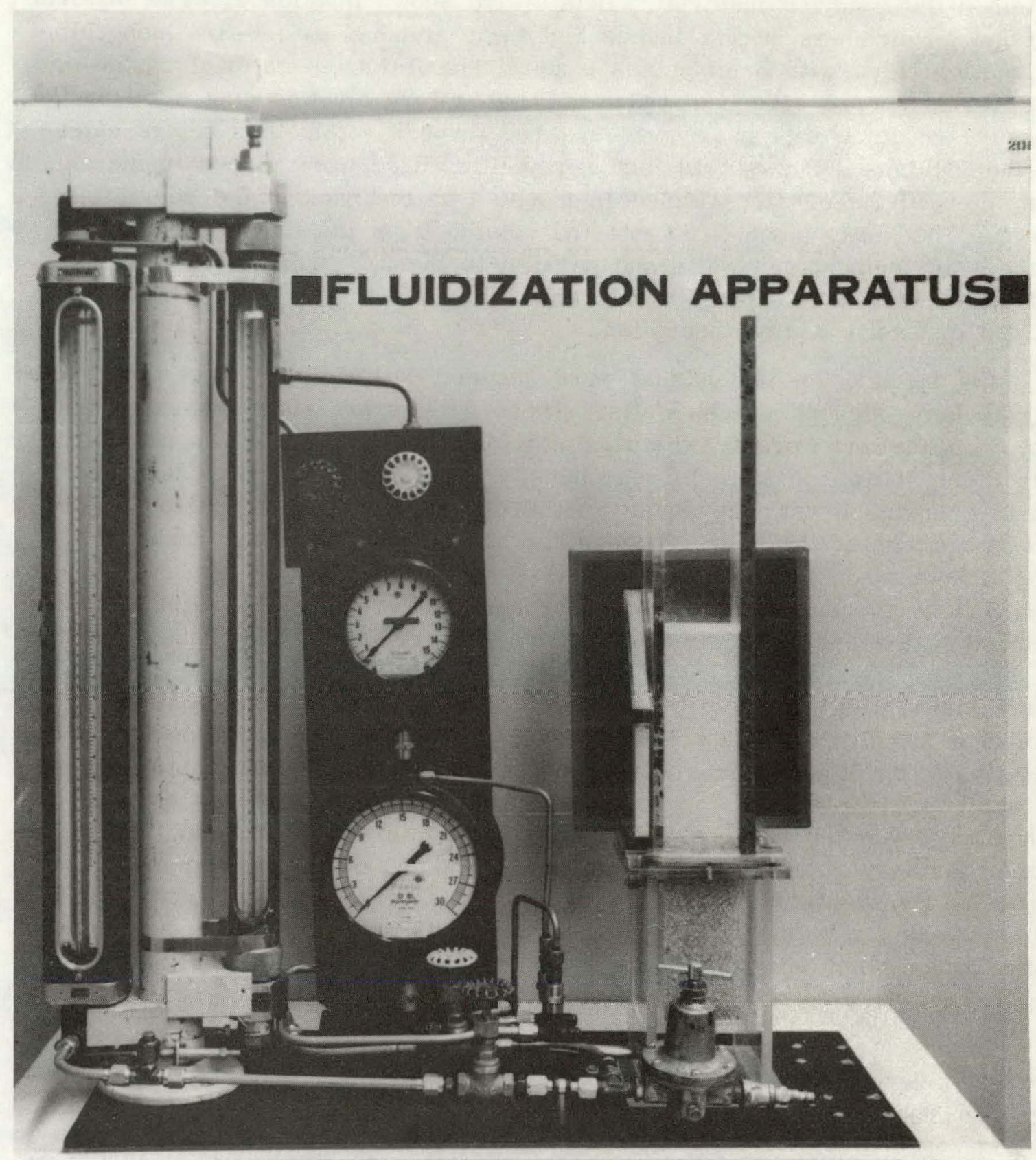

Slide 1 
contained the fluidized material. Details are shown in Slide 2. The material container, which was 20-1/4 inches high, was attached to a $6-3 / 8$ inch calming section filled with $5 \mathrm{~mm}$ glass beads. The fluidized material rested on a 300 mesh bronze screen distributor. Laboratory air was controlled by the pressure reducing valves and was metered through rotameters before entering the columns. The $8 \times 10$ inch x-ray films used were placed inside a light intensifier cassette, which was mounted on the back of the column as shown. The $x$-ray beam fired into the column from the front perpendicular to the $1 / 16$ inch column walls and parallel to the $3 / 8$ inch walls. The $3 / 8$ inch walls served as a density reference while the thin $1 / 16$ inch walls avoided excessive $\mathrm{x}$-ray absorption.

On the sides of the column were mounted calibration wedges constructed of $1 / 16$ inch thick plexiglass walls. These wedges are right triangular 3 by 4 by 5 inches and were filled with fluidizing material to a known density. With them, it was possible to read density as a function of film darkness and to compare it with the density within the bed, making it possible to map density throughout the bed. To read the darkness of the photographs, a special homemade densitometer was devised. It consisted of a photo pick-up cell, a light source, and a carriage for holding the film. The film was mounted in the carriage, which has provisions for lateral and longitudinal movements. The light source illuminated the film from below, and the transmission was picked up by the photocell, whose output operated the $\mathrm{X}$ axis of a Moseley autograph $\mathrm{X}-\mathrm{Y}$ recorder. The carriage position operated the $\mathrm{Y}$ axis, and thus film darkness and position were recorded automatically. To determine bubble sizes, a procedure was developed using a ping pong ball imbedded within the bed operated just below minimum fluidization. Photographs of the ping pong ball were obtained and the densitometer output recorded in the region of the ball. By theoretical arguments we were able to relate the known volume of the ping pong ball to any other volume as integrated from densitometer output curves.

Properties of the fluidizing material are shown in Slide 3. All data were taken on a white silica sand which was approximately spherical. The particle size was mostly in the 150 - 200 Tyler mesh range; the true arithmetic average diameter was 0.0028 inch.

\section{RESULTS AND DISCUSSION}

Slide 4 is a photographic sequence showing some of the more general characteristics observed in x-ray photographs of fluidized beds. This is a sequence for one particular bed operated at flowrates from 1.0 to 5.0 times minimum fluidization. Visual inspection of photographs of the nature show 


\section{COLUMN DETAILS}

(DIMENSIONS IN INCHES)
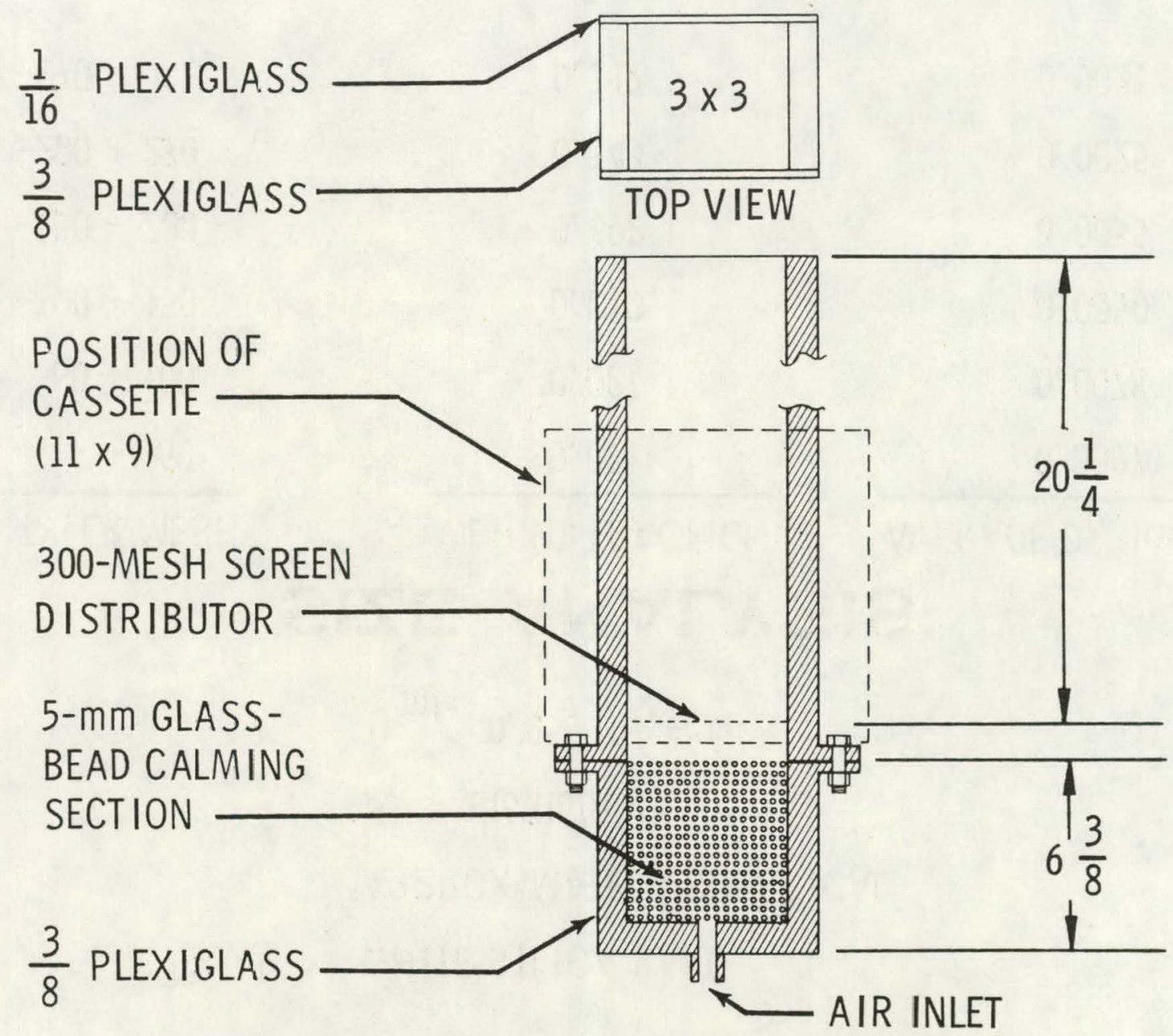

Slide 2 


\section{MATERIAL PROPERTIES}

WHITE SILICA SAND

APFROXIMATELY SPHERICAL

$\rho_{S}=146\left(\mathrm{lb} / \mathrm{ft}^{3}\right)$

$U_{m f}=0.056(\mathrm{ft} / \mathrm{sec})$

\section{SIZE ANALYSIS}

\begin{tabular}{l} 
TYLER MESH \\
\hline+60 \\
$-60+100$ \\
$-100+150$ \\
$-150+200$ \\
$-200+270$ \\
-270
\end{tabular}

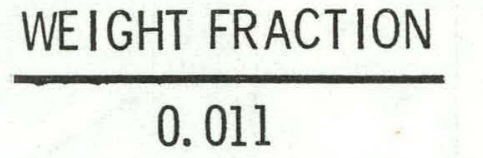

AVERAGE $D_{p}$ (inches)

TRUE AR ITHMETIC AVERAGE DIAMETER $=0.0028$ (inches) 


\section{- BUBBLE SIZES}

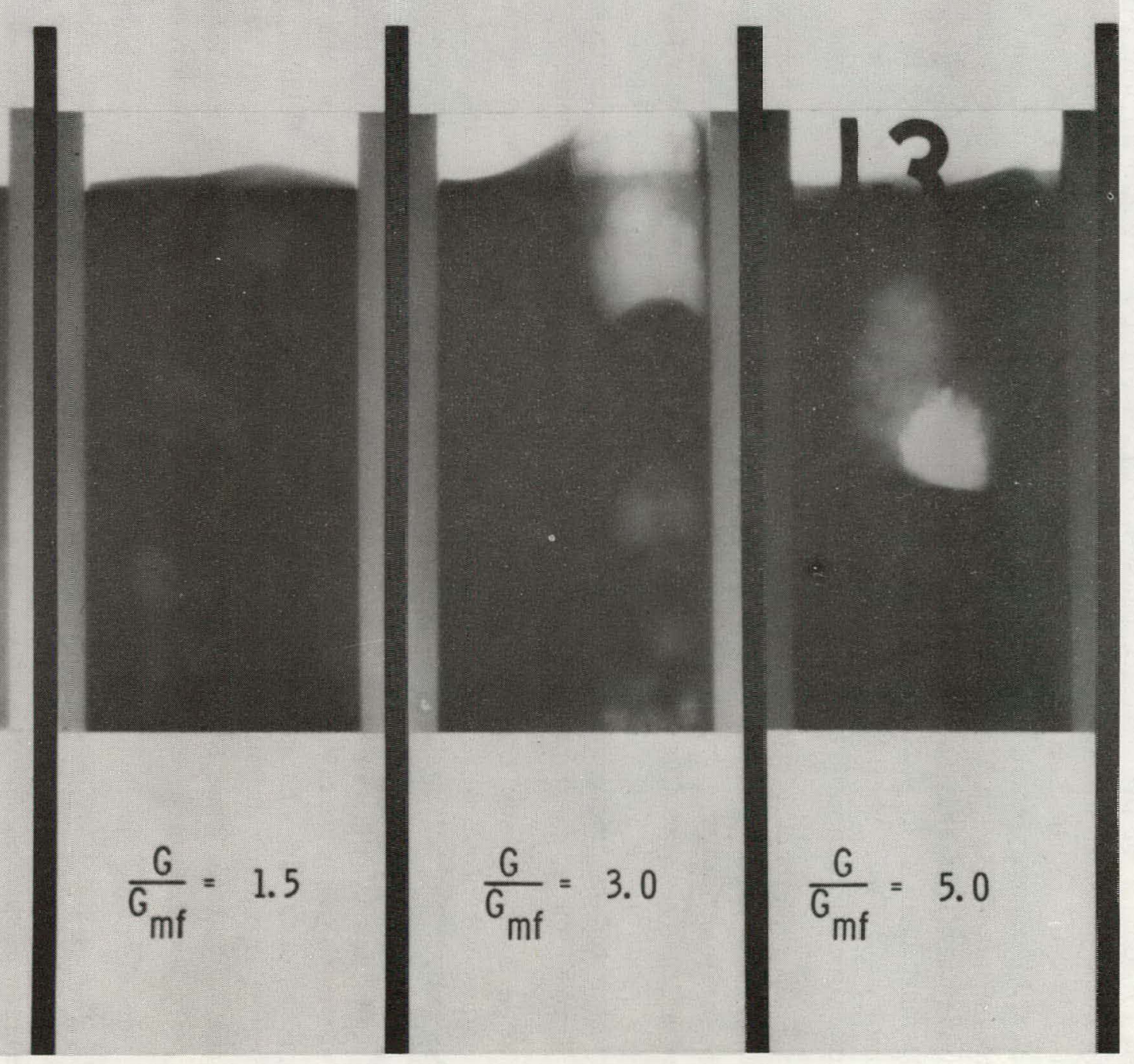

Slid $\equiv 4$ 
the bed to consist of two distinct phases over most of its length as had previously been postulated. One of the striking characteristics is, however, that near the bottom of the bed there is less definition between phases and no distinct bubbles are found. Apparently this region is quite turbulent, and the flow is influenced by particle concentration changes. Rowe ${ }^{3}$ has shown from drag considerations that the bed is very unstable to concentration changes, and a small change in particle concentration can cause large changes in flow or velocity. In this region, the flow may be adjusting to these changes and the flow alterations may result in bubble formation.

Another characteristic that can be observed is the increase in bubble size with flow rate, a result which is qualitatively in agreement with the two-phase flow theory. Inspection of many photographs of bubbles also showed in many cases the convex or flat shaped bottom surface of bubbles, which is predictable from liquidlike theories proposed for fluidized beds. Notice also on the photograph at the left that even at minimum fluidization a few tiny bubbles, perhaps $1 / 8$ to $1 / 4$ inch in diameter, are apparent.

Slide 5 shows some results of the density analysis. The chart on the right shows a typical densitometer output obtained for the calibrating wedge and the bed. The triangular wedge output is used as a reference to determine the density of the bed at any point. The bed is scanned longitudinally every $1 / 4$ inch by the densitometer in alternating directions. The baseline is offset a constant amount after each scan to prevent lines piling on one another. Notice the change in output intensity in the region of a bubble. Interpreted in actual density as given by the numbers superimposed on the photograph, you see that there is little variation of bed density in the dense phase. In the region of a bubble, however, there is considerable variation.

By averaging the bed dense phase densities, obtained from many photographs at a given flowrate, we obtain the plot shown in Slide 6. This slide shows the variation of average dense phase bulk density with tlowrate. For comparison purposes, the expected density variation when $10 \%$ and $100 \%$ of the excess gas passes through the dense phase is also shown. This calculation was based on the theory of Ergun and Orning, 4 assuming that the bed expands in accordance with the flow through the dense phase. It is seen from these data that our results indicate at least more than $90 \%$ of the excess gas by-passes as bubbles even at the high flowrate. Thus, the dense phase density is independent of flowrate even at a flowrate five times minimum. This appears to constitute good evidence for the validity of the Two-Phase Flow Theory.

3. Rowe, P. N., Trans. Inst. Chem. Engrs., 39, 175 (1961).

4. Ergun, Sabri and A. A. Orning, Ind. Engr. Chem., 41, 1179 (1949). 


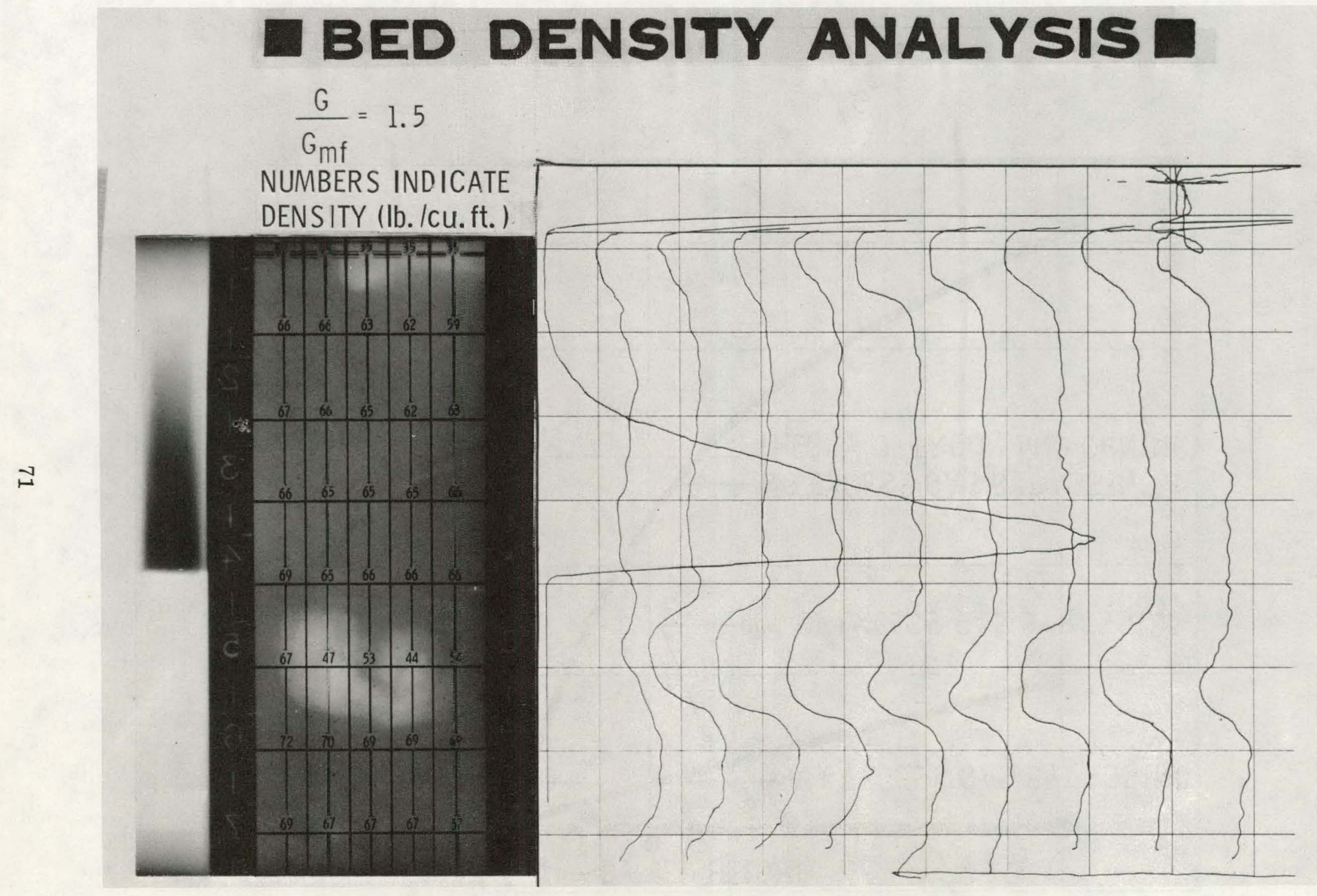

Slide 5 


\section{DENSE PHASE AVERAGE DENSITIES}

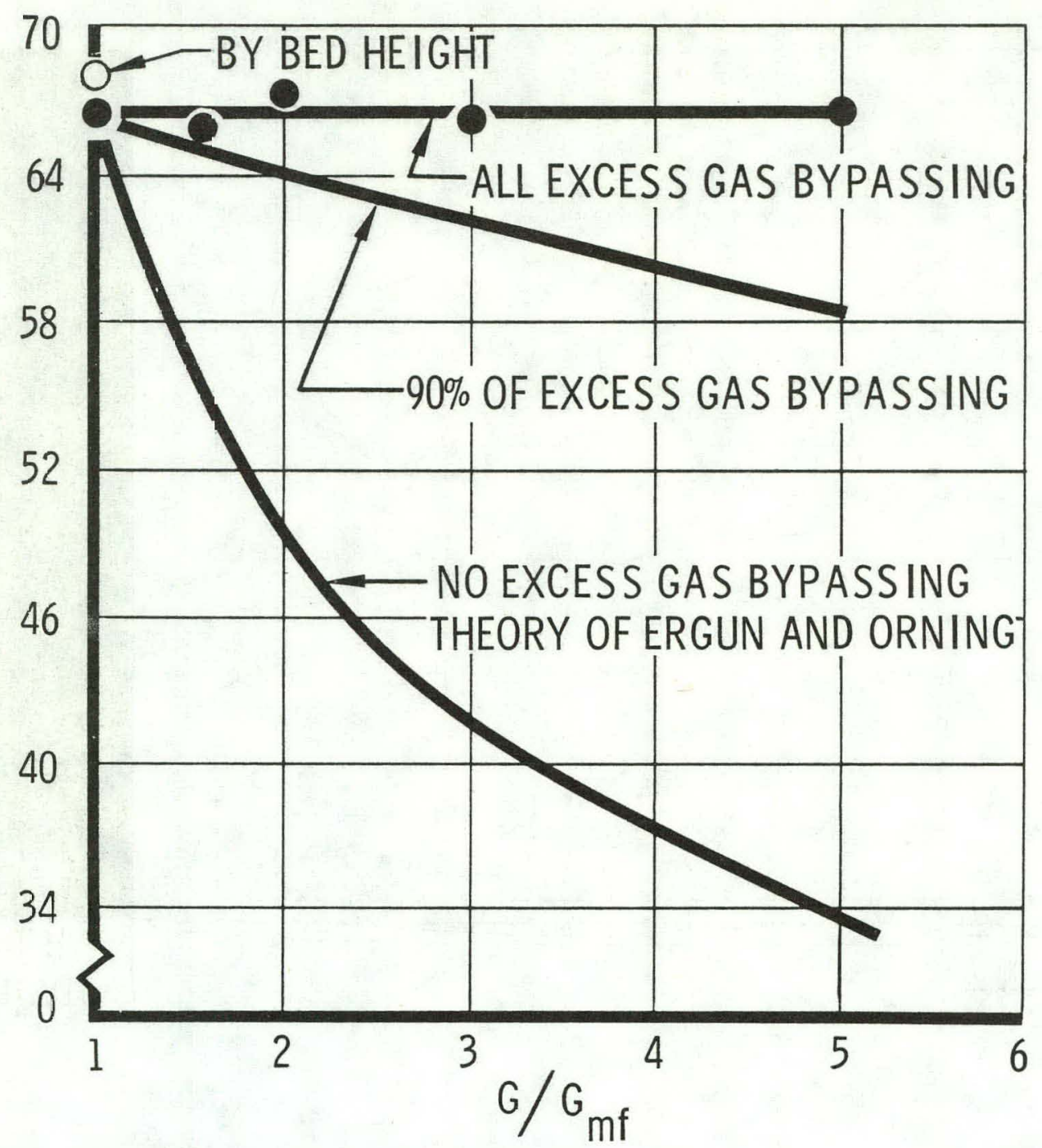

Slide 6 
Slide 7 shows the densitometer output obtained by scanning horizontally every $1 / 8$ inch through a ping pong ball. Notice the symmetry of the output corresponding to the spherical shape of the ball. Slide 8 shows a similar scan through an actual bubble, which is much more irregular. By integrating over these outputs, we were able to compute average bubble sizes, some values which are tabulated in Slide 9 for the various flowrates studied. As the flowrate is increased, the bubble volume increases--in qualitative agreement with the Two-Phase Theory. Using this bubble volume and Two-Phase Flow Theory, we can calculate bubble frequencies which are also shown. The frequency also increases with flowrate, and is in the range of $100-300$ per second per square foot.

In previous work, ${ }^{5}$ we were able to correlate bubble sizes by plotting dimensionless diameter (diameter divided by height above support) versus flowrate. The results obtained in this work are plotted in Slide 10 in this fashion, and are some five orders of magnitude larger. This discrepancy is probably due to the difference in measurement technique used. The previous data were obtained by probe methods and probably did not measure the maximum bubble size. A further complication may be the different bed supports used. This latter problem could adversely affect bubble sizes.

The typical time sequence photographs used for measuring bubble velocity are shown in Slide 11. These photographs show a large bubble photographed as it passed through two sections of the bed by two x-ray units fired 0.303 seconds apart. During this time, the bubble travelled 0.71 feet, and thus its velocity was 2.3 feet per second. The bottom photograph is of interest since there appears to be a combination of two bubbles in progress. The combination apparently succeeds, since the upper photograph shows only one bubble, and its volume is about equal to the combined volume of two bottom ones.

From theoretical grounds, Davies and Taylor ${ }^{6}$ have determined the expected velocity of bubbles in fluidized beds. This theory predicts that the bubble velocity, $\mathrm{U}_{\mathrm{B}}$, should vary with sixth power of its volume, $\mathrm{V}_{\mathrm{B}}$. Slide 12 compares velocities measured in our experiments with this theory; the bubble velocity is plotted versus the sixth root of bubble volume. Our data, in general, follow the theoretical predictions quite closely plotting only slightly higher. To a good approximation, our data can be correlated by the equation $\mathrm{U}_{\mathrm{B}}=\mathrm{g}^{1 / 2} \mathrm{~V}_{\mathrm{B}}^{1 / 6}$. This might be compared to the relationship

5. Romero, J. B., and L. N. Johanson, Chem. Engr. Sym. Ser. 58, 28 (1952).

6. Davies, R. M., and Sir Geoffrey Taylor, Proc. Roy. Soc. (London), A200, 375 (1950). 


\section{"BUBBLE SIZE ANALYSIS}
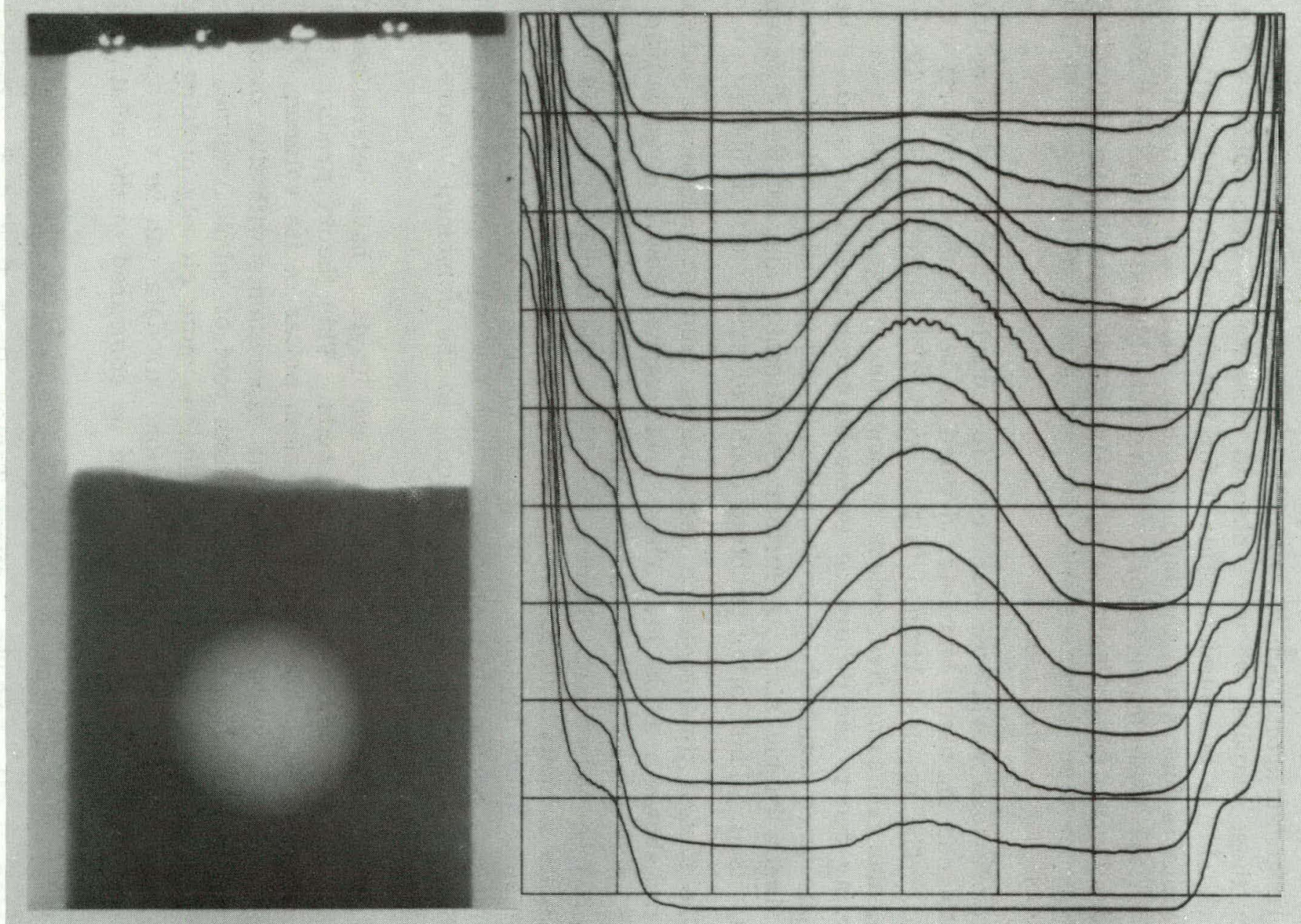

Slide 7 


\section{aUBBLE SIZE ANALYSIS}
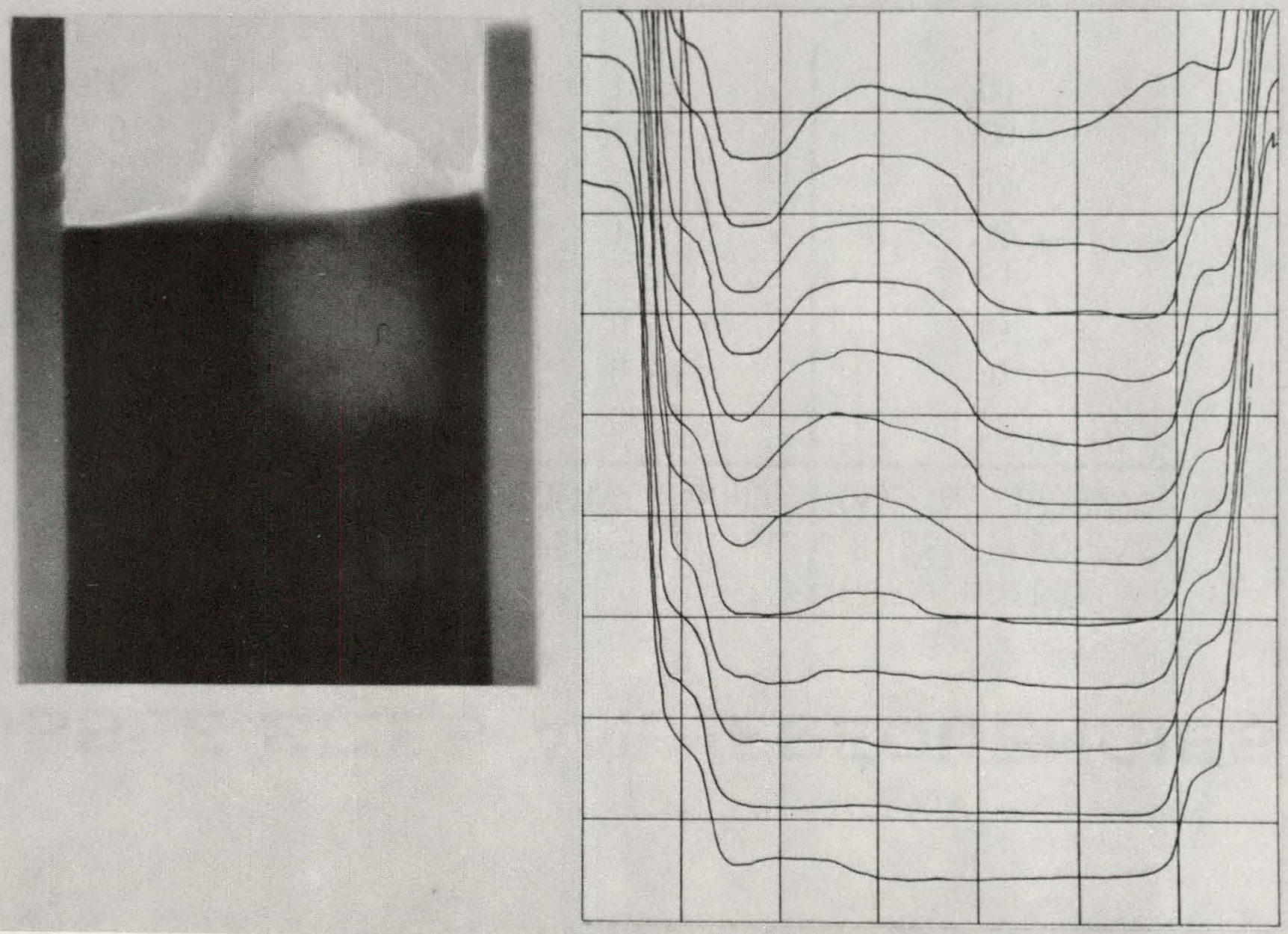

Slide 8 


\section{BUBBLE SIZES AND FREQUENCIES}

\begin{tabular}{c|l|c|c}
$G / G_{m f}$ & $\begin{array}{c}L_{m f} \\
\text { (in.) }\end{array}$ & $\begin{array}{c}\text { AVERAGE BUBBLE } \\
\text { VOLUME (cu.in.) }\end{array}$ & $\begin{array}{c}\text { (TWO-PHASE FLOW) } \\
\text { BUBBLE FREQUENCY } \\
\text { (no./sec. - ft?) }\end{array}$ \\
\hline 1.5 & 3.1 & 0.68 & 72 \\
1.5 & 5.9 & 0.50 & 97 \\
1.5 & 10.8 & 0.51 & 97 \\
3.0 & 3.1 & 2.3 & 85 \\
3.0 & 5.9 & 1.3 & 149 \\
3.0 & 10.8 & 1.7 & 114 \\
5.0 & 3.1 & 1.3 & 300 \\
5.0 & 5.9 & 1.9 & 200
\end{tabular}




\section{BUBBLE SIZE CORRELATION}

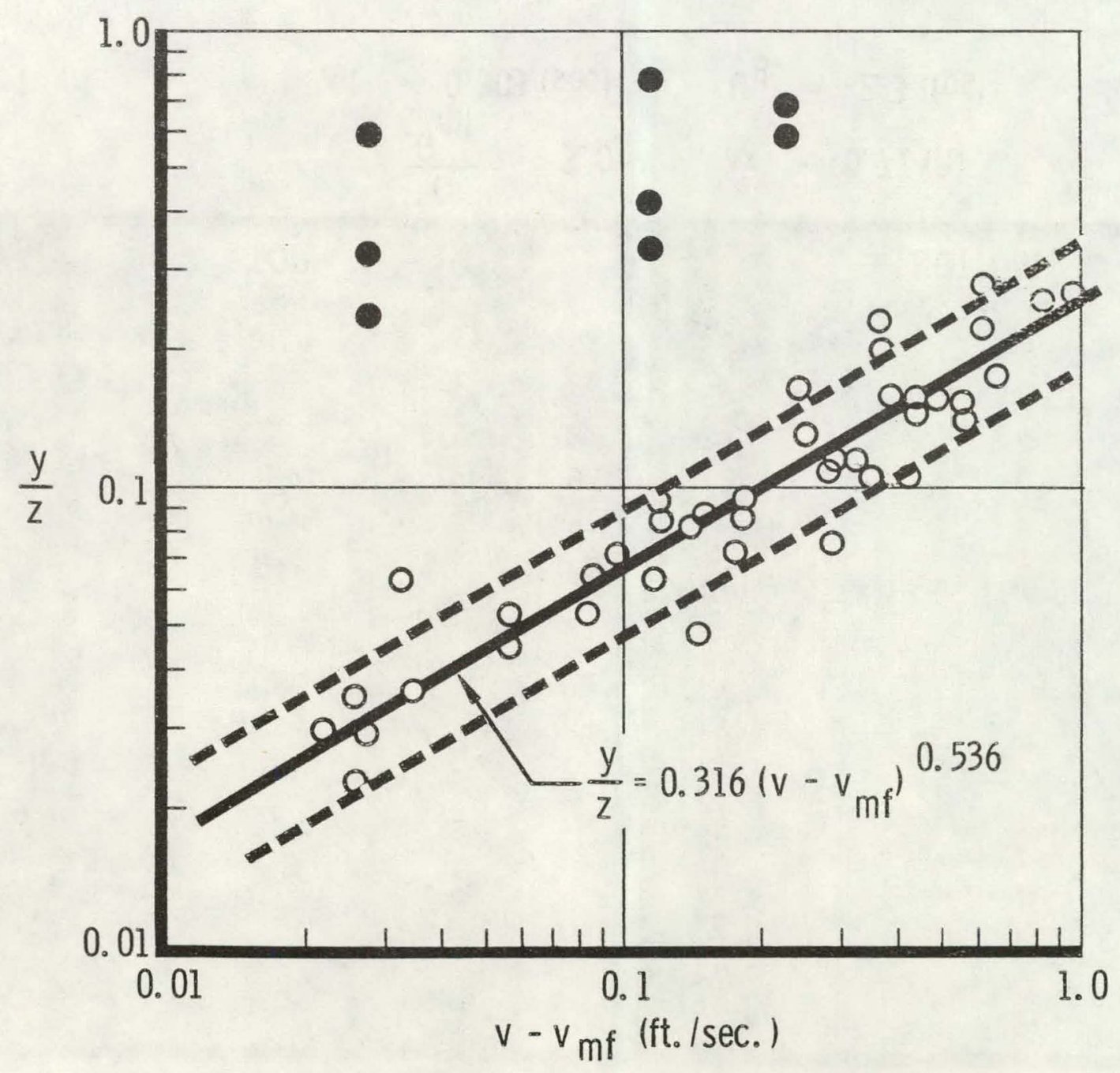

Slide 10 


\section{IBUBBLE VELOCITY MEASUREMENTE}

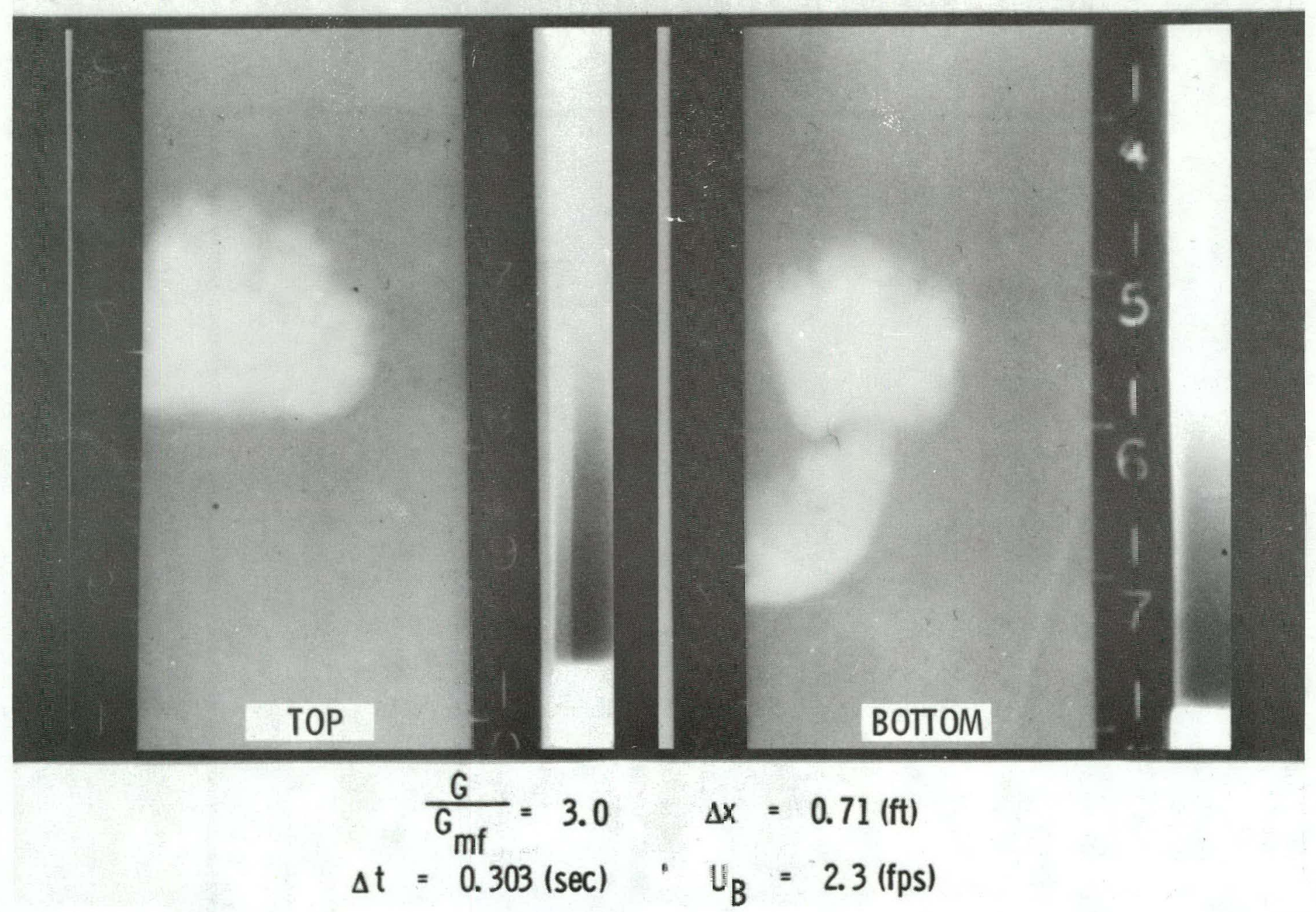

Slide 11 


\section{BUBBLE VELOCITY CORRELATION}

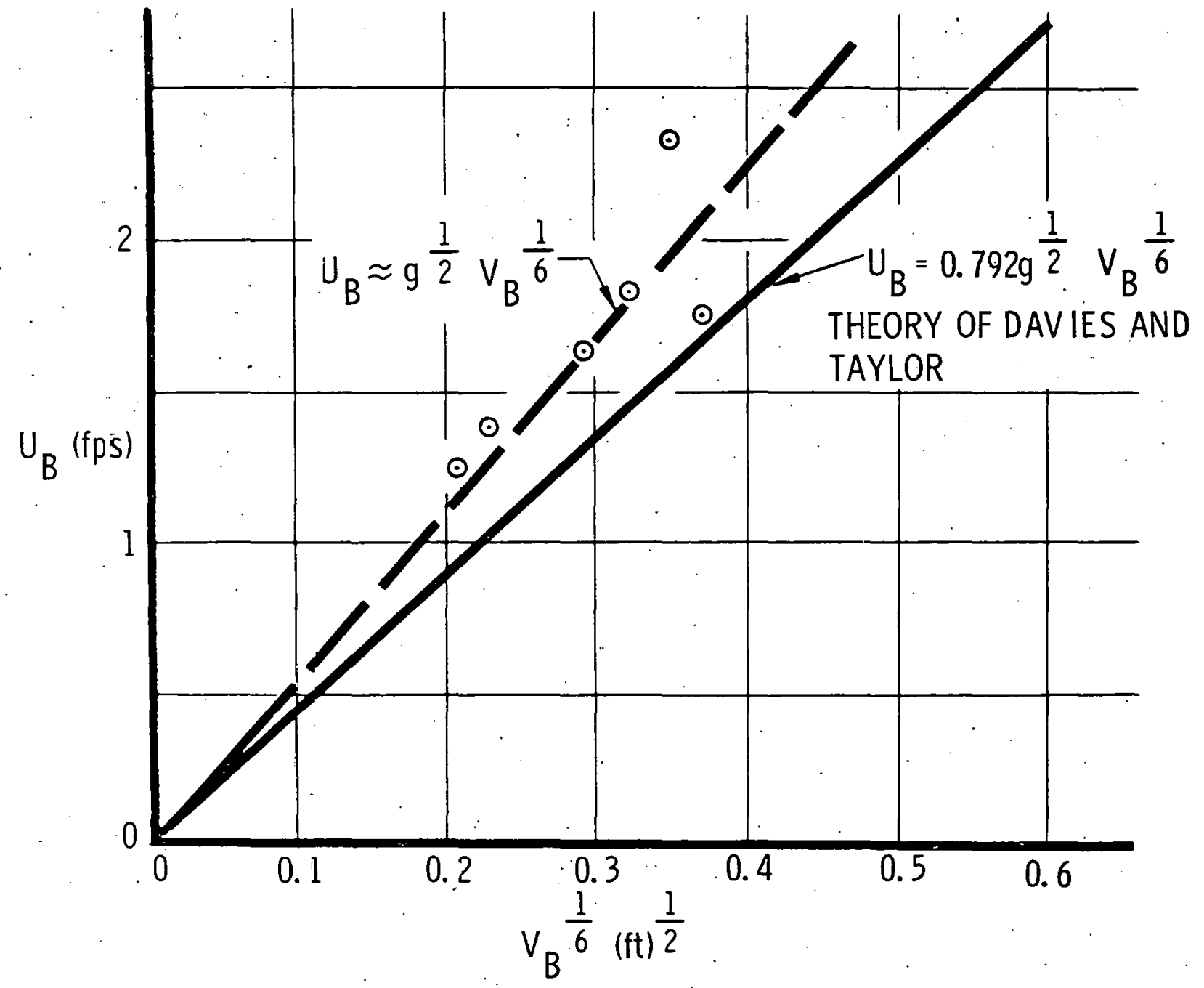

Slide 12 
suggested by Davies and Taylor, $\mathrm{U}_{\mathrm{B}}=0.792 \mathrm{~g}^{1 / 2} \mathrm{~V}_{\mathrm{B}}^{1 / 6}$ (g is the gravitational constant).

\section{CONCLUSIONS}

The flash x-ray machine has been used to study the internal structure of fluidized beds and to make basic measurements on their properties. The most important results obtained indicate that the fluidized bed consists of distinct dilute and dense phases, with the latter maintaining constant properties - particularly density. The flow through the dilute phase is in the form of gas bubbles, whose sizes and frequencies increase with flowrate and whose rise velocities follow closely the theory of Davies and Taylor. ${ }^{6}$ The constancy of dense phase bed density with flowrate provides good evidence on the validity of the Two-Phase Theory of Fluidization. Observations of bubble sizes, shapes, and velocities strongly suggest a liquidlike nature for the fluidized bed as has been suggested by some investigators.

The flash $x$-ray unit has proven an invaluable tool for studying flow, and I believe that if we are seriously considering designing reactors of this type, much more work of this nature is necessary. I hope the results presented here will be of value for reactor design and also chemical applications.

Question: Were you able to distinguish individual particles in the x-ray photographs, or is the bed so thick that you just get the average density?

Answer: No, we were not able to distinguish individual particles, so we read an average density. We could distinguish bubbles somewhere in the order of $1 / 8$ inch diameter.

Question: In the first photograph in Slide 4 when $G$ is equal to $G_{m f}$, is there a difference in density in the bed? There seems to be a gradation.

Answer: I'm sorry that some photographs appear lighter or seem to have a gradation, but in reproducing the slides they were doctored somewhat, and it is impossible to make comparisons just by looking at them. Actually, it is even somewhat impossible to compare the actual $\mathrm{x}$-ray photographs just by sight alone since there are some differences between them due to several sources of error, such as variations in the voltage at which the machine fired. This is the reason that we calibrated cach photograph individually. You will remember that we placed a wedge on the side of the column which was used for this purpose. In this manner, each photograph could be calibrated to a known reference.

Remark from Audience: There is a point which some people may not have gotten; that is, the bubbles move much more slowly than the gas, so that 
the se are not slugs coming up, but these bubbles have a lot of flow through them. Much of the gas, even though it passes through the bubble, also passes through the dense phase, and this is the point many people may not have realized.

Remark of Author: If all the gas passed through the dense phase, the bed would expand somewhat the same as that observed in liquid fluidization.

Question: The thing you just mentioned raises a point that has always interested me. Years ago, I worked on the partial theory of flow of liquid fluidization, and when gas fluidization came along some years later, about 1940 's, they adopted the same equations. Now, the equations I was involved with go into flow through a capillary in which there must be a purely uniform suspension of particles, otherwise it was chaos. But the chemical engineers adopted these equations, and I fail to see why there is any resemblance, one to the other, in the theoretical sense. Yet, those equations are all the same apparently; so it seems to me the gas going through the dense phase is not changing as you force more gas through. Why does the expansion of the bed resemble the expansion of a liquid fluidized bed?

Answer: The bed expansion in general does not resemble that of liquid fluidized systems. The curve I showed in Slide 6 in which all the gas passes through the dense phase was shown simply for comparison purposesto compare the expansion we actually obtained to what would be expected if all the gas passed through the dense phase. There is some expansion in a gas fluidized bed, however; but this is mostly due to the volume of the bubbles which are present within the bed at any one time. As you force more gas through the bed, the size of these bubbles also increases and so does the expansion.

Question: Do the newer theories on fluidization involve the assumption that the gas in the bubble is rising at the bubblc vclooity?

Answer: No, the bubble as an entity is rising at this velocity, but the gas in the bubble which may be circulating may have a different velocity.

Question: Could you define bypassing for me; specifically, what is it?

Answer: Bypassing is the amount of gas that flows in the form of bubbles. Now it has nothing to do with the circulation through the bubble we talked about. It is simply the amount of gas flow we would observe, if we measured the flow of bubbles, withoul regards to circulation.

Question: When you say $90 \%$ bypassing, does this mean that $90 \%$ of the gas flow appears in the form of bubbles? 
Answer: This means that $90 \%$ of the flow above minimum fluidization appears as bubbles. I am not considering the flow required to fluidize the material.

Question: In a liquid fluidized system with solid particles, generally no bubbling occurs. As you increase the flow rate, you tend to spread the particles more and more so they become less dense. The point is that apparently in the gas fluidized bed this does not matter. The density of the fluidized particles stays the same. The extra fluid appears as bubbles. What you are saying is that for some reason or other the dense phase is a very stable configuration, and it tends to keep constant properties?

Answer: Yes, precisely.

Question: What do you mean by bubble frequency?

Answer: It's hard to define frequency, because there are some bubble combinations, but the frequency talked about here is that which you would observe if you counted the number of bubbles bursting at the top surface of the bed.

Question: Were you able to follow a single bubble all the way through the bed?

Answer: We were able to identify it at two places since we only had two $x$-ray units. In principle, we could follow it all the way through if you had 10 or $12 \mathrm{x}$-ray units which you fired in sequence.

Question: Do you think that the aspect ratio of the bed would influence the data that you obtained?

Answer: Yes, I believe it would, since we know definitely that we had wall effects in many cases, especially when the bubbles were large. Also, if we had very shallow beds, the influence of the distribution might predominate. As I showed before, near the distribution there are some anomalies. You could, for instance, have different heat transfer and flow characteristics.

Question: In analyzing the heat transfer data which you briefly mentioned, did you use a two-phase model?

Answer: No, because we used a transient scheme which measured an overall coefficient. In any case, whatever parameter we varied we measured a coefficient which we could not distinguish from inlinity.

Question: If I interpret the bubble comments right, if you follow a bubble up through the bed, it is not always the same gas which is in the bubble?

Answer: Yes. In other words, there is circulation taking place transferring gases from the dense phase through the bubble and out again. 


\section{FLUIDIZED BEDS}

A. C. Juveland, H. P. Deinken, and J. E. Dougherty

Los Alamos Scientific Laboratory

Los Alamos, New Mexico

The early work at LASL on fluidized beds was done by G. Grover, E. Salmi, and J. Todd, all of $\mathrm{N}-5$. Many qualitative experiments were done at room temperature, using stationary and also rotating systems. They also found the copper and chromium particles could be heated by induction to essentially the melting point before agglomeration of the particles took place.

The work done at $\mathrm{W}-3$ can be divided into three parts: heat transfer, evaporation losses at high temperature, and mechanical properties. The heat transfer measurements were made by a steady-state method. The particles were heated by induction, and they were fluidized by helium or argon. The particle temperatures were measured by an optical pyrometer, and the exit gas temperatures were measured by a high-speed thermocouple probe. From the measured gas flows, heat transfer coefficients could then be found assuming that the particle temperature was essentially constant throughout the bed (verified by experiment) and gas was in piston-type flow. According to these assumptions, the gas temperature should rise exponentially from the bed inlet to the exit, and should be constant across the bed at any given height. The heat transfer coefficients obtained in this manner were much less than one would expect from boundary layer theory. The reason for these low coefficients is that there: are probably preferential flow paths around aggregates of particles. The gas within the aggregates flows more slowly, and its temperature rises within a small fraction of the bed depth to the particle temperature after which no more heat is transferred. The gas flowing around the aggregate sees a much larger average temperature driving force, but the surface which transfers the heat is much smaller. As the Reynolds number becomes smaller, the boundary layer also increases, and the aggregates become larger and more surface area is by-passed. Finally the gas flows mainly in the regions of nonoverlap of the aggregates, and the flow can be described as channeling. Indications are that if the beds 
can be agitated in such a way as to break up aggregates, the amount of heat transferred can be increased.* However, indications are that no special effort will be required, since even though the heat transfer coefficient is low, the total particle surface area is extremely large even in a shallow bed. The results of our measurements agree with other experimenters who used high-speed thermocouples to measure gas temperatures.

The material losses were measured in a resistance heated high temperature furnace. The heating element, which also served as the fluidized bed container, consisted of a graphite tube with a centrally located perforated graphite disc. Both the tube and the disc were coated with $\mathrm{NbC}$ by $\mathrm{CMB}-3$. Solid solutions of $\mathrm{ZrC}-\mathrm{UC}$ were usually used for the particle material.

The losses were definitely not seriously limited by diffusion of gaseous material in the boundary layer of gas around the particles. The large variation of the losses with temperature precludes this possibility. In fact, calculations show that if the bed is thick enough or efficient enough to transfer heat well, the vapor pressure of the gaseous products at the surface of the particles will be in equilibrium with their partial pressures in the bulk of the exit gas stream.

The losses of $\mathrm{Zr}$ could be explained as being mainly due to the reaction

$$
2 \mathrm{ZrC}+\mathrm{H}_{2} \uparrow \rightleftarrows \mathrm{C}_{2} \mathrm{H}_{2} \uparrow+2 \mathrm{Zr} \uparrow
$$

when $\mathrm{H}_{\mathrm{B}}$ was the fluidizing gas or the reaction

$$
\mathrm{ZrC} \rightleftarrows \mathrm{7r} \uparrow+\mathrm{C} \uparrow
$$

if He were the fluidizing gas. These reactions were assumed to have come to equilibrium at the bed exit, and the partial pressures of the reactants in the bulk of the gas stream could be calculated from the free energy change in these reactions. The losses calculated from this partial pressure and the fluidizing gas flow rate agreed well with the measured losses in the beds.

The losses of uranium scemed to be controlled by diffusion in the particles. The loss rate of uranium should be much larger than for zirconium on the basis of their vapor pressures. Hence the surface becomes depleted in uranium and the loss is regulated partly by diffusion and partly by the rate which zirconium evaporates and exposes uranium at the surface.

*Instabilities such as bubbling and slugging are known to increase gas-solid contact in chemical reactors. 
There were no indications of chemical reaction rate control in these experiments. If the loss rates were higher, this mechanism might become important.

During these runs, some of the samples agglomerated. This occurred at very near the melting point of the particular solid solutions used. The highest temperature achieved without agglomeration was obtained using $\mathrm{ZrC}_{0.92}$ particles. These particles remained well fluidized for 10 minutes at $3050^{\circ} \mathrm{C}$ using $\mathrm{H}_{2}$ as the fluidizing gas. The temperature of $3050^{\circ} \mathrm{C}$ agrees well with the melting point of $\mathrm{ZrC}_{0.92}$.

Both rotating, multi-gravity systems and normal gravity systems were used to study the mechanical properties of fluidized beds. In multi-gravity systems minimum fluidization flow rates were measured at up to $150 \mathrm{~g}^{\prime} \mathrm{s}$ and Reynolds numbers of up to 12,000. Thesc data agree with extrapolations of the data of other experimenters. The rotating systems tended to become more unstable as the number of $g^{\prime}$ 's were increased. Several varieties of normal gravity systems were tried in order to attempt to achieve a stable bed. It was found that if directed inlets were used, the beds became more stable. The increased particle velocity in this case may have made the transit time of the particles across the bed depth small compared to the period of any instabilities, thus reducing their growth.

Other schemes of increasing the particle motion might be variation of the flow across the inlet, additional gas inlets within the bed itself, or vibrating the bed. Unfortunately none of these schemes were exploited in the rotating system.

The results of our work are described in more detail in the following reports:

A. C. Juveland, H. P. Deinken, and J. E. Dougherty, LAMS-2994 (Aug. 1963), "Loss of Z1rconlum and Uranium from Fluidized Beds of $\mathrm{ZrC}$ and UC-ZrC Particles at High Temperatures."

A. C. Juveland, H. P. Deinken, and J. E. Dougherty, LA-3061 (Jan. 1964), "Particle-to-Gas Heat Transfer in Fluidized Beds."

H. P. Deinken, A. C. Juveland, and J. E. Dougherty, LAMS-3012 (Sept. 1963), "Experimental Studies Relating to Rotating Fluidized Bed Reactors." 


\title{
FLUIDIZED BEDS
}

\author{
Ralph Cooper \\ Los Alamos Scientific Laboratory \\ Los Alamos, New. Mexico
}

I have examined a number of aspects of the fluidized bed concept, applying the recent literature and doing some calculations on criticality, heat transfer, and fuel loss. Certain crucial areas are apparent, and particular data are necessary for a better evaluation.

The fluidized bed should be able to give significantly higher specific impulse than solid core reactors. In moderate or large size reactors one can use a carbide fuel with a few percent of uranium in $\mathrm{ZrC}$ or $\mathrm{NbC}$. The good heat transfer and small fuel size should allow close approach to the melting points, and hydrogen dissociation heat capacity should reduce the hot spot factors. This might give $I_{s p}$ 's of 1000 to 1100 seconds for the above fuels and perhaps up to 1200 with $\mathrm{HfC}$ in a fast reactor. The unknown area here is the metal carbide-uranium carbide melting points and vapor pressures. The metal carbides have melting point maxima at compositions of $\mathrm{MC}_{0.9}$, which are several hundred degrees above the stoichiometric values. The maxima occur at 3400 to $3500^{\circ} \mathrm{C}$ for $\mathrm{ZrC}_{0.9}$ and $\mathrm{NbC}_{0.9}$. However at these compositions the carbon activity is probably low, and thus the uranium loss might be relatively high compared to the stoichiometric compositions. Very little data have been obtained on depression of the melting points by addition of UC (which itself melts at $2500^{\circ} \mathrm{C}$ ), but a linear mole fraction effect is a fair guess. Exit gas temperatures of 3000 to $3200^{\circ} \mathrm{C}$ might be possible.

The vaporization loss is more difficult to estimate because the data are more sparse and the loss is sensitive to the pressure, composition, mechanism, and degree of equilibrium. Assuming the vacuum vaporization rate of UC diluted to a few percent leads to very small loss rates, but the experiments of Juveland et al. ${ }^{1}$ indicate much higher losses in flowing

1. A. C. Juveland, H. P. Deinken, and J. E. Dougherty, Loss of Zirconium and Uranium from Fluidized Beds of $\mathrm{ZrC}$ and $\mathrm{UC}-\mathrm{ZrC}$ Particles at High Temperatures, LAMS-2994 (Aug. 1963). 
hydrogen. Should the surfaces of the particles become depleted of uranium, then the uranium diffusion through the solid or the diluent metal carbide vaporization could become the loss limiting mechanism.

The criticality of this type of reactor is fairly well understood. Critical masses of $\mathrm{UC}-\mathrm{Z} \mathrm{rC}$ and $\mathrm{UC}-\mathrm{NbC}$ fuels are quite $10 \mathrm{w}(10-30 \mathrm{~kg})$ and low percentages of UC can be used in thermal spectra. The reactors are somewhat sensitive to poisoning effects of impurities and structure, and the critical mass of the $\mathrm{NbC}$ system rises rapidly as the spectrum becomes harder. The $\mathrm{HfC}$ system would have to be a fast reactor, with 20 to $30 \%$ $\mathrm{UC}$ as a minimum. Separated $\mathrm{HC}^{180}$ behaves like $\mathrm{Nb}$ in an intermediate spectrum and is much worse in a thermal spectrum.

The radial power profiles are either flat or decreasing radially inward which is as desired for achieving high exit gas temperature. The axial profile is flat in small reactors, with thermal end peaks that can be removed by appropriately placed absorbers. In larger reacturs some flux shaping might be desired. This might be accomplished by shaping the reflector.

The greatest uncertainty lies in the area of the stability of the flow. Recent work by the British ${ }^{2}$ has shown that all fluidized beds are unstable in the sense that fluctuations in the bed density will propagate upward with increasing amplitude. In deep beds, bubbles (particle-poor spaces) will be formed; but, generally, the rest of the bed will be maintained in a fluidized state. Thus even though the bed may be unstable it can be possible to have adequate heat transfer throughout the bed to prevent local overheating and meltdown of the fuel. Large area shallow beds might possess other modes of instability, such as channeling, which could make the concept unfeasible; and it is necessary to examine the flow under appropriate conditions.

Carbide particles have a density of the order of $10 \mathrm{gm} / \mathrm{cm}^{3}$, and material of this density would be carried out by relatively modest flow rates. It is necessary to increase the effective particle mass by centrifugation to reach high flow rates. The limit is not imposed by structural considerations as much as by the pressure drop through the bed. A bed of a few centimeters thickness has a mass of $\sim 10 \mathrm{gm} / \mathrm{cm}^{2}$ which corresponds to $0.13 \mathrm{psi}$ at normal gravity. At a rotation giving $1000 \mathrm{gm}$ (about $1000 \mathrm{rpm}$ for a 4foot-diameter cavity) the pressure drop is 130 psi, which is approaching the system pressure for typical reactor rockets. Furthermore the criterion for instabilities to appear gets worse as the acceleration and pressure drop are increased. The heat transfer has not been measured under these conditions;

2. R. Jackson, The Mechanics of Fluidized Beds, Trans. Inst. Chem. Engrs., 41, 13 (1963) 
but extrapolating from data at lower Reynold's numbers, and using results for fixed beds at high Reynold's numbers, indicates that the heat transfer will be quite adequate under normal flow conditions. Nonnuclear experiments could provide data in this regime, possibly by using the mass transfer-heat transfer analogy. Some experiments are needed with internal heating, since the gas in the rocket will increase in temperature, markedly changing its properties in passing through the bed. Normally fluidized beds have uniform particle temperatures throughout, but this may not be the case at very high flow rates. Also it appears that the state of fluidization will change through the bed, which is likely to be unfluidized at the inlet.

We have examined two methods of startup which appear feasible, and once in operation the power and thrust should be variable over a factor of ten. If release of the fuel is permissible, it can be easily accomplished and would be of great operational value. It would eliminate aftercoolant requirements and would leave the vehicle with an only slightly radioactive engine, permitting rendezvous, reuse, and perhaps even some engine repair. 
THE LIQUID CORE NUCLEAR ROCKET

\author{
W. Louis Barrett, Jr. \\ The Boeing Company \\ Seattle, Washington
}

The work reported at the symposium was published in two papers ${ }^{1,2}$ which are abstracted here by $R$. Cooper

\begin{abstract}
The liquid core nuclear rocket engine is examined with regard to the specific impulse available, heat transfer and fluid flow, containment and nucleonics.

The $I_{S p}$ was found to be limited by vaporization of the high atomic weight fuel to values below 1500 seconds. For undiluted $\mathrm{UC}_{2}$ as fuel the maximum $\mathrm{I}_{\mathrm{sp}}$ is at $7000^{\circ} \mathrm{R}$, at which point the fuel loss rate is quite large. It can be reduced a factor of 10 by going down to $6000^{\circ} \mathrm{R}$.

Under typical conditions the bubbles travel through the liquid at terminal velocity. The heat transfer coefficient necessary to reach approximate equilibrium between the gas and liquid has been computed and was found to be less than that observed in experimental studies. The liquid is contained in a rotating cylinder. At reasonable rotation rates the thrust is limited to relatively low values by the rate of bubble rise through the liquid. Typical engine thrust/weight ratios are in the range of $10^{-3}$ to $10^{-1} \mathrm{~g}$.
\end{abstract}

1. W. L. Barrett, Jr., "Specific Impulse of a Liquid-Core Nuclear Rocket," ALAA Journal, 1, 2649 (1963).

2. W. L. Barrett, Jr., "Liquid Core Nuclear Rocket," AIAA Paper 64-541, presented at Cleveland, May 1964. 


\section{THE LIQUID-CORE NUCLEAR ROCKET* \\ Jerry Grey \\ Princeton University \\ Princeton, New Jersey}

\section{INTRODUCTION}

The concept of a liquid-core nuclear rocket was first introduced by McCarthy ${ }^{1}$ in 1954. The basic idea of McCarthy's configuration was that of a single large hollow cylinder in which the molten fissionable material is contained by the centrifugal force resulting from rotation of the cylinder. This is the fundamental concept presented by Barrett ${ }^{2}$ in a previous paper in this volume, who brought out the fundamental limitations of the liquid-core concept when restricted to the McCarthy geometry. However, there are a number of improvements which can be made in the liquid-core rocket performance by consideration of these limitations and by analytical studies directed at their minimization or elimination.

The fundamental limitation of the liquid-core nuclear rockct, as indicated by Barrett, is that the specific impulse is limited by vapor loss of the liquid fissionable material. Barrett stated that the limiting specific impulse was 1300 seconds, whereas our estimate is approximately 1550 seconds, the increase resulting from the dilution of the fissionable material by a relatively low-vapor-pressure moderator. Also; the low thrust-to-weight ratio and the extremely high fissionable material investment indicated by Barrett can be

*The initial presentation of this paper included a computational error which resulted in a maximum value for spccific impulse of 1250 seconds. This error has been corrected in the present paper as published in this volume.

1. McCarthy, J., "Nuclear Reactors for Rockets," Jet Propulsion 24, 1954, p. 36 .

2. Barrett, W. L., "Liquid-Core Nuclear Rocket," AIAA Paper No. 64-541, May 4-6, 1964 (also appearing in this volume). 
improved considerably by introducing several major system configuration changes.

The fundamental variation we have examined is that of utilizing a multiple arrangement of fuel elements, as shown in Figure 1, instead of the single large rotating element suggested by McCarthy, ${ }^{1}$ Barrett, ${ }^{2}$ and others. ${ }^{3}$ Although this concept of the multiple-element reactor was introduced some time ago, ${ }^{3}$ an additional variation, discussed below, was not incorporated into Reference 3; and consequently although the performance indicated by Reference 3 was somewhat better than that of Barrett's, the fissionable material investment was still quite large, as was the overall dimension of the thrust chamber. This second major performance improvement was obtained by considering not only the use of a low-vapor pressure moderator mixed with the fuel (as indicated in Reference 3), but also the introduction of a low-temperature, highly efficient hydrogenous moderator in the cooler portions of the reactor. This final configuration change, together with the use of multiple elements, was effective in reducing the critical mass by several orders of magnitude, reducing the dimensions of the system by almost an order of magnitude, and increasing the thrust-weight-ratio by several orders of magnitude. Both of these system improvements will be discussed in some detail in the present paper.

\section{DESCRIPTION OF THE MULTI-ELEMENT LIQUID-CORE NUCLEAR ROCKET}

The fundamental configuration of the multi-element reactor system is shown in Figure 1. The operation of each individual fuel element is exactly the same as that of the single large fuel element configuration; that is, the rotating element is surrounded by a manifold into which the cold propellant is introduced. The propellant then flows radially inward through small holes in the outer portion of the rotating element. As the propellant flows inward, its temperature is increased by the absorption of nuclear fission energy from the fueled element until eventually the propellant is hot enough to exceed the melting point of the solid fuel element material. At this point a liquid interface is formed, and the propellant then continues to flow inward, but now in the form of bubbles flowing through a liquid-phase fissionable material. The latter is, of course, retained in the cylindrical configuration by the centrifugal force due to rotation of the element. When the hot propellant bubbles issue from the surface of the liquid, they then flow axially down the

3. Nelson, S. T., \& Grey, J., "Conceptual Design Study of a Liquid-Core Nuclear Rocket," Princeton University, Aeronautical Engineering Report No. 665, September 1963. 


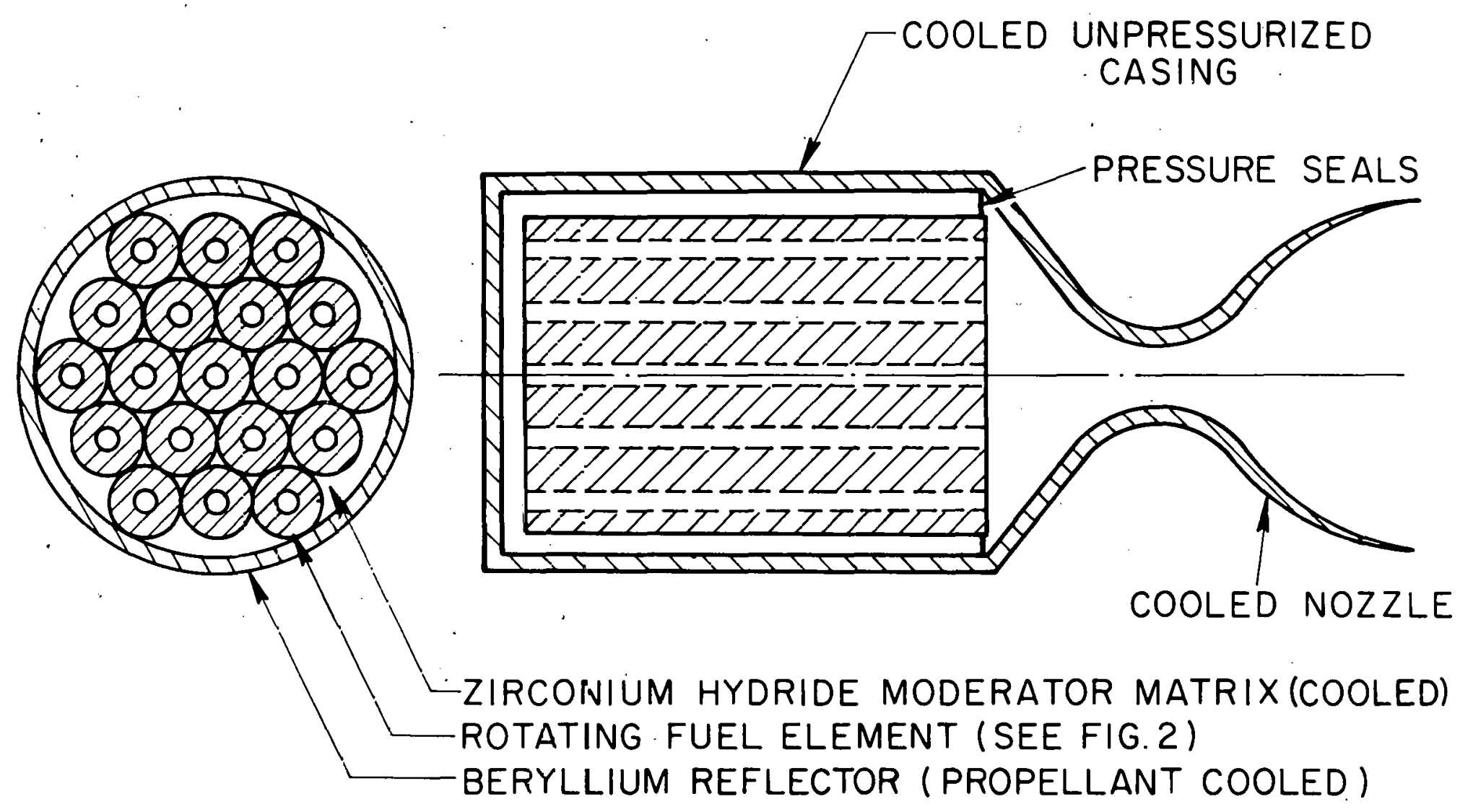

Figure 1. The Multi-Element Liquid-Core Nuclear Rocket 
cavity in the center of the element until they reach the nozzle chamber, at which point they are collected in the nozzle plenum and exhausted in the usual way through a supersonic de Laval nozzle.

The familiar-looking design of Figurc 1, which appears in concept to be quite similar to that of the conventional solid-core nuclear rocket, provides a number of fundamental improvements over the single large element discussed in previous work. First, the nucleonic efficiency of such a reactor, which approaches the "homogeneous core," is far greater than that of the cavity-type reactor required for the single rotating element design. Furthermore, the flow area, which in the case of the liquid core reactor is that of the total inner surface of the rotating cylinders, is considerably increased over that of the single element system.

Improvements resulting from the use of a moderator are also significant. First, by using a very low vapor pressure, high melting-point moderator as part of the fuel element in which melting takes place, we inhibit the vapor loss of the fissionable fuel mixture. In one sample design, the high-temperature material selected was zirconium carbide, with uranium carbide or uranium dicarbide as the fuel. By using large dilution ratios ( $\mathrm{ZrC} / \mathrm{UC})$, therefore, the low vapor pressure of zirconium carbide will reduce the overall vapor loss from this system by approximately a factor of 20 , as compared to that of pure uranium carbide. Second, in order to obtain significant improvements in critical mass investment, a much more efficient low-temperature moderator can be introduced into the interstitial spaces between the rotating fuel elements, since this region is completely in contact with the cold propellant at all times. In one typical design the material selected for this purpose was zirconium hydride. The resulting improvement in critical behavior, as will be indicated later, has produced a net reduction in critical mass by several orders of magnitude and almost an order of magnitude in reactor dimensions. The final result of both the utilization of the multi-element design and thermalization of the core permits the use of individual pressure shells on each fuel element, providing far higher pressure capability than the: single large-cylinder unmoderated reactor would be capable of. As a result the overall thrust-to-weight ratio, which, as will be shown later, is specifically dependent on the chamber pressure, can be extended to far higher values. Thus the performance prediction for the liquid-core reactor may be estimated, on the bases of analytical studies, as nearly double the performance of the solid-core reactor, by achieving the order of 1500 seconds specific impulse at a thrust-to-weight ratio for the engine which will be at least of the order of unity. The liquid-core therefore fits into the category of a major improvement on solid-core nuclear rocket performance rather than that of a competitor for the much higher specific impulse gaseous-core nuclear rocket systems. 
A second major area of improvement offered by the multi-element configuration is that of engineering feasibility of the concept. First, the entire structure of the multi-element reactor is cool, since the outer portion of each fuel element cylinder is exposed to incoming cold propellant. Thus the high-temperature region is limited to the central core of each element, which has essentially no structural requirement. Further, rotation of the small cylinders, necessary to retain the molten fuel mixture, is obtained quite simply by the use of individual turbines on each element or, if the radiation field permits, electric motors. Because of this low temperature nature of the entire structure and relatively small size of the minimum system, and the high pressure attainable with the individually pressurized rotating elements, the engineering problems confronting the liquid-core reactor appear to be no worse than those of the solid-core system, although they are largely different in quality. Typical designs of the fuel elements are discussed in a later paper. ${ }^{4}$

\section{RESULTS OF SYSTEM ANALYSIS}

A detailed discussion of a typical liquid-core nuclear rocket design, together with an outline of the analytical techniques used, is presented in Reference 4. For the purposes of the present brief paper only the results of the analysis 'will be indicated.

Figure 2 shows the critical mass of fissionable material (assumed in the present case to be uranium-235 in the carbide form) as a function of the reactor diameter. The two curves indicate (a) the case in which no zirconium hydride is used, and (b) the case in which zirconium hydride is used in the interstitial spaces between the fuel elements shown in Figure 1. This significant reduction in the critical mass represents one of the major improvements discussed previously. Note that the final values of critical mass are of the order of 4 or 5 kilograms, and occur at reactor diameters of the order of 3 feet. Thus, we are now discussing an engineering problem of at least reasonable magnitude rather than one which will require major "state-of-the-art" technological improvements.

Figure 3 shows the results of specific impulse optimization studies. This figure plots the specific impulse as a function of the maximum core temperature, which, of course, would occur at the inner surface of the liquid

4. Nelson, S. T., Grey, J., \& Williams, P. M., "Conceptual Study of a Liquid-Core Nuclear Rocket," Accepted for publication in the Journal of Spacecraft and Rockets, 1965. 


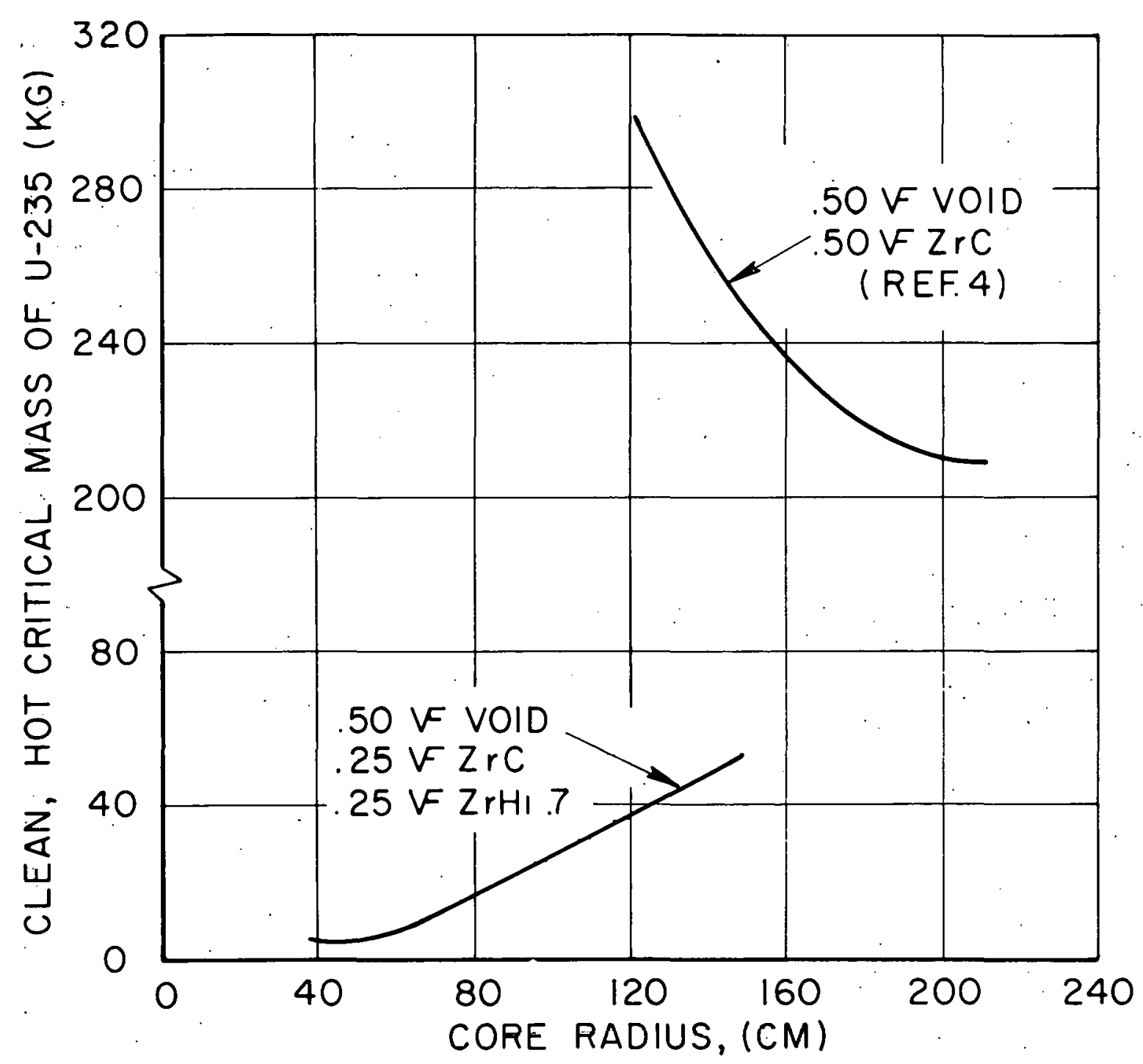

Figure 2. Comparison of Criticality Requirements for Moderated and Unmoderated Multi-Element Liquid-Core Reactors 


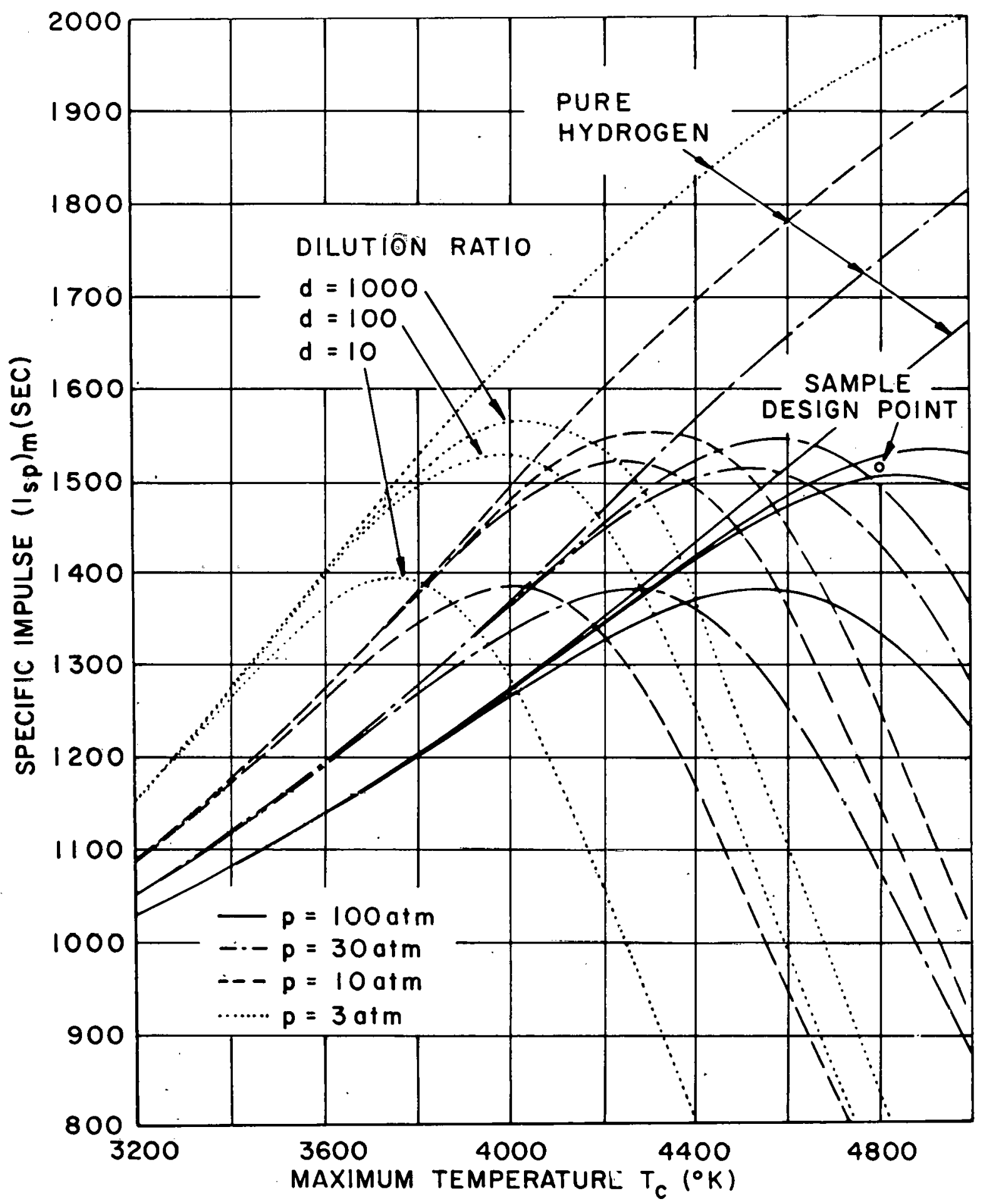

Figure 3. Specific Impulse Optimization of Liquid-Core Nuclear Rocket (Pressure Ratio $=3000)$ 
in each fuel element. There are several curves plotted for different pressures and for different dilution of uranium carbide by zirconium carbide in the hot portion of the reactor. Data for pure hydrogen are also shown, as obtained from the real-gas calculations of Reference 5. The peak value of specific impulse obtainable appears to be between 1500 and 1550 seconds. The peak, of course, as was indicated by Barrett, ${ }^{2}$ results from the increasing molecular weight of exhaust due to vapor loss of the fuel mixture, and therefore represents an essential limitation on the specific impulse obtainable. This behavior is strongly dependent on the material selected, as was shown by Barrett. ${ }^{2}$ In the present case, the selection of zirconium carbide and uranium carbide was based on a preliminary survey of the unclassified literature. It is, of course, conceivable that other combinations might be found which would provide lower overall vapor pressure and therefore higher specific impulse. This general subject, which includes not only material selection but also detailed vapor-pressure studies of molten materials of this type in various mixtures, represents a major area for research in the future of the liquid-core nuclear rocket concept.

The optimization curves of Figure 3 are shown in a slightly different form in Figure 4, which plots specific impulse as a function of pressure with temperature as a parameter. The significant feature of Figure 4 is that the optimum specific impulse remains relatively constant with increasing pressure. Thus, increases in thrust-to-weight ratio can be obtained simply by increasing the propellant pressure in the fuel element, without sustaining a comparable loss in specific impulse. As pointed out earlier, the mechanical structure necessary to contain this pressure is not only at low temperature but is also relatively small in dimension (in the detailed design of Reference 4, typical fuel element diameters of the order of 5 inches were postulated). It is therefore not unlikely that pressures in range of 100 to 1000 atmospheres can be considered for the liquid-core nuclear rocket, providing an engine thrust-to-weight ratio ranging from approximately 1 to 10 .

It was shown in Reference 3 that heat transfer is not a fundamental limitation on the performance of the reactor; that is, by the use of a rather simple calculation for each individual fuel element, it was shown that the incoming cold propellant reaches thermal equilibrium with the molten portion of the liquid fuel element by the time it has traversed one-quarter of the distance from the solid-liquid interface to the inner surface of the hot liquid. Thus, increases in flow rate achieved through possible increases in chamber pressure, as discussed earlier, should not introduce any heat transfer

5. King, C. R., "Compilation of Thermodynamic Properties of Theoretical Rocket Performance of Gaseous Hydrogen," NASA TN D-275, April 1960. 


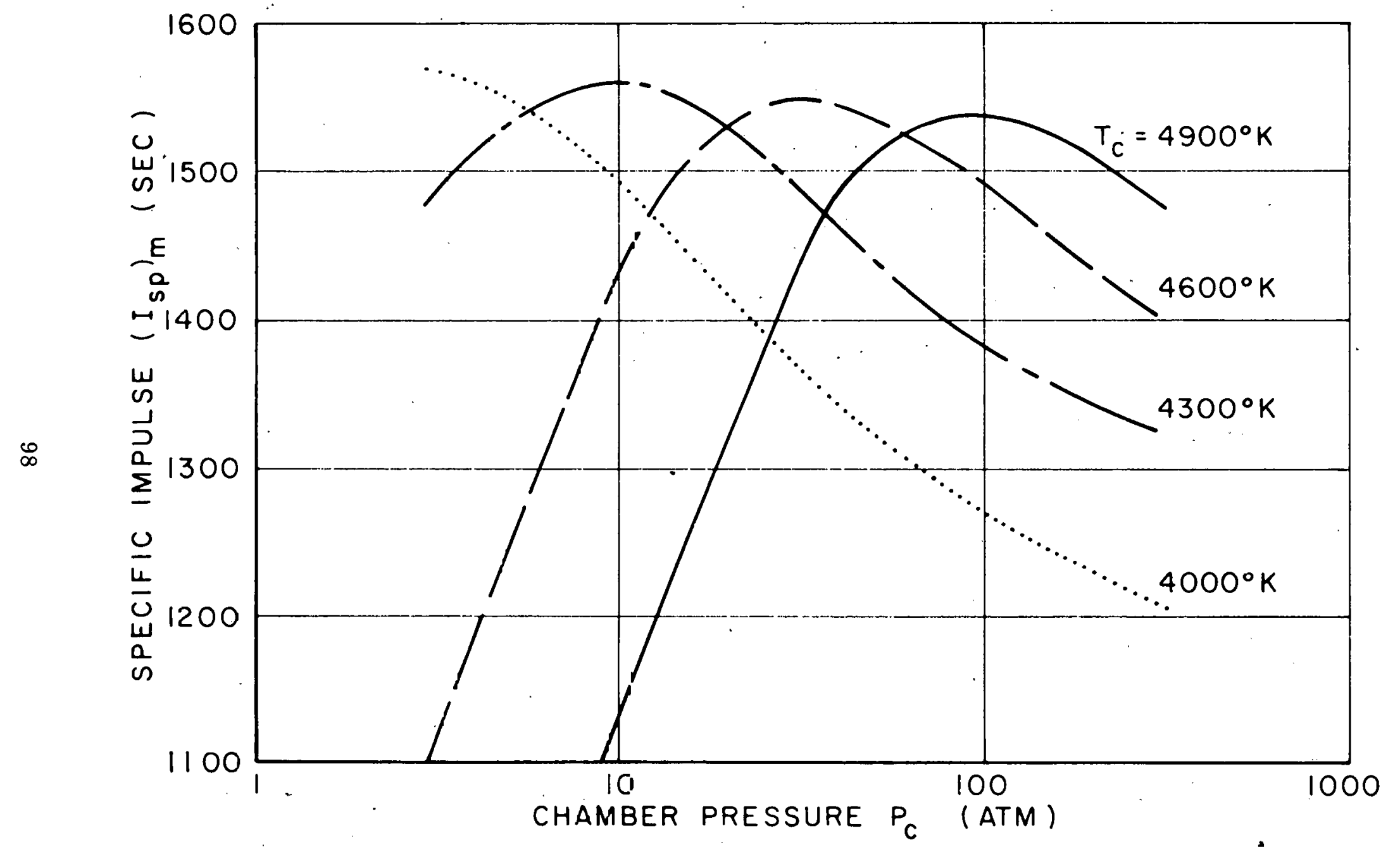

Figure 4. Dependence of Specific Impul:se on Chamber Pressure (Dilution Ratio $=1000$, Pressure Ratio $=3000$ ) 
limitation. The basic limitation on the liquid-core nuclear rocket remains the flow restriction due to limited liquid surface area for the bubbles to flow through. The entire field of bubble flow and heat transfer under accelerations of the order of 1000 earth gravities represents a second major area for feasibility study, which is now undergoing experimental research at Princeton and which will be discussed briefly later in this paper.

The other feasibility areas which will eventually require study are those of control, startup and shut-down, as well as time-dependent behavior resulting from the loss of fissionable material. Because of the small size and the relatively homogeneous nature of the core, however, it is not likely that any of the control problems should be any more serious than those of conventional solid-core systems. The two major areas which may introduce difficulty are (a) hot spots in the liquid, because vapor loss is extremely sensitive to small changes in temperature, and (b) shut-down and restart of the reactor, that is, the freezing-in of liquid passages for the propellant such that on restart these liquid passages will provide adequate flow area for the cold incoming propellant gas.

\section{DISCUSSION OF EXPERIMENTAL PROGRAM}

Experimental work accomplished to date has been performed in a onegravity environment in order to establish the experimental techniques which will be necessary for the projected high-gravity experiments. All of these studies at the present time are related to the fundamental problem of bubble flow and heat transfer in configurations resembling those of the liquid-core nuclear rocket fuel element.

The first consideration is the selection of a bubble flow regime optimum for liquid-core nuclear rocket requirements. Figure 5 shows vertical bubble velocity as a function of bubble dimension, which is equivalent to the bubble Reynolds number. This figure indicates the various regimes of bubble flow. The lower regime corresponds to very small bubbles rising essentially as solid spheres in a liquid (Stokes flow). This has been dealt with extensively in the previous literature, as have several extensions which include "deformable" bubbles, bubbles with interior gas circulation, and bubbles in which the exterior liquid interaction is affected by surface-active agents. The next range of bubble flows is that of the ellipsoidal bubbles, which are strongly deformed by the hydrodynamic forces resulting from their velocities in the liquid. Finally, as the bubble dimension increases, we reach a class of bubble flows called "spherical-caps," which are discussed in some detail in. Reference 3 . 


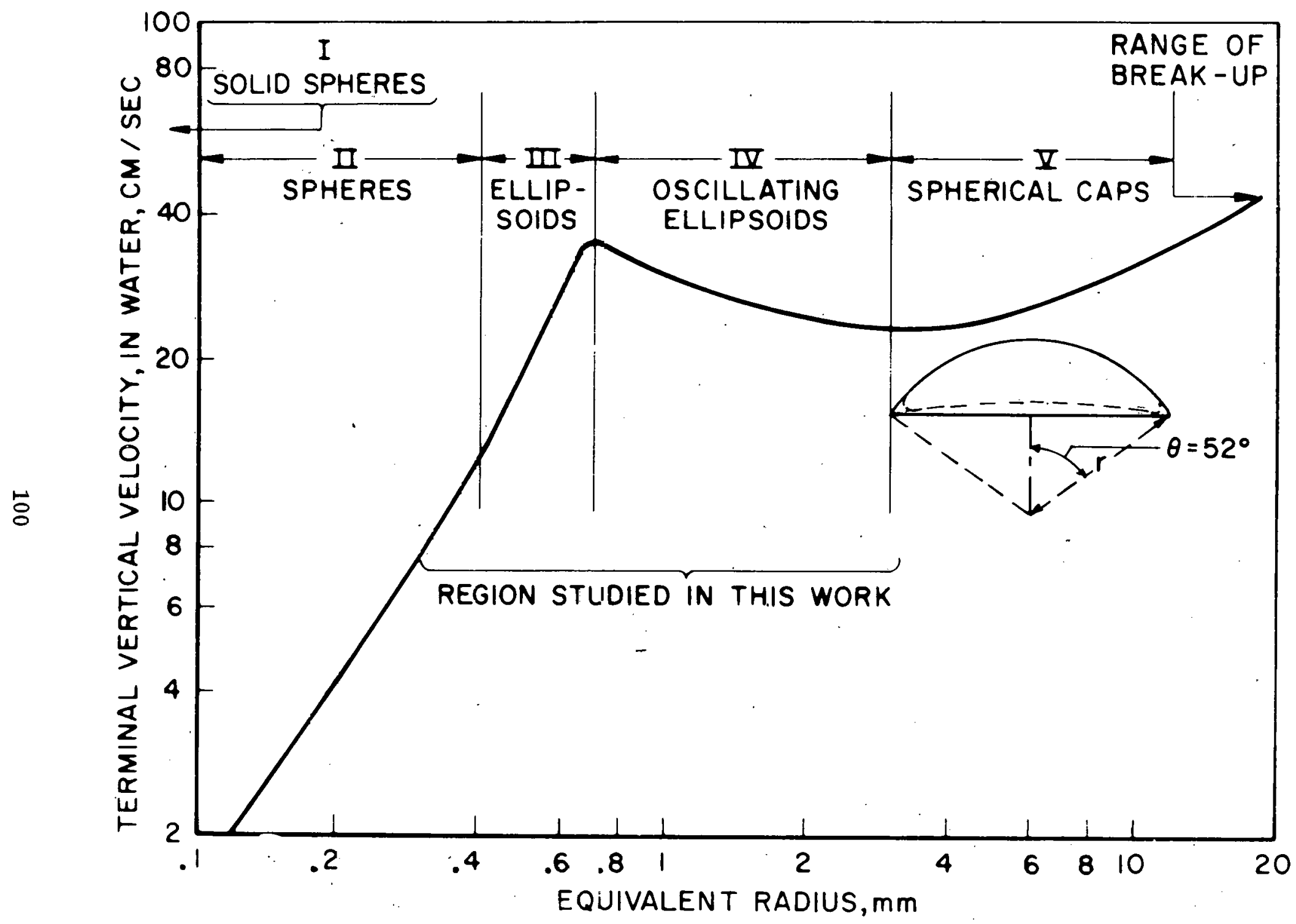

Figure 5. General Regimes of Bubble Shape and Velocity 
The experimental work to date has been in the unstable ellipsoidal bubble regime rather than with the spherical-caps, since the photographic technique was more suitable to this class of bubbles at the one-gravity level. At the higher gravities, however, it is planned to extend these studies through the round and ellipsoidal range into the spherical-cap regime. Experimental results of the one-gravity studies are illustrated in Figure 6, which shows rather good experimental correlation up to the point at which oscillating ellipsoidal bubbles were encountered. The broad scatter observed for e1lipsoidal bubbles in the unstable region was found to be due partly to contamination, which has been previously observed in the literature, and partly to the effect of a lateral as well as a vertical bubble velocity, as has been clearly defined for the first time in Reference 6.

The technique utilized in obtaining experimental data on these bubble flows is that of a double-exposure photograph in two dimensions. That is, as the bubbles rise approximately vertically in the gravity field, two microsecond-duration photographs are made by a high-intensity spark source flashing twice, with known intervals between flashes. This is done simultaneously on two cameras mounted at right angles to each other. Thus, knowing the intervals between the flashes, the dimension between the two successive images of a bubble on each photograph can be translated directly into the velocity components in both the vertical and the horizontal planes, and the actual motion of the bubble resolved. Photographs of this type (typical of those shown in Figures 6 and 7) were extremely useful not only in determining the velocities, but also the shapes, sizes, and various other effects in the bubbling flow.

These lateral velocity and bubble shape effects are, of course, not particularly significant at one gravity, but when amplified by a factor of the order of 1000 , as they would be in the ellipsoidal bubble regime at 1000 gravities, or hy the square root of 1000 , as theoretically indicated for the spherical-cap regime, any distortions or transverse velocity effects will become quite significant. An example of one effect is that of bubble oblateness, which is shown in Figure 8. The theory shows a sharp peak at the critical point, corresponding to the peak in the velocity curve of Figure 5. This effect was clearly observed experimentally but not nearly as sharply as predicted by the theory.

Figure 9 shows the ratio of horizontal to vertical velocities in the

6. Lieberherr, J. F., Williams, P. M., \& Grey, J., "Bubble Motion Studies for the Liquid-Core Nuclear Rocket," Princeton University Aeronautical Engineering Report No. 673, December 1963. 


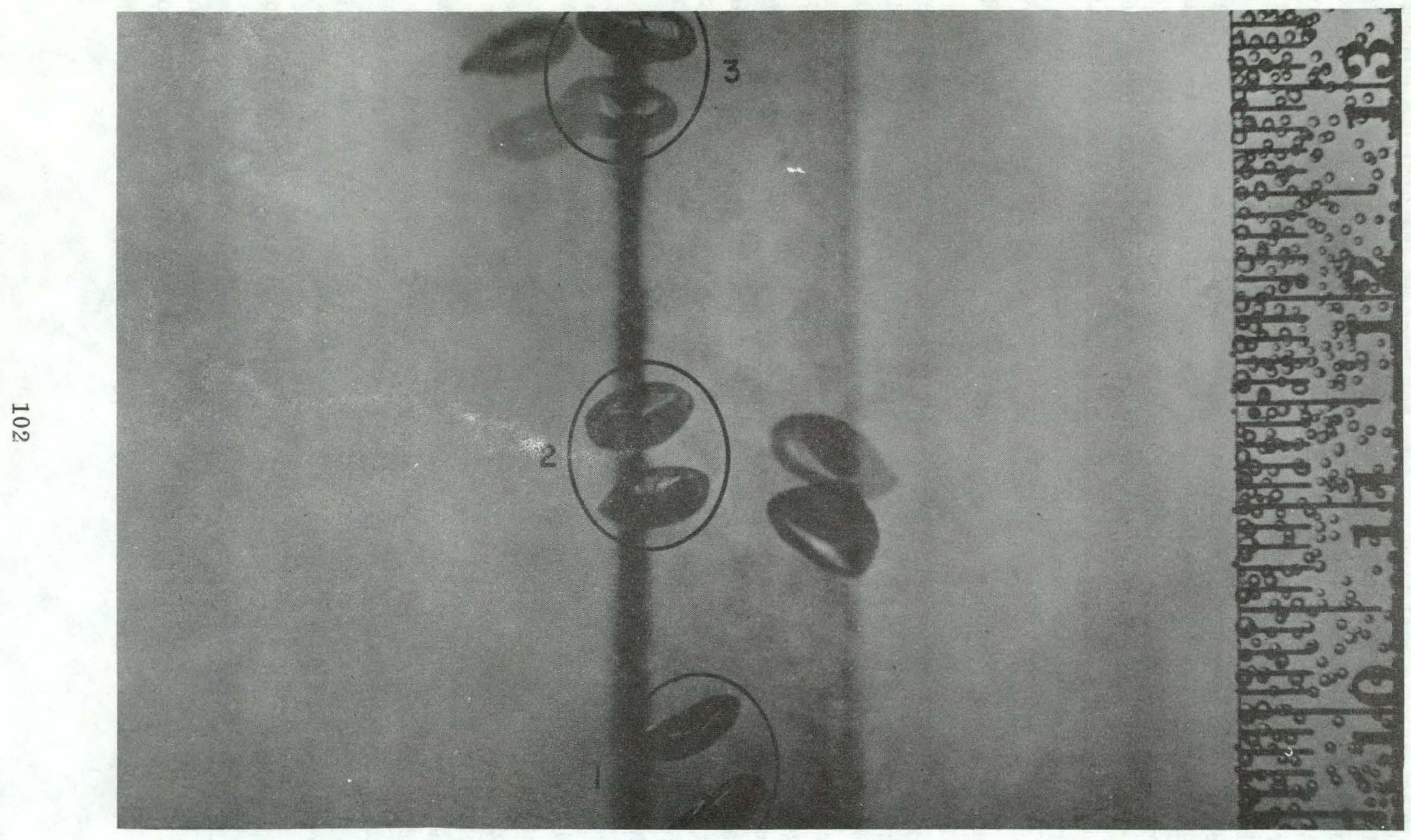

Figure 6. Large Oblate Bubbles, View A 


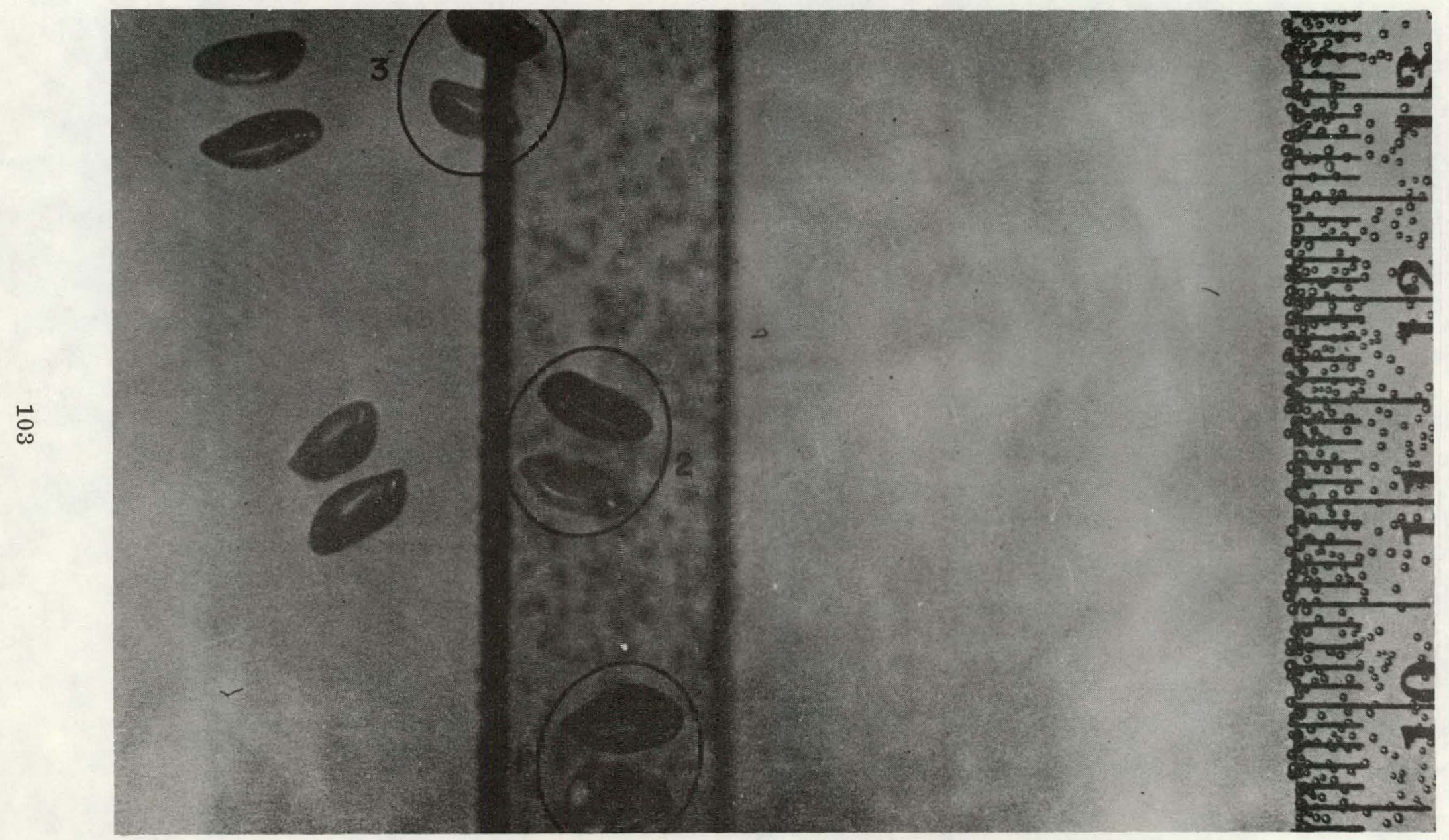

Figure 7. Large Oblate Bubbles, View B 


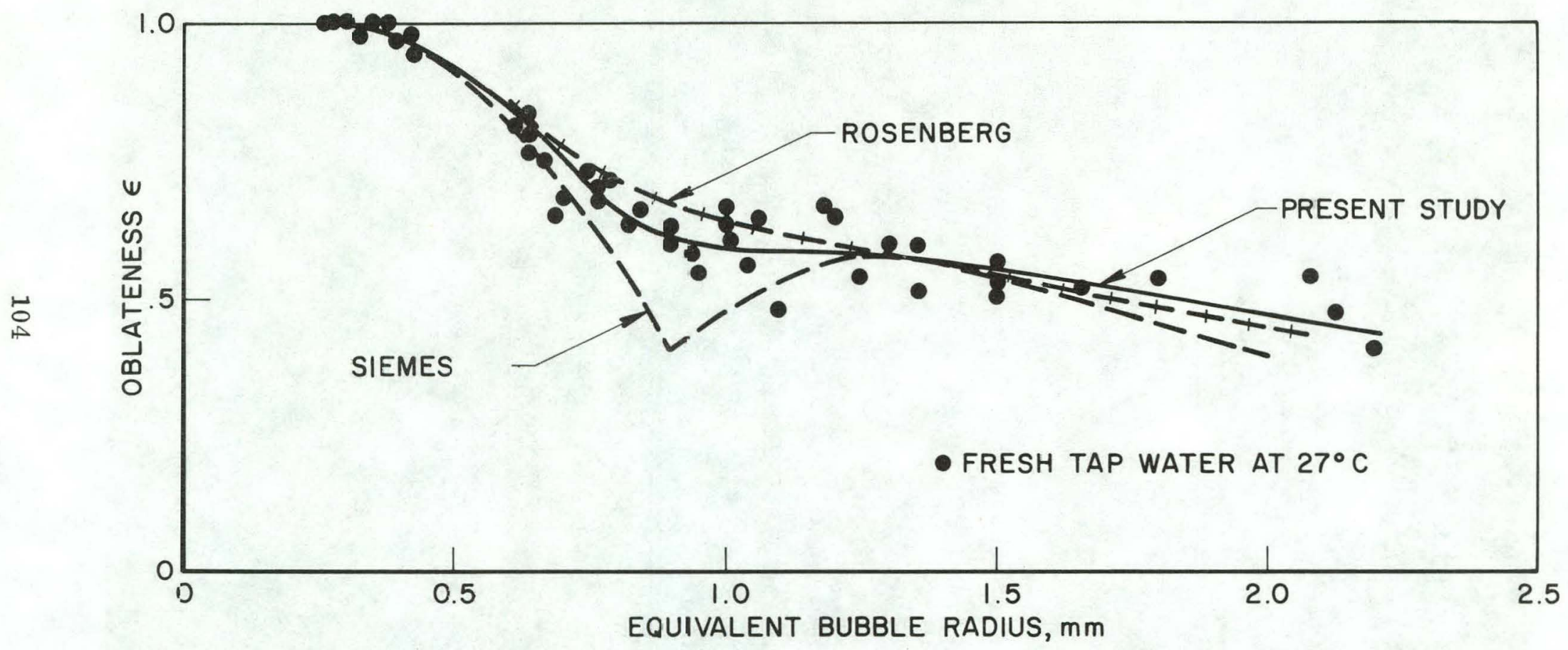

Figure 8. Bubble Oblateness versus Equivalent Radius 


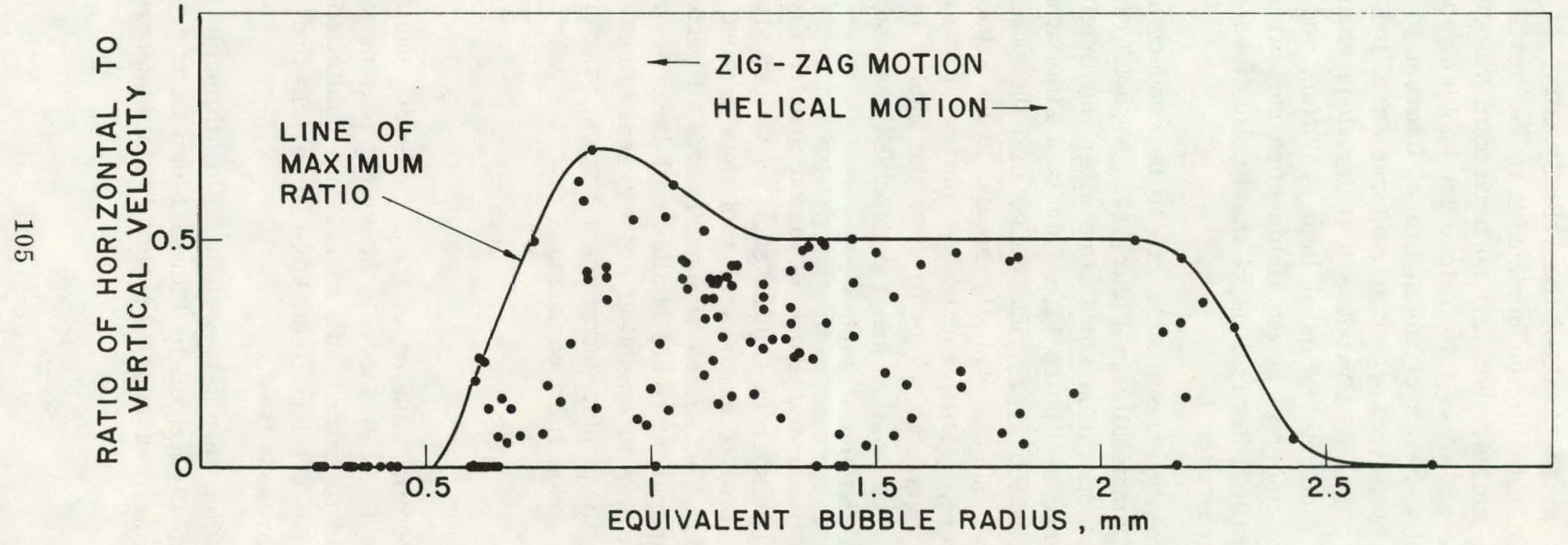

Figure 9. Ratio of Horizontal to Vertical Velocity vs Equivalent Bubble Radius 
unstable regime near the peak of the velocity curve of Figure 5, indicating the envelope of the ratio of the horizontal to the vertical velocities as a function of bubble radius. We find no horizontal velocities in the spherical regime, but as we progress into ellipsoidal bubbles, and extrapolating to the spherical-cap bubbles, both of which are of interest to the liquid-core system, we begin to observe horizontal components of the order of $50 \%$ of the vertical velocity. This will produce a tremendous change in effective heat transfer and flow characteristics at high gravities, and must, of course, be integrated into any analysis of the liquid-core nuclear rocket system. Explanations and detailed discussions of the implications of this lateral motion are included in Reference 6.

The high-gravity experiments are to be conducted in the rotating apparatus shown schematically in Figure 10. A photograph of this apparatus, which was put into operation some time after the original presentation of this paper, is shown in Figure 11. This is a simulation of the liquid-core nuclear rocket in which a gas, introduced into the outer annulus around a rotating liquid bed, bubbles radially inward to an exhaust duct in the axis of the rotating element. Multiple-exposure photographs are taken by a camera rotating with the apparatus so as to view the bubble by a high-intensity spark backlight. The system has the capability of operating at up to 1500 earth gravities at the liquid surface.

The system discussed above is slated specifically for optical work; that is, utilizing transparent liquids such as water, alcohol, and glycerol; and is therefore more of a research tool than a close simulation of a liquidcore fuel element. The series of experiments projected beyond the completion of the transparent liquid studies will be done with low-melting-point liquid metals in order to establish overall heat transfer behavior and certain problems related to droplet entrainment losses, startup and shut-down, and freezing-in of the propellant passages.

\section{CONCLUSIONS}

(1) The specific impulse of the liquid-core nuclear rocket is limited to the range 1500 to 1550 seconds, assuming that no new material technology will be discovered to extend this range. Its limitation is due principally to the vapor pressure of those fissionable materials and moderators which are available at the present time.

(2) The engine thrust-to-weight ratio attainable with the multi-element liquid-core design illustrated in Figure 1 can be approximately unity for a fuel-element pressure of approximately 100 atmospheres. Extending the 


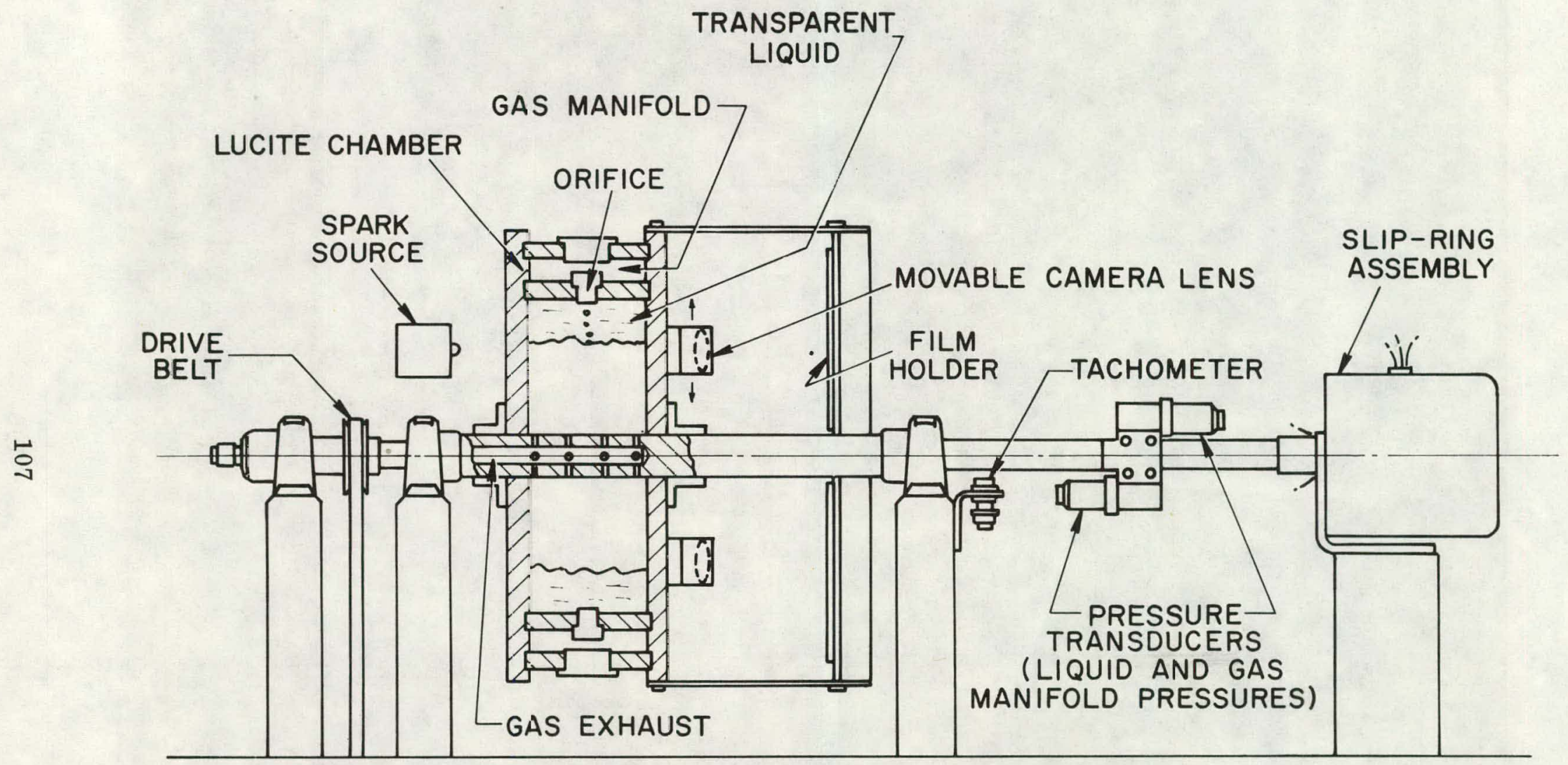

Figure 10. Apparatus for High-Gravity Bubble Flow Studies (Liquid-Core Nuclear Rocket) 


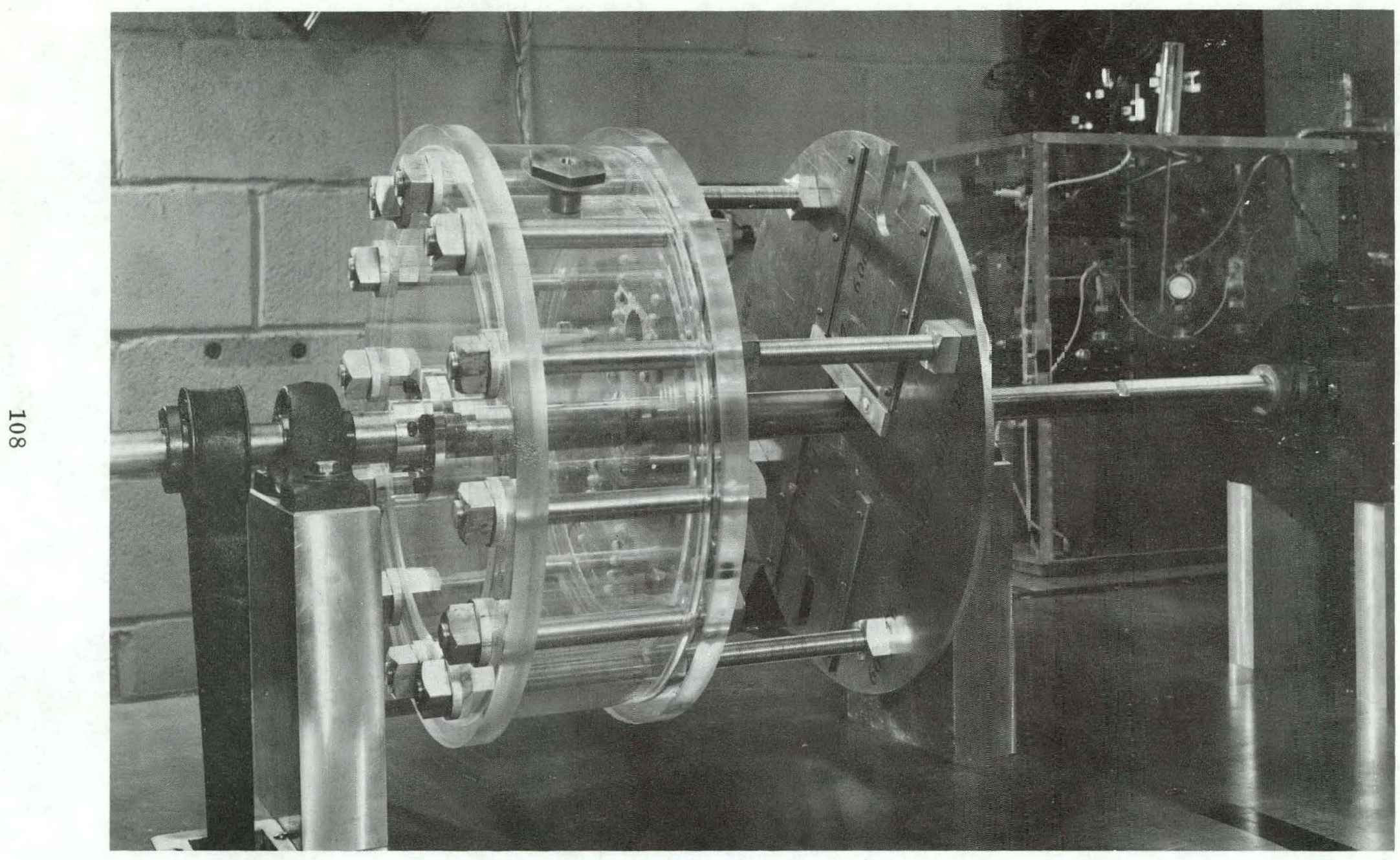

Figure 11. Apparatus for High-Gravity Bubble Flow Studies (Liżuid-Core Nuclear Rocket) 
fuel-element pressure to 1000 atmospheres provides improvement by a factor of nearly 10 .

(3) Heat transfer does not appear to be a limitation on the performance of the liquid-core nuclear rocket. Rather, the thrust is limited by the available flow area, and can be improved directly by either increasing the relative surface area of the fuel element or increasing the chamber pressure of the system.

(4) The structural design and mechanical operation of the multi-element liquid-core reactor system is considerably simplified over configurations employing a single large fuel element, in that the small size of the individual fuel elements, which can be individually pressurized, provides far greater capability for the high chamber pressures necessary to achieve high thrustto-weight ratios. Furthermore, the entire structure of the reactor is at incoming propellant temperatures, the only hot regions being restricted to the structurally unloaded material at the center of each of the fuel elements. Rotational speeds for these small elements required to achieve the gravity levels necessary for proper retention of the fissionable material will be of the order of several thousand rpm, and can be driven by either small hydrogen turbines operated by incoming propellant or, should the radiation field prove nonprohibitive, by small individual electric motors. The power consumption of these drives is negligibly small.

(5) The principal feasibility areas are those of bubble flow and heat transfer in liquid annuli at high gravities, a problem now under study at Princeton University from an experimental point of view, and determination of the vapor pressures and "metallurgy" of the various phase mixtures of refractory compounds suitable for reactor core materials.

Question: What is the effect of the flow of propellant down the central core of each fuel element? Won't this propellant shear off some of the liquid from the internal surface of the element and carry it out the nozzle?

Answer: The diameter of the central cavity of each fuel element is sufficiently large that the Mach number of the flow is extremely small, so that the resulting shear force on the liquid surface is several orders of magnitude smaller than the centrifugal acceleration force tending to maintain it within the element. This has been discussed in detail in Reference 4. The actual flow limitation, in terms of the possibility for liquid entrainment, occurs at the surface of the liquid when the bubble breaks through. The surface shattering effect there might tend to carry off small droplets of the liquid which then may be swept out with the gas before they are returned by the centrifugal field to the liquid surface. This is one of the problems 
which will be studied in the rotating bubble apparatus, both photographically and by quantitative measurements of the loss.

Question: What is the loss involved in providing the hydrogen propellant with angular momentum within the fuel element?

Answer: Much less than 1\% of the overall power. This is discussed in Reference 4.

Question: What about the effect of the axial forces on the liquid within the fuel elements? Won't you obtain a pileup of material at the aft end of the reactor due to the fact that there is a longitudinal acceleration as well as a radial acceleration in each element?

Answer: We have checked the effect of longitudinal acceleration, which is extremely small compared to the centrifugal acceleration field proposed. For a vehicle thrust-to-weight ratio of one gravity, which is perhaps as high as this system would ever provide, at a centrifugal field of approximately 1000 gravities, the half-cone angle of the resulting surface will be only a degree or two. This has been calculated in Reference 4, and does not represent a significant problem.

Question: How much flow area do you have in the system; is it comparable to that of conventional solid-core rocket?

Answer: The total void fraction of all the central gas passages in the fuel $\overline{\text { elements }}$ is of the order of $35 \%$ of the overall reactor, and the void fraction of all the radial gas passages and the bubbles within the liquid is about $15 \%$. Thus the total void fraction considered is in the neighborhood of $50 \%$. This was, incidentally, arbitrarily selected as a limiting value. It is conceivable that in subsequent designs void fractions may run considerably below this.

Question: The point of maximum specific impulse which you indicated was gnverned by the molecular weight of the exhaust. What is the ratio of the propellant flow rate to the total flow rate?

Answer: For the mission considered in Reference 4, which corresponded to a velocity increment of about $37,500 \mathrm{ft} /$ second, the propellant mass flow rate was $6.5 \mathrm{lb} /$ second and the uranium loss rate about $0.0012 \mathrm{lb} /$ second. This corresponds to a separation ratio of over 5000, which is considerably higher than any of the gaseous-core-rocket goals. 


\section{EXPERIMENTAL WORK IN RELATION \\ TO THE GLOW-PLUG CONCEPT}

J. W. McKee

General Electric Company

Santa Barbara, California

A central problem in the application of gaseous fission reactors to space vehicle propulsion is to reduce the amount of fissionable material which leaves the system with the propellant. Several years ago at Douglas Aircraft Company, Max Hunter and the author (Reference 1) suggested that a possible approach to this problem is to partition the fissionable material from the propellant by a transparent wall which is regeneratively cooled. This is the basic idea of the glow-plug propulsion system shown in Figure 1. In this particular configuration an elongated toroid forms the fuel container. Regenerative cooling of nozzle, reflector-moderator, and container is accomplished by propellant before it is seeded to increase opacity and heated by radiative transfer.

Experimental and analytical work required to determine feasibility and expected levels of performance of this and similar concepts is in progress in several organizations. A small program within the General Electric Company to explore the concept has been divided into three basic parts: (1) simple experiments on measurements of transparency of container materials during irradiation, (2) experiments on the chemical compatibility of container materials with potential fuels, and (3) planning of an experiment employing the TREAT reactor to obtain radiant emission from gaseous fissionable material.

In conjunction with optical experiments in the GETR, a sample of high purity fused silica (Corning 7090) was simultaneously irradiated at $10^{14} \mathrm{n} / \mathrm{cm}^{2}$ sec and measured for transparency using light from a Xenon flash lamp.

1. McKee, J. W., "The Glow-Plug Gas Core Reactor," presented at Symposium on Gaseous Fission Reactors, April 26-27, 1962, California Institute of Technology, Pasadena, Calif. 


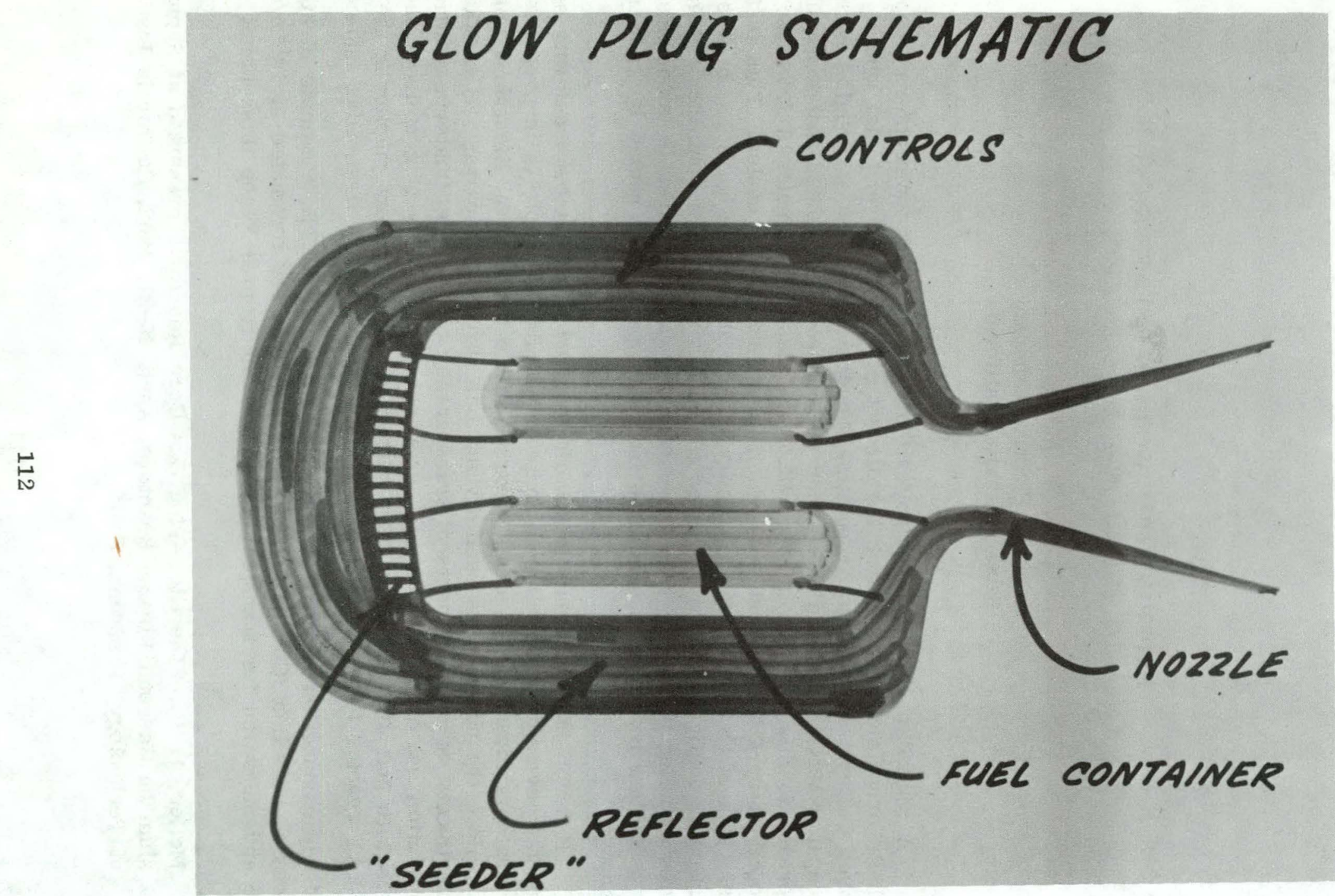

Figure 1. Schematic Diagram of the Glow-Plug Concept 
The sample temperature was between 100 and $150^{\circ} \mathrm{F}$. After 10 minutes the absorption was approximately $1 \%$ per $\mathrm{cm}$.

Work is currently in progress at the Nuclear Materials and Propulsion Operation at Evendale involving the chemical compatibility of container materials with several potential fuel carriers. The objective is to gain information on the corrosion up to temperatures of $1500^{\circ} \mathrm{C}$ of these materials with the tetrabromides, tetraiodides and tetrachlorides of uranium and uranium hexafluoride and fluorine. Past experience (Reference 2) has shown that severe corrosion is minimized if a nonvolatile layer of material is formed at the interface. In the case of aluminum oxide the material formed is aluminum fluoride which remains on the surface at temperatures of $1800^{\circ} \mathrm{F}$ for two days.

In Figure 2 the third area of activity is shown. This is a planned experiment in which a transparent envelope containing a fissionable material is introduced into the TREAT reactor (Reference 3). The reactor can be pulsed to about $10^{16} \mathrm{n} / \mathrm{cm}^{2} \mathrm{sec}$ (unperturbed) on a period of about 80 milliseconds. The transparent envelope is placed near the center of the core within another fail-safe container which has a geometry as shown in the figure. The optical path is arranged to allow radiant energy to leave the reactor, allowing a variety of measurements to be performed. Measurements of pressure, inner wall temperature (or heat flux), and electrical conductivity are also contemplated. An objective in addition to data collection is the demonstration of the ability to produce under controlled conditions a radiant, fissioning gas. Figure 3 shows what may be expected in terms of temperature and pressure within the inner envelope. These estimates are based on an equilibrium between energy derived from the fission process and energy radiated from the gas in an envelope consisting of a sphere of $10 \mathrm{~cm}$ diameter. The temperature is, therefore, the maximum equivalent blackbody temperature which occurs at the peak of the neutron flux during the reactor transient. Equivalent blackbody temperatures of $4000^{\circ} \mathrm{K}$ appear reasonable at pressures of approximately 100 atmosphere if $\mathrm{U}^{235}$ is used as fuel.

2. Hale, C. F., Barber, E. J., Bernhardt, H. A., and Rapp, K. E., "High Temperature Corrosion of Some Metals and Ceramics in Fluorinating Atmospheres," AEC Research and Development Report K 1459.

3. Freund, G. A., Iskenderian, H. P., and Okrent, D., "TREAT, A Pulsed Graphite-Moderated Reactor," Peaceful Uses of Atomic Energy, Vol. 10, United Nations, Geneva, 1958. 


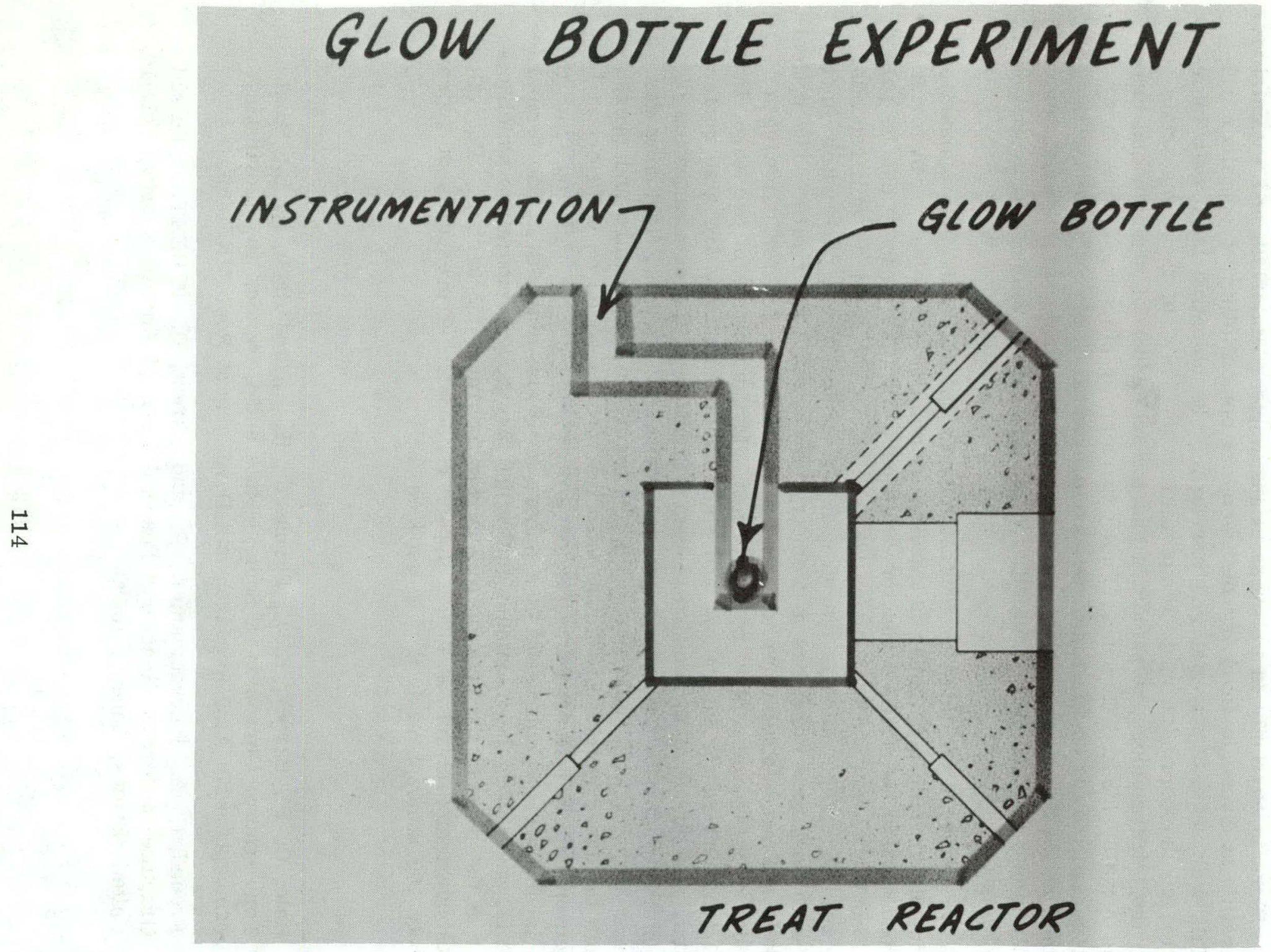

Figure 2. Diagram of the TREAT Reactor Showing Container of Fissionable Gas in Core 


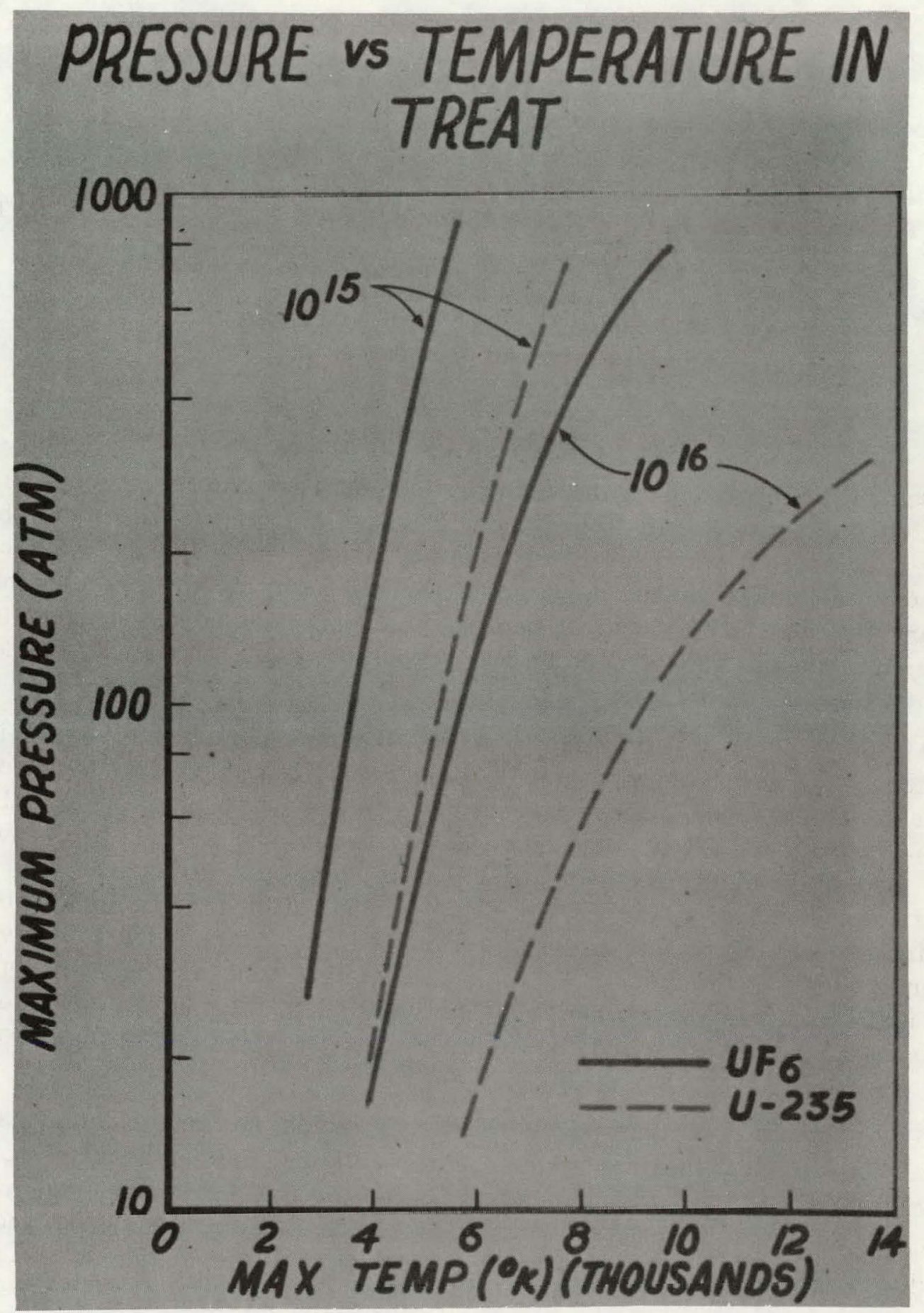

Figure 3. Pressure vs Temperature Within Fissioning Gas for a Thermal Neutron Flux of $10^{15}$ and $10^{16} \mathrm{n} / \mathrm{cm}^{2} \mathrm{sec}$ 
THE TRANSPARENT PARTITION GASEOUS CORE REACTOR CONCEPT

\author{
D. E. Knapp \\ Douglas Missile \& Space Systems Division \\ Douglas Aircraft Company, Inc. \\ Santa Monica, California
}

Our initial approach to the transparent partition reactor concept is illustrated in Figure 1. Hydrogen from a propellant tank is heated in three steps: first as it flows through the cooling passage in the double walled tubes containing fuel, second as it cools the reflector moderator, and third absorbing thermal radiation as it passes outside the fueled transparent tubes. Between the second and third steps the propellant must, of course, be seeded to absorb thermal radiation.

A typical configuration might consist of 3-inch-diameter tubes 12 inches on center. For a maximum chamber temperature corresponding to specific impulse of 1600 seconds, convective heating on the outer tube surface limits the flow velocity to $2-3 \mathrm{ft} / \mathrm{sec}$. This is turn limits the engine thrust so that engine thrust to weight ratios of about 0.1 are expected (Reference 1). Significant improvements in engine thrust to weight ratios may be achicvable by transpiration cooling of the outer tube surface. However, I don't believe refinement of these estimates is as important at this time as the basic question of the ability of transparent materials to function in the manner required by this concept. I want to spend the remainder of the time on this latter question.

The properties of transparent materials which are important are their thermal conductivity, thermal stress characteristics, optical absorption, and chemical stability. The thermal conductivities of BeO, sapphire, and fused quartz are shown in Figure 2. Magnesium oxide is also of interest and has thermal conductivity comparable to that of $\mathrm{Al}_{2} \mathrm{O}_{3}$. Fused quartz is the most

1. F. A. Ross, "Conceptual Design Study of the Glow Plug Gaseous Core Reactor," Douglas Report SM-44042, November 1963. 
TRANSPARENT

$\Xi \quad$ PARTITION

\section{EVALUATION}

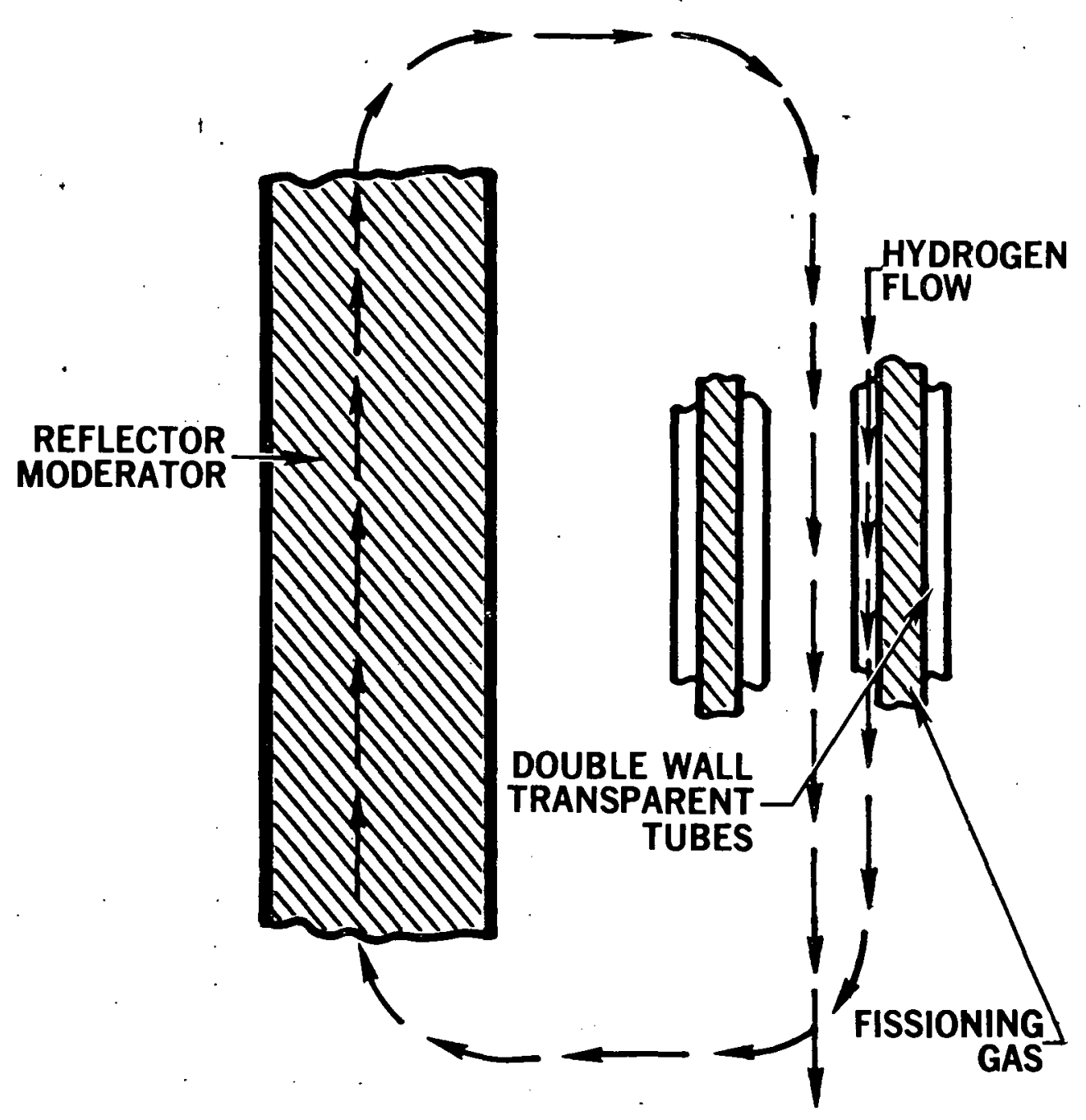

Figure 1 
THERMAL CONDUCTIVITY OF TRANSPARENT MATERIALS

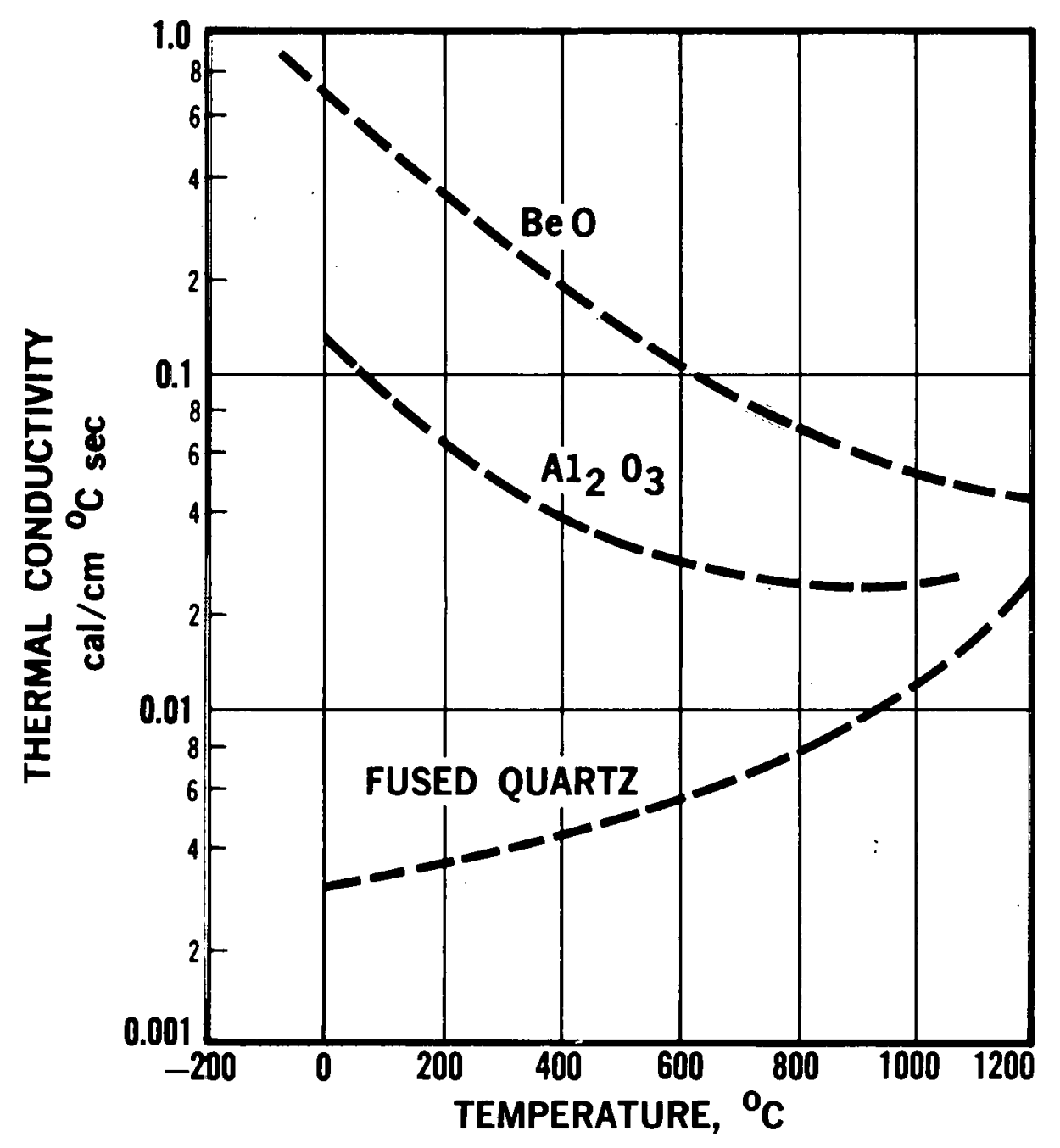

Figure 2 
interesting material so far for this concept. Its thermal conductivity is relatively low at room temperature and rises at higher temperatures due to the contribution of radiative transfer in the solid. This problem is dealt with in detail by Lee and Kingery (Reference 2).

The thermal conductivity and thermal stress properties of candidate materials can be expressed by two figures of merit. The first is the temperature difference at which the thermal stress is approximately equal to the rupture modulus. The second is a measure of the amount of energy that can be conducted out of the solid by this temperature difference. The se quantities are tabulated below for the oxide ceramics indicated along with a representative alkali halide $\mathrm{CaF}_{2}$.

\begin{tabular}{|c|c|c|}
\hline & $\Delta \mathrm{T}=\mathrm{R} / \alpha \mathrm{E}$ & $\mathrm{K} \Delta \mathrm{T} T=1000^{\circ} \mathrm{C}$ \\
\hline $\mathrm{BeO}$ & $49^{\circ} \mathrm{C}$ & $2.8 \mathrm{cal} / \mathrm{cm} \mathrm{sec}$ \\
\hline $\mathrm{MgO}$ & 36 & 1.3 \\
\hline $\mathrm{Al}_{2} \mathrm{O}_{3}$ & 103 & 2.5 \\
\hline $\mathrm{SiO}_{2}$ & 2730 & $1.9 *$ \\
\hline $\mathrm{CaF}_{2}$ & 6.3 & 0.03 \\
\hline
\end{tabular}

*for $\Delta \mathrm{T}$ arbitrarily set at $200^{\circ} \mathrm{C}$.

It is clear that for quartz the $\Delta T$ calculation from thermal stress limits is not meaningful because it exceeds the melting point of quartz. For this reason the quantity $\mathrm{K} \Delta \mathrm{T}$ is calculated for a $\Delta \mathrm{T}$ arbitrarily set at $200^{\circ} \mathrm{C}$. $\mathrm{BeO}$ and $\mathrm{Al}_{2} \mathrm{O}_{3}$ are next in their capacity to remove energy by conduction. $\mathrm{CaF}_{2}$, which has favorable optical properties, has a very stringent thermal etress limit and is ruled out for that reason.

From this brief summary of thermal and mechanical properties, I want to go on to the question of optical absorption and how it is modified by neutrons, gamma rays, and fission fragments. We obviously have a substantial amount of data from the solid state physicists about this problem, but much of the data is not quantitatively applicable to the present concept. For this reason we embarked on a program of material irradiations and optical measurements.

2. D. W. Lee and W. D. Kingery, "Radiation Energy Transfer and Thermal Conductivity of Ceramic Oxides," Jnl. of the Amer. Ceram. Soc. 43, 594 (1960). 
Optical measurements are made in a $40 \mathrm{~cm}$ vacuum ultraviolet spectrometer. A stabilized hydrogen discharge lamp with a lithium fluoride window was used for most of the measurements. Samples were held in the reflectometer shown in Figure 3. The rotating light pipe allows measurements of transmitted, reflected, and scattered light. The sample holder holds both a standard quartz sample and the sample to be tested. All measurements are then made relative to the standard sample. In this way any drift over a period greater than a few seconds is eliminated. Figure 4 illustrates the accuracy achieved to date in calibrating the quartz standard. It's very easy to achieve accuracies of a percent or less in transmission. Comparable accuracies in reflection are more difficult.

Subsequent measurements are then characterized by the accuracy of the standard sample calibration. In evaluating the transparent partition concept we have found that about $3 \%$ absorption of thermal radiation to the wall is tolerable. Thus attainment of meaningful positive data (with respect to the concept) requires accuracy of $1 \%$ or better.

Typical transmission measurements for the materials that we've been concerned with are shown in Figure 5. On this basis quartz seems to be superior. Magnesium oxide is not particularly interesting while sapphire may be somewhat better. In addition to these materials which formed the basis for irradiation testing, we were able to obtain a very small single crystal specimen of beryllium oxide. The sample shows a cutoff somewhat below $2000 \AA$ (Figure 6). If in the future anyone were to make tubes of beryllium oxide, this would turn out to be interesting. The indication of about $80 \%$ transmission relative to the quartz standard is very likely due to instrumental errors resulting from the small sample size or due to scattering from imperfections in the single crystal.

One of the next features of interest is the effect of temperature on the transmission of these materials. For a good dielectric material a physical argument suggests that both the ultraviolet and infrared absorption processes are broadened in such a way as to reduce the width of the transmission window. Infrared measurements by Lee and Kingery of sapphire (Figure 7) behave in just the way I think they should, namely, there is effectively no difference in the transmission except near the infrared cut off. The shift. in the ultraviolet is expected to be even smaller so that this should not be a problem. The same data for quartz (Figure 8) is somewhat puzzling and less optimistic. We suspect that the shift in transmission far from the infrared cut off may be due to a structural transition or some other unexplained phenomena which does not represent an intrinsic property of quartz. We have not really designed our apparatus to make measurements on samples 


\section{REFLECTOMETER SCHEMATIC}

芯 (SMITH, R.S.I. 50, 862 (1960)

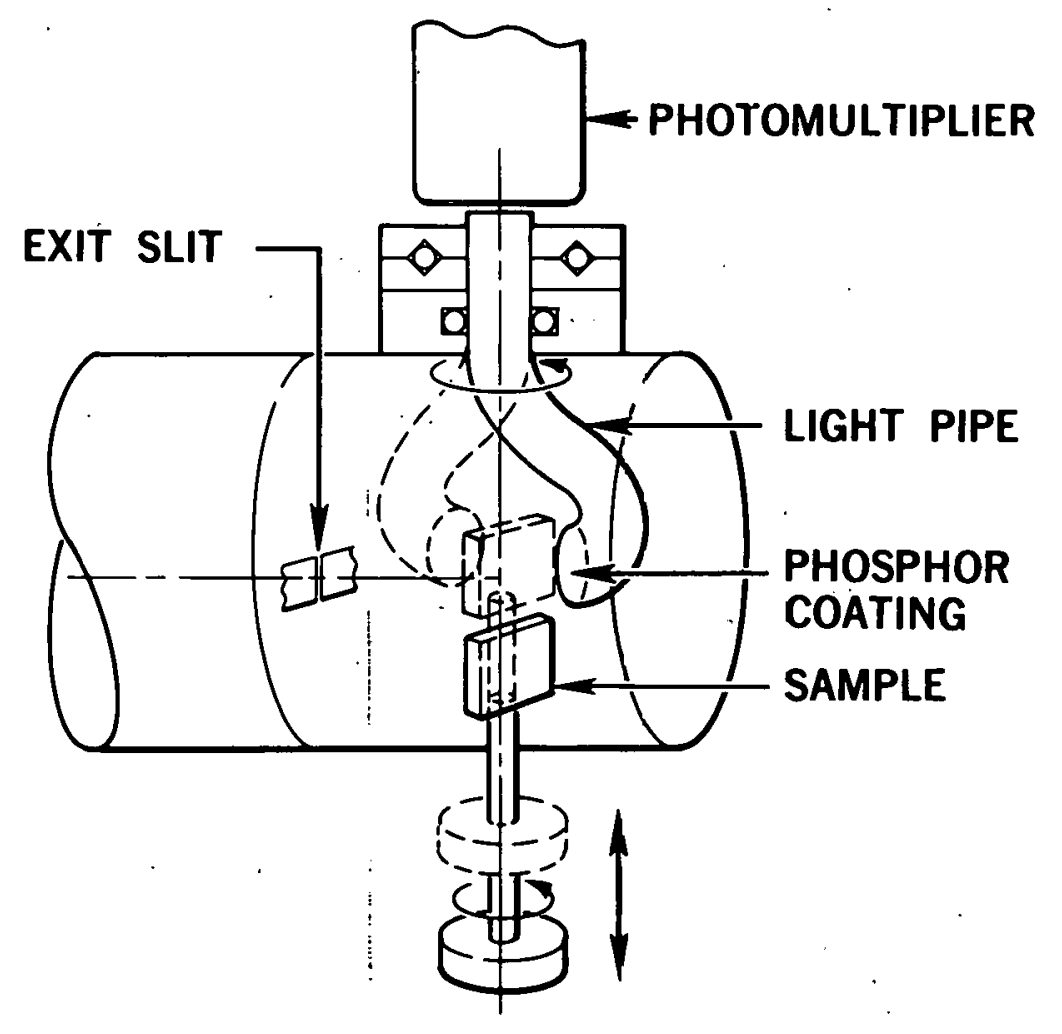

Figure 3 


\section{QUARTZ STANDARD \\ ¿ CALIBRATION}
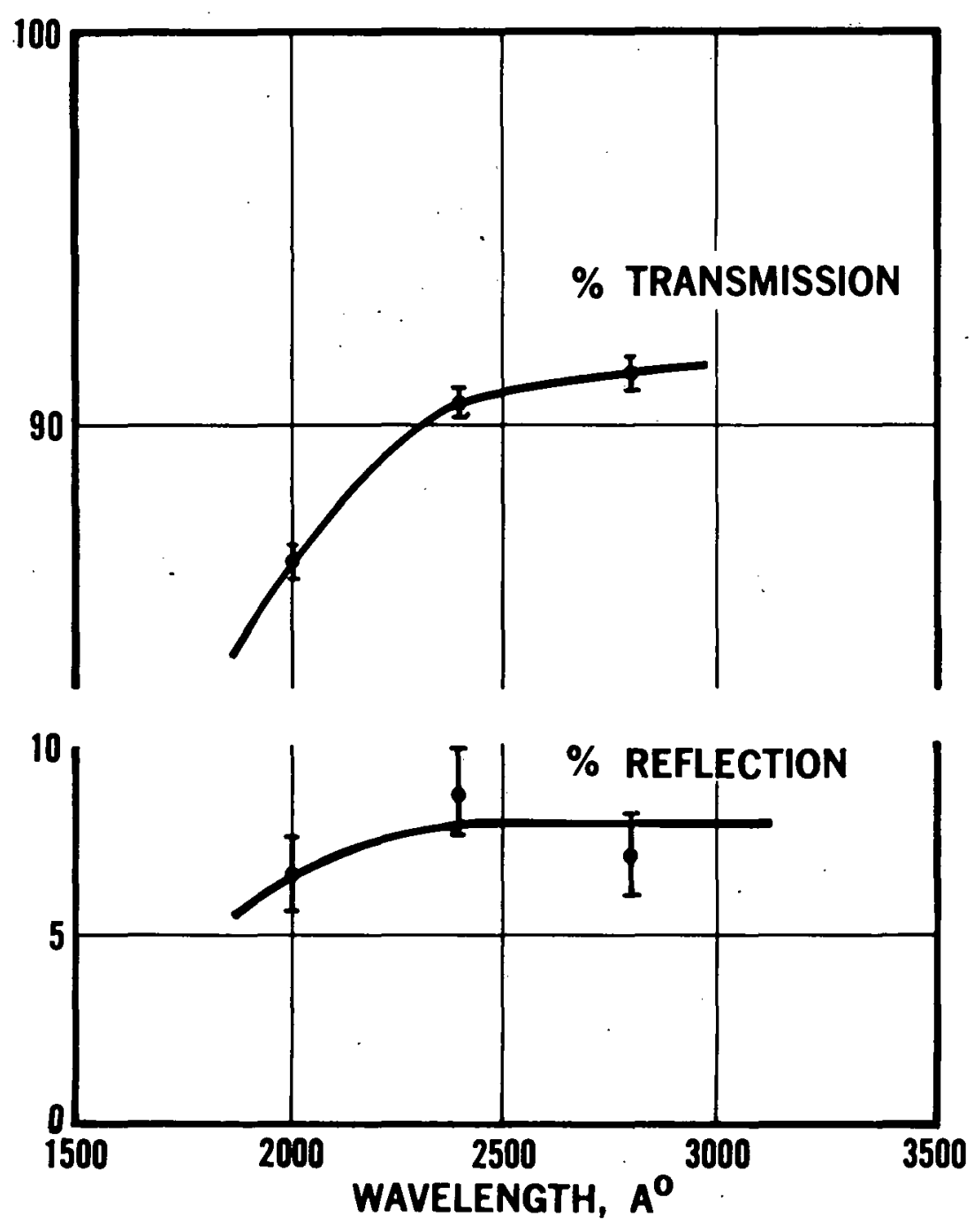

Figure 4 


\section{TRANSMISSION OF UNIRRADIATED SAMPLES}

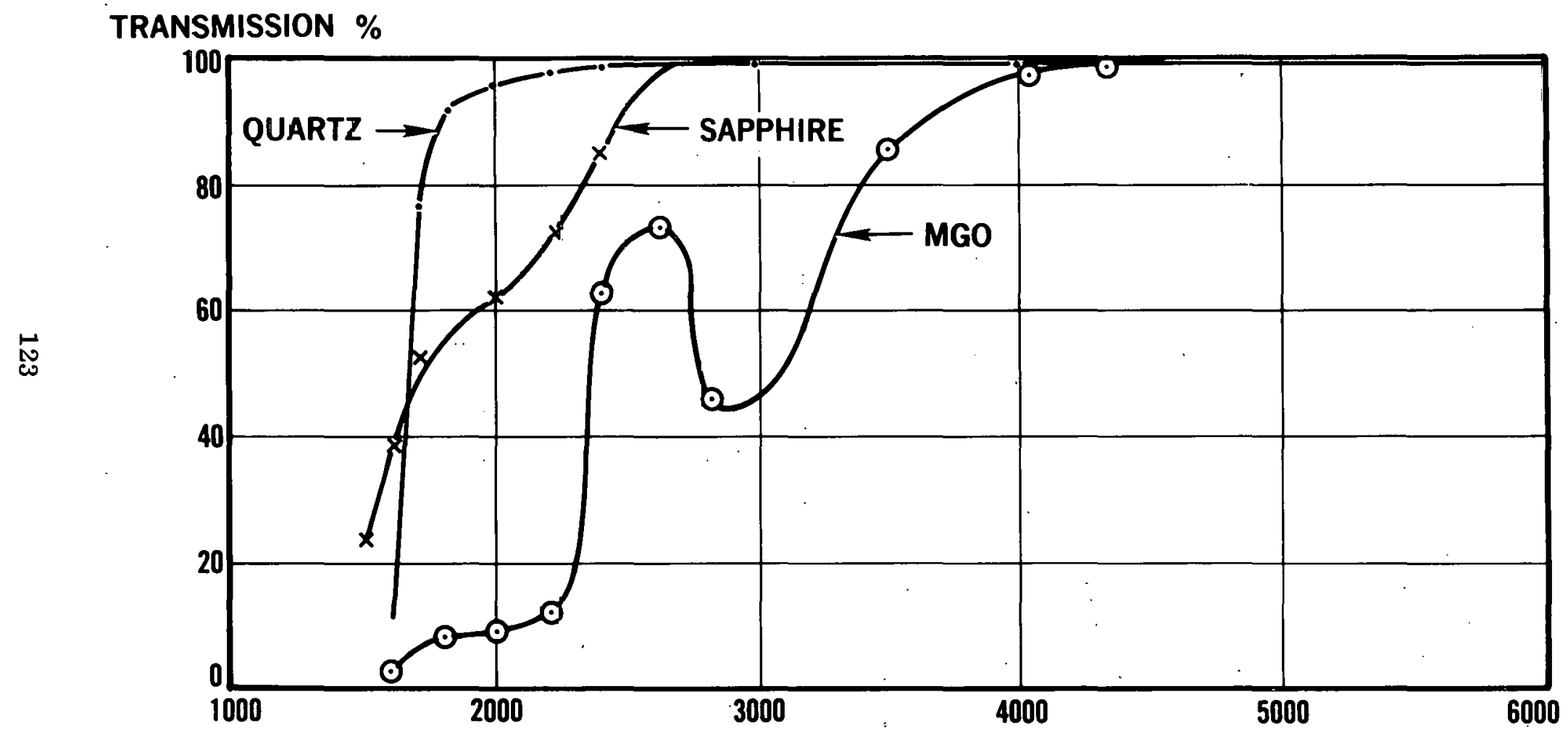

Figure 5 


\section{OPTICAL TRANSMISSION OF SINGLE CRYSTAL BeO}

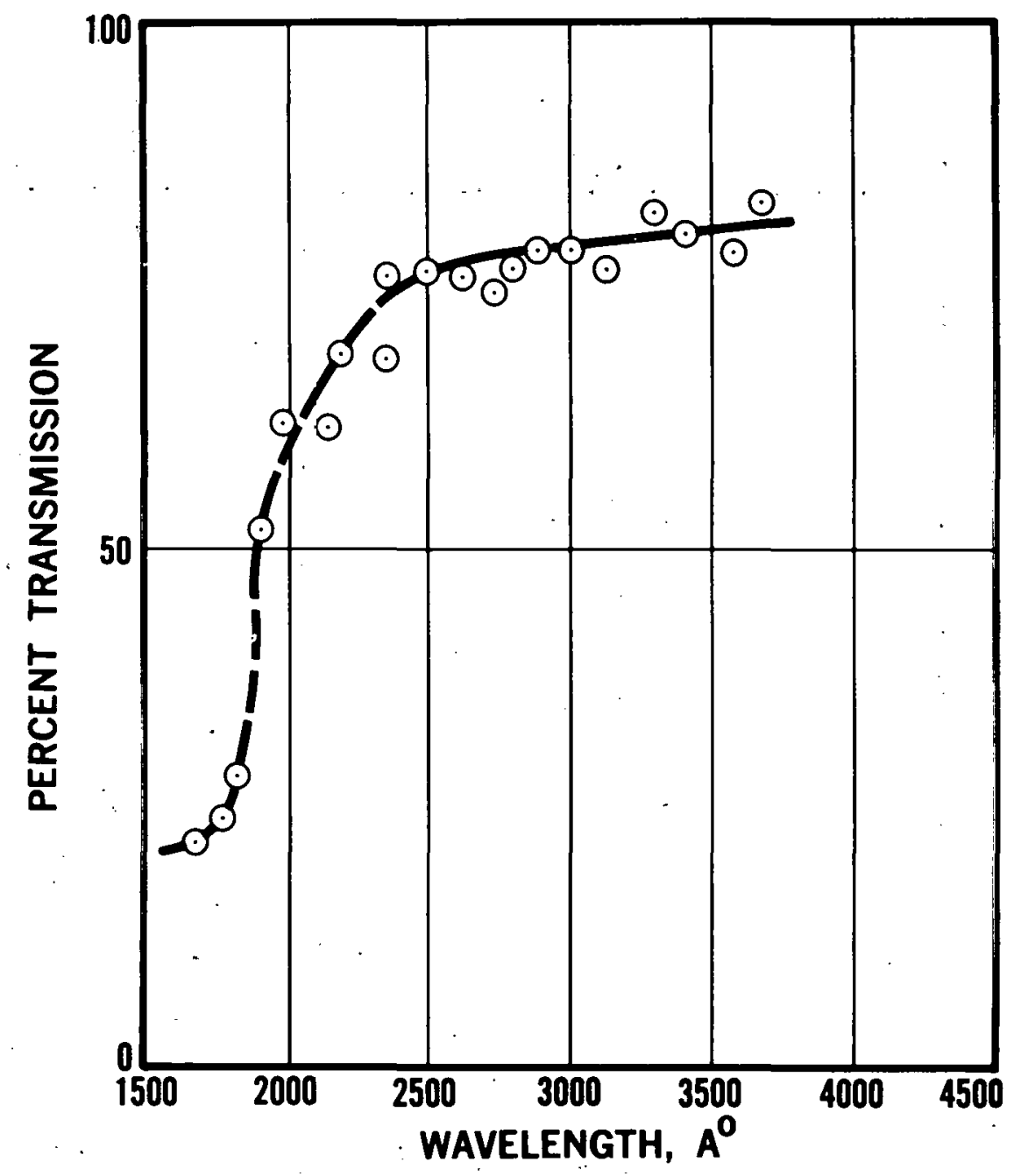

Figure 6 


\section{INFRARED TRANSMISSION OF SAPPHIRE (LEE \& KINGERY)}

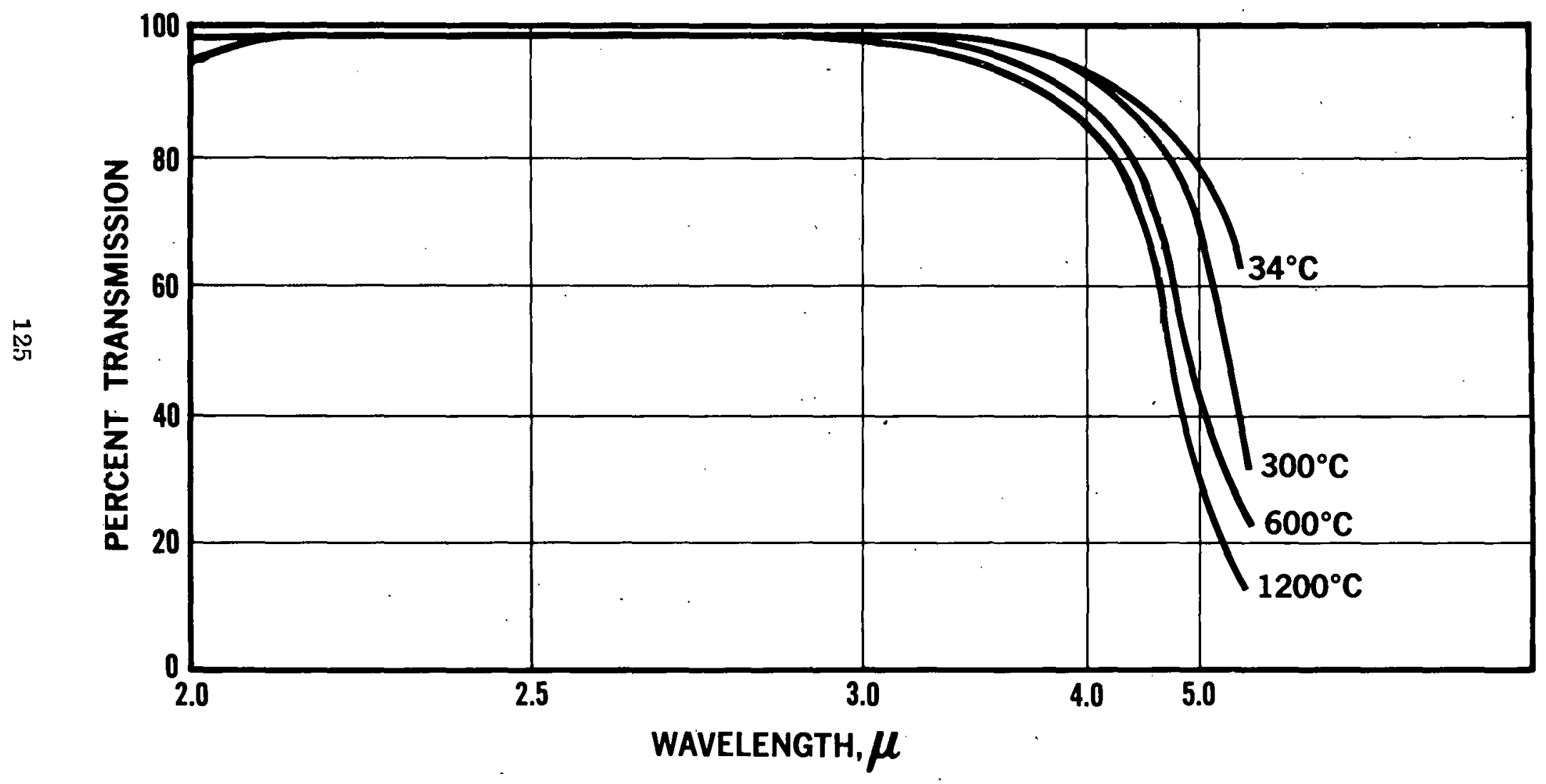

Figure 7 


\section{INFRARED. TRANSMISSION OF FUSED QUARTZ (LEE \& KINGERY)}

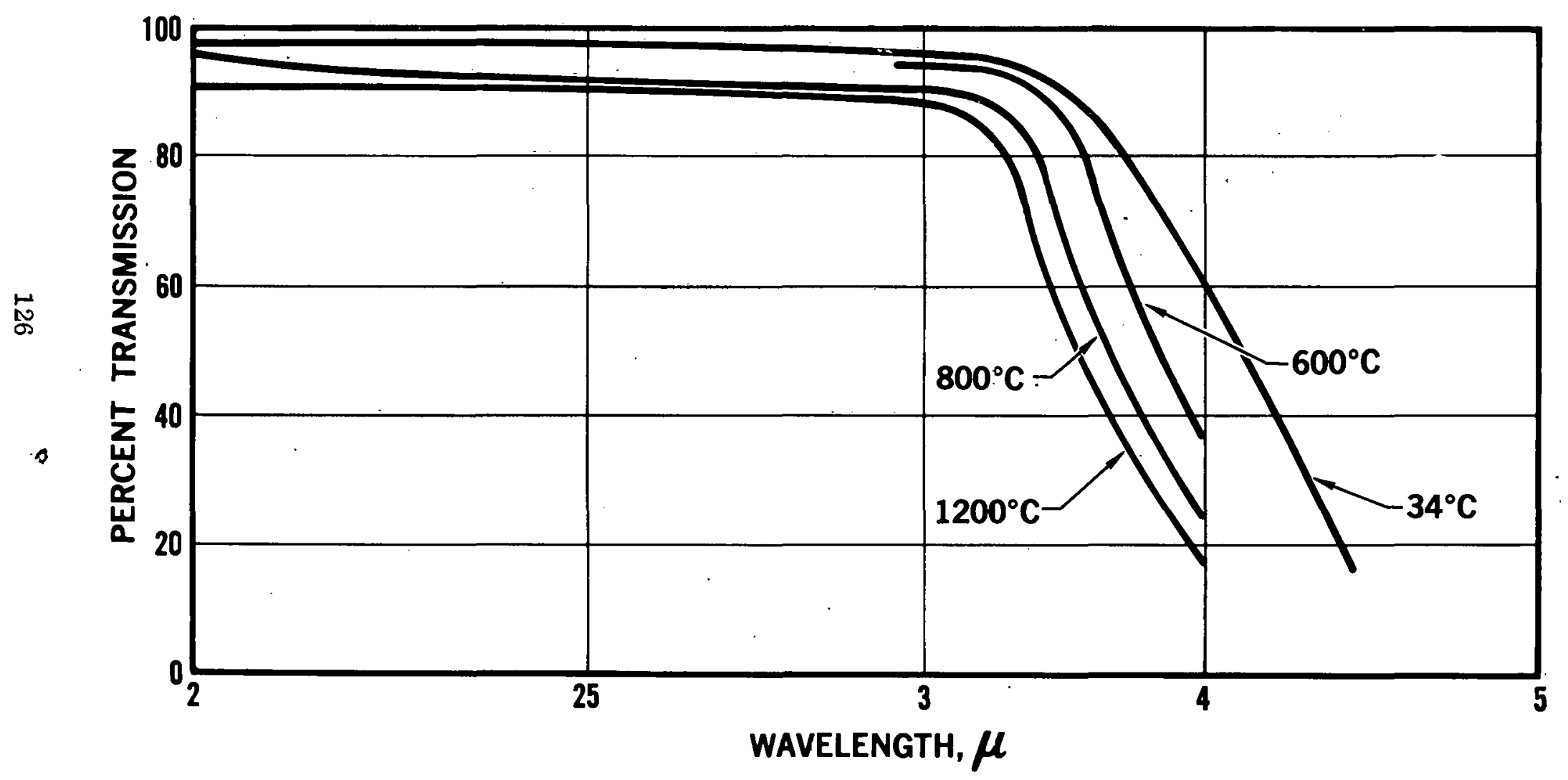

Figure 8 
at high temperature, but we have made some measurements between $4000 \AA$ and $6000 \AA$. To an accuracy within $0.5 \%$ we can observe no change in the transmission of fused quartz between room temperature and $500^{\circ} \mathrm{C}$. Thus we do not see the effect observed by Lee and Kingery in the infrared being propagated into this region of the spectrum. Perhaps George McLafferty will have some comment on this subject in a few minutes.

The first material irradiations which we made in this study were carried out at the GETR under conditions summarized below:

\begin{tabular}{cllc} 
Capsule & Position & $\frac{\text { Flux }}{\text { Integrated Flux }}$ \\
\cline { 3 - 4 } 1 & Shuttle & $2.3 \times 10^{13}$ & $9.7 \times 10^{16}$ \\
2 & Shuttle & $2.3 \times 10^{13}$ & $9.7 \times 10^{17}$ \\
3 & Shuttle & $2.3 \times 10^{13}$ & $9.6 \times 10^{18}$ \\
4 & Pool & $6.9 \times 10^{12}$ & $1.6 \times 10^{19}$ \\
5 & Pool & $3.0 \times 10^{13}$ & $7.0 \times 10^{19}$ \\
6 & Pool & $1.5 \times 10^{13}$ & $3.6 \times 10^{19}$
\end{tabular}

The attempt here was to cover a range of flux levels and integrated fluxes to establish boundaries on the radiation damage problem. Upon visual inspection the quartz samples remained transparent while the magnesium oxide turned deep blue and the sapphire pale yellow after irradiation. Transmission measurements for quartz are shown in Figure 9. There are three elements of interest here. First the absorption builds up with dose showing some spectral structure near the ultraviolet cut off but saturating near nvt $=10^{18}$. Second no effect of dose rate is detectable. Third, the region between 4000 and $6000 \AA$ shows a gradual rise in transmission loss with increasing neutron exposure. This latter transmission loss is very rapidly annealed out and may be due to an as yet unidentified scattering process. Annealing studies of the most persistent ultraviolet absorption center at $1650 \AA$ are shown in Figure 10. The indicated transmission in excess of $100 \%$ is due to absorption in the quartz standard sample which can be reduced by annealing at elevated temperature. An important feature to keep in mind in this data is that at $500^{\circ} \mathrm{C}$ this particular absorption process is not significantly annealed even after extended periods of time.

Following these initial screening measurements we felt it important to irradiate materials at elevated temperature in order to establish whether or not the damage and annealing processes could be uncoupled for separate study. The answer turns out to be no. We arrived at this conclusion after 


\section{FUSED QUARTZ TRANSMISSION VS WAVELENGTH FOR DIFFERENT FAST NEUTRON DOSES}

\% TRANSMISSION RELATIVE TO UNIRRADIATED QUARTZ

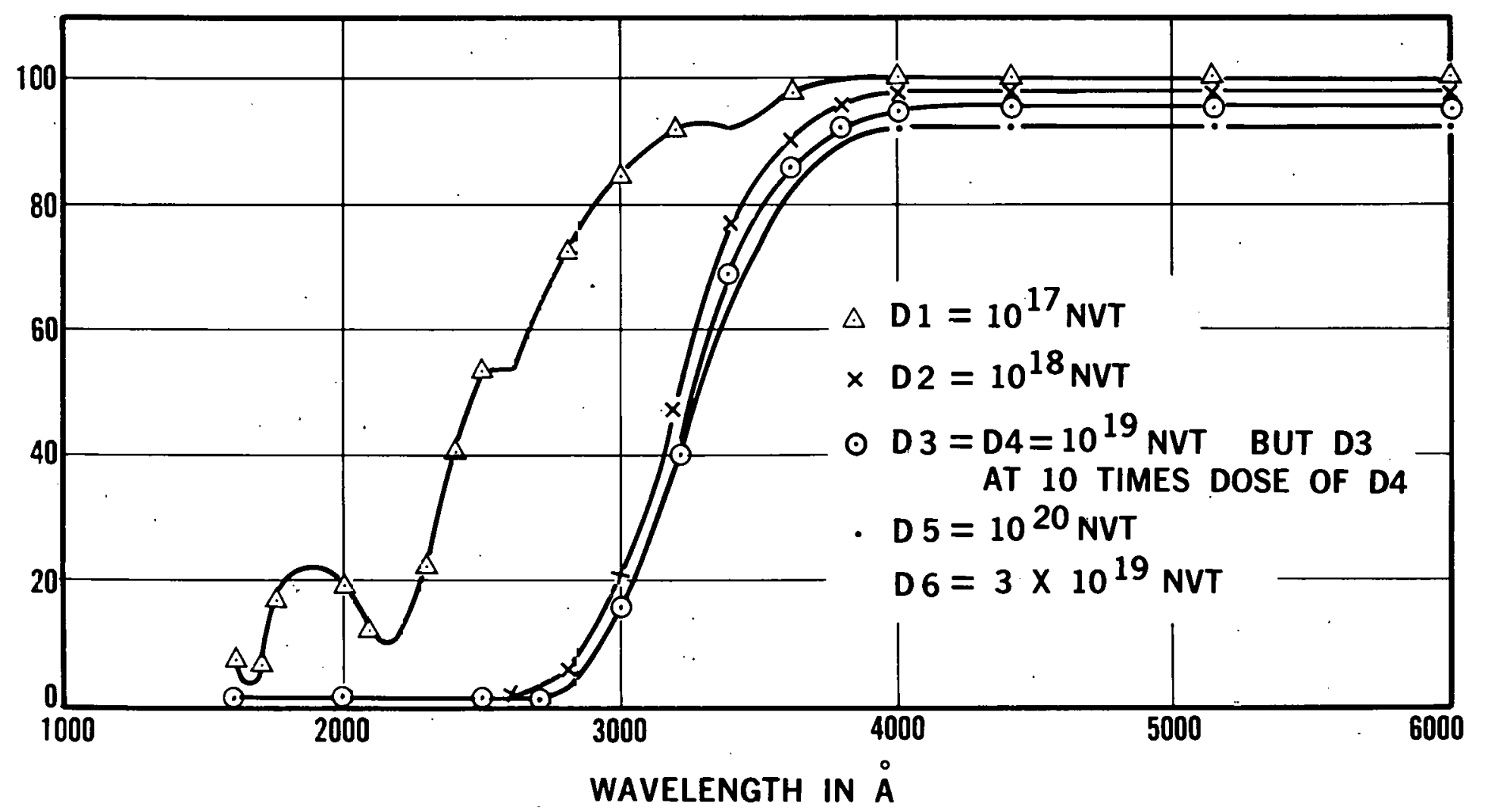

Figure 9 


\section{ANNEALING $1650 \AA$ ABSORPTION PEAK VS TIME AT ANNEALING TEMPERATURES}

RELATIVE TRANSMISSION \%

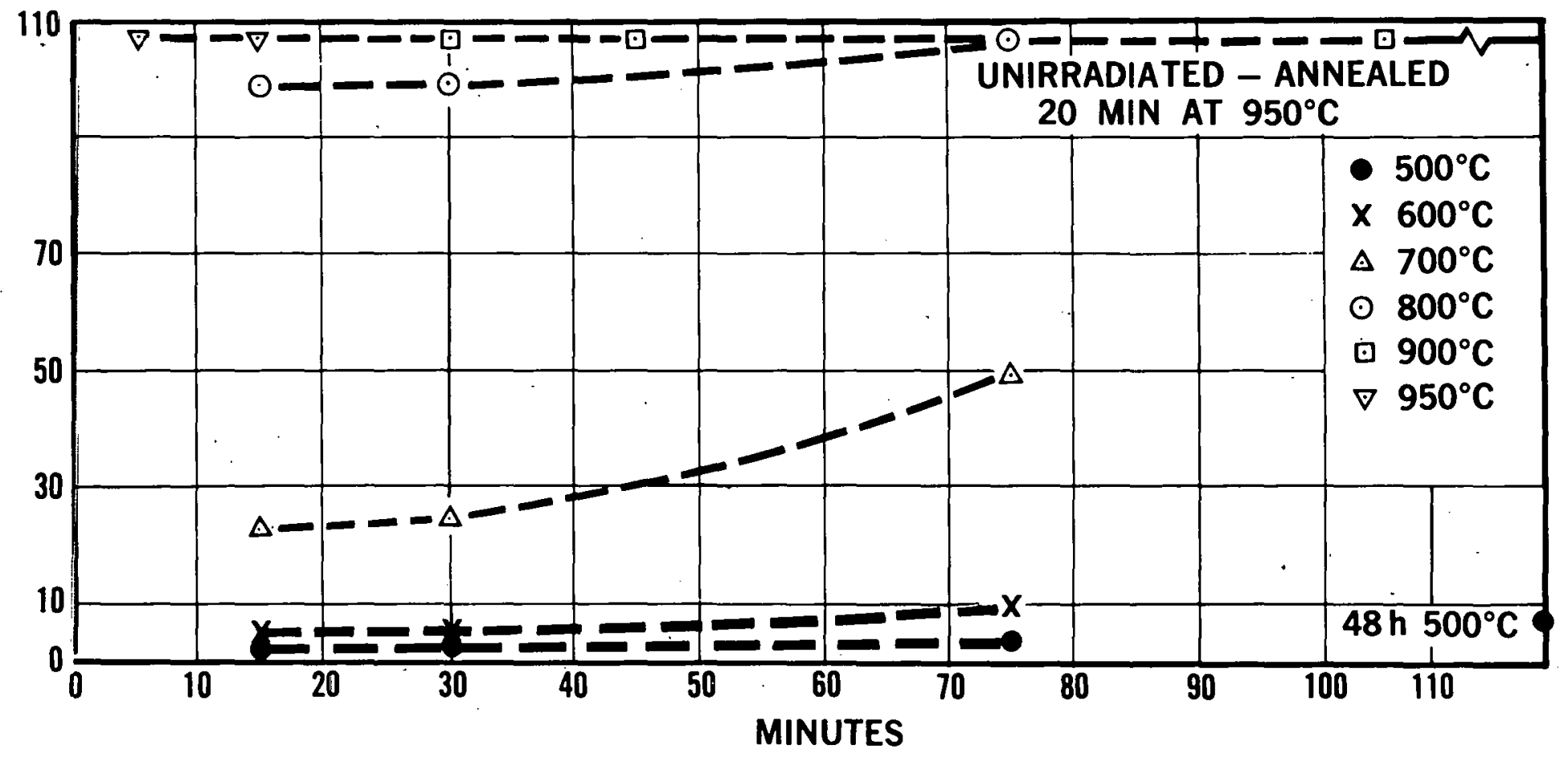

Figure 10 
an irradiation made in the capsule shown in Figure 11. It is essentially a Thermos bottle configuration with a vacuum jacket and low conductance supports at each end. The sample holder consists of an invar sleeve surrounding a graphite cylinder in which the samples are imbedded with tightly packed copper foil. This insures an isothermal zone for the center region of the capsule. This region is heated by neutron and gamma ray fluxes and cooled by radiation. As a result the temperature is only slightly sensitive to reactor power level. The design temperature was confirmed at carefully sealed Tempilaq indicators.

Transmission results for quartz irradiated at $500^{\circ} \mathrm{C}$ in this capsule are shown in Figure 12. Also shown on the same figure are transmission measurements for samples irradiated at low temperature and later annealed for the indicated time and temperature combinations. We can see that there is a distinct difference between low temperature irradiation with subsequent annealing and samples irradiated at elevated temperature. Furthermore, we found that when we began to anneal samples irradiated at elevated temperatures, the previously tenacious absorption band at $1650 \mathrm{~A}$ almost completely disappeared as did the induced absorption at higher wave lengths. Apparently the residual absorption after irradiation at elevated temperature is much more susceptible to annealing than absorption due to low temperature irradiation.

A possible explanation of this phenomenon consists of two parts. First the elevated temperature capsule was held in the reactor during the reactor shutdown operation. During this sequence the neutron flux stopped abruptly while the gamma ray flux fell slowly over an extended time period. Thus we postulated that the residual absorption may have been due to gamma irradiation during reactor shutdown. To test this hypothesis quartz samples were irradiated at the $\mathrm{Co}^{60}$ facility at the UCLA Medical Center. The similarity of absorption spectra for gamma irradiation, low level neutron irradiation at low temperature, and high level neutron irradiation at elevated temperature is shown in Figure 13. In addition the gamma ray induced absorption showed the same rapid annealing characteristics found for the elevated temperature neutron irradiated samples. The inference is that if we were to repeat this experiment by cutting off both the gamma and the neutron source at the same time, we would find an absorption spectrum which would be rather close to the unirradiated samples. This effect was not observed for sapphire or magnesium oxide.

The second part of an explanation of this behavior in quartz must treat the question of why neutrons are so much less effective in inducing absorption. We don't know the answer to this question. However, the measurements 


\section{TEST SPECIMEN HOLDER AND CHAMBER}

$$
500^{\circ} \text { CAPSULE }
$$

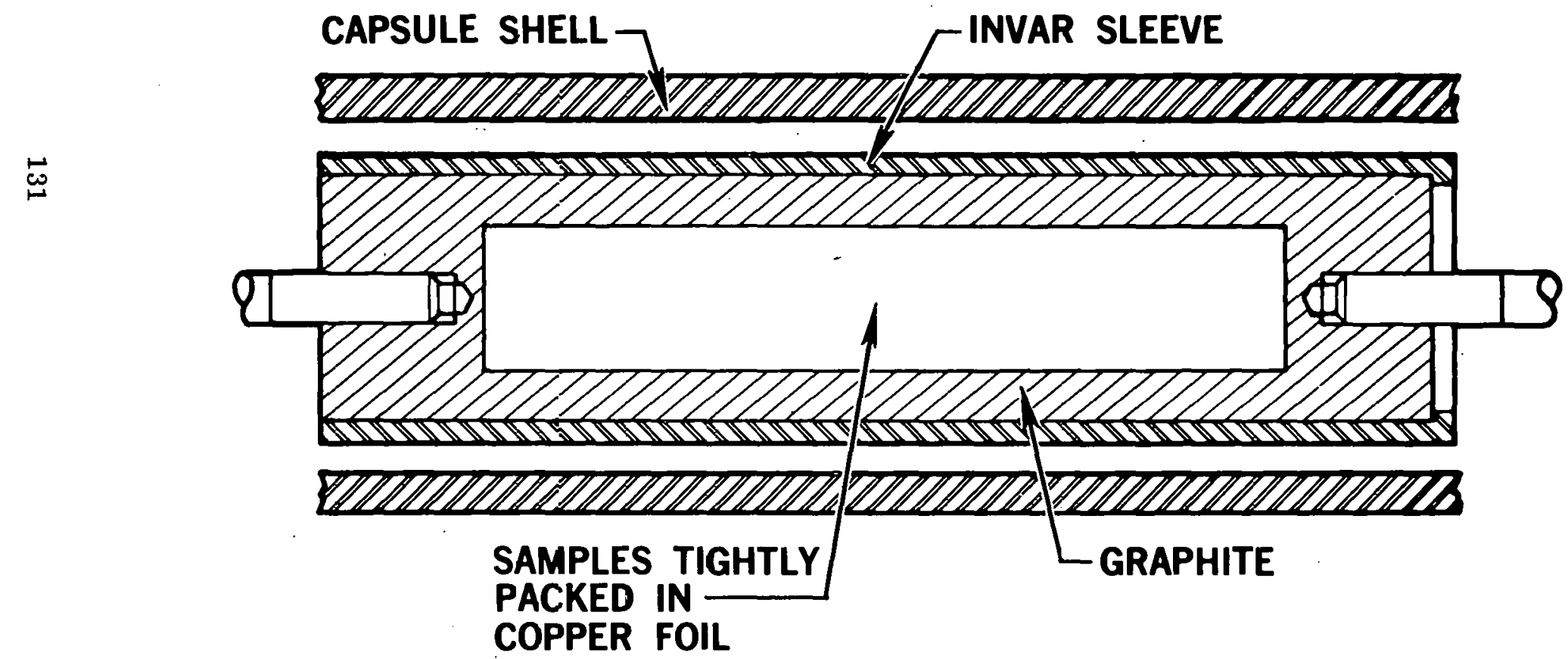




\section{SIMULTANEOUSLY ANNEALED QUARTZ COMPARED TO LABORATORY ANNEALED SAMPLES}

\% TRANSMISSION PRELATIME TO STANDARD

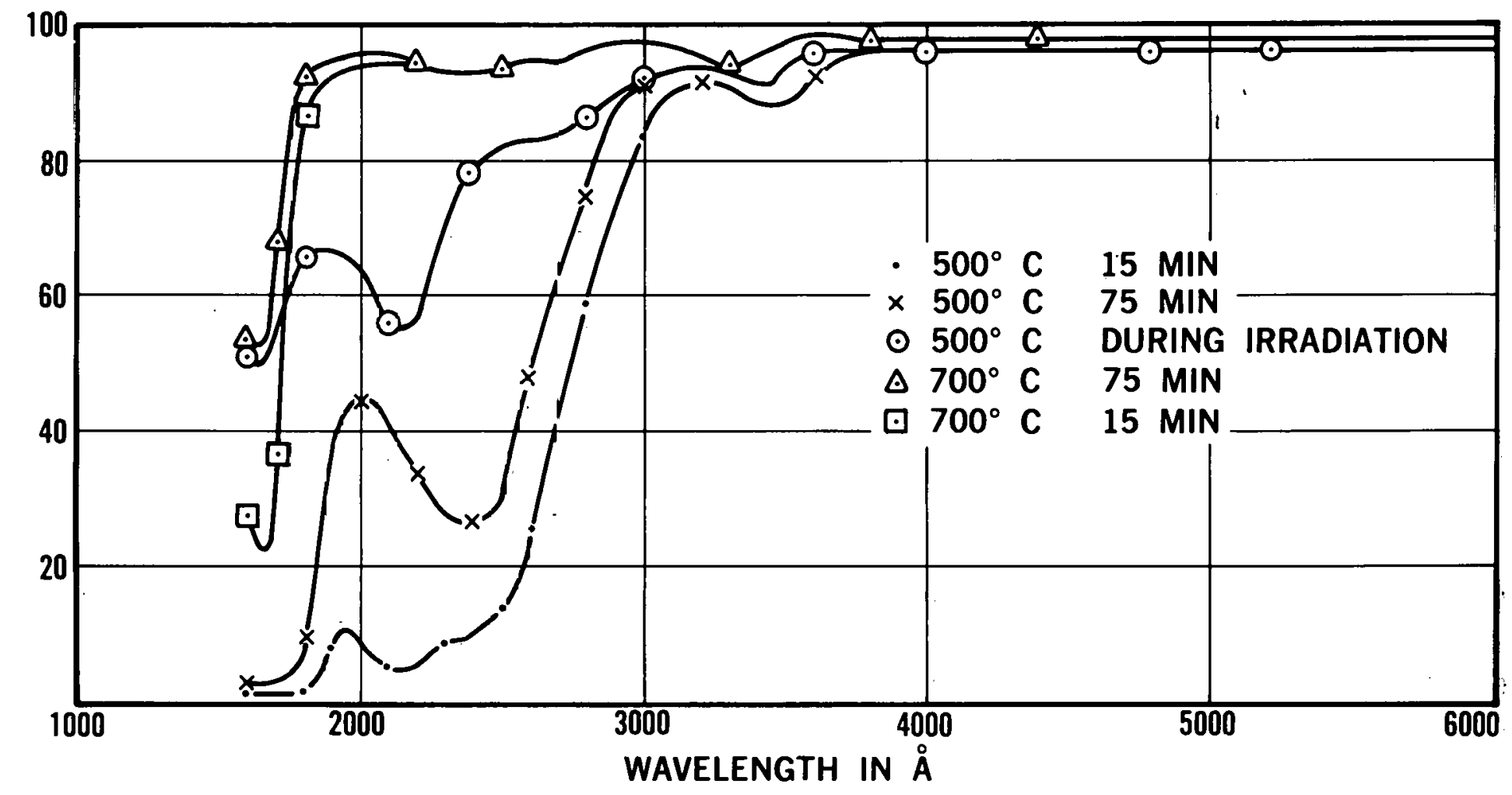

Figure 12 


\section{ABSORPTION COEFFICIENT FOR QUARTZ}

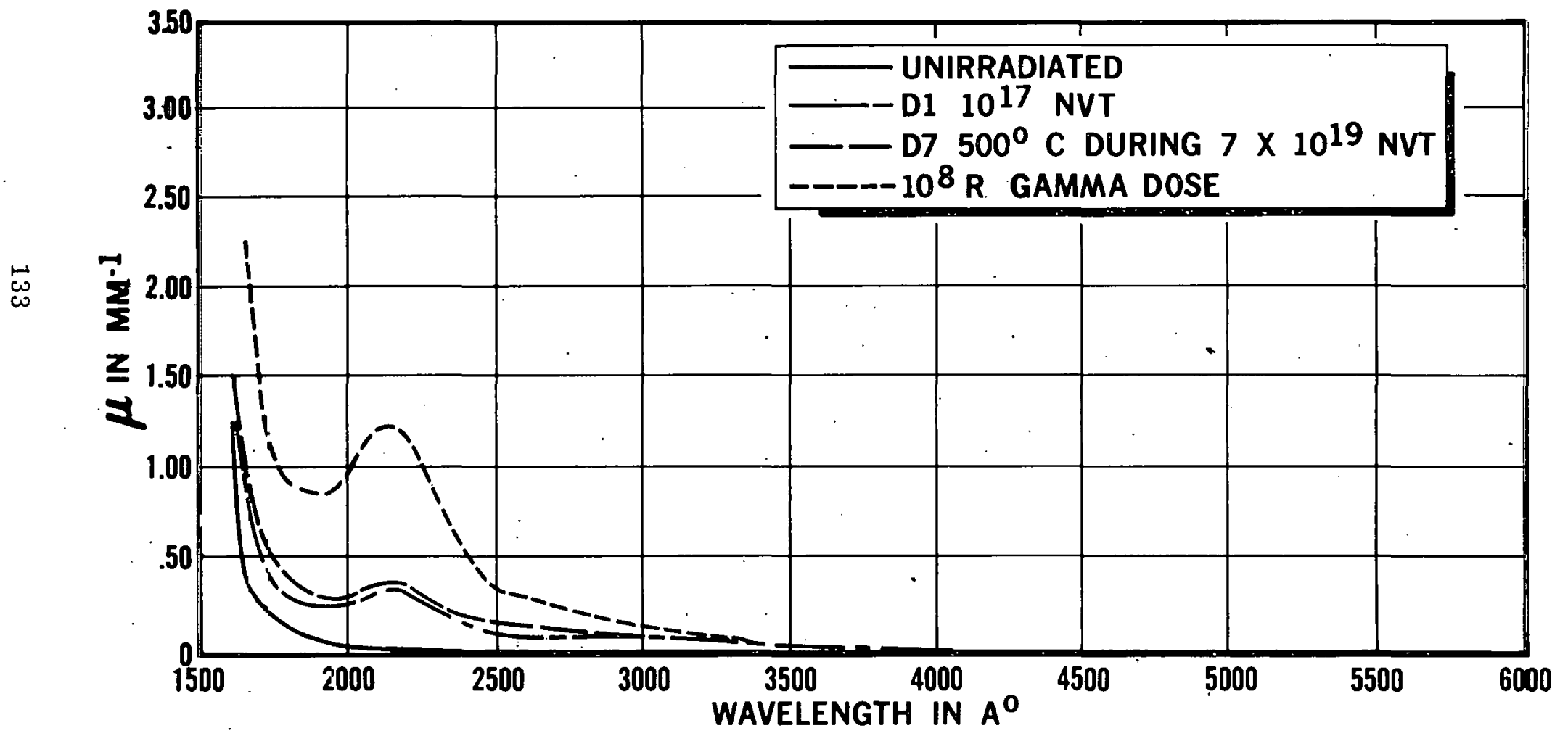

Figure 13 
of Weissmann (References 3 and 4) during the past year provide an interesting clue as to how this might occur. For a long time people have known, or thought they knew, that if crystalline quartz were irradiated at sufficiently high dose, the crystalline structure would be completely destroyed. In some very careful measurements Weissmann has shown that there is a microcrystalline structure remaining in what would have otherwise been characterized as fused quartz. Apparently the same group has also demonstrated the converse situation, that is formation of very small sciale structured regions in neutron irradiated fused quartz. I suggest that an understanding of the effect of temperature on this latter process may provide an explanation for our observation of low or readily annealed absorption due to neutron irradiation at $500^{\circ} \mathrm{C}$. Further measurements confirming our initial observations and extending them to lower temperatures during irradiation would certainly be of value.

The next element of concern about radiation damage is that of damage due to fission fragments. The gross heating effect is manageable under selected design conditions, but optical or mechanical changes in transparent materials subject to fission fragment bombardment may be significant. We have done no experimental work on this question to date, but we can get a fairly interesting idea of what should be done from the work of Elleman, Price, and Sunderman (Reference 5) who were concerned about properties of ceramic oxides for fuel coating. They have measured expansion coefficients per fission atom at an exposure level of $10^{14}$ fission fragments $/ \mathrm{cm}^{2}$. Typical expansion coefficients and tensile stress resulting from $10^{14}$ fission fragments $/ \mathrm{cm}^{2}$ from their work are illustrated below:

$\begin{array}{lll}\mathrm{MgO} & 1.2 \pm 1.2 \times 10^{-21} \mathrm{~cm}^{3} / \text { atom } & \frac{\text { Tensile Stress }}{1.9 \times 10^{4} \mathrm{psi}} \\ \mathrm{SiO}_{2} & 3.3 \pm 1.1 \times 10^{-21} & 1.3 \times 10^{3} \\ \mathrm{BeO} & 3.9 \pm 0.3 \times 10^{-21} & 5.9 \times 10^{3} \\ \mathrm{Al}_{2} \mathrm{O}_{3} & 4.6 \pm 1.7 \times 10^{-21} & 9.6 \times 10^{3}\end{array}$

3. S. Weissmann and K. Nakajima, Jnl. Appl. Phys. 34, 611 (1963).

4. S. Weissmann and K. Nakajima, Jnl. Appl. Phys. 34, 3152 (1963).

5. T. S. Elleman, R. B. Price, and D. N. Sunderman, "Fission-FragmentInduced Stresses in Ceramic Materials," Battelle Memorial Institute Report BMI-1635, June 1963. 
Now, the experiments were carried out at very low levels of fission fragment exposure with an indicated nonlinear behavior in the expansion coefficient, i.e., a five fold increase in exposure produced only a two fold increase in expansion. Based on our experience with the optical measurements we would expect these results to be substantially changed for fission plate experiments carried out at high temperature. An indication of the appropriate temperature range for such experiments is provided by the strain annealing measurements by Elleman, Price, and Sunderman shown in Figure 14.

I would like to conclude with a summary of the experiments which provide critical data on radiation damage for the transparent partition concept and with some comment on the future of the concept. The desirable experiments fall into two categories. The first consists of neutron and gamma irradiation of quartz samples to an integrated fast flux of $2 \times 10^{18}$ with prompt irradiation cutoff over a temperature range between 200 and $500^{\circ} \mathrm{C}$. These measurements will confirm the interpretation of the data we have presented and will establish the temperature below which radiation induced absorption becomes serious. The second consists of fission foil experiments over an exposure range of $10^{14}$ to $10^{16}$ fission fragments $/ \mathrm{cm}^{2}$ and a temperature range of 600 to $1000^{\circ} \mathrm{C}$. These results will indicate the extent of optical and physical property degradation produced to be expected from fission fragment exposure.

As we presently visualize the concept, it has the following nominal characteristics:

\section{Specific Impulse \\ Engine thrust to weight ratio at a thrust level of $50,000 \mathrm{lb}$}

Fucl Loø8

\section{0 seconds}

0.1

nil

The work accomplished to date indicates that radiation damage to quartz is not the limiting factor for the concept. Aside from very formidable mechanical design problems, the major performance limit seems to be the low engine thrust to weight ratio resulting from convective heating on the outer tube surface. This limit may yield to a more sophisticated approach such as film or transpiration cooling. If this problem can be satisfactorily overcome, the prospect of negligible fuel loss would make the transparent partition concept extremely attractive. 

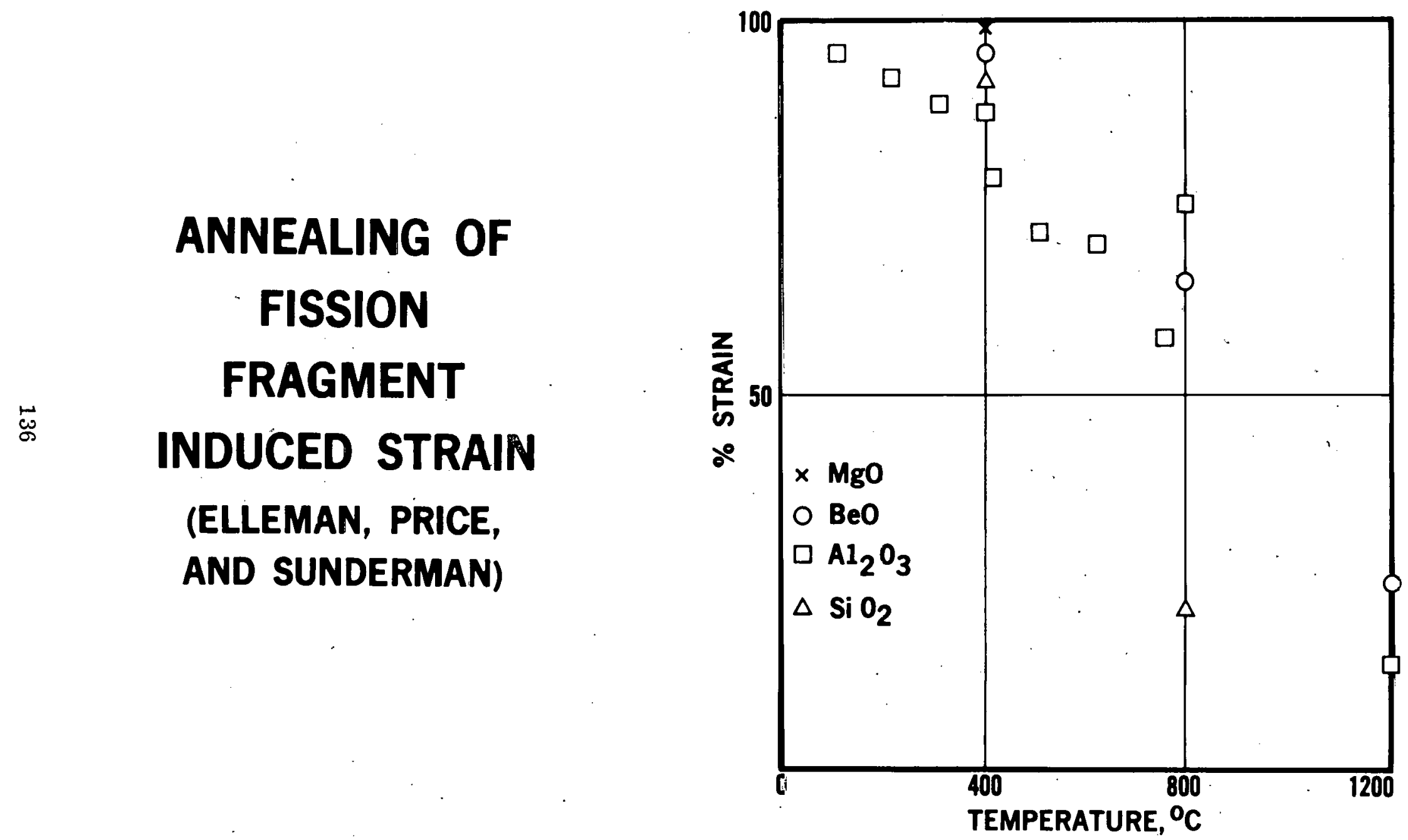

Figure 14 


\title{
WORK ON NUCLEAR LIGHT BULB. AT UAC RESEARCH LABORATORIES
}

\author{
George H. McLafferty \\ United Aircraft Corporation Research Laboratories \\ Hartford, Connecticut
}

We at the United Aircraft Corporation Research Laboratories have been working on gaseous nuclear rockets for approximately five years. Most of this work has been devoted to a concept which we call a vortex-stabilized gaseous nuclear rocket and which we will describe in detail tomorrow. The discussions will cover radiant heat transfer, fluid mechanics, and a number of other subjects. A fairly small percentage of our work has been devoted to a concept that we call a nuclear light bulb and that Douglas and G.E. call a glow-plug. We applied the name of nuclear light bulb when we started work on this concept about four years ago. The results which we will describe today were obtained under contract with the Space Nuclear Propulsion Office.

The particular form of the concept that we have been investigating is illustrated in UAC Slide 1. The configuration is symmetrical about the center line. Gaseous nuclear fuel at a high temperature radiates thermal energy to seeded hydrogen passing axially along a passage on the outer side of the transparent wall. The transparent wall is composed of a double layer of transparent material with a coolant, possibly helium, passing between the two layers and with helium injected inside the wall to keep the gaseous nuclear fuel away from the wall. We decided that a helium film was needed for three reasons. First, a reason mentioned in a preceding discussion, that any gaseous nuclear fuel near the wall would be so opaque that it would be very difficult to pass radiant energy through it. Second, and more important, the fission fragments from fissions occurring near the wall would have an adverse effect on the wall transparency because of the resulting damage to the wall material (which possibly can be annealed out) and because of the resulting coating on the surface (which cannot be annealed out). On this basis, we decided that we would have to employ a film thickness on the 


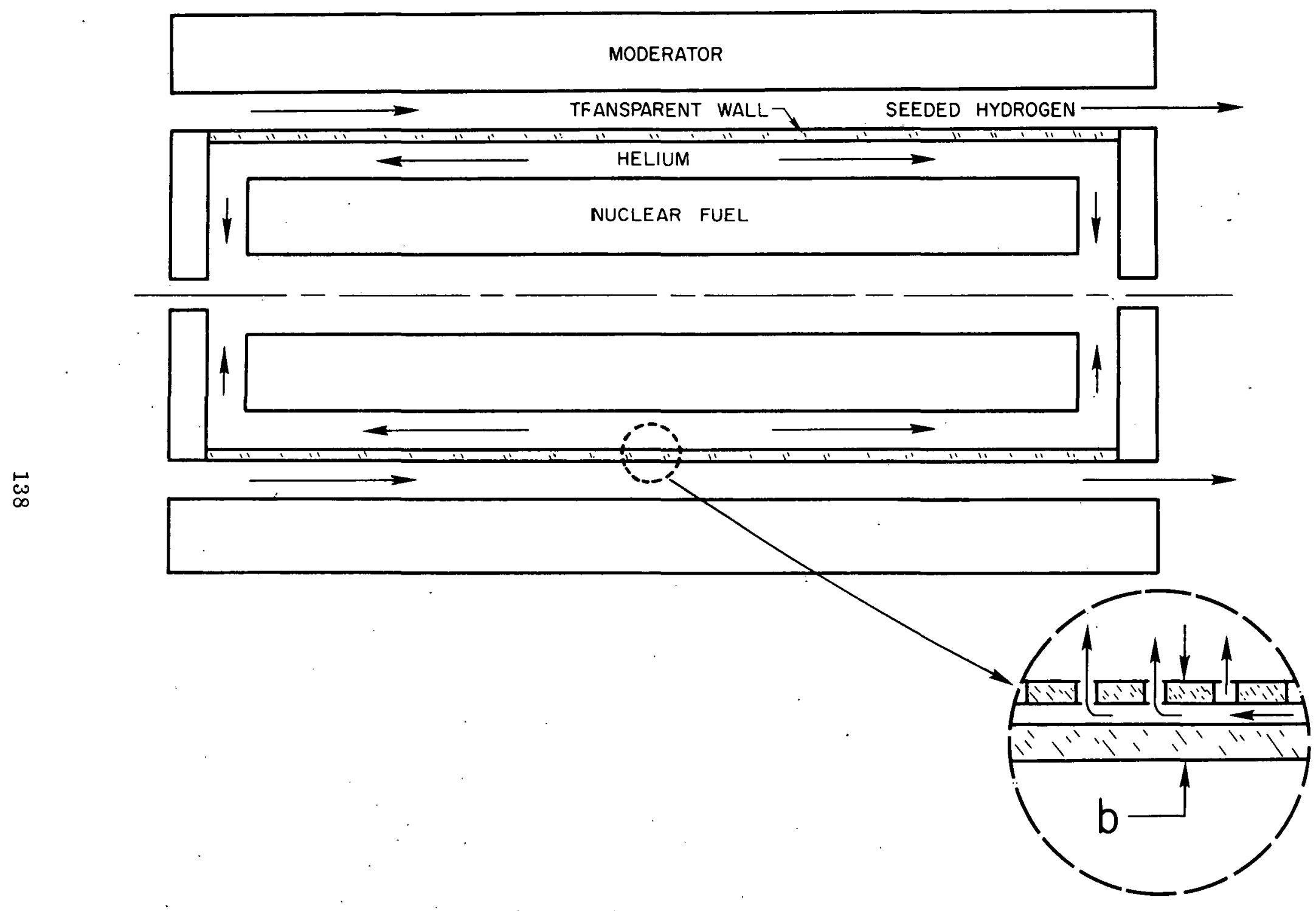

Slide 1 
order of one fission fragment range in depth to keep the fission fragments from hitting the wall. Third, a film coolant would avoid the corrosion of the transparent wall by the gaseous fissionable fuel. We will talk about the fluid mechanics of the flow within the transparent wall and the problem of seeding the hydrogen to make it opaque in tomorrow's sessions.

The main problem in a nuclear light bulb which is different from other gaseous nuclear rocket concepts is the problem of annealing the radiationinduced coloration in the transparent wall material. We've gone through the same literature as Douglas, and have also concluded that the most promising wall material to work with, of those which transmit over a wide range of wavelengths, is undoubtedly fused silica. Therefore, our initial investigations have been concerned with this material. However, we have set ourselves a somewhat higher goal than other investigators as regards minimization of light absorbed. We think that the absorption of light energy in a transparent wall might be on the order of a quarter to a half percent of the energy radiated through the wall. Because of this, we have spent a lot of effort trying to get detailed measurements of the amount of absorption of light energy in several commercial samples of fused silica. We've done this with two spectrophotometers. The first of these is a standard Cary model 14 which we have used only at room temperature, and the second is a spectrophotometer which we've assembled using company funds and which permits us to make measurements from about $1500 \AA$ out to approximately 6 microns at sample temperatures up to 1000 to $1200^{\circ} \mathrm{C}$.

Typical data from one of these spectrophotometers is shown in UAC Slide 2, where we have plotted optical density as a function of wavelength for one sample of Amersil Suprasil taken over a period of three days. The height of the vertical lines represents the scatter of the individual data points. You can see that the scatter of data obtained from our instrument is quite low, which allows us to obtain an extremely good measure of just how much light is actually absorbed in the transparent wall. If we had an average absorption coefficient of $0.01 \mathrm{~cm}^{-1}$ and an effective wall thickness of 0.1 inch $(0.25 \mathrm{~cm})$, approximately 0.25 percent of the light that's passing through the wall would be absorbed.

Both instruments that we use give good repeatability of data, although we had our doubts several months ago because of occasional erratic results. Some of these erratic results are shown on UAC Slide 3. Here we have data from five different samples from the same batch of Amersil Suprasil. There is obviously considerable difference from sample to sample. Some of this is due to strain in the material; we can sometimes anneal out quite a bit of the strain simply by heating the material before we run any tests on it. 
SUPRASIL AMERSIL

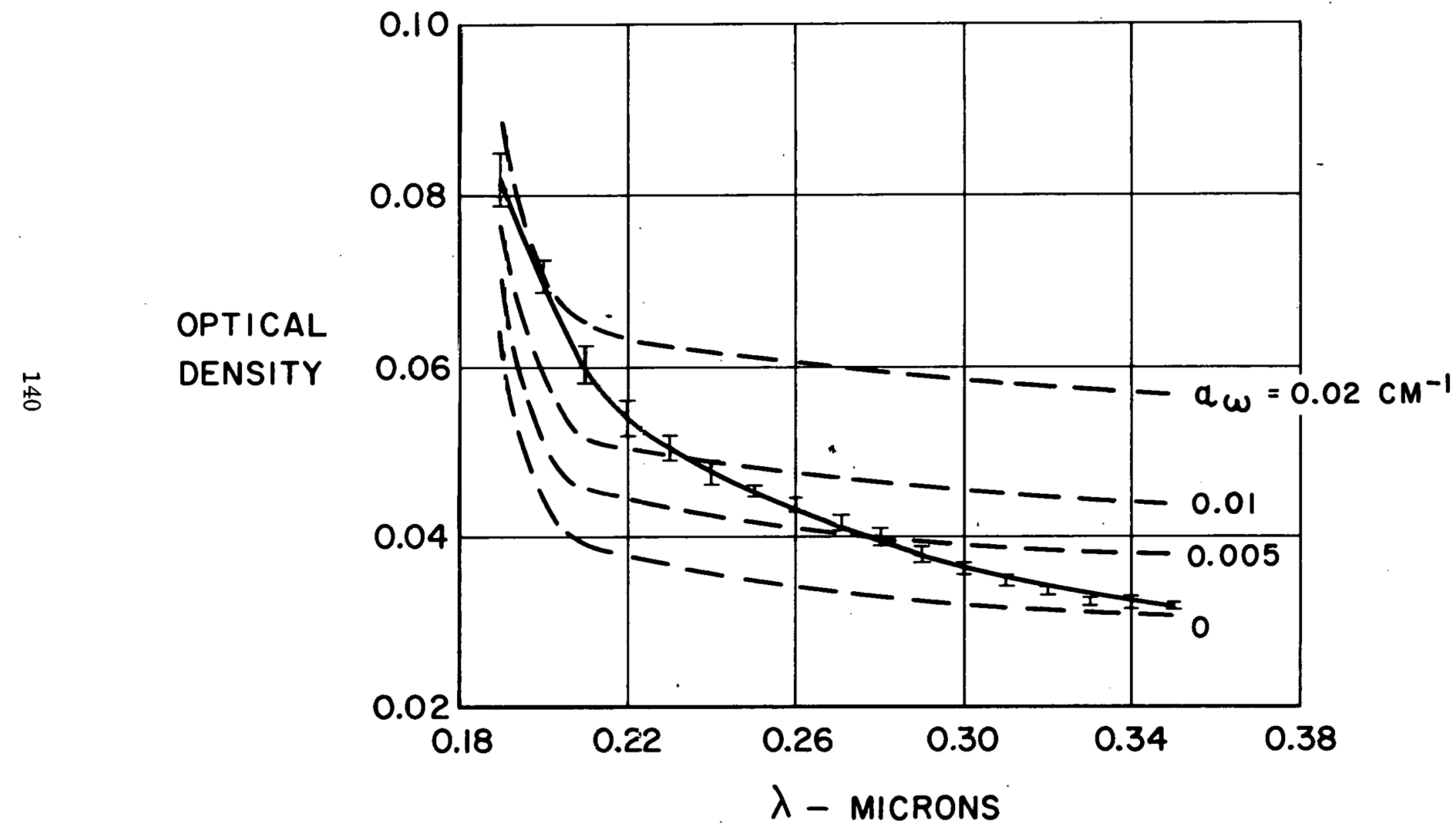

Slide 2 


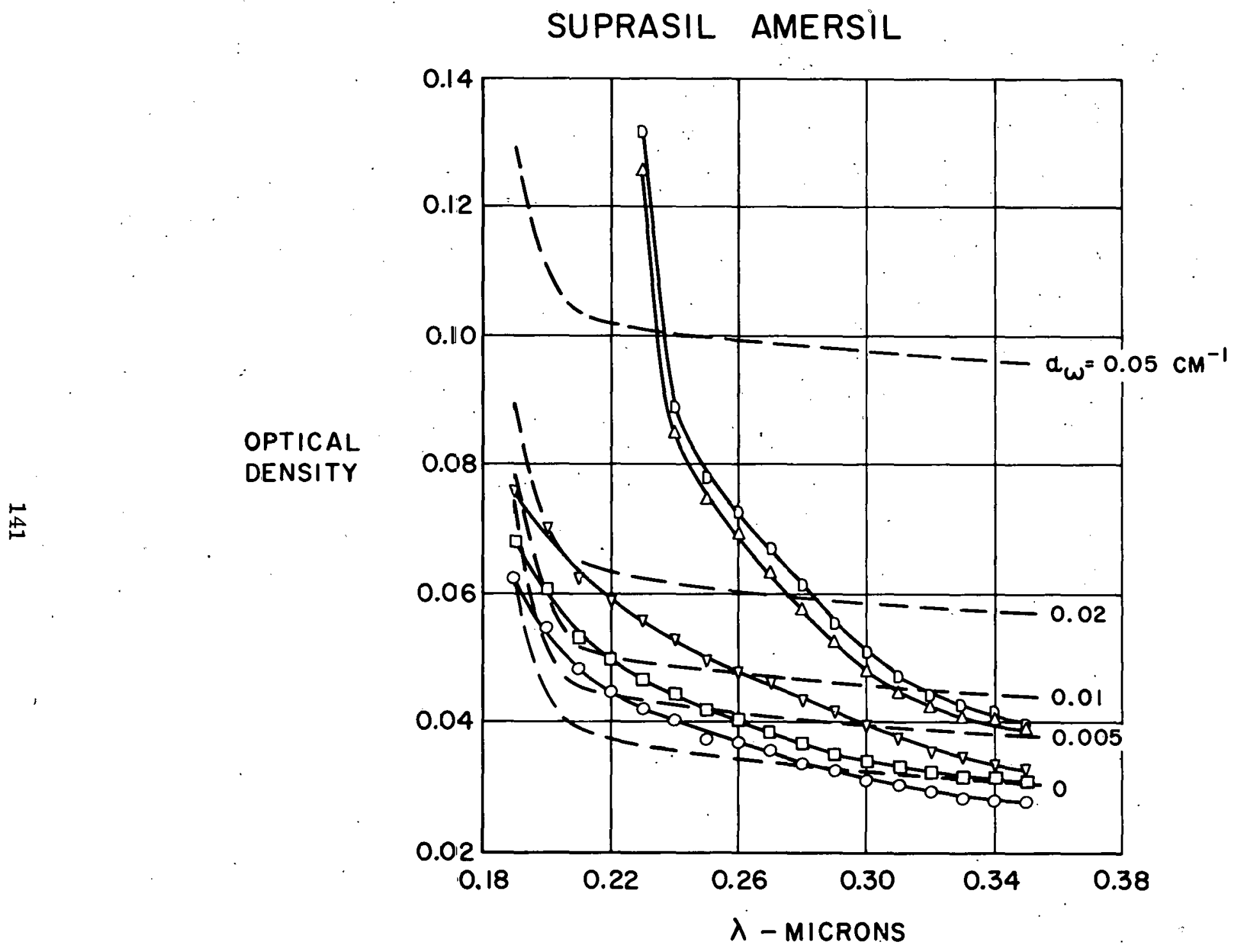

Slide 3 
Sometimes polishing the samples will change their absorption coefficients. We ran similar repeatability tests using five samples of Corning 7940. These results were more repeatable, but still scattered much more than was desirable. The most repeatable samples we have tested were obtained from Thermal American.

On UAC Slide 4 we show results from tests of an unirradiated Infrasil sample in three different parts of the wavelength spectrum. An increase in sample temperature creates absorption in the cutoff regions of both the ultraviolet and the infrared. There appears to be an upward shift in the visible of less than $0.01 \mathrm{~cm}^{-1}$ in absorption coefficient, which represents a change of about a quarter of a percent in the light absorbed in a transparent wall having an effective thickness of 0.1 inch. We have some new data which we didn't have a chance to get in slide form which shows less effect of temperature on the absorption in the visible region than the data in UAC Slide 4. It can be seen from UAC Slide 4 that the sample is quite transparent from approximately 0.3 to 2 microns, which is the region where we have a large fraction of the energy radiated from the fuel in a nuclear light bulb.

UAC Slide 5 shows data similar to Dave Knapp's data on annealing of radiation-induced coloration in transparent materials. We again have plotted optical density as a function of wavelength in microns. After irradiation and annealing only at room temperature, we measured values of absorption coefficient of approximately $0.9 \mathrm{~cm}^{-1}$ in the visible. If you look at a sample, it will look colored and opaque. We also annealed the sample at $500^{\circ} \mathrm{C}$ and $925^{\circ} \mathrm{C}$, and after each anneal brought the sample back down to room temperature and moacured its opaoity. After annealing at $925^{\circ} \mathrm{C}$, the absorption coefficient for wavelengths from 0.3 up to over 2 microns is less than $0.01 \mathrm{~cm}^{-1}$, which means that for a 0.10 -inch-thick wall, the absorption of light is on the order of a quarter of a percent. We think that this data is much more accurate than other available data of this type. Besides the data that Douglas Aircraft has obtained, there has been a lot of this type of data obtained at Brookhaven, Oak Ridge, and in England which shows the same general effect. If a sample is annealed at high temperature, and brought back to room temperature, the induced coloration will be annealed out.

We knew when we built the high-temperature spectrophotometer that, on the basis of available data, the samples should be transparent after being annealed, but did not know that the post-annealing absorption was going to be as. low as we have measured. However, the primary reason we built this spectrophotometer was to obtain data on the rate of annealing of radiationinduced coloration. In the wall of the nuclear light bulb, there is a continual 


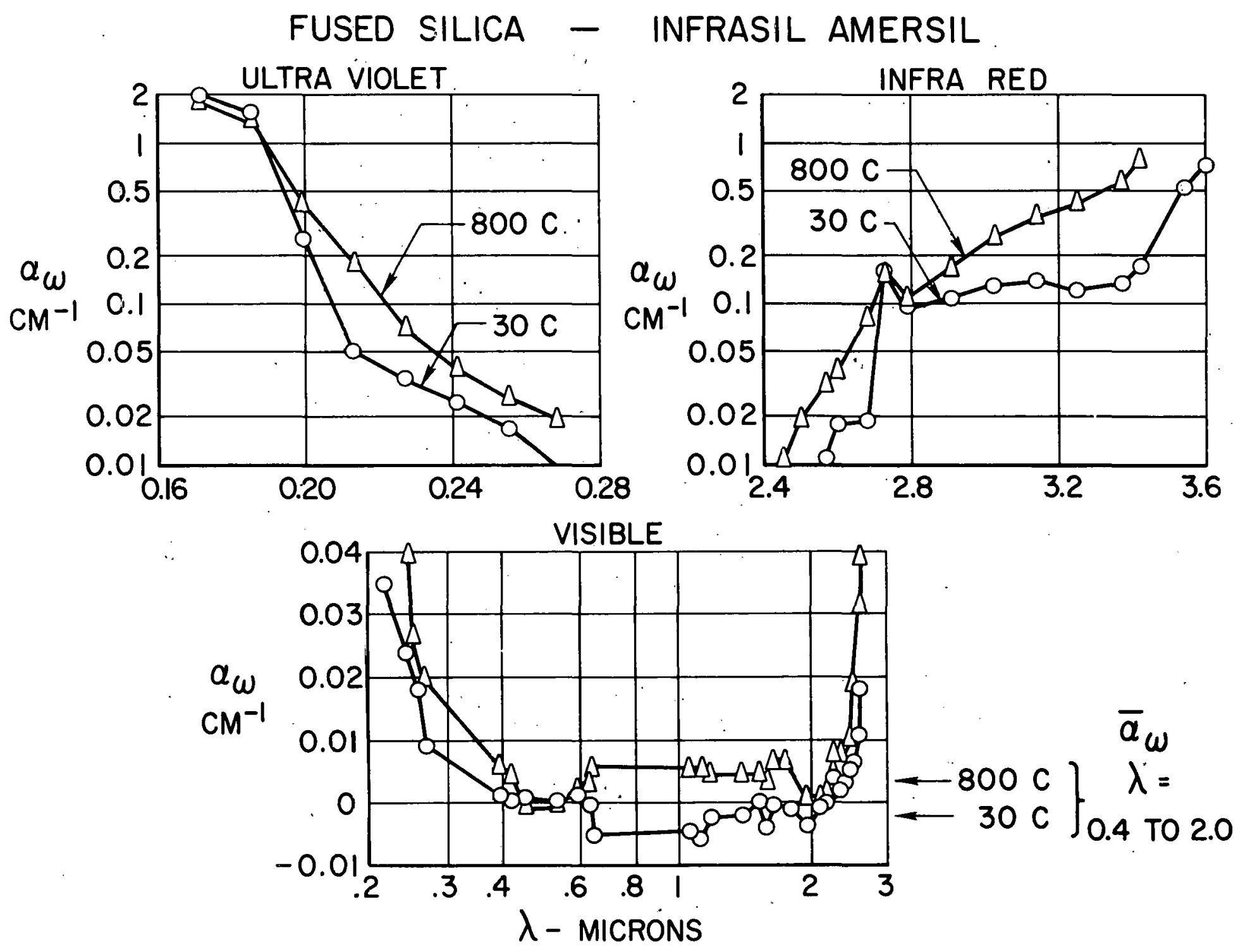

Slide 4 


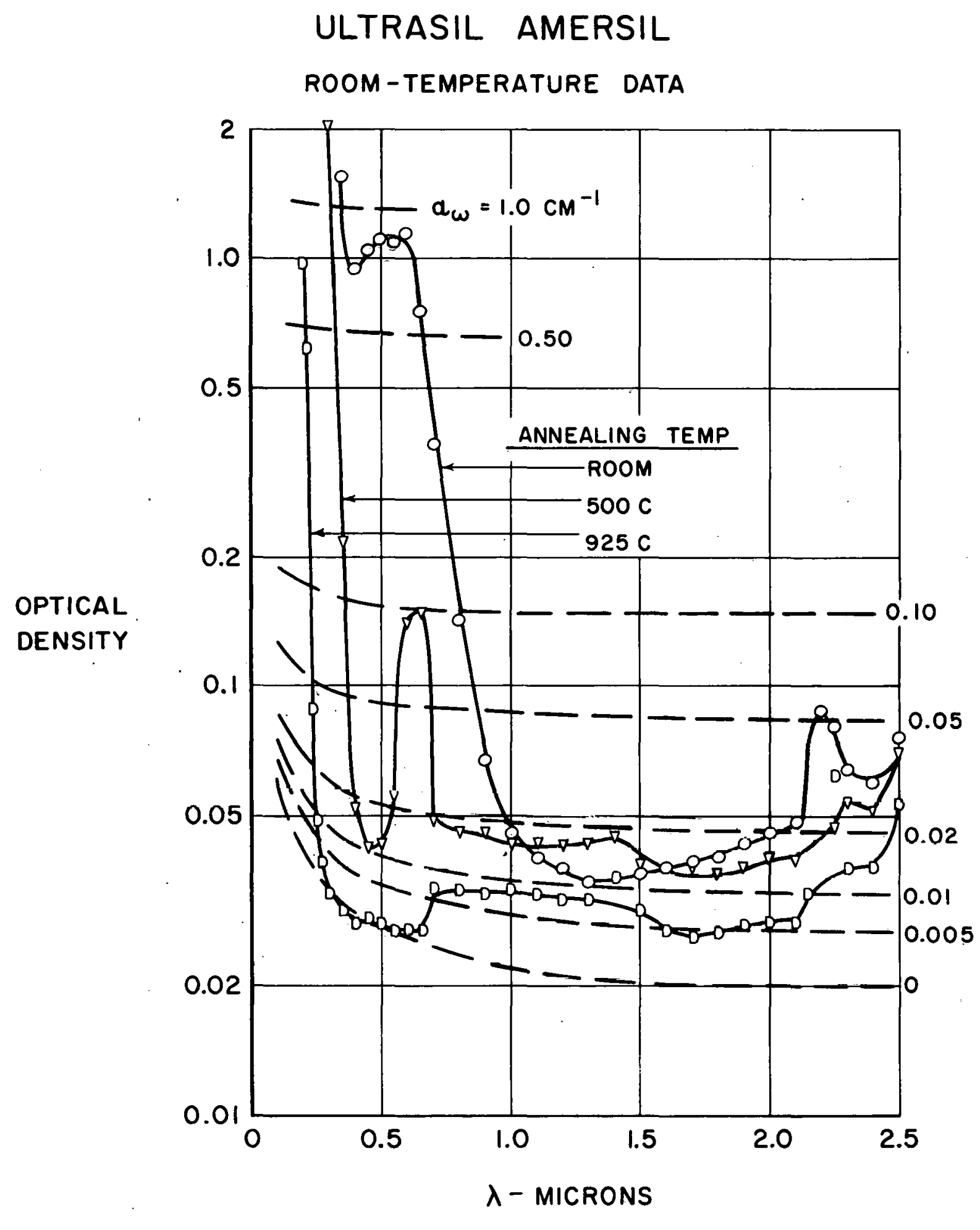

Slide 5 
process of creating color centers and a continual process of annealing these centers. The rate of creation of color centers can be calculated from the data on total coloration fairly accurately. We plan to use two different techniques for determination of annealing rate. The first technique involves step changes in temperature as a function of time. The second technique involves a ramp change in temperature as a function of time. We will then fit the absorption coefficient decay curves with a series of exponentials which will tell us the activation energies associated with the annealing process. Techniques of this kind have been used for obtaining activation energy in other solid materials. Once we have determined activation energies, we can use the activation energy spectrum to calculate the equilibrium amount of absorption that will exist in the wall of a nuclear-light-bulb gaseous nuclear rocket engine. We believe that the use of one of these techniques is much more desirable than trying to measure light absorption in a reactor at the present time. Reactor measurements would be extremely expensive, particularly if high accuracies were desirable. We have found in our three years of experience with this one spectrophotometer that it is extremely difficult to obtain accurate measurements of light absorption in transparent materials.

Question (Holl): For much of your application, don't you have at least one percent of blackbody energy beyond the ultraviolet cutoff? If this is true, what is the point of one-quarter of one percent accuracy in the visible range?

Answer: The answer to this question depends on the radiating temperature of the nuclear fuel. If this temperature is equal to the sun's surface temperature of approximately $10,000^{\circ} \mathrm{R}$, less than one-tenth of one percent of the blackbody energy is at wavelengths shorter than 0.2 microns. A number of materials have ultraviolet cutoffs well below this wavelength. For higher fuel radiating temperatures, the fraction of energy which would be encountered at wavelengths near the ultraviolet cutoff increases. In such an instance, it may be possible to reduce the radiant energy approaching the wall at low wavelengths by seeding the helium coolant region shown in UAC Slide 1. Such a seed would act in a manner similar to air in a spectrophotometer which absorbs ultraviolet radiation and prevents measurement of light transmission in this region.

Question (Taylor): Isn't even a small amount of absorption of radiation going to cause ablation in the transparent wall?

Answer: Any deposition of heat in the wall from any source will eventually cause the wall to ablate or melt if the wall were not continually cooled. The wall must reach a steady-state temperature in which the heat deposition from various sources is equal to the heat removal by the coolant passing through 
the double transparent wall of the nuclear light bulb. We have analyzed the various causes of heating of the transparent wall. Some of these causes of heating can be influenced by the design of the nuclear light bulb. For instance, fission fragment impingement can be eliminated by the use of a film coolant between the gaseous nuclear fuel and the transparent wall. Conduction and convection of heat to the wall also can be substantially reduced by the use of a film coolant. However, certain causes of wall heating can be influenced to little or no extent. For instance, we can do nothing about neutron and gamma heating of the wall. We may or may not be able to do anything about wall heating due to absorption of thermal radiation at certain wavelengths. We have analyzed the various sources of wall heating and have also analyzed the problem of conducting the heat through the wall to a transparent coolant fluid. Dave Knapp in a preceding slide showed values of thermal conductivity of various transparent wall materials. Using such values of thermal conductivity, we have calculated the temperature differences across the transparent wall and the allowable wall thicknesses which will permit us to remove the heat deposited in the wall from various phenomena on a continuing basis.

Question (Taylor): So you're saying that you never actually raised the temperature of the quartz beyond the melting point?

Answer: Correct. We control the temperature of the quartz within a specified range. The transparent wall must be hot enough so that the radiationinduced coloration will be continuously annealed out. However, it must be cool enough so that the transparent wall will not absorb because of opacity induced by its own temperature. In addition, it must be cool enough so that it retains structural strength. These factors determine the permissible range of steady-state operating temperatures for the transparent wall.

Comment (Holl): Our results indicate that several percent of the heat can be removed by conduction through the transparent wall. It does not particularly have to be at the 0.1 percent level.

Comment (Taylor): Let me rephrase what I am worried about. If you subject a piece of transparent material to the light coming from an argon flash, which is at an effective blackbody temperature something like 20,000 or $30,000^{\circ} \mathrm{K}$, you observe that a large part of the energy goes into ablating away this transparent material.

Answer: The distribution of energy for a blackbody radiating temperature of 20,000 to $30,000^{\circ} \mathrm{K}\left(36,000\right.$ to $\left.54,000^{\circ} \mathrm{R}\right)$ would undoubtedly contain a large fraction of energy beyond the cutoff of transparent materials. However, we are talking of lower temperatures where the fraction of the energy in the hard ultraviolet is much less than for temperatures of 20,000 to $30,000^{\circ} \mathrm{K}$. 
Question: Is there any problem of getting the thermal radiation out of the nuclear fuel region?

Answer: We do not have a complete solution of the radiation transport problem in the nuclear light bulb. However, we will talk about solutions of the radiant heat transfer problem in a different kind of gaseous nuclear rocket in tomorrow's classified session.

Comment (Cooper): Let me make several additional comments about this system. $I^{\prime} \mathrm{m}$ almost willing to concede that you can remove a few percent of the energy through the transparent wall, but this may be a crucial problem. There are a number of other problems that bother me and I think bother a number of other people who, we'll say, are more engineering-minded or material-minded. There is a nuclear criticality consideration which is going to force you to a pressure of hundreds of atmospheres, particularly where you have a single cavity.

Answer: The pressure would be approximately the same on both sides of the wall.

Comment (Cooper): Yes, but you have to control the pressure difference between the inside and outside to something like two atmospheres. I think that this is a very difficult problem.

Answer: That's a mechanical problem.

OK, that's all, if that's all that's holding us down, we can fly one next year.

Question: Would you say that the crucial question is in the material? Answer: Yes

Question: What temperature are you talking about getting as a maximum hydrugen temperature?

Answer: This depends on how low an absorption we can get in the transparent wall. The lower the absorption in the transparent wall, the higher the permissible temperature of the nuclear fuel and the higher the temperature of the hydrogen propellant. 


\title{
A TRANSPIRATION-COOLED NOZZLE AS APPIIED TO A GAS CORE NUCLEAR_PROPULSION SYSTEM
}

\author{
Elliot L. Kramer, Research Engineer \\ Sig Gronich, Deputy Manager
}

Presented at Los Alamos Symposium by Dr. D. E. Knapp

Douglas Aircraft Company, Inc.

Santa Monica, California

Published as AIAA Paper No. 64-544, Presented at Cleveland, Ohio, May 1964

\begin{abstract}
The use of mass injection through a porous wall as a cooling technique for axisymmetric nozzles is investigated. The effect of homogeneous mass injection on the heat transfer in a turbulent, compressible boundary layer is analyzed using an integral momentum approach. Approximate formulations of the gaseous thermal radiative and recombination energy contributions are included in the analysis. Coolant requirements are determined for a conical nozzle associated with a million pound thrust gas core system operating at a 300 atmosphere chamber pressure. Hydrogen is assumed to be used for the propellant and coolant. Chamber temperatures range from $7500^{\circ}$ to $20,000^{\circ} \mathrm{R}$, and wall temperatures range from 2000 to $3000^{\circ} \mathrm{R}$. The degradation of specific impulse, which is a result of expelling the coolant at a lower total temperature than that in the chamber, is found to be as high as $7 \%$ at. $20,000^{\circ} \mathrm{R}$ for a wall temperature of $3000^{\circ} \mathrm{R}$.
\end{abstract}




\section{HIGH-TEMPERATURE GAS INTERACTIONS \\ Jerry Grey \\ Princeton University \\ Princeton, New Jersey}

\section{INTRODUCTION}

All concepts in the field of gaseous-core nuclear rockets deal with high-temperature gas interactions in one form or another. Experimental and theoretical studies of the interaction between dense, partly ionized gases has been under way at Princeton since 1957, in the areas of high-temperature gas mixing in the both laminar and turbulent modes, heat transfer, transport properties, radiation, and the general problem of experimental techniques in this regime. The range of parameters has extended to temperatures of about $26,000^{\circ} \mathrm{F}$ at pressures up to one atmosphere using argon, helium, and nitrogen as test gases. Although the general problem is of direct relevance to all gaseous-core rockets, the particular experimental device used on these studies has special significance in the coaxial geometry of the gaseous-core system discussed in Reference 1.

The principal contribution of the research tasks to be discussed in this paper has been in the field of high-precision experimental measurements under previously unmeasurable conditions. These measurements are designed to provide much-needed evaluation of the volumes of theoretical work now available in this operating regime, which has heretofore had no experimental substantiation. Many of the results of this program have been discussed in previous publications, and the purpose of the present paper is to summarize briefly the problems, methods, and results. Specific references cited in each area may be consulted for more detailed information.

1. Weinstein, H., and Ragsdale, R. G., "A Coaxial Flow Reactor--A Gaseous Nuclear-Rocket Concept," ARS Paper No. 1518-60, December 5-8, 1960. 


\section{DESCRIPTION OF FACILITIES}

The high-temperature gases required for these studies were produced by an $80-\mathrm{kw}$ DC arcjet torch manufactured by Thermal Dynamics, Inc. This device is used to generate a high-temperature jet of argon gas in either the laminar or turbulent mode at very low subsonic speeds (Mach number <0.1, or velocities of the order of 300 to $1000 \mathrm{ft} / \mathrm{sec}$ ). Surrounding the hot argon core is an annular passage through which a cool gas may be injected coaxially with the argon jet, as shown in the configuration diagram of Figure 1. This annular flow may consist of either helium or nitrogen, and in the past only very low velocities (up to the order of $50 \mathrm{ft} / \mathrm{sec}$ ) have been used. However, forthcoming tests in which coaxial gas velocities of the same order as those of the hot central core are being considered.

The entire flow field exhausts into a test chamber equipped with transparent windows for viewing and photography, and then into a 1000-gallon pressure tank which can either be evacuated to a pressure of approximately 1 inch $\mathrm{H}_{\mathrm{g}}$ absolute or filled with any desired gas environment.

The arcjet was powered by a $150 \mathrm{KVA}$ marine diesel generator operating four Westinghouse RA-2, 800-ampere selenium rectifiers. Water delivered by a Pesco gear pump at a pressure of 300 psi was used to cool the torch and leads. The system was fully instrumented to provide net coolant power as well as instantaneous current and voltage readings.

Special-purpose instrumentation used with this device consisted of an optical system operating on the schlieren principle, using a high-power spark flash source capable of overcoming the luminosity of the arcjet "flame," a water-cooled calorimetric sampling probe having its own pressurized cooling system and two-dimensional drive, and a water-cooled total radiation probe collimated to provide local measurements at any point within or external to. the plasma region. Both the calorimetric probe and radiation probe are capable of steady-state operation in the arc-heated gas, as will be discussed in further detail below.

Utilizing this equipment it was possible to obtain and survey laminar flows of argon at peak temperatures of approximately $25,000^{\circ} \mathrm{F}$ and 1 atmosphere, corresponding to about $20 \%$ ionization, at velocities up to $500 \mathrm{lt} / \mathrm{sec}$ with a jet diameter of approximately 1 centimeter. Turbulent jets of approximately 2 centimeters in diameter at approximately the same conditions were also obtained.

Although argon, helium, and nitrogen are not the gases intended for use with most gaseous-core nuclear rocket concepts, they nevertheless provided 


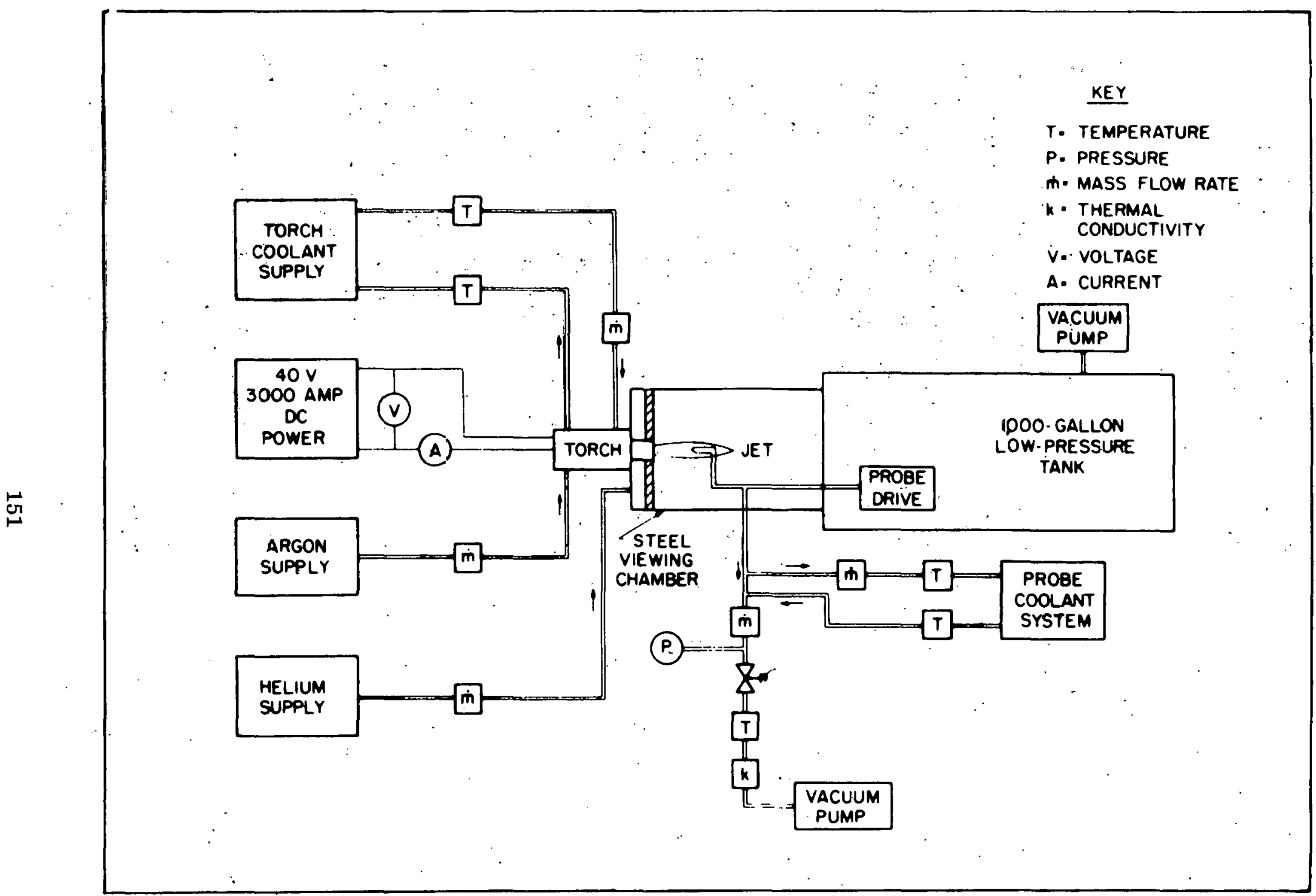

Figure 1. Diagram of Overall Mechanical System 
the simplest possible theoretical description of the flows under consideration with regard to transport properties, excited states, ionization, etc., and therefore were best suited to the evaluation of theoretical descriptions of the interactions considered.

\section{CALORIMETRIC SAMPLING PROBE}

The fundamental item of instrumentation most essential to the study of the pressure and temperature regimes of interest is the multi-purpose probe $^{2,3}$ shown in Figure 2. This is a water-cooled probe, having an overall outer diameter as small as $1 / 16$ inch, which is used to measure the enthalpy, velocity, and chemical composition of the gases inside the arcjet exhaust. Enthalpy is measured by a simple calorimetric principle: the probe is water-cooled, with high-pressure coolant entering the outer jacket of the probe shown in Figure 2, proceeding down to the probe tip, around a baffle, and returning through the iriner jacket. The central tube is open to the hot gas flow. Thermocouples are provided at the water inlet and outlet as shown in the figure. The central tube may be opened or shut by a valve located downstream, thereby permitting a gas sample either to flow through the probe (drawn through by a vacuum pump) or to be stagnated within the probe. In order to measure the enthalpy, the valve is first closed and measurements of water flow rate and water temperature rise are made with the probe inserted into the hot gas stream. The valve is then opened, permitting the gas sample to flow through the probe; and the same measurements are repeated, together with the enthalpy of the cooled gas sample as it leaves the probe (using the thermocouple shown in Figure 2) and the flow rate of the gas sample. The difference between the energy extracted from the probe between the "flow" and "no-flow" cases (referring to the flow of the gas sample) then provides a measurement of the energy extracted from the gas sample. itself. This technique of using a tare measurement thus eliminates errors due to both the external cooling requirements and radiation. The only requirement is that the flow configuration at the tip of the probe not be significantly different for the "flow" and "no-flow" cases, a condition which has been verified adequately by a number of experiments. ${ }^{4}$. The impact

2. Grey, J., Jacobs, P. F., \& Sherman, M. P., "Calorimetric Probe for the Measurement of Extremely High Temperatures," Rev. Sci. Instr. $\underline{33}$, July 1962, pp. 738-741.

3. Grey, J., "Sensitivity Analysis for the Calorimetric Probe," Rev. Sci. Instr. 34, August 1963, pp. 857-859.

4. Grey, J., "Thermodynamic Methods of High Temperature Measurement," to be published in ISA Transactions, January-February 1965. 


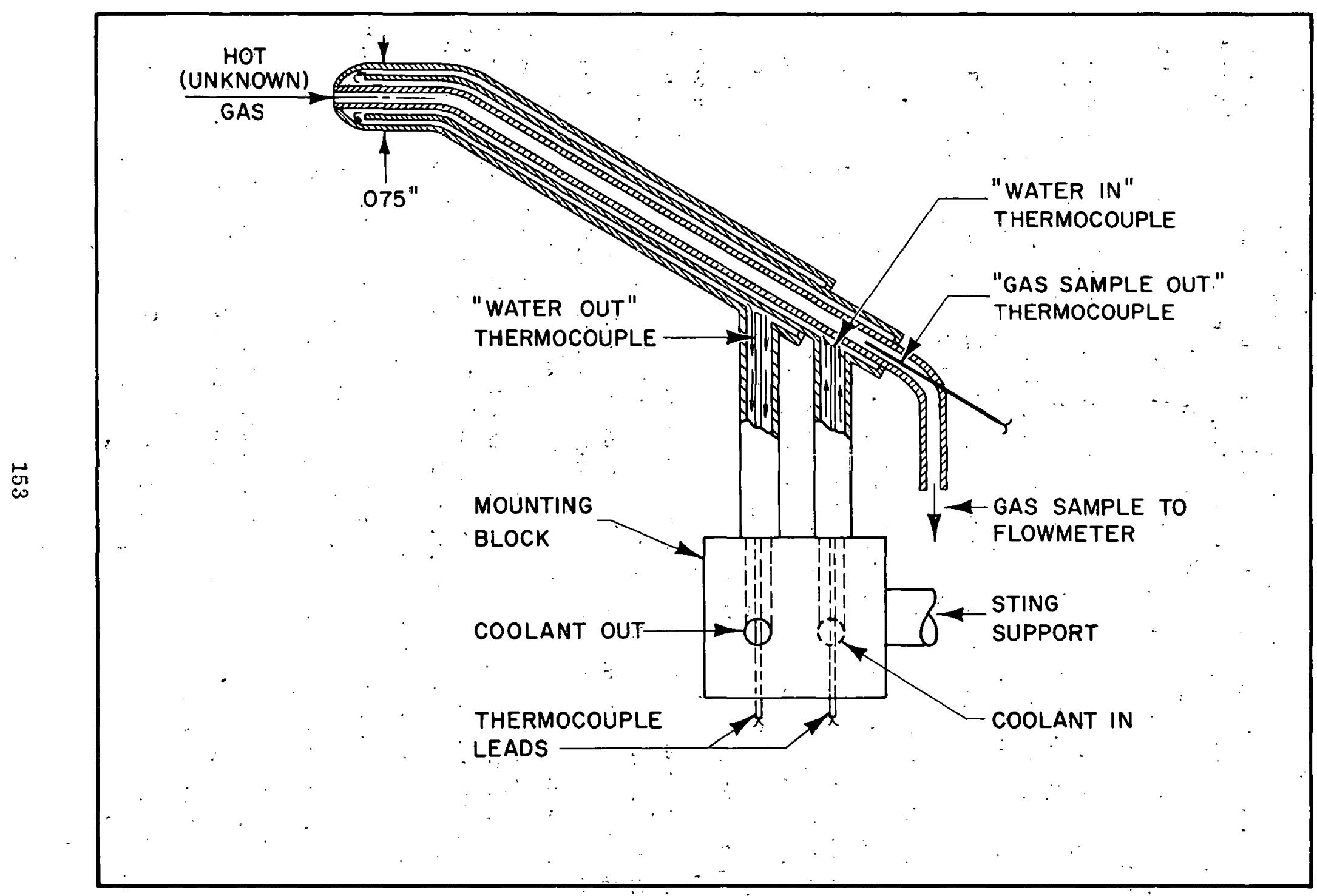

Figure 2. Calorimetric Probe 
pressure of the gas at the probe tip may be measured during the "no-flow" point; and when the gas sample is extracted during the "flow" point, its composition may be measured by a suitable device. A typical instrumentation system for use with this probe is shown in Figure 3, and a photograph of the probe facing a 3/4-inch-diameter arcjet nozzle is shown in Figure 4. Other configurations, including up to $90^{\circ}$ bends in the probe itself, are shown in Figure 5.

Calibrations of this probe are performed by energy balance methods across an operating arcjet; that is, the net power of the gas issuing from the arcjet nozzle was compared with the result of integrating a 15-point radial survey across the exit plane of the arcjet nozzle using the probe. Results are shown in Figure 6, indicating a $3 \%$ standard deviation for the $1 / 8$-inch probe with an average error of about $0.5 \%$. Details of this work are reported in Reference 2 .

Application of this probe to a typical turbulent arcjet mixing case is shown in Figure 7, in which each curve represents a radial profile of enthalpy (converted to temperature by the assumption of equilibrium flow). Each curve on Figure 7 represents one radial survey, the various curves being made at different axial positions. Note that the gradients of Figure 7 are on the order of $50,000^{\circ} \mathrm{F}$ per inch, indicating the excellent resolution possible even with the comparatively large 1/8-inch probe. Another example of the resolution capability of this probe is shown in Figure 8, which illustrates the mixing boundaries between a hot turbulent argon core with a cool coaxial turbulent helium flow. Note the clear definition of the different boundarles, corresponding to concentration, momentum, and energy. The data of Figure 8 were obtained from a series of radial surveys of the type indicated in Figure 7. The solid lines on Figure 8 represent the results of a simple integral analysis based on the experimental nozzle exit-plane profiles and propagated downstream by conventional analytical techniques. Details of this turbulent study, carried out over the rangc 12,000 to $26,000^{\circ} \mathrm{F}$, are discussed in References 5 and 6.

The 1/16-inch probe shown in Figure 5 has been used to conduct similar studies in a 1-centimeter-diameter laminar arcjet with approximately the same type of behavior as indicated in the turbulent studies of References 5

5. Grey, J., and Jacobs, P. F., "Turbulent Mixing in a Partially Ionized Gas," Princeton University Aeronautical Engineering Report No. 625, September 1962.

6. Grey, J., and Jacobs, P. F., "Experiments on Turbulent Mixing in a Partly-Ionized Gas," AIAA Journal 2, March 1964, pp. 433-438. 


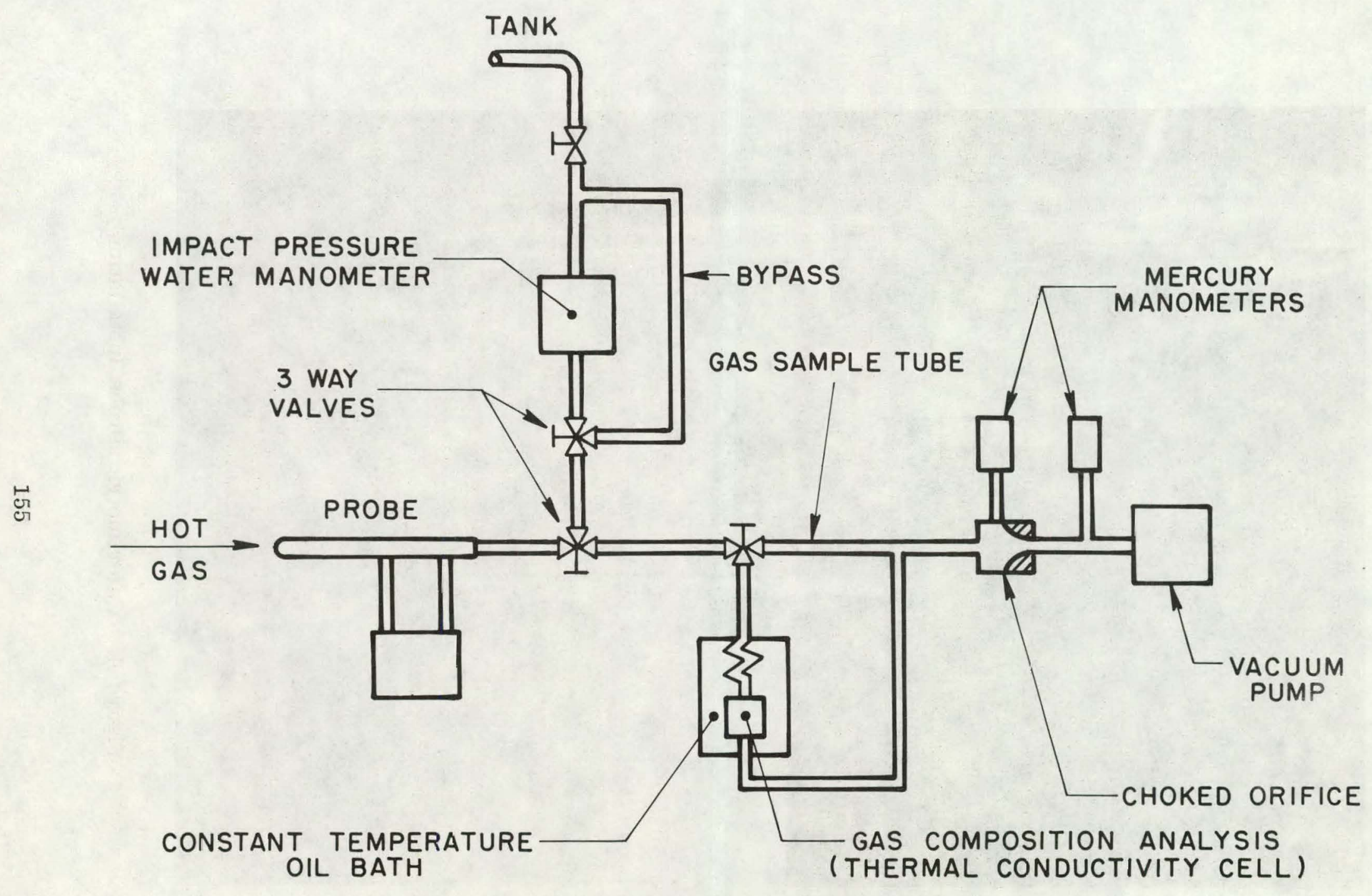

Figure 3. Calorimetric Probe Gas Sample System 


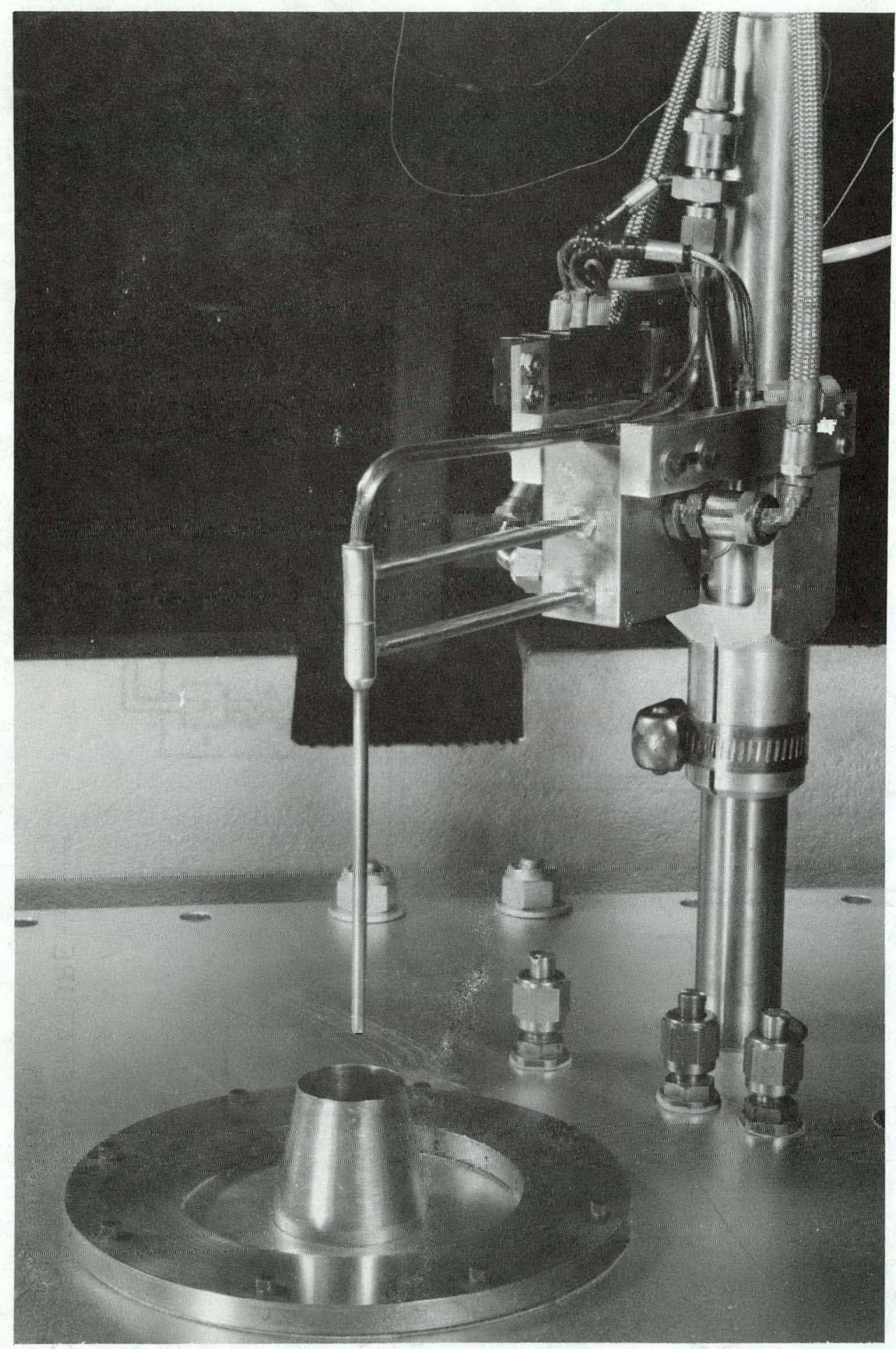

Figure 4. Calorimetric Probe Installation 

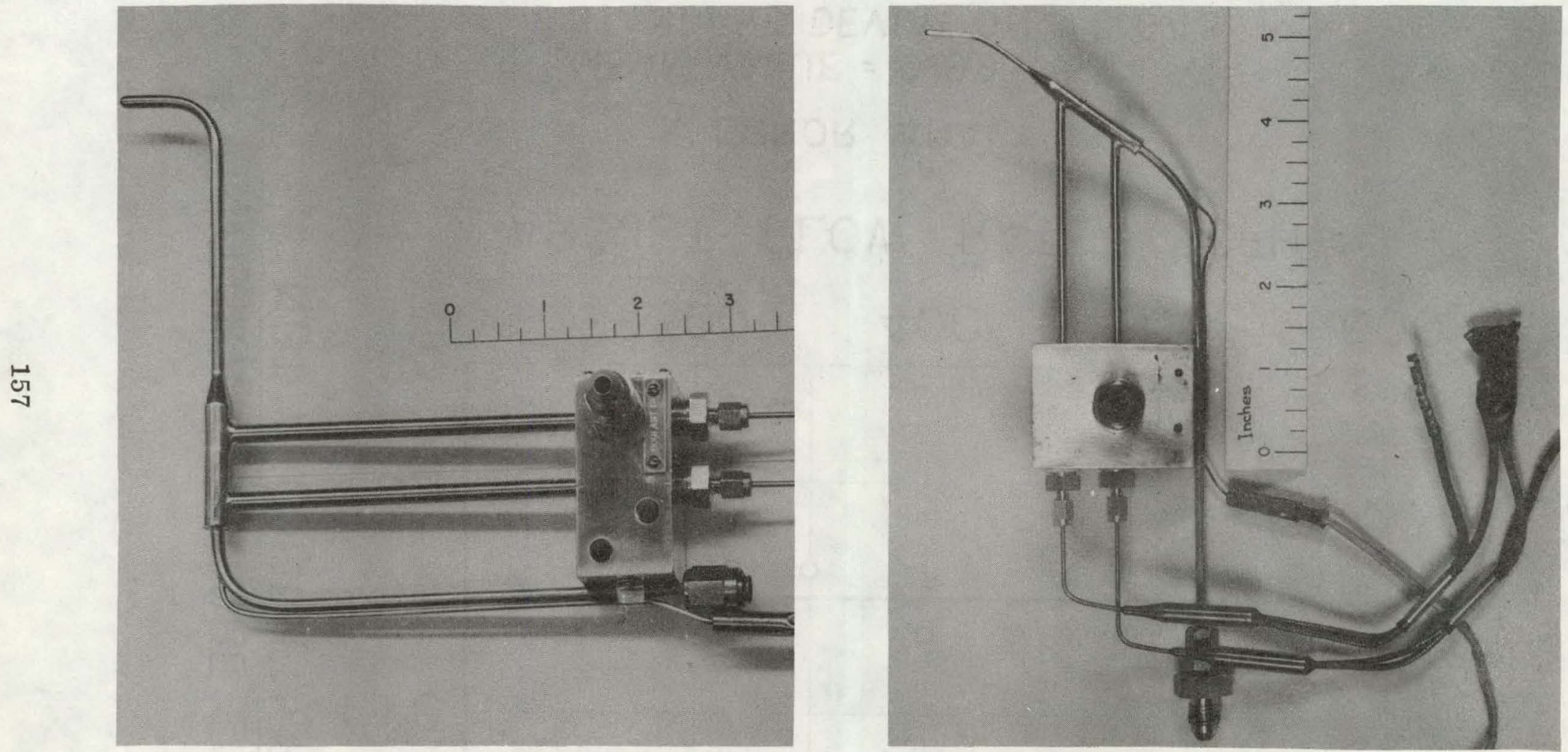

Figure 5. Bent Configurations of Tare-Measurement Calorimetric Probe 


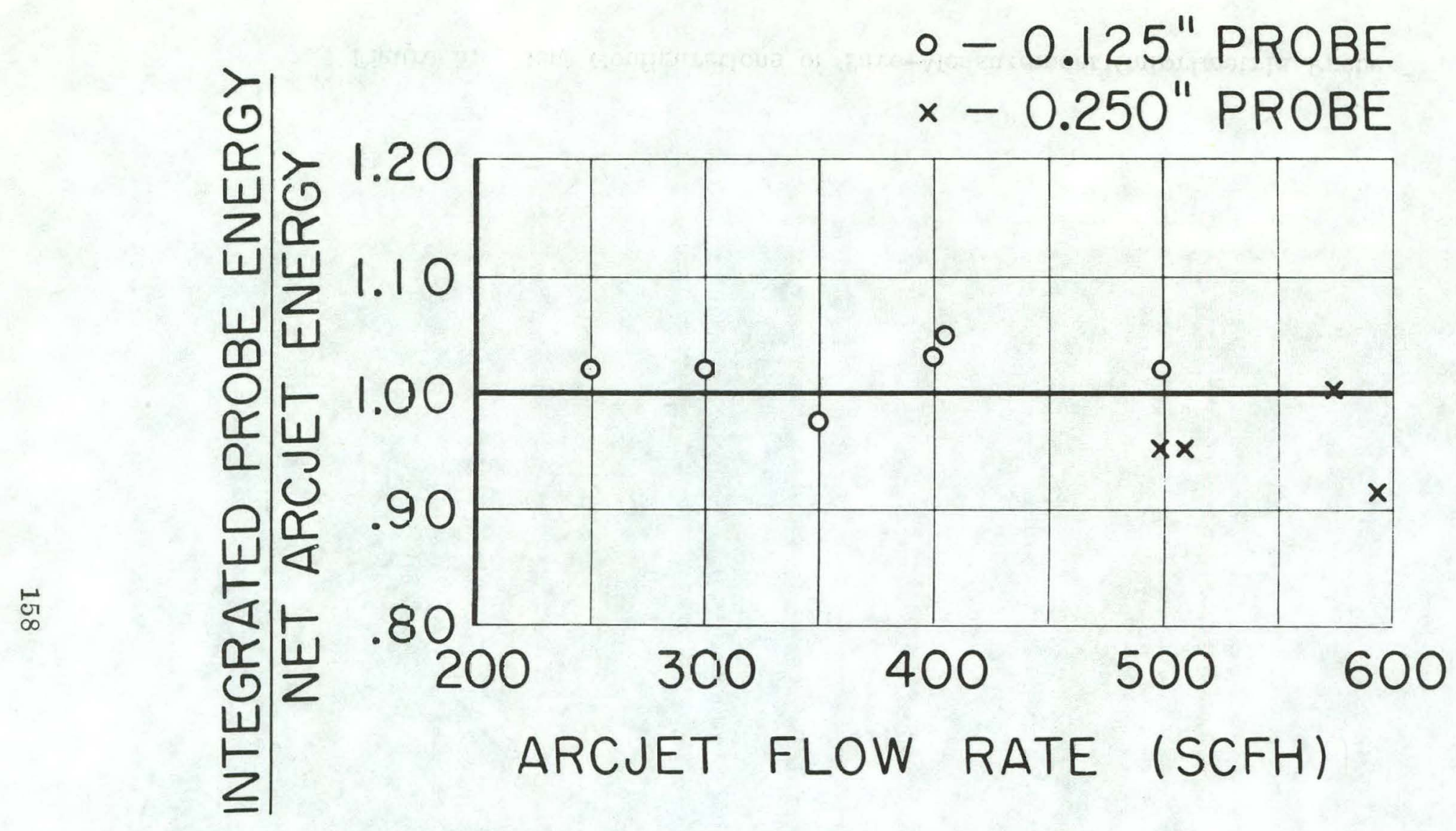

ERROR ANALYSIS:

MEAN VALUE $=0.993$

STANDARD DEVIATION* $=0.043$

* 0.03 FOR 0.125 "PRÖBE ALONE

O.IO FOR 0.250"PROBE ALONE

Figure 6. Results of Energy-Balance Calibrations 


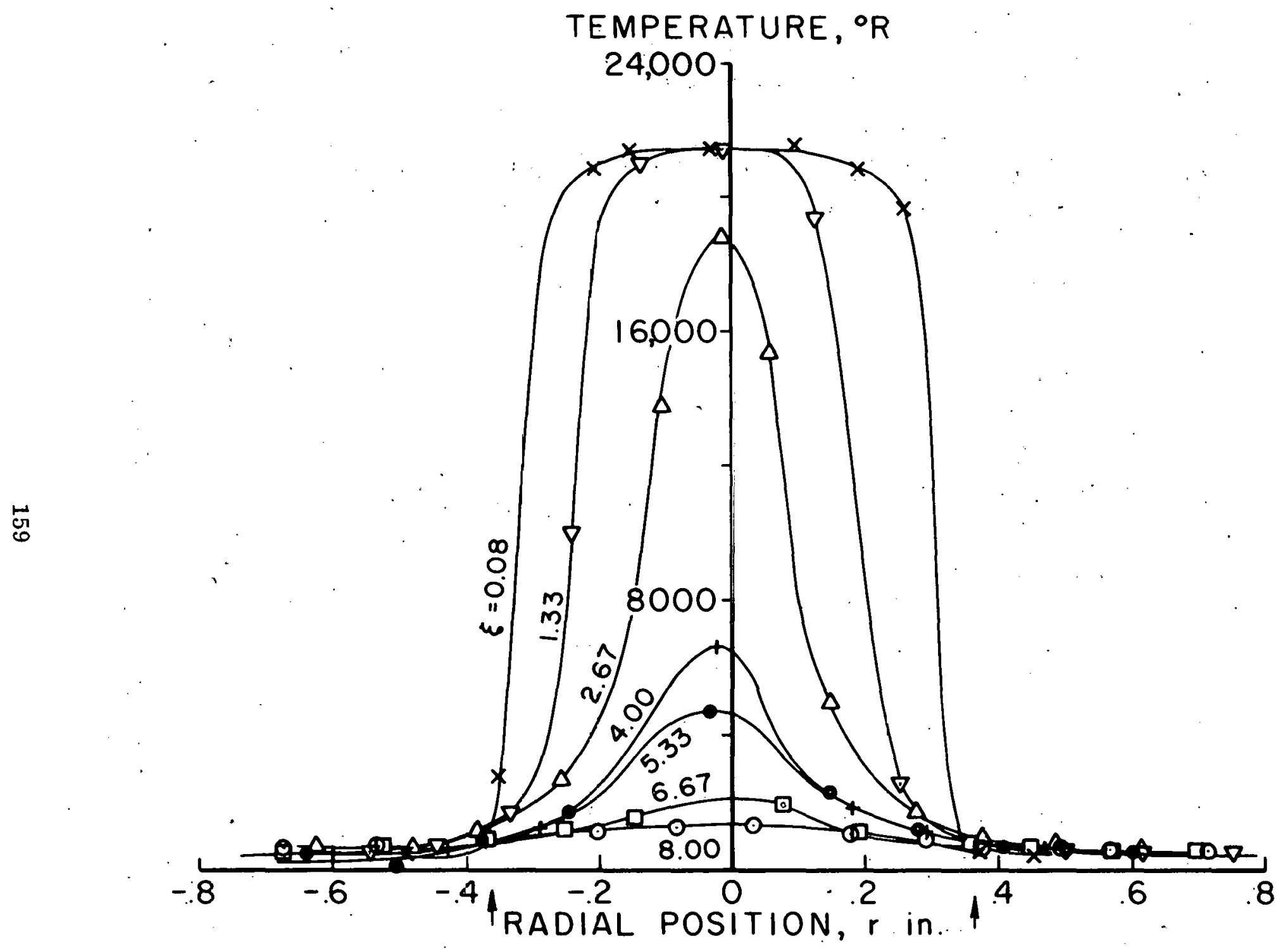

Figure 7. Typical Radial Temperature Profiles (Experimental) 


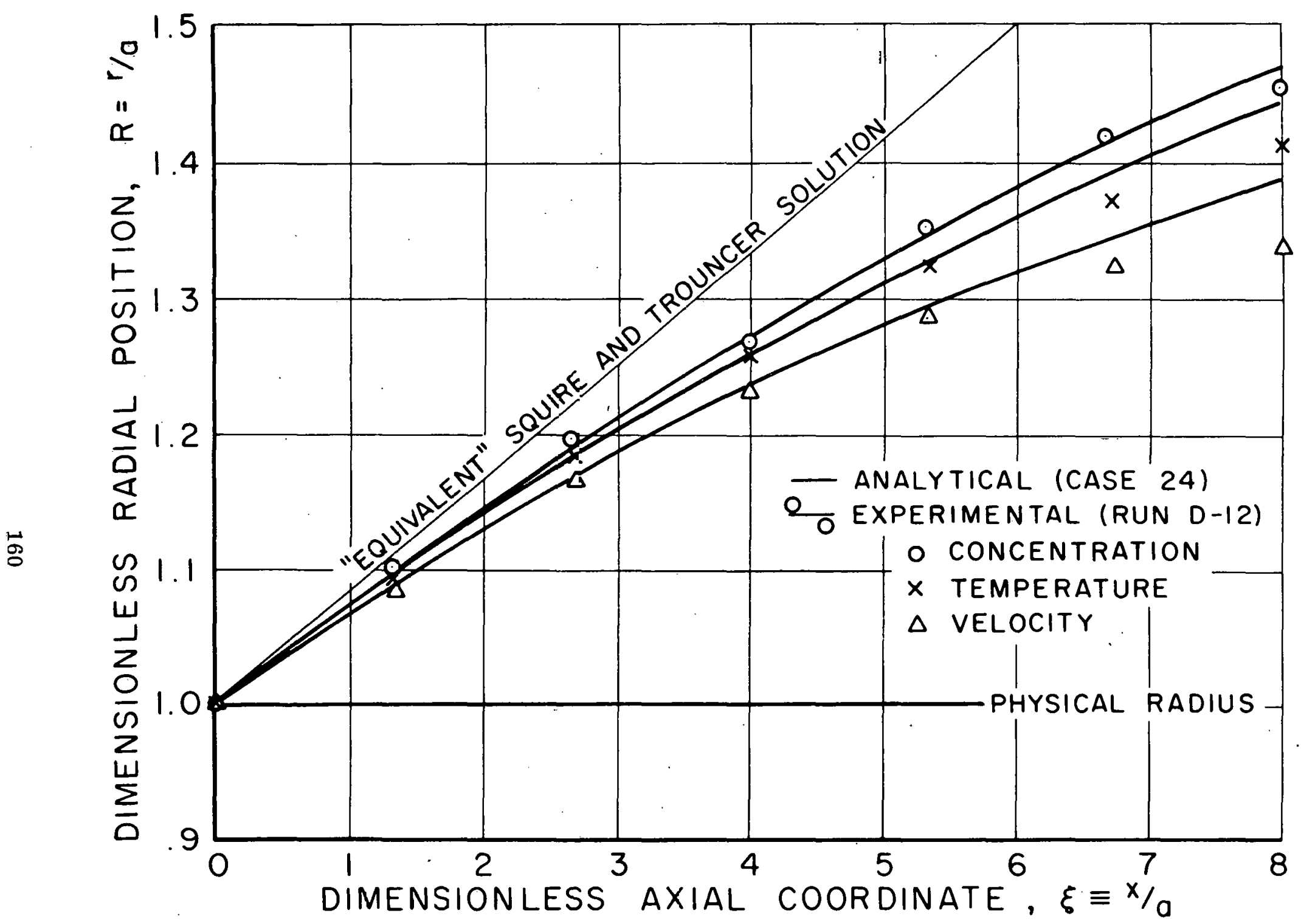

Figure 8. Typical Radial Spreading of Velocity, Concentration, and Temperature With Respect to Axial Position 
and 6. The theoretical development of laminar flow, of course, is more readily obtainable than for turbulent flow; and reasonably good correlation with theory was obtained, based on transport property calculations in the extremely high-density gases under consideration here. The method of calculating transport properties of 1-atmosphere mixtures of cool helium and partly ionized argon are described in detail in Reference 7, and results of the first set of laminar mixing studies are reported in Reference 8 . A marked improvement has been recently obtained in the quality of laminar data by utilizing a more modern arcjet generator incorporating much more accurate control of both electrode relative positions and location of the anode contact point. These studies, which show far better consistency than those discussed in Reference 8 , are presented in detail in Reference 9.

\section{TOTAL RADIATION MEASUREMENTS}

One of the major problems encountered in the study of extremely hightemperature gases, as indicated by a number of the papers in the present volume, is that of radiation. Even with the argon gas used in the Princeton facility, which is far simpler than the uranium or other fissionable gases required for the various gaseous core concepts, the problem is extremely complicated from an analytical point of view. In fact, calculations of radiated energy from gases in this temperature and pressure range have often differed by more than an order of magnitude. It was of some interest in the Princeton program, therefore, to determine the amount of energy radiated from the arcjet in order to more accurately define its effect on the detailed laminar and turbulent mixing processes; and, consequently, both theoretical and experimental studies were conducted to determine the absolute magnitude of this radiated energy.

Considering first the possible energy exchange process that can occur in a cooling argon plasma, we have, as illustrated by Figure 9, a number of

7. Sherman, M. P., and Grey, J., "Calculation of Transport Properties of Mixtures of Helium and Partly-Ionized Argon," Princeton University Aeronautical Engineering Report No. 673, December 1963.

8. Sherman, M. P., and Grey, J., "Interactions Between a Partly-Ionized Laminar Subsonic Jet and a Cool Stagnant Gas," Princeton University Aeronautical Engineering Report No. 707, November 1964.

9. Grey, J., Williams, P. M., and Fradkin, D. B., "Mixing and Heat Transfer of an Argon Arcjet with a Coaxial Flow of Cold Helium," Princeton University Aeronautical Engineering Report No. 710, November 1964. 


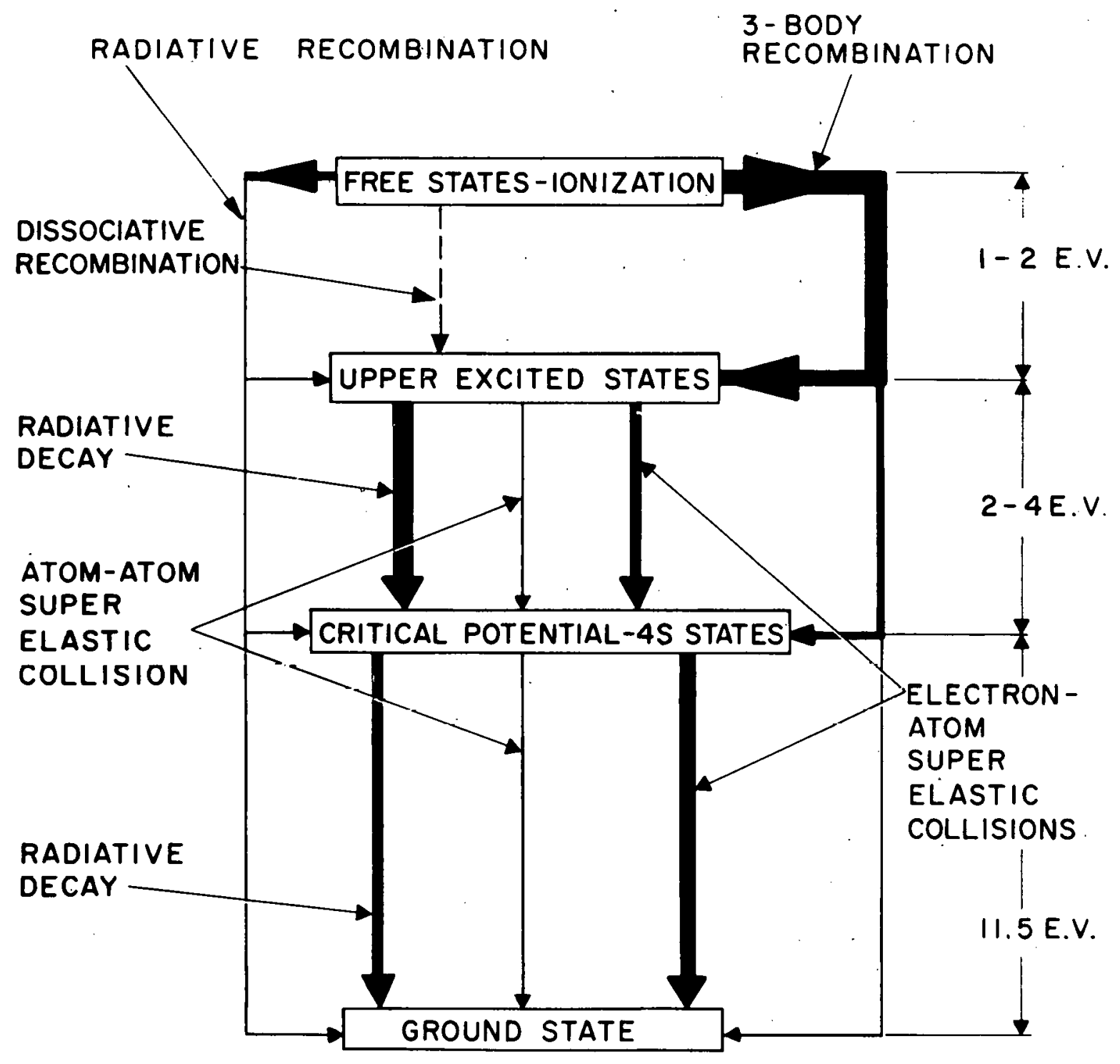

Figure 9. Net Recombination Processes in a Cooling Argon Plasma 
different mechanisms. It is clear from this figure, which is self-explanatory, that even in a comparatively simple gas such as argon the radiation problem is not at all simple in terms of the energy transport mechanisms. Utilizing the most up-to-date information on cross sections for the various recombination and energy decay processes, it was found that the uncertainty in calculating the total energy radiated from argon is perhaps a factor of three. It might be expected that in much more complicated gases composed of fissionable materials and their compounds, this uncertainty could be considerably larger.

Theoretical calculations of the radiated energy from the particular case of the argon arcjet used in the Princeton facility are discussed in Reference 10. These calculations were checked by a series of experimental measurements made with a simple water-cooled radiation probe (Figure 10) capable of insertion directly into the hot arcjet region. This probe consists of a water-cooled tube with a collimating orifice located just inside its open end and a radiation measurement device (in our case, a commerical vacuum thermopile) at the other end, to measure the magnitude of the radiated energy incident upon its face.

Since we are concerned with processes radiating strongly in the ultraviolet (e.g., high-energy free-bound transitions), it was necessary to use a fluid within the collimating tube, as well as a cover plate for the thermopile, which would not absorb in the ultraviolet range. A helium bleed was therefore introduced into the collimator, since helium has extremely low absorption in the ultraviolet, and a lithium fluoride sealing window was used in the thermopile. With this configuration, a transmissivity of the probe device was achieved which extended down to approximately 1100 angstroms, thereby including much of the dominant regime of free-bound transitions.

Water-cooling of the probe provided the capability for insertion directly into the arcjet, thereby permitting determination of the optical thickness of the gas being observed. Note that with the collimating tube, the thermopile "sees" only a thin pencil of space directly facing the end of the collimator, and therefore can be used quite well for localized measurements of radiation.

Figure 11 shows the various types of radiation surveys made in the arcjet environment. The first series of surveys, in which the collimating tube was mounted at right angles to the "flame" and was traversed in the axial direction, provided an axial survey of the radiation being emitted

10. Grey, J., Sherman, M. P., and Jacobs, P. F., "Measurements of Arcjet Radiation with a Cooled Collimated Probe," IEEE Trans, on Nuclear Sci., Vol. NS-11, January 1964, pp. 176-186. 


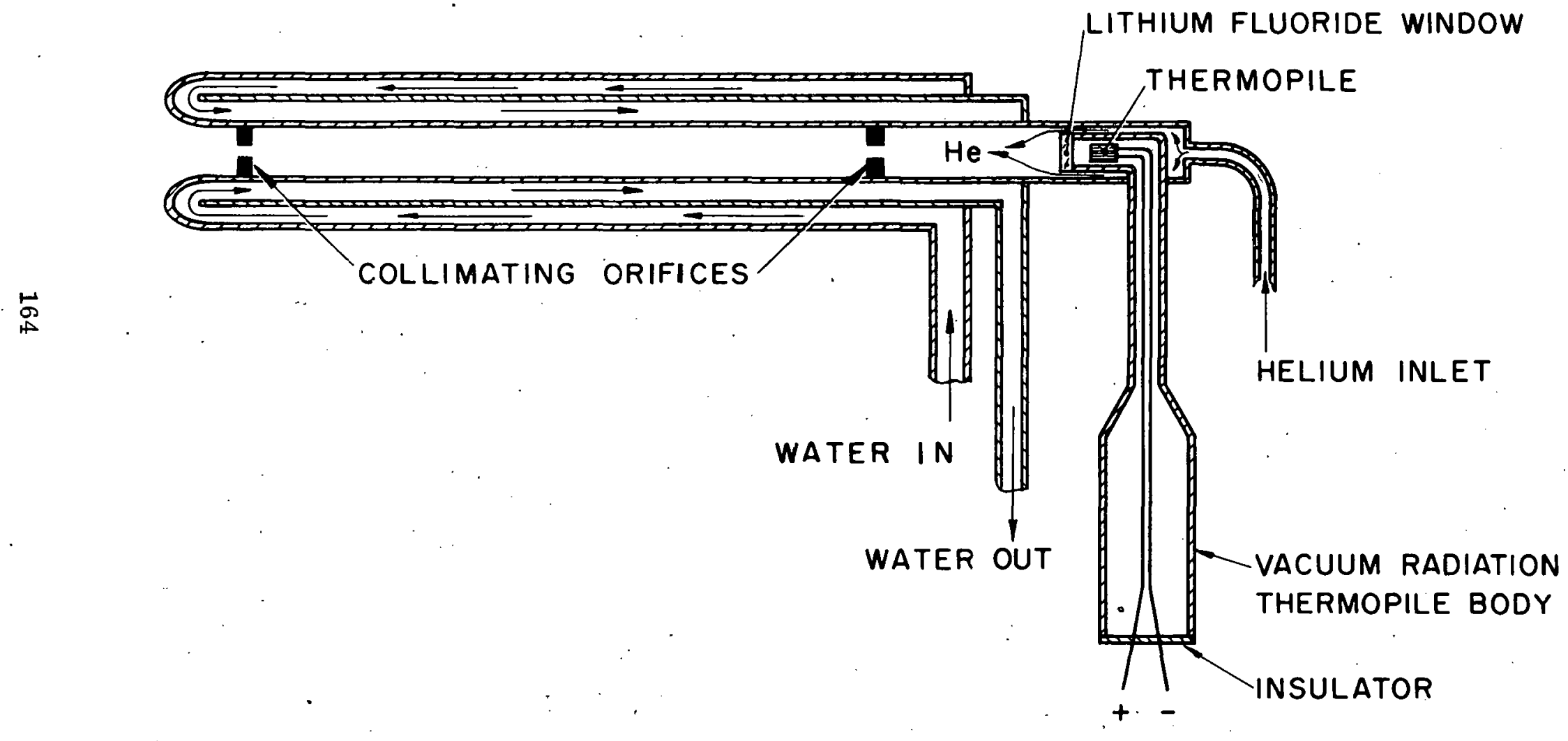

Figure 10. Diagram of Radiation Probe 


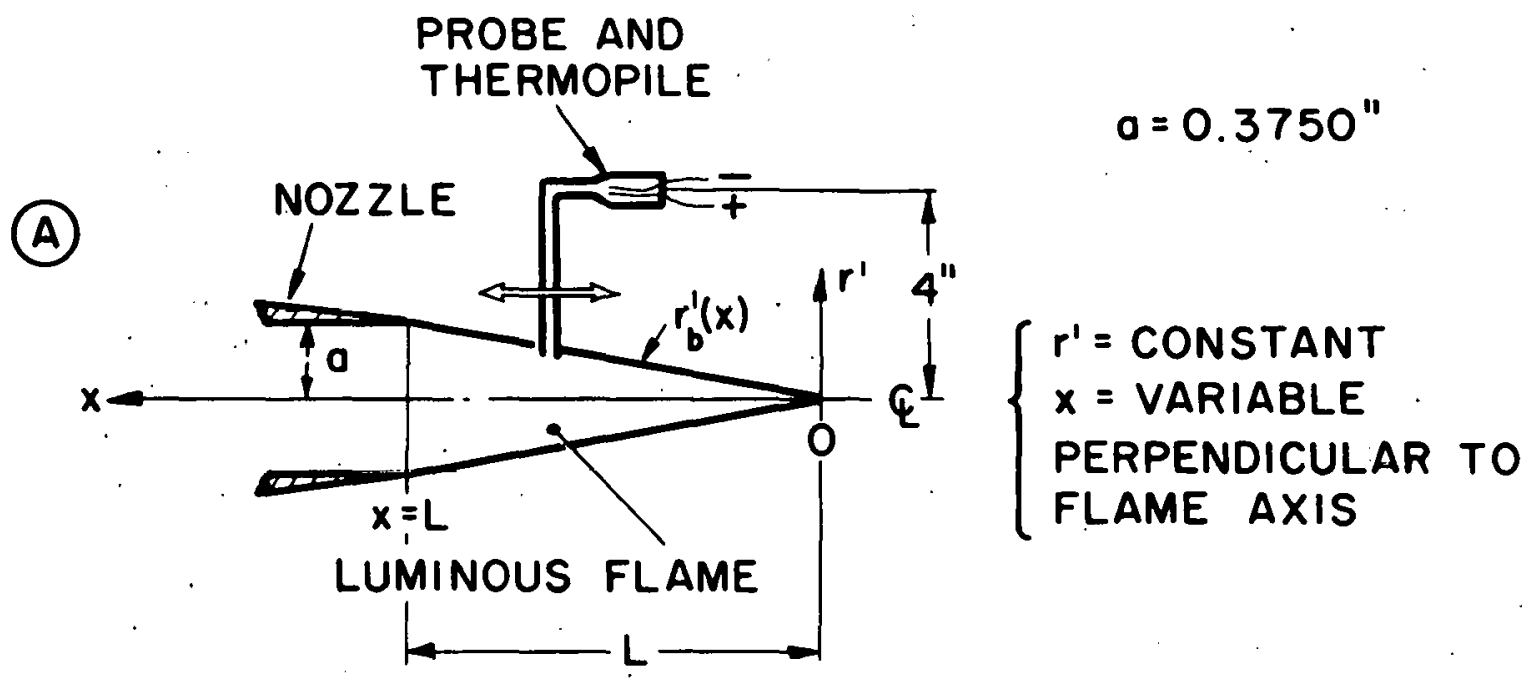

(B)

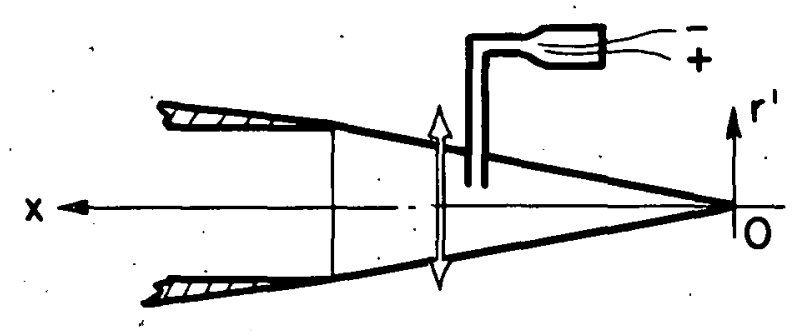

$\left\{\begin{array}{l}r^{\prime}=\text { VARIABLE } \\ x=\text { CONSTANT } \\ \text { PERPENDICULAR TO } \\ \text { FLAME AXIS }\end{array}\right.$

(C)

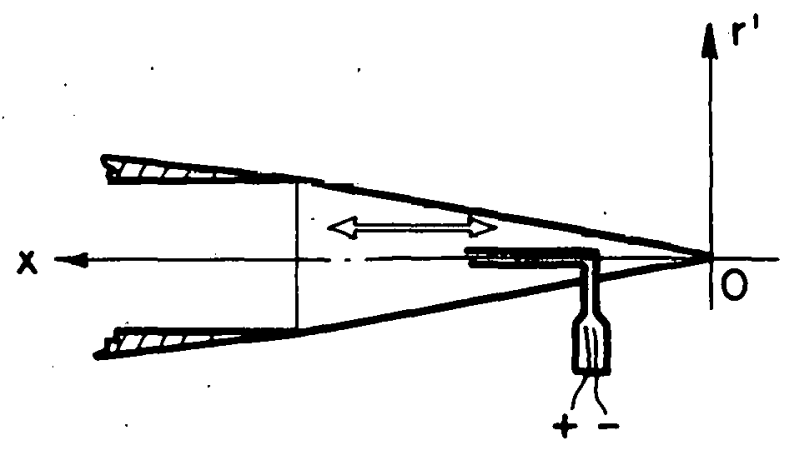

Figure 11. Probe Orientations and Traverse Modes 
transversely from the flame as a function of distance from the arcjet nozzle. The second series of tests was made with the probe in the same position relative to the flame, but traversing radially so as to pass through the flame. This series permitted direct determination of the optical thickness, since with the probe inserted all the way through the flame there will be no radiation; and as it is retracted radially, there will be observed an increasing level of radiation depending directly on the optical thickness of the path being viewed. Finally, in order to get some idea of the radiation intensity issuing from the interior of the arcjet itself, a series of measurements was made with the radiation probe facing directly upstream, traversing also in the axial direction so as to record the intensity of radiation from the interior of the nozzle at various axial positions. This radiation consisted primarily of the energy issuing from the hot cathode face and arc column region 10cated inside in the arcjet nozzle, less any absorption in the gas jet itself. Detailed results of these studies are reported in Reference 10, but it is of interest to observe the experimental results of these surveys, shown here in Figures 12, 13, and 14. Figure 12 indicates the axial decay of radiation in the arcjet, and shows that at a temperature of approximately $7000^{\circ} \mathrm{K}$, the radiated energy has dropped to essentially zero. Figure 13 indicates a parabolic distribution of radiated energy, corresponding exactly that which would be expected from the previously measured temperature distribution if the gas were completely transparent. Figure 14 shows that the intensity of radiation issuing from the almost blackbody neighborhood of the nozzle interior is far more intense than that issuing from the jet itself, and again shows that the jet is essentially transparent.

Integrating the total energy issuing from the arcjet, as discussed in detail in Reference 10, it turns out that at a temperature of approximately $24,000^{\circ} \mathrm{R}\left(12,600^{\circ} \mathrm{K}\right)$, the total amount of radiated energy is only of the order of $12 \%$ of the net arcjet power. This radiation, however, was found 10 to increase as approximately the seventh power of the temperature, and therefore at only slightly higher temperatures radiation can become dominant. These conclusions were subsequently verified quite closely by other investigators.11

Further experiments comparing the radiation intensity of a much hotter laminar core, as compared to the relatively cool turbulent flame measured as described above, will be conducted next year.

11. Tankin, R. S., and Berry, J. M., "Experimental Investigation of Radiation from an Argon Plasma," Phys. of Fluids 7, October 1964, pp. 16201624. 


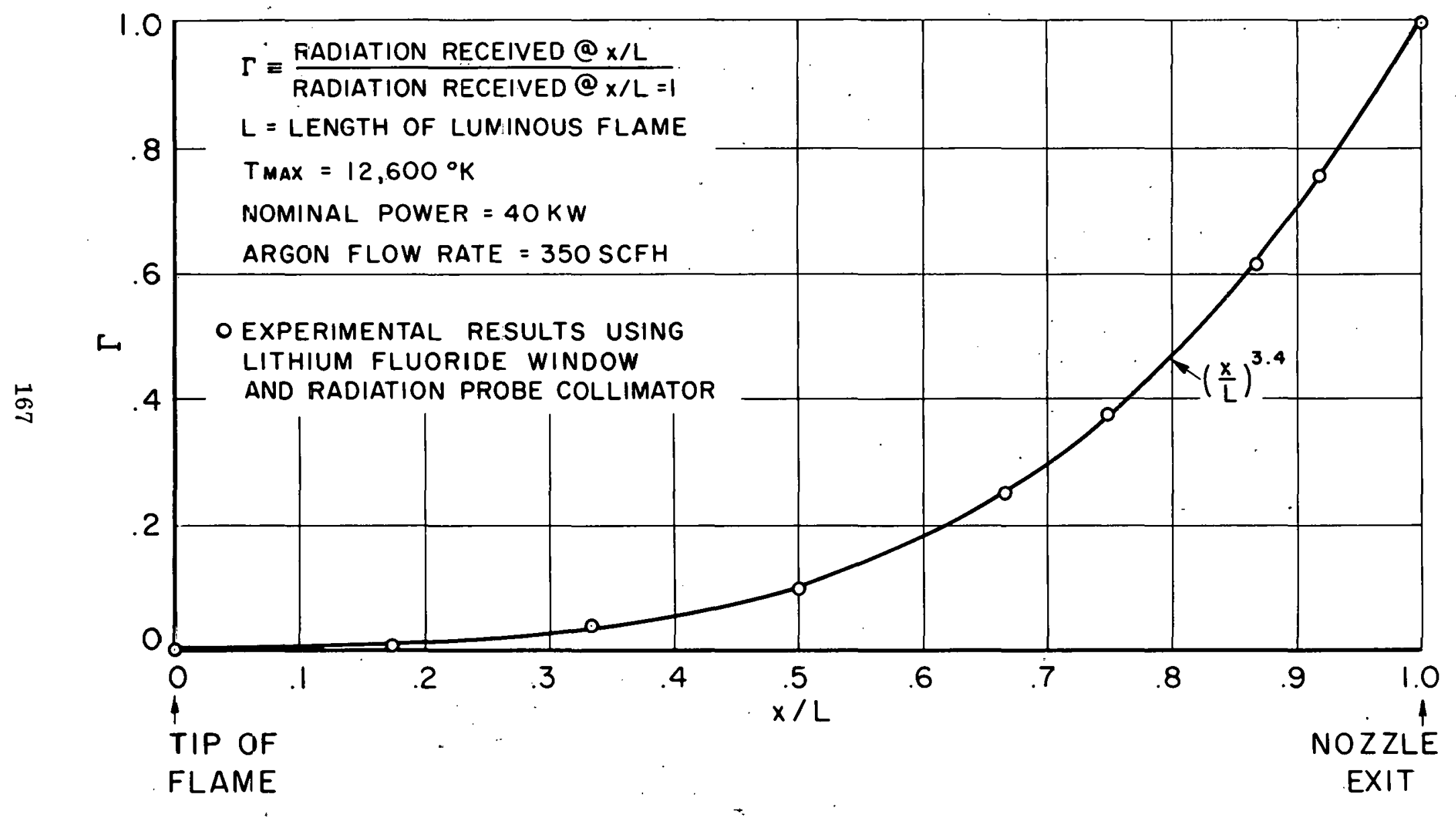

Figure 12. Axial Radiation Attenuation 


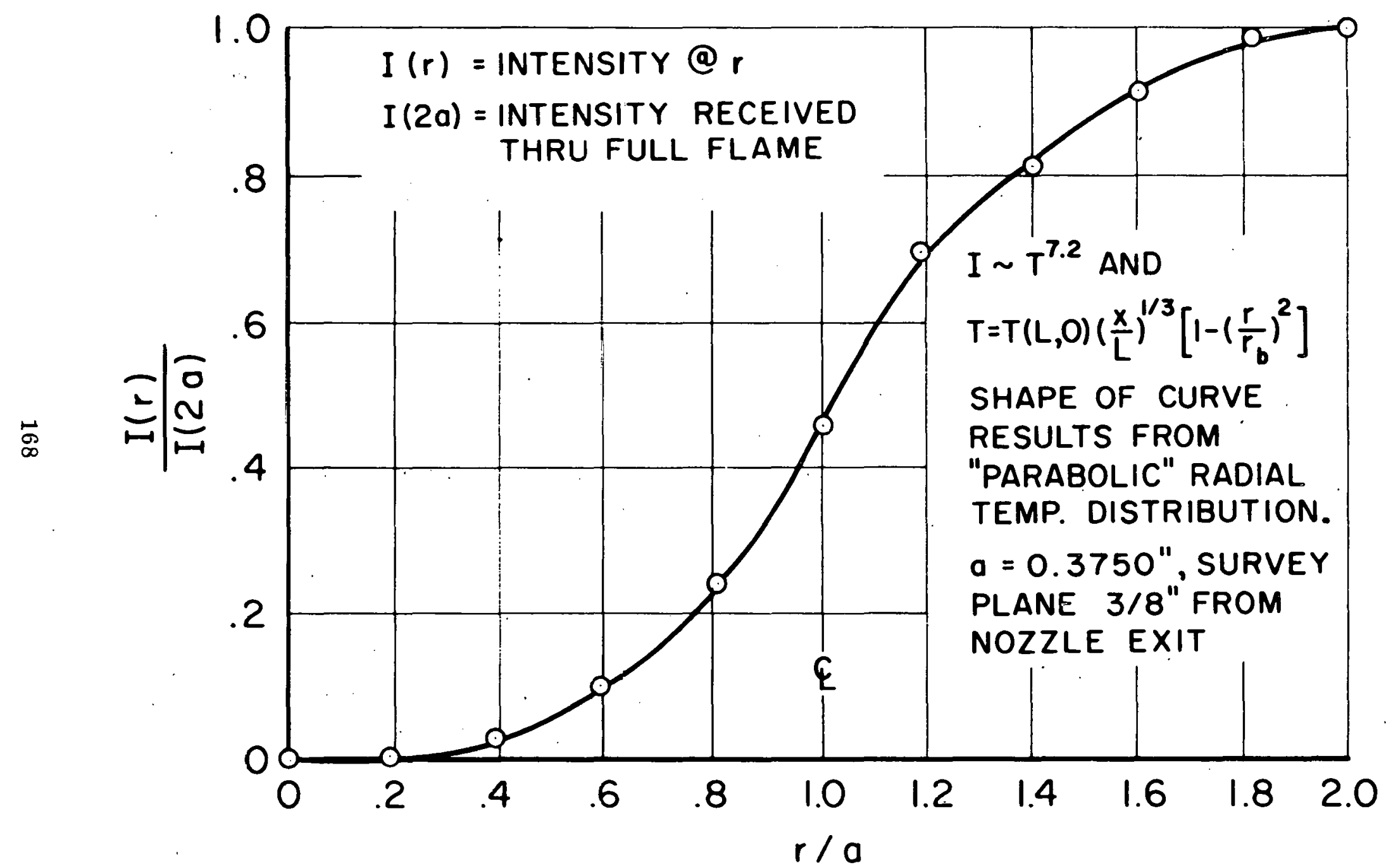

Fisure 13. Radial Survey 


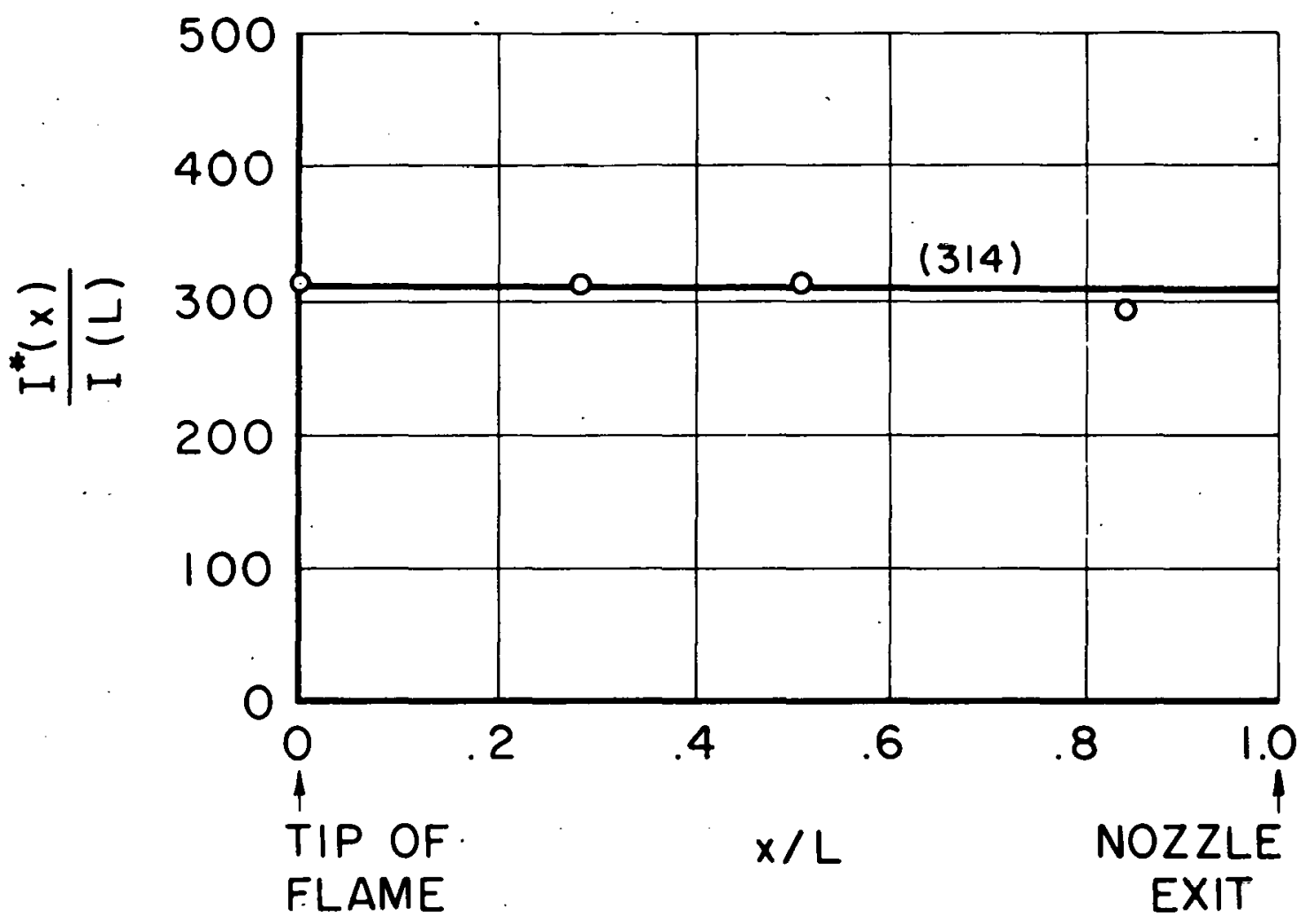

$\begin{aligned} I^{*}(x)= & \text { TRANSFERRED INTENSITY } \\ = & \text { INTENSITY MEASURED AT } x \text { AND REFERRED TO } \\ & \text { NOZZLE EXIT } \\ I(L)= & \text { INTENSITY MEASURED AT NOZZLE EXIT } x=L\end{aligned}$

Figure 14. Axial Survey on Centerline 
DUCTED MIXING OF A PARTLY IONIZED GAS

WITH A COOL COAXIAL FLOW

A series of measurements was made to determine the mixing characteristics between an initially laminar core flow of hot argon at temperatures ranging up to $25,000^{\circ} \mathrm{F}$ with a cool coaxial turbulent flow of helium issuing from an annular nozzle surrounding the arcjet nozzle. This configuration represents a rather idealized version of one gaseous-core nuclear rocket scheme. The matter of greatest interest concerned the effect of coaxial gas velocity upon the transition from laminar to turbulent flow of the core jet. This characteristic was determined by both visual and detailed survey techniques.

The visual experiments were run in a rectangular duct, to facilitate schlieren photographs, which provided an indication of the effects of both different gases and the relative flow velocities on the location of the transition region. Typical photographs of this type are shown in Figures 15 and 16 , in which the peak exit plane velocity of the central arcjet core was of the order of $500 \mathrm{ft} / \mathrm{sec}$ (Mach number less than 0.05) and the coaxial jet velocity (nitrogen) was of the order of only a few feet per second. Figure 15 shows typical laminar and turbulent jets, and Figure 16 consists of a series of photographs showing the transition to turbulence, which occurred in this case about 12 inches from the exit of the 1-centimeter-diameter argon nozzle. Figure 17 summarizes the results of the visual surveys, plotting the Reynolds number of the coaxial gas as a function of the location of the transition region, that is, the length of the laminar jet from the nozzle exit to the onset of turbulence. This was dunt for two duot cross sertions, one round and one rectangular, and it was: found that the transition behavior was approximately the same for both ducts. Figure 17 also includes the results for a free jet, i.e., a jet of argon issuing into a coaxial helium flow of low velocity, but not surrounded by a duct. The important conclusion illustrated by Figure 17 is the extremely low coaxial gas Reynolds number required to produce the transition. It is bclieved that this early transition and very rapid decay of the central core jet results principally from the turbulence level of the coaxial flow, which could not be controlled on these experiments, rather than from the shear: between the coaxial and central core flows. This conclusion is based on the fact that increasing the coaxial velocity even in the extremely low velocity range of 0.2 to $3 \mathrm{ft} / \mathrm{sec}$ (performed in a later series of experiments as described in detail in Reference 9) reduced rather than increased the distance to transition. This would indicate that since the shear between the two flows decreases as the velocities reach equality, the turbulence level of the coaxial jet, which increases as its velocity increases, must have dominated the mixing process. 


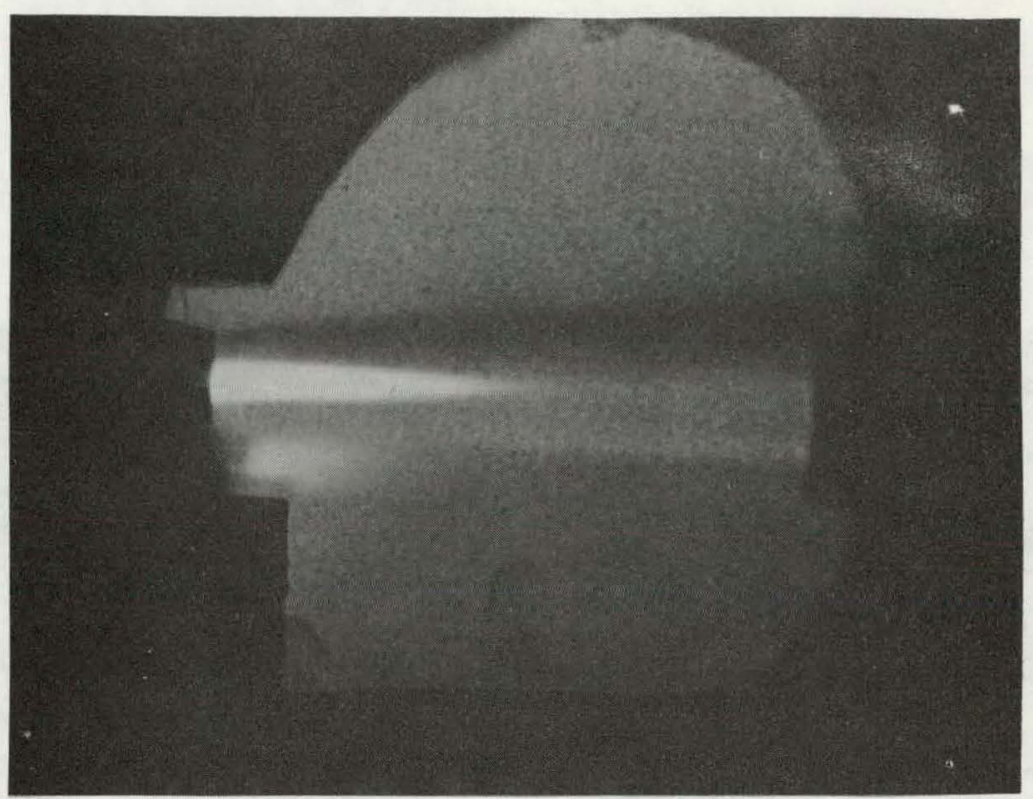

$3 / 8$ TYPICAL LAMINAR FLOW

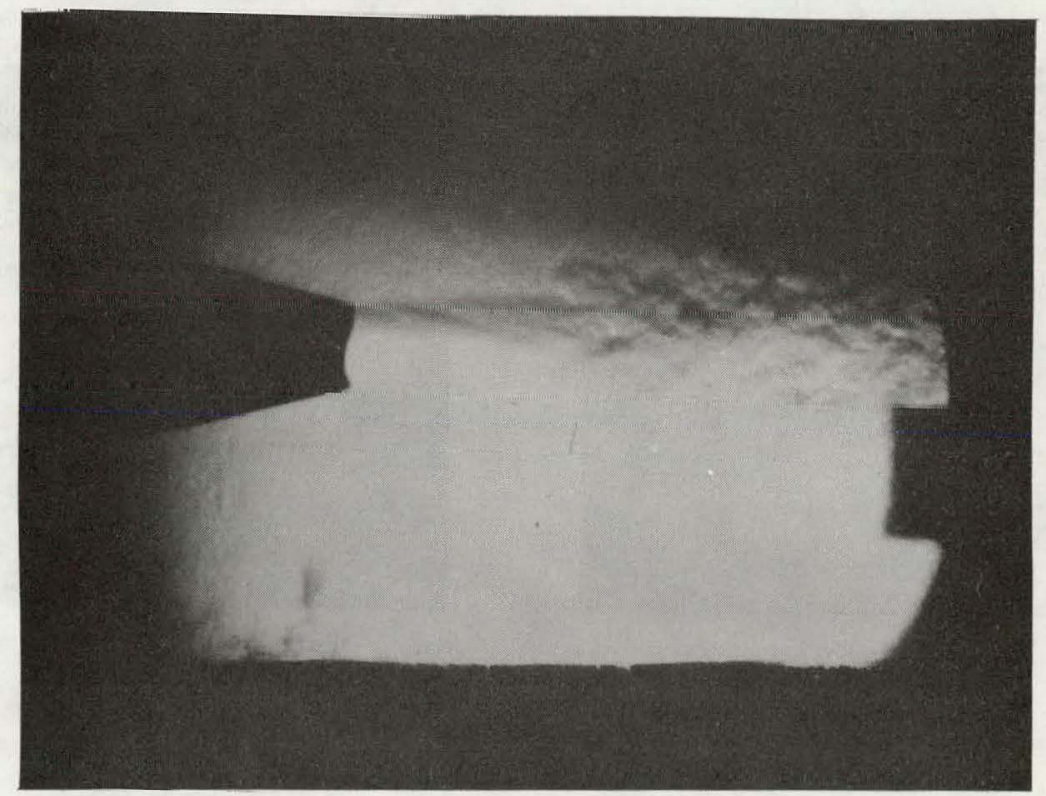

TYPICAL TURBULENT FLOW

Figure 15. Schlieren Photographs of Argon Plasma Jets in Helium 

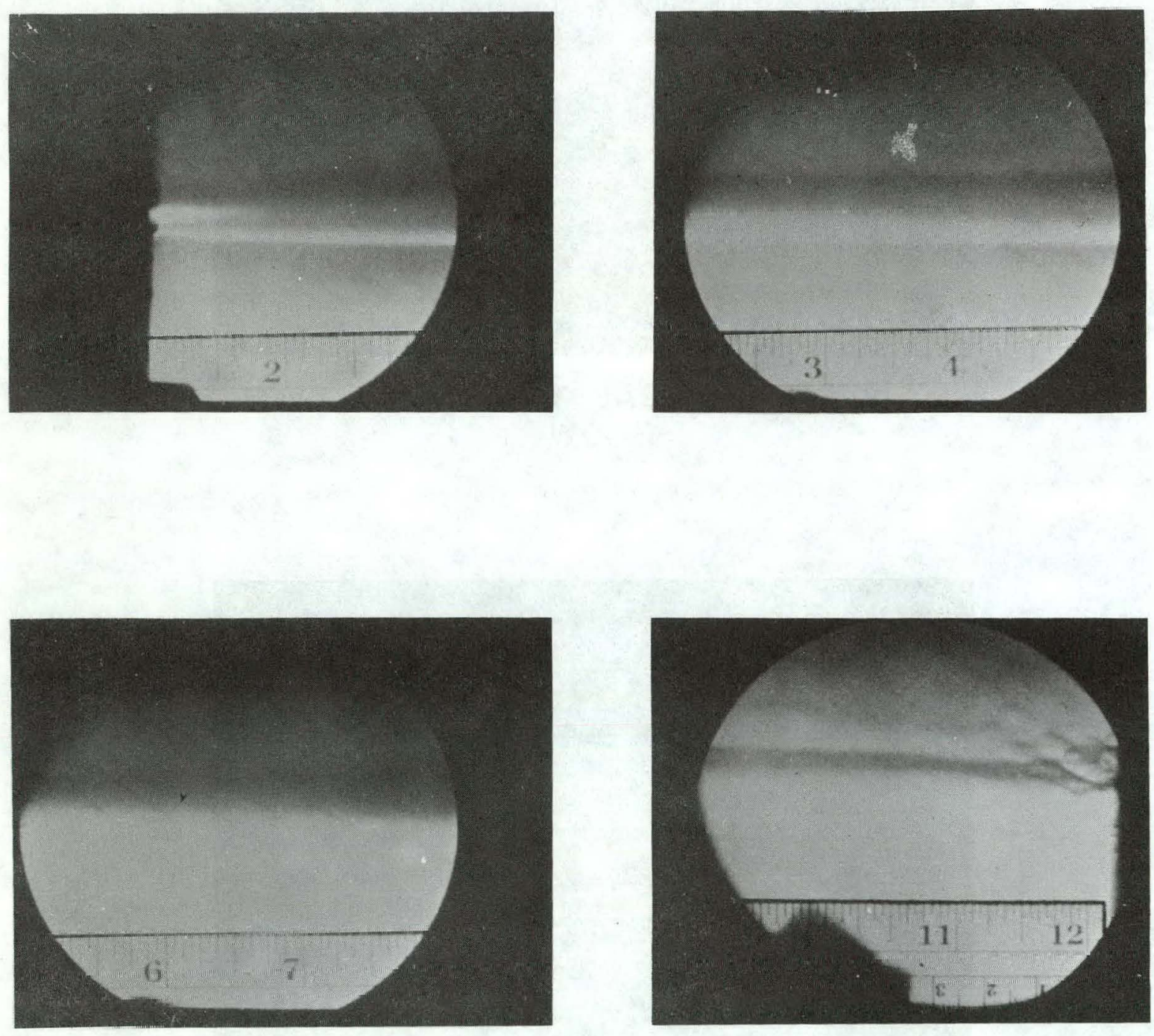

Figure 16. Schlieren Photographs of Free Laminar Argon Arcjet in Nitrogen at 1 Atmosphere (3/8" Diameter Nozzle) 


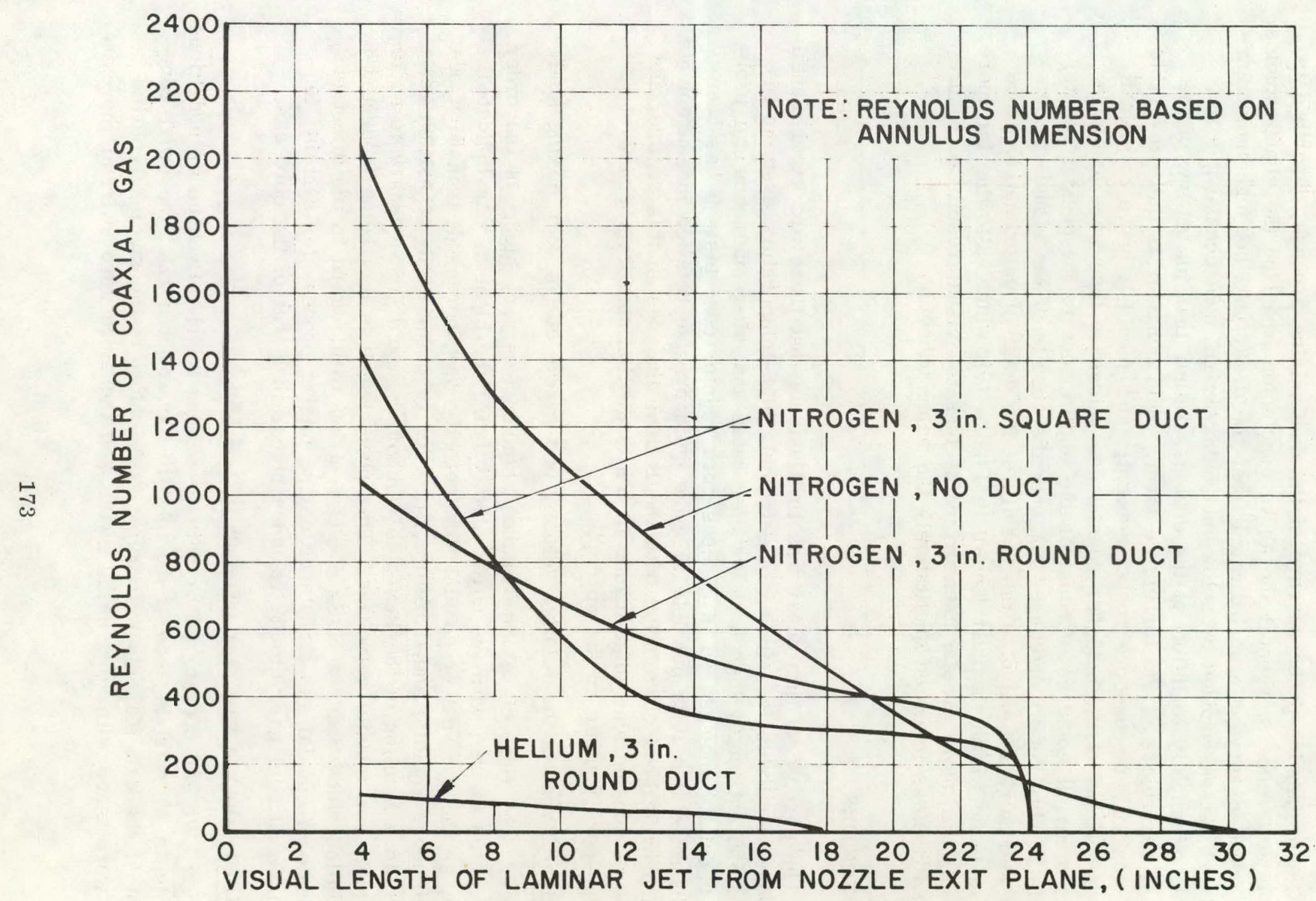

Figure 17. Visual Length of Laminar Arcjet Versus Coaxial-Flow Reynolds Number 
Comprehensive probe surveys of the duct mixing process, described in detail in Reference 9, support the above conclusion based on the visual studies. These detailed surveys also indicated ${ }^{9}$ that the decay of a hot high-molecularweight gas jet surrounded by a low-molecular-weight cool coaxial gas flow was due principally to inflow of the cool gas rather than an outflow of the hot gas, regardless of the turbulence level. This behavior is quite favorable to the coaxial gaseous-core rocket concept; ${ }^{1}$ and should it prove to prevail under conditions of much higher coaxial gas velocities than were available on this study, it could be of considerable significance to the coaxial reactor program. This report discusses not only the results of the visual studies shown in Figure 17, but also the results of the detailed calorimetric probe surveys made within the duct (shown in Figure 18), which, allowing far more detail and far more precise measurements than the visual results of Figure 17, nevertheless indicated qualitatively the same behavior.

\section{CONCLUSIONS}

The purpose of this paper was to illustrate that there are experimental techniques available for studies of extremely high-temperature systems involving the extremely high heat transfer rates and other environmental problems normally encountered in gaseous-core reactor concepts. It has been demonstrated that it is possible to make quite precise detailed measurements which provide good correlation with theoretical analyses in this regime, and can be of considerable significance in the evaluation and development of gaseous-rnre nuclear rocket concepts.

Question: What is the relative velocity of the two jets in your mixing study? Answer: The ratio of velocity in these studies was very high: of the order of 25 or 50 to one, with the core velocity being the highest. As indicated above, as the external or coaxial flow velocity was increased, transition occurred more rapidly; and therefore, in order to observe any transition behavior as a function of the Reynolds number, it would be necessary to go to extremely low coaxial velocities. This phenomenon is now being subjected to detailed study under a NASA SNPO contract with regard to the effect of the scale of coaxial gas turbulence on the mixing process for systems in which the coaxial gas velocity is much higher than that of the core gas.

Question: Do you have any swirl at the jet exit?

Answer: We can operate either with or without swirl. Even under conditions in which a $6^{\circ}$ swirl is used at the inlet to the arcjet nozzle, the swirl component at the exit plane is well below $1 / 2^{\circ}$. Most of the studies reported here were made with zero initial swirl, and therefore zero swirl at the exit. 


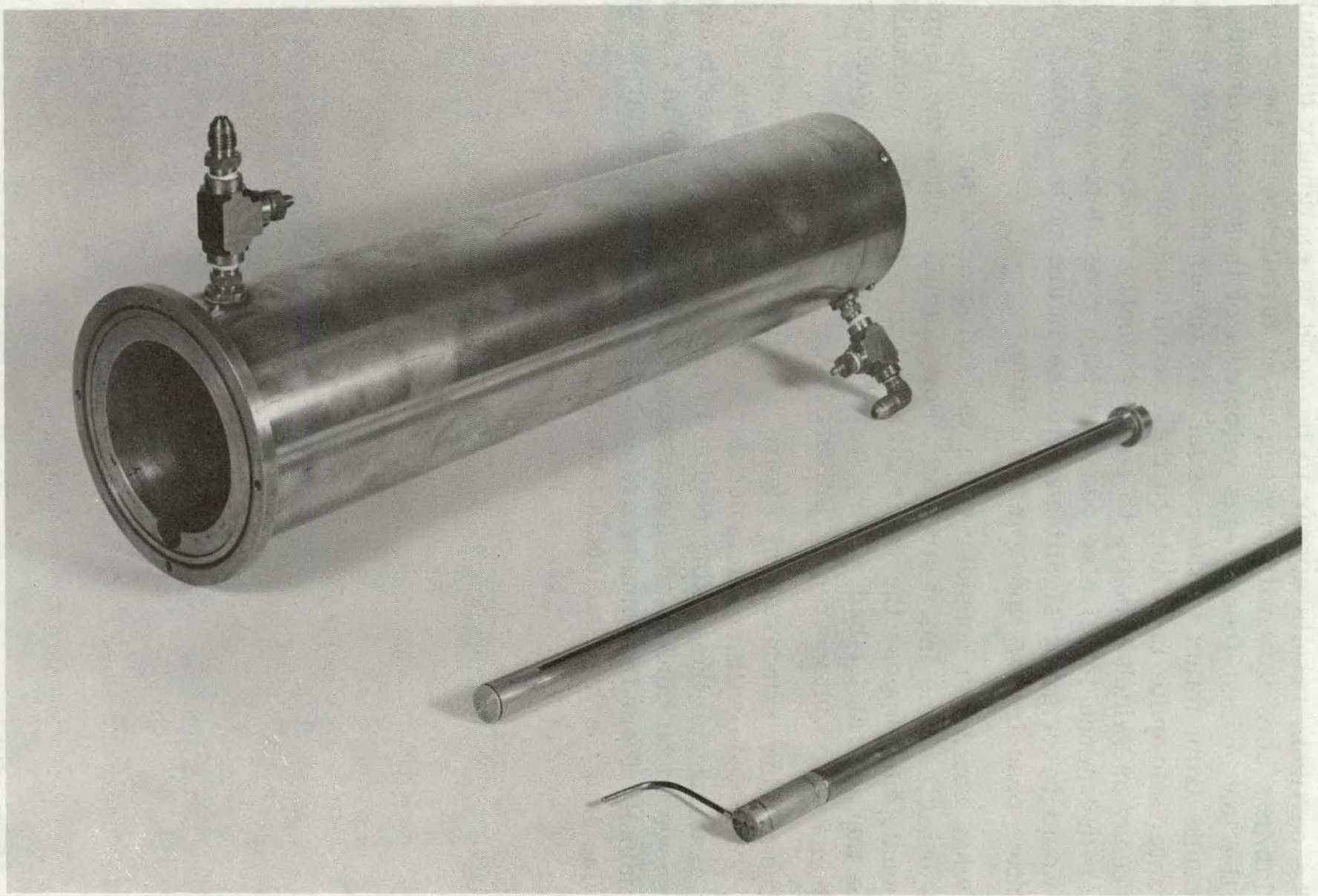

Figure 18. View of Calorimetric Duct Used for Detailed Coaxial-Flow Mixing and Heat Transfer Measurements 
Question: What influence does this work have on the coaxial gaseous core reactor concept?

Answer: The pertinent results appear to be (a) it is extremely difficult to maintain a laminar core flow with any degree of turbulence in the outer flow and (b) for the low coaxial-to-core-gas velocities of these experiments, core jet decay results from influx of the coaxial gas rather than efflux of the core gas, a characteristic quite favorable to the coaxial gaseous-core concept. Should this behavior (which appears to be only weakly dependent on scale of turbulence or turbulence level) also occur at high coaxial-to-core-gas velocities, it would be of great significance to the future of the coaxial reactor. The latter case is now under investigation.

Question: What would you consider to be the influence of the density ratio between the outer and the inner stream? For example, when the temperatures are equal, and suppose the outer stredm had a vory low molecular weight while the central jet had a very high molecular weight, wouldn't the mixing phenomena be quite strongly influenced?

Answer: The mixing boundaries of Figure 8 indicate a strong degree of curvature. For gases with equal molecular weight, that is, gases in which the density gradient for the mixing region is not important, these boundaries have been shown to be conical. ${ }^{12}$ The strong curvature shown in Figure 8 results principally from the density gradient of the low-molecular-weight helium flowing into the high-molecular-weight argon.

12. Forstall, W., and Shapiro, A. H., "Momentum and Mass Transfer in Coaxial Gas Jets," J. App. Mech. 10, 1960, p. 339. 


\title{
COAXIAL FLOW GASEOUS NUCLEAR REACTOR CONCEPT
}

\author{
Frank E. Rom \\ NASA-Lewis Research Center \\ Cleveland, Ohio
}

In addition to research being carried out in general support of gaseous reactors, Lewis is attempting to determine the feasibility of a particular class of gaseous nuclear reactors - the coaxial flow type. In this concept, as shown in Figure 1, separate coaxial streams of uranium (or plutonium) and hydrogen are introduced into a reflector moderated cavity. The hydrogen flows coaxially and concentric with the uranium core. The uranium flows at a lower velocity than the hydrogen to maintain an acceptable ratio of uranium-to-hydrogen mass flow ratio. The resulting mixture of hydrogen and uranium is exhausted through a nozzle to produce thrust. An acceptable value of the uranium-to-hydrogen mass flow ratio is about 1:35 which results from dividing the cost of hydrogen in orbit (approximately $\$ 200 / 1 b$ ) by the cost of uranium (approximately $\$ 7000 / 1 b$ ). This assumes that we are willing to allow the cost of fuel to equal the cost of hydrogen. The hydrogen is heated by thermal radiation from the fissioning central zone of uranium. The hydrogen contains seeding material which renders it opaque to thermal radiation from the core.

In this concept the most important factor to determine is the number of uranium atoms that are in the core at any given time, considering the fact that mixing occurs between the coaxially flowing streams. Our hydrodynamic analyses are aimed at predicting the concentration profiles that exist both in the axial and radial direction following injection of the uranium and hydrogen. In other words, we attempt to determine what nature will give us in such a situation. We are talking in general about turbulent systems with lots of mixing and consequently relatively small zones of undisturbed uranium.

The gas core program at Lewis is divided into three fundamental areas which are: 


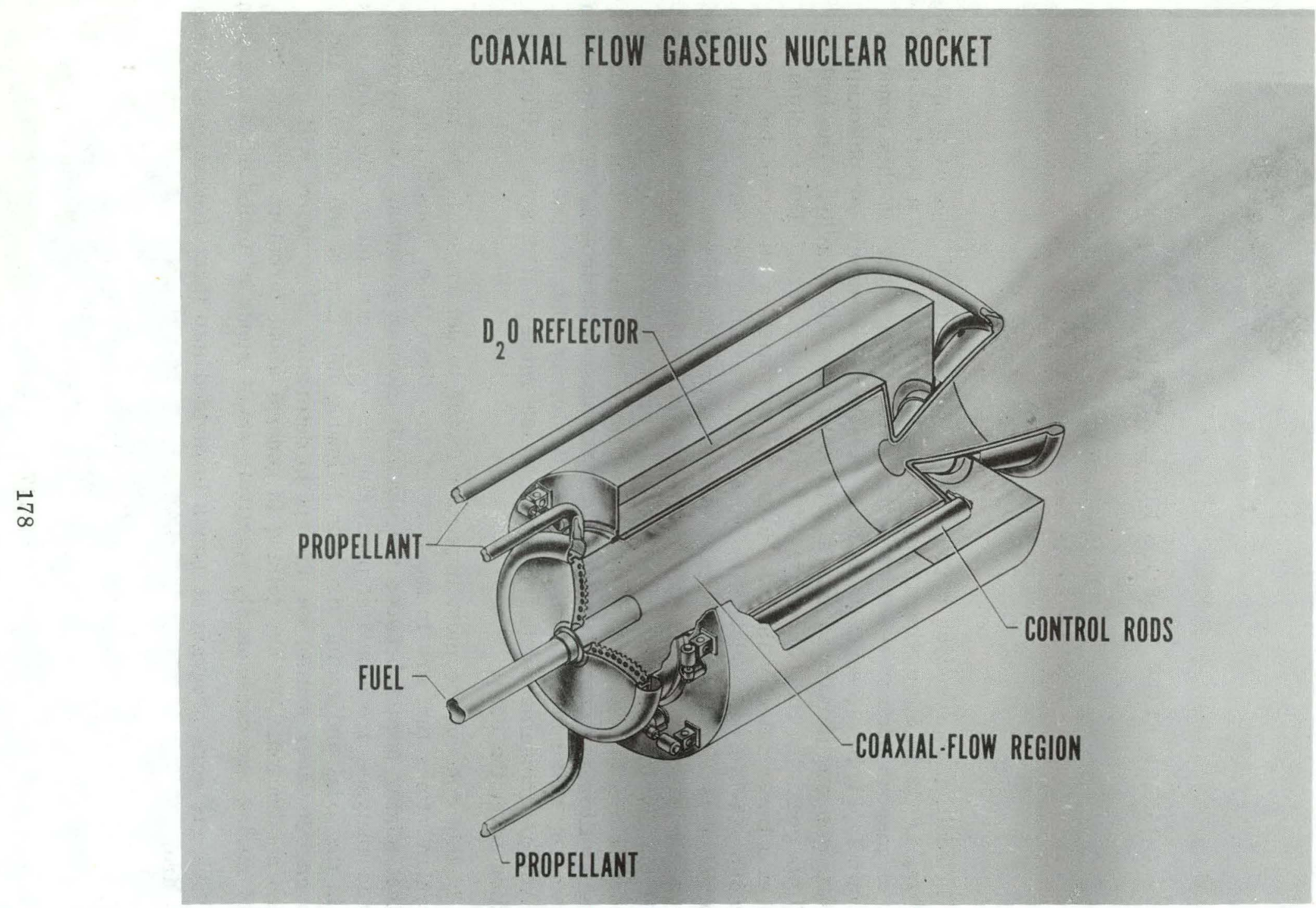

Figure 1 


\section{Hydrodynamics}

2. Heat transfer

3. Nucleonics

The first area, hydrodynamics, has as its goal the determination of experimentally verified analyses which determine the amount of uranium which is within the reactor cavity at any given time as a function of flow rates, velocity ratios, and diameter ratios of coaxially flowing systems. The heat transfer area is concerned with the determination of the temperatures that exist throughout both zones of the coaxial system as a function of relative flow rates, reactor power, amount of seeding, velocity ratios, etc. The third area, nucleonics, is of extreme importance inasmuch as the amount of uranium required for criticality directly determines operating pressure levels. In gas cores the operating pressures tend to be high; therefore, anything which can be done to reduce critical mass is of major interest.

In all three areas the emphasis has been the establishment of experimentally verified analytical solutions so that analytical extrapolation to real systems can be made with confidence. Not much time has been devoted to systems analysis or engine design studies. Only a limited amount of such studies has been or will be undertaken until a firm foundation on which to base them has been established.

Bob Ragsdale will discuss the Lewis hydrodynamic and experimental heat transfer programs. In addition, he will briefly mention some of our work in the nucleonics area. Al Kascak will talk in detail on our latest radiation heat transfer analysis which represents we feel significant progress in this field and should therefore be of general interest. 


\title{
COAXIAL FLOW RESEARCH STUDIES
}

\author{
Robert G. Ragsdale \\ NASA-Lewis Research Center \\ Cleveland, Ohio
}

Figure 1 shows the topics to be discussed in this paper; fluid mechanics, heat transfer, and nucleonics. Under fluid mechanics, we will discuss the analysis, which is of a coaxial flow system, and is therefore not general, but directly related to our concept. Under heat transfer, I will describe the experimental program; it is primarily aimed at radiation heat transfer to a transparent gas made opaque by the addition of small solid particles. The heat transfer analysis will be discussed in the following paper. Briefly, the analysis is of radiation heat transfer with temperature and region dependent opacities. Nucleonics will be discussed to a lesser extent than the first two. items. There will be no discussion of analysis since little has been done beyond that which has already been published on some calculations made with one and two dimensional diffusion codes. After discussing the status of the various research studies, we will indicate the kind of performance that can be predicted by applying the present knowledge in these three areas to a coaxial flow gaseous reactor system.

\section{FLUID MECHANICS}

Figure 2 illustrates the fluid mechanics problem that was outlined in the preceding introduction; the flow pattern involves the injection of a lowvelocity gaseous fissioning fuel into a surrounding, high-velocity propellant, hydrogen. The problem here is one of coaxial mixing, or more basically, one of free-turbulence between two coaxial jets of dissimilar fluids. As was suggested in the introduction, what we want is poor mixing; more exactly, what we want is no mixing. If the fuel were to proceed through the reactor at its initial low velocity, surrounded but unaffected by the high velocity hydrogen, this would automatically provide any desired residence time ratiowhich is what is desired in any gas-core system. Of course that does not 


\section{RELATED RESEARCH}

FLUID MECHANICS

COAXIAL MIXING (FUEL LOSS RATE)

HEAT TRANSFER

THERMAL RADIATION (SPECIFIC IMPULSE AND WALL HEATING)

NUCLEONICS

EXTERNAL MODERATION (REACTOR PRESSURE AND WEIGHT) 


\section{CO-AXIAL FLOW IN REACTOR CAVITY}

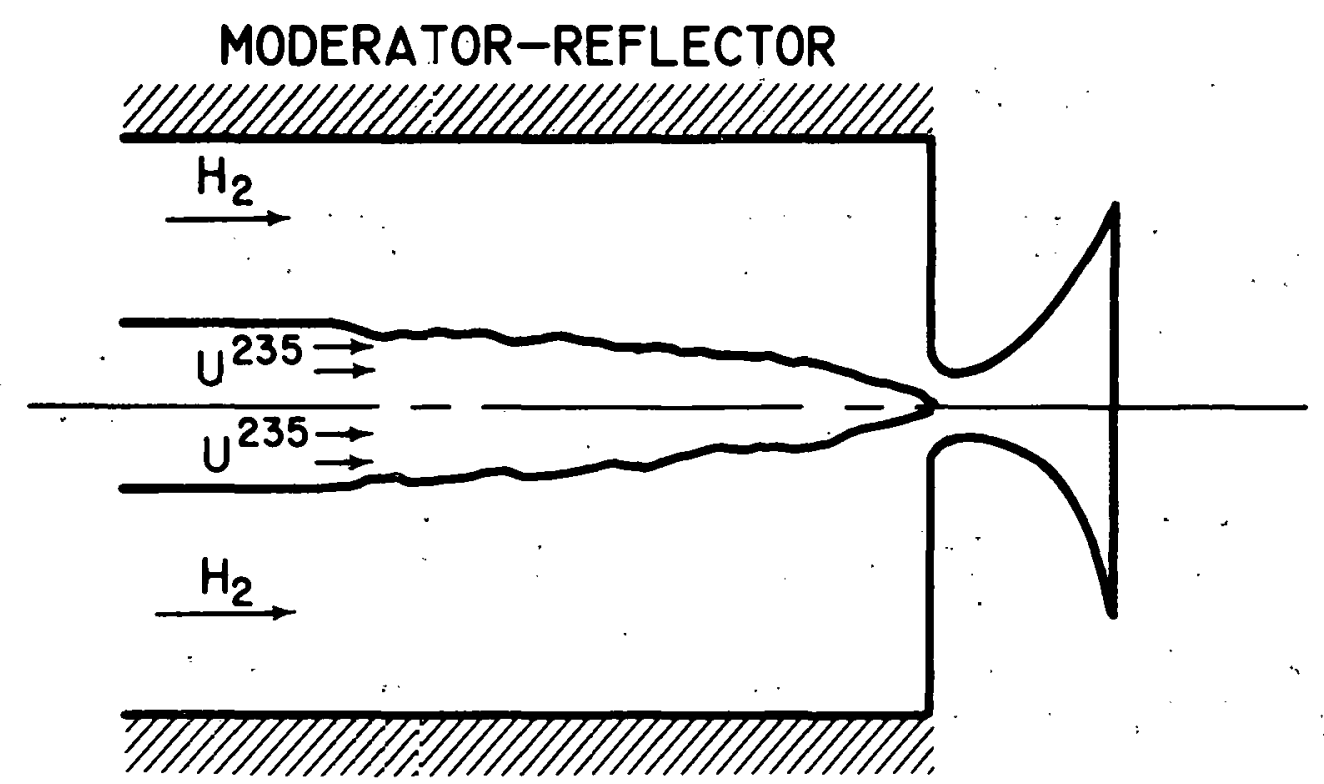

(A) REACTOR FLOW SCHEMATIC

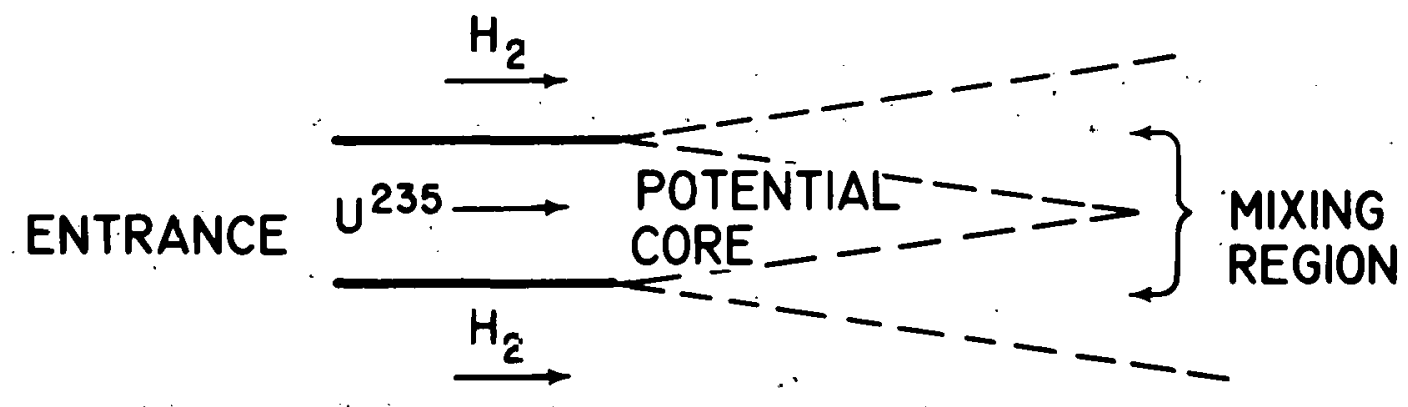

(B) FLOW REGIONS

Figure 2 
occur in the real case, there is momentum and mass transfer between the two streams so that the inner one is then accelerated. The analytical problem is to predict the rate at which it is accelerated and the rate at which it diffuses into the outer stream; it is a combined momentum and mass transfer problem.

The model that was analyzed is as shown in Figure 2. The analysis begins at time zero at the injection point; the solution of the momentum and diffusion equations from this point downstream has been formulated as a computer program which gives, as a function of initial conditions, radial concentration and velocity profiles at various distances downstream from the injection point. The basic equations are for laminar flow; thus they contain binary and molecular diffusion coefficients for mass transfer and viscosities for the momentum transfer. In the usual manner, we apply these equations to a turbulent flow situation by saying that basic processes are the same, and it is sufficient to simply add on a contribution of turbulence. Thus in the analysis, where we have a viscosity term, we add to it a turbulent viscosity; similarly, to the binary diffusion coefficient we add an eddy diffusivity, and then use the program to describe the turbulent coaxial mixing process. This procedure introduces one more unknown into the program; the ratio of turbulent to laminar viscosity. This is not easily obtained and is what necessitates an experimental study of turbulent coaxial mixing. By making measurements and comparing the analysis to the experiment, one can inductively obtain an empirical relation which expresses the turbulence level in a real system.

Figure 3 shows a flow model that has been analytically studied, and which will be experimentally investigated in the near future. It is the flow pattern which would most likely be utilized in a coaxial flow reactor, since it tends to minimize mixing. This is the flow problem with a buffer layer. which was mentioned in the introduction. The goal here is to reduce the turbulent mixing by introducing a buffer region of intermediate-velocity hydrogen between the slow moving fuel and the fast moving hydrogen. This effectively forms a momentum buffer between the inner and outer streams. Although this does in fact tend to improve the situation, there is a limit to the usefulness of a buffer region. This limit is incurred as follows. The average hydrogen velocity must remain constant for a fixed channel dimension and a fixed thrust; a decrease in buffer-hydrogen velocity must be accompanied by an increase in outer-hydrogen velocity in order to maintain a constant propellant flow rate. Thus at the inner boundary the turbulence is reduced, but at the outer boundary a new source of turbulence is created; the ultimate balance of this situation is that there is an optimum combination of buffer layer thickness and velocity. 


\section{FLOW PROBLEM WITH BUFFER LAYER}

$\Rightarrow \quad$ LIGHT FAST MOVING

$\Rightarrow$ PROPELLANT

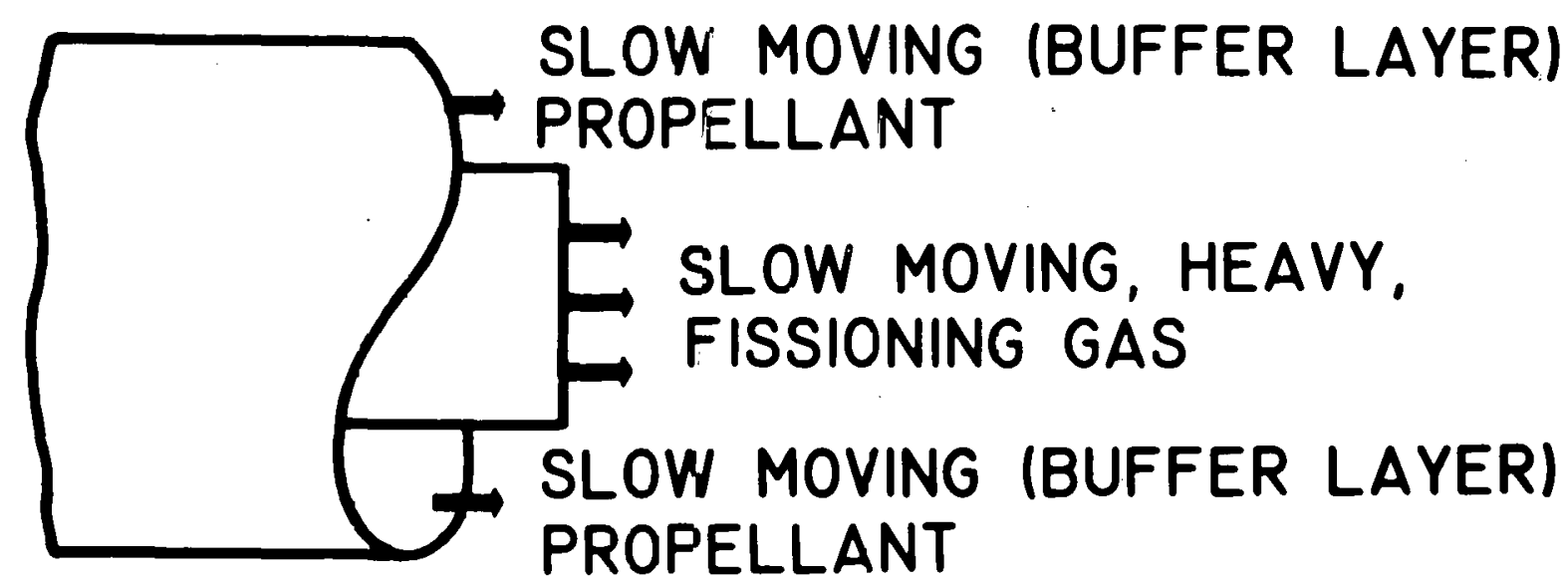

$\Rightarrow \quad$ LIGHT FAST MOVING

$\Rightarrow$ PROPELLANT 
The fluid mechanics experiment was conducted on a basic coaxial flow system with no buffer region present. Air and bromine were used as a low molecular weight gas and a high molecular weight gas, respectively; mixing rates were experimentally determined.

Figure 4 is a photograph of the experimental setup; the flow is from top to bottom in a 5 inch by 5 inch lucite channel. Bromine is injected at a relatively low velocity through a center monel tube that is a half inch in diameter. Flowing around it, also from top to bottom, is a higher velocity air stream. Experimental dimensions and flow rates were selected to cover a range of from laminar to turbulent Reynolds numbers. Experimental data was obtained for velocity ratios of outer-to-inner stream from about one up to 49 to one. Measurements were made of the average concentration of this bromine stream at different positions downstream from the injection point. Because the outer stream is going faster than the inner one, the bromine is accelerated and diluted; the net result is that the bromine concentration decreases with distance downstream from the injection point. The concentration measurements were obtained by measuring the attenuation of light beams that were passed through the bromine stream.

Figure 5 illustrates the variation of bromine concentration with axial position. Theoretical curves for laminar and turbulent flow are also shown. The curve for turbulent flow was obtained by selecting a ratio of turbulentto-laminar viscosity ratio that gave the best fit to the data. For this particular run the value of $\epsilon^{+}$was 30 . The fact that the general shape of the curve conforms to that of the data indicates that the basic mixing process is properly described by the equations, since varying $\epsilon^{+}$only affects the level of the curve. This same procedure was used to obtain values of $\epsilon^{+}$for a range of Reynolds numbers and velocity ratios.

Figure 6 shows the final correlation of the $\epsilon^{+}$values thus obtained. The turbulence factor, $\epsilon^{+}$, was found to be a function of the initial bromine Reynolds number and the initial air-to-bromine velocity ratio. This information is then applied to a gaseous reactor situation as follows. The turbulence factor, $\epsilon^{+}$, is obtained from the correlation equation for the fuel Reynolds number and the initial hydrogen-to-fuel velocity ratio selected. This turbulence factor, the velocity ratio, and estimated hydrogen and fuel properties are used as input to the fluid mechanics program, which then computes the concentration and velocity profiles throughout the reactor.

One limitation of the coaxial mixing study is that only radial average concentrations were measured. In addition to these average values, it would be desirable to measure the actual radial profiles. This is the next step of the experimental program. As illustrated schematically in Figure 6, radial 


\section{COAXIAL FLOW EXPERIMENT}

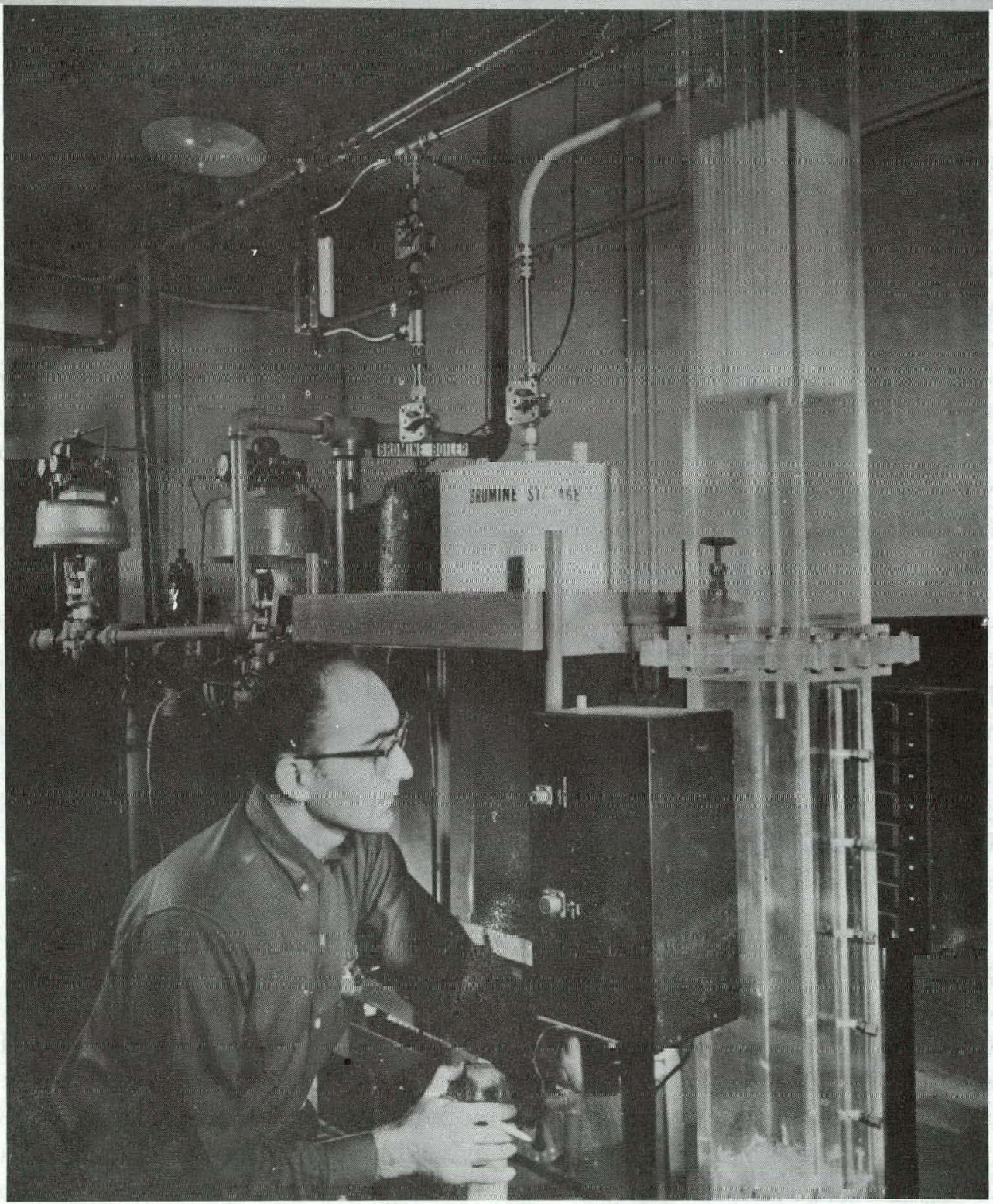

Figure 4 


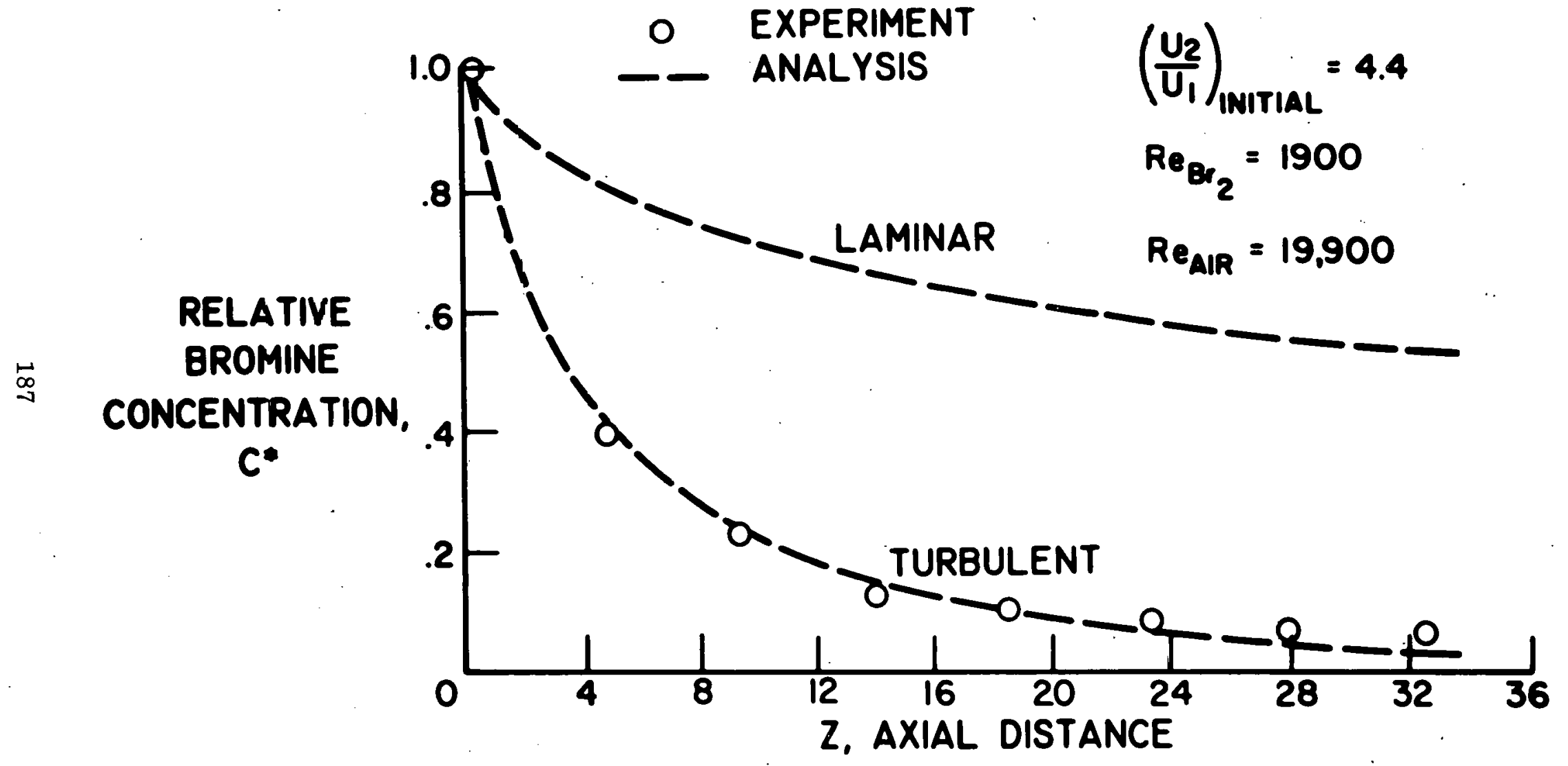

Figure 5 
FLUID MECHANICS

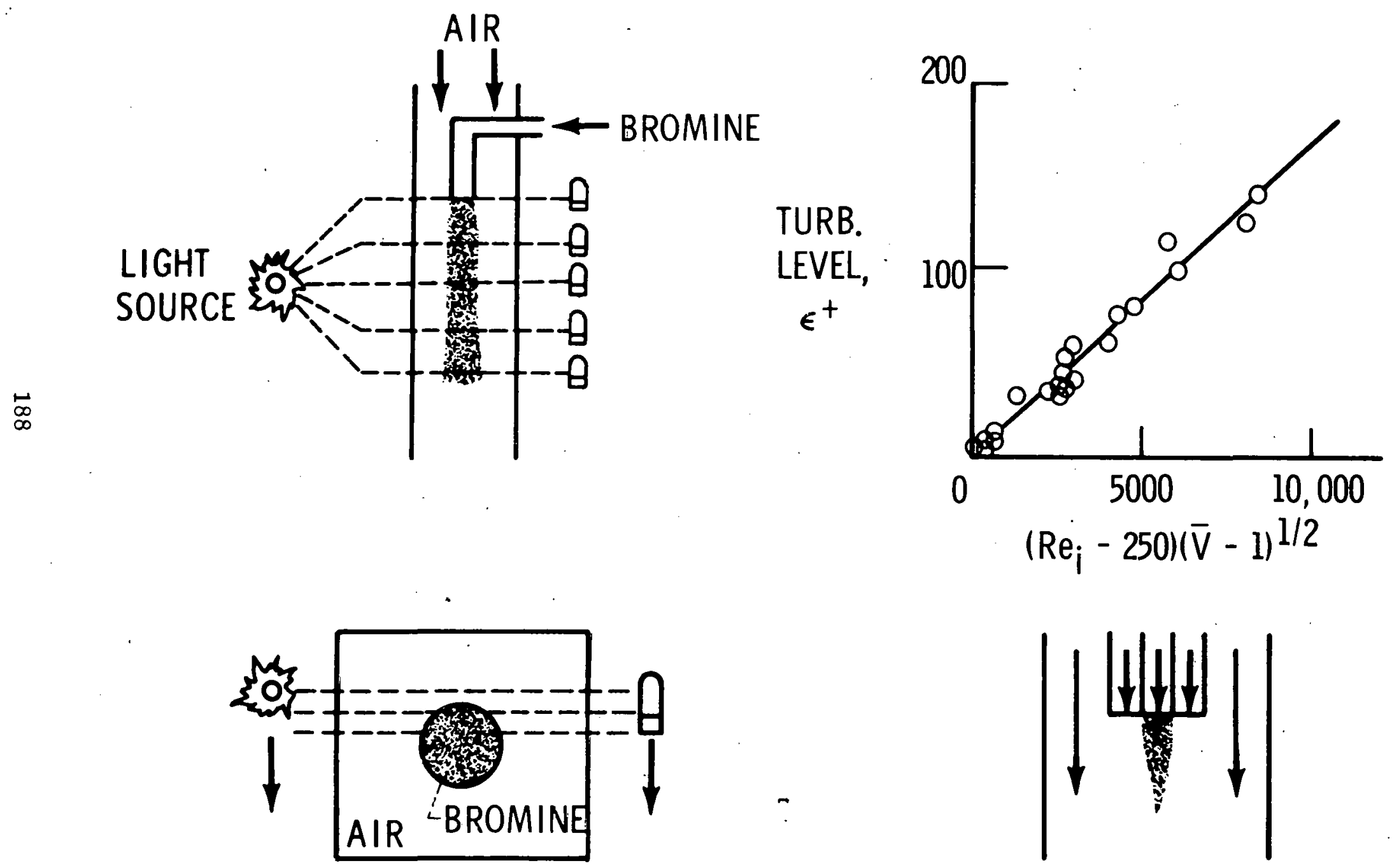

Figure 6 
concentration profiles will be measured by traversing a light beam across the bromine stream at various axial positions. This experimental setup will be similar to the first one, except that the bromine stream will be of greater diameter and the light beam will be smaller. With this more detailed information, it should be possible to treat the spatial dependence of $\epsilon^{+}$in a more sophisticated fashion than was done in the first experiment. After this study, experimental measurements will be made for a buffer flow pattern. Again, bromine will be used as the inner fluid and air in the buffer and outer regions.

This concludes the discussion of coaxial flow fluid mechanics studies being conducted in-house at the Lewis Research Center. Some additional fluid mechanics studies are being supported at the Catholic University under Dr. C. C. Chang, at the nlinois Institute of Technology under Dr. H. Weinstein, and at Princeton University under Dr. J. Grey.

\section{HEAT TRANSFER}

The experimental heat transfer work is primarily concerned with radiation to a transparent gas that contains small solid particles. One setup involves radiation from an electric arc to air that contains sub-micron carbon particles. The other study is of heat transfer from an electrically heated tungsten tube to helium seeded with sub-micron tungsten particles. Both of these experiments are illustrated schematically in Figure 7.

The arc facility employs an electric arc maintained by vortex-type flow of nitrogen gas within the inner tube of an annular glass heat exchanger. For typical conditions, 700 kilowatts from the power supply delivers 170 kilowatts to the nitrogen plasma. From 10 to 20 percent of this power is released as radiant energy; thus, typically, about 27 kilowatts of thermal energy is radiated from the inner glass tubo.

The tungsten tube test will be conducted using an electrically heated tungsten tube. A transparent gas, helium, will flow through the tube. The tube dimensions and helium flow rates will be selected to give a low thermal efficiency for forced convection heating. Thus the addition of tungsten particles to render the gas opaque should cause a significant increase in heat transfer. The maximum tube wall temperature of $5900^{\circ} \mathrm{R}$ is large enough to afford a significant amount of radiant energy. For the conditions of this test, we expect an outlet helium temperature of about $1500^{\circ} \mathrm{R}$ with no particle addition. The addition of tungsten particles to the helium stream should result in an outlet temperature of approximately $2000^{\circ} \mathrm{R}$. Though these numbers are estimates, they indicate that the increase in heat transfer due to particle seeding should be quite measurable. 


\section{RADIATION HEAT TRANSFER}
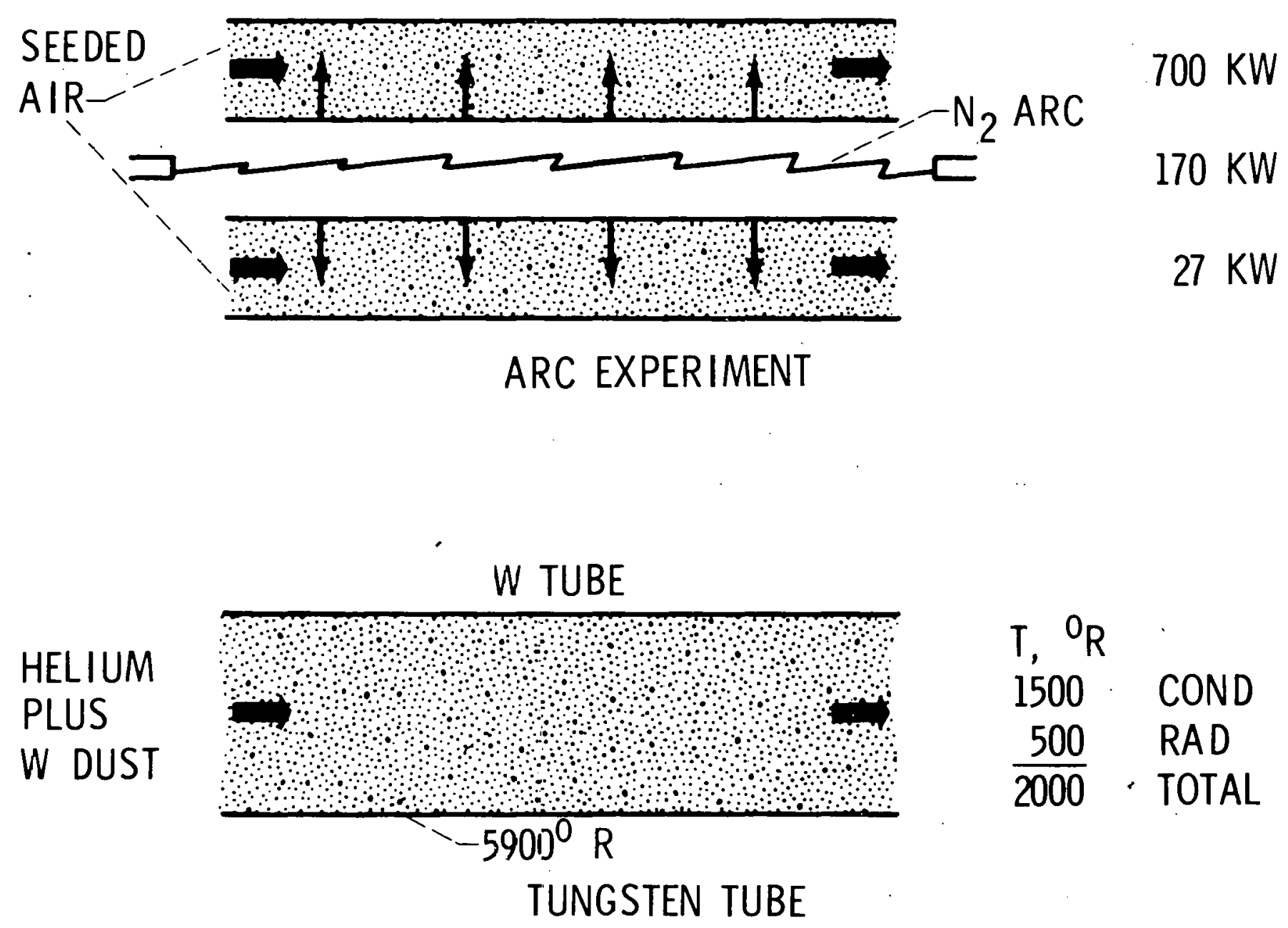

Figure 7 
The glass heat exchanger test section of the arc facility is shown in Figure 8. The arc is contained in a 54-millimeter (O.D.) quartz tube having a wall thickness of 4 millimeters. Tube lengths of 18 and 28 inches were used. The arc is maintained between fixed-upstream and movabledownstream water-cooled copper electrodes. The arc is stabilized by metered nitrogen gas that is introduced through a vortex generator at the fixed-electrode end. The nitrogen exhausts into an insulated collector where it is mixed with ambient air. The grounded downstream electrode is mounted on a movable carriage. The arc is initiated by a pneumatic electric servosystem that drives the downstream electrode into the quartz tube until contact is made with the fixed electrode. Then the movable electrode is withdrawn to a position 2 inches beyond the end of the quartz tube. The entire starting sequence is accomplished in about 10 seconds.

The variable-frequency alternating-current power supply available provided continuous variation of voltage from a minimum of 540 up to 6480 volts with an associated frequency of 1 cycle per second for each 54 volts. A maximum power of 7 megawatts was available at the limiting current of 1125 amperes. A bank of water-cooled stainless-steel tubing was used as a $1.6-\mathrm{ohm}$ pure resistance in series with the arc to provide electrically stable operation.

Seeded air is introduced into the annulus of the heat exchanger through an entrance nozzle attached to the front of the vortex generator. The air flow is metered with a rotameter before the carbon particles are added to it. The mixture passes through the heat exchanger and is then exhausted to a vacuum system. The pressure in the heat exchanger was maintained at 1 atmosphere.

Figure 9 shows a typical set of data; these are time dependent measurements of temperatures. The arc is initiated at time zero, and the unsecded air temperature in the annulus comes up to a steady state value of about $540^{\circ} \mathrm{F}$. After steady state is achieved in about 2 minutes, carbon particles are added to the air stream. When the seed material is added, the air absorbs radiant energy causing the outlet temperature to increase to $740^{\circ} \mathrm{F}$. When the seed injector is turned off, the air outlet temperature returns to its previous value of $540^{\circ} \mathrm{F}$.

The intensity of the arc radiation was measured with a thermopile detector located external to the heat exchanger; this is also shown in Figure 9. The ratio of the arc intensity readings obtained with and without seeding of the air stream is a measure of the attenuating ability of carbon particles. Thus in addition to heat transfer data, the arc tests afford some information on the extinction coefficient of carbon. 


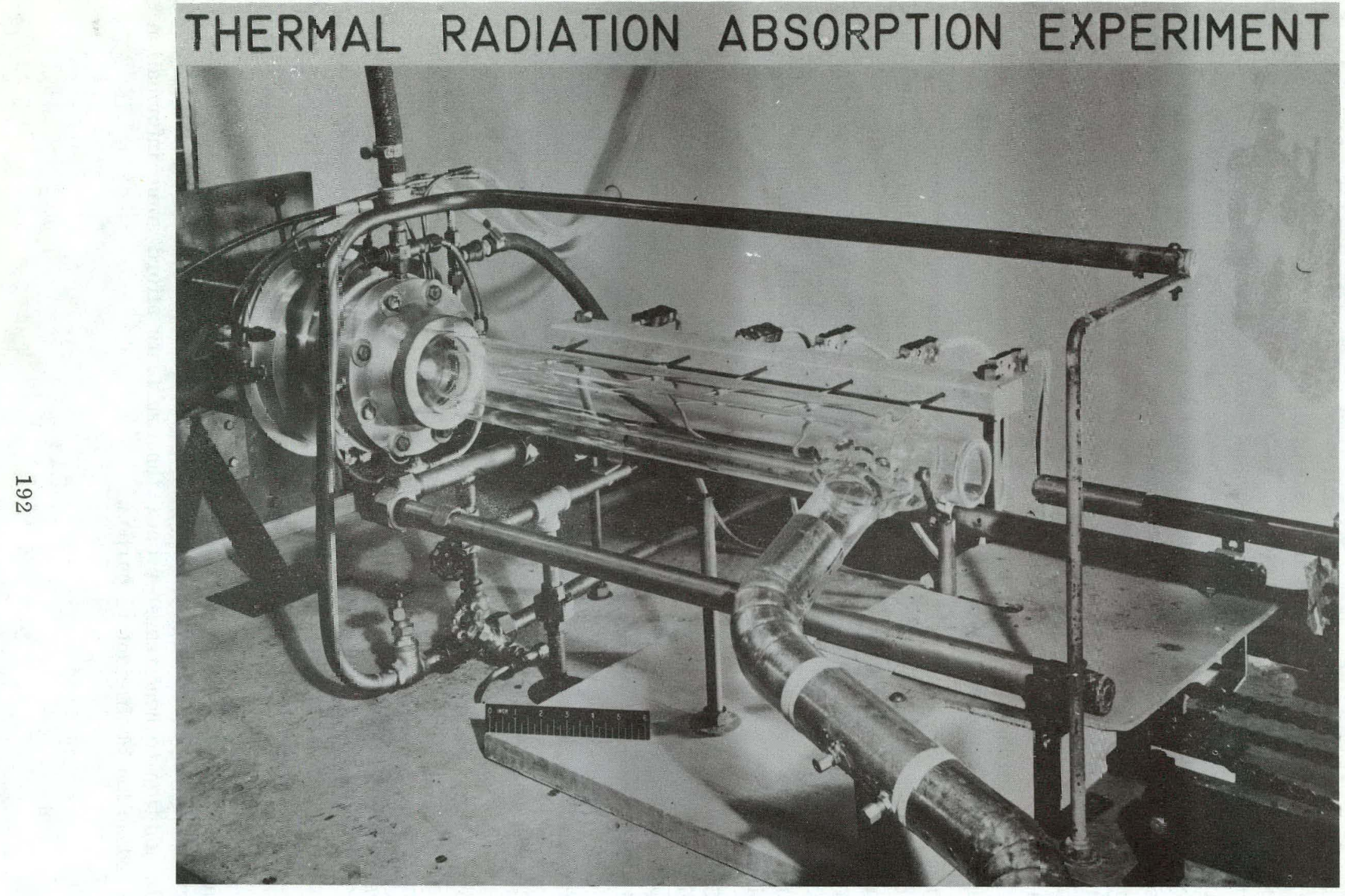

Figure 8 


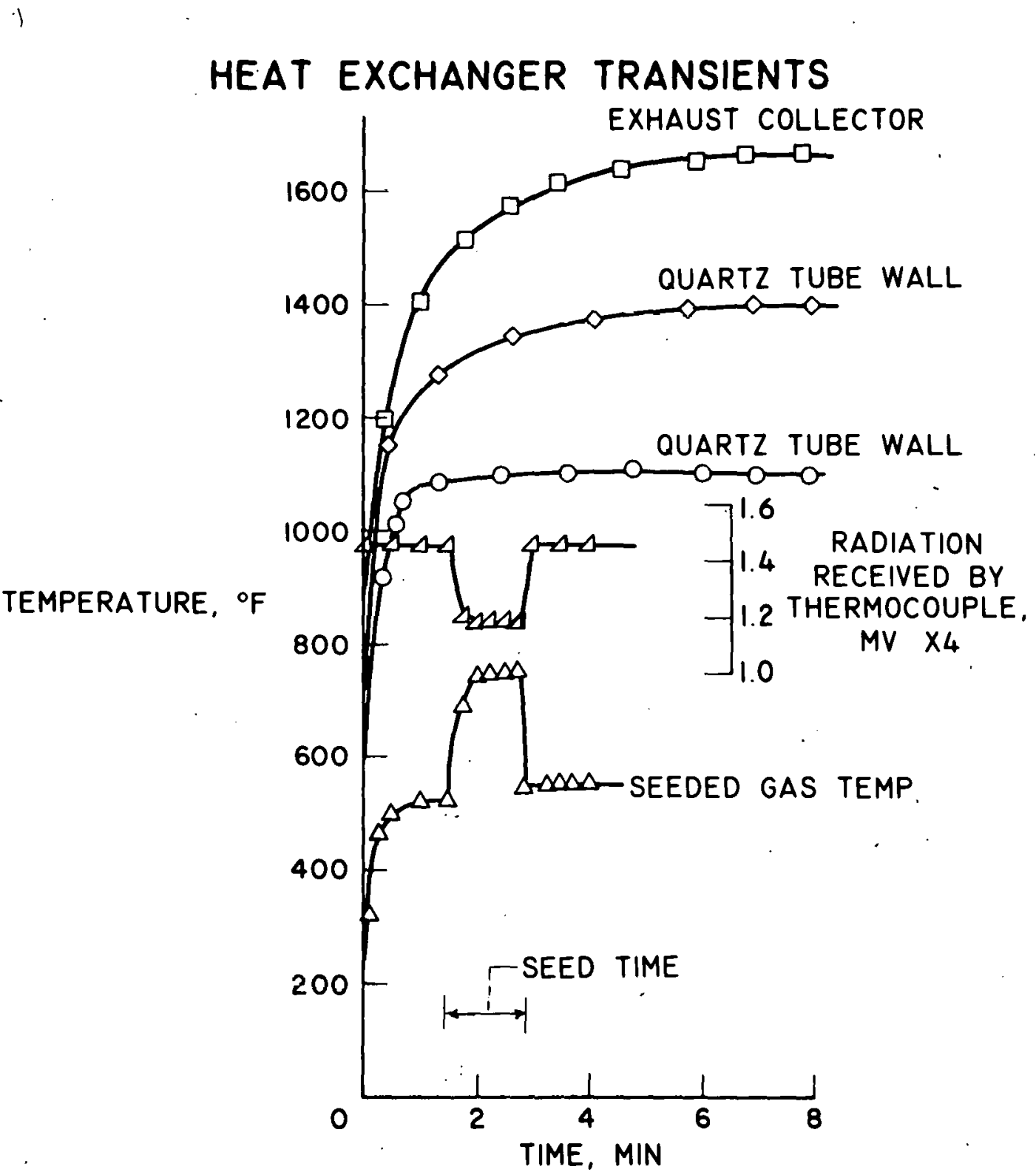

Figure 9 
The variation of the extinction cross section per particle $\epsilon / \mathrm{N}$ of carbon particles with particle size is shown in Figure 10 . Theoretical values taken from Barré and Stull and Plass are shown, as well as the geometric cross section. The data of this report are less than theoretical values but are considerably higher than the measurements of carbon particles dispersed in water.

The fact that the present data are higher than previous measurements and closer to theoretical estimates is attributed to better particle separation. It is felt that the extinction cross section data indicate that the particle injection system reduces particle agglomeration. Obviously, there are many factors involved in data such as these, but the conclusion seems justified. Further studies of particle size distribution, wavelength, and temperature effects are necessary to disclose whether complete particle separation exists.

The two primary conclusions of the arc tests are as follows:

1. A quartz-tube-contained electric arc is a useful research device for radiation heat transfer studies. A radiation flux of 305 watts per square inch was obtained at the surface of a 54-millimeter-diameter tube; this is the radiant flux that would be emitted by a $3050^{\circ} \mathrm{R}$ blackbody surface.

2. The addition of a small weight fraction of solid particles to a flowing transparent gas can significantly increase the total heat transfer in a system where both forced convection and radiation heat sources are present. For example, a 65-percent increase in heat transfer was obtained by adding less than 3 weight percent of carbon particles to an air stream flowing parallel to a quartz tube containing an electrio arc.

Figure 11 illustrates two additional heat transfer experiments which are in preparation. The first of these is intended to provide measurement of the opacity of particle-hydrogen mixtures. This experiment is shown schematically in the upper portion of Figure 11. A hydrogen plasma will be produced by a modified plasma torch designed to achieve hydrogen temperatures in the range of $20,000^{\circ} \mathrm{R}$. Seed materials such as carbon particles will be added to the hydrogen. A xenon arc lamp will be used as a light source, and a spectrophotometer will be used to measure the attenuation of a beam as it passes through the seeded plasma. Spectral opacities will bc measured over a wavelength range from 0.16 to 3.5 microns.

It is anticipated that this experiment will provide opacity measurements from room temperature up to a temperature above the sublimation or boiling point of the seed material. It is not likely, however, that this initial experiment will provide conditions such that the hydrugen itself contributes to the absorption process. To study hydrogen absorption, it will be necessary to: 


\section{EXTINCTION CROSS SECTION PER PARTICLE OF CARBON}

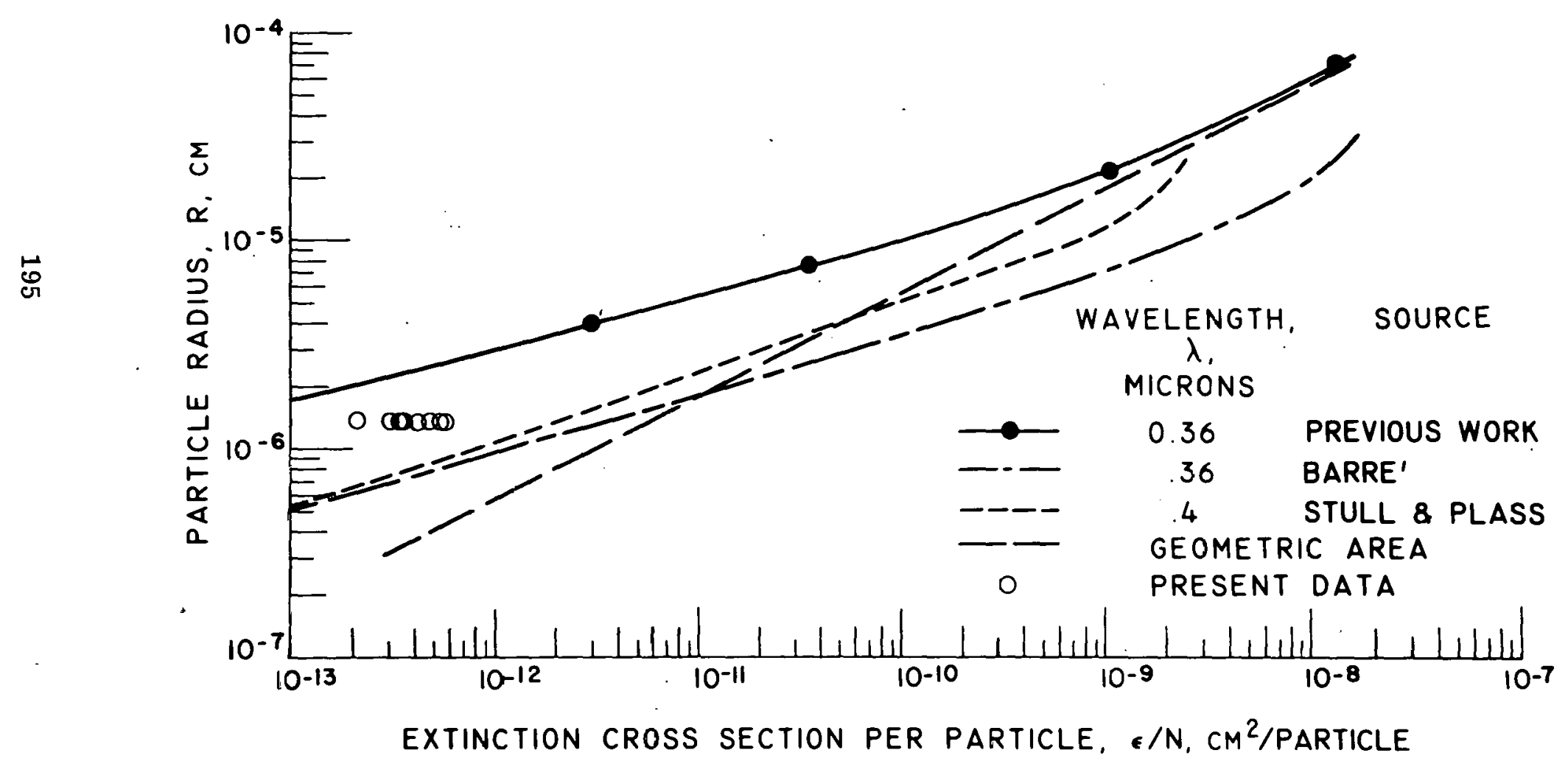

Figure 10 

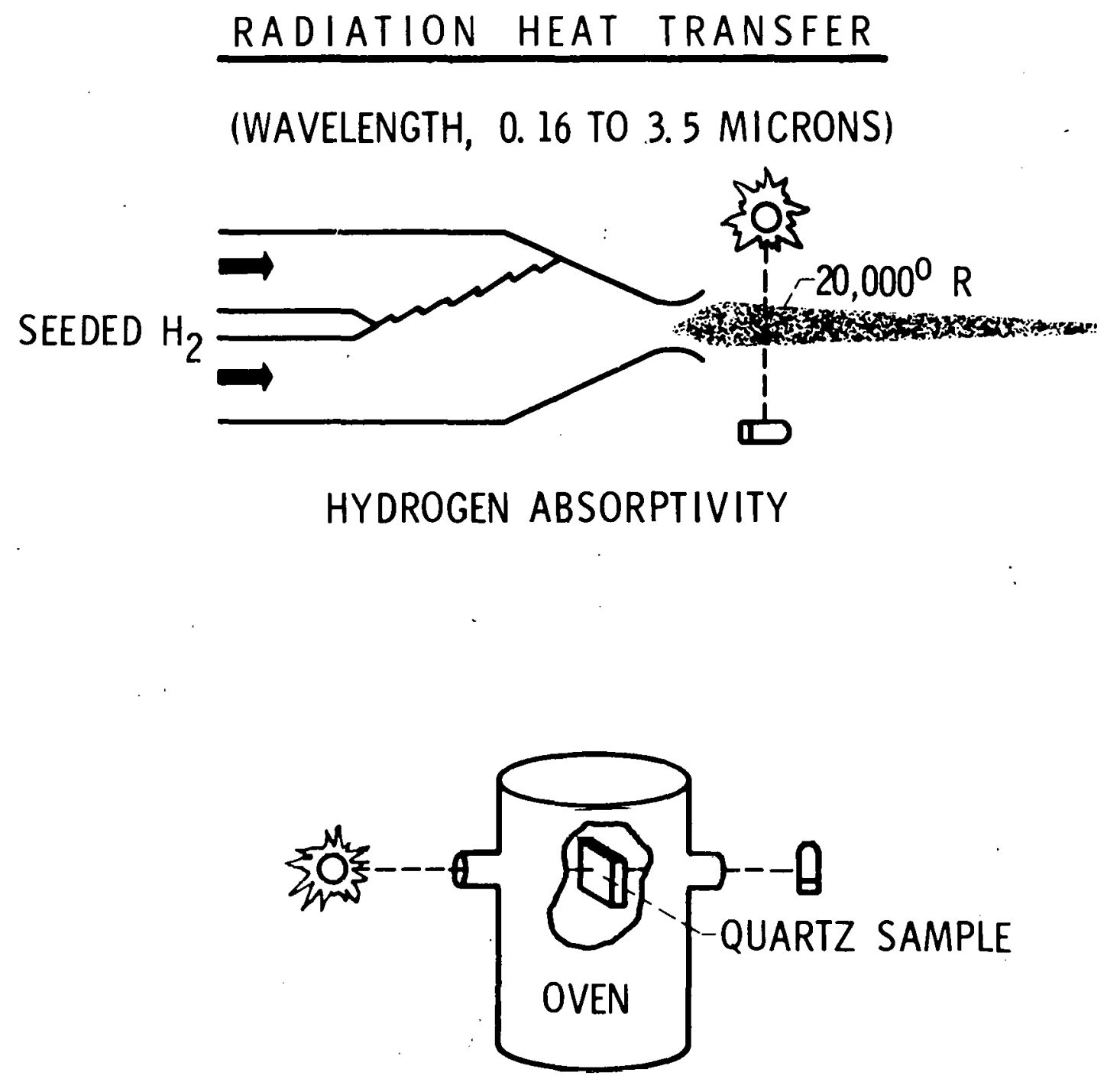

WINDOW TRANSMISSIVITY

Figure 11 
(1) extend the wavelength range of the instrumentation down to 800 angstroms or up to 10 microns, (2) extend the hydrogen temperature up to about $26,000^{\circ} \mathrm{R}$, or (3) extend the hydrogen pressure up to about 20 atmospheres. Such an experiment would be quite difficult, however, and would therefore be preceded by a comparison with other experimental approaches.

The lower portion of Figure 11 illustrates an experiment on the spectral transmissivity of transparent materials at elevated temperatures. Measurements will be made for wavelengths from 0.16 to 3.5 microns and sample temperatures up to $1200^{\circ} \mathrm{C}$. Materials such as quartz, fused silica, and sapphire will be investigated.

A heat transfer study is being funded at Georgia Institute of Technology under the direction of Dr. C. Orr. This work is an analytical and experimental study of heat transfer to clouds of small particles.

\section{NUCLEONICS}

A neutron diffusion experiment on a cavity reactor geometry that is underway at Lewis Research Center is shown in Figure 12. The apparatus consists of two concentric aluminum drums. A plutonium-beryllium neutron source moves along the central axis of the inner cylinder to simulate a line source. Flux distributions measured by foil activation will be compared with various analytical formulations. Geometry and moderator material effects will be studied. Similar work on a water-reflected spherical cavity is being supported at Case Institute of Technology under Professor F. Miraldi.

\section{COAXIAL FLOW ENGINE PERFORMANCE}

Some exploratory calculations of the performance characteristics of a coaxial flow nuclear engine have been made hy utilizing the current information on turbulent mixing, radiation heat transfer, and reactor criticality. In order to make the calculation, a number of engine parameters were assigned constant values. A reactor cavity diameter of 10 feet and a specific impulse of 1500 seconds were chosen. The total moderator thickness was taken to be 3 feet; it is composed of a thin ( 4 to 8 centimeters) liner of $\mathrm{D}_{2} \mathrm{O}$, a $\mathrm{BeO}$ region, and an outer $\mathrm{D}_{2} \mathrm{O}$ region. The entire reactor is enclosed by a pressure shell. Plutonium is the nuclear fuel used. A schematic view of the engine is shown in Figure 13.

For a selected thrust level of one million pounds $(694 \mathrm{lb} / \mathrm{sec}$ hydrogen flow), consideration of turbulent mixing, heat transfer, and reactor criticality 


\section{NUCLEONICS RESEARCH}

1. ANALYSIS - GEOMETRY, MATERIALS, TEMPERATURE

2 NEUTRON DIFFUSION EXPERIMENT:

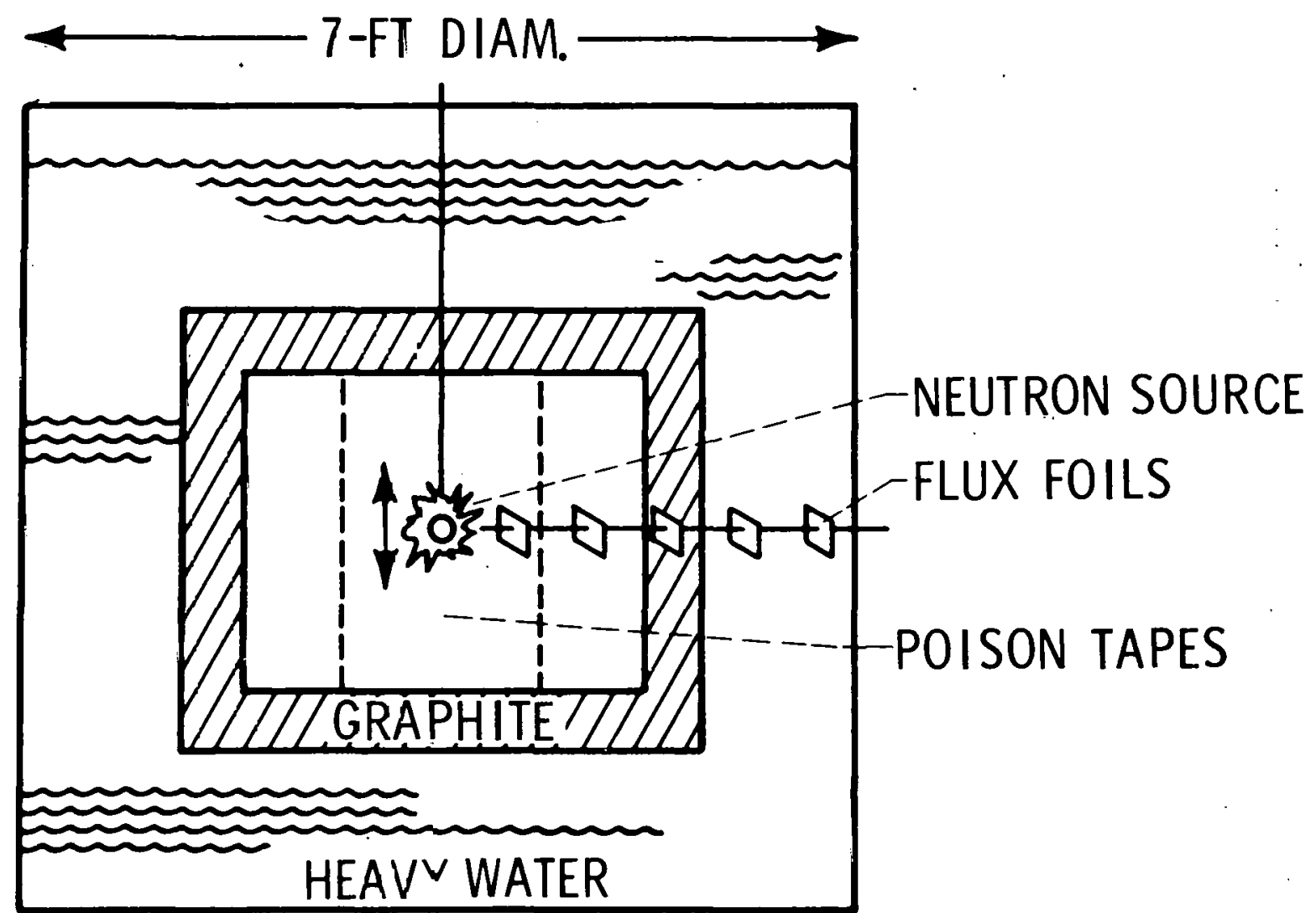

Figure 12 


\section{COAXIAL FLOW GNR-CONCEPTUAL SCHEMATIC}

$$
I_{S P}=1500 \text { SEC } F=10^{6} \mathrm{LB}
$$
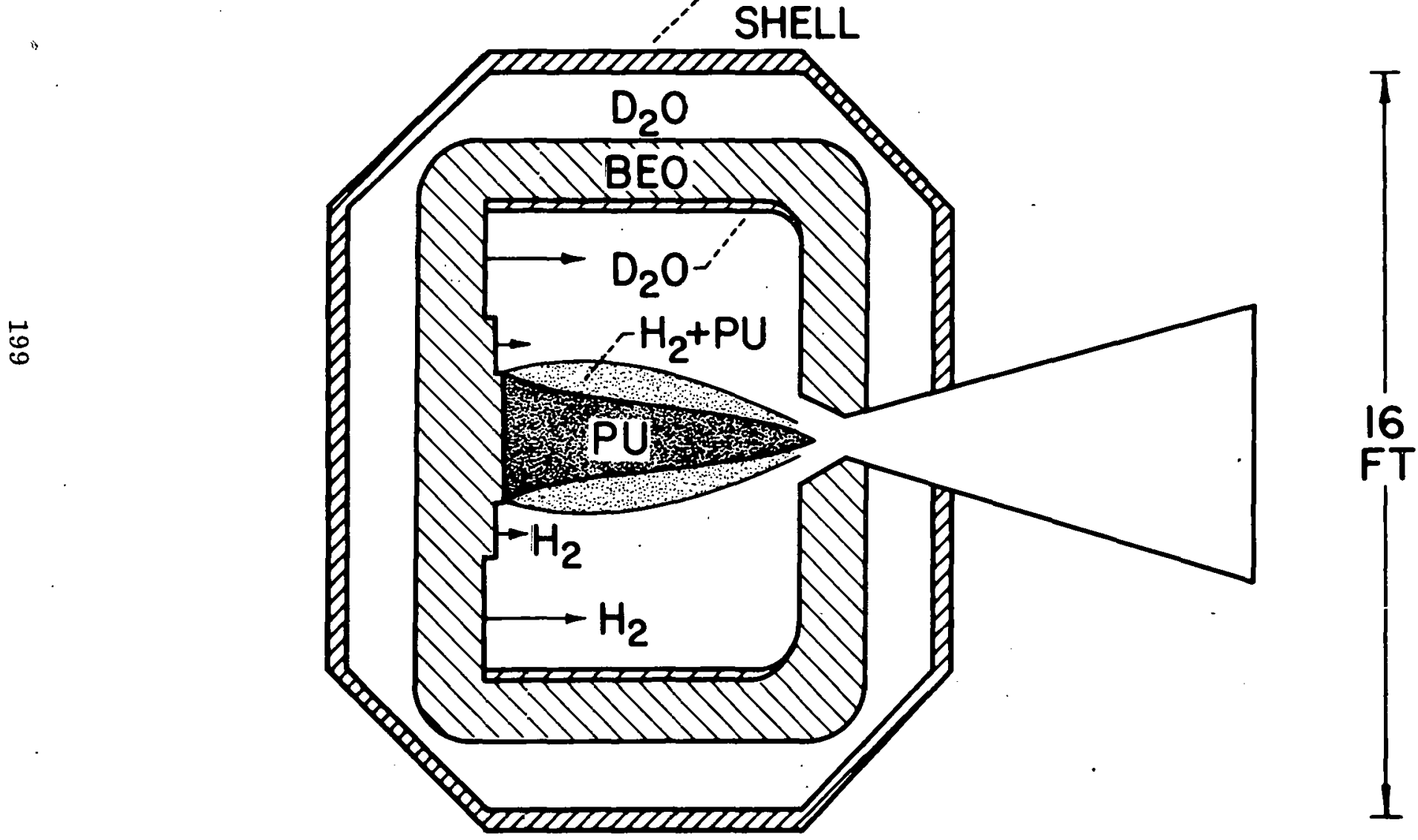

Figure 13 
yields the following quantities. The fuel injection diameter is 3 feet, and the initial velocity is $0.8 \mathrm{ft} / \mathrm{sec}$. The buffer region hydrogen is injected at a velocity of $17 \mathrm{ft} / \mathrm{sec}$, and is 1.5 foot thick. The initial velocity of the outer hydrogen is $40 \mathrm{ft} / \mathrm{sec}$. The reactor cavity is 7.5 feet in length. The average fuel temperature is $40,000^{\circ} \mathrm{R}$, the reactor pressure is 586 atmospheres, and the fuel is $79 \%$ singly ionized. The hydrogen-to-fuel flow rate ratio for this case is 41 . The moderator weight is $169,000 \mathrm{lb}$., and the pressure shell weight is $152,000 \mathrm{lb}$. This gives an engine thrust-to-weight ratio of 3.2 .

As the fuel flows through the reactor it is accelerated by the faster moving hydrogen. The fuel is injected at a velocity of $0.8 \mathrm{ft} / \mathrm{sec}$; the reactor exit, it is moving at an average velocity of $5.6 \mathrm{ft} / \mathrm{sec}$. The fuel residence time is 2.6 seconds, as compared to 0.23 seconds for the hydrogen. Initial and final velocity distributions for this case are shown in Figure 14.

The reactor pressure required for criticality can be reduced by increasing the fuel flow rate. Conversely, a higher ratio of hydrogen-to-fuel flow ratio can be obtained by operating at a higher reactor pressure. This ability to decrease the fuel loss at the expense of pressure is adversely affected by thrust level. These engine characteristics are shown in Figure 15. The three-way trade-off between fuel loss, reactor pressure, and engine thrust is further affected by many other factors not considered as variables here. The general trend indicated in Figure 15 is probably valid, however; an increase in engine thrust requires an increase in either reactor pressure or fuel loss, but this penalty diminishes rapidly at higher thrust levels.

Although the performance culculation involves many assumptions, and the engine shown is not an "optimum" one, it is consistent with current information. Further, such a calculation serves to illustrate some interesting characteristics of a gaseous fueled nuclear engine operating at a 1500 second specific impulsc:

1. Reactor moderator, reflector, and structural materials are unfueled and can be operated at a relatively modest temperature of $3200^{\circ} \mathrm{R}$.

2. Hydrogen enters the reactor cavity at $3000^{\circ} \mathrm{R}$ and is cxhaustcd at an average temperature of about $8700^{\circ} \mathrm{R}$.

3. Reactor criticality can be maintained by a pressure of 500 to 800 atmospheres.

4. The fuel loss rate can be maintained sufficiently low that: (1) the cost incurred will be less than that of the hydrogen propellant for a given mission, and (2) there is no appreciable degradation of specific impulse. 


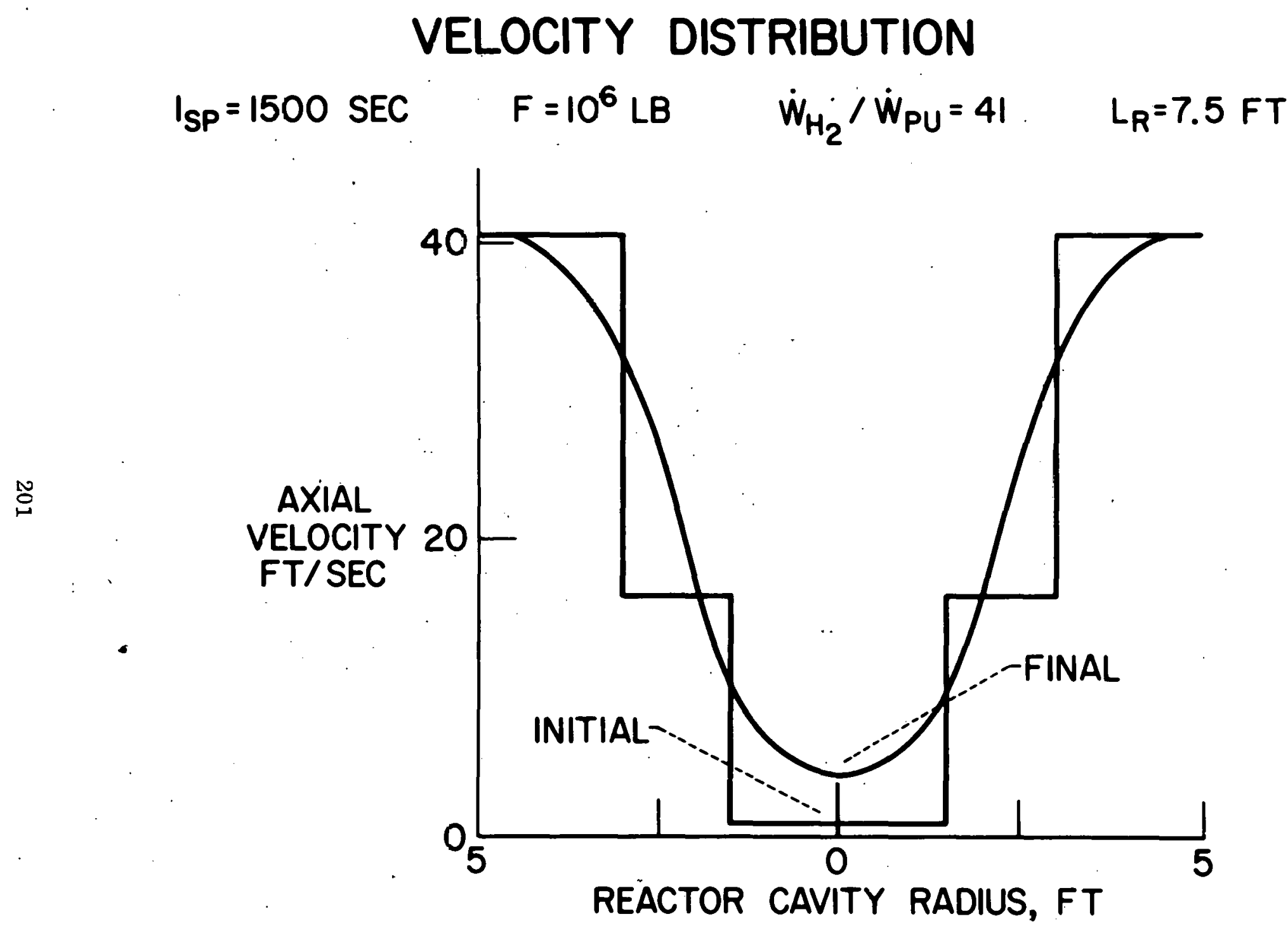

Figure 14 
EFFECT OF THRUST LEVEL ON FUEL LOSS RATE

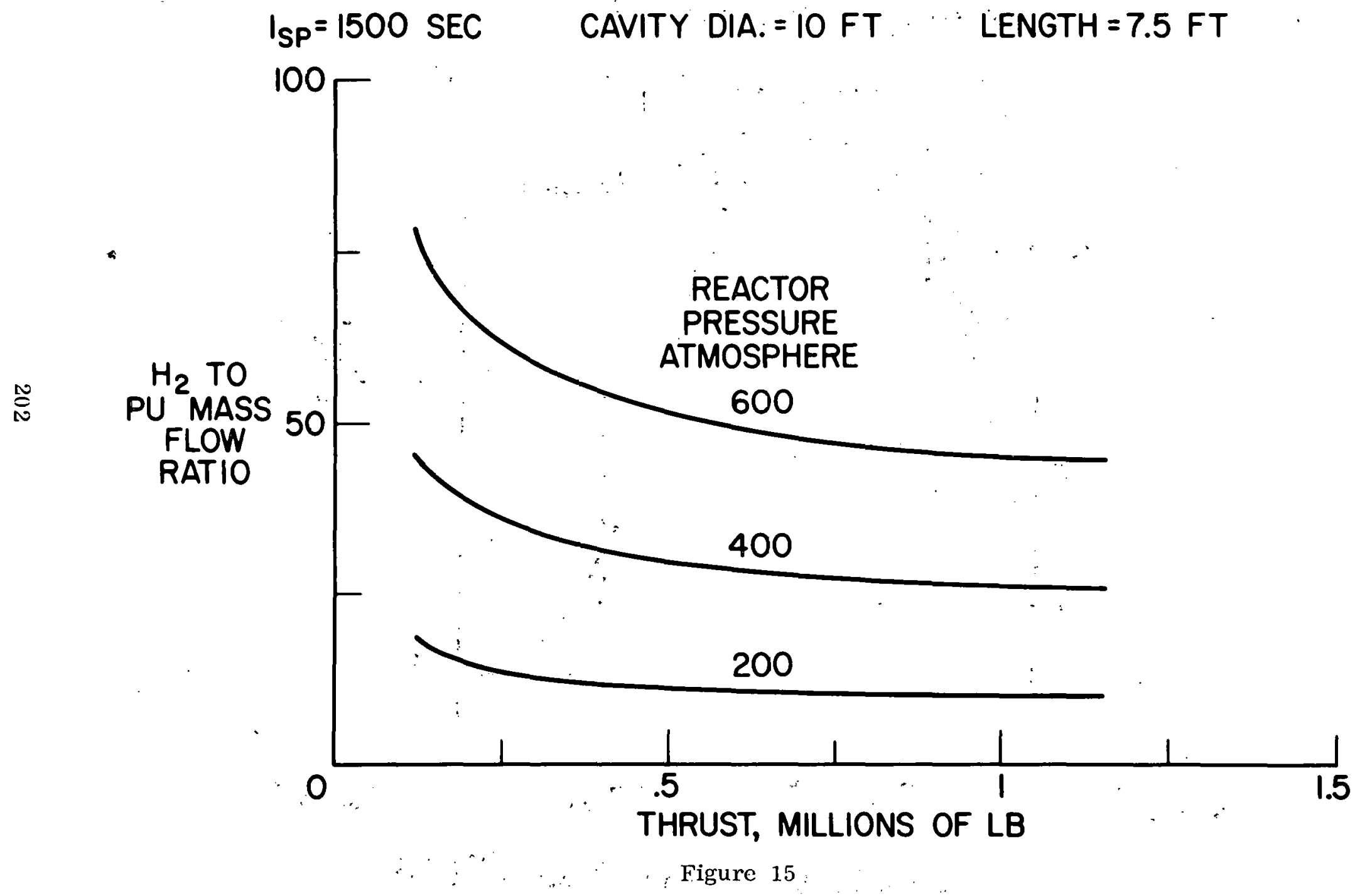


5. Engine thrust-to-weight ratios greater than one are possible, and no severe penalty results from increasing the thrust level. Figure 16 summarizes the probable range of pertinent engine parameters.

Although the work on gaseous-fueled nuclear rocket engines to date can be interpreted as encouraging, it has served to define additional problems as well as solve preliminary ones. Some important problem areas that will require future attention are listed in Figure 17 . With the possible exception of items 2 and 6 ; these problems are of a general nature and can be usefully studied independent of a specific concept.

Question: Why do you use plutonium, with its contamination problem?

Answer: It requires a lower critical mass.

Question: By how much?

Answer: It depends on the particular case, but maybe by a factor of two to four.

Question: Is that because of the low energy resonance peak?

Answer: Yes. Of course, that also offers a potential disadvantage, since there is a resonance peak in the absorption cross section as well as the fission. So, for some cases, the advantage of plutonium may be lost because of self-shielding.

Question: Are you doing any.criticality correlations?

Answer: We have made some two dimensional diffusion calculations comparing plutonium and uranium. These were reported in NASA TN D-1575. These calculations showed that if the plutonium fuel was compressed to too small of a radius inside the cavity, the critical mass increased rapidly.

Question: Of course, then the reactor is boooming fast.

Answer: Well, that is the direction of the trend, but the reactor is still 95 to 99 percent thermal at the point where the critical mass begins to increase.

Question: What is a typical critical mass of plutonium?

Answer: Twenty kilograms, although I'm not sure just how typical it is. This might be reduced to 10 kilograms if it were possible to select the most favorablc oombination of factors. This is one area that needs additional work; this is extremely important, since criticality is the reason that these reactors are so large.

Question: Has anyone considered reactor kinetics? For example, what happens if the plutonium is displaced a little? Is that a stable situation? 


\section{PROBABLE PERFORMANCE RANGE}

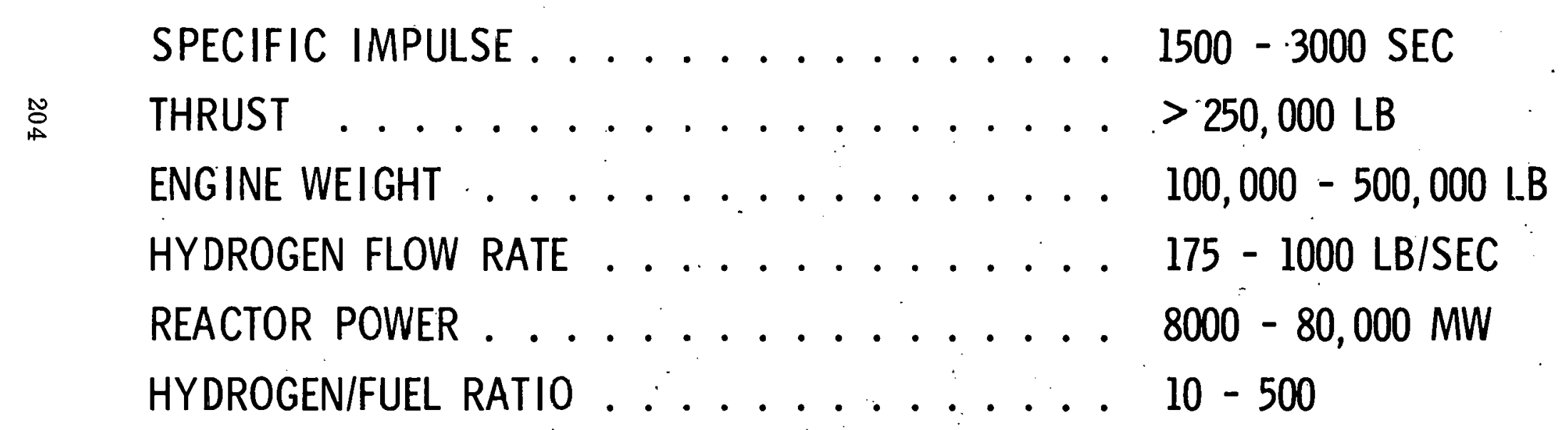

Figure 16 


\section{FUTURE PROBLEMS}

1. SEED-HYDROGEN OPACITY WINDOW

2. COMBINED FLOW AND HEATING

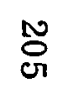

3. CRITICAL EXPERIMENTS

4. NOZZLE HEAT TRANSFER

5. STABILITY AND CONTROL

6. FUEL STORAGE AND INJECTION 
Answer: Very little has been done as yet on this subject. It is a difficult problem because it involves the interaction of heat transfer, nucleonics, and fluid mechanics.

Question: What is the residence time of fission products?

Answer: Again, there is no "typical" answer, but it could be on the order of 2 seconds.

Question: How is the reactor controlled then, if all of the delayed neutrons are removed?

Answer: This is another problem area that will require study. Even if some of the delayed neutrons are lost, control may not be a serious problem because neutron lifetimes are considerably longer in cavity reactors than in a more conventional geometry.

Question: In your experimental flow studies; you have not simulated the $\overline{\text { presence }}$ of an end wall deflecting the flow radially inward toward a nozzle. Are you concerned about this?

Answer: This too is a subject for future investigation. To date, this effect is not included in either the experiment or the analysis. The analysis is written for a flow field that is infinite in both the radial and downstream directions; there are no boundary or end effects. The coaxial turbulent mixing analysis is quite complicated when treated as an initial value problem; as a boundary value problem it is even more difficult.

Question: Have you considered the effects of simultaneous heat generation in the plutonium and radiative heating of the hydrogen on the hydrodynamic mixing?

Answer: No. At least we have not investigated this effect yet. We do have a version of the flow analysis which contains arbitrarily distributed heat sources. As an approach to this problem, we will be able to assign heat source distributions which yield temperature profiles that are the same as those obtained from the radiation heat transfer analysis. Similarly, the heat transfer code can handle arbitrary velocity profiles, which can be adjusted to agree with those from the flow code. This is an empirical procedure, but the problem is a difficult one.

Question: What is the reactor chamber pressure?

Answer: This depends on so many unknowns and arbitrary trade-offs that there is no one answer, but 500 atmospheres is a reasonable guess. It will be as low as is possible without exceeding some limiting quantity such as fuel loss rate. It should fall within the 300 to 800 atmosphere range. 
Question: Do you think that the mixing between the plutonium and hydrogen is sensitive to the initial conditions of the two streams?

Answer: Yes, because the turbulence level is, and that is governing factor in the mixing process.

Question: Are the mixing calculations based on the assumption of ideal injection of plutonium and hydrogen?

Answer: No. The coaxial mixing calculations are made for turbulent flow, not laminar. The turbulence level is computed from a correlation based on turbulent mixing data. It includes effects of fluid properties, initial stream Reynolds number's, and the initial velocities of both streams. For reactor conditions, the turbulent transport properties are 3000 times as large as the laminar values. Laminar flow calculations would give reactor pressures about 5 to 10 times lower than what we get for turbulent mixing. 
COAXIAL FLOW RADIATION HEAT TRANSFER ANALYSIS

\author{
Albert F. Kascak \\ NASA-Lewis Research Center \\ Cleveland, Ohio
}

Most gaseous core rockets use the same mechanism to transfer heat to the propellant. In the reactor energy is released in the core (due to nuclear reaction). The core temperature increases until it is high enough to radiate the heat to the propellant.

Since most gaseous core reactors have cylindrical geometry, the problem was formulated, and a computing program written to solve a radiating and convecting gas in a cylindrical geometry. The analysis was written to include both radial and axial flow. The present discussion is limited to axial flow problems only.

The specific rocket concept being examined is the coaxial flow system. The first slide shows a simplified diagram of this model. The flow enters the reactor from the left and exits to the right. (The arrnws are in the wrong direction at the exit.) The central cylinder represents the gaseous nuclear core and the outer annulus represents the propellant region.

Besides the highly idealized physical model, there are certain assumptions made in the formulation of the problem. These are:

1. Velocity profiles are known functions of position.

2. Viscous and conduction effects are neglected.

3. Inlet and side wall temperatures are known.

4. Walls are black and follow the cosine law.

5. The gas is gray but optically temperature dependent.

6. The exit wall is at the local gas temperature.

The first three assumptions are common and, therefore, no conment 


\section{PHYSICAL MODEL}

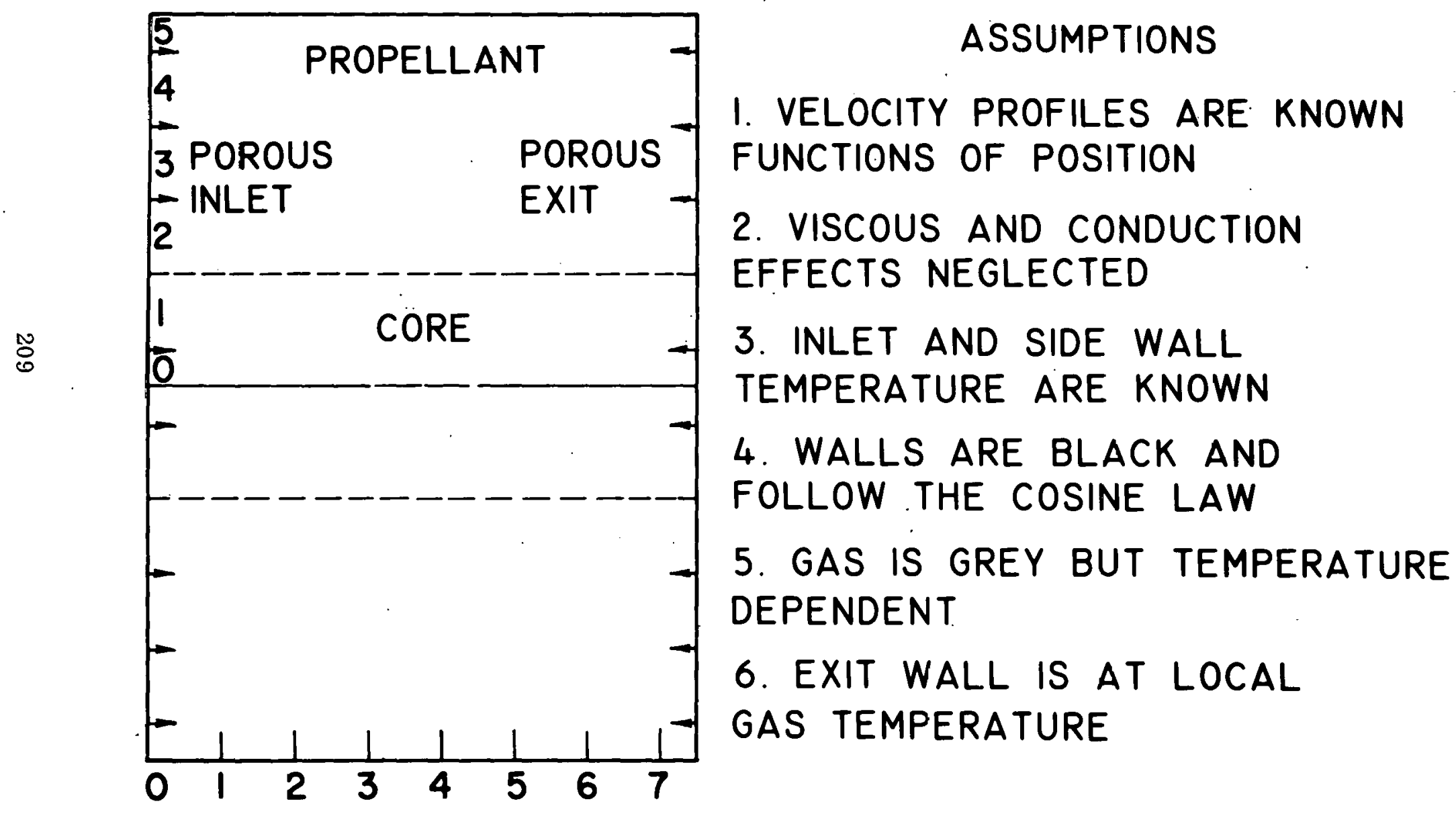

Figure 1 
will be made on them. The fourth assumption was made because better knowledge was not available and was justified because back radiation from the wall was relatively small.

The fifth assumption is tantamount to using a frequency averaged absorption coefficient, but yet temperature dependent. The proper averaging technique is perhaps debatable; but the difference between Rosseland and Planck mean is not too great for the propellant.

The sixth assumption was made for two reasons. (1) The back radiation from the nozzle region was not known and the exact exit details were not of interest at the present time. (2) This specific choice was made to eliminate large amounts of heat transfer across the exit wall.

Assumptions 1 and 2 leave only the energy equation to be solved. The second slide shows this nonlinear integral-differential equation. The first term is the convective term. The second is the internal heat generation term; it is a known function of position. The third term is the radiation being emitted. The fourth term is the radiation being absorbed from the surrounding gas and walls. The unknowns in the equation are the temperature distribution and any property which depends on the temperature. This includes the absorption coefficient.

The basic method of solution was to assume a temperature distribution, then evaluate the radiation integrals as a function of position, and then to solve the resultant nonlinear differential equation for a new temperature distribution. The process was repeated until the temperature distribution did not change by some specified amount.

The second slide also shows the general technique for evaluating the radiation integrals. The field point lies on a typical representative plane. The integrals are first evaluated in the radial direction and terminated at the wall or when the contribution to the total radiation becomes negligible. This allows us to calculate the optical distance between the field and source point and its contribution almost at the same time. The surface term is then just added to the total.

The angular integration was weighted in specific directions. The weighting function was chosen so that direction with high heat flux could be sampled with smaller increments. In this case the core direction was weighted the heaviest.

Question: How many angular groups are you using?

Answer: About every five degrees. This is all right if the gas if not too clear. 


\section{ENERGY EQUATION}

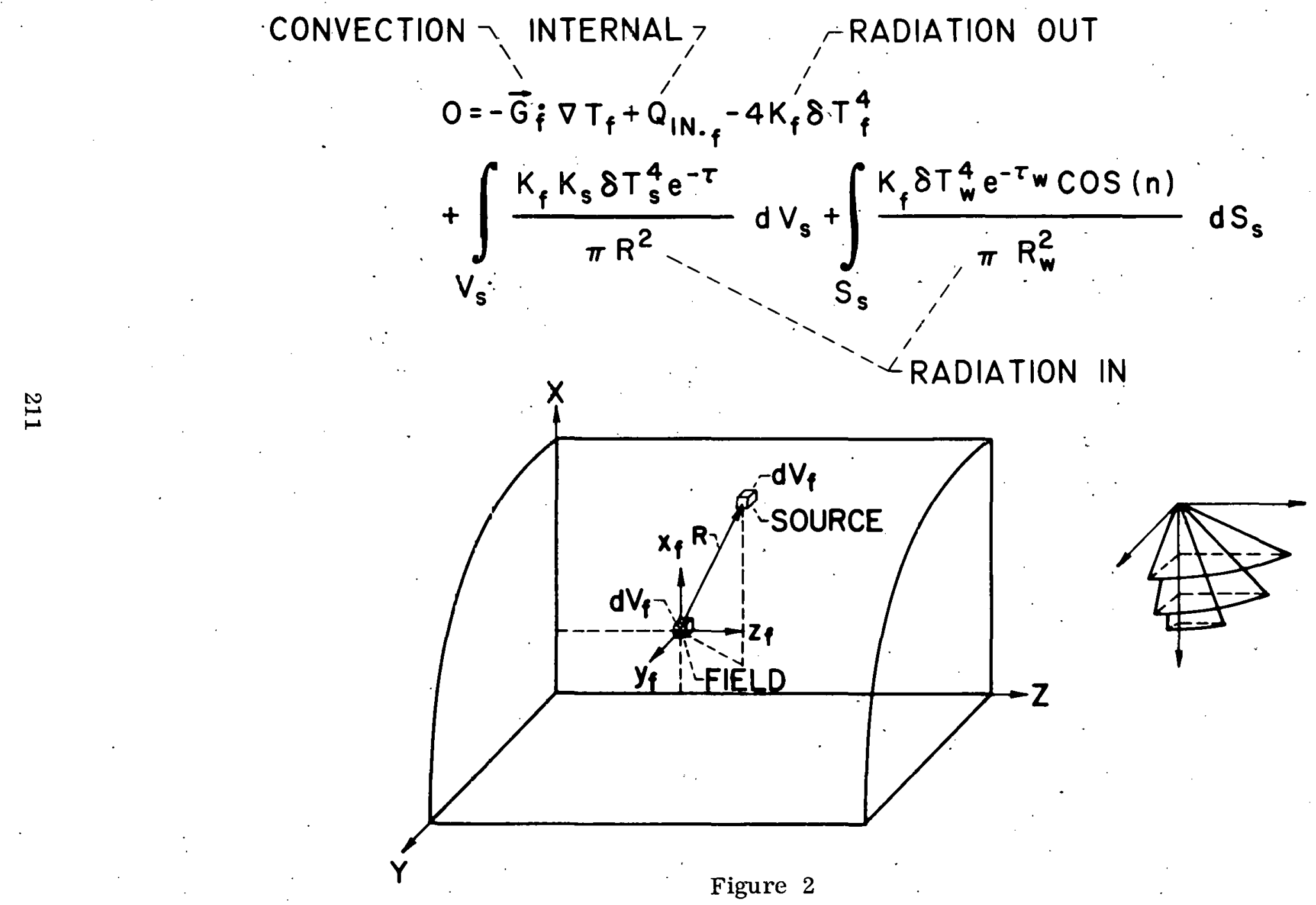


The field points were chosen so that a two dimensional interpolator could be used to find intermediate values. Since the amount of radiation coming into a point is a space integral, it would be a smooth function of position. The smoother the function the easier it is to interpolate.

The physical dimensions of the reactor under study are: a length of 7.5 feet, a cavity diameter of 10 feet, and a fuel-core diameter of 3 feet. Physically the reactor is more like a pancake than a tube. Therefore, end effects can be expected to be large.

The physical parameters of the core are: an absorption coefficient of $100 \mathrm{ft}^{-1}$, a specific heat of $0.06 \mathrm{Btu} / \mathrm{lb}$, a mass flow rate of $2.83 \mathrm{lb} / \mathrm{sec} / \mathrm{ft}^{2}$, and an internal heat generation rate of $498,000 \mathrm{Btu} / \mathrm{sec} / \mathrm{ft}^{3}$. In the propellant region the absorption coefficient is $2 \mathrm{ft}^{-1}$, the specific heat is $6 \mathrm{Btu} / \mathrm{lb}$, the mass flow rate is $9.72 \mathrm{lb} / \mathrm{sec} / \mathrm{ft}^{2}$, and there is no internal heat generation.

The third slide shows some typical radial temperature profiles. Near the entrance, the core temperature is much higher than the propellant temperature. This results in a large gradient between the two regions. Further into the reactor the propellant temperature near the core builds up like a boundary layer. This continues until the propellant near the core is in thermal equilibrium with the core. The boundary region then begins to thicken. This boundary region then ceases to be a heat sink and becomes a transmitter of energy.

Question:. Do you allow your absorption coefficient to be a function of temperature?

Answer: Yes, in one of the later slides.

Question: Do yoü allow your power profiles to vary axially?

Answer: In the general program the internal heat is a function of both radial and axial positions; but for the present example it is assumed constant in the axial direction.

The fourth slide shows some typical axial temperature profiles. The temperature of the fuel increases very fast upon entering the reactor. In this region, convection is the primary mode of heat transfer. The rest of the way down the reactor, the temperature of the core is relatively constant. In this region radiation is of prime importance. The propellant has the same type of temperature profile, except that it lags that of the fuel.

The fifth slide shows the effect of varying the fuel absorption coefficient. As the absorption coefficient increases, the exit temperature of the core decreases. For a transport type of process this is true. An absorption 


\section{RADIAL TEMPERATURE DISTRIBUTIONS}

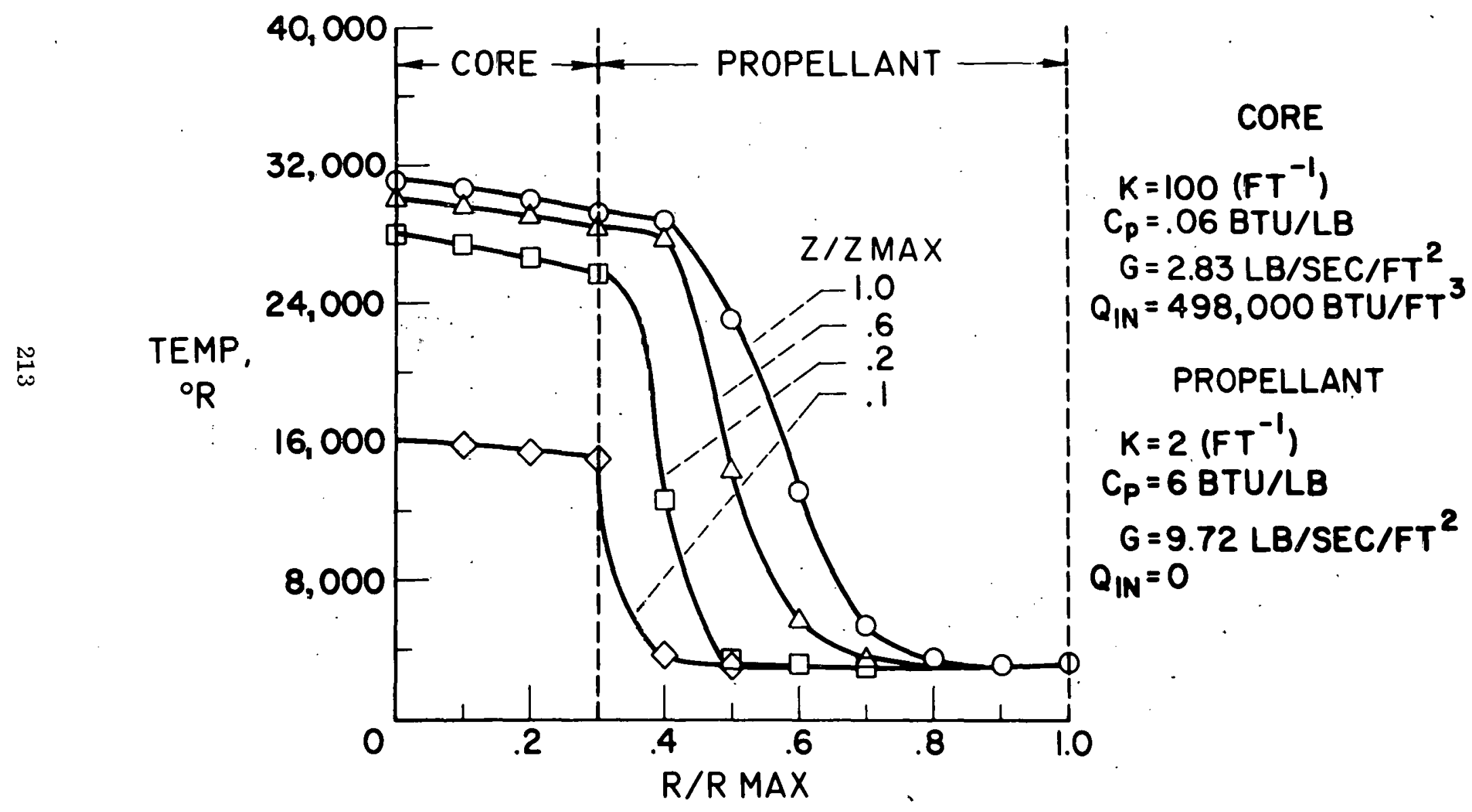

Figure 3 


\section{AXIAL TEMPERATURE DISTRIBUTIONS}

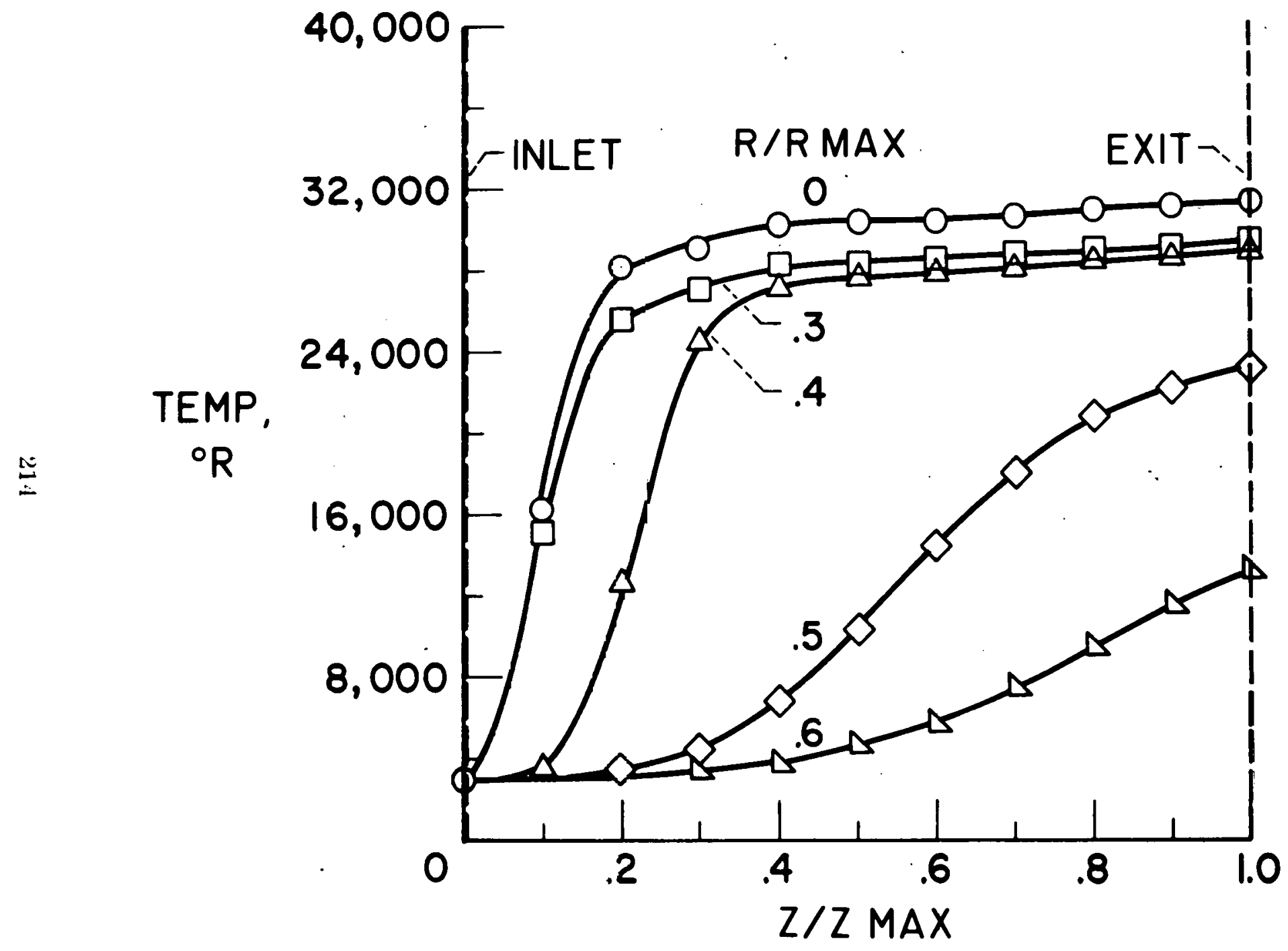

Figure 4 


\section{VARYING CORE ABSORPTION COEFFICIENT}

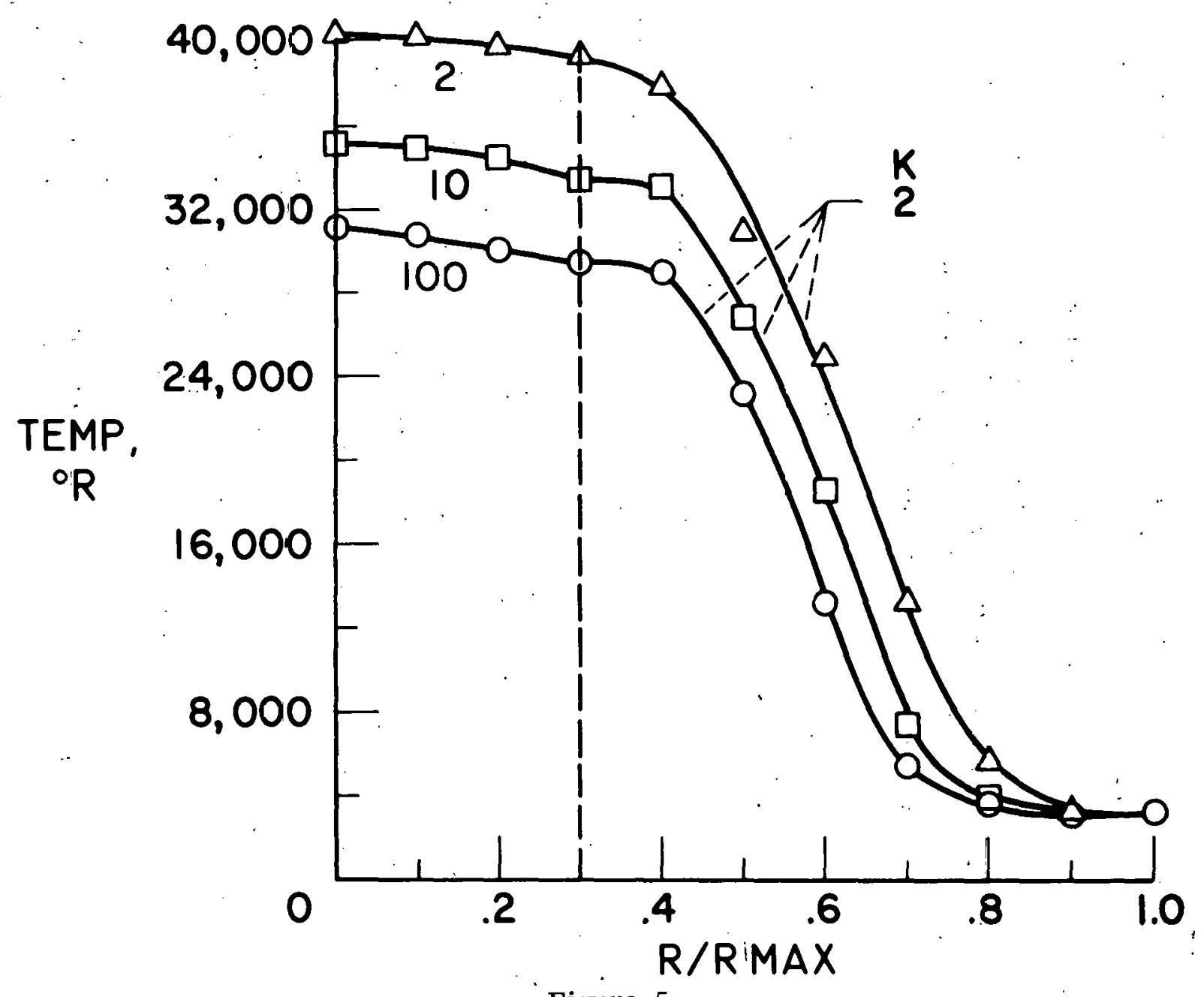

Figure 5 
coefficient of $2 \mathrm{ft}^{-1}$ or maybe even $10 \mathrm{ft}^{-1}$ might be considered a transport type process, but $100 \mathrm{ft}^{-1}$ is not. One reason for this is the increase in end losses as the absorption coefficient of the core increases.

Question: What is the density in the core?

Answer: It was not used anywhere in the calculation, so it was not calculated for this analysis.

Question: The absorption coefficient of the fuel seems low.

Answer: An analytical estimate of the fuel absorption coefficient was not used in this calculation. Arbitrary values, higher than the absorption coefficient of the propellant, were chosen. The primary purpose of the study was to look at the propellant, not the core.

Question: Why is not there a sharp temperature drop at the edge of the fuel considering the very short radiation path length in the core?

Answer: At the inlet there is a large gradient at the core-propellant interface; but axial convection eventually reduces this gradient.

Question: Is most of the energy transferred by radiation?

Answer: Yes, most of the energy in the core is radiated to the propellant, and then convected away.

Question: At a radius ratio of point three what is the radiant heat flux as compared to the blackbody radiant flux?

Answer: The program does not use this ratio, and it was never calculated. Question: Where is the heat flux less than blackbody heat flux?

Answer: What do you mean by blackbody heat flux?

Question: Well if $I$ took the heat flux passing a radius ratio of point three, radially outward (Btu per second per square foot), and then calculate blackbody heat flux at that position (which is $\sigma \mathrm{T}^{4}$ ), and compare the two.

Answer: The program did not calculate these quantities, so I don't have that comparison here.

Question: What is the mechanism of getting energy out of the center of the fuel? How does the energy in the center of the core get out so well? It seems that the center of the core ought to be much hotter than the edge. It is just like the sun, much hotter at the center than at the edge. The sun is opaque to its own radiation.

Answer: The absorption coefficient of the fuel was assumed to be $100 \mathrm{ft}^{-1}$. 
The fuel temperatures shown are consistent with this value. Higher opacities would give high fuel centerline temperatures. The main purpose of this study was to look at propellant region temperatures.

Question: What is the energy creation rate at the edge of the core as compared to that of the center of the core?

Answer: The energy profile was flat across the core.

Question: The exit plane has the same temperature profile as the gas. Can this exit temperature distribution radiate as though a spatial source was there?

Answer: Yes. At the exit, energy is transported into the reactor in certain regions and transported out in others.

Question: Isn't the profile in the propellant going to be greatly affected by both the detail of the fuel temperature profile and the frequency effects?

Answer: Since convection in the core is small, most of the heat must leave by radiation. The amount of radiation leaving the core must be almost constant, and equal to the total internal heat. Therefore the amount of radiation coming from the fuel should be relatively constant, and the temperature profiles of the propellant should be almost independent of the core temperature profiles. Wavelength dependence of opacity has not yet been included in this analysis, so no conclusion can be drawn as to its relative importance.

The sixth slide shows a temperature dependent absorption coefficient. In the fuel the absorption coefficient is constant at $100 \mathrm{ft}^{-1}$. In the propellant the absorption coefficient is $2 \mathrm{ft}^{-1}$ for temperatures less than $7200^{\circ} \mathrm{R}$. This region represents the seeded gas. At $7200^{\circ} \mathrm{R}$ the seed vaporizes; for temperatures between $7200^{\circ} \mathrm{R}$ and $10,000^{\circ} \mathrm{R}$ the absorption coefficient is $0.02 \mathrm{ft}^{-1}$. This region represents a window. For temperatures greater than $10,000^{\circ} \mathrm{R}$ the absorption coefficient is $2 \mathrm{ft}^{-1}$. This absorption coefficient approximates the real: absorption coefficient of the propellant.

The seventh slide shows radial temperature distributions at the exit with and without the window. The general effect of the window is to flatten the propellant temperature distribution; that is, the gas heats up, becomes transparent, and transmits energy through itself.

The big question in this regard is whether the propellant will become totally transparent and the energy in the core will be deposited on the wall; or will the propellant bridge the gap and still shield the wall. The eighth figure answers this question. It shows constant temperature lines on a representative plane. The $10,000^{\circ} \mathrm{R}$ line starts in the propellant nearest the fuel 


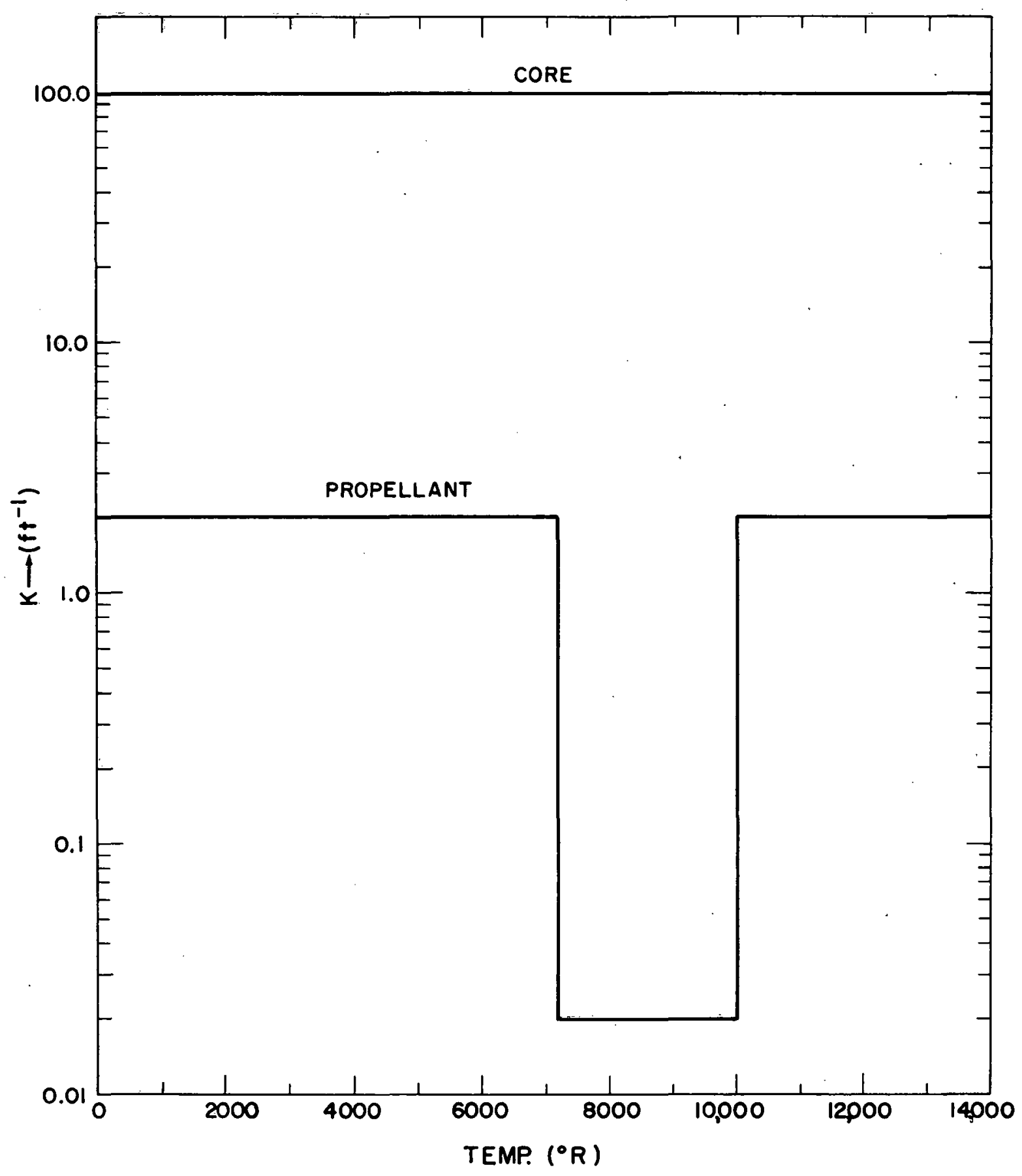

Figure 6. Absorption Coefficient vs. Temperature 


\section{RADIAL TEMPERATURE DISTRIBUTIONS}

AT EXIT

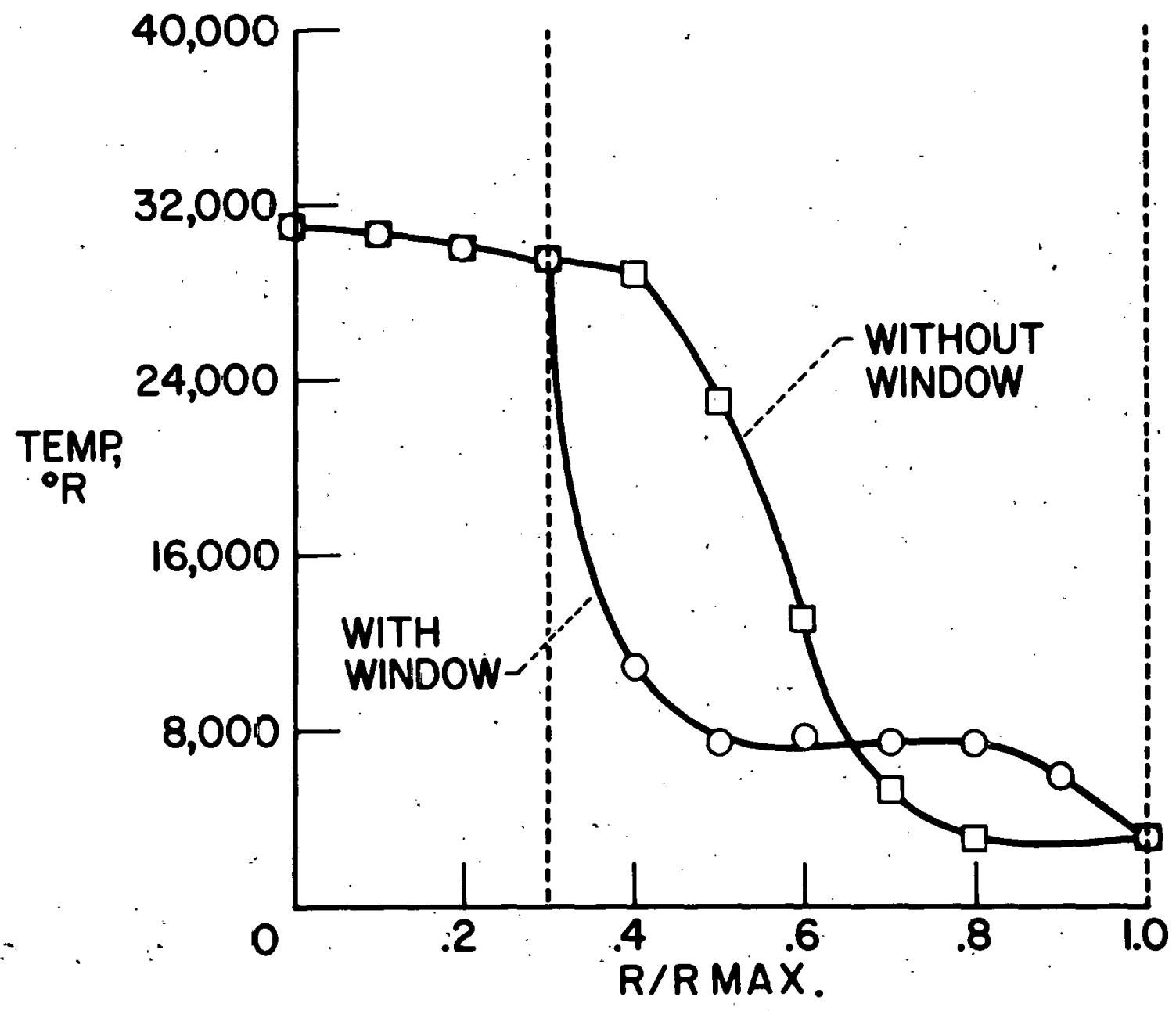

Figure 7 


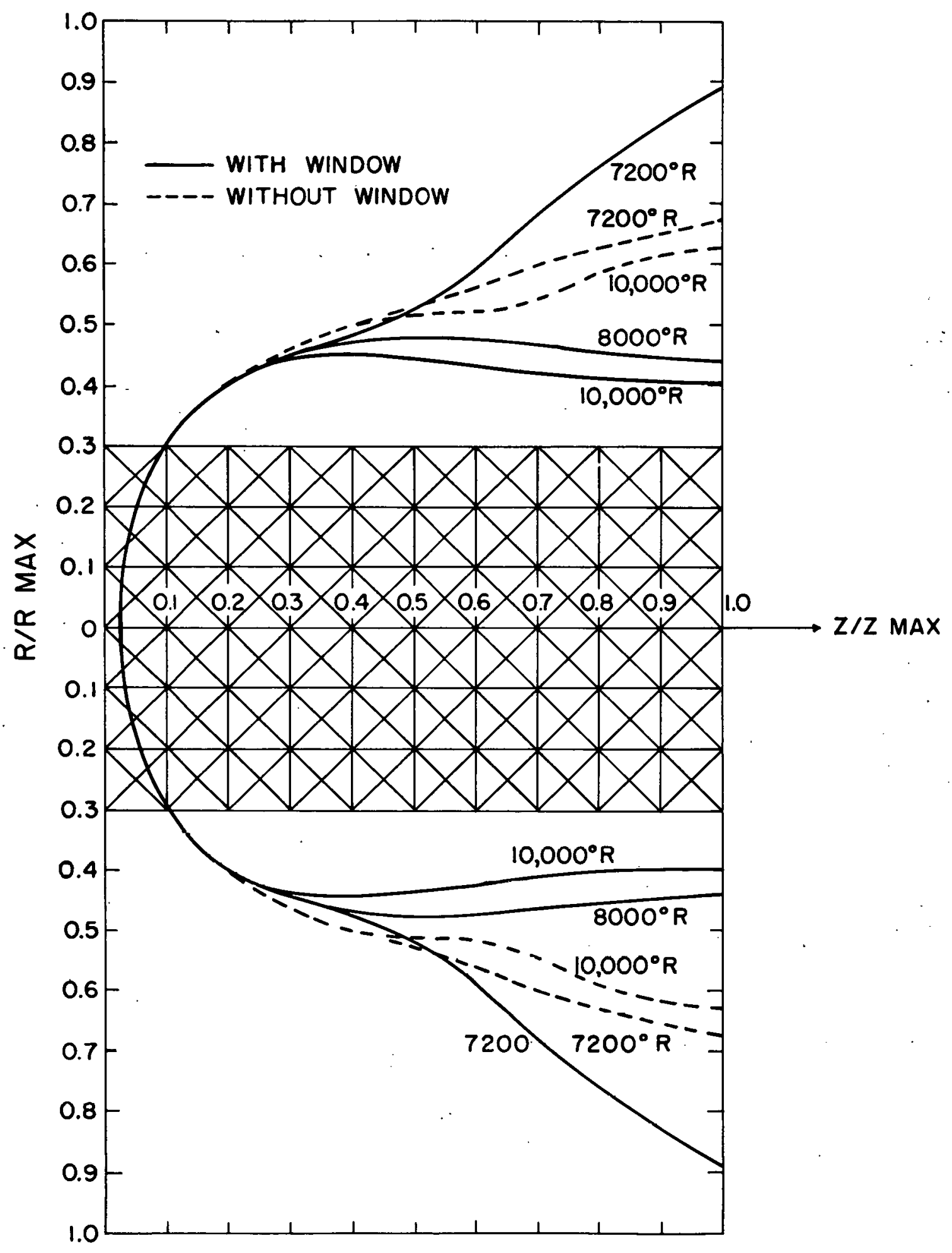

Figure 8. Constant Temperature Lines 、 
before the $7200^{\circ} \mathrm{R}$ line reaches the wall. This indicates that the wall can be shielded at all times from the hot core.

Question: With the window what is the amount of radiated flux at the wall as compared to the amount at the core?

Answer: The amount of heat that reaches the wall is about 2 percent of the total heat generated.

Question: To save running time, why don't you let the radiation come from a shell at the edge of the core?

Answer: Since the core diameter is about one-third the diameter of the reactor, the time saved is about one-third the total running time. The problem is actually two dimensional in the sense that convection is also present. This would result in a temperature distribution in the shell which is a function of the propellant temperature distribution.

This summarizes the present work on radiation heat transfer. Future work will be to investigate some characteristics of gas-core radiation theat transfer such as the seed-hydrogen opacity window, and temperature dependent opacities. The present analysis can also be used to explore the usefulness of radially dependent seeding and seeded transpiration cooling of the cylindrical wall. 


\title{
GAS CORE REACTORS
}

\author{
Henry J. Stumpf \\ Jet Propulsion Laboratory \\ California Institute of Technology \\ Pasadena, California
}

The two major problems confronting the gaseous vortex scheme are containment of the fuel and heat deposition in the tube walls.

An experimental program oriented toward a better understanding of the fluid dynamics of vortex flows has been underway for several years at the Jet Propulsion Laboratory. The effort at present consists of a gas separation device which simulates a single vortex tube and a flow visualization device.

A detailed discussion of this program and the problems presently plagueing us will be given by Paul Massier. I should just like to make a few brief comments about the two problem areas as they affect the system.

\section{FUEL CONTAINMENT}

To obtain some quantitative feeling for the containment problem it is necessary to define several parameters. It is convenient to speak in ternts of a containment factor $\psi$, which is defined as the ratio of the average density of fuel to propellant in the reactor cavities divided by the ratio of the average densities in the rocket exhaust. The quantity $\psi$ gives a direct measure of the excellence of the fuel-containment mechanism; the larger this factor, the more effective the containment mechanism. If $\rho_{\mathrm{FC}}$ and $\rho_{\mathrm{PC}}$ denote, respectively, the average densities of nuclear fuel and propellant in the cavities, and $\rho_{\mathrm{FRC}}$ and $\rho_{\mathrm{PRC}}$ those in the rocket chamber (and exhaust), then

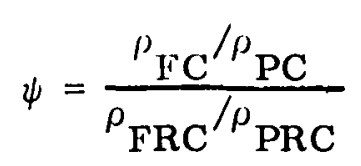


It is implied in this formulation that the fuel-propellant mixture leaving the reactor cavities passes into a rocket motor chamber and thence out the exhaust nozzle. Thus whatever fuel leaves the cavities is lost to the system.

Another quantity of interest is the average concentration ratio of nuclear material to propellant in the rocket exhaust $n_{\mathrm{FP}}$ :

$$
\mathrm{n}_{\mathrm{FP}}=\frac{\left\langle\mathrm{N}_{\mathrm{FRC}}\right\rangle}{\left\langle\mathrm{N}_{\mathrm{PRC}}\right\rangle}
$$

where $\left\langle\mathrm{N}_{\mathrm{PRC}}\right\rangle$ is the concentration of the propellant and $\left\langle\mathrm{N}_{\mathrm{FRC}}\right\rangle$ the concentration of the fuel in the rocket chamber.

On the basis of performance alone we can compute a maximum allowable value for $n_{\mathrm{fp}}$. This constraint arises because of the great disparity in molecular weight of nuclear fuels and efficient propellants. For plutonium and hydrogen this ratio $\left(\mathrm{A}_{\mathrm{F}} / \mathrm{A}_{\mathrm{P}}\right)$ is about 120 to 1 so that the addition of one part of plutonium to 120 parts of hydrogen would double the effective molecular weight of the exhaust and reduce the specific impulse by about $25-30 \%$. To prevent the specific impulse from being seriously degraded it is necessary to have the fuel propellant ratio in the exhaust small. Hence $n_{\mathrm{fp}}\left(\mathrm{A}_{\mathrm{F}} / \mathrm{A}_{\mathrm{P}}\right) \ll 1$ or $\mathrm{n}_{\mathrm{fp}} \leq 10^{-3}$. But this is no constraint at all since it results in a fuel loss $\sim 12 \%$ of the propellant expelled - a very large amount indeed.

To find the relation between containment factor and fuel expended we write

$$
\psi=\frac{\left\langle\mathrm{N}_{\mathrm{FC}}\right\rangle}{\left\langle\mathrm{N}_{\mathrm{PC}}\right\rangle_{\mathrm{FP}}^{\mathrm{n}_{\mathrm{F}}}}
$$

and since

$$
n_{f p}=\frac{W_{F}^{e x}}{W_{P}}\left(\frac{{ }_{P}}{A_{F}}\right)
$$

we must compute $\mathrm{w}_{\mathrm{F}}^{\mathrm{ex}} / \mathrm{W}_{\mathrm{P}}$, where $\mathrm{w}_{\mathrm{F}}^{\mathrm{ex}}$ is the total weight of nuclear fuel expended per propulsion period and $W_{p}$ is the total weight of propellant discharged during this same period. We will use the criterion of relative cost of the vehicle; i.e., what fraction of the total vehicle cost will we be willing 
to allow for fuel loss. If we set the fuel cost equal to the rest of the vehicle cost minus payload then

$$
\frac{\mathrm{w}_{F}^{\mathrm{ex}}}{\mathrm{w}_{\mathrm{P}}}=\frac{\mathrm{p}_{\mathrm{P}}}{\mathrm{p}_{\mathrm{F}}}\left[1+\mathrm{s} \frac{\mathrm{p}_{\mathrm{T}}}{\mathrm{p}_{\mathrm{P}}}+\frac{\mathrm{p}_{\mathrm{N}}}{\mathrm{p}_{\mathrm{p}}}\left(\frac{\mathrm{a}}{\lambda_{\mathrm{e}}}\right)\right]
$$

where $p_{i}$ is the cost per pound of component $i, \lambda$ is the propellant-to-vehicle gross weight ratio, $a_{e}$ is the rocket engine thrust-to-weight ratio, $a$ is the vehicle acceleration at the start of the propulsion period, and $S$ is the tankto-propellant weight ratio; the subscript $\mathrm{T}$ denotes tank and $\mathrm{N}$ reactor. In the examples which follow, the following values are used: $p_{F} / p_{P}=40,000$, $\mathrm{p}_{\mathrm{N}} / \mathrm{p}_{\mathrm{P}}=800, \mathrm{p}_{\mathrm{T}} / \mathrm{p}_{\mathrm{P}}=400, \mathrm{p}_{\mathrm{F}}=10,000$ dollars $/ \mathrm{lb}$ and $\mathrm{s}=0.05$.

In order to indicate some typical values of containment factor and of the corresponding fuel lost per propulsion period two examples will be considered. The first is that of a single stage booster to place a 100,000 lb satellite in Earth orbit, and the second is a low-thrust interplanetary vehicle to transfer a 400,000 $\mathrm{lb}$ payload from Earth orbit to a circular orbit about Mars. We will use $f$, the fraction of the total power produced in the solid region of the reactor, as the independent variable and $\Pi_{\mathrm{PL}}$, the payload ratio, as a figure of merit for the vehicle. The objective is to decrease $f$ to zero in order to obtain the highest specific impulse ratio possible but this requires large concentrations of fuel in the gas phase and therefore large containment factors. Thus the efficiency of the containment process dictates the maximum value of $f$, hence vehicle performance.

Consider first the satellite mission. In Figure 1 it is seen that as $f \rightarrow 0$, containment becomes more severe due to higher fuel concentrations in the gas phase and smaller mass or cost of the vehicle. In this example $\psi$ varies from unity for $\mathrm{f} \sim 1$ to over a thousand for systems with considerable gas phase heating. Regardless of the value of $\left\langle\mathrm{N}_{\mathrm{PC}}\right\rangle \psi$ must be at least 10 to give a $10 \%$ increase over Rover performance. Even then $1000 \mathrm{~kg}$ of fuel is lost per propulsion period. Experimental results to date have yielded values of $\psi \sim 1.1$.

The low thrust application of gaseous core reactors appears to be most promising for missions to the near planets. Again $f$ is taken as the independent variable but now the specific impulse can be optimized for each $f$ value because of the addition of the radiator. Figure 2 shows the results for the low thrust mission. Again fuel loss increases as $\dot{\psi}$ decreases. At the low value of $\left\langle\mathrm{N}_{\mathrm{PC}}\right\rangle, \psi=2.6 \times 10^{22}$; and the fuel loss in a single propulsion period is $6000 \mathrm{~kg}$. To reduce this by an order of magnitude the 


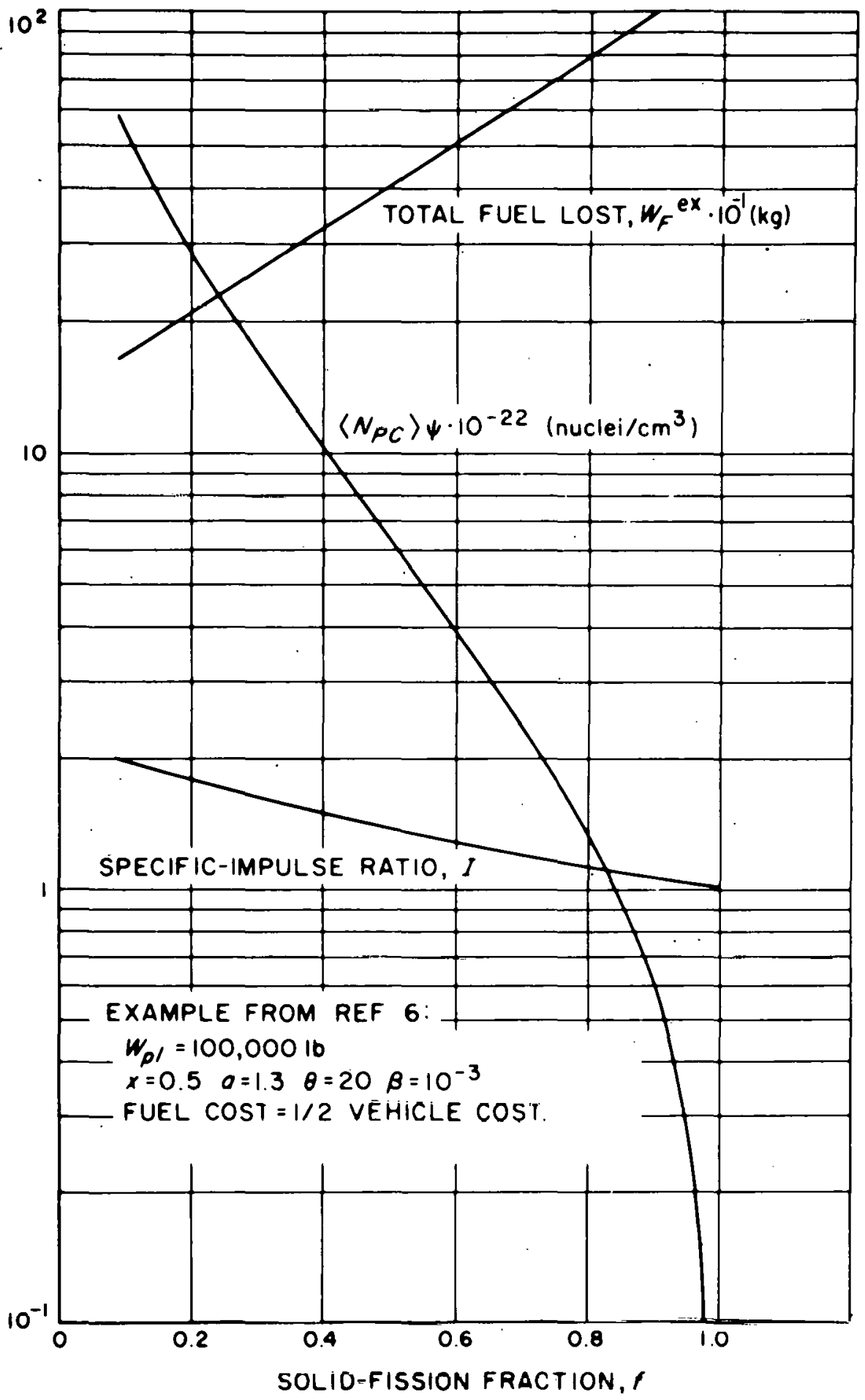

Figure 1. Fuel-Containment Requirements for Single-Stage Satellite Booster $(\mathrm{V}=1.14)$ 


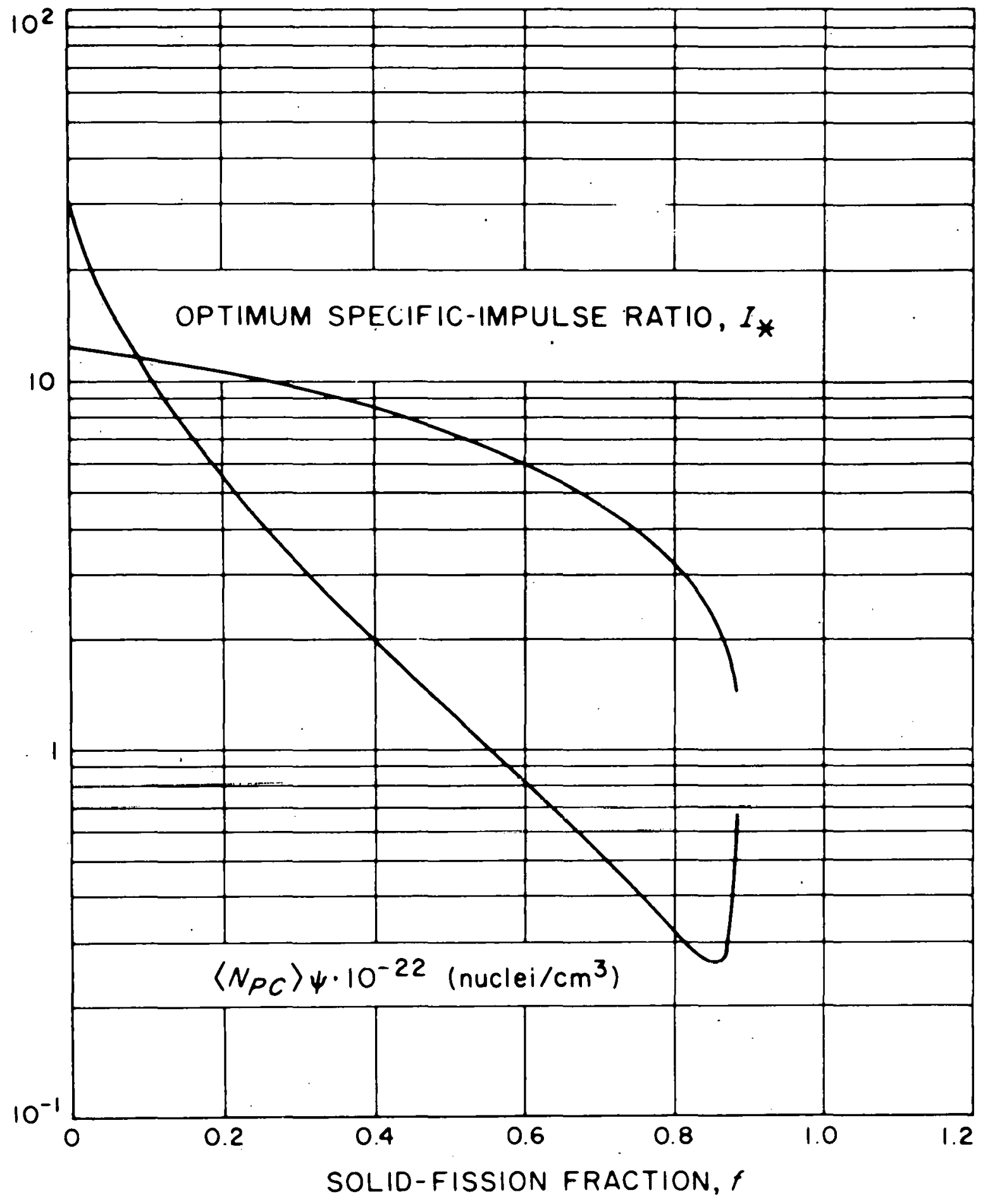

Figure 2. Fuel-Containment Requirements for Equivalent Mars Orbiter $(\mathrm{V}=2)$ 
containment factor must be increased by a factor of 20. Even this, however, results in a cost of some 12 million dollars per flight for fuel alone.

Some general observations can be made from this analysis.

1. The primary constraint in determining containment requirements is the total fuel loss allowed in one propulsion period.

2. In high thrust applications the gain over conventional nuclear systems is significant when $\psi>50$.

3. For low thrust systems $\psi \sim 50$ is required to compete with nuclear electric. For $\psi<50$ specific impulse is still high so if fuel loss is acceptable the system may still be attractive for lower energy missions.

\section{HEAT DEPOSITION IN THE SOLID REGIONS}

Since the energy deposition rate in the solid members of a gaseous reactor has a direct bearing on the ultimate performance potential of these systems, I should like to examine this problem more closely. The physical processes which contribute are:

1. Direct nuclear radiation in the form of gamma rays and neutrons which originate in the gaseous region and are absorbed in the solid region,

2. fission fragments born in the gaseous region which reach the tube wall with some residual kinetic energy, and

3. radiation from the hot gases in the vortex tubes which is absorbed in the solid regions.

The direct nuclear losses are insensitive to tube radius and propellant temperature and pressure and may be assumed to be some constant fraction of the total power generated in the vortex tubes (i.e., $7.5 \%$ ).

A crude estimate of the fission-fragment heat load can be obtained by considering a very simplified model of the vortex tube. The simplifying assumptions are:

1. Each fission results in the production of one heavy and one light fission fragment which are released isotropically.

2. The properties of the gaseous mixture in the vortex tube are taken to be those of hydrogen, and appropriate average values are used to correct the ranges of the light and heavy fission fragments from the values at standard conditions. 
3. The power density in the vortex tube is constant, the energy loss of the fission fragments is a known function of the path length, and the length of the tube is large compared to the fission fragment ranges.

The results of this simple analysis show that for tube diameters of the order of the fission-fragment range a relatively large portion of the fission-fragment energy can be deposited in the tube wall. It is obvious that infinitely large tubes would be required to reduce this energy loss to a negligible portion of the total power generated in the vortex tube, but for tube diameters larger than $20 \mathrm{~cm}$ at an exhaust pressure of $100 \mathrm{~atm}$ and larger than $8 \mathrm{~cm}$ at 300 atm this heat load can be reduced to a few percent of the total power. Of course this model gives very conservative results. A more realistic calculation taking into account the peaked distribution of the fission fragments would yield lower limits on the tube diameters.

The thermal radiation from the gas mixture in the cavities presents a very difficult problem. Without detailed knowledge of the composition, temperature, distribution, and densities of the gas mixture, it is, of course, impossible to determine precisely the thermal radiation flux at the solid boundaries. The problem is further complicated by the fact that the presence of fissionable species, such as uranium and plutonium halides, even at small concentrations will most likely affect the radiative characteristics of the mixture.

Another complication even less well understood at this time is the role of the various metastable species produced in the mixture by the slowing down of the tission tragments. It is expected that these too will intluence the radiative transfer phenomena. The futility of attempting a detailed treatment of the problem in the absence of experimental facts about the nuclear fuel carrier, the separation process, and fission-fragment physics is therefore apparent.

In spite of these difficulties it is possible to extract some additional information about the thermal radiation heat load by attempting to bracliet the actual physical situation with two limiting cases. At one extreme the gas mixture in the cavity is considered to be entirely opaque, and at the other, essentially transparent. In the first case both the gas and the solid boundaries are assumed to radiate as blackbodies, the effective radiating temperature of the gas being some intermediate value between the wall temperature and the central or maximum temperature of the gas in the cavity. In the transparent case, the gas mixture is taken to have a very low emissivity, zero opacity, and to radiate at its maximum temperaturc. 
When the gas is opaque, the shape of its outer boundary does not enter into the analyses and the system can be represented by a pair of infinite, flat surfaces. One surface is the gas, which is taken in general as a gray body with emissivity $\epsilon_{\mathrm{c}}$ and radiating temperature $\mathrm{T}_{\mathrm{g}}$, and the other is the solid boundary with emissivity $\epsilon_{S}$ and temperature $T_{S}$.

When the gas is transparent the actual geometry of the cavity wall must be considered. To obtain the net radiation passing from gas to wall, the difference between the total radiation incident upon the wall and that incident upon the gas is required. This approach, however, would only be valid if the emissivity of the gas were independent of temperature. To go one step further we can consider the temperature dependence of the emissivity and compute the thermal radiation heat load using a highly simplified model of the physical processes involved.

We will make the following simplifying assumptions:

1. The gas in the vortex tube is hydrogen.

2. The emissivity of hydrogen as a function of temperature is given by

$$
\begin{array}{rlrl}
\epsilon_{\mathrm{I}} & =7.30 \times 10^{-2} & \ell^{-1.17 \times 10^{-3} \mathrm{~T}} & 2000^{\circ} \mathrm{K} \leq \mathrm{T} \leq 4100^{\circ} \mathrm{K} \\
\epsilon_{\mathrm{II}}=7.98 \times 10^{-8} & \ell^{2.18 \times 10^{-3} \mathrm{~T}} & 4100^{\circ} \mathrm{K} \leq \mathrm{T} \leq 7500^{\circ} \mathrm{K} \\
\epsilon_{\mathrm{III}}=1.00 & & \mathrm{~T} \geq 7500^{\circ} \mathrm{K}
\end{array}
$$

where the data given in Figure 18 of Reference 1 have been approximated by the above expressions.

3. The radiation per unit volume from the gas is

$$
4 k(T) \ell_{g}(T)=4 k(T) \sigma T^{4}
$$

where $\mathrm{T}$ is the gas temperature, $\sigma$ is the Stefan-Boltzmann constant, $\ell_{\mathrm{g}}(\mathrm{T})$ is the emissive power of the gas, and $k(T)$ is the absorption. coefficient.

4. The tube wall acts like a blackbody.

1. Olfe, D., Equilibrium Emissivity Calculations For a Hydrogen Plasma at Temperatures Up To $10,000^{\circ} \mathrm{K}$, Technical Report 33 , California Institute of Technology, May 1960. 
5. The tube is long, and end-effects are negligible.

6. The temperature distribution of the gas is

$$
T(R)=T_{s}\left\{I^{2}-\left(I^{2}-1\right)\left(\frac{R}{R_{v}}\right)^{2}\right\}
$$

where $T_{S}$ is the tube wall temperature, $R_{V}$ is the tube radius, $\mathrm{I}^{2}=\mathrm{T}_{\mathrm{c}} / \mathrm{T}_{\mathrm{S}}$, and $\mathrm{T}_{\mathrm{c}}$ is the gas temperature at the center of the tube $(\mathrm{R}=0)$.

7. The radiation flux in any given direction is attenuated according to a simple exponential law

$$
q(r)=q(0) \exp \left[-\int_{0}^{r} k\left(r^{\prime}\right) d r^{\prime}\right]=q(0) e^{-\tau(r)}
$$

where $q(0)$ is the thermal radiative flux at $r=0, q(r)$ is the thermal radiative flux at $r$, and $\tau(r)$ is the optical thickness.

The results of this analysis are shown in Figure 3 . It can be seen that the heat flux increases with radius and exhaust temperature $T_{c}$. For a fixed value of $I^{2}$ (or equivalently $T_{c}$ ) the rate of increase decreases as the radius increases since we approach the case of an infinite body of gas radiating to a flat plate. For a fixed tube radius the heat flux increases rapidly for core temperatures much greater than $7500^{\circ} \mathrm{K}$ because the gas in the vortex tube is then radiating as a blackbody at a temperature substantially above that of the wall.

Having made a crude estimate of the various heat losses from the vortex tube a power balance can be made to determine the fraction of the total power that is utilized in heating the propellant and the required power density in the gaseous region of the reactor. It will be assurned that a particular central or exhaust temperature is to be maintained in the vortex tube. These results are shown in Figures 4, 5, and 6. At $I=1.43$ and $R_{V}>5 \mathrm{~cm}$ about $90 \%$ of power goes to hoating the propellant. The increase with $R_{V}$ is due to decreasing fission fragment losses.

At $I=1.94$ the fraction of power used to heat the propellant first increases to about $86 \%$ and then decreases. The fission fragment losses decrease rapidly at first but then fail to compensate for rapidly rising thermal radiation losses. 


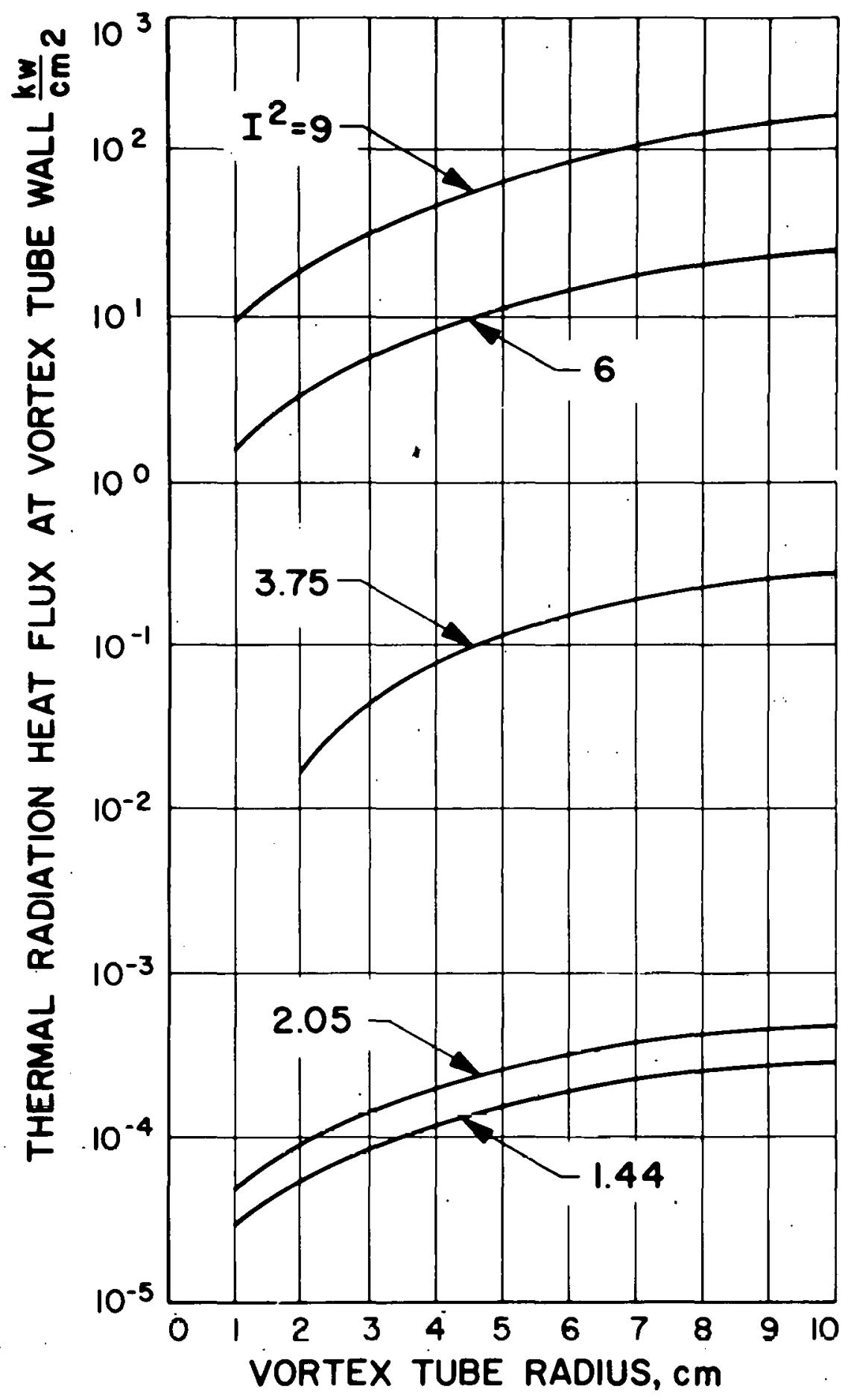

Figure 3: Thermal Radiative Heat Flux at Vortex Tube Wall as a Function of Vortex Tube Radius 


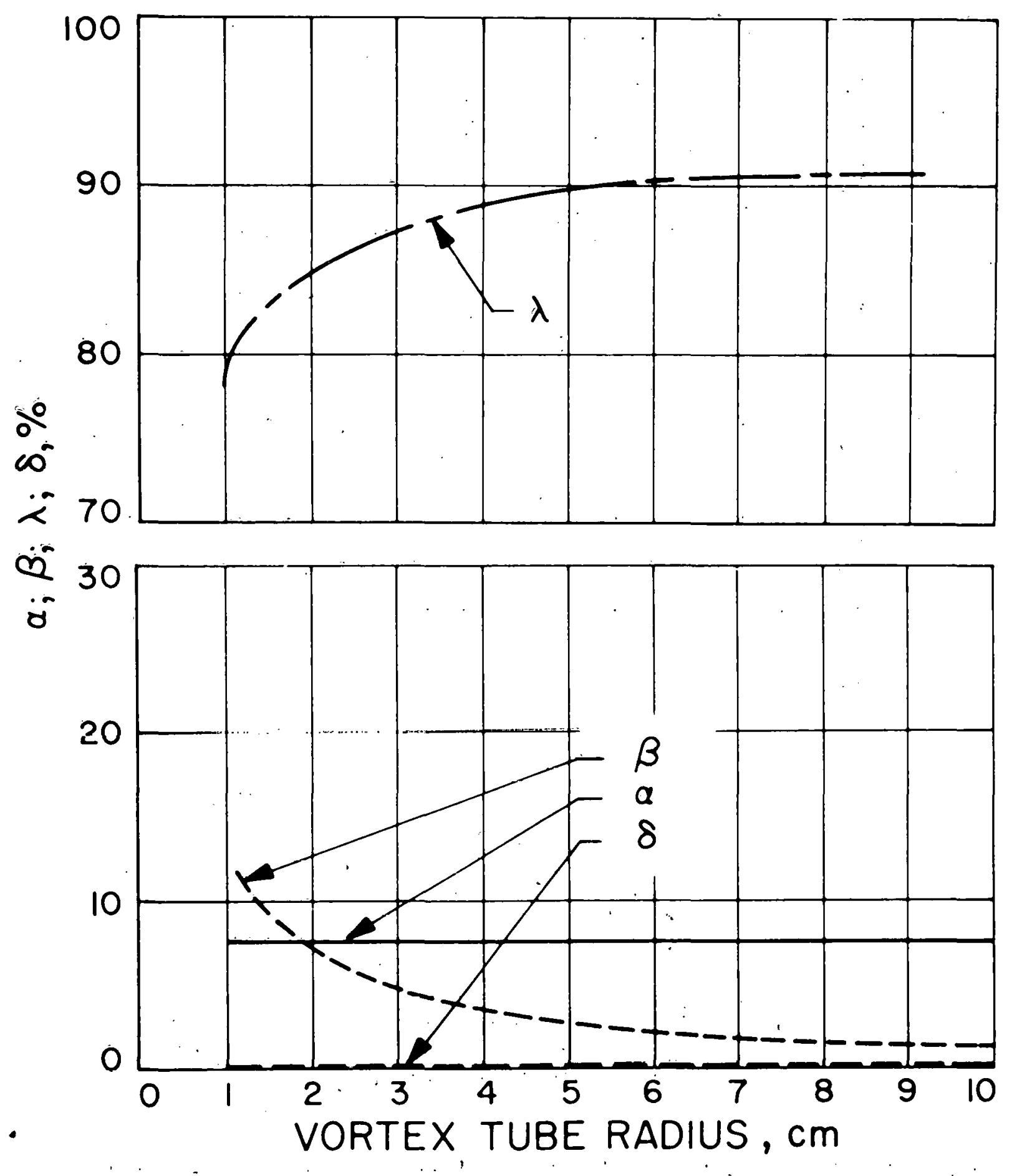

Figure 4. Power Balance for Vortex Tube, $I=1.43$ 


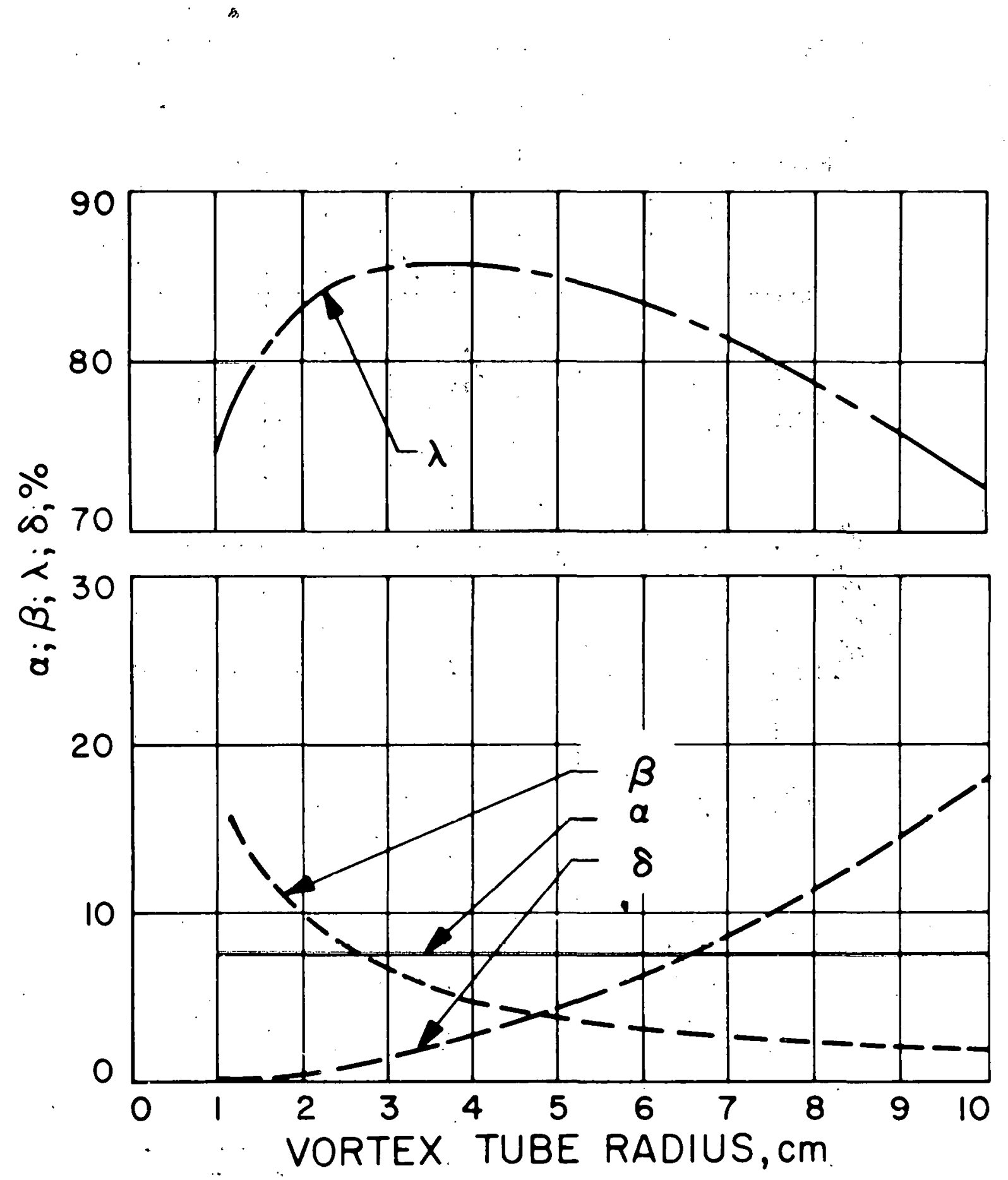

Figure 5. Power Balance for Vortex Tube, $\mathrm{I}=1.94$ 


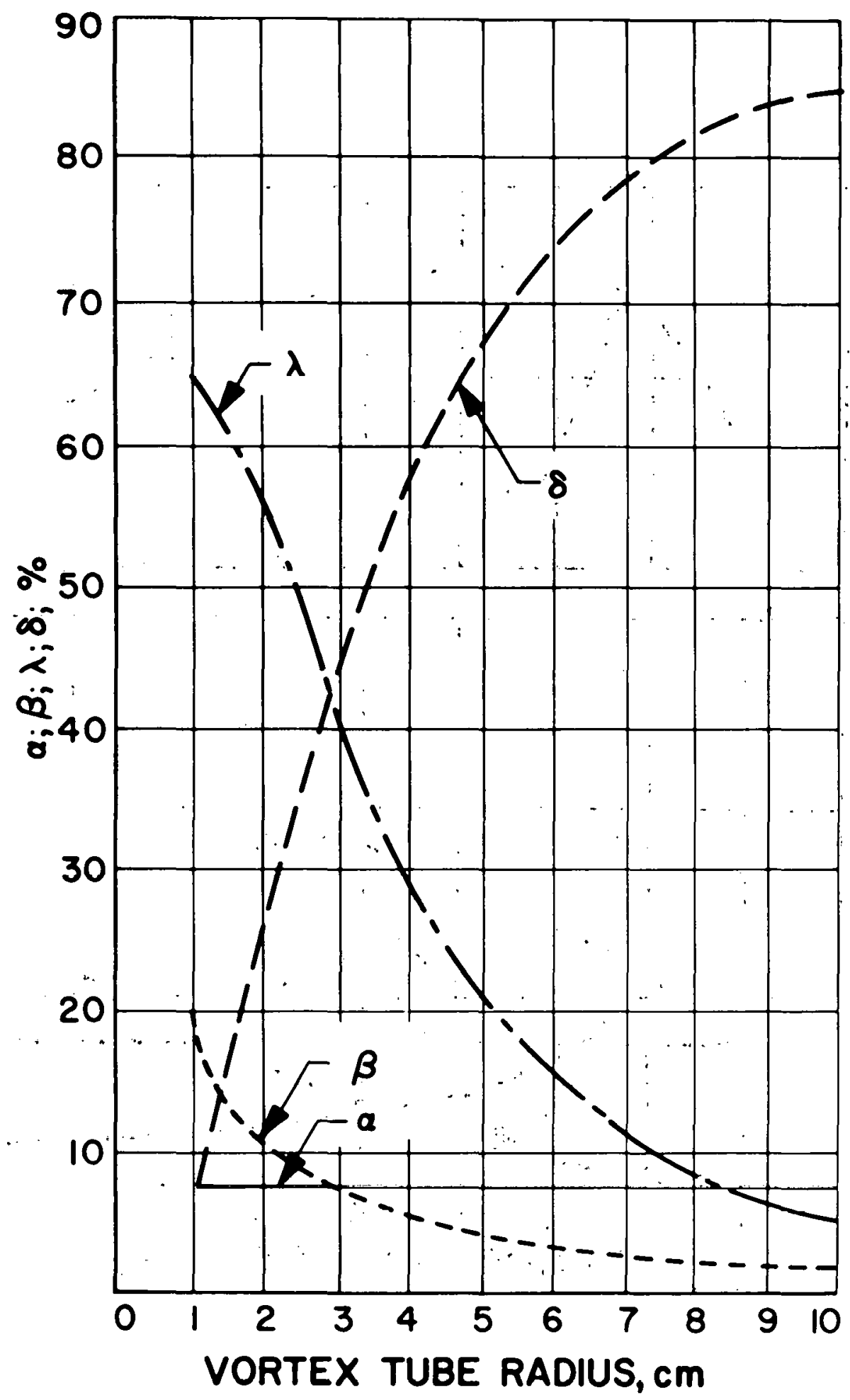

Figure 6. Power Balance for Vortex Tube, $I=2: 45$ 
At $I=2.45$ the propellant absorbs less than two-thirds the total power even at small $R_{v}$ and rapidly decreases to less than $10 \%$ for the larger radii. This is again due to reason given above plus the fact that for $\mathrm{T}_{\mathrm{c}} \gg 7500^{\circ} \mathrm{K}$ we have essentially blackbody radiation at a temperature $\gg \mathrm{T}_{\mathrm{S}}$.

Figure 7 shows the required power density and fuel particle density and flux in the cavities. It can be seen that for power densities in excess of $1 \mathrm{KW} / \mathrm{cm}^{3}, \mathrm{~N} \sim 10^{18} \mathrm{part} / \mathrm{cm}^{3}$ and $\phi \sim 10^{16}$ neutrons $/ \mathrm{cm}^{2}$ - sec.

\section{CONCLUSIONS}

As a candidate to propel future generation rockets the gaseous-fuel reactor may outperform solid fuel propulsion reactors by a factor of two. A number of very formidable problems must be solved, however, before this goal can be attained. The potential certainly seems to warrant extensive study of these problems. A major shortcoming at the moment is the lack of experimental data on the fluid dynamics and radiative properties of the complex gaseous mixtures that will occur in the vortex tube.

These systems hold promise for high performance for missions to nearby planets although their performance cannot equal that of the nuclear electric system for missions to the far planets. 


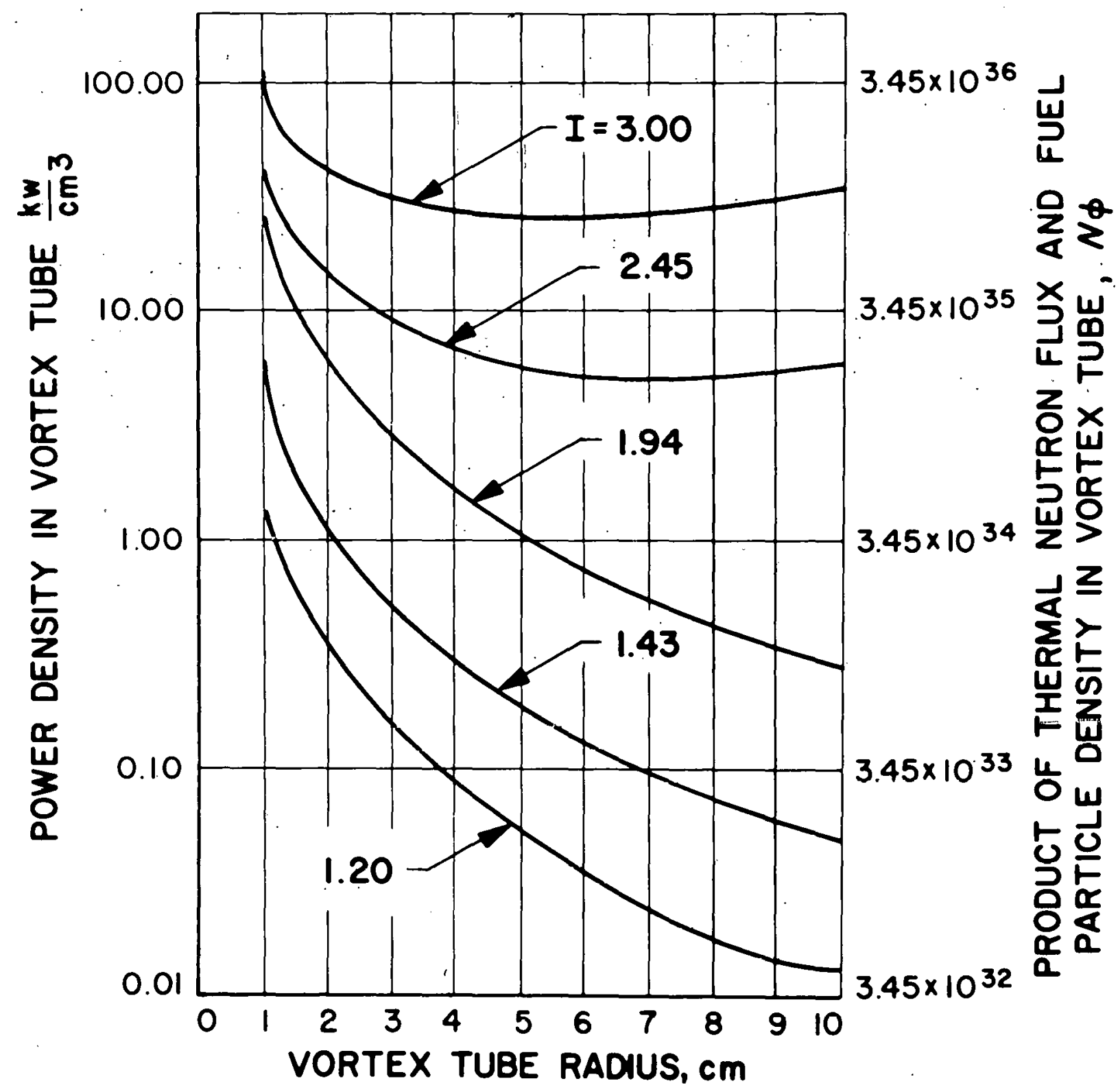

Figure 7. Power Density in Vortex Tube as a Function of Vortex Tube Radius $P_{T}=3.45 \times 10^{34} \mathrm{~N} \phi$ 


\title{
SEPARATION MEASUREMENTS IN \\ BINARY SPECIES GASEOUS VORTEX FLOWS AND \\ FLOW VISUALIZATION STUDIES IN A WATER VORTEX
}

\author{
T. J. Pivirotto and E. J. Roschke \\ Presented by Paul F. Massier \\ Jet Propulsion Laboratory \\ California Institute of Technology \\ Pasadena, California
}

\section{INTRODUCTION}

A gaseous-core nuclear reactor propulsion concept will be reviewed briefly, and some of the major fluid dynamics problems associated with this concept will be discussed. Following this, some results of the experimental binary separation and flow visualization studies that have been performed at the Jet Propulsion Laboratory will be presented. Analytical studies of various types have also been made. So far, in our fluid dynamics experiments we have made use of fluids at essentially room temperature. No tests have been conducted on flows in which there were reactions or heat transfer.

\section{VORTEX GASEOUS CORE CONCEPT}

Figure No. 1 is a drawing that demonstrates the vortex concept. A binary mixture of gases which have significantly different molecular weights is introduced tangentially into a cylindrical container at its periphery. Both fluids spiral inward; however, the fluid of greater molecular weight forms into a rotating annular cloud as a result of the forces introduced by the rotation. Ideally this cloud would be retained within the container, and the light fluid would diffuse radially inward through the cloud and be discharged through an orifice in the end wall as shown. Once the cloud of heavy fluid has been formed, injection could be limited primarily to the light gas. In a propulsion device the gas of high molecular weight would be the nuclear fuel 


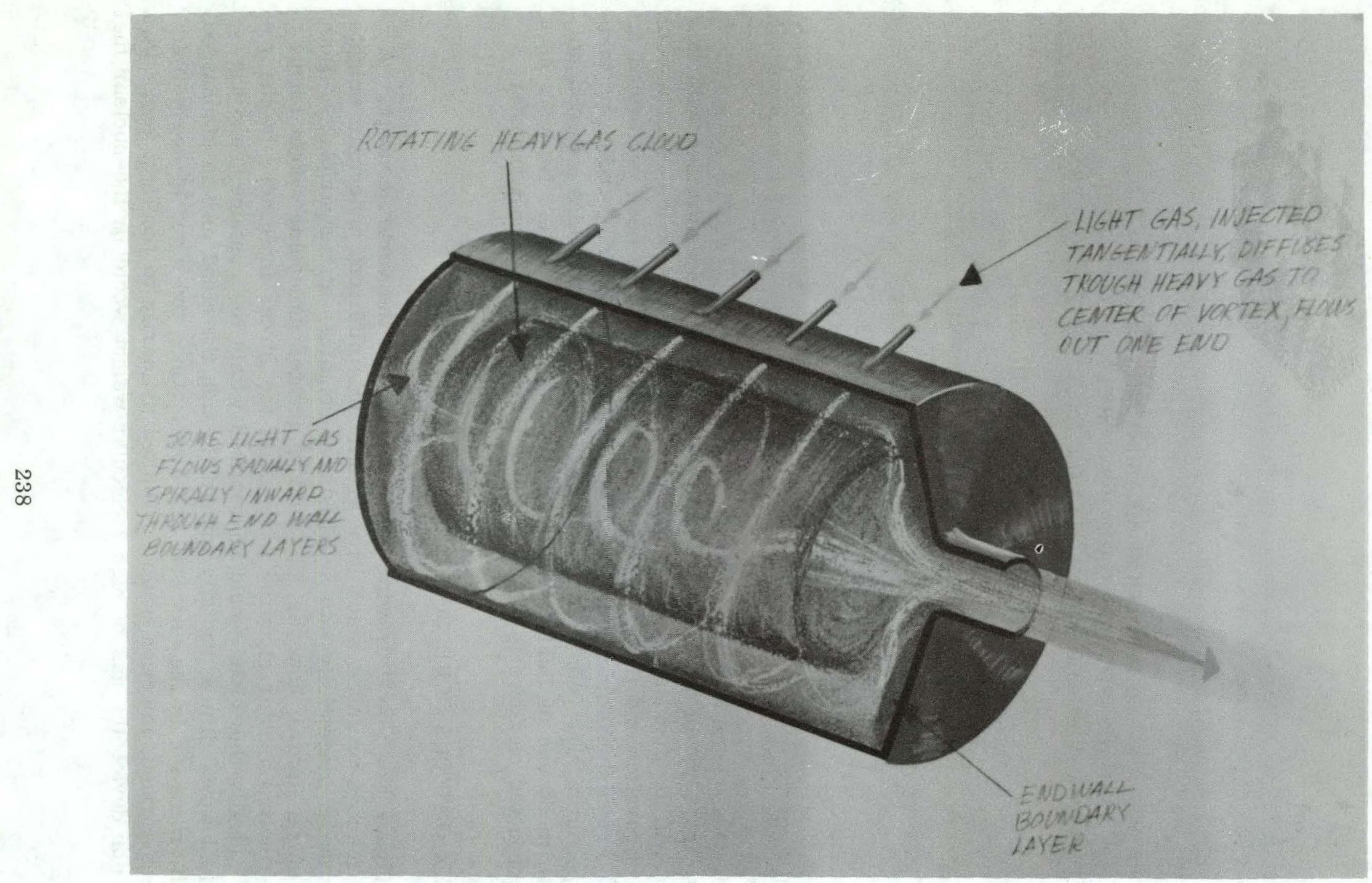

Figure 1. Fluid Dynamics Study of Binary Vortex Flows 
and the gas of low molecular weight the propellant. Energy would be transferred from the fissioning gas to the propellant primarily by collisions during the diffusion process and also by thermal radiation. A rocket engine would presumably consist of numerous vortex cells like this, each one discharging into a chamber from which the propellant would be ejected through a nozzle to produce thrust.

It is apparent that this concept could be used as a propulsion device only (1) if a rotating heavy gas cloud of sufficient density can be sustained at the desired radii without a significant loss of fuel through the exit orifice and (2) if a sufficient lraction of the injected light gas will diffuse through the heavy gas so that an adequate amount of energy transfer can occur by direct particle interactions. These requirements are fluid dynamic in nature; consequently, most of our small effort has been oriented toward the study of the fluid dynamic behavior of vortexes.

\section{OBSERVED DETRIMENTAL FLUID DYNAMIC BEHAVIOR}

From the standpoint of the diffusion concept, certain detrimental flow effects have been observed. One of these which has been found to occur in a confined vortex driven exclusively by peripheral tangential injection is that a large fraction of the injected fluid flows axially toward the end walls. It then flows radially inward through the end-wall boundary layers and axially through a cylindrical region, along the centerline, and is discharged through the exit orifice. Associated with these flows recirculating radial and axial flows have also been found. The injected fluid is drawn toward the end walls because of viscous shear at the vortex tube end walls. This shear causes the fluid to slow down, forming end-wall boundary layers; and this flow, which has lower tangential velocity, then does not have sufficient tangential momentum to support the radlal pressure gradient impressed on the boundary layers by the body of the vortex. Consequently, these boundary layers offer the path of least resistance to a large portion of the injected fluid. Movies of dye injected into a water vortex which show some of the secondary flows that can occur in a vortex will be shown at the end of the presentation.

\section{SEPARATION MEASUREMENTS IN BINARY GASEOUS VORTEXES}

In gaseous vortex flows of the type that have been described, ineasurements of both the radial and axial distributions of the ratio of heavy-to-light gas mass densities have bcen made for injected mixtures of freon-13 and hydrogen as well as mixtures of argon and hydrogen. Freon-13 has a 
molecular weight of 104.5. These measurements were made by sampling the flow with a small probe that extended diametrically across the tube and then analyzing the sample with a thermal conductivity cell. The flow through the vortex tube was always steady; i.e., the flow rate of the mixture entering was always equal to the flow rate of the mixture discharging through the exit orifice. Therefore, the local concentration, as a function of time under conditions for which only hydrogen was injected after a heavy gas cloud was established, has not been determined. To perform a transient experiment of this type one must shut off the flow of heavy gas after steady-state conditions are established and then use a measuring technique which has a faster response than the present sampling method, e.g. infrared absorption. Presently the development of such a method is being considered.

Figure No. 2 shows a cross section of the vortex tube in which the steady-state concentration measurements were made. The gas mixture was injected approximately tangentially through 804 jets 0.007 in. in diameter distributed as shown. Six axial rows of jets arc spaced equally around the circumference. The internal diameter of the tube is $4.5 \mathrm{in}$., and the length is approximately 24 in. Various exit orifice configurations have been tested, but for the tests reported here the one shown on this figure was used. The sampling probe, which is moveable in the radial direction, has an external diameter of $0.010 \mathrm{in.,}$ an internal diameter of 0.005 in., and a sampling orifice of 0.005 in. diameter. End wall pressure distributions were obtained with a moveable wall pressure tap which has a diameter of $0.010 \mathrm{in}$.

Figure No. 3 shows radial distributions of heavy gas enrichment at five different axial pusitions. 'The ratio $\mathrm{Z} / \mathrm{D}$ indicates these positions in terms of tube diameters from the closed end wall. The ordinate is the concentration ratio of heavy to light gas compared to the concentration ratio that existed in the manifold from which the fluid was injected. The abscissa is the local radius normalized to the radius of the tube. Notice that near the closed end wall there is little change in concentration ratio with radius. This implies that (1) either the radial pressure gradients are not high enough to produce much separation at that axial location or (2) that the strong secondary flows that exist at the end walls are affecting the density ratio distribution. No significant diffusion can occur in the secondary flow along the end walls because of their high radial velocities. Thereforc, this secondary flow will transport fluid from the vortex periphery, where the concentration ratio is very nearly equal to the manifold value, directly into the vortex core, a region in which maximum enrichment was found to occur at the opposite end. This results in a flattened radial profile near the closed end wall.

For this set of results notice that the concentration ratio in the manifold was different for the distribution measured near the closed end wall, 


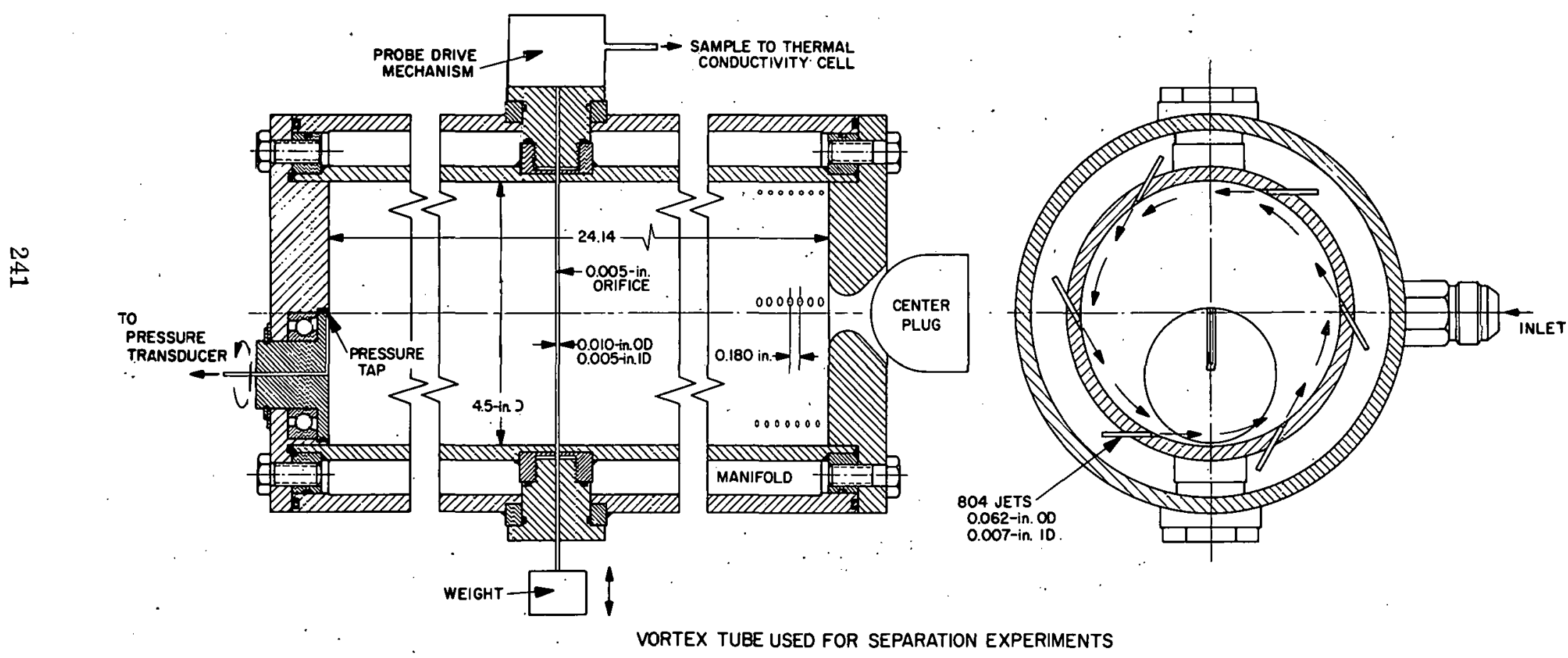

Figure 2 


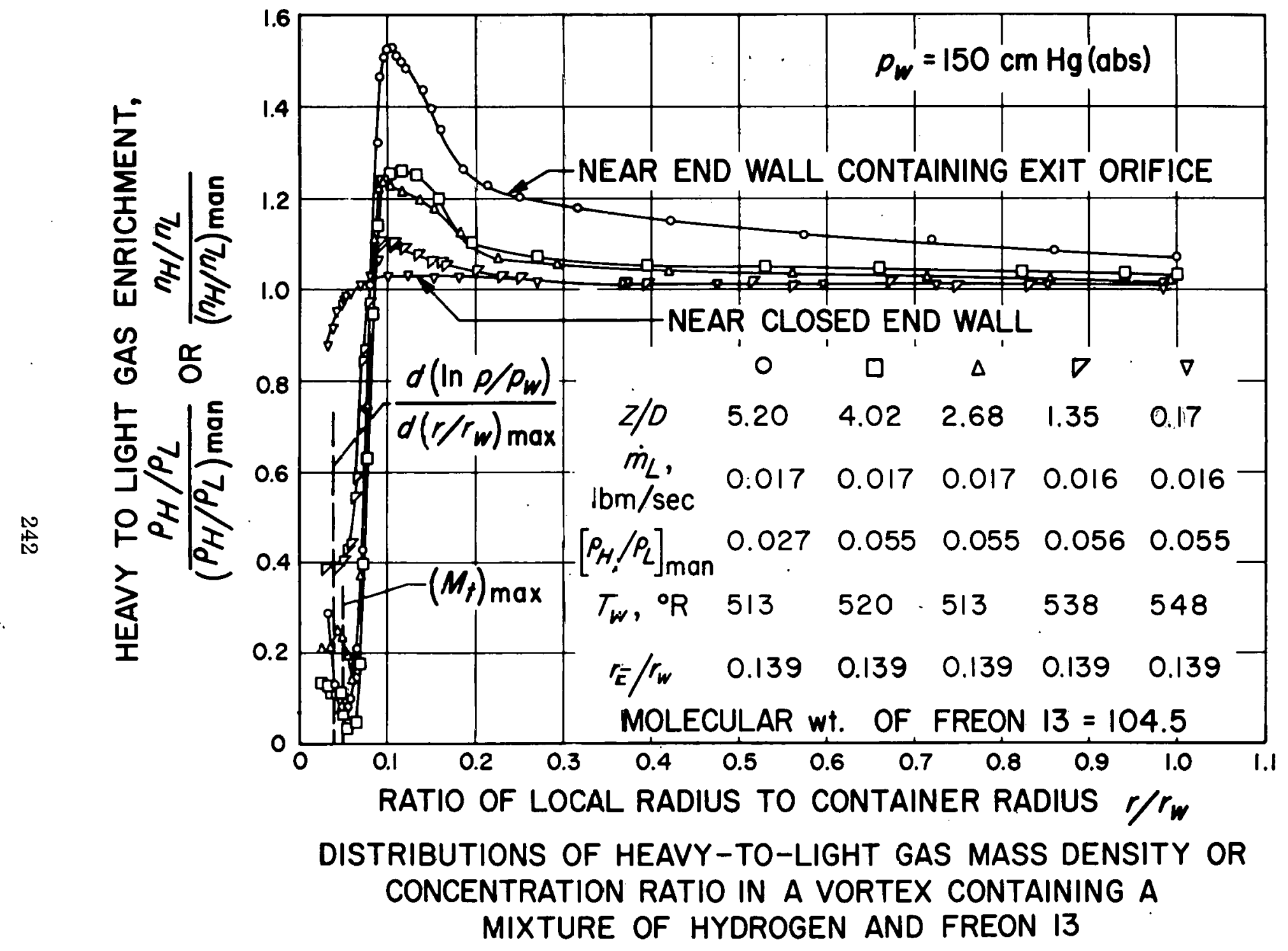

Figure 3 
than at other axial locations. It has been found that in a plane midway between the two end walls the manifold concentration ratio had little effect on the normalized distributions measured at that location, and here it is assumed that it does not have much of an effect near the end wall containing the exit orifice. Each one of these curves represents an individual test after which operation of the unit was terminated so that the axial position of the probe could be changed.

At other axial positions notice that the peak enrichment gradually increases from the closed end-wall value toward the end wall that contained the exit orifice. This trend may result to a large extent from the influence of the fluid that flows radially inward through the boundary layer adjacent to the closed end wall. As this fluid, in which the concentration of heavy to light gas is probably about the same as that of the injected fluid, approaches the center of the container, it must turn and flow axially toward the exit orifice. As this boundary layer fluid flows axially within the container, the radial pressure gradient, which results from the fluid's angular momentum, causes the heavy species to diffuse radially outward. 'This separation continues as the boundary layer fluid moves progressively down the tube. Hence, if this is a correct description of the flow process, the greatest enrichment would be found near the exit end wall. There are other factors that also have an influence on the concentration distributions, and in an accurate description of the flow in the body of the vortex, the fluid that flows through the boundary layer on the end wall containing the exit orifice must also be accounted for. The discussion given here is limited to what is presently considered to be a possible primary influence. The maximum enrichment of the heavy gas that was measured was $53 \%$ above the manifold value. This occurred approximately $0.76 \mathrm{in}$. from the end wall that contained the exit orifice. The radial location of the peak enrichment did not change much with axial location and was located inside the exit orifice radius.

Inside of the radius of maximum donsity ratin the density ratio decreases very rapidly with decreasing radius. In some cases a minimum is attained before the curves rise again. This large change in density ratio occurs in a region in which the Mach number changes rapidly with radius. The Mach number attains a maximum value approximately where the density ratio is a minimum. It is at this approximate radius that the radial pressure gradient is also a maximum, and hence it is at this location that the highest diffusion velocities occur forcing the heavier gas particles to larger radii just as the measurements indicate. The magnitudes of the Mach number and the radial pressure gradient reduce below the peak values at smaller radii; consequently, not as much separation can occur at these smaller radii, and as is shown the concentration ratio again increases as the radius approaches zero. 
Figure No. 4 shows the radial distributions of tangential Mach number and static pressure for a comparable test in which the probe was located near the closed end wall. The Mach number and pressure distributions were found, by measurement, to be very nearly independent of probe axial position. The Mach number was computed from the end wall static pressure measurements; hence, for this calculation it was assumed that the axial and radial velocities were zero. Also this test was made with pure hydrogen since the small amount of heavy gas that is introduced for separation studies does not influence the pressure distribution at the end wall. The locations of the maximum density ratios as shown on the previous slide are also shown here.

These tests have demonstrated that separation does occur in a vortex of this type. Of major importance, however, to the propulsion concept are the actual particle concentrations computed from the measurements by assuming an isothermal process and shown in Figure No. 5. Notice that the maximum concentration of the heavy species occurs at the periphery which in a propulsion device would be particularly undesirable because of the consequent high heat transfer rate to the vortex tube cylindrical wall. As was mentioned before, all of these tests were performed under conditions for which a steady mixture of heavy and light gas was being injected continually; and therefore, one would expect the concentration ratio at the periphery to be approximately the same as the concentration ratio in the manifold.

\section{INFLUENCE OF PROBES}

Since all of the concentration measurements have been made by sampling quantities of gas through a probe that was located within the vortex flow field, comments on the influence of probes on the flow field are in order. Measurements of end-wall pressure distributions in gaseous vortexes have been made under conditions for which probes of various diametcrs have been inserted diametrically through the vortex at the mid-axial position. Figure No. 6 shows the influence of probe diameter on the static pressure drop between vortex tube periphery and centerline. The trend shown was found to be about the same with the probe located at other axial positions. The ordinate is the difference in pressure between the cylindrical wall value and the value at the centerline divided by this difference for conditions under which there was no probe in the vortex. The abscissa is the probe diameter divided by the vortex tube diameter. The probe diameters investigated varied from 0.003 to $0.042 \mathrm{in}$. The location of a $0.010 \mathrm{in}$. diameter probe which was used for sampling in a similar vortex tube is indicated. These tests were performed for a given exit orilice which has a radius ratio, $r_{E} / r_{w}$, of 0.083 . 


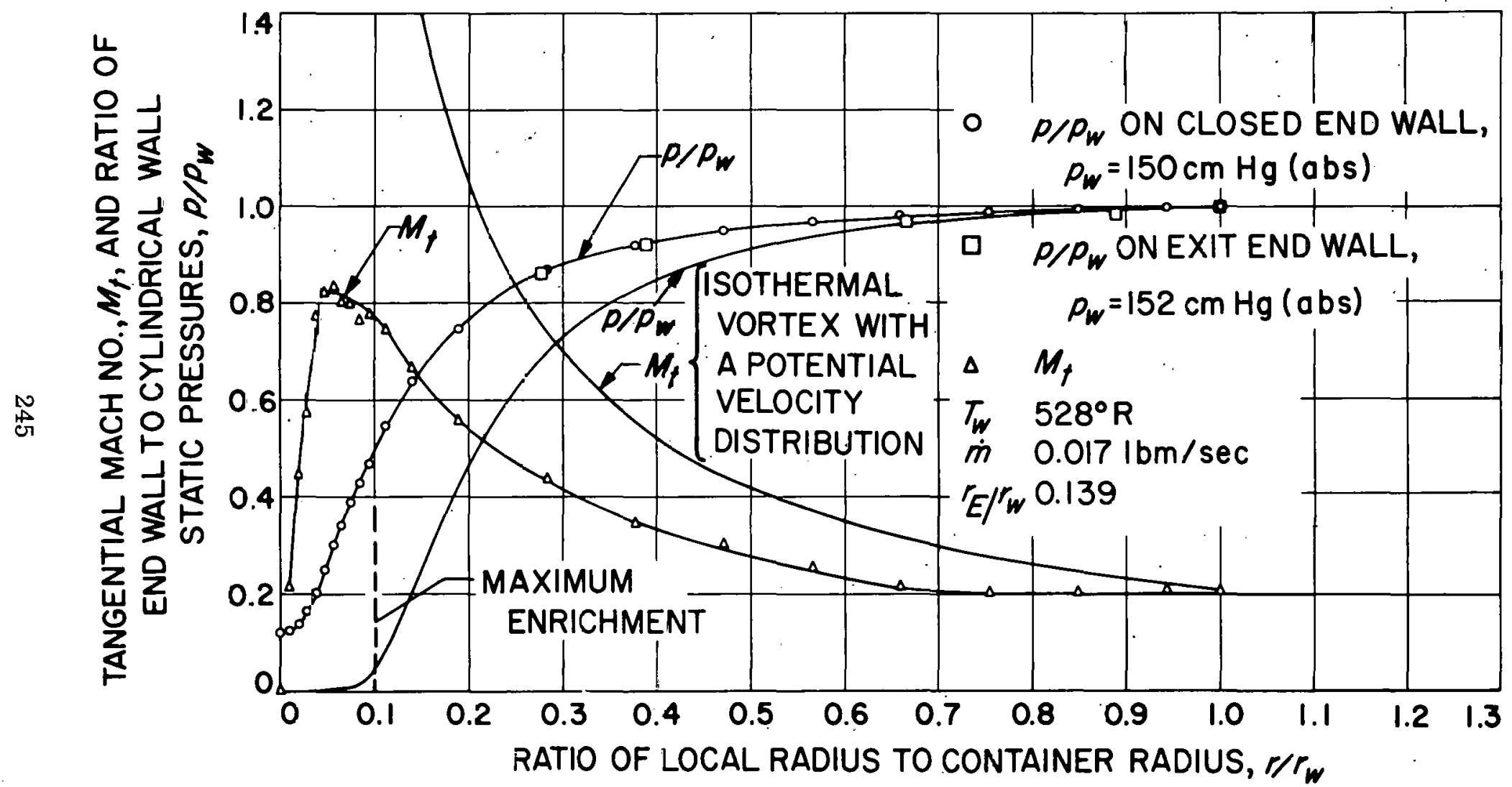

TANGENTIAL MACH NO. AND RADIAL STATIC PRESSURE DISTRIBUTIONS ALONG END WALLS OF A HYDROGEN VORTEX 

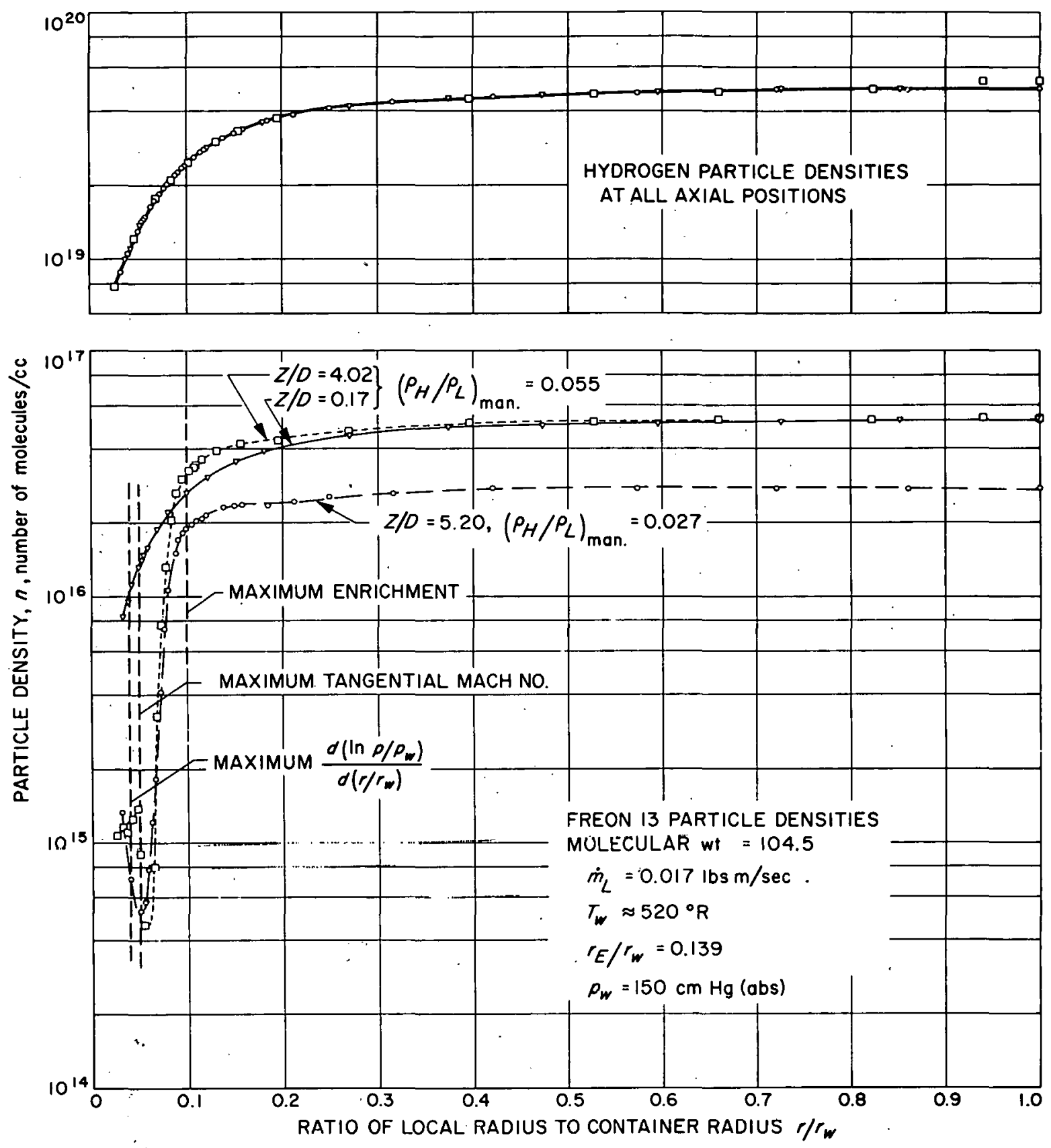

PARTICLE DENSITY DISTRIBUTIONS IN A VORTEX CONTAINING A MIXTURE OF HYDROGEN AND FREON 13

Figure 5 


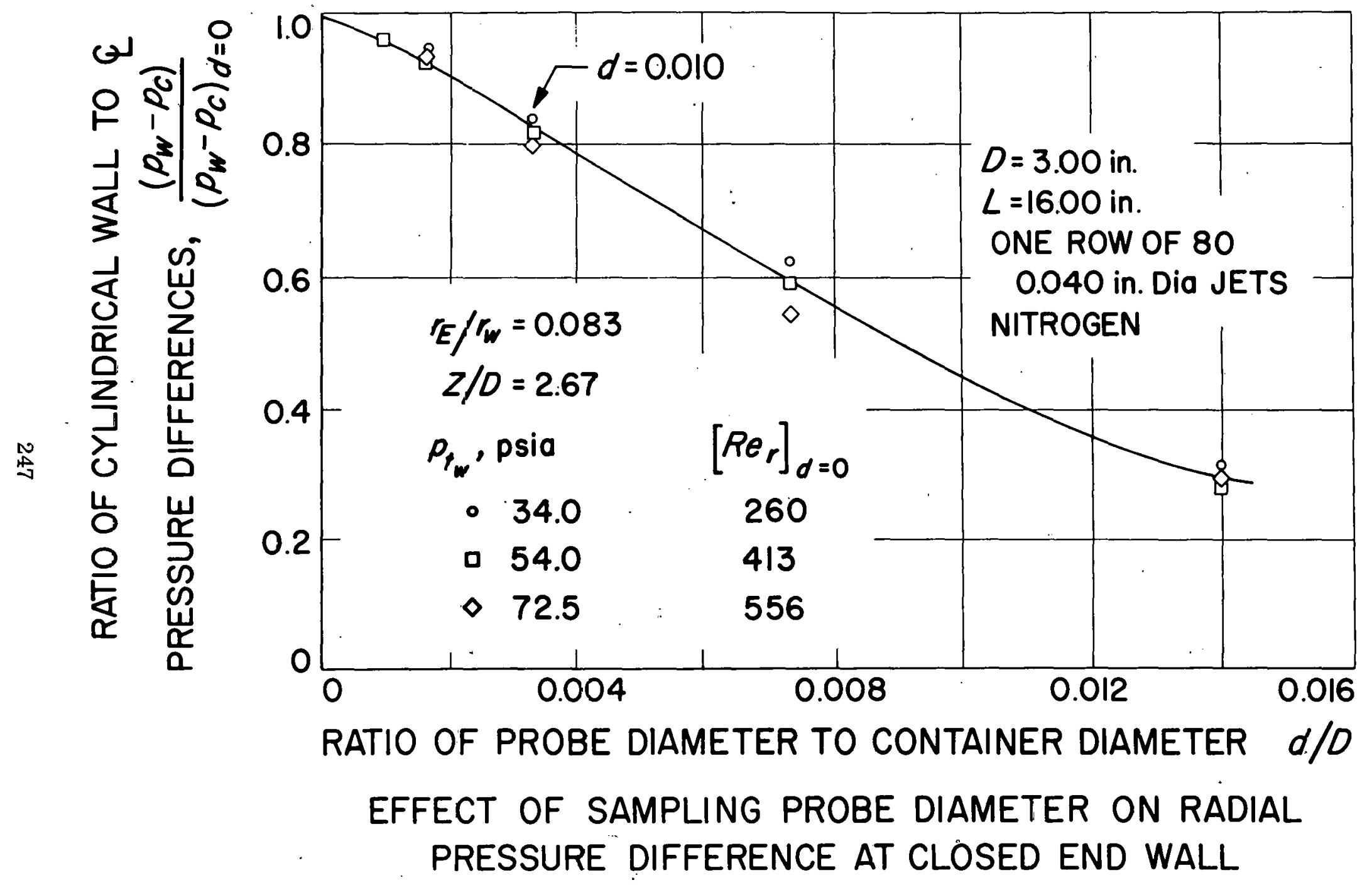

Figure 6 
Notice that the data lie approximately on one line for the pressure range covered. This reduction in pressure difference probably results in a decrease in the maximum pressure gradient, and hence, also in the maximum Mach number which would result in a reduction of enrichment. These detrimental effects are reduced somewhat for larger exit orifices and larger mass flow rates but nevertheless are still present.

\section{WATER VORTEX MOTION PICTURES}

Perhaps of greater significance is the influence of the probe on the entire flow field of the vortex, and some of these effects can be seen in a movie that shows dye patterns in a water vortex. These movies are shown at the same frame speed at which they were taken. The general conclusions deduced from the dye patterns in water vortexes are that axial flows occur and that concentric annuli are sometimes formed in which axial velocities occur in opposite directions. Furthermore, the simultaneous introduction of probes across the diameter at different axial locations produces cells between probes with noticeable differences in flow patterns from those observed when there are no probes in the flow fields. Visually, dye does not appear to flow axially from one cell to another through the wake introduced by the presence of a probe; however, there must be flow across the shear layers to maintain continuity. This flow occurs in a region near the vortex axis. Eventually the dye does disappear. It is evident from these visualization studies that probes can have a significant influence on the flow pattern which in turn can influence the concentration distribution in a binary vortex. The extent to which this occurs has not yet been established.

\section{ADDITIONAL REMARKS}

In addition to optical absorption measurements, we wish to inject fluid at the end walls in order to accelerate the boundary layers and thereby attempt to prevent a considerable amount of the injected gas from flowing through the end wall boundary layers.

The experimental resuits that have bccn presented as well as additional work has been published in Volume IV of several JPL Space Programs Summaries listed as references. 


\section{REFERENCES}

1. T. J. Pivirotto, "Binary Diffusion in a Compressible, Three-Dimensional Turbulent Vortex", Space Programs Summary 37-15, Vol. IV, pp. 159-162, Jet Propulsion Laboratory, California Institute of Technology, Pasadena, California, June 30, 1962.

2. T. J. Pivirotto, "Binary Diffusion in a Compressible, Three-Dimensional Turbulent Vortex", Space Programs Summary 37-18, Vol. IV, pp. 129-132, Jet Propulsion Laboratory, California Institute of Technology, Pasadena, California, December 31, 1962.

3. E. J. Roschke, "An Examination of Vortex Strength in a Compressible Viscous Vortex", Space Programs Summary 37-20, Vol. IV, pp. 73-76, Jet Propulsion Laboratory, California Institute of Technology, Pasadena, California, April 30, 1963.

4. E. J. Roschke, "Some Gross Effects of Cylindrical Wall-Friction and End-Wall Boundary-Layer Flow on Confined Vortex Flows", Space Programs Summary 37-21, Vol. IV, pp. 102-108, Jet Propulsion Laboratory, California Institute of Technology, Pasadena, California, June 30, 1963.

5. T. J. Pivirotto, "Binary Diffusion in a Compressible, Three-Dimensional Turbulent Vortex", Space Programs Summary 37-23, Vol. IV, pp. 119-127, Jet Propulsion Laboratory, California Institute of Technology, Pasadena, California, October 31, 1963.

6. E. J. Roschke, "Comments on the Stability of Curved Compressible Flows", Space Programs Summary 37-24, Vol. IV, pp. 109-114, Jet Propulsion Laboratory, California Institute of Technology, Pasadena, California, December 31, 1963.

7. T. J. Pivirotto, "Binary Diffusion in a Compressible, Three-Dimensional Turluulent Vortex", Space Programs Summary 37-25, Vol. IV, pp. 81-88, Jet Propulsion Laboratory, Callfornia Institute of Technology, Pásadena, California, February 29, 1964.

8. E. J. Roschke, "Preliminary Experiments on a Jet-Driven Torroidal Vortex", Space Programs Summary 37-25, Vol. IV, pp. 76-81, Jet Propulsion Laboratory, California Institute of Technology, Pasadena, California, February 29, 1964. 


$\begin{array}{ll}\text { NOMENCLATURE } \\ \mathrm{d} & \text { Probe diameter } \\ \mathrm{D} & \text { Vortex container diameter } \\ \mathrm{L} & \text { Length of vortex container } \\ \mathrm{M}_{\mathrm{t}} & \text { Tangential Mach Number } \\ \dot{\mathrm{m}} & \text { Mass flow rate } \\ \mathrm{n} & \text { Particle density } \\ \mathrm{p} & \text { Static pressure } \\ \mathrm{r} & \text { Radius } \\ \mathrm{Re} & \text { Radial Reynolds Number } \\ \mathrm{T} & \text { Temperature } \\ \mathrm{Z} & \text { Axial distance from closed end wall } \\ \rho & \text { Mass density }\end{array}$

\section{Subscripts}

$\begin{array}{ll}\text { c } & \text { Condition at container centerline } \\ \text { d } & \text { Probe diameter } \\ \text { E } & \text { Conditions at exit orifice } \\ \text { H } & \text { High molecular weight gas } \\ \text { L } & \text { Low molecular weight gas } \\ \text { man. } & \text { Manifold condition } \\ \text { t } & \text { Stagnation condition (except } \mathrm{M}_{\mathrm{t}} \text { ) } \\ \text { w } & \text { Condition at inner wall of cylindrical container }\end{array}$


VORTICES AND VORTEX MATRICES

\author{
Martin L. Rosenzweig \\ and \\ Steve Lewellen \\ Aerospace Corporation \\ Los Angeles, California
}

Well, we've all seen in the last few days how important vortex flow configurations are to any of the schemes that have been presented, and of course we've been in the vortex business for about five years now. We began by looking at the same types of problems that people at JPL have in terms of the diffusion concept-that is, utilizing pressure diffusion to separate heavy and light gas in vortex flow. We have a relatively small effort, and have concentrated on the fluid dynamics of vortex flows. Most of our work is with a single fluid. We have. not studied separation at all but have really tried to get an understanding of why jet-driven vortices, or those generated in rotating containers, do what they do, with the idea of getting to the point where some day we can perhaps sit down with paper and pencil and design a vortex tube which will do what we predict apriori.

It seems like a very far-sighted view at the present time but we'll see how far along we've come in this respect. A lot of our work is similar in some nature to the work that was reported by JPL and also by Bill Foley. That is, we've made flow visualization studies; we have also varied quite a few things geometrically; and we have undertaken end-wall blowing, as Paul mentioned they were going to do at JPL. We are also working on a device which you have heard about no doubt-a matrix device, which many people have laughed at. I have myself at times.

I'm going to briefly describe our program, and then I will make a few comments as to how we presently feel with regard to an engine which might work on the basis of fission. fragment energy absorbtion rather than radiation and thereby, perhaps, alleviate some of the problems associated with the very high temperatures and high pressures that we are forced to in a 
radiation system. I'm going to try, if $I$ can, to dissociate in your minds the probliems of pressure diffusion and those of fission fragment heating. We'll get into that in more detail after our discussion.

First I'm going to introduce Steve Lewellen who is going to tell you a little bit about our theoretical work concerning the end-wall boundary layer growth in these vortex tubes and the interaction with the primary vortex flow, in an attempt to give you: a little better theoretical feel for why we have these very unusual flow situations that we have seen in the past few days. When he's finished, I'll come back and very briefly go through our experimental program including the flow visualization experiment, some comments on the turbulence experiment that we have been involved with in the last few years and, finally, some comments about our matrix. I also have a short movie which will demonstrate some of the things that we ve done in the lab. Steve:

Most of our theoretical work has been aimed at trying to solve the Navier Stokes equations for a flow model of a vortex in a container. This rather basic flow model is applicable for several of the different concepts described in the preceding presentations.

Figure 1 gives the fundamental equations in the form we have used as a starting point. We have been willing to make several simplifying assumptions to make the analysis tractable. One of our principal assumptions is the assumption of laminar flow, although at points in our work we have included the effect of turbulence in a semi-empirical fashion. Also, we have assumed the flow to be incompressible in most of our work.

One important point to be obtained from these equations is the fact that flow in strong vortices always tries to be uniform in the axial direction; that is, the flow tends to be two dimensional. The tangential velocity $v$ is only a function of radius. The reason for this is that in any system in which the tangential velocity, that is the rotation, is much larger than the mass flow through it, the radial pressure gradient must be much larger than the axial pressure gradient. From the radial momentum equation you can see that the radial pressure gradient is determined by the centrifugal force term $v^{2} / r$, while from the axial momentum equation the axial pressure gradient is dependent upon the radial velocity $u$ and the axial velocity $w$. So necessarily, the radial pressure gradient will be much larger than any axial gradient. But then, since the pressure gradient depends upon $v, v$ must also be independent of $z$; that is, it must be almost a function of $r$ only. This is a point that all of our experiments demonstrate quite clearly. Regardless of the shape of the container the flow turns out to be essentially independent of $\mathrm{z}$ 
BASIC EQUATIONS

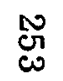

$$
u \frac{\partial u}{\partial r}+w \frac{\partial u}{\partial z}-\frac{v^{2}}{r}=-\frac{1}{\rho} \frac{\partial p}{\partial r}+\nu\left(\frac{\partial^{2} u}{\partial r^{2}}+\frac{1}{r} \frac{\partial u}{\partial r}-\frac{u}{r^{2}}+\frac{\partial^{2} u}{\partial z^{2}}\right)
$$

$$
\begin{aligned}
& u \frac{\partial v}{\partial r}+w \frac{\partial v}{\partial z}+\frac{u v}{r}=v\left(\frac{\partial^{2} v}{\partial r^{2}}+\frac{1}{r} \frac{\partial v}{\partial r}-\frac{v}{r^{2}}+\frac{\partial^{2} v}{\partial z^{2}}\right) \\
& u \frac{\partial w}{\partial r}+w \frac{\partial w}{\partial z}=-\frac{1}{\rho} \frac{\partial p}{\partial z}+v\left(\frac{\partial^{2} w}{\partial r^{2}}+\frac{1 \partial w}{r} \frac{\partial}{\partial r}+\frac{\partial^{2} w}{\partial z^{2}}\right)
\end{aligned}
$$

Figure 1 
over the main flow region. This cannot usually hold throughout the flow. Whenever any boundary conditions occur in the real flow to destroy the two dimensionality of the flow, shear zones are formed in the flow where the radial velocity $u$ and/or the axial velocity $w$ become of the same order as $v$.

In Figure 2, I have indicated the simple two-dimensional solution that can be obtained from the equations for the main part of the flow (Reference 1 ), outside of any shear zone (i.e. outside of any end-wall boundary layers, etc.). This is valid whenever $Q \boldsymbol{\ell} / \Gamma_{\mathrm{O}} \mathrm{r}_{\mathrm{O}} \ll 1$, where $Q \boldsymbol{\ell}$ is the flow through the device, and $\Gamma$ is the circulation $2 \pi \nu \mathrm{r}$. This type of parameter is quite common to meteorologists who have dealt:with rotating systems for some time. They call it a Rossby number. In this limit, the solution for the velocities is quite simple. The axial velocity is given as a linear extrapolation between two boundary values. The radial velocity and the tangential velocity are given by simple quadratures and are both functions of radius. only.

The end-wall boundary layer, which is necessitated by the fact that the tangential velocity must go to zero on the end wall, has been studied quite a bit by several people, e.g., at UAC, JPL, and our place. We have obtained exact similarity solutions for certain particular cases (Reference 2), and we have obtained approximate integral solutions for more practical cases (References 3,4 , and 5). Some of these results are given in Figure 3. This is an approximate solution for the volume flow $Q_{b .1}$. in the end-wall boundary layer. I have written it in a form which is valid for both laminar and turbulent flow. In this formula, the boundary layer begins to grow at some outer radius $r=1$ with the flow in the boundary layer directed toward

1. Lewellen, W. S., "A Solution for Three-dimensional Vortex Flows with Strong Circulation", Journal of Fluid Mechanics, Vol. 14, Part 3, November 1962.

2. King, W. S. and W. S. Lewellen "Boundary Layer Similarity Solutions for Rotating Flows with and without Magnetic Interaction", 1963. The Physics of Fluids, Vol. 7, October 1964.

3. King, W. S., "Momentum-Integral Solutions for the Laminar Boundary Layer on a Finite Disk in a Rotating Flow", American Society of Mechanical Engineers Paper No. 64-FE-14, June 1964.

4. Rott, N., "Turbulent Boundary Layer Development on the End Walls of a Vortex Chamber", Aerospace Technical Report ATN-62(9202)-1, July 1962.

5. Rott, N. and W. S. Lewellen, "Boundary Layers in Rotating Flows", Aerospace Technical Report ATN-64(9227)-6, 4 September 1964. 
APPROXIMATION FOR STRONG CIRCULATION

$$
\text { AS } \begin{aligned}
& \frac{Q}{\Gamma} \frac{\ell}{r_{0}}-0 \\
& w=w_{1}(r)+\frac{z}{l}\left[w_{2}(r)-w_{1}(r)\right] \\
& u=-\frac{1}{r l} \int\left[w_{2}(r)-w_{1}(r)\right] r d r \\
& v=\frac{c_{1}}{r} \int r \exp \left(\int \frac{u}{v} d r\right)+\frac{c_{2}}{r}
\end{aligned}
$$

Figure 2 


$$
Q_{b .1 .}=2 \pi C \Gamma^{b}\left[\int_{r}^{1} r^{a-1} \Gamma^{2-a(1+b)} d r\right]^{1 / a}
$$

LAMINAR:

$$
C=2 \sqrt{2 v_{1} r_{1}} r_{1} ; a=4 / 3 ; b=2.3
$$

TURBULENT:

$$
C=0.135 \nu^{1 / 5}\left(v_{1} r_{1}\right)^{4 / 5} r_{1} ; a=1 ; b=4
$$

Figure 3 
decreasing $r$. (Note that $r$ and $\Gamma$ have been made dimensionless by dividing by $r_{1}$ and $2 \pi v_{1} r_{1}$, respectively.) From this formula, we can see how the boundary-layer mass flow will initially increase regardless of what the outside distribution is; and then at a smaller radius, where the circulation has been sufficiently decreased, the mass flow in the boundary layer will be ejected out of the boundary layer. This will give rise to the secondary flows which we have seen in the experiments. We can see from the value of the constants just what the mass flow in the boundary layer is dependent upon; that is, it is proportional to the square root of the kinematic viscosity $v$ times the circulation in the laminar case and proportional to the fifth root of $"$ times the four fifths root of the circulation in the turbulent case. Of course, this sort of analysis assumes that the tangential velocity outside the boundary. layer is a given quantity; but in a true case, there will usually be an interaction between the boundary layer and the outside flow. If the mass flow is being detoured through the boundary layer, then this must affect the mass flow in the outer flow, i.e., the mass flow driving the outer flow. The mass flow in the outer flow is reduced which causes the circulation to fall off, and in turn causes the boundary layer mass flow to decrease and bring the flow back out of the boundary layer.

We have at hand all of the necessary tools to consider the interaction between the primary flow and the boundary layer. We have the simple twodimensional solution for the flow outside of the shear zones (Figure 2) and also we have a solution for the flow in the boundary layer (Figure 3). All we have to do is place the two together to get the solution of the interaction problem. Figure 4 shows the geometry which we used in doing this interaction problem (Reference 6). We assume that the flow is divided up into three zones. The first zone (I) is the main body of the flow which is, as previously noted, two dimensional. The second zone (II) is the end-wall boundary layer where the axial shear is high and the equation determining the end-wall boundary layer flow is valid. In the third zone (III), what happens depends very strongly upon conditions in the exhaust system. For simplicity we have considered the flow out the exhaust to be uniformly distributed.

There is one more point to be brought in here in setting up the interaction problem. In line with this statement that the flow tries to be twodimensional, outside of any shear zone, it is to be expected that any little disturbance occurring at one place in the flow will propagate across the flow.

6. Rosenzweig, M. L., W. S. Lewellen and D. H. Ross, "Confined Vortex Flows with Boundary-Layer Interaction", Aerospace Technical Report ATN-64(9227)-2, 20 February 1964. 


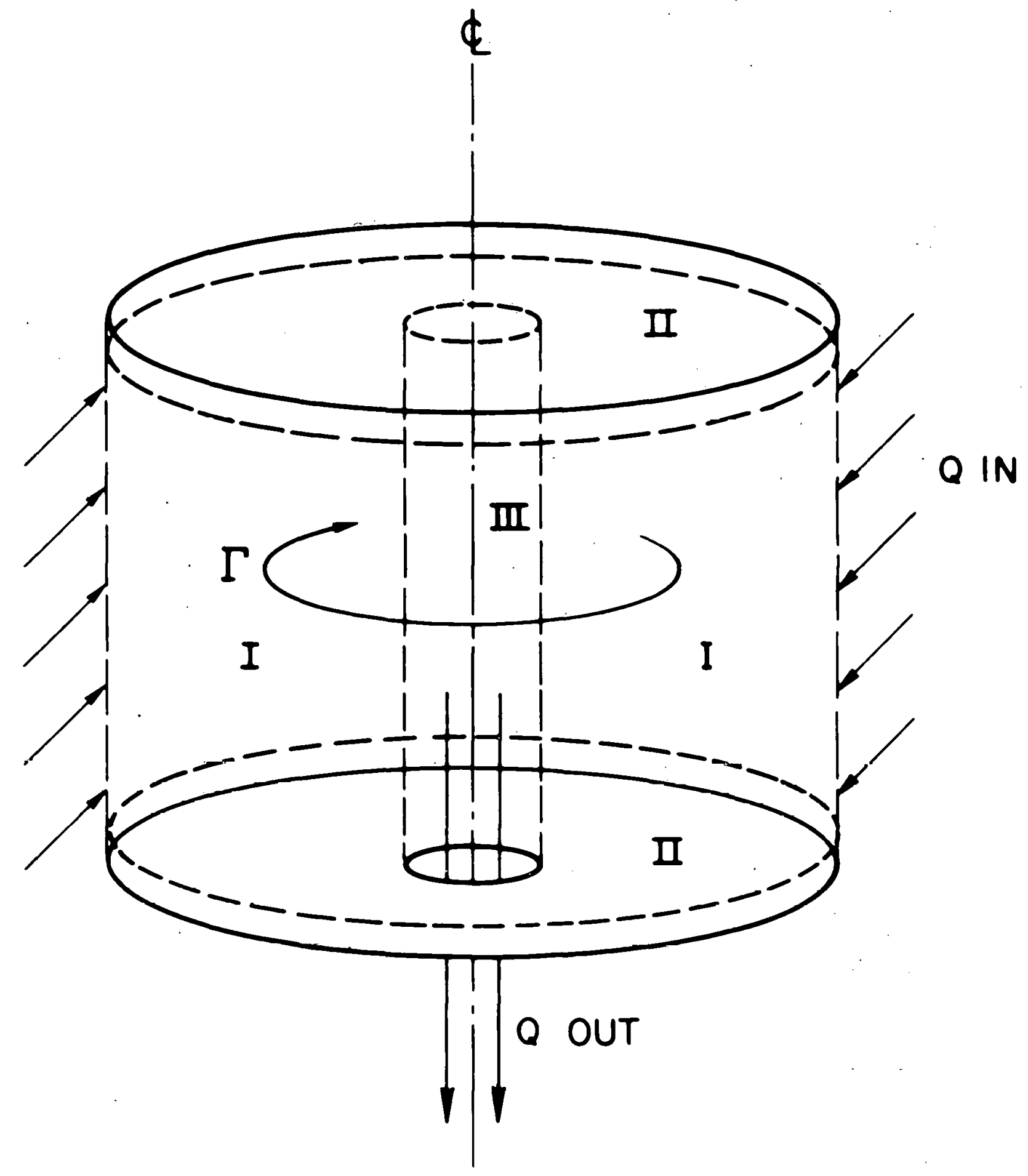

Figure 4 
That is, a local disturbance on the boundary layer, because of the twodimensionality of the flow, propagates in the $z$ direction across the flow. We have included an extra factor in the analysis to determine the effect of the boundary at the edge of the exhaust. This local disturbance forces some fraction $(\zeta)$ of the flow out of the boundary layer and back into the main flow. In the experimental work to be presented by Marty this will be demonstrated quite clearly.

Figure 5 gives some results of our interaction studies. The principal parameters in the study are of course the radial Reynolds number $\operatorname{Re}_{r}$ which is dependent upon mass flow $\left(\operatorname{Re}_{\mathbf{r}}=\mathrm{Q} / 2 \pi \nu\right)$, and the interaction parameter $\mathrm{A}$ which determines the effect of the end-wall boundary layer on the total flow. We see appearing in this interaction parameter the ratio of circulation to mass flow which is the reciprocal of the Rossby number. The particular form of the interaction parameter given in Figure 5 is based upon a turbulent boundary layer. The results shown are for two different values of zeta, zero and one, because there is an uncertainty as to exactly what it should be. The true value of zeta must lie somewhere between zero and one, so this brackets its effect.

This particular figure shows results of an interaction parameter of $A=1$. Both the circulation distribution $\Gamma$ and the dimensionless mass flow $f$ are plotted as function of the radius. The circulation at the relatively modest Reynolds number of -10 is still fairly uniform outside the radius of the exhaust. We have to go to low Reynolds number (such as -3) before the performance in circulation falls off. The mass flow in the boundary layer, of course, is greater in cases in which the circulation remains almost constant; and since the mass flow running through the device is that which is left after a fraction has been detoured through the boundary layer, the case of $R e_{r}=-10$ represents flow with the larger flow in the boundary layer.

Figure 6 gives the result for a slightly higher $A=2.7$. In the case of Reynolds number of -50 the circulation remains fairly constant outside the radius of the exhaust. Yet, at $r / r_{0} \approx 0.5$, all the mass flow has gone to the boundary layer, since the mass flow on the outside has gone to zero. In fact, for zeta equal to one, i.e., when the flow in the boundary layer is forced to come back out at the edge of the exhaust, there is actually a recirculation. In this region, the flow outside the boundary is outward; the flow in the boundary layer is greater than the total.

At $r / r_{0}=0.4$, the oase for $R e_{r}=-50$ shows a region of zero radial flow. This corresponds to regions where experimentally one can get dye to stay for indefinite periods of time since there is no through flow to carry it out. 


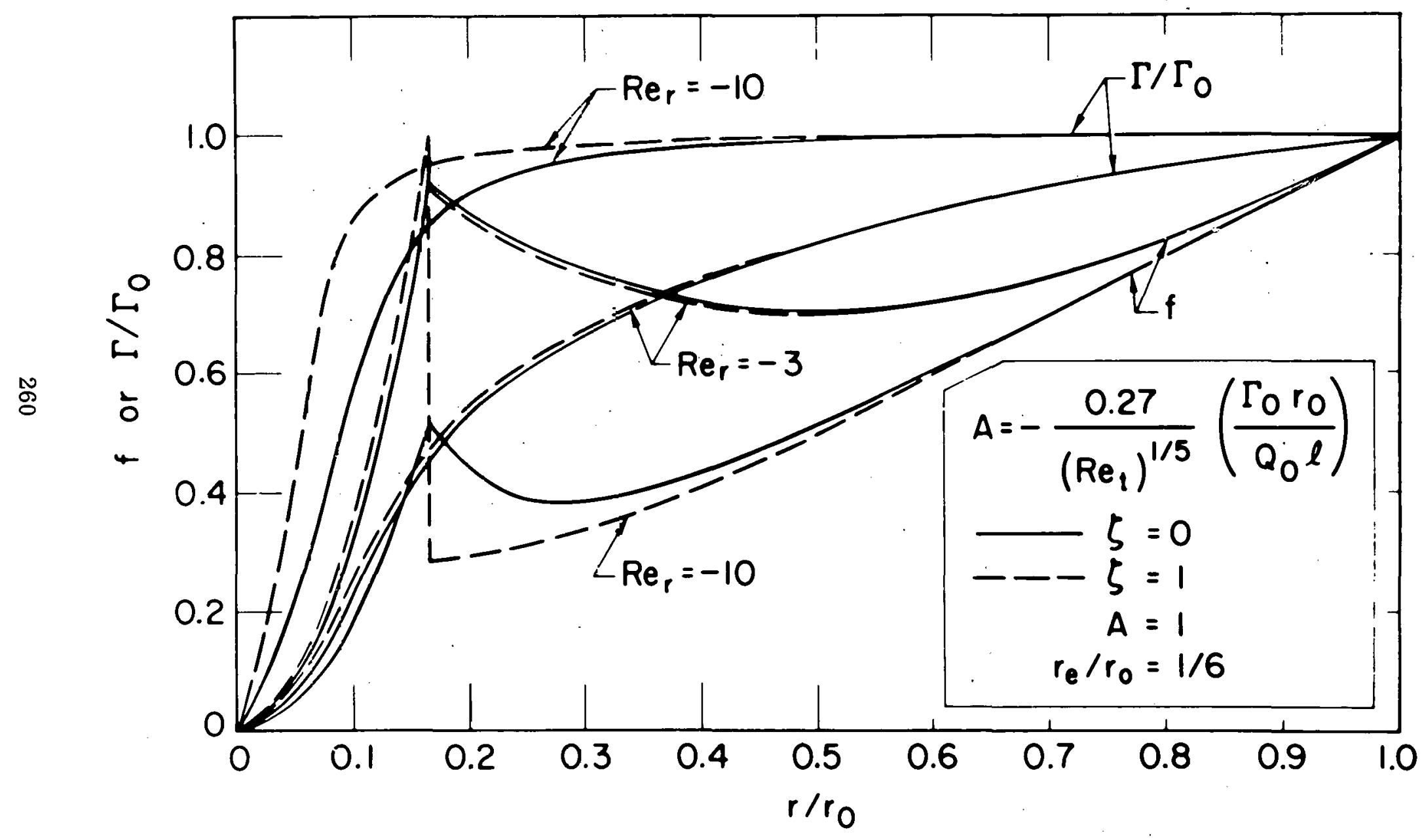

Figure 5 


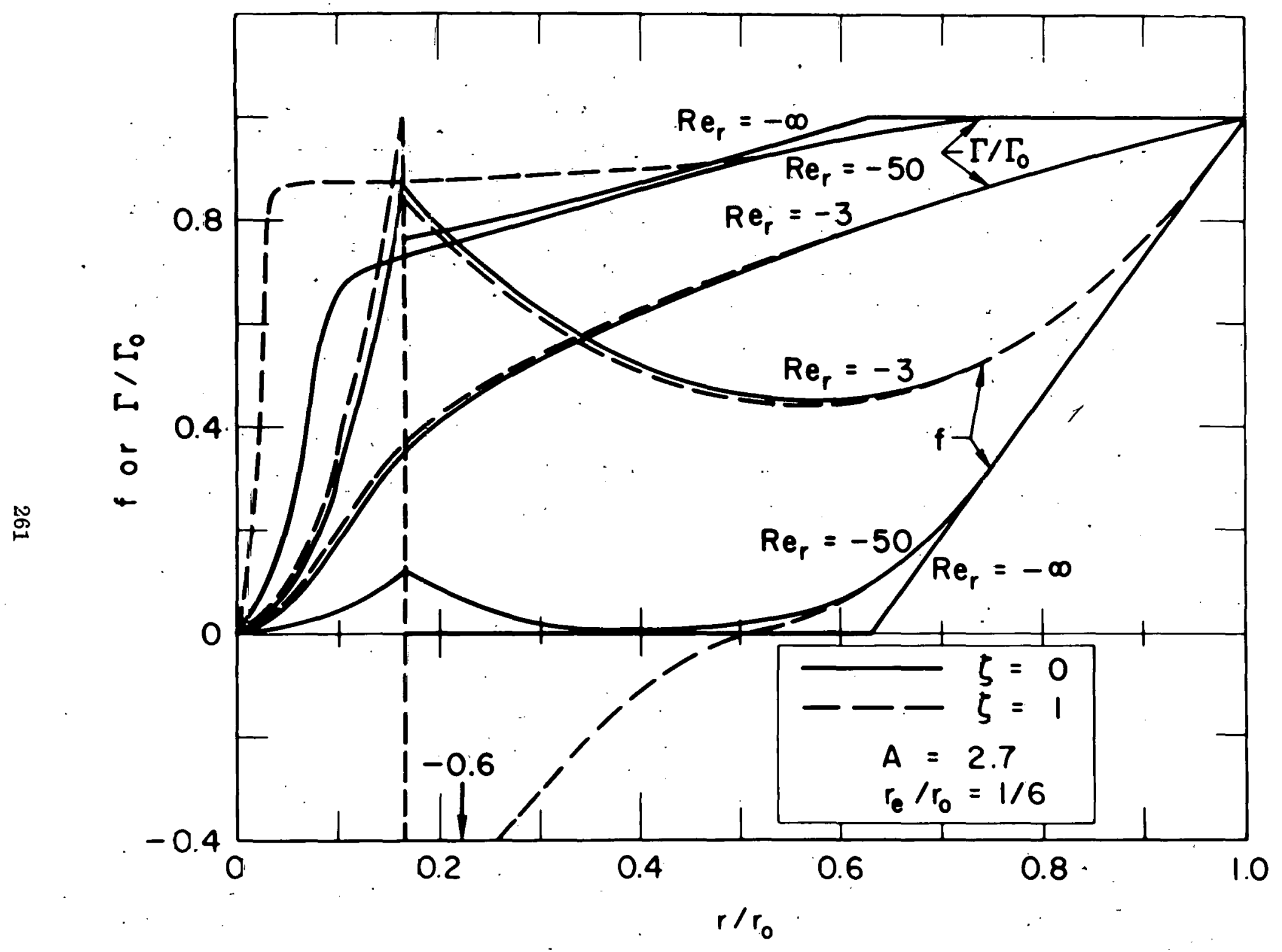

Figure 6 
Question (McLafferty)'s How long. would that dye stay there if it weren't turbulent?

Answer; Off course, turbulent diffusion will affect the decay rate of the dye cells. All we are saying here is that this is the way in which this zone is created. That is, such a zone can occur when all the flow has been bypassed through the boundary layer, and this is the order of parameters for which this will occur. This can occure whether there is turbulence in the system or not. One can actually get just as good a circulation profile in the turbulent case as in a laminar case by juggling the mass flow. Marty will say more on turbulence when he describes our turbulence experiment.

This then shows the way in which the dye cells remain intact. They can oecur at any position of zero radial and axial velocity; the position depends on the different values of these parameters-you can shift the position of zero radial flow to almost any position.

In Figure 7 as a summary figure of merit, the circulation at the exhaust radius divided by that at the outside is plotted as a function of $\mathrm{A}$ and of Reynolds number. I should add here that, of course, all of this is a function of radius ratio, the ratio of the exhaust radius $r_{e}$ to the radius $r_{0}$ at which the flow enters. All of the results given here have been for a radius ratio of $1 / 6$.

In Figure 7 you see that rather large values of $A$ are required before there's any appreciable fall off in the circulation ratio $\Gamma_{e} / \Gamma_{0}$ for rather modest values of Reynolds number. Even at Reynolds numbers of -10 , whether you're talking about zeta equal to zero or zeta equal to one, there is still a pretty good vortex at $A=1$. As George just pointed out, we are usually doing the experiments at laminar Reynolds number of around 100, and sometimes over a thousand or so. You can see that for practical purposes such flows are quite close to the limiting curve of $\operatorname{Re}_{r} \rightarrow \infty$. In this limit the full analysis can be simplified substantially. The circulation remains constant up to A's of somewhere between one and two depending upon the parameter zeta. The rate at which the circulation decreases for large $A$ in this limit is quite interesting. It occurs that at a certain radius depending upon $A$ all of the flow has been detoured to the boundary layer. Then at smaller values of the radius the circulation falls off at just the rate which will be required for all of the flow to stay in the boundary layer. This flow pattern is shown in Figure 8 for a large A. This is the flow pattern in the limit of large radial Reynolds number.

Figure 8 also gives the pattern of flow that occurs in a solid-body rotating system with a small flow through it. In the prcceding presentations 


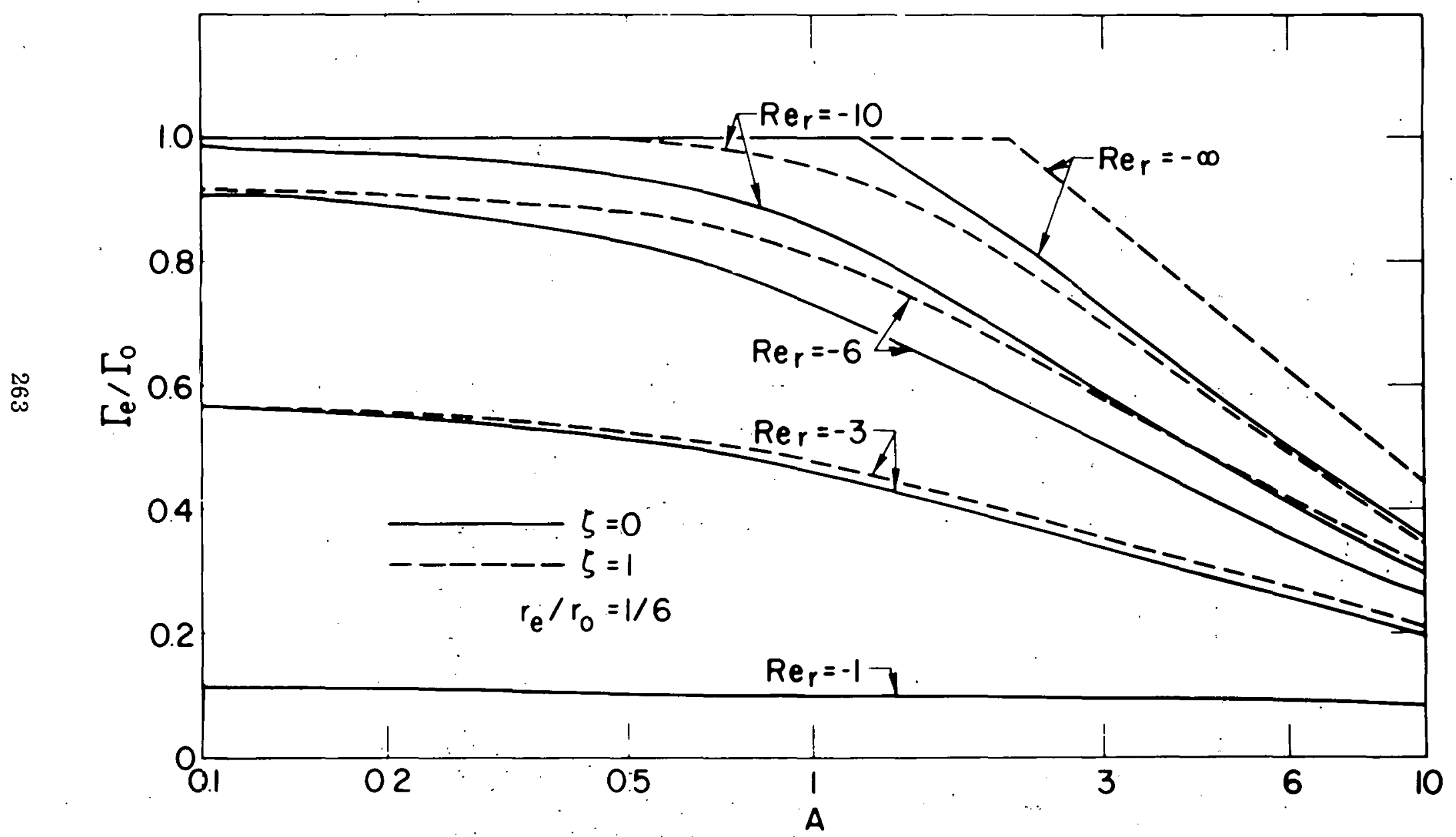

Figure 7 


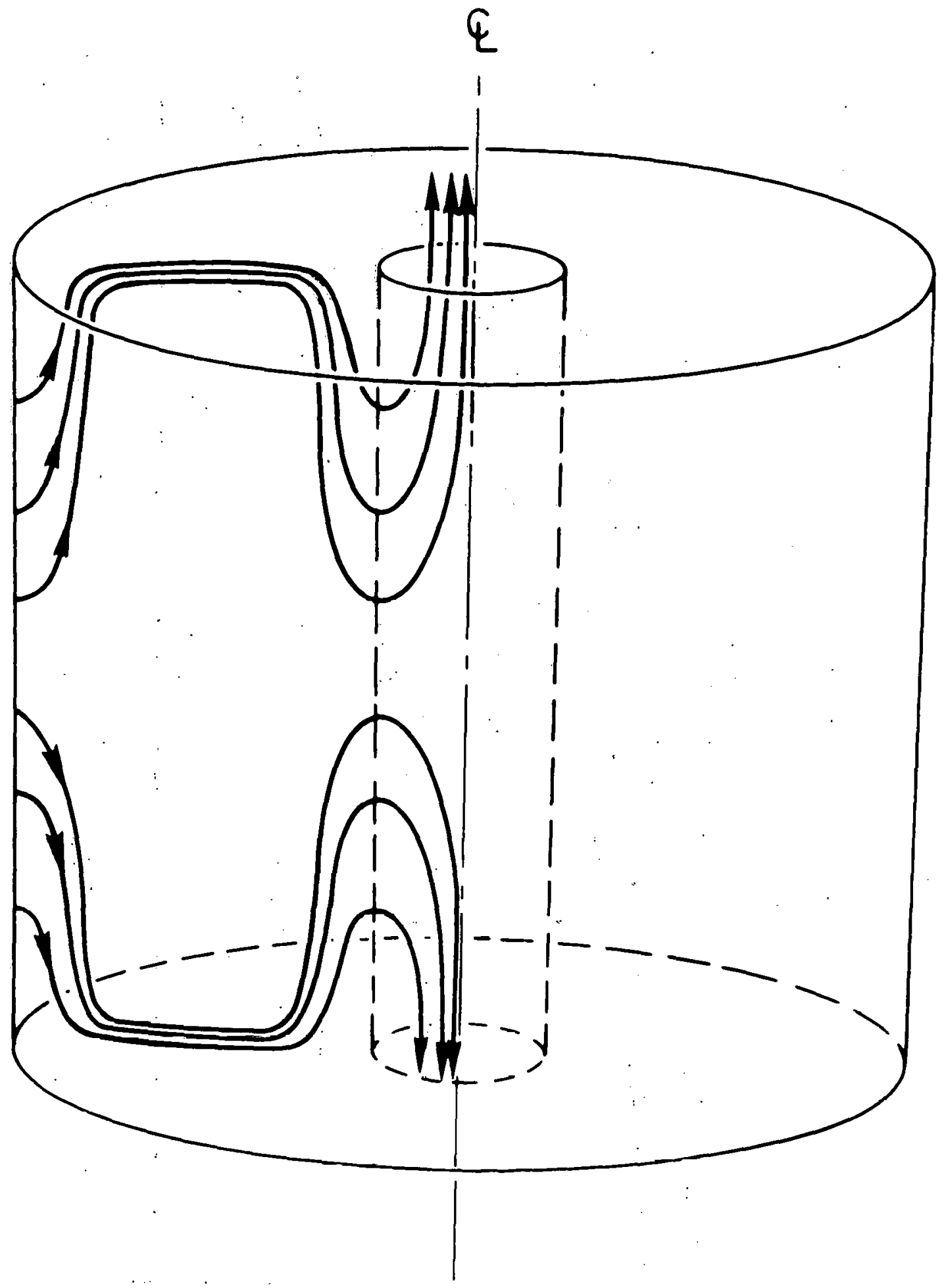

Figure 8 
the re have been several concepts of dust bed or liquid core systems which depend upon a rotating container. The same sort of flow pattern as given in Figure 8 occurs in the rotating container if the mass flow going through it is small. The requirement for this type flow pattern is that the Rossby number be less than the reciprocal of the square root of the tangential Reynolds number (Reference 7).

In conclusion, the vortex can provide three mechanisms which may be useful in a gas core reactor. One, it can stabilize the flow and permit high radial gradients to occur in the flow. Second, it sets up strong secondary flows which may create zones of stagnant fluid. And third, the vortex provides a pressure diffusion phenomena which, as discussed by the JPL representatives, can set up a favorable concentration distribution. We feel that by better understanding these problems, that we can actually make all of these things work for us. In our present theoretical studies we are trying to combine the flow picture described here with diffusion for the case of two species flow in order to see what conditions will give the most favorable concentration distributions.

Marty will now describe our experimental studies.

(Most of the material covered in this talk is included in References 6 , 8, 9, and 10.)

First I'm going to show you some of the slides of the flow-visualization experiment, which was performed in a single vortex tube. We're going to see what the influences are of various perturbations in the end-wall geometry. As you have seen, by varying the parameter $A$ in the interaction theory, one should be able to change the type of secondary flow distribution to the point

7. Lewellen; W. S., "Linearized Vortex Flows", Aerospace Technical Report ATN-64(9227)-4, 24 April 1964.

8. Rosenzweig, M. L., D. H. Ross and W. S. Lewellen, "On Secondary Flows in Jet-Driven Vortex Tubes", J. Aerospace Sci., Vol. 29, No. 9, Sept. 1962.

9. Ross, David H., "An Experimental Study of Secondary Flow in JetDriven Vortex Chambers", Aerospace Technical Report ATN-64(9227)-1, 27 January 1964.

10. Ross, David H., An Experiment Investigation of Turbulent Shear in JetDriven Vortex Chambers", Aerospace Technical Report ATN-64(9227)-5, September 1964. 
where, by raising A high enough, you should be able to achieve the stationary cylinders which we've seen in other people's slides.

Now, actually, I'll just sum up very briefly what we found with geometrical perturbations. We found that the basic flow pattern, which you'll see on the first couple of slides in a moment, is very difficult to perturb, even with very radical changes in the end walls. There were, however, two changes that did make a difference. One was a very highly curved end wall, in which the end wall was faired into the exit hole in a continuous curve. With this type of arrangement, you increase the flow in the end-wall boundary layer, because for a given projected area normal to the axis, you have a much higher total shear area to suck mass flow into the boundary layer. We found, in this case, with two curved end walls, that we raised A sufficiently so that we produced a stable cylinder. When this occurs, all of the through-flow goes into the boundary layers and comes out again near the axis to produce the recirculating counter-flows that we've seen in other people's work. Even in this case, where apparently there's a very favorable gradient for the flow to get out of the exit hole, we see that there are recirculations occurring outside the radius of the exit hole.

The second change in the end wall that produced a large effect is related to what steve was talking about before. That is, that any perturbation in the end-wall geometry which is of the order of magnitude of the boundary layer thickness will produce mass ejection from the boundary layer which will then propagate clear across the device. We actually tried an end wall with a circular step at about mid-radius. Even though the step is stepped downward with respect to the direction of the flow, we did get mass ejection, and you will see this on the slide.

We have also done the end-wall blowing case with tangential slot injection and have verified indeed that by supplying the momentum defect in the boundary layer with the end-wall blowing, one can reduce the secondary flow, and by blowing hard enough, you can actually cancel it entirely or reverse it and make the secondary flow go toward the outside. This is the same type of device that Dr. von Ohain was talking about yesterday. I have motion pictures of the end-wall blowing case. I don't have them documented on a slide. Let's go through the first few slides and I'll show you the apparatus that we're talking about.

Most of you have probably seen this--it is simply a single vortex tube (Figure 9). We use water with different types of dye. In some slides you'll see ink, and in some slides you'll see fluorescein dye with ultraviolet illumination. In some cases, we have a fine probe which is used to inject dye at various places; and in most of the cases which you see here, there is no 


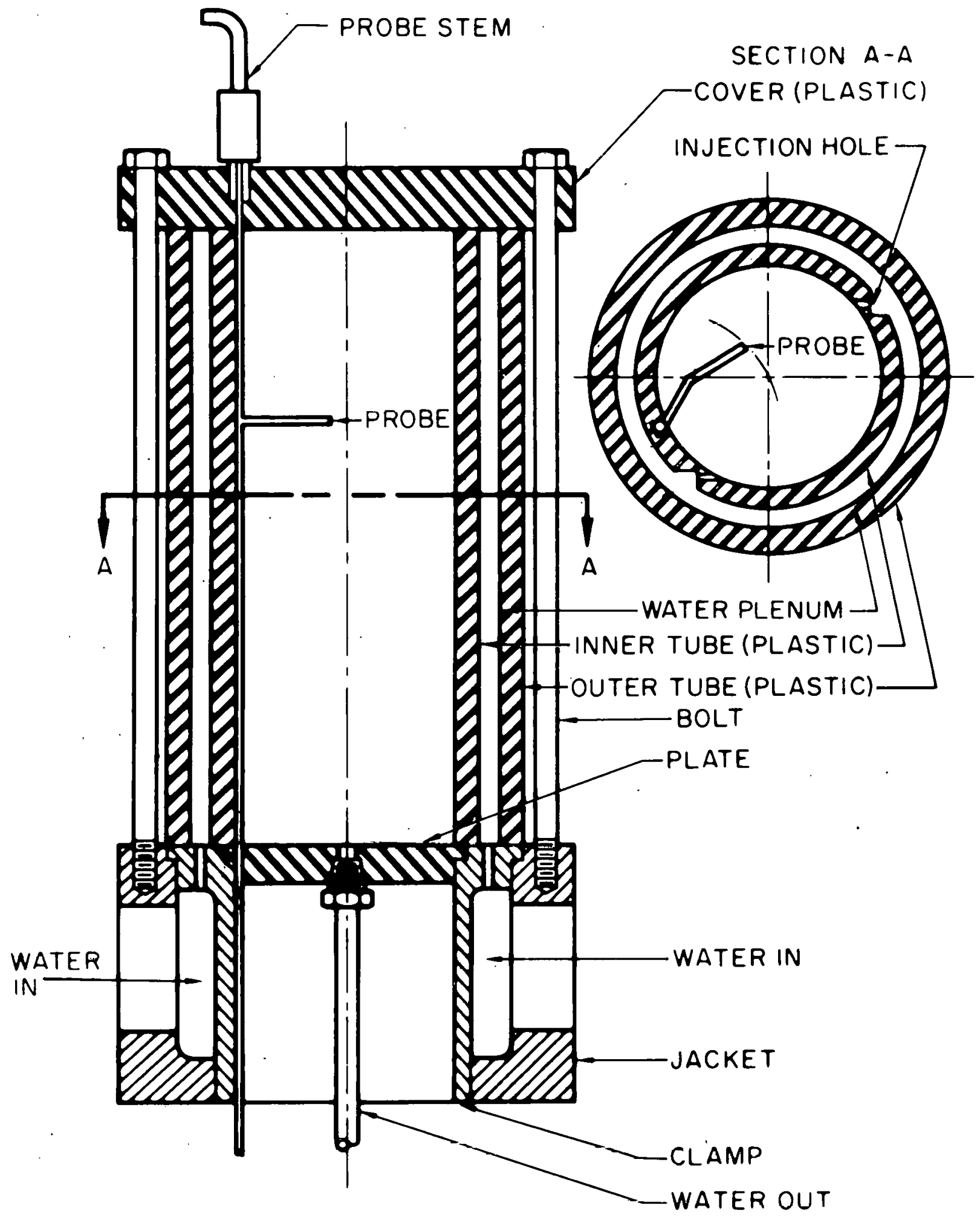

Figure 9 
probe, but rather dye is injected through one or both end walls. Ordinarily we take the flow out at one end wall, but we also have the capability to take flow out both ends. This is just a plenum chamber between two plexiglass cylinders. Let's see the next slide, please (Figure 10). This is a typical result with planar end walls, no geometrical perturbations, or blowing. Here's the probe in this case, and flow is coming in through these little tangential jets. The exit hole is on the bottom. We have a core of flow that originates from the bottom boundary layer and proceeds upward. The ejection occurs just outside the radius of the exit hole. The radius at which this ejection takes place appears to scale with the exit hole radius which leads us to believe that it's probably due to a combination of both drop-off of circulation in the main flow and perturbation produced by the sharp-edged orifice. We see the flow coming out of the boundary layer proceeding all the way across the tube. Next slide, please (Figure 11). This is an asymmetric situation. We have an exit hole on the bottom, and we have a boundary layer, of course, on the top. These tubes don't want to be symmetrical at all, as you probably know. Here we have the probe near the upper end wall. Some dye comes down a central core and goes right out the hole. There's a clear region immediately surrounding that, which is fed from the bottom boundary layer, which we saw in the previous slide. We see dye also propagating down from the top boundary layer in an annulus around this inner core region which appears light.

Next slide, please (Figure 12). This is a fluorescein dye picture, and this is the stepped end wall that I just drew to my right. Here we have an end wall which has a step down. This is the radius of the step. We see with dye injected through the end wall that there is mass ejention for this cuse at the radius of the step. We can also verify, although $T$ don't believe I have a slide to show here, that if you inject dye inside the radius of the step you can observe ejection outside the radius of the exit hole as we did in the preceding slide. So here is a case in which you can artificially trigger the zeta parameter and force some mass out of the boundary layer. How much or how little, we are not in a position to say at the present time.

Next slide, please (Figure 13). This is a dye picture which shows the same configuration as before, but with dye injection from both end walls. Both end walls are stepped in this case, and you can see some of the structure that exists inside the radius of the step. Actually, this is about as symmetrical a situation as we've ever been able to produce in this particular geometry, but even then, it's not entirely symmetrical.

Next slide, please (Figure 14). This shows the device with the highly curved end walls. You can just barely see some of the curvature through 


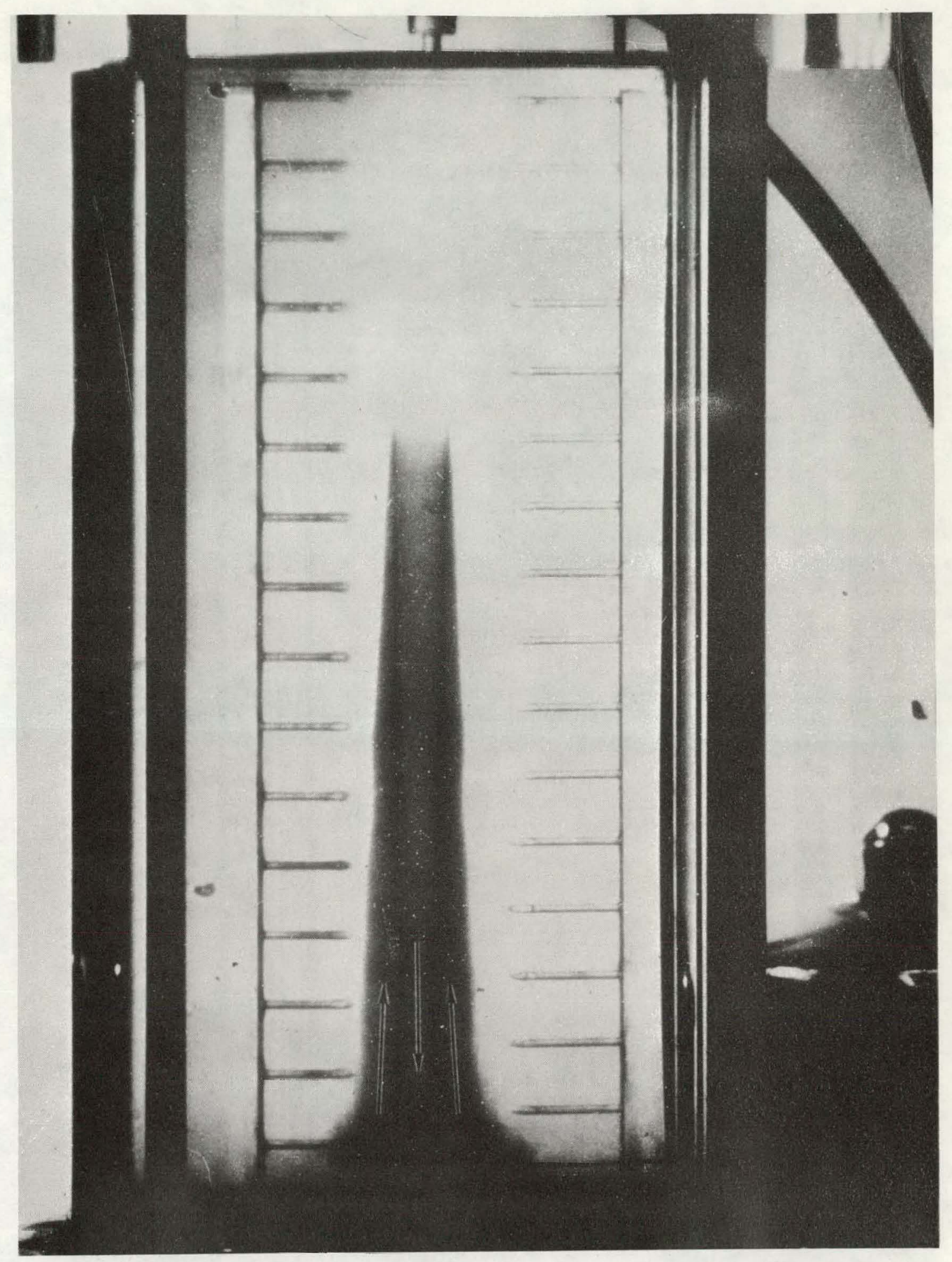

Figure 10 


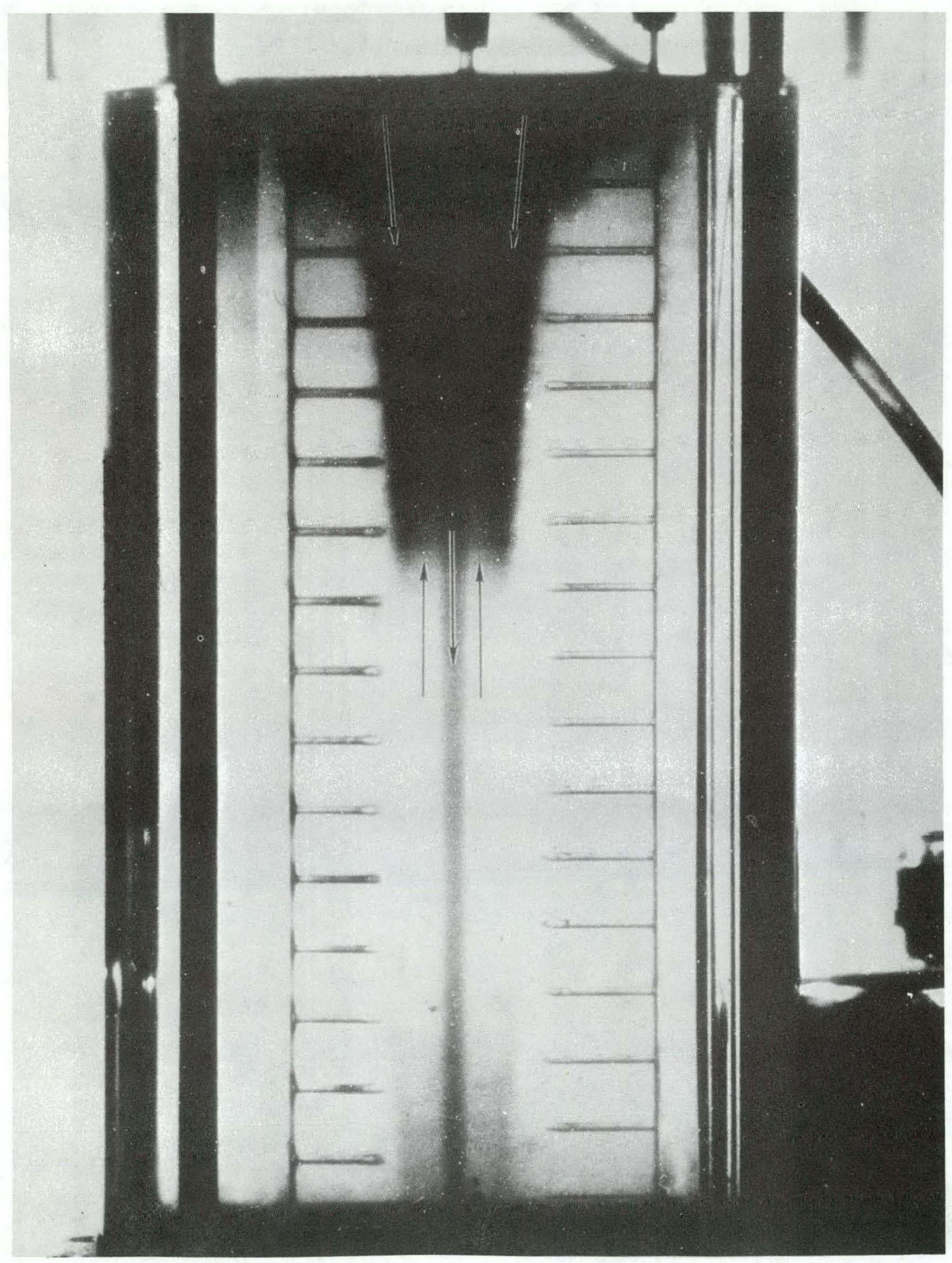

Figure 11 


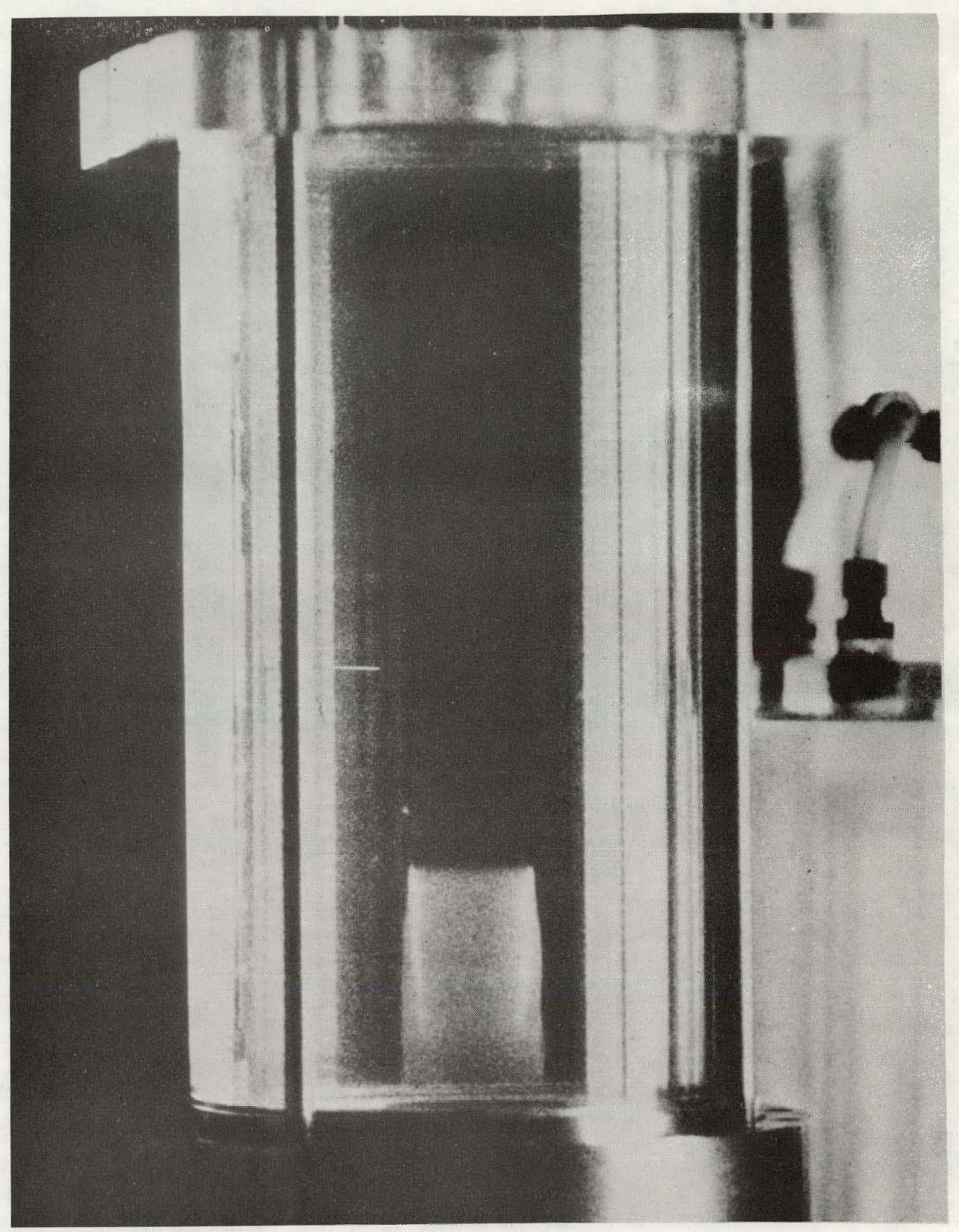

Figure 12 


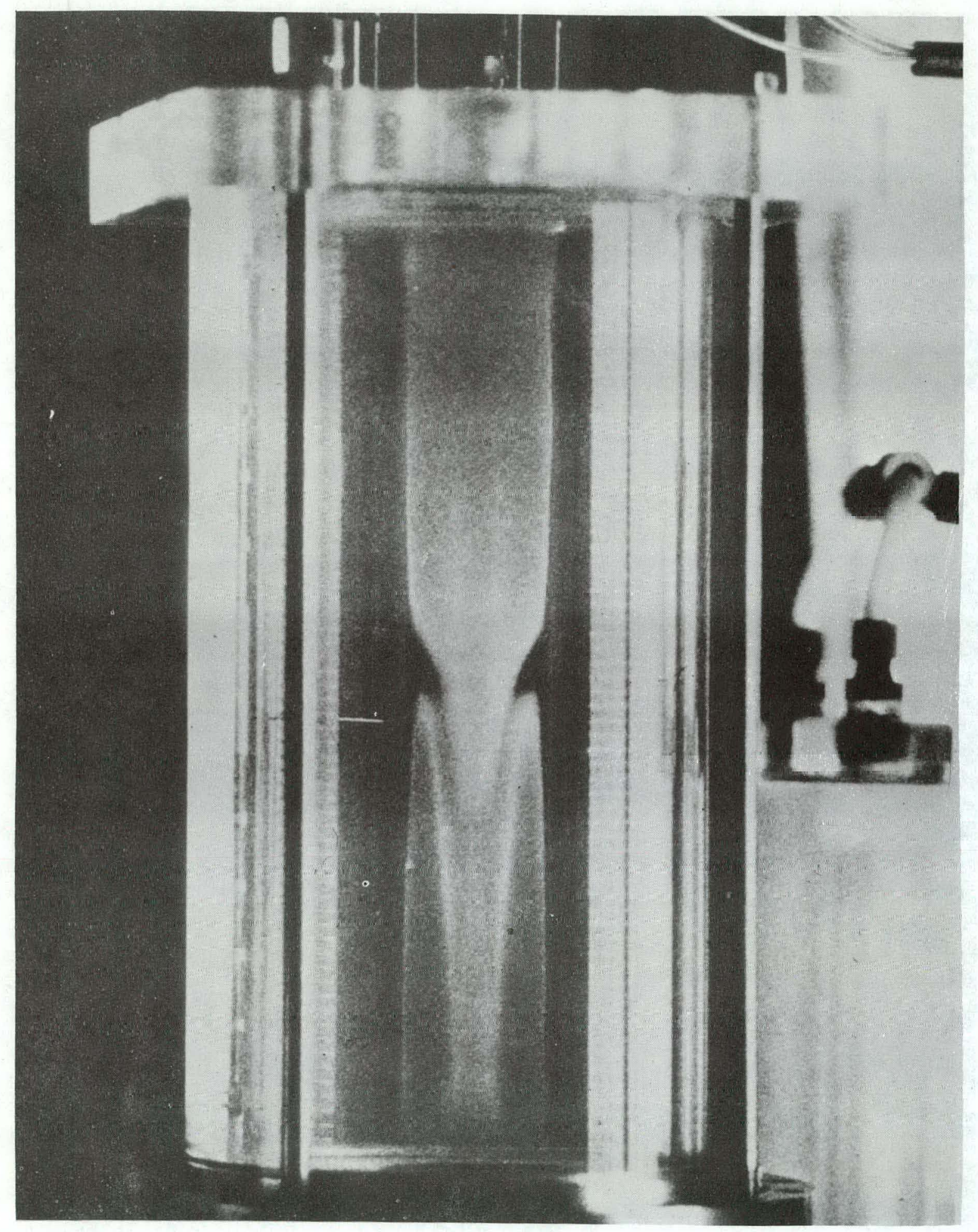

Figure 13 


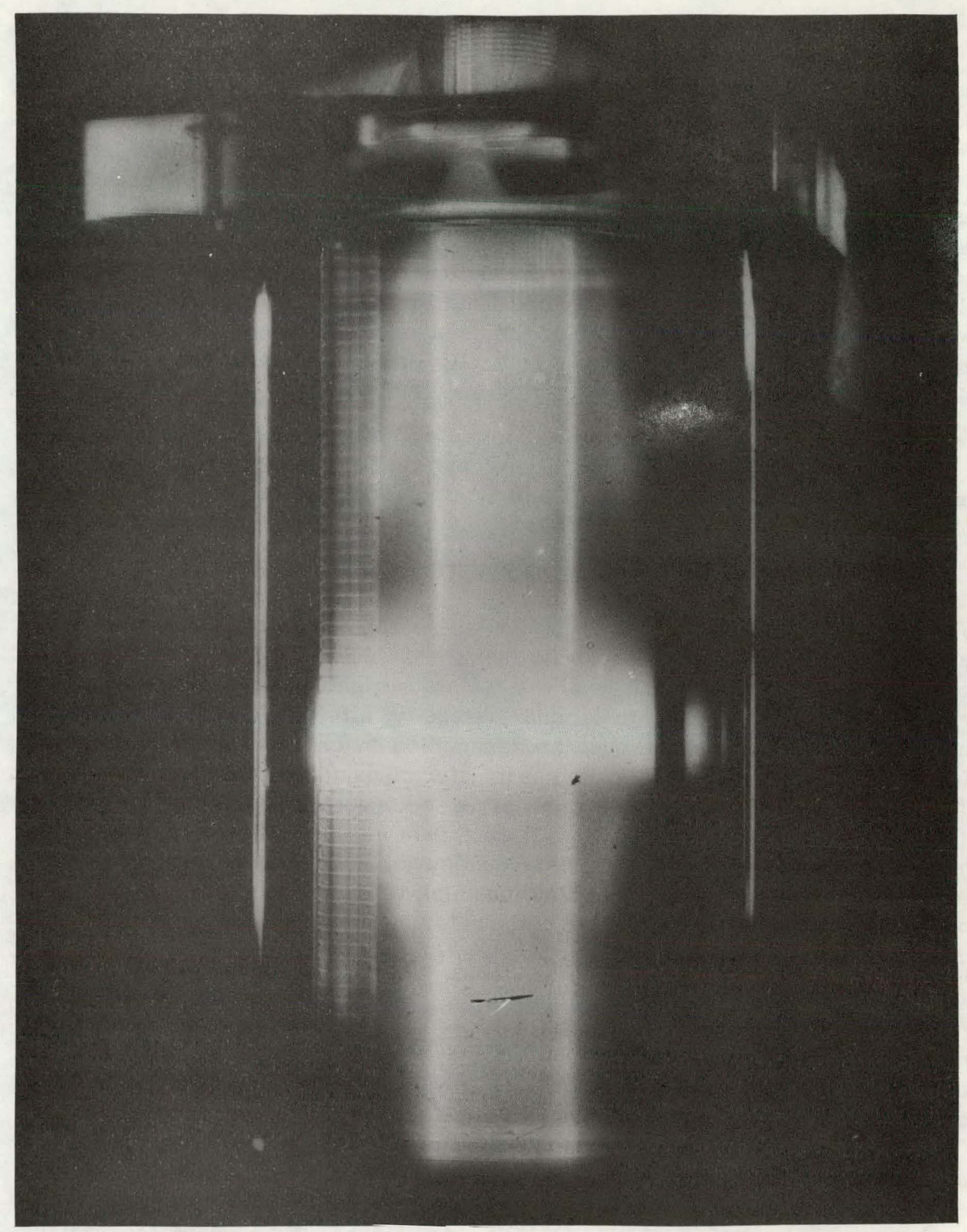

Figure 14 
the distortion of the plexi-glass end plates. In this case the dye is being injected by a probe located at the periphery midway between the end walls. This photograph was taken during the dye injection, and you see that the dye proceeds almost immediately to the cylinder shown here. The radius of the exit hole is very small in comparison to the dye-cylinder radius. This is very analogous to the photographs that we saw this morning on the iodine containment. Actually, this stable dye cylinder remains, as previously mentioned, for a very long period of time under maximum flow conditions. The next slide was taken about 10 or 15 minutes later and it's still there (Figure 15). The through-flow is going directly to the end walls and then travelling up and down in the interior region and finally leaving through the exhaust holes.

Now as I mentioned before, I have the result of the blowing on the movie, but I'd rather save that for 1ast, after I've finishcd the other part of my presentation.

Now I'd like to talk very briefly about a turbulence experiment that we have been working on for the last few years. It's a device which enables us to measure the radial distribution of tangential shear stress in a vortex tube geometry. If you want to make some extrapolations it is possible to consider the ratio of measured shear to laminar shear stress to be the ratio of turbulent eddy viscosity to laminar viscosity, although I wish to emphasize it's not necessary to make such an assumption in order to interpret the data from our experiment. Our experiment determines the actual tangential shear. The next slide (Figure 16) shows an artist's conception of the apparatus. The device is actually somewhat different than as shown here. It doesn't have rounded corners, and the latest configuration that we have run has the porous tube, through which the flow must leave, suspended from the bottom, outside the vortex chamber. Then, the interior of the device is similar to everyone else's vortex tube.

I'll just give you the principle of the experimont, and not go lliruugh it in detail. What we do is as follows. We know the angular momentum that we put in, and we measure the angular momentum that the fluid has as it leaves the system, by measuring the torque on the porous tube. We can calculate the shear losses on the end walls theoretically, or we can measure them by means of another device attached to the porous tube. By maliing an angular momentum balance at any radial station-using a cylindrical control surface-one can then deduce how much shear must have been transferred back to the side walls in order to have equilibrium. By varying the radial position of the (hypothetical) control surface, we can calculate what the distribution of shear has to be without assuming it is laminar or turbulent. 


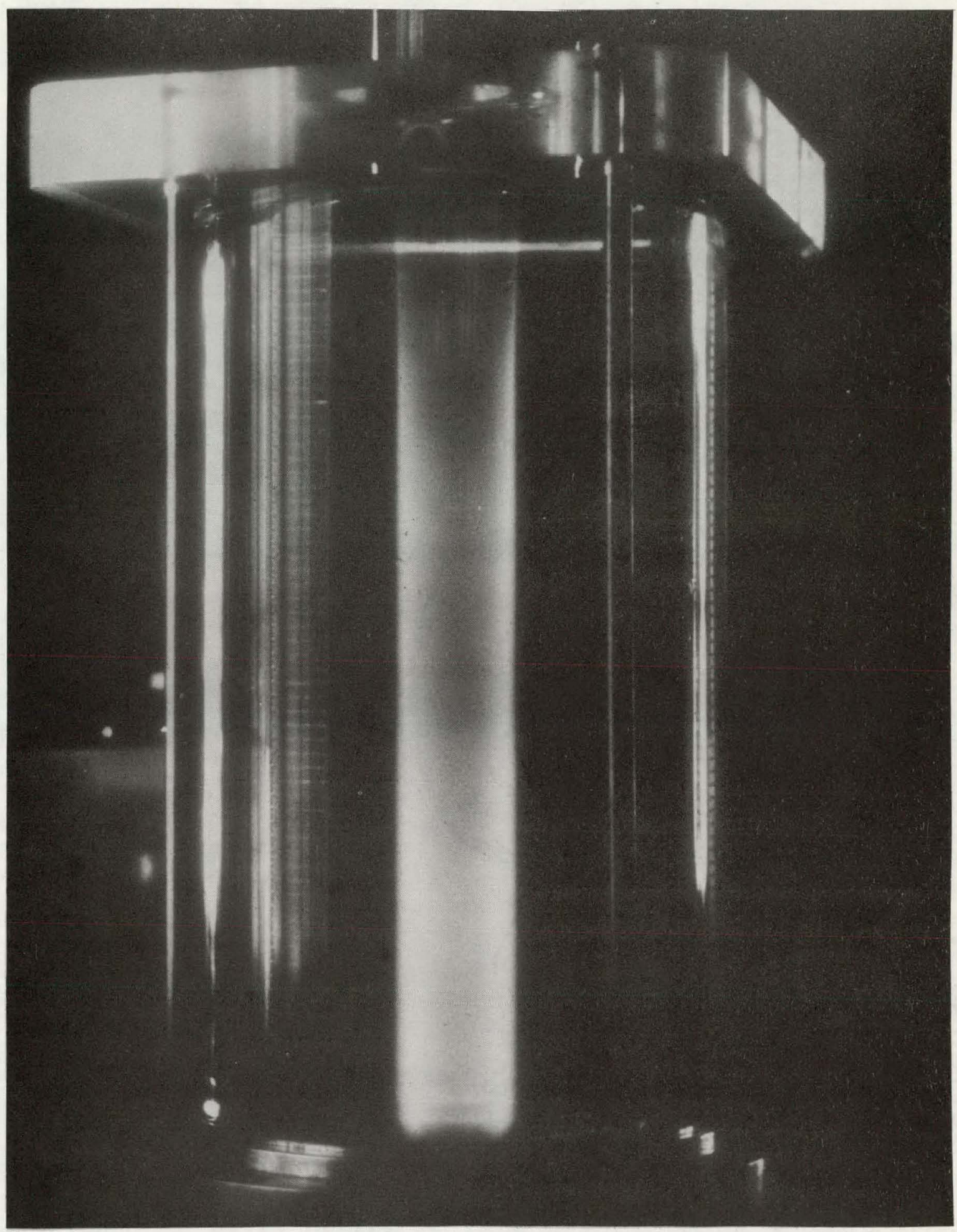

Figure 15 


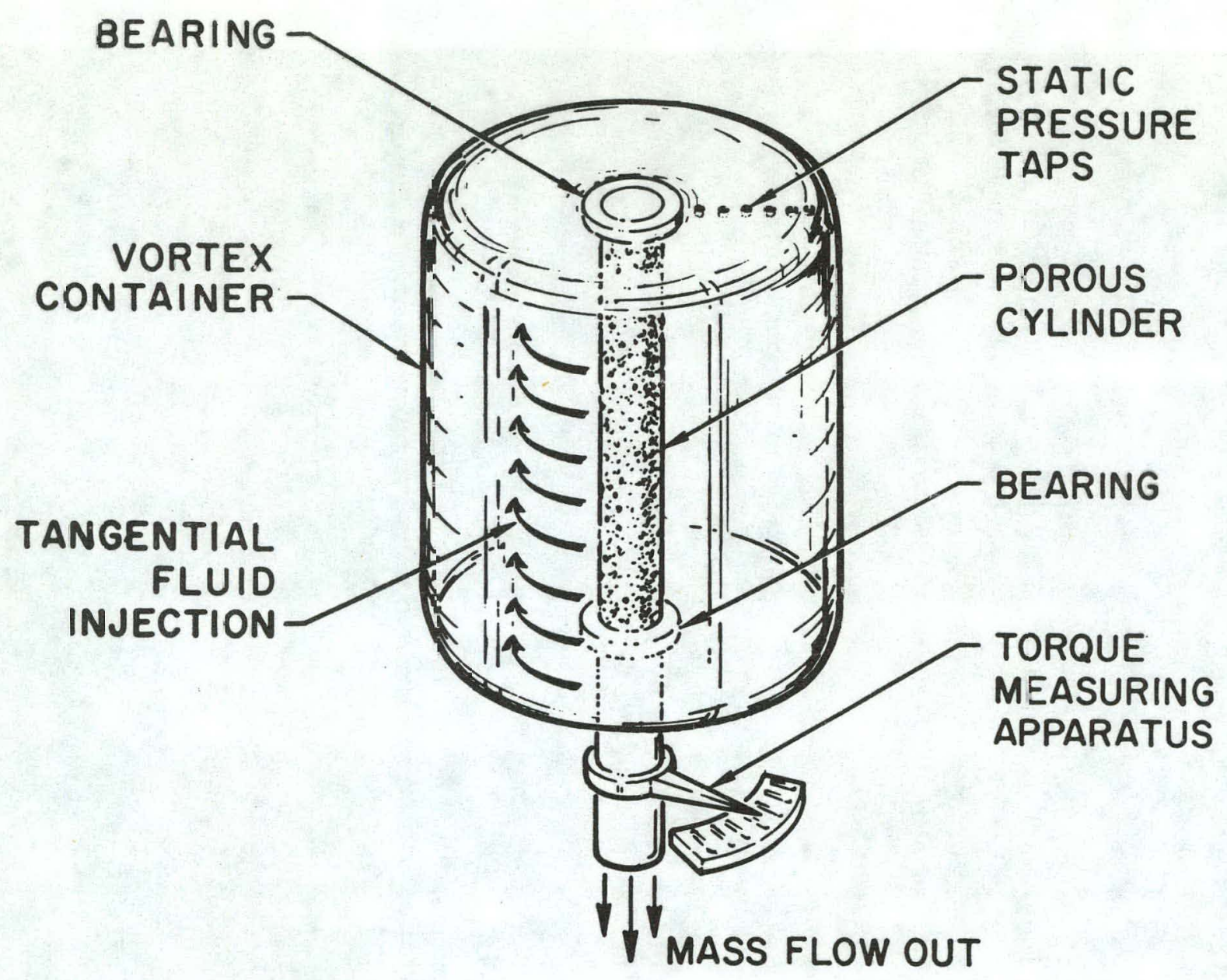

\section{VORTEX TURBULENCE EXPERIMENTAL APPARATUS}


Then, of course, we can always calculate what the laminar shear would be because it's simply $u[(d v / d r)-(v / r)]$. We know what the tangential velocity distribution is by our pressure measurements. We can then compare the measured shear with the calculated laminar shear based on the measured pressure distribution. This procedure is completely independent of our interaction theory that Steve Lewellen has described. We can compare the results of the two methods, and we'll show some of the results on our next slide (Figure 17).

Now you probably all remember John Keyes' work at Oak Ridge. We have plotted on this slide a ratio of actual radial Reynolds number based on the measured mass flow, divided by an effective radial Reynolds number, based on the interaction theory. The latter is the Reynolds number which is necessary to cause agreement between the theoretical and the measured velocity distribution, say at the edge of the exhaust hole." Keyes' curve looked like this. It caused great dismay to a lot of people, and many people said that they didn't believe it. His data were reduced on the basis of the two-dimensional analysis. There was no consideration given to three-dimensional flows which occur as a result of the boundary layers. As we've seen, most of that flow that gets diverted into the boundary layer gets out of the boundary layer again before it leaves the system and, as a matter of fact, on the average, only a fraction of its angular momentum is lost in the boundary layer. Consequently, it is still possible to support a fairly strong velocity distribution. It turns out that if you make the full three-dimensional calculation on the basis of our interaction theory and some other experiments that we've made, you get the points shown on the slide. There are two points at each tangential Reynolds number. One corresponds to a value of zeta of one and the other to zeta of zero. As you can see in most of the cases, it doesn't make a great deal of difference whether zeta is zero or one. This is primarily because we use the value of gamma at the edge of the exit hole as the criteria from which to calculate $\operatorname{Re}_{\mathrm{r}}^{*}$; and gamma, as we've seen, is not very sensitive to the value of zeta. It's very hard to calculate, in this way, what the actual value of zeta is. If we had a measurement of the mass flow in the boundary layer, we could make a much better estimate of the value of zeta, because this quantity is much more sensitive to zeta than gamma is.

Well, the important thing here is that these are the results from our interaction theory for the various experimental apparatuses that we have in our laboratory, using very low and intermediate Reynolds numbers. These very low Reynolds number points were obtained using helium. The straight line is, of course, an extrapolation of Keyes' curve. We find that the data rounds off something like this. These points, with the solid dots in the 


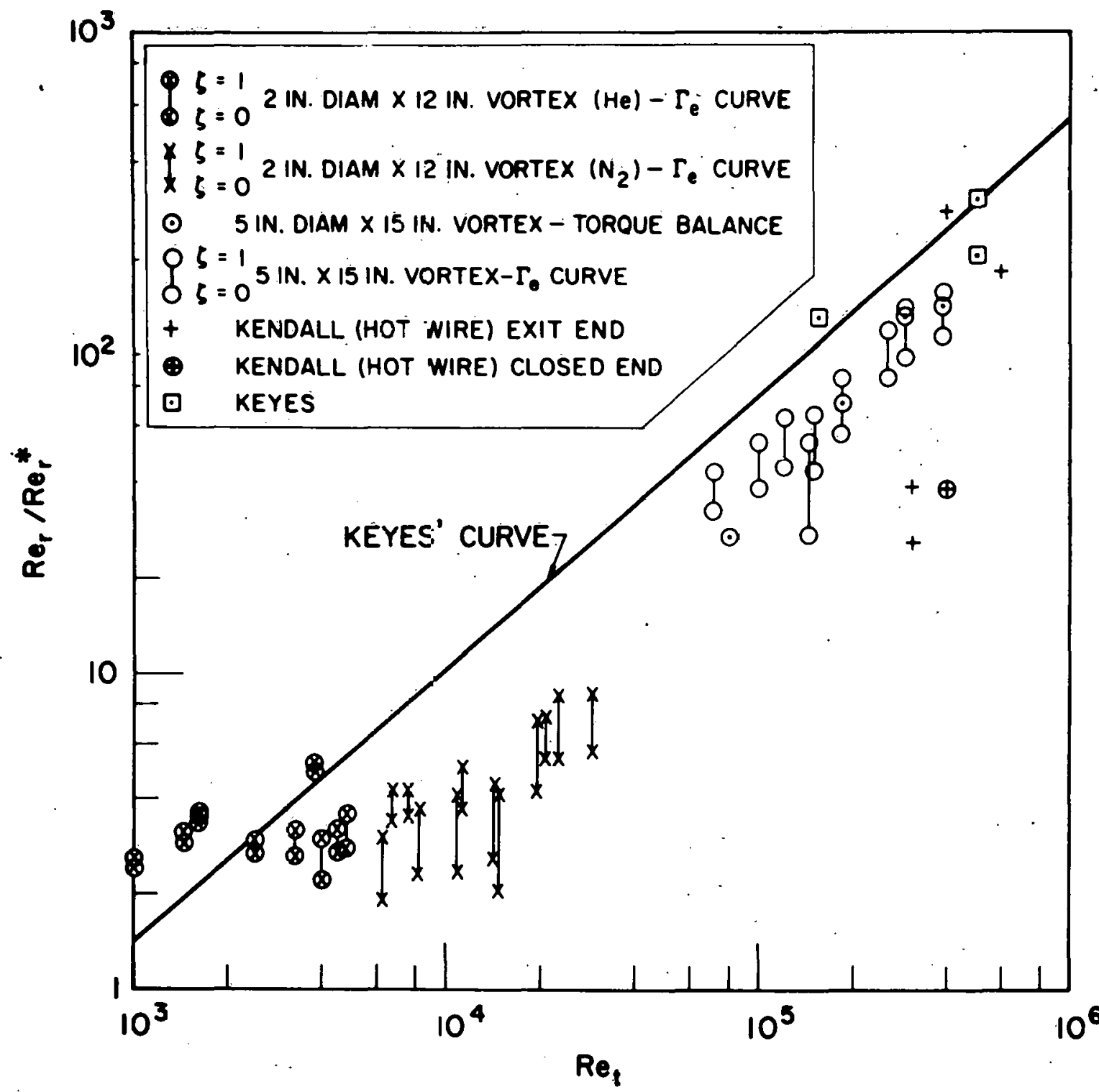

Figure 17 
center, are the independent corroborations from our turbulence experiment, which do not depend upon our interaction theory at all. We've also plotted some other people's points here. Kendall at JPL has measured some turbulence levels with a hot-wire anemometer in a vortex tube, and these points are included in this chart as well. Three of Keyes' actual data points are also shown. The interesting thing is that the new points are calculated with full three-dimensional effects included, and we still see very high ratios of the Reynolds numbers. Whether or not you can ever get much lower than this in a jet-driven vortex tube is a moot point. The only time we've ever gotten measurements that are considerably below these lines was in the matrix geometry, where we did reach, in this region, something like an order of magnitude below what this curve shows.

But, I'm not presenting this to be unduly pessimistic. I think that, as other people's work has shown, for example the measurements of Pivirotto at JPL, and the work at UAC, that if the flow is turbulent, and I believe it is-I don't believe that the turbulence is isotropic or homogeneous in any sense-it's definitely oriented in the axial direction, because, again, there's no resistance to axial motion in these devices, but a very strong resistance to radial motion. I think this is what enables us to see these very steep gradients that people have shown in the slides before.

Question (Bill Foley, UAC): One of the characteristics of your slide is, I think, it looks like the turbulence level stays constant out to a Reynolds number approaching $5 \times 10^{5}$ then all of a sudden it curves up. It remains horizontal over one and a half orders of magnitude in tangential Reynolds number. This is where we have made most of our measurements concerning the turbulence level, so there may not be an inconsistency between our data and yours.

Answer: No, I don't think there is any inconsistency, but I would like to insert a word of caution here; and that is as you extrapolate up in Reynolds number, if this trend is indeed a fact, then you can anticipate runniny into some additional difficulties. Whether it will mean anything with regard to the pressure or concentration diffusion or in excessive loss is another point, because I really don't know how this result is translated in terms of diffusion phenomena. Somehow or other, this experiment tells us that this much shear must be transmitted back to the side walls. How it is done, with fluctuations oriented in what direction, $I^{\prime} \mathrm{m}$ in no position to say. I can only present the results of our work. The important thing is that these results were obtained for a very wide variety of the parameter $A$, so that we were not in just one regime where the end-wall effect is not important. By the way, that's the reason Keyes' curve isn't so bad. If you evaluate his value 
of A you find that it was of the order of 1 , and in that regime the effect of the end=wall boundary layer on the gamma distribution is not that strong. His results are a little bit high compared to the full three-dimensional case, but they're not that far off.

This is the point in my discussion where I will ask you to abide with what Dr. Cooper said in his letter and open your minds to some speculation on my part as to what all this might mean in terms of propulsion systems. Actually, we have given very little consideration to actual propulsion concepts in our work. Because we have such a small manpower effort, we felt we should concentrate on those areas where we felt most competent. But, as you are aware, we have been experimenting with a device called the matrix, which was originally conceived in order to get around some of the difficulties associated with the single vortex tube and diffusion concept, specifically, the fact that the mass flow per unit length of vortex is limited by the diffusion phenomena. I think this is what has frightened most people away from this idea; that is, if you relied only on pressure diffusion to separate a heavy and light species, you might have to limit yourself in mass flow per unit length of vortex. The matrix was an idea to get around that, that is, to provide many linear feet of vortices through which you could have diffusion. I think it's clear at the present time from the results that wc've just seen from JPL, and from other people's results, that the simple elementary scheme, as proposed by Kerrebrock and Meghreblian in 1957, is not going to be able to do the job by itself. You just have too many spurious effects which you have to pay some attention to. Now, how can you possibly get around it? We have seen various ways of influencing secondary flows, influencing them so as to make these flows do what we want them to do. Let's see what might be possible, on the basis of the idea of fission-fragment heating, that is, still relying on molecular collisions to heat the gas rather than radiation. It's true that in any system that requires pressure diffusion as the primary separation mechanism, you are going to be limited to a mass flow restriction per unit length of vortex. Now, the molecular interaction heating does require a more intimate contact of propellant and fuel. But, let's look again at the matrix device and see if we can conceive of a useful system. For those of you who don't know what a matrix is, I think $I$ have a picture on the next slide of an experimental device that we've run in the laboratory (Figure 18). This is a 25-cell vortex matrix. The idea is that you attempt to minimize, to the best of your ability, the wall area per vortex by generating a large number of vortices in a single container. This is a working drawing of an actual device that we've built in our lab. If you inject tluid tangentially through little tubes that are placed at the corners of the adjacent vortices, it's possible to generate very strong vortices, very 


\section{SKETCH OF VORTEX MATRIX}

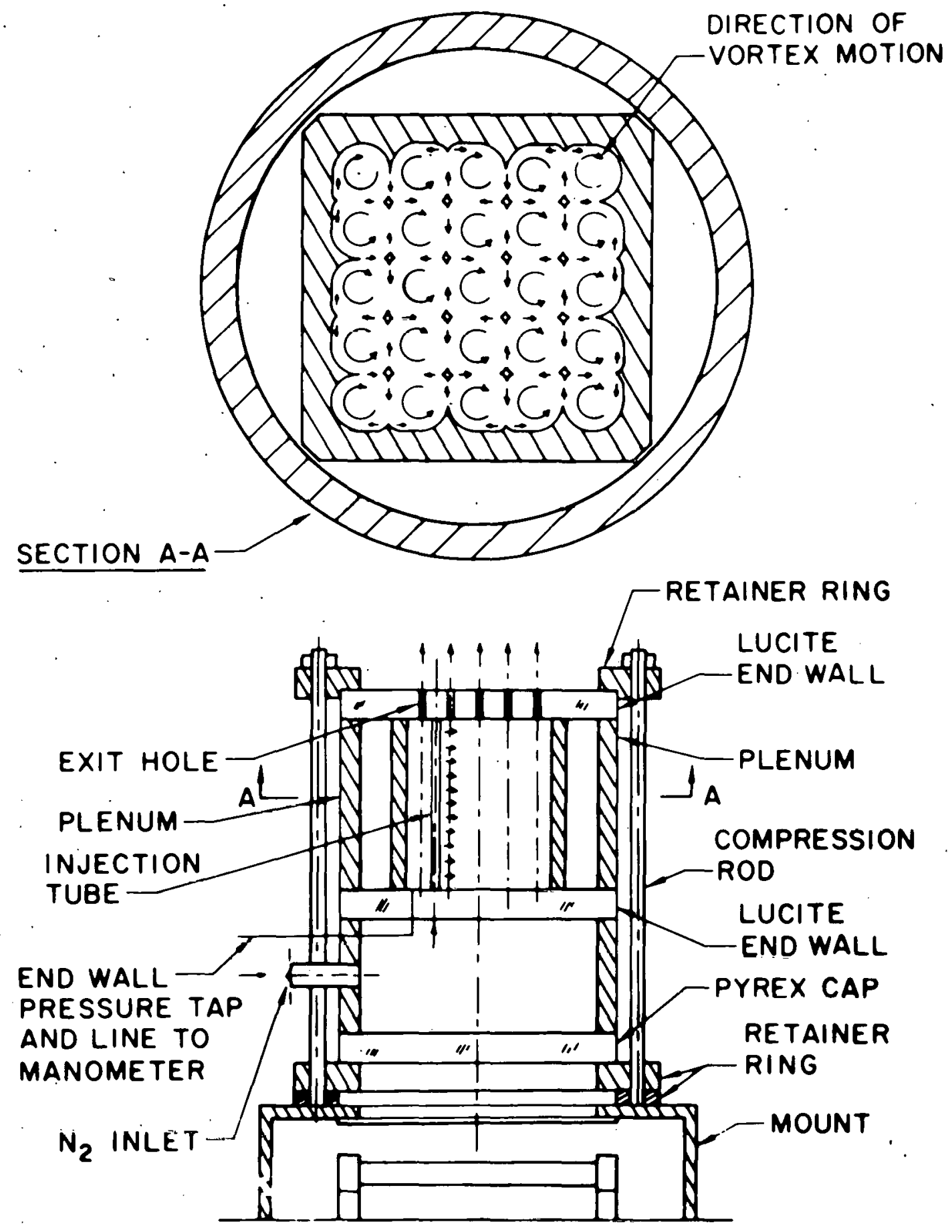

Figure 18 
stable vortices, and as many as you like. There's no limitation. This one generates 25. We have verified that in this device; the turbulence levels in the center cell are lower than those in a comparable single vortex tube built to the same geometry.

Question (Hatch): What were the measured turbulence levels in the matrix?

Answer: Well, as I indicated, they were about an order of magnitude below the general values that I showed on the last slide, and this was the only geometry that we can say that about.

In each of these individual vortex cells, you have exactly the same kind of secondary flow pattern as we have seen in the single vortex. Indeed, you have the same problems of secondary flow, but what isn't clear necessarily, is what is the degree of communication from one cell to the next? Actually, what we've verified is that the interior cells require a much lower mass flow to generate the same strength vortex as the side or corner cells. In fact, the way these things communicate is that the mass flow distributes itself such that the pressures at the boundaries of eâch cell are equal. So there is a possibility of communication. That means that the mass flow has to distribute itself so that in the corner cells and the side cells you have to have higher mass flow to overcome the larger dissipation, than you do in the interior cells, where the only viscous loss is as the result of the small tubes. Since the adjacent vortices roll on one another, there is no shear at these boundaries.

Originally, as I indicated, the matrix was devised for the purpose of generating a large number of vortices because that's what it appeared was needed for a pressure diffusion device. But there are other things that one might do with it. For instance, suppose one was to close off the exhaust in one of these vortices, and just let the fluid in it roll like a solid body being driven by its neighbors. You would essentially, then, create a region of relatively high pressure, locally, in the device. The tangential velocities in the closed cell would be on the average less than in the adjoining cells, and it might be possible, perhaps, to contain or confine a particular fluid in there, if the secondary flows were self-contained. This is an idea we've been playing with lately. We've been examining what the interaction of one cell is with the next, and seeing how much mixing there is. If you could have a number of cells closed off, and if you could produce a closed secondary flow pattern, you could, conceivably, place a heavy species in the closed cells and transfer energy into adjoining cells (if the pressure of the system were suitable) by fission fragment absorption. You'll notice that a lot of the objections to direct fission heating that Henry Stumpf brought up, that is, with regard to energy deposition in the structure, are eliminated in 
this device. The characteristic dimension for fission fragment energy absorption in the solids is no longer the diameter of one cell, but rather the diameter of the overall device, which can be many times the diameter of one cell. So the possibility exists of using secondary flows to create closed streamlines and still utilize pressure diffusion in the sense that the pressure diffusion will always tend to make the heavy species move to the regions: of higher pressure, which are the closed regions. It is conceivable that one might have alternating rows, or every third or fourth cell, full of fissionable gas, still have molecular collision heating, and avoid the mass flow limitations inherent in the diffusion process because there's no reason why the mass flow through the other cells could not be considerably higher than the diffusion-limited value. Actually, in the experiments we've run, which are shown at the end of the movie that we will see in a moment, we have allowed a very small amount of mass flow to go through the center cell in a 9-cell matrix geometry. This is being operated with water using dye (fluorescein dye, again); and we have found in that particular device (which was actually the earliest matrix device we ever built), that we have quite a bit of wall area in having only 9 cells. There's quite a bit of viscous drag, so in this system we were forced to have some flow through the center cell in order to generate vortices of sufficient strength to produce the secondary flow : pattern that we want. It may be that this is a favorable situation, or it may be that in an actual design, you could eliminate the secondary flow altogether.

Now, I want to describe very briefly what you're going to see on the movie. The movie is in three parts. The first part involves the end-wall blowing. The film shows the effects of tangential injection in the end walls with increasing fraction of mass injected through the end wall as a function of time. You will see the secondary flows gradually disappear and finally reverse in direction. The second part of the movie is concerned with the highly-curved end walls, and shows the formation of the stationary cylinder that we saw on the slide earlier. The third part of the movie is concerned with the matrix experiment and ... well, I'll narrate it as we go along.

Narration of movie followed.

I would like to conclude with the statement that we feel there may still be quite a few experiments that are worth doing regarding vortex flow fields and, obviously, it is going to be important with regard to many concepts. I wouldn't want to close my line on any particular concept on the basis of the information that is available to us at the present time. Thank you.

Question (Colgate): What would you guess is the ratio of the viscous friction of the vortices in the vortex matrix to that in an ordinary vortex tube? 
Answer: That, of course, will be a function of how many cells you would have in a given device. Since the viscous dissipation is proportional to the wall area, you would expect it to be reduced in the ratio of wall area per vortex. In a single vortex the wall area per unit length of vortex is just the circumference of the tubes. In a matrix device, if you go to an infinite number of cells, essentially the wall area per vortex is reduced to the circumference of one of the small injection tubes, so that is, in essence, the type of advantage you can possibly hope to achieve. This can be on the order of 100 perhaps. The injection tubes are cooled by the flow coming through them.

Remark (Foley): There are a couple of comments, Marty, that are pertinent. We have been, of course, concerned with the uniformity of $v$, the circumferential velocity, in the axial direction. Of course, we've assumed the same thing in our theoretical work, and we've looked to see if we could find anything experimentally with our particle traces of any axial velocity gradient; and within the scatter of our data, we certainly cannot see it. Bruce is smiling because he says there is a fair amount of scatter in the data; but even in the axial flow case where there is a rather substantial amount of flow going axially, we do not see a circulation gradient with $\mathrm{z}$ position, which is, I think, kind of interesting. We have also solved the three-dimensional flow problem for compressibility now, which is somewhat interesting in that if you go supersonic in the flow, the basic streamline patterns don't change from those that Steve has been showing here. They're very, very similar even though you go supersonic in the flow.

Answer: We've made estimates of that, too, by considering the isothermal vortex case, which is, for this type of flow about the worst possible type of situation, that is where compressibility will have the largest influence on the velocity distribution. For Mach numbers of the order of a quarter on the outside, which is characteristic of the type of thing that we've been dealing with, the corrections were only a few percent, is that right Steve?

Steve: Yes, we found a 5\% correction, and it was going supersonic inside the flow.

Remark (Foley): Here's another thing. We got some heat transfer results across the end-wall flow, under such circumstances that we wernn't so sure we believed from our integral analysis. Olof Anderson has been doing the work, and he thinks he can solve the boundary layer point by point, and actually calculate the profiles. This should be interesting, and we'll look at heat transfer in some detail to see what the temperature distributions in the end wall are. This may give a lot of input to the theory of the Ranque-Hilsch tube. It may change some of the thoughts on what goes on there. 
We've tried a similar thing with this curved end wall that you show, and of course, you still get cells. I think Bruce Johnson, over there, who has made some calculations on how much skin friction it takes to hold that flow on the end wall boundary layer, will have some input because you really have to add some additional friction on the end wall to prevent out flow.

Remark (Bruce Johnson): It's on the order of about a thousand times as much as the naturally occurring skin friction coefficient at the inside radius. We tried putting probes in, and the probe stuff is pretty crude, but it works. Remark (Foley): We just can't get enough additional surface by curving the end wall.

Answer (Rosenzweig): Well, of course, it depends on what regime you're in. Remark (Bruce Johnson): That's true. 


\title{
MHD NUCLEAR PROPULSION SCHEMES USING MAGNETOHYDRODYNAMIC CONVERSION TECHNIQUES 9
}

\author{
Richard J. Rosa \\ and \\ Thomas R. Brogan \\ Avco-Everett Research Laboratory \\ Everett, Massachusetts
}

I hope this discussion of MHD energy conversion will not seem out of place in a meeting on advanced reactor concepts. The fact is that to be useful in space, the MHD generator needs high temperatures of the sort that can only be produced by advanced types of reactors. I hope that today I can make the case, or at least establish a reasonable possibility, that to be useful in space the advanced reactor concepts in turn need the MHD generator.

Although I think many of you know how an MHD generator works, let me review the basic principles briefly. Figure 1 illustrates the principle and compares it to that of a turbine generator. The basic principles are the same in the two cases. These are that expansion of a gas produces motion of a conductor and the motion of a conductor through a magnetic field generates an electromotive force. In the case of the MHD generator, the gas is itself an electrical conductor and is moved through the magnetic field. Observe that the MHD generator performs the function of both a turbine and a conventional generator. The function that it performs best is that of the turbine. In fact, it is really more useful to think of it as a high temperature turbine rather than as an electrical generator. In practice, an MHD generator would resemble a rocket nozzle with a field coil wrapped around it. It would have no hot, highly stressed, moving parts; no close tolerances; and the only solid parts, namely the walls, are readily accessible for external cooling, as are the walls of a conventional rocket. As a result, it can handle temperatures and pressures like a rocket nozzle and can stand erosive and corrosive atmospheres which would completely destroy any other type of energy conversion device in a very short time. Also as we will see 


\section{TURBO GENERATOR

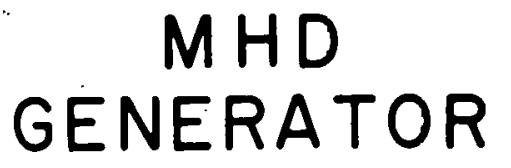

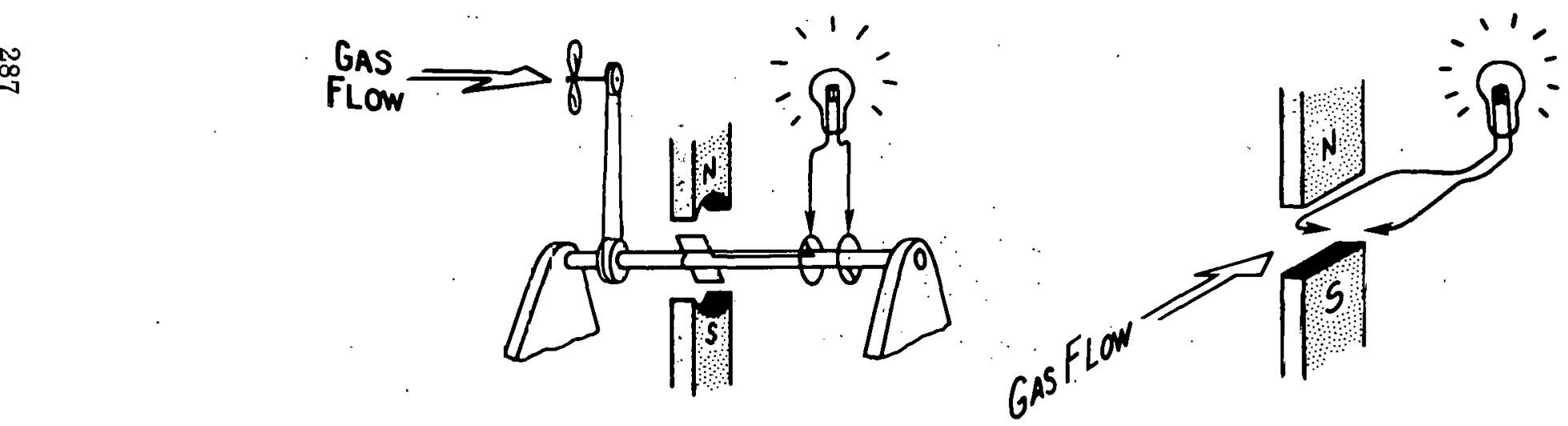


later, it can produce very large amounts of power per unit volume and per unit weight.

The primary limitation on how and where one uses an MHD generator is a low temperature limit. This is because at the present time the only way that we are sure is practical for rendering a gas conducting is introducing into it a few tenths of a percent of an alkali metal seed material and then heating it. Results obtained in combustion products, but typical for all gases, are shown on Figure 2. The points are experimental; the solid line is theoretical. Observe that the conductivity is a very steep function of temperature. In practice we find that below about $2000^{\circ} \mathrm{K}$, the exact value depending upon just what gas is used, the conductivity becomes too 1ow to be useful. Observe that a few hundred degrees change in temperature can bring about an order of magnitude change in conductivity. This in turn can bring about order of magnitude improvement in the performance of an MHD converter.

This illustrates why you should not be misled by statements in the literature (or photographs of our devices) into assuming that MHD generators are intrinsically very large and heavy. In our struggle to fit MHD generators into existing technology, we do indeed make them as large and as heavy as the traffic will bear. But the technology which you people are discussing here can move us a very long way up this exponential curve. For example at just about $4000^{\circ} \mathrm{K}$ in hydrogen an MHD generator containing. one cubic meter of volume could generate as much electric power as the sum total of all of the power plants in this country, i.e., about 200,000 megawatts.

Now given a turbine which has no temperature or power limit and can handle any atmosphere, the next question is, how exactly can it be usefully employed in space? The answer (as is usual for questions of this nature) is that there are a virtual infinity of possibilitics. The real problem of course is trying to decide which, if any, of these possibilities are really worth pursuing. I obviously should not take the time here to discuss them all. So I will discuss rather briefly a few schemes which, I hope, illustrate the range of possibilities.

In devising these propulsion schemes, one of the ground rules has been that it should not be necessary to retain fuel within the reactor. Desirable perhaps, but NOT necessary. Figure 3 illustrates a system in which it is obviously not necessary. What we have here is essentially a conventional closed thermodynamic power cycle which is using the propellant as its heat sink. In effect what happens is that heat is trunslerred between the reactor and the propellant by means of solid surfaces up to the maximum temperature 
ELECTRICAL CONDUCTIVITY OF SEEDED JP4 OXYGEN FLAMES

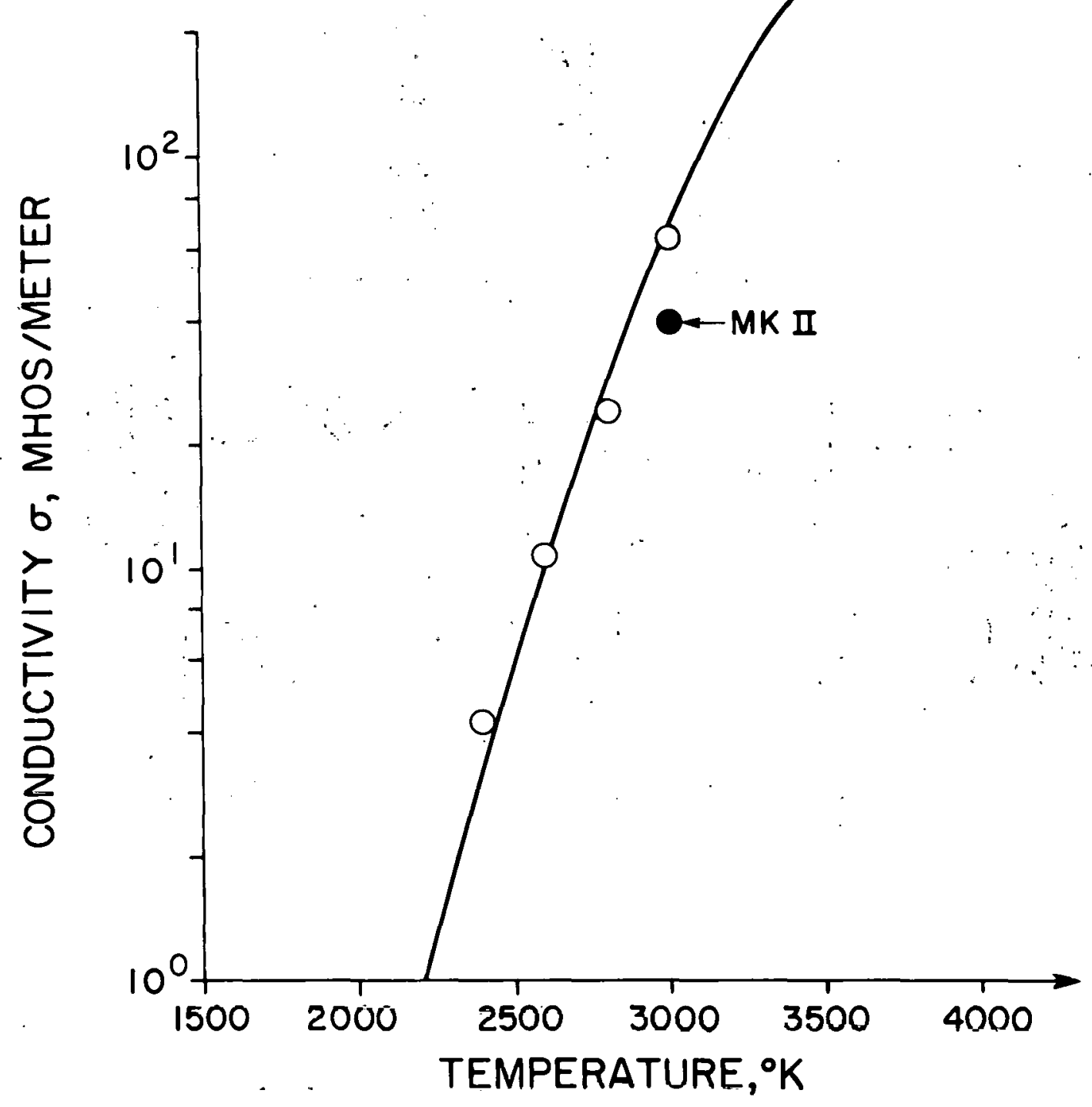

Figure 2 
ENERGY EXCHANGE BY MHD FROM A GAS CORE REACTOR TO PROPELLANT

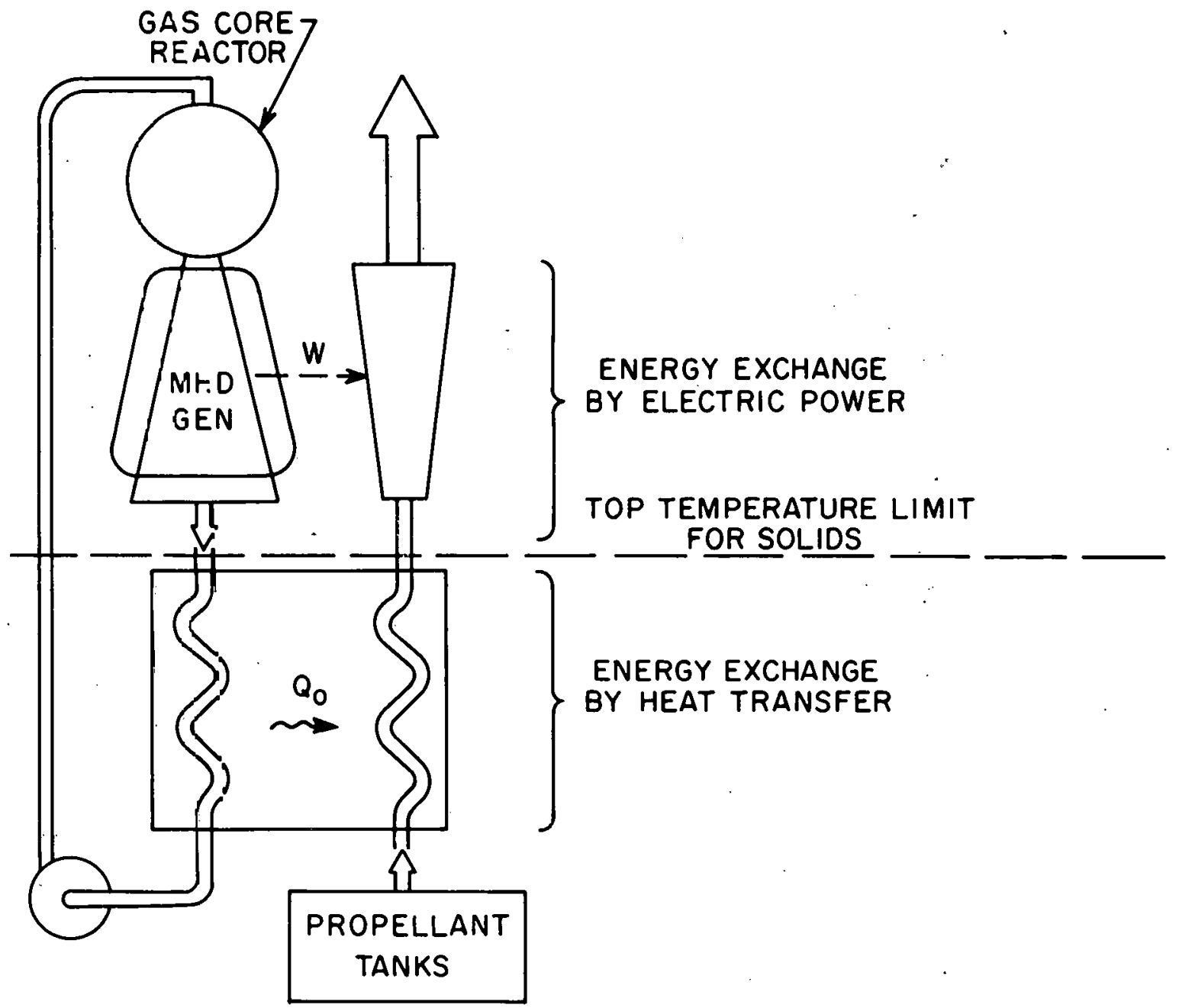

Figure 3 
that solids can be used, and above that it is transferred by means of the MHD generator and the accelerator. That is, energy is transferred by convection and conduction up to perhaps $2000^{\circ} \mathrm{K}$; and above that, energy and also momentum is transferred electrically. Compare this with concepts such as the glow plug and the coaxial jet in which radiation is used to transfer energy at temperatures above the solids limit. In this respect, I think the MHD scheme has two things to its advantage. First of all, without trying very hard one can make the encrgy delivered at the electrode wall of an MHD generator at a given temperature be orders of magnitude greater than the energy per unit area delivered by even blackbody radiation. The second advantage of the MHID scheme is that wall materials and structures which are transparent to DC electric power arc a great deal easier to find than materials which are transparent to optical electromagnetic frequencies.

Figure 4 is a simplification of the scheme shown in Figure 3. Here the same gas is used as working fluid in the reactor and MHD generator and as the propellant. As a result, the compressor and the heat exchanger are eliminated. Here we depend upon the fact that at a generator exit temperature of 2000 to $2500^{\circ} \mathrm{K}$ practically all of the fuel will be condensed and can be recovered by a gas-liquid separation technique without cooling the gas any further.

Figure 5 illustrates the point that use of an MHD converter can do more than simply provide a way around the fuel containment problem. Shown here is the open cycle propulsion scheme illustrated in Figure 4, except that the power output of the generator is not put back into the propellant but rather used in an extcrnal air accelerating device. Obviously this is not a propulsion system for deep space. What we have here is the nuclear MHD analog of a turbo-rocket. The propulsive efficiency of such an arrangement is much higher than that of a rocket alone, assuming you are in the appropriate range of flight velocity through the atmosphere. In the case of a nuclear MHD turbo-rocket this appropriate velocity range could be from zero right up to the satellite velocity. Moreover, an electric ram jet might turn out to be a much easier device to get good performance out of than a comparable chemically fueled ram jet when operating in the hypersonic velocity range. There are a number of reasons for this, but what they all boil down to is just that electricity is a more highly organized or available form of energy than is chemical energy.

Figure 6 shows the kind of speoific impulse one might expect to get from the type of propulsion systems shown in Figures 4 and 5. This is shown as a function of the pressure ratio across the generator and the top temperature produced by the reactor. "Unaugmented rocket" corresponds to 


\section{OPEN CYCLE - GAS CORE REACTOR}

옹

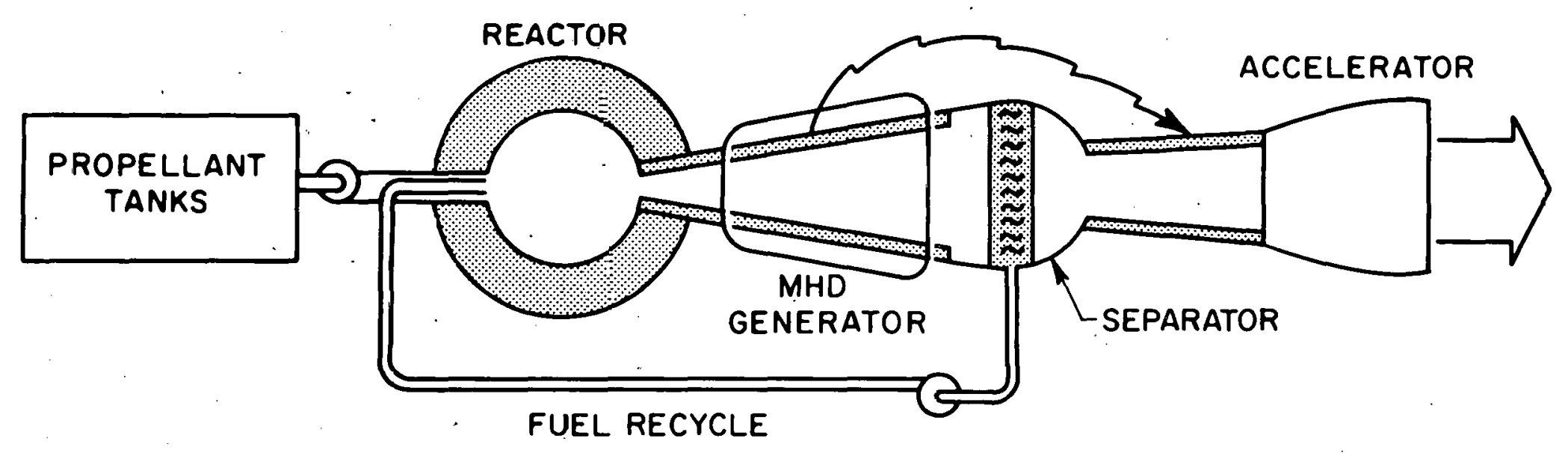

Figure 4 
OPEN NUCLEAR MHD PROPULSION CYCLE

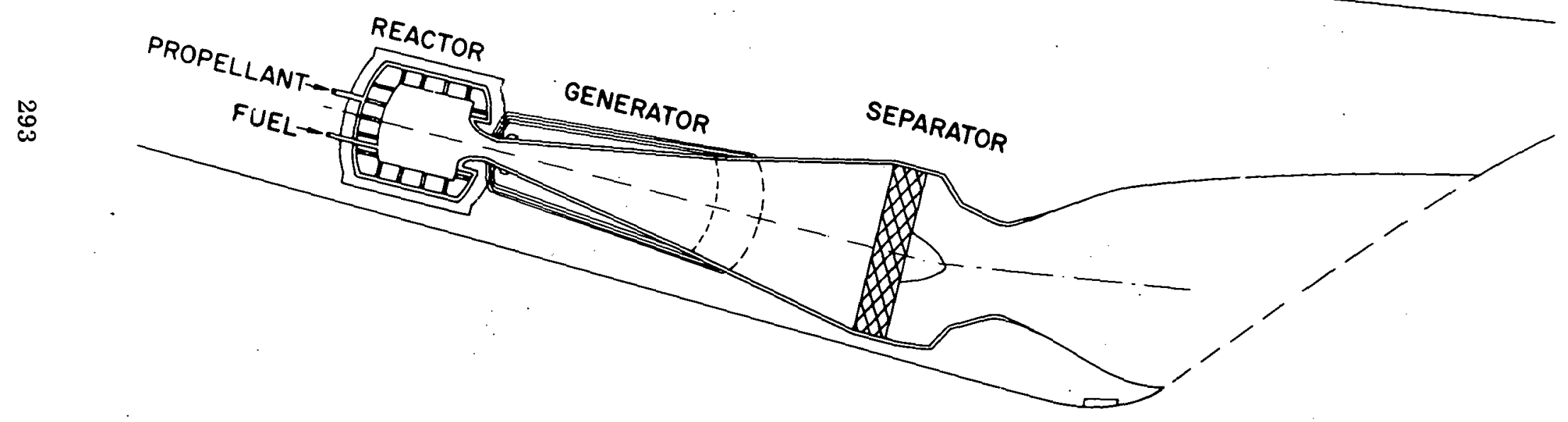

ELECTRIC RAM JET

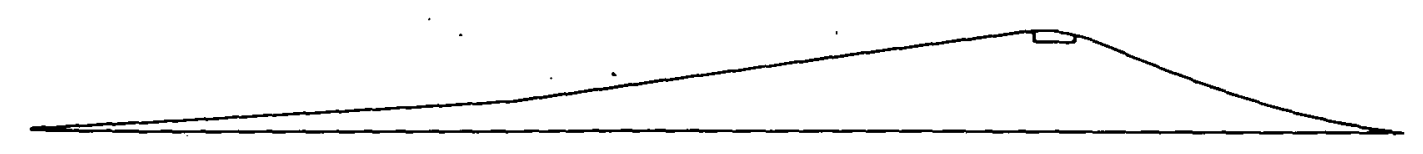

Figure 5 


\section{AVERAGE SPECIFIC IMPULSE-OPEN CYCLE}

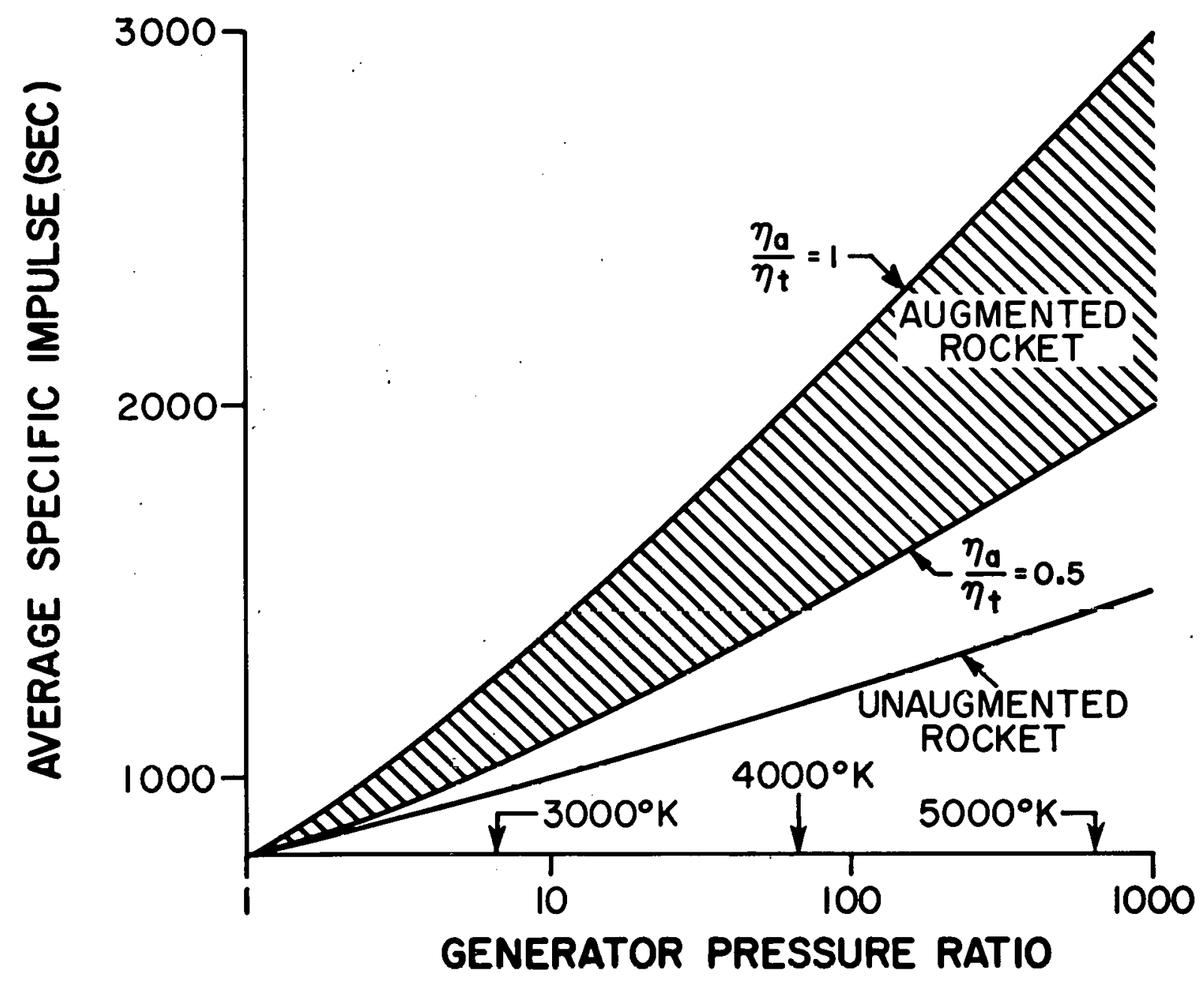

Figure 6 
the system shown on Figure 4. The "augmented rocket" corresponds to the system shown on Figure 5. For the latter, the specific impulse shown is a weighted average over the flight velocity from zero up to the satellite velocity, and a range of values is shown corresponding to a range of assumptions about the efficiency of the electric air accelerator, or electric ram jet. $\eta_{\mathrm{a}}=\eta_{\mathrm{t}}$ corresponds to an accclerator efficiency equal to the thermal efficiency of a rocket nozzle, that is about $70 \%$; then $\eta_{a}=0.5 \eta_{t}$ corresponds to an efficiency of about $35 \%$. The closed cycle shown in Figure 3 may also be operated either as a pure rocket or as an air augmented rocket, and the performance that would result is shown in Figure 7 . The closed cycle would be a good deal heavier than the open one. However, I believe that in sizes corresponding to a thrust level of 100 tons and up, both systems could be made to have a thrust to weight ratio substantially in excess of one.

In order to get a specific impulse greater than 2000 to 3000 seconds in space it is necessary to consider a system which uses a radiator. This is true whether one is considering a nuclear-MHD scheme or a nuclear reactor working alone. As is well known, a key, if not the key, to making a system of this type with a reasonable thrust to weight ratio is attaining a high heat rejection temperature. In addition, gains of up to a factor of five can be made simply by making the cycle more efficient. Presently conceived space electric power supplies have a temperature limit set by their reactor and conversion device. By using a gas core reactor and an MHD generator there would no longer be a limit on top cycle temperature. Then the compressor and the radiator temperature could rise accordingly to what is now the top cycle temperature. Eventually it should be possible to also make an MHD compressor, and then only the radiator would be a solids limited device. However, even with "solids limited compressors" we can do orders of magnitudes better than presently conceived electric power systems as is shown on Figure 8. Here power pex unit radiator area is displayed vs top cycle temperature for a variety of radiator and generator temperatures. It shows that we ought to be able to do at least a hundred times better than SNAP 8 in terms of power per unit radiator area. Assuming that the weight of all cycle components scales by the same factor, and there is reasonable grounds for supposing that it might, the result would be a propulsion system for interplanetary flight which would be very hard to heat.

Now except for paper work, the amount of actual work that we at Avco have done on any of these propulsion schemes is exactly zero. However, we do have a very vigorous program going to develop these generators for commercial use. The funding level of this program is comparable to, and in fact possibly somewhat in excess of, the total amount being spent on gas 


\section{EFFECTIVE SPECIFIC IMPULSE, PROPELLANT REHEAT CYCLE, CYCLE GAS HYDROGEN}

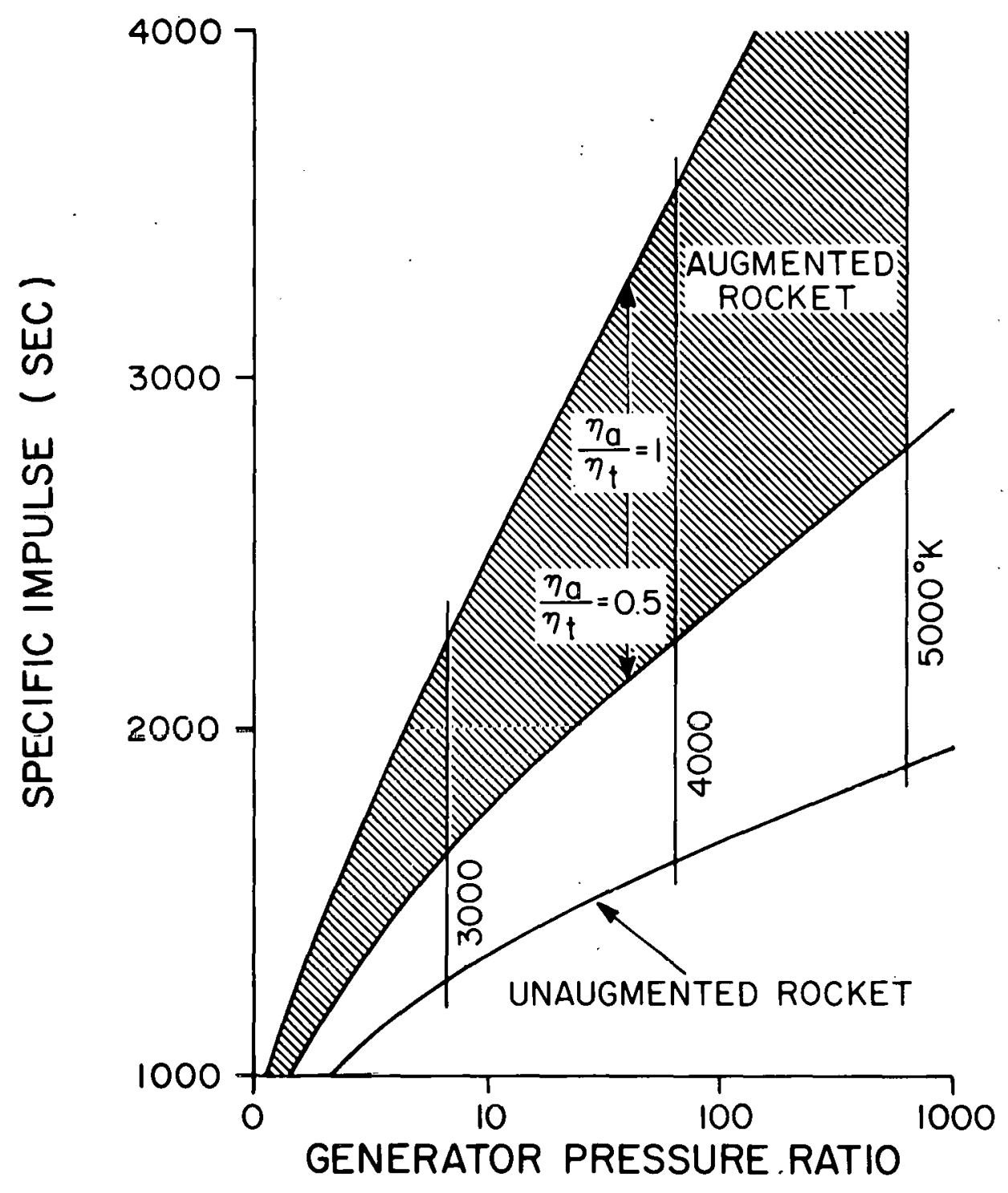

Figure 7 


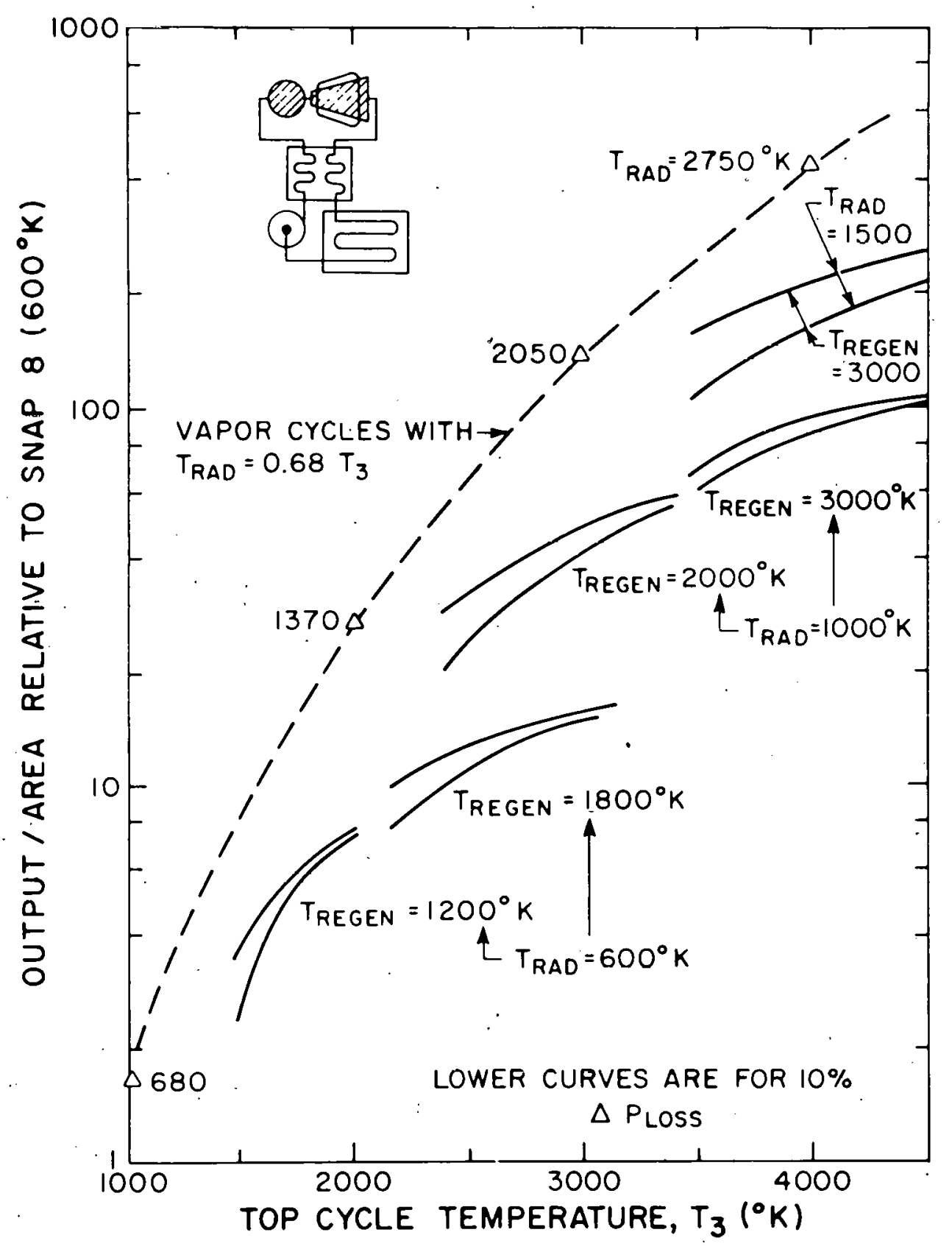

Figure 8 
core reactors. Although the work is directed primarily toward chemically fueled power plants, it is developing the art of MHD energy conversion as a whole and a large amount of this work is applicable to nuclear space applications. Hence I would like to have Tom Brogan, who runs this program, tell you something about its present status.

\section{MHD GENERATOR DEVELOPMENT - STATE OF THE ART}

Except for some early speculation, the real development of the MHD generator began about in 1958. Since then there have been quite a few programs going on in our Laboratory to take care of the various objectives of MHD development. In the first place there is a program sponsored by the Avco Corporation and a group of 11 electric utility companies headed by the American Electric Power Service Corporation to evaluate the MHD generator for commercial purposes. Here its ability to handle high temperatures and high temperature working fluids can lead to thermodynamic cycles of high efficiency. Secondly there are the programs sponsored by ARPA to develop the combustion MHD generator for delivering high power for relatively short periods of time. Also there are other Air Force applications which I will talk about.

Since the MHD generator is an expansion engine just like a turbine, we would like to be able to predict and calculate the performance of the MHD generator much as we do in the case of a conventional turbine. In order to investigate this, some years ago we built the Mark II MHD generator, which is shown in Figure 8. Tho MID cliaumel is in the foreground. It has an active length of 5 feet and an average cross section of about threetenths of a squarc foot. This cross section is required in order that the MHD effects shall be dominant over the effects of wall friction and heat transfer. The channel is pushed into the gap in the magnet in the background. This magnet is driven by a battery bank and provides a field of 33,000. gauss. A combustion chamber bolted on to the flangc in the foreground provides seeded combustion gases which drive the MHD generator. The combustion heat source is very convenient, of course, in that the combustion chamber wall can be at a temperature much lower than that of the working fluid. At the inlet of the channel a nozzle expands the gas to supersonic speed. Mass flows up to about 4 kilograms per second can be handled.

We have conducted a long series of experiments with the Mark $\Pi$ generator, and a kind of synopsis of the work is shown in Figure 10, which plots the total power output as a function of the total current for various mass flows at constant magnetic field strength. We have plotted both 


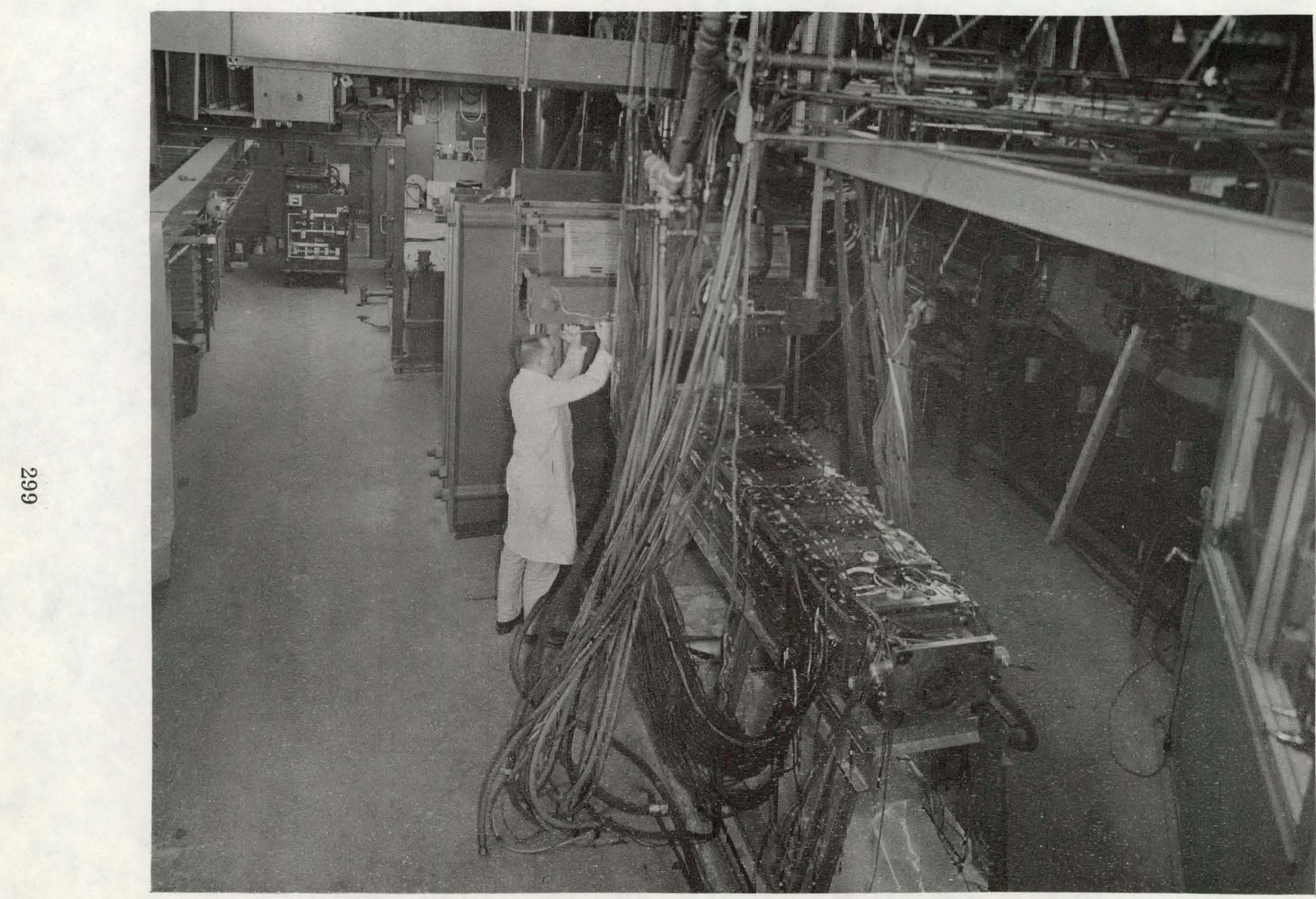

Figure 9. Mark II 1.5 Megawatt Experimental MHD Power Generator 


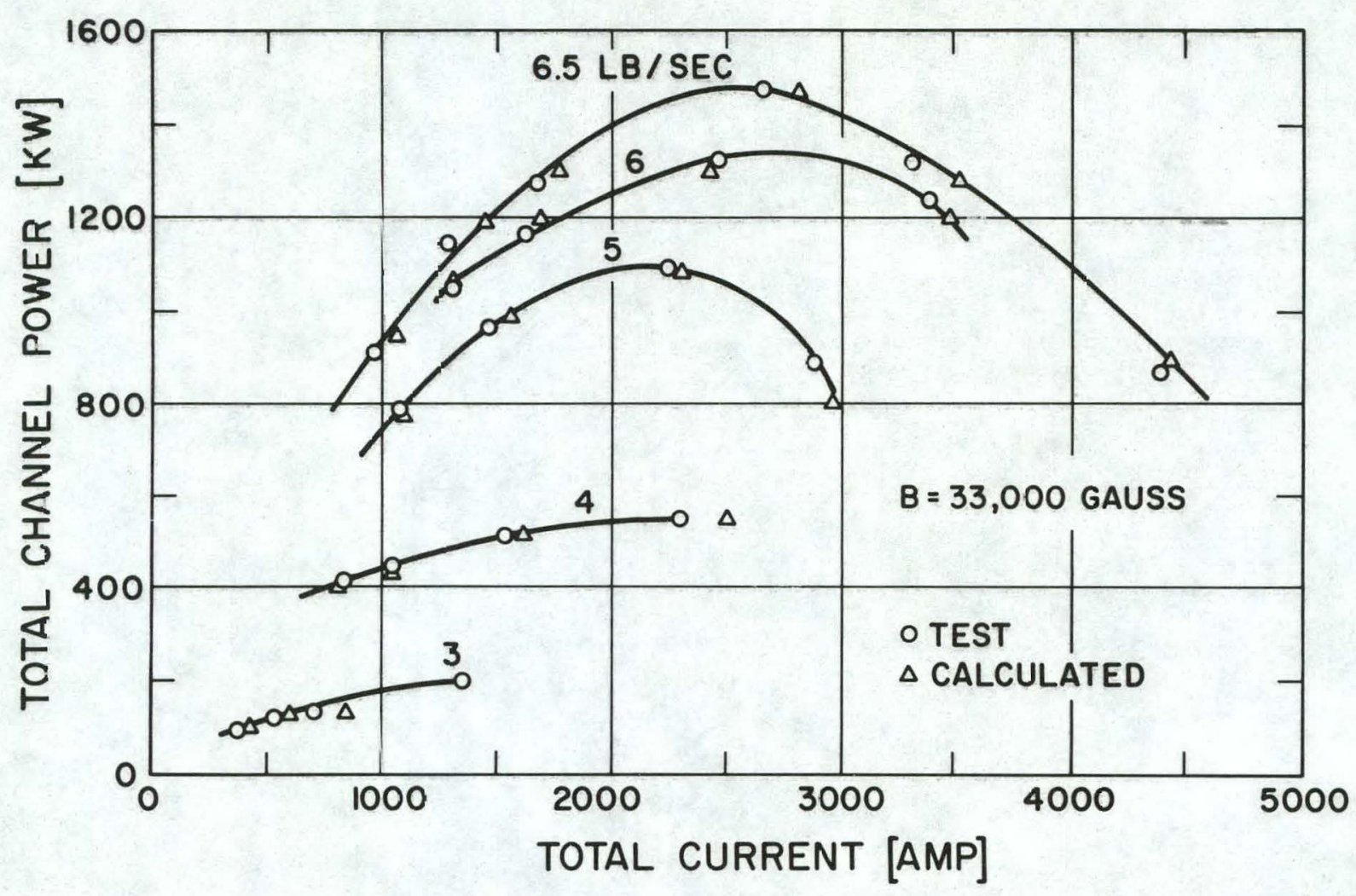

Figure 10. Mk II Overall Performance at 33,000 Gauss 
observed points and calculated values determined from a detailed theoretical analysis. It can be seen that we are capable of calculating the flows in detail and accurately. I might add that over the range covered by these curves, the flow changes from subsonic to supersonic, and in general goes through a wide variety of different regimes. The important point is that the performance is predictable. You will note that powers as high as 1.5 megawatts have been developed in this device. Presently, experiments regarding a Hall generator are being carried out with the Mark II. The experiment was designed specifically to evaluate the fluid mechanics of the MHD generator; so we are not interested in duration or in making net power, but rather in studying the details of the gas-field interaction. The Mark II is designed for short duration operation, since the fluid mechanics comes to equilibrium very quickly. We have, however, put a good deal of effort into the development of long duration MHD generator components on a smaller flow scale than is the case with the Mark II.

A water cooled insulating wall for an MHD channel developed at Avco is shown in Figure 11. It is composed of isolated metallic elements separated by thin sections of refractory material which stays cool because it is thin. Coolant fluid is circulated through these pegs. These and similar walls have been run for hundreds of hours in the products of combustion gases of commercial fuel at $3000^{\circ} \mathrm{K}$ and with their ash, seed, and sulphur. This and similar work has established the fact that MHD channels will be operable for long periods of time under the conditions required for the MHD generator.

One of the reasons the MHD generator has to be big in order to work is that if the magnet is made of room temperature material the generator must supply a good deal of power for its own magnet. This means that the channel cross section must be bigger than a certain minimum in order to supply the power. We have a contract with ARPA in which we have built and are testing a self-excited MHD generator for military applications. This generator is a prototype of a class of device to supply a large burst of power for relatively short periods of time. The picture of the apparatus is shown in Figure 12. This is a so-called Mark V generator. The combustion chamber on the left can burn up to 130 pounds per second of rocket propellant and seed. The copper magnet is of air core design and is inside the steel structure. It is driven by approximately one-half the output of the MHD generator.

A schematic of the Mark $\mathrm{V}$ generator system is shown in Figure 13. Most of the equipment here is what might be contained in a rocket engine test stand. A battery is used to pre-excite the MHD generator magnet after 


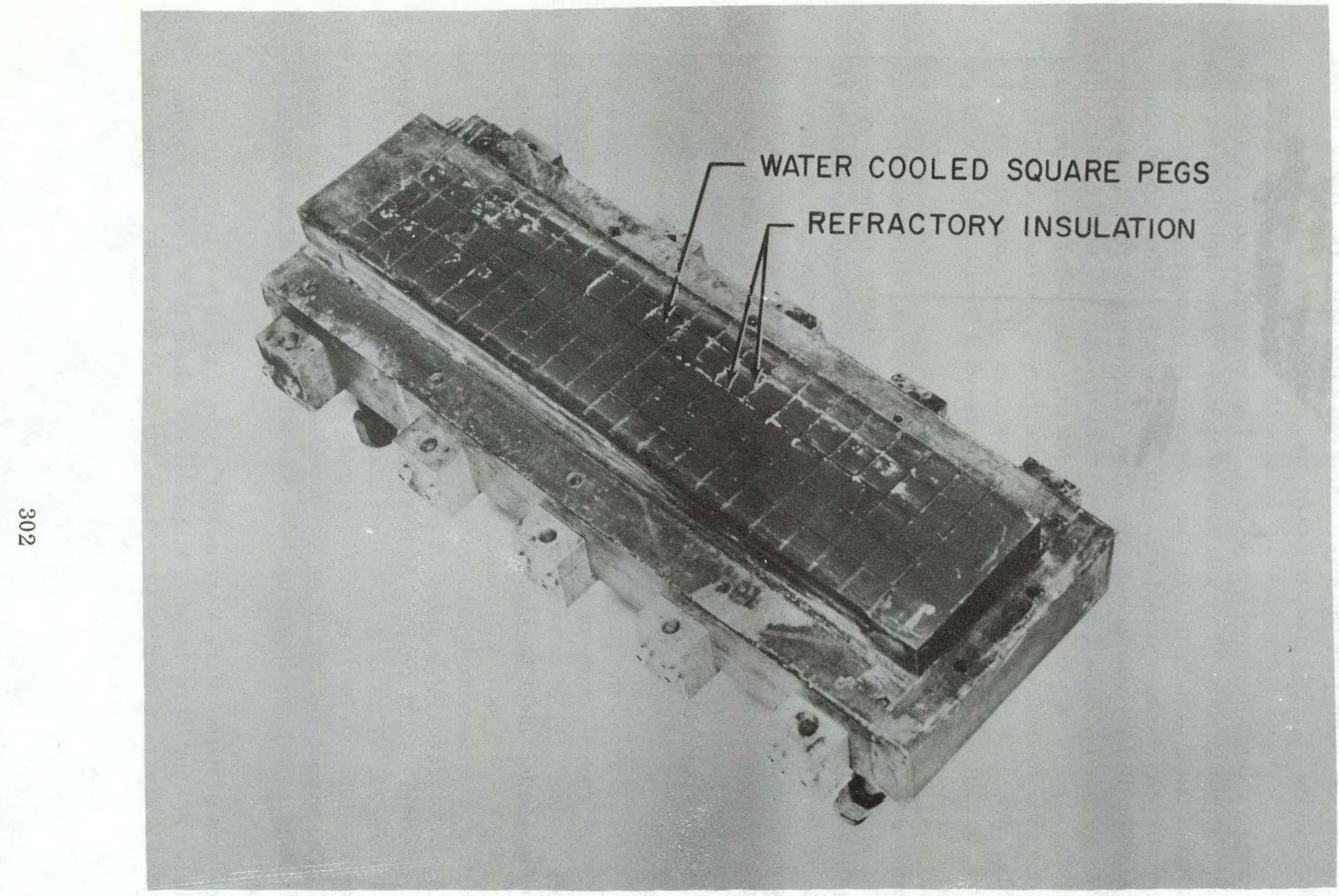

Figure 11. Example of MHD Gererator Insulating Walls Which Have Been Operated for Several Hundred Hours in Seeded, Ash Laden Combustion Gases 


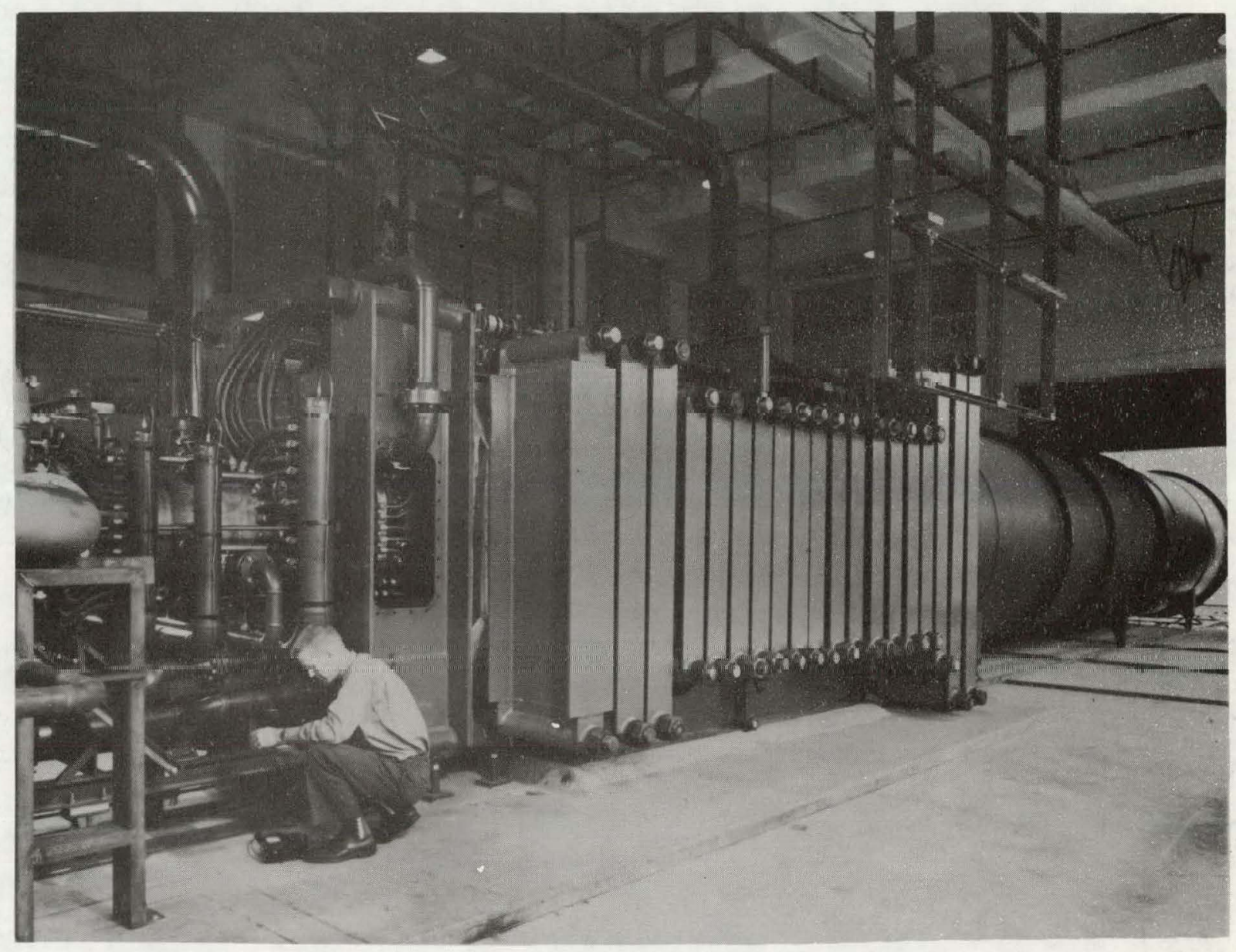

Figure 12. Mk V Self-Excited Rocket Driven MHD Generator 


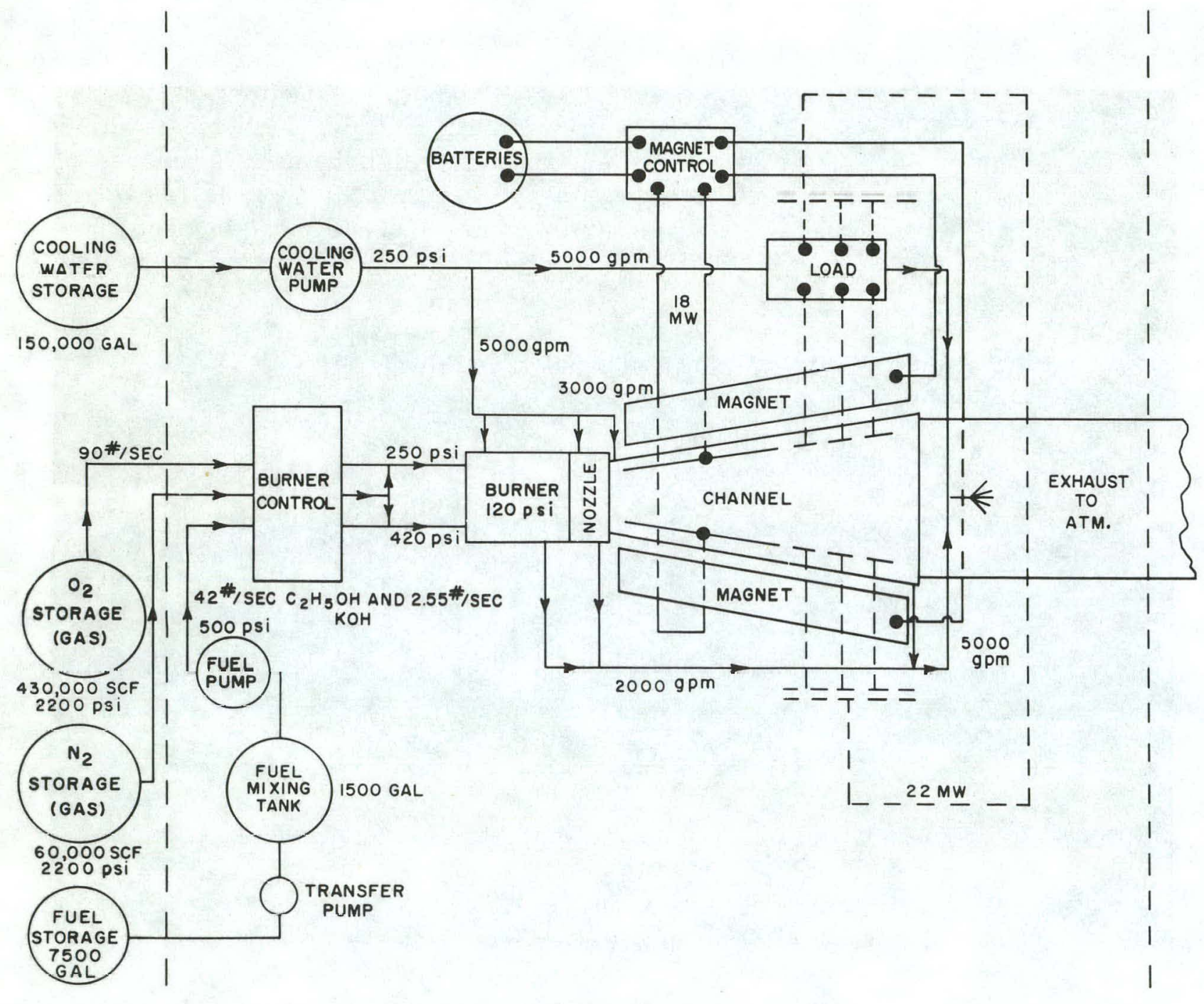

Figure 13. Mk V Generator System Schematic 
which the generator itself is fired and the generator and battery bank work in parallel on the magnet. When the battery current drops to zero the batteries are disconnected and the generator continues alone and self-excited. Additional power is delivered to the loads. The performance in a typical run is shown in Figure 14, where we have plotted the current and voltage across the magnet as a function of time, along with the $\mathbb{R}$ drop in the magnet. The batteries provide an initial current of about 4000 amperes to the magnet. After about 7 seconds, the burner is fired. Generator and battery continue to power the magnet until after about 12 scconds, when the batteries are disconnected. The magnet current continues to climb, and the excess of magnet voltage above the IR drop supplies the excitation power, i.e., L di/dt. Although we are experiencing the usual bugs expected in a development of this kind, the generator gross output has reached 11.2 megawatts and is expected to go to 35 megawatts eventually.

The Arnold Engineering Development Center of the U. S. Air Force has engaged us to provide them with a device to accelerate air to a very high velocity for a wind tunnel. This device is shown in Figure 15. It is an MHD motor-generator, in which the power output of a 20-megawatt MHD generator is put into an MHD accelerator. The accelerator takes air from a conventional arc heater (which is limited in effective reservoir pressure and enthalpy) and accelerates that air to much higher conditions of effective reservoir pressure enthalpy and velocity: This device, scheduled to be operational in June 1965, is the prototype of a 300-megawatt version proposed by the Air Force. This device is an example of the type of energy transfer process which might be used in some of the propulsion devices described by Dr. Rosa. It uses MHD to exchange momentum and energy between two gas streams under conditions which cannot possibly be handled by any other means. The MHD motor-generator which we are building will increase the effective reservoir pressure from 5 atmospheres to 660 atmospheres. and triple the gas enthalpy.

Recently we have been carrying out some work in connection with our commercial program which may have some importance in the context of the subject of this meeting. In the commercial power plant we must add seed to the gas and for economic reasons we must recover a large fraction of it and recirculate it. The way this is accomplished is shown in Figure 16, which is a schematic diagram of a seed recovery test apparatus we have been operating at our Laboratory. Fuel and oxidizer are burned in the combustion chamber along with seed, simulated coal ash, and sulphur as appropriate to a common coal. Leaving the combustion chamber the gas passes through a water-cooled tailpipe which simulates both the temperature drop and transit time of an MHD generator. In the combustion chamber all 
POWER TEST \# 10

VOLTAGE $\varepsilon$ CURRENT VS TIME

$\dot{m}=86$ LB/SEC, $2 \%$ SEED BY VOLUME

$\stackrel{\mathscr{C}}{\mathscr{C}}$

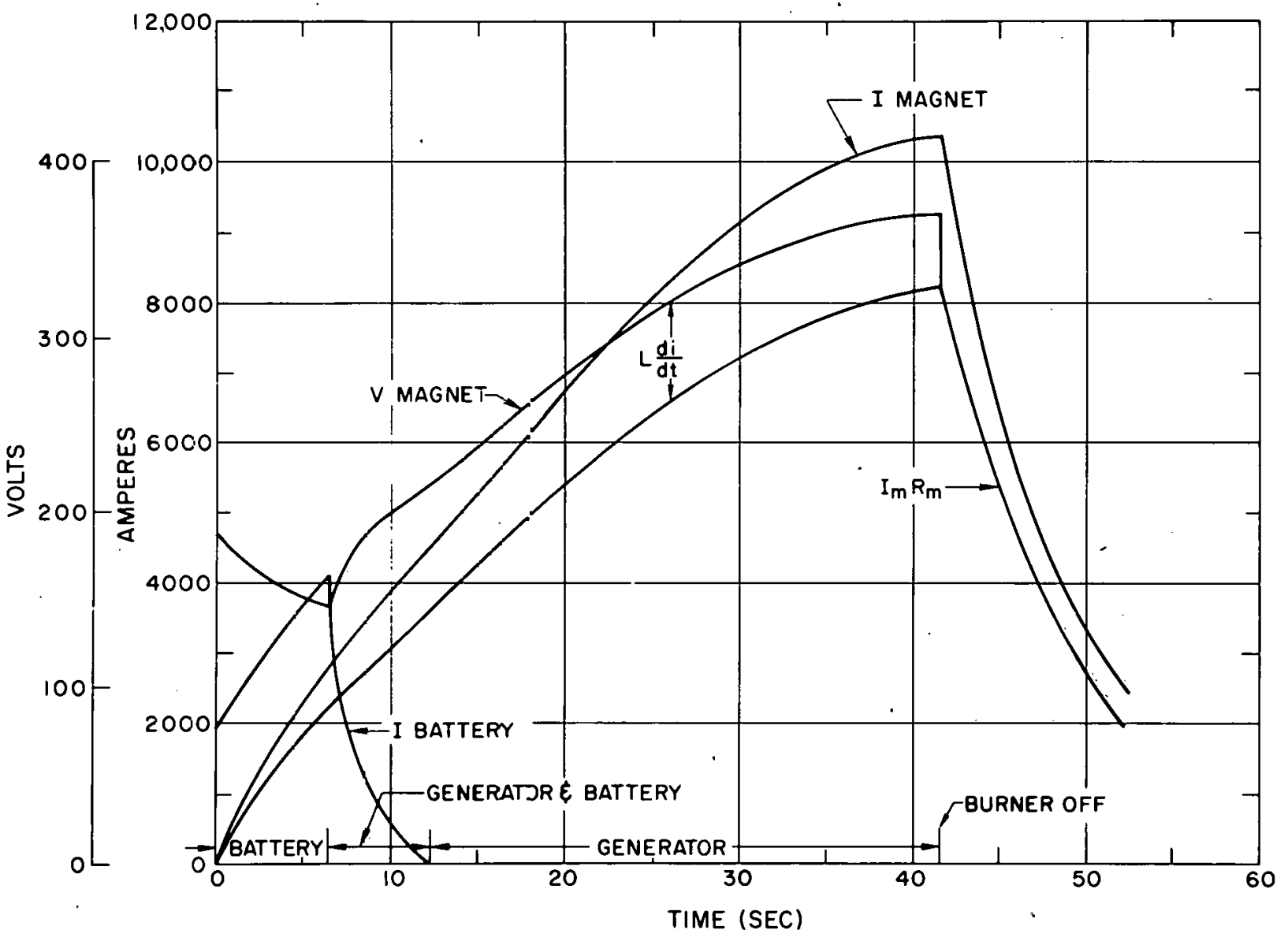

Figure 14. Typical $\mathrm{Vk} \mathrm{V}$ Self Excitation Characteristic 


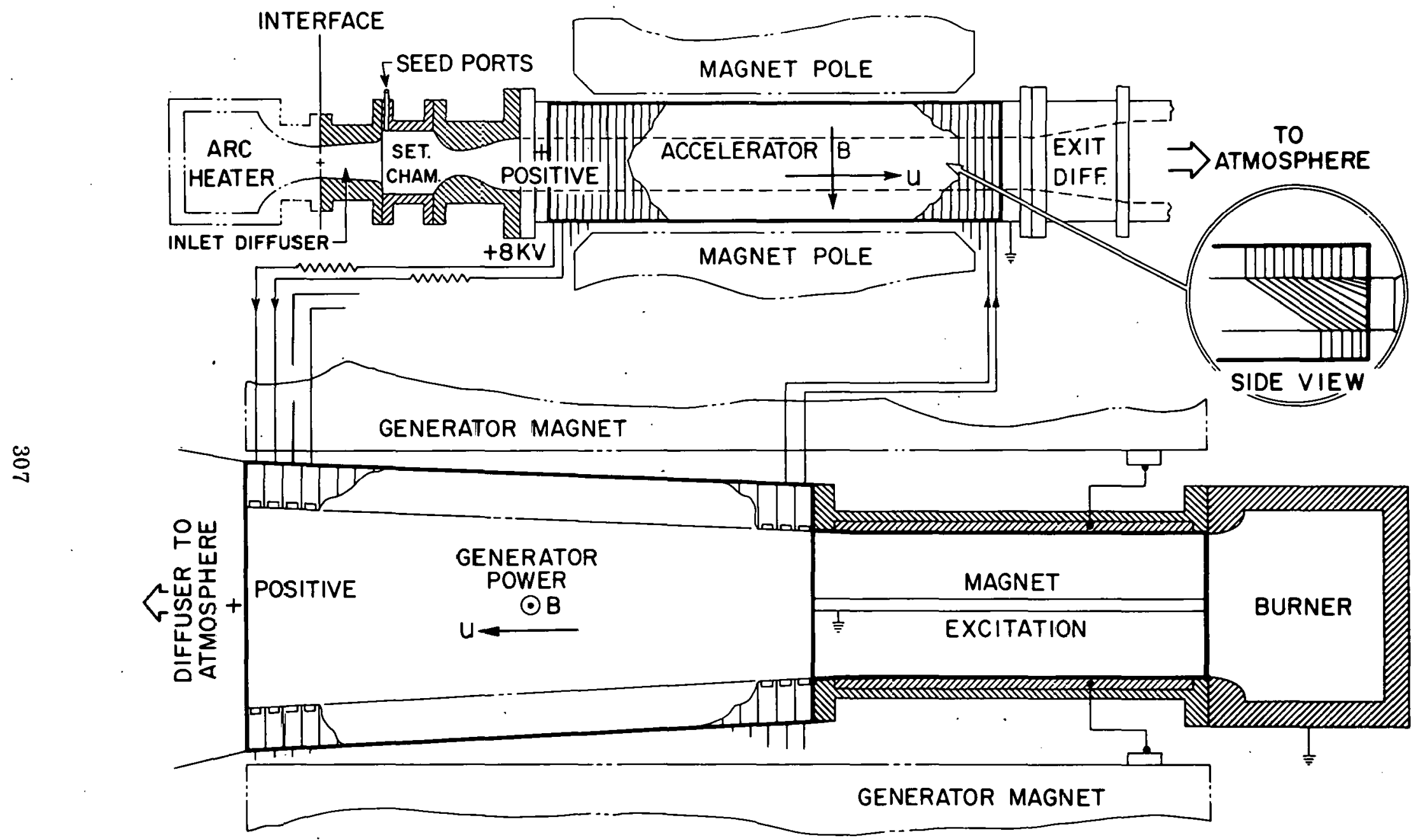

Figure 15. 20 Megawatt LORHO Pilot MHD Generator-Accelerator being Built for Hypersonic Wind Tunnel at the USAF Arnold Engineering Development Center 


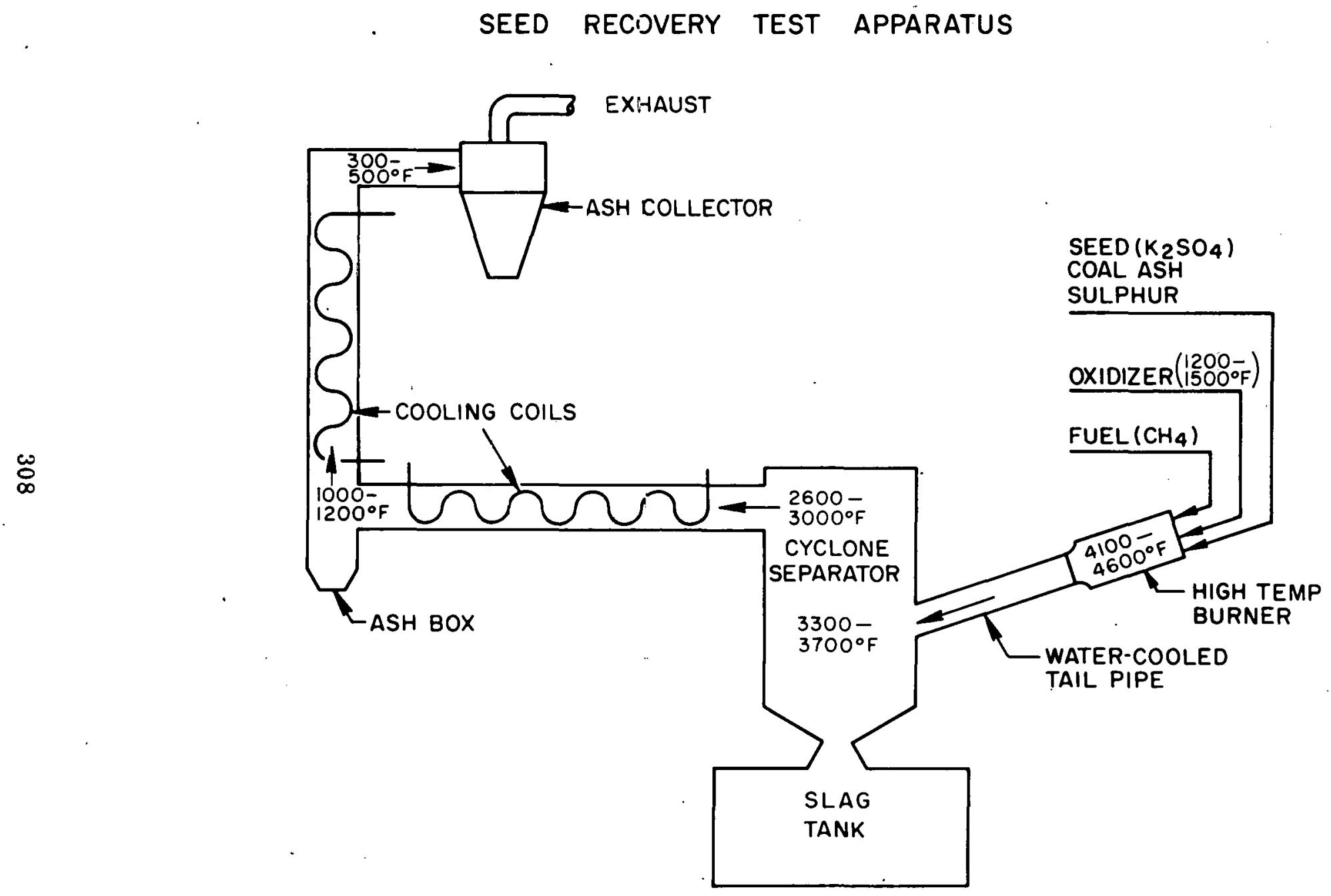

Figure 16. Schematic of Apparatus Used in Studying Separation of Condensing Metal Oxides from Combustion Gases 
of the seed and most of the coal ash has been vaporized. The ash, however, condenses during the transit through the MHD generator; but because of higher volatility the seed remains in the vapor form. Upon leaving the generator, the gases are cycloned and the condensed ash is separated from them. This leaves the flue gas entering the coolers highly enriched in seed, which is then picked up in the precipitators at the end and recirculated. The work with this equipment has demonstrated very effective and efficient seed recovery. The vaporization of the coal ash in the combustion chamber and condensation during the transit time through the generator and subsequent separation by a cyclone is rather similar to the process which would be encountered in the propulsion systems discussed by Dr. Rosa. It is very encouraging that with this crude apparatus it is possible to get the ash content, that is the content of the low volatile oxides, down to approximately $2 \times 10^{-4}$ parts by weight.

Now I would like to turn the discussion back to Dr. Rosa for summary. Thank you.

\section{CONCLUSION}

Now that Tom Brogan has shown you something of our present. work, I would like to make one or two further comments and then summarize.

First of all I imagine that in the figures that were shown you observed the very massive field coils in our present devices. I would like to assure this audience again that this is not an inevitable feature. First, the devices you have seen were designed for combustion product gases which produce a rather well defined temperature and hence conductivity. Now as we saw earlier, conductivity is an exponential function of temperature, and the size of the generator is pretty much proportional to the gas conductivity. Secondly, very large reductions in the size and weight of the magnet can be made by cryogenic cooling, and most of these propulsion systems would have an abundance of hydrogen available for this purpose. Figure 17 illustrates these points. On it coil mass is plotted as a function of the size of the generator in terms of gross megawatts of output. The top curve is for a combustion fired generator in which the coil dissipates $30 \%$ of the gross power or $6 \%$ if the coil is liquid oxygen cooled. The Mark $\mathrm{V}$ generator which Tom Brogan discussed falls slightly below this curve because its dissipation is closer to being $50 \%$. You observe that it is a break-even generator. If it had been made much smaller, all of the copper in the world would not have made it self-excite. The lower curve shows what would happen if the gas conductivity and velocity is increased as it would be in a 


\section{COIL MASS}

COMBUSTION; JP4 - LOX $+1 \% K_{i} \sigma u^{2}=4 \times 10^{7} \frac{\text { mho meter }}{\mathrm{Sec}^{2}}$

NUCLEAR; $r_{2}+0.1 \%$ Cs AT $2500^{\circ} \mathrm{C}, 3$ atm; $\sigma u^{2}=4 \times 10^{8} \frac{\text { mtio meter }}{\mathrm{sec}^{2}}$

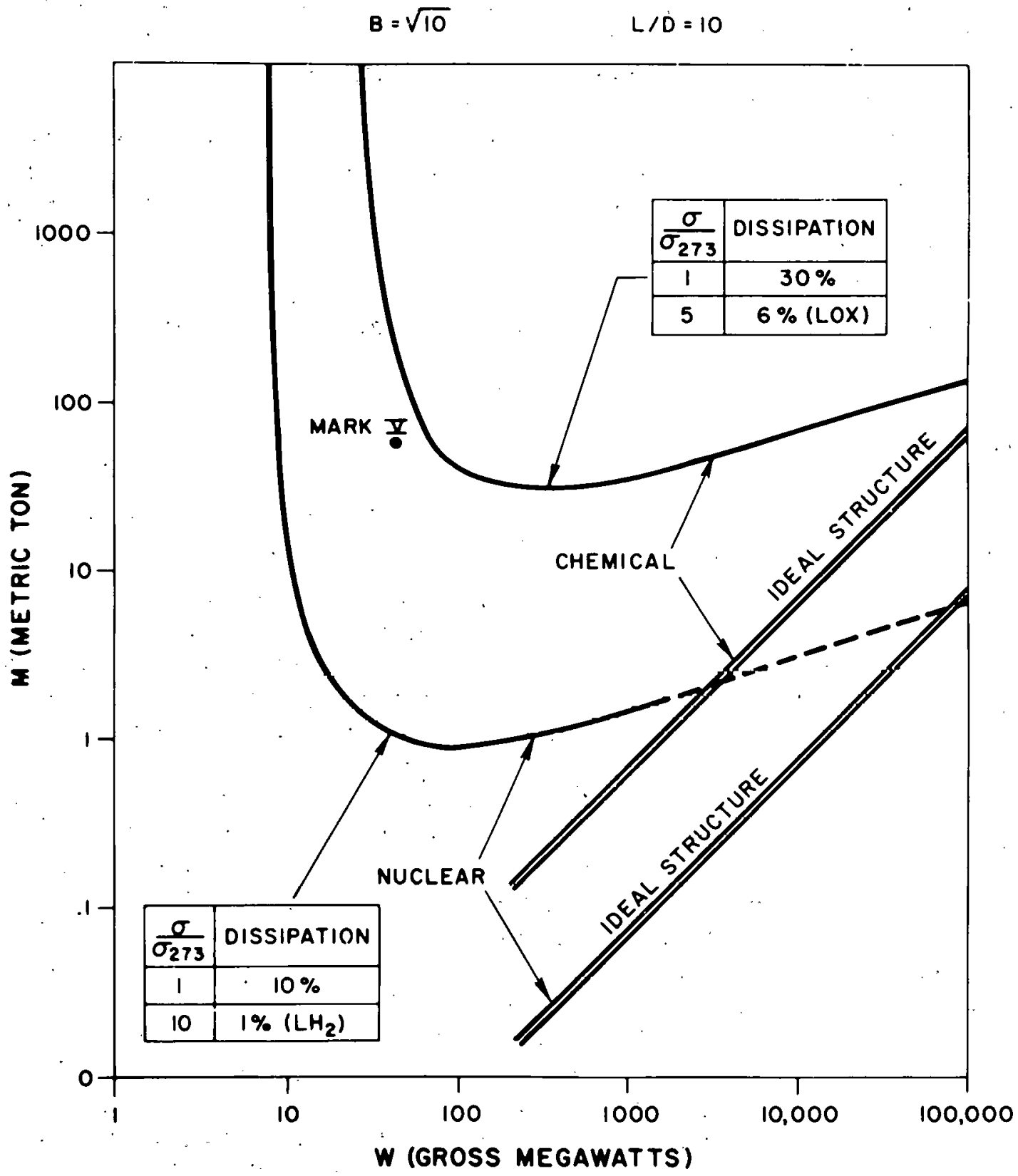

Figure 17 
nuclear system at $2500^{\circ} \mathrm{C}$ using hydrogen as the working gas. Here the dissipation is $10 \%$ at room temperature, or $1 \%$ if the coil is cooled enough to produce a factor of 10 increase in conductivity. This could easily be accomplished using liquid hydrogen. In fact, much greater gains should be possible. Observe here that as long as the power level is greater than 10 megawatts the coil will weigh on the order of 1 ton. At power levels on the order of 1000 megawatts and up, this corresponds to an extremely small weight per kilowatt of energy handled.

In summary then, there is no reason why an MHD generator cannot be made light enough for the kind of high thrust propulsion systems which we have been discussing here.

Figure 18 attempts to summarize the kind of systems that we think we can build using an MHD generator and advanced reactors on a map of specific impulse vs engine thrust to weight. The curve labeled "gas core propellant cooled" corresponds to systems as illuistrated in Figures 3 and 4. The curve labeled "air breathers" corresponds to a system such as that shown on Figure 5, but includes also schemes using a closed as well as an open cycle. The vertical lines labeled "radiators" correspond to systems such as were discussed in connection with Figure 8. This figure gives the impression that for boosting off the surface of the earth, or any other body, air (or "atmosphere") breathers are hard to beat, and that for interplanetary flight into space, radiating systems are hard to beat if you can get up to power to weight ratios equal to, or exceeding 1 kilowatt per kilogram. However, the main point that I want to make with this curve is just, that by combining an MHD generator with advanced high temperature reactors, we can make propulsion systems whose performance is comparable to what you can hope to get in any other way. In particular they are comparable to, or perhaps better than, what you could hope to get with a gas core reactor alone. . . . and you do not have to solve the fuel containment problem in order to get it! 

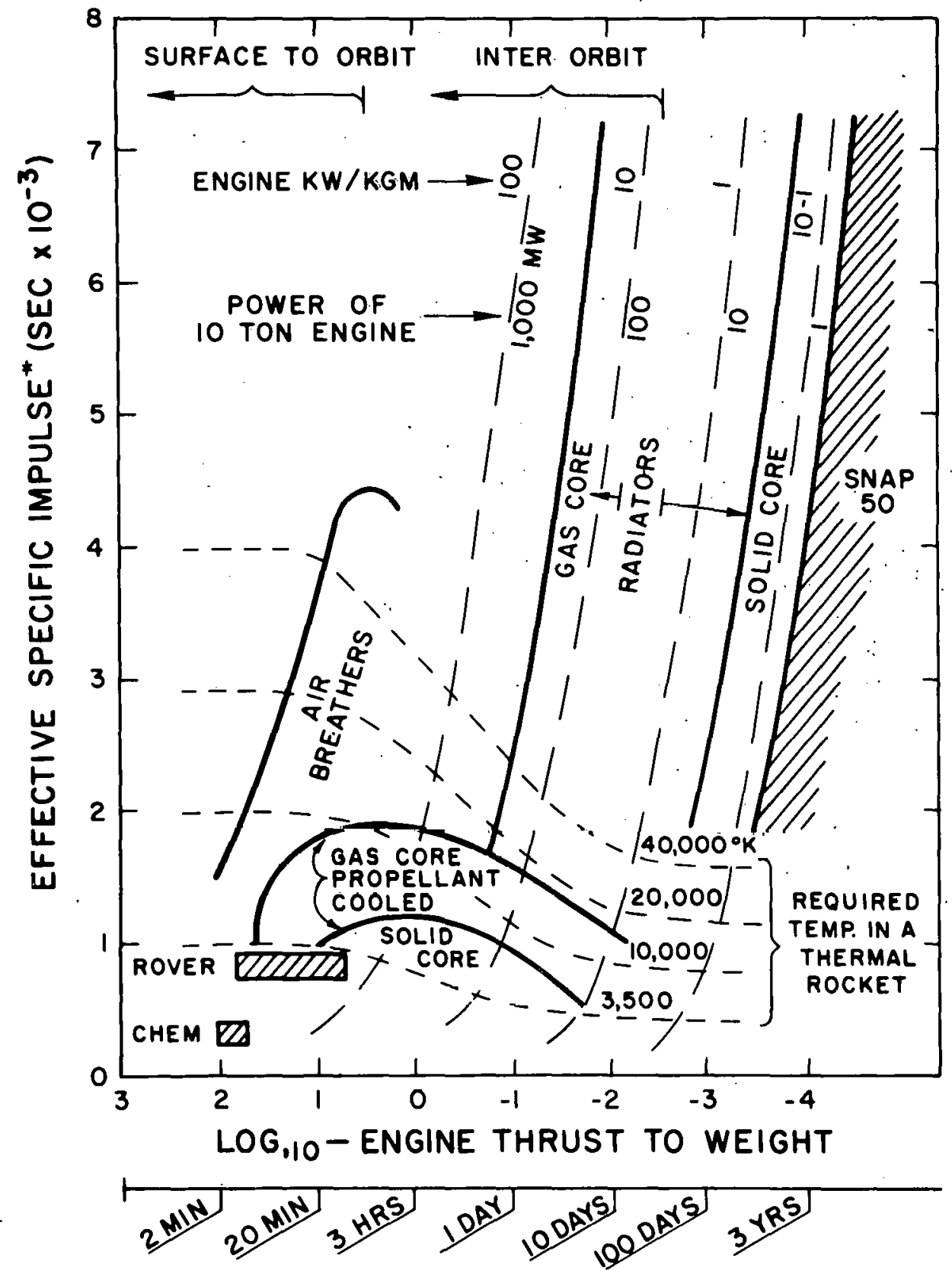

TIME TO ACCELERATE THRU $10 \mathrm{kM} / \mathrm{SEC}$ *

- VEHICLE WEIGHT ASSUMED = $10 \times$ ENGINE WEIGHT

Figure 18 


\title{
VORTEX GAS ACCELERATOR
}

\author{
Stirling A. Colgate \\ University of California \\ Livermore, California
}

Published in AIAA Journal, 2, 2138 (1964)

\section{ABSTRACT.}

The exhaust velocity of a propellant gas from an isothermal cavity can be significantly increased by a factor of approximately 1.7 . above that corresponding to the usual isentropic expansion by utilizing multiple reheat by blackbody radiation from the walls during expansion. In order to reduce the necessary volume and the frictional drag with the walls during this reheat and expansion process, the gas is wrapped up in a rotational flow pattern in which angular momentum per unit mass is constant along all streamlines. As a consequence, the expansion in the nozzle corresponds to the conversion of rotational to linear momentum, rather than the usual thermal to linear. In order to absorb the radiant energy from the walls, the gas must have a sufficient optical opacity (achieved by a small contaminant addition to hydrogen) such that the gas in the cavity is approximately one radiation mean free path thick. 


\title{
FUEL CONTAINMENT IN THE GASEOUS-CORE NUCLEAR ROCKET BY MHD-DRIVEN VORTICES
}

\author{
Jacob B. Romero \\ The Boeing Company \\ Seattle, Washington \\ Published in AIAA Journal, 2, 1092 (1964)
}

\begin{abstract}
ABSTRAC'T
A gaseous fission propulsion engine is considered in which an electromagnetic vortex is employed for fuel retention. The pertinent continuity, momentum, energy, diffusion, and electromagnetic equations are derived for steady-state, laminar, two-dimensional flow and are solved for several cases. Economical fuel retention is considered from the point of view of rocket performance and design. Two basic design concepts are considered: a single critical chamber and multiple vortex tubes. Engine thrust-to-weight ratios range from ten-thousandths for the single vortex designs to tenths for the multiple vortex designs. Bypass flow. systems are suggested as a means of obtaining performance improvement.
\end{abstract}




\section{CRITICALITY OF GAS CORE REACTORS \\ R. J. Holl \\ Duuglas Aircraft Company \\ Santa Monica, California}

I will describe some of the nuclear calculations performed at Douglas. We feel that nuclear analysis is important for these systems for several reasons. First, there will be a large number of combinations of the materials, dimensions, configurations, and temperatures that can be eliminated on the basis of criticality within a reasonable pressure. This should help in limiting the number of cases considered for containment. Secondly, a knowledge of the required constituents and conditions is important in defining meaningful experiments. For instance, containment experiments with heavy-to-light gas density ratios of about two were described in another paper. In the design, the ratio is nine for a hydrogen core. If this critical mass were to increase by an order of magnitude, this ratio would be 90 . These considerations must motivate the range of conditions studied. We have tried to generalize the nuclear analysis to make it applicable to any gas core concept. Specifically, it will apply to the particular concept shown in Figure 1, which is typical of a number of vortex devices. It has a central fuel cell containing uranium with an annular hydrogen propellant zone surrounding it and contained within the moderator-reflector. At one end of this device is a nozzle. The physical phenomena occurring here are that neutrons produced in the fuel cell will have to be moderated in the reflector and returned to the core to produce fission. Competition for neutrons will be between three basic processes-core absorption, reflector absorption, and leakage. This competition for neutrons should be kept in mind as we examine the different materials and configurations.

Our first effort was devoted toward establishing calculational methods. Initially, the only method that can be used with confidence is multidimensional, transport theory calculations. Everything that I will describe today has at least been verified by one- or two-dimensional transport calculations. However, we found a large number of systems for which diffusion theory is 


\section{TWO CELL VORTEX REACTOR}

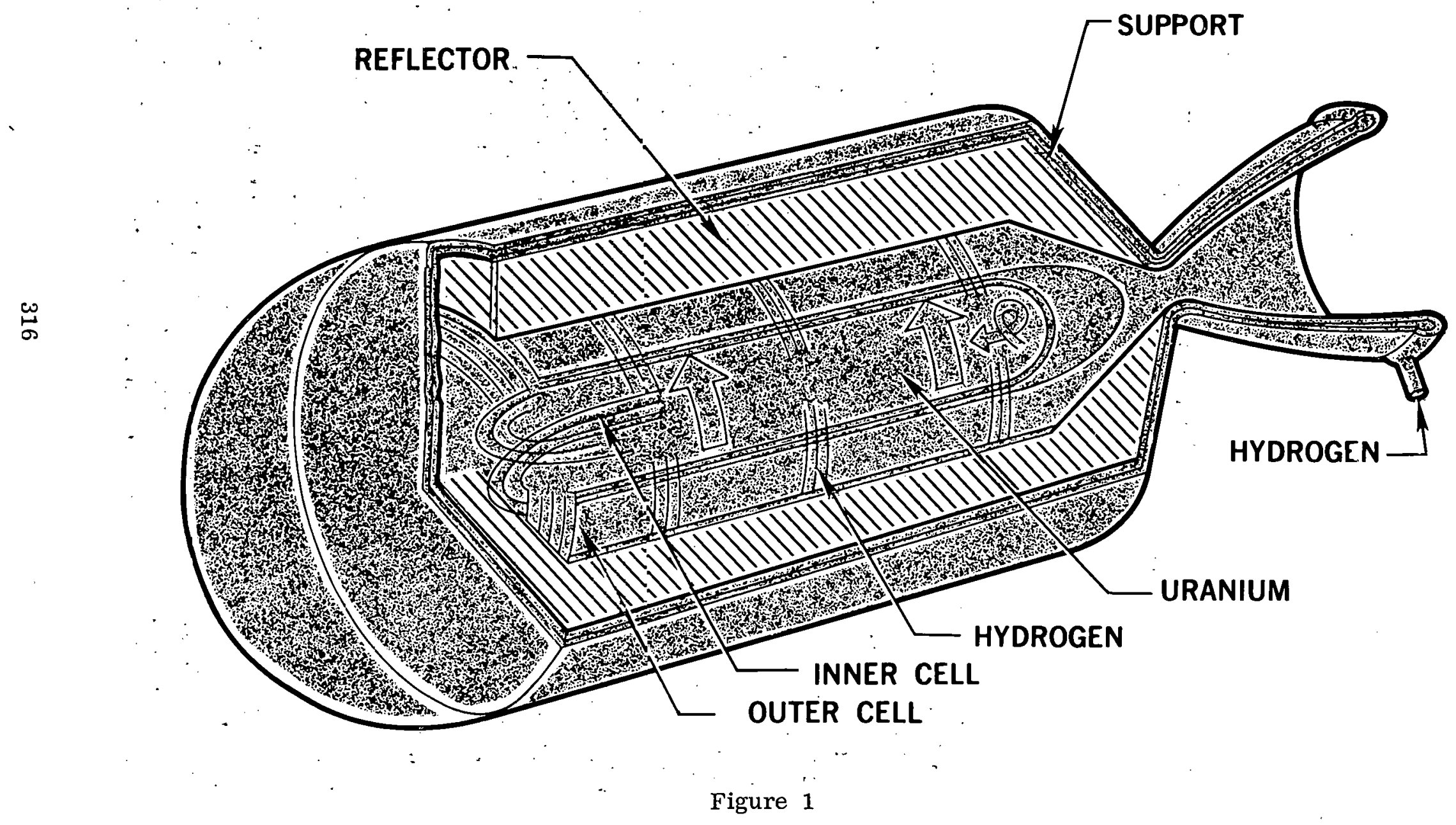


adequate. A typical case is shown in Figure 2 having spherical geometry with a cavity radius of $80 \mathrm{~cm}$, a fuel radius of one-half of the cavity radius, and a graphite reflector thickness of $100 \mathrm{~cm}$. It 18 interesting that the factors involving reactivity of the system are nearly the same for the diffusion and $S_{8}$ calculations. The absorptions in the reflector are proportional to the integral of the neutron flux which is very nearly the same with the two methods. Core absorptions, proportional to the integral of the flux in the core, are virtually the same. The leakage is indistinguishable between the two calculational methods. This yields a reactivity comparison, in this case, of 0.0005 difference between the reactivity eigenvalue of the diffusion and $S_{8}$ approximations. This is with a multigroup treatment-this particular case used 14 groups. Most of the calculations were done with multienergy groups. The results will be quite comparable if one used a few group treatment with flux averaging over the fast neutron spectrum-especially spatial dependent flux averaging in the moderator reflector region. We find it more efficient, especially in one-dimensional calculations, to do the averaging within every calculation itself, by using multigroup calculations, rather than performing separate spectrum and averaging calculations. However, if one were to do a large number of design calculations for systems that did not vary greatly in the effect on the fast neutron spectrum, one could very reasonably employ something like four-group calculations.'

These methods were used to survey fuel materials, reflector materials, core dimensions, and reflector thicknesses. Figure 3 shows the critical mass in kilograms of U-235 versus core size for graphite and beryllium oxide reflectors with variable reflector thicknesses. A reflector temperature of $2800^{\circ} \mathrm{K}$ was used.

Beryllium did not have sufficient nuclear advantage to offset its lower temperature limit. Hydrogenous materials have excessive absorption. $D_{2} \mathrm{O}$ was not considered because the systems are very sensitive to any extraneous absorber, and substantial containment would be required to separate it from the core. We did some calculations on the effect of a thermal absorber at the moderator cavity interface and: near the cavity in the reflector, and it was very deleterious. In the graphite system, the minimum critical mass occurs for cavity dimension of about $80-\mathrm{cm}$ radius. For beryllium oxide, this optimum occurs at a much lower value, about $40-\mathrm{cm}$ radius. Beryllium oxirie also gives lower critical mass for nearly infinite reflectors. This is due to the fact that it is a superior moderator which slows down the neutrons closer to the core so they have more chance to cause fission rather than to leak out of the system. The graphite and beryllium oxide comparison is also characterized by beryllium oxide having greater reflector absorption, while graphite, even with large reflectors, has most loss through leakage. 


\section{COMPARISON OF DIFFUSION AND $S_{8}$ APPROXIMATIONS FOR A SPHERICAL CAVITY REACTOR}

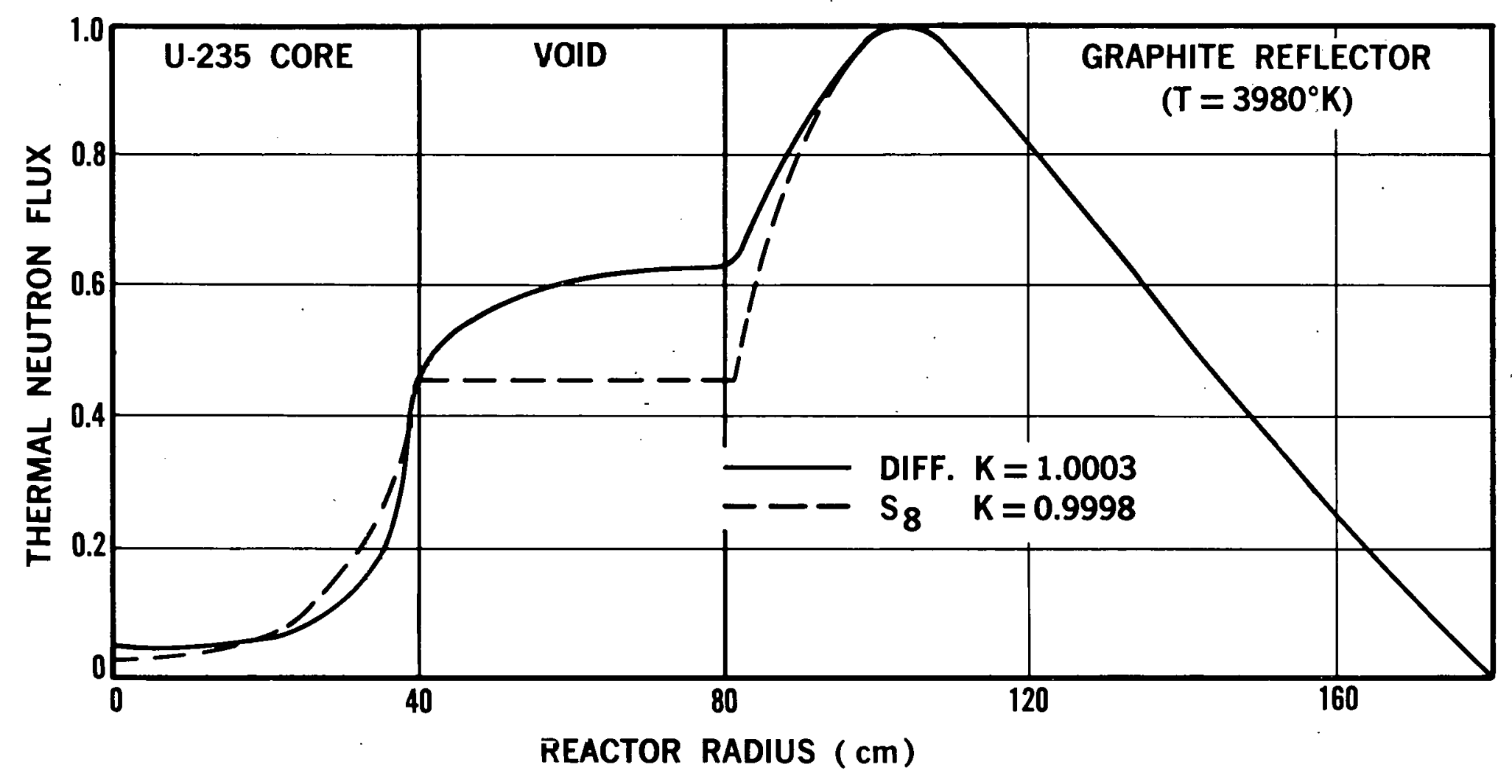

Figure 2 


\section{CRITICAL MASS OF SPHERICAL CAVITY REACTORS}

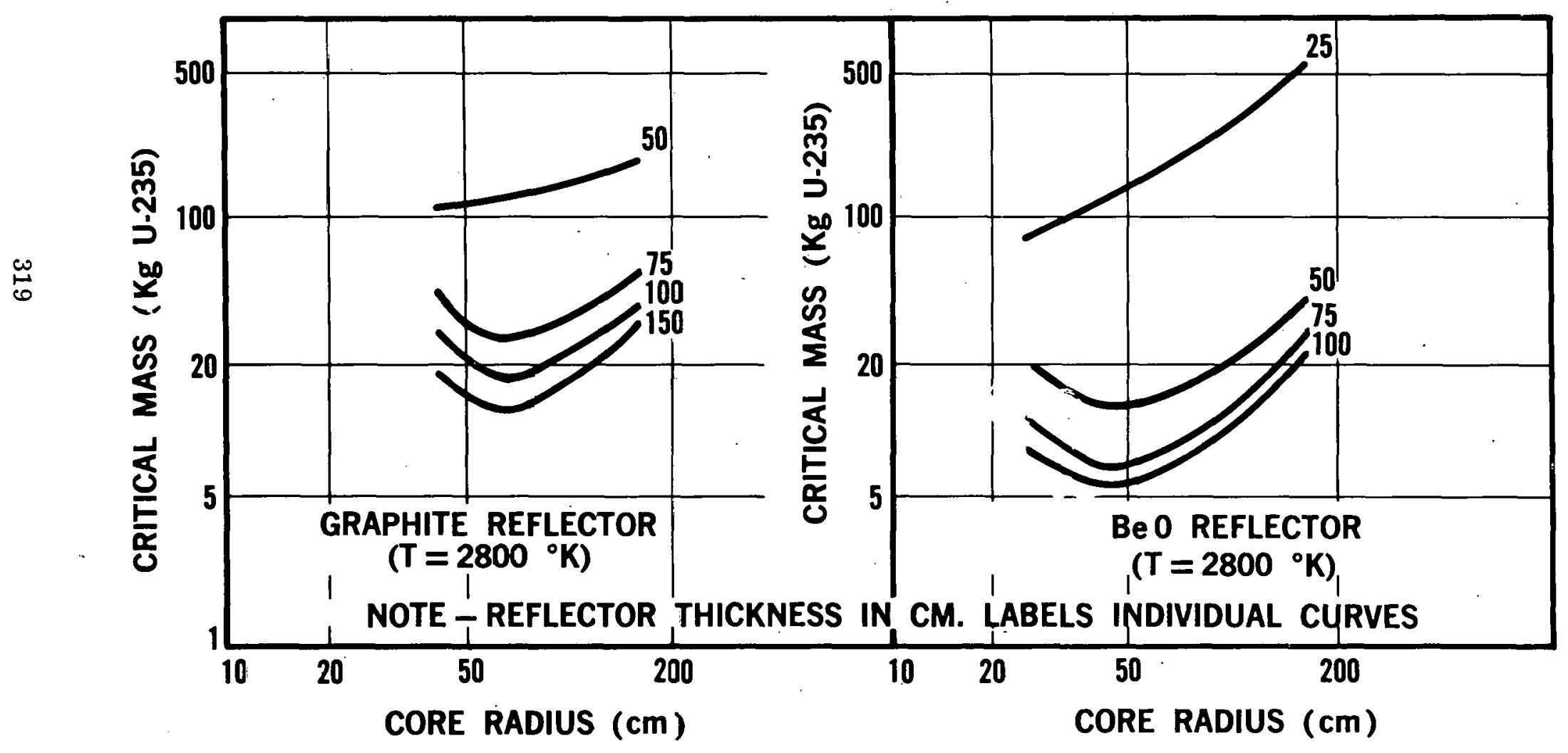

Figure 3 
Of course, with a core $\eta$ of a little over two, about one-half of the neutrons will have to be absorbed in the core and the other half will be divided between reflector absorption and leakage. Figure 4 shows the effects of temperature and fuel species combined. This is for the case at the optimum core radius with a $100-\mathrm{cm}$ graphite reflector. So the se are minimum critical masses for that particular system as a function of temperature and fuel. Of course, Pu-239 and U-233 are superior fuels to U-235. As the temperature increases, Pu-239 approaches the critical mass of U-233. However, the two other fuels have a different basis for their superior characteristics compared with U-235. Even though the ratio of fission to absorption in $\mathrm{Pu}-239$ is inferior to $\mathrm{U}-235$, its cross sections are just so much greater that for a fairly lightly fueled system, such as this, it has a much lower critical mass. The macroscopic cross section of the core is larger for $\mathrm{Pu}-239$ than for $\mathrm{U}-235$. This should be kept in mind when considering characteristics which will make the fuel more concentrated. The increase in the plutonium concentration will be greater than in U-235 because plutonium will become "black"-the fuel will become self-shielded much more rapidly. Plutonium is not available in quantity as pure $\mathrm{Pu}-239$. Material produced in a power reactor with an irradiation of $0.5 \times 10^{21}$ thermal nvt was evaluated. This fuel contained about $8 \% \mathrm{Pu}-240$. This modest amount of $\mathrm{Pu}-240$ caused the critical mass of plutonium to exceed that of U-235. We feel that $\mathrm{U}-233$ is the best fuel. Its superiority is due to its better multiplying properties rather than extremely large thermal cross sections. As the fuel zone becomes black, U-233 will be the most reactive fuel. Configurations can be made critical with U-233 which could not be with U-235 or $\mathrm{Pu}-239$. However, due to cost and availability, we will use U-235 for the rest of the results. We can keep in mind that U-233 has probably a factor of two lower critical mass. For economic considerations, at least for the foreseeable future, U-233 will be more than a factor of two more expensive than $\mathrm{U}-235$.

We will now show a two-dimensional analysis of a device which might be used in space propulsion. Selecting dimensions near the optimum, the transformation from a spherical to a cylindrical geometry, with a length to diameter ratio of unity, can be made by keeping the fuel density constant. Thus, a cylinder with radius equal to that of a sphere will have an increase of critical mass of about $50 \%$ corresponding to the increase in volume. This is only true for a near-optimum system, on the basis of minimum critical mass. We will then determine the effect of some of the necessary geometric characteristics of a propulsion device on this system.

Figure 5 shows the critical mass as a function of the ratio of fuel radius to the cavity radius with variable graphite reflector thickness. Again 


\section{CRITICAL MASS FOR SPHERICAL CAVITY REACTORS WITH REFLECTOR TEMPERATURE VARIATION}

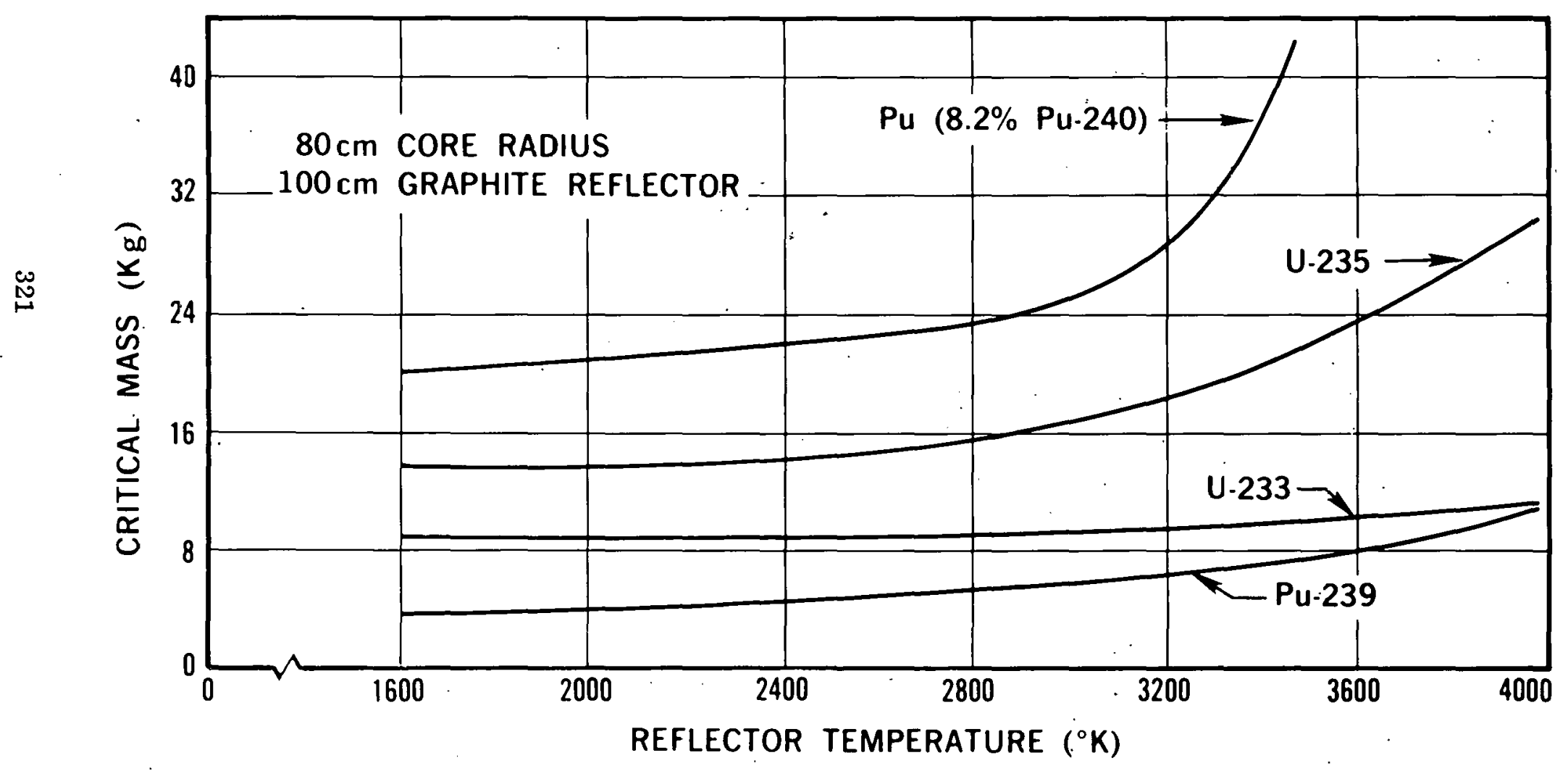

Figure 4 


\section{EFFECT OF REDUCED FUEL RADIUS FOR VARIABLE REFLECTOR THICKNESS}

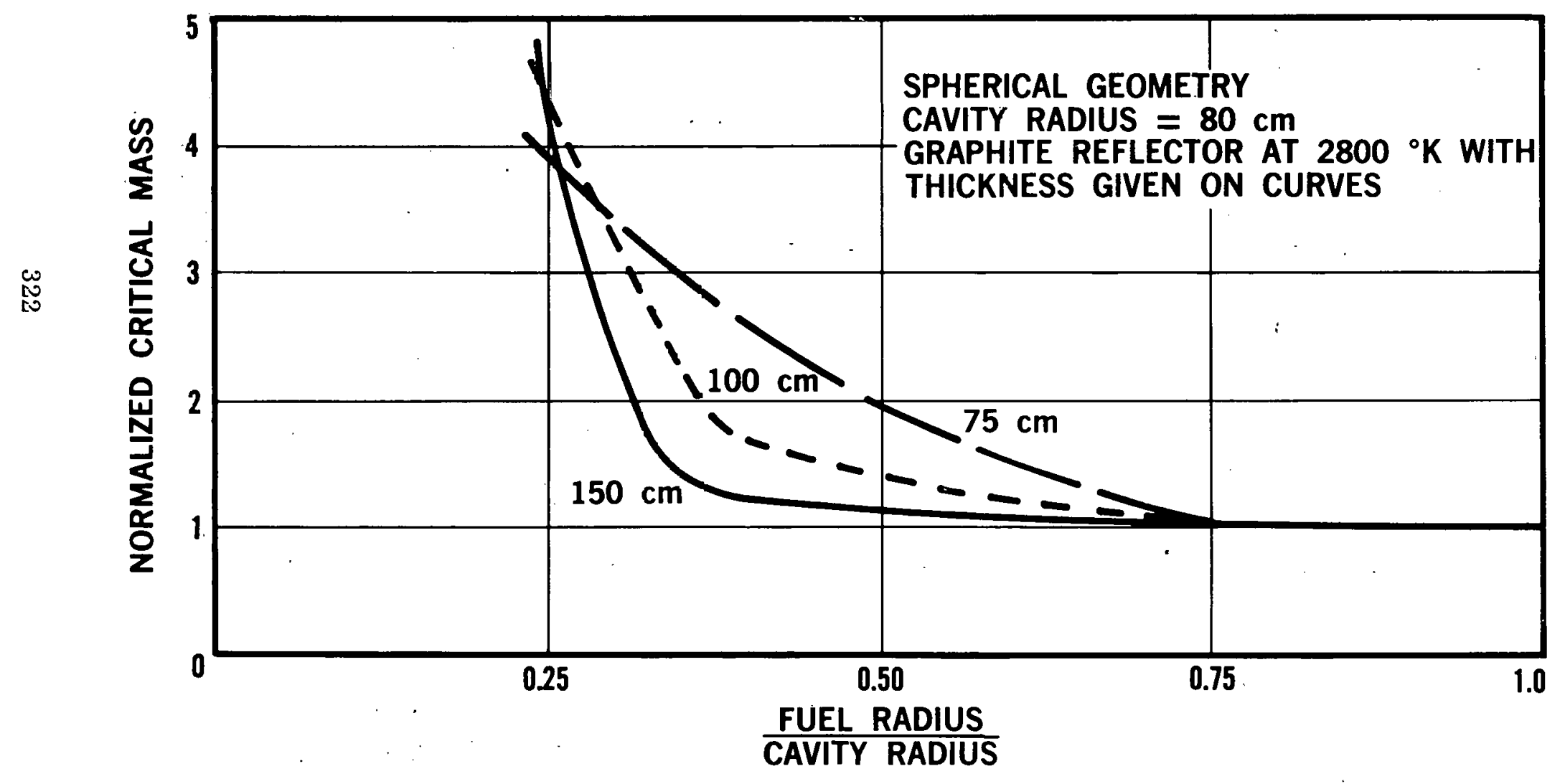

Figure 5 
spherical geometry and optimum cavity dimensions are used. It can be seen that for a nearly infinite reflector, the curve is first flat and then increases for smaller fuel dimensions. For thinner reflectors the curve starts increasing much sooner. What occurs with reduced fuel radius is that some of the neutrons that left the reflector entering the cavity missed the fue 1 and passed into the reflector on the other side, giving them a chance to be absorbed or to leak out of the system. Of course, a thin reflector increases the chance of leakage. Now consider the characteristics of an infinite reflector. The location of the knee of the curve will be determined by the absorption in the reflector. Thus, there are two characteristics seen in the curves. One, the effect of leakage is shown by the parameter, reflector thickness. The other one, the effect of reflector absorption, is shown by the knee on the curve for the effectively infinite reflector. Figure 6 shows the effect of geometry on the same factor for spherical, infinite cylindrical, and finite cylindrical geometry with cavity $\mathrm{L} / \mathrm{D}$ of unity. For the finite cylinder the fuel extends the entire length of the cavity. The curves show the ratios of the critical mass to the critical mass for the fully fueled cavity. The dependence on geometry is quite striking. In this case, the sphere is similar to Figure 5, except that the reflector is composed of an outer region of $70 \mathrm{~cm}$ of $\mathrm{BeO}$ with $15 \mathrm{~cm}$ of graphite immediately surrounding the core. For this reflector, which is also effectively infinite, the knee occurs at a slightly larger fuel radius than for graphite due to the fact that the beryllium oxide has somewhat more absorption. The effect of reducing the density of the reflector is shown in Figure 7. The model is a right circular cylinder, fully fueled. The normalized critical mass is plotted as a function of the void fraction in the reflector. For orientation, at about $25 \%$ void fraction, there is a $50 \%$ increase in critical mass.

Figure 8 shows the effect of a nozzle hole as the increase in critical mass as the ratio of exhaust nozzle diameter to cavity diameter is increased. For interesting systems this ratio is 0.15 and higher. This gives an increase in critical mass in excess of $50 \%$.

Figure 9 shows the composite of all these effects. The fuel radius is reduced by a factor of two, there is a $10 \%$ void fraction in the reflector, and a nozzle hole which corresponds to the throat of a nozzle with one million pounds thrust. These represent minimum effects on the critical mass. Certainly, it would not be possible for the propellant to traverse the entire reflector in an area no larger than the throat of the nozzle. We have selected temperatures of $3000^{\circ} \mathrm{K}$ in graphite and $2000^{\circ} \mathrm{K}$ in $\mathrm{BeO}$. This gives an estimated neutron temperature in the core of about $2800^{\circ} \mathrm{K}$. We applied this throughout the entire reactor. This case has a critical mass of $58 \mathrm{Kg}$ of $\mathrm{U}-235$. The basic model as a fully fueled, finite cylinder at temperature 


\section{EFFECT OF REDUCED FUEL RADIUS WITH GEOMETRY VARIATION}

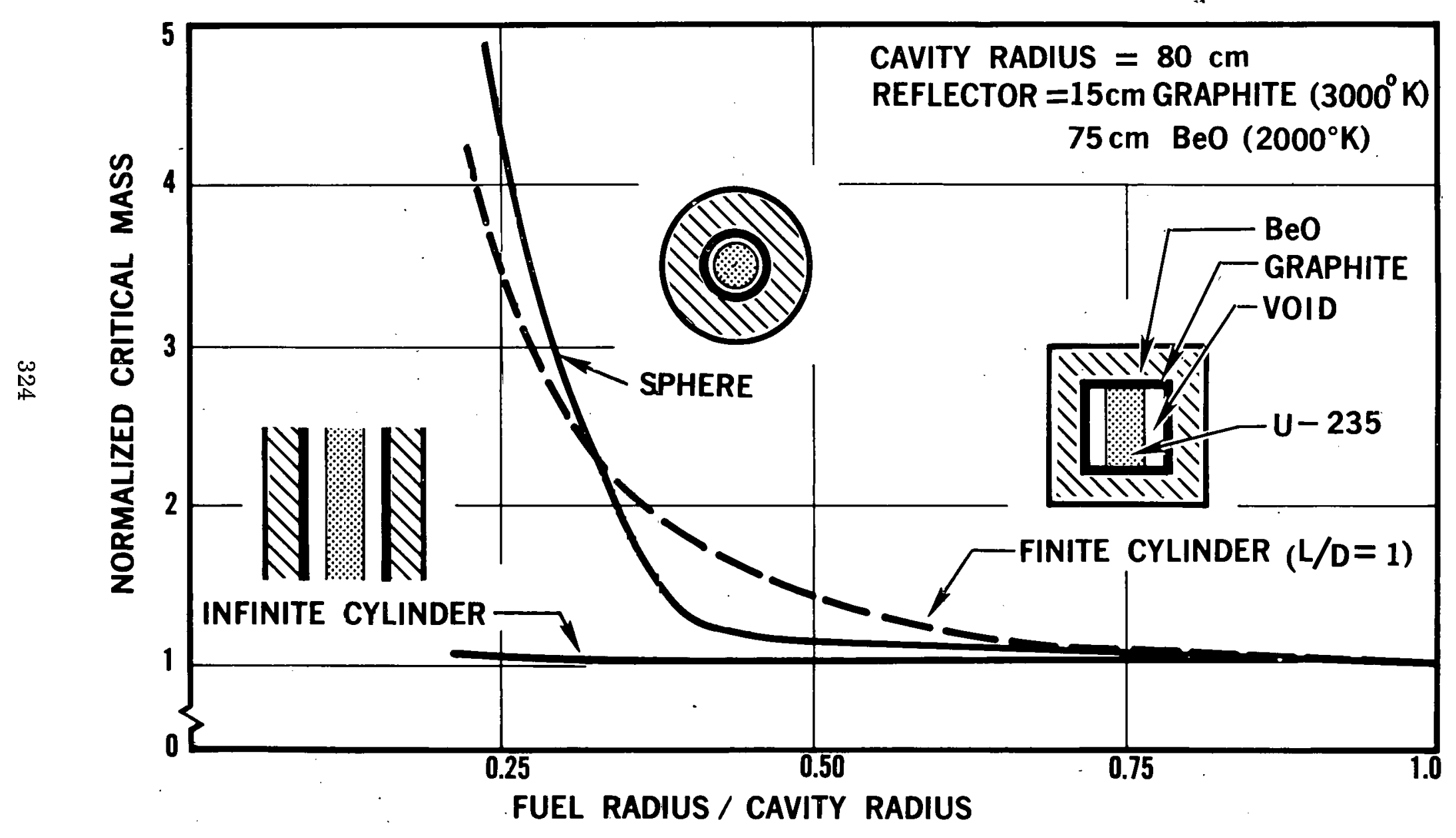

Figure 6 


\section{EFFECT OF VOID FRACTION ON CRITICALITY. FOR A CYLINDRICAL CAVITY REACTOR}

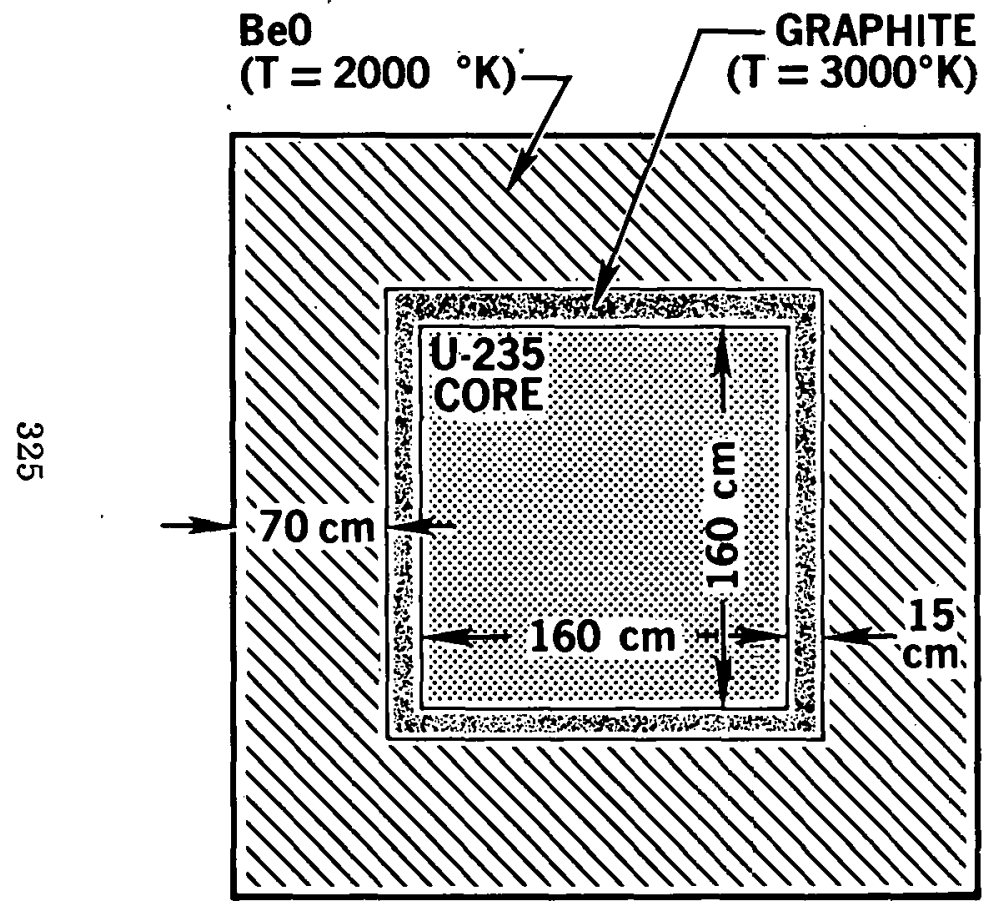

EFFECTIVE NEUTRON TEMPERATURE $=2800^{\circ} \mathrm{K}$

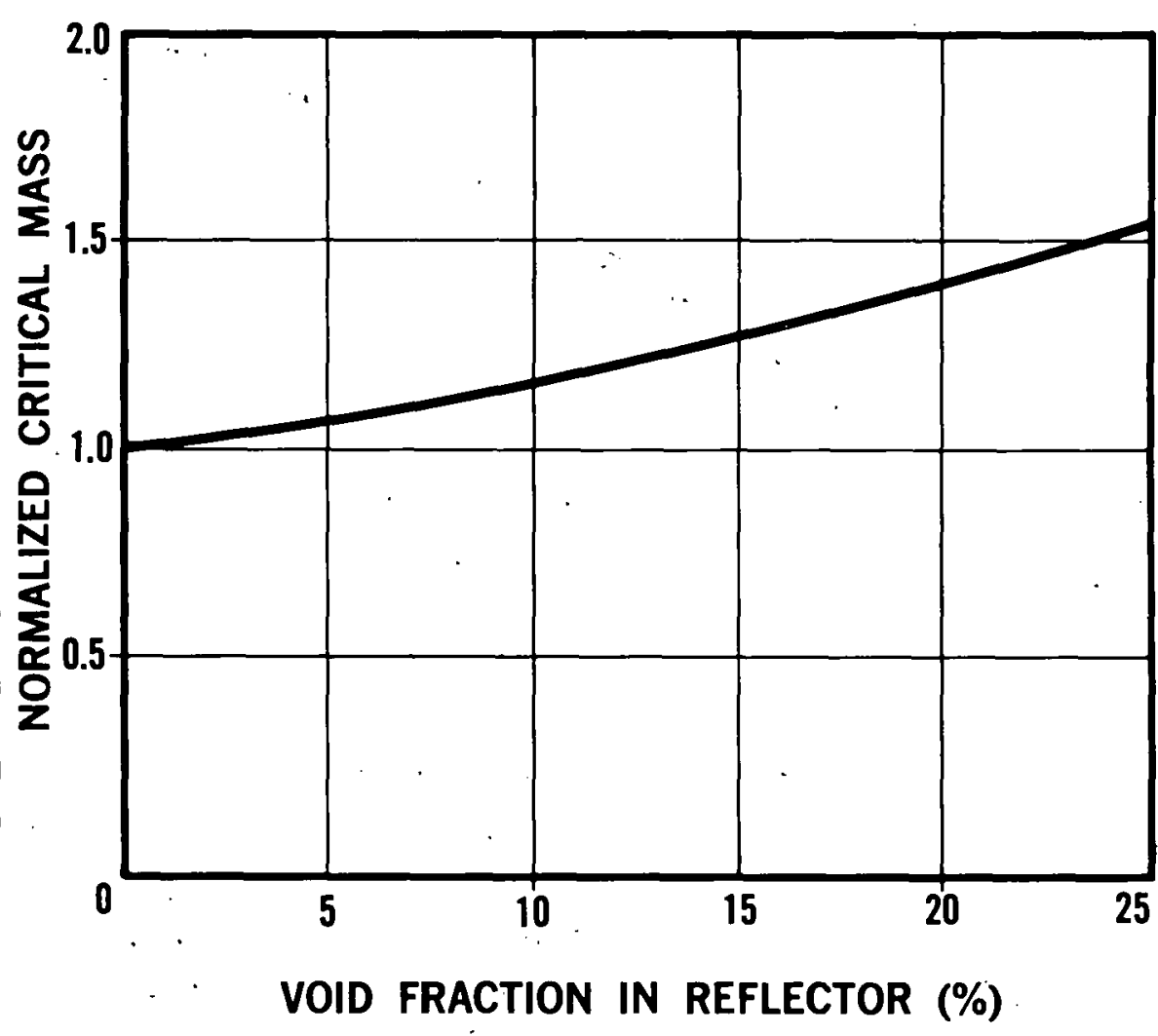

Figure 7 


\section{EFFECT OF EXHAUST NOZZLE ON CRITICALITY FOR A CYLINDRICAL CAVITY REACTOR}

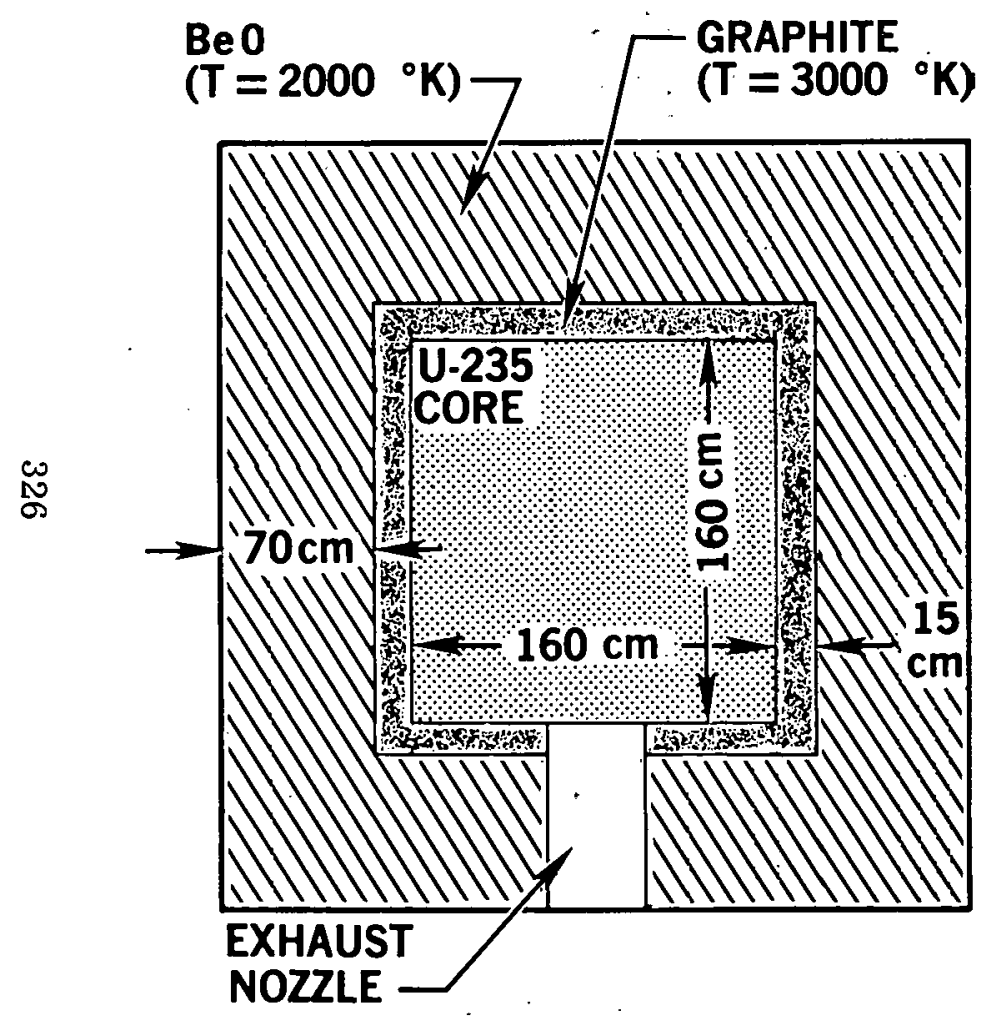

$T_{\text {NEUTRON }}=2800^{\circ} \mathrm{K}$

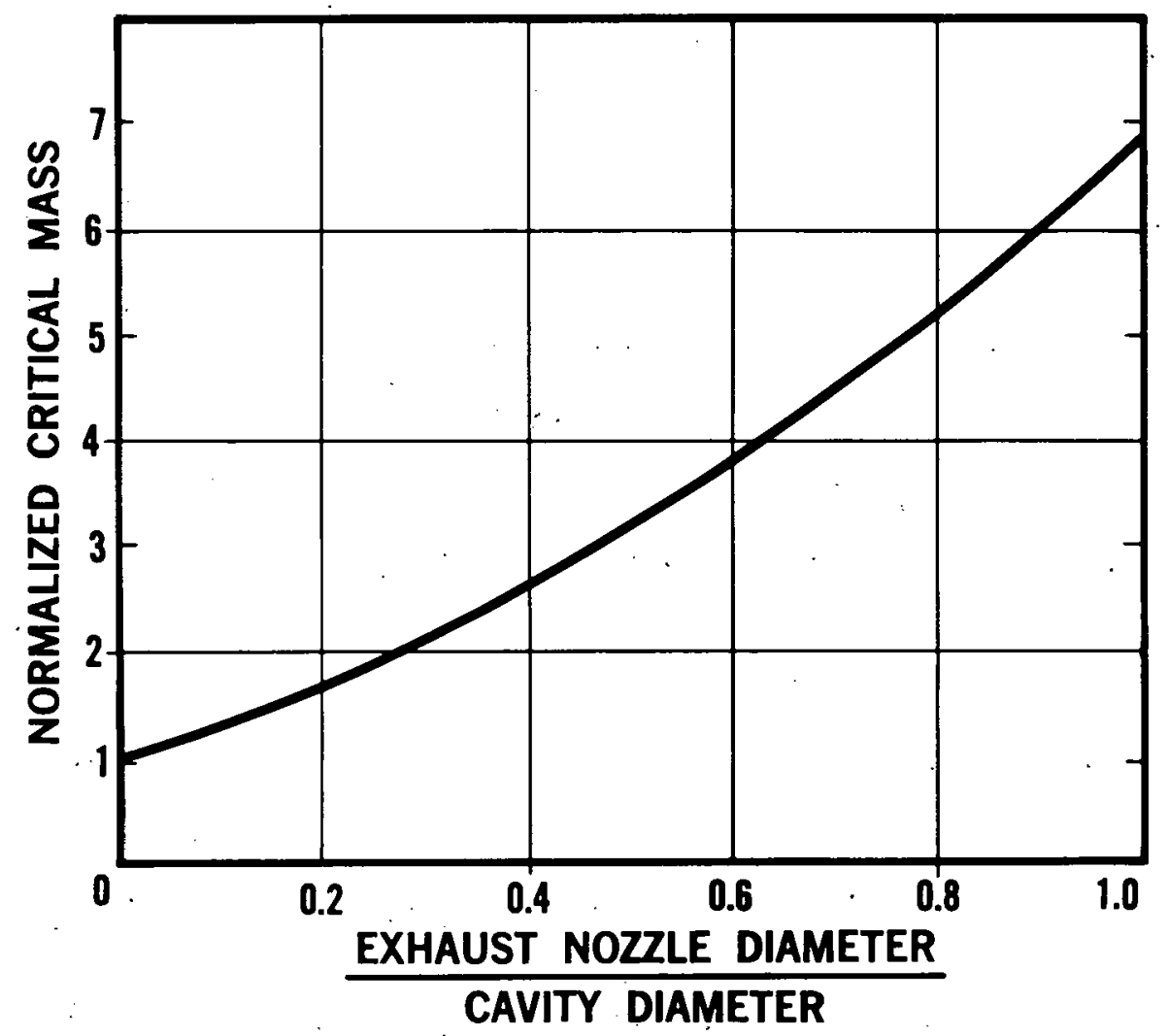

Figure 8 


\section{DESIGN MODEL FOR TWO-DIMENSIONAL ANALYSIS OF CAVITY REACTORS}

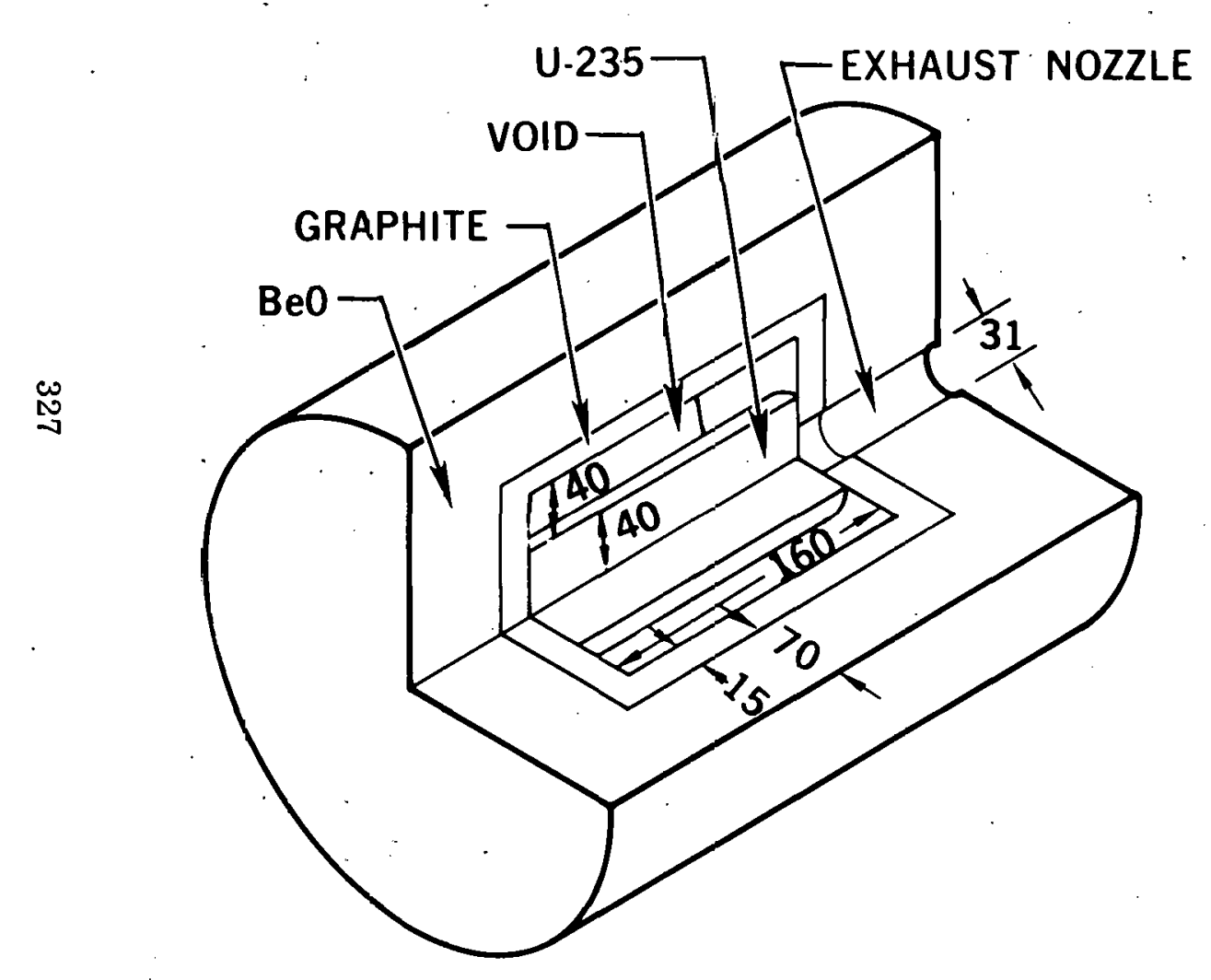

\section{REACTOR DESIGN SPECIFICATIONS}

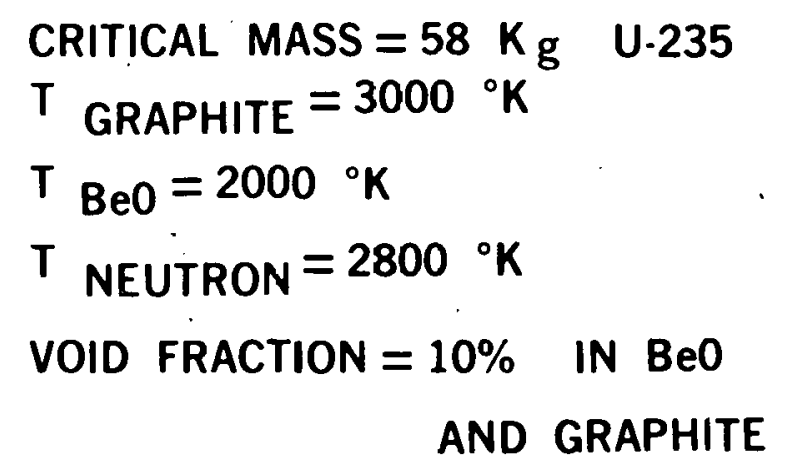

ALL DIMENSIONS IN CENTIMETERS

Figure 9 
had a critical mass of about $20 \mathrm{Kg}$ of U-235. If we applied the multiplicative superposition of the factors shown in normalized fashion, $37 \mathrm{Kg}$ of U-235 would have been the prediction. Of course this difference is due to the fact that as more fuel is added, self-shielding is increased, and the reactivity worth of fuel decreases very rapidly. This mode does not include provisions for any additional absorber. Any absorber in the reflector near the core would cause a further increase in critical mass. Are there any questions here?

Question: Have you any estimate of how -much increase there would be for more absorber?

Answer: For this particular case, a product of thermal absorption cross section times thickness of 0.025 yields an increase in critical mass of a factor of five. Now, this is very nonlinear, as I indicated. Smaller amounts of absorber would have proportionately less effect.

Question: Is this thermal absorption?

Answer: This is purely thermal absorption. Most of the leakage in these systems occurs at thermal energy.

Question: What happens if you go up to a temperature of $100,000^{\circ}$ for your fuel?

Answer: A $100,000^{\circ}$ fuel temperature would not reflect very greatly in the neutron temperature. The neutron temperature will be dominated by the reflector temperature. We did look at the Doppler effect for elevated core temperatures for the reaction of neutrons with uranium, and we found that this was a very minor effect.

We tried to establish the critical mass in order to know how much fuel was in the system. This yields the potential fuel loss for a given fuel loss time constant. Another factor will be the operation time needed in order to accomplish a given mission. If we are losing a core loading in $12 \mathrm{sec}$, for instance, and we can operate at a very high power level so that we can accomplish the mission in a few hundred seconds, it is much less serious than if a longer operation time was required. So in an attempt to define reasonable operating times in order to establish a criterion for an interesting hydrodynamic separation efficiency, we have looked at the limitation imposed by reflector heating. This was the basis in our nuclear analysis of selecting a reflector which had graphite as the inner liner and beryllium oxide outside of it. This is because the heating rate in the reflector will be almost proportional to the density of the reflector material.

Figure 10 shows the heating rate in the reflector as a function of 

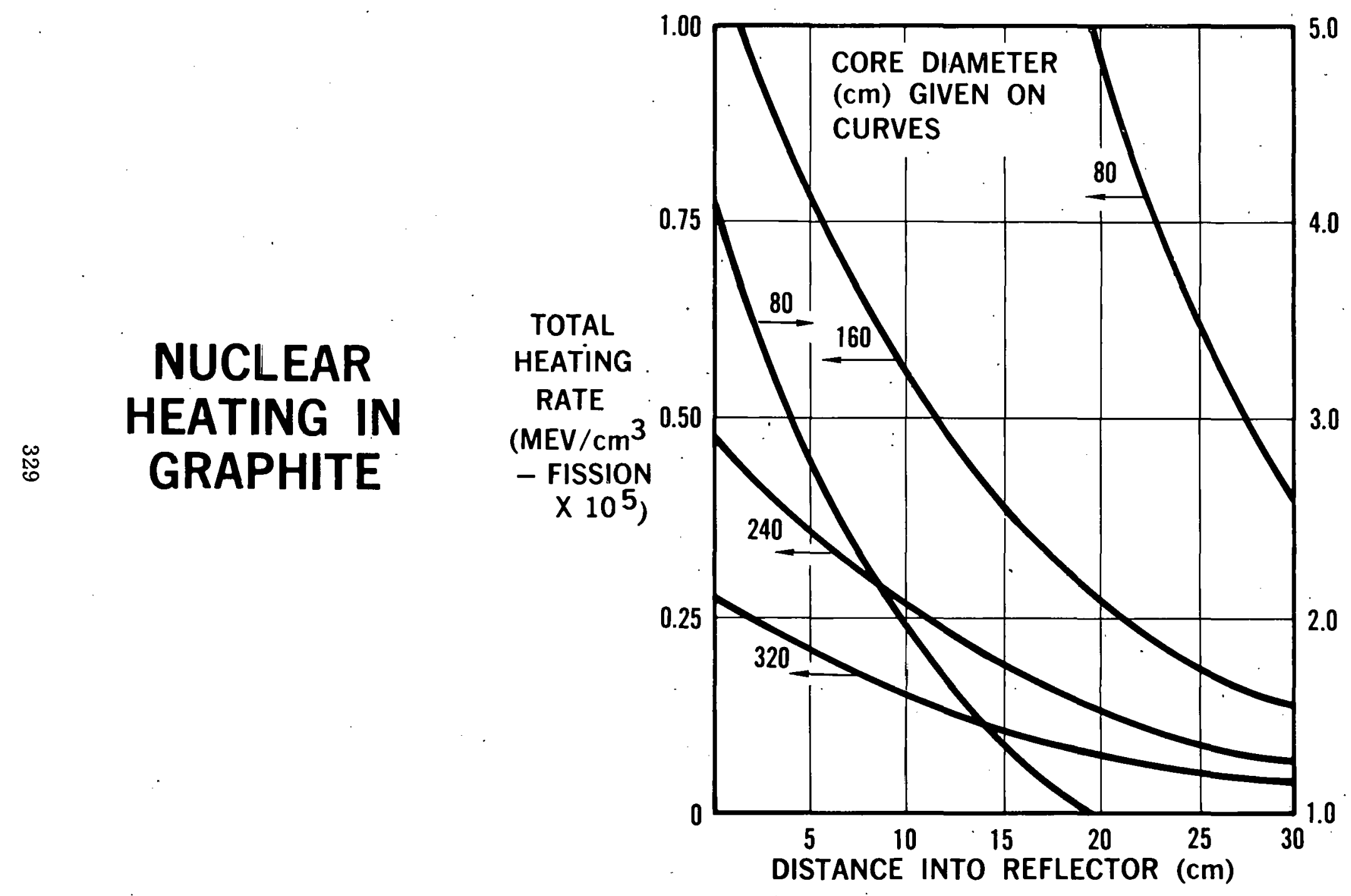

Figure 10 
distance into the reflector for different core diameters. This is done to see how the heating rate in the reflector affects the system as a function of size. This is presupposing at the moment that reflector heating will be the quantity limiting the maximum power of the device. The same analysis was also performed for the beryllium oxide reflector. Considering the heating due to neutrons and gammas and neglecting contributions due to thermal radiation, conduction, or convection and applying a reasonable capability for cooling the reflector allows us to determine the maximum reactor power as a function of core size. We also will evaluate the composite reflector, having a $15-\mathrm{cm}$ liner of graphite within $70 \mathrm{~cm}$ of beryllia. The reflector heating problem for that system will be taken to be the same as for graphite reflectors because in $15 \mathrm{~cm}$, heating has generally dropped off by about a factor of three which makes the heat removal problem in beryllia about comparable to that in the graphite liner.

Figure 11 shows this reactor power as a function of core size. The basis for reflector cooling capability was a preliminary design which was able to remove $2.2 \mathrm{KW} / \mathrm{cm}^{3}$; this maximum heating rate at the core reflector interface resulted from heat loads of 25 to $30 \mathrm{KW} / \mathrm{cm}^{2}$ on the reflector surface. Since this is very close to the Phoebus design, it was selected as a reference. So the rest of the result will be normalized to $2.2 \mathrm{KW} / \mathrm{cm}^{3}$. The maximum reactor power can be changed in proportion to the reflector cooling capability for other values. Selecting one value of specific impulse, here $2000 \mathrm{sec}$, and using the reactor power just found, Figure 12 shows the maximum thrust as a function of the core diameter. These devices run from something under a half million pounds to something around two million pounds thrust over the range of core diameters evaluated $(80-320 \mathrm{~cm})$. In order to establish the operation time required to accomplish a mission, it is necessary to calculate the propellant weight required to accomplish a given impulsive velocity mission.

Figure 13 shows the engine weights for a large number of systems that carry the reflector thickness as a parameter. This engine weight is only the weight of the moderator-reflector plus the weight of the pressure shell. Over most of the range, however, the pressure shell is not a large fraction.

Figure 14 shows the thrust-to-engine weight for these systems as a function of core diameter. For the beryllium oxide-graphite composite, the range is from one to four.

Figure 15 shows the propellant weight required for an impulsive velocity of $90,000 \mathrm{ft} / \mathrm{sec}$ with a specific impulse of $2000 \mathrm{sec}$. A modest payload was added to the engine weight corresponding to a particular core size. The payload used was $84,000 \mathrm{lb}$. This was held constant. These systems will all 


\section{MAXIMUM REACTOR POWER}

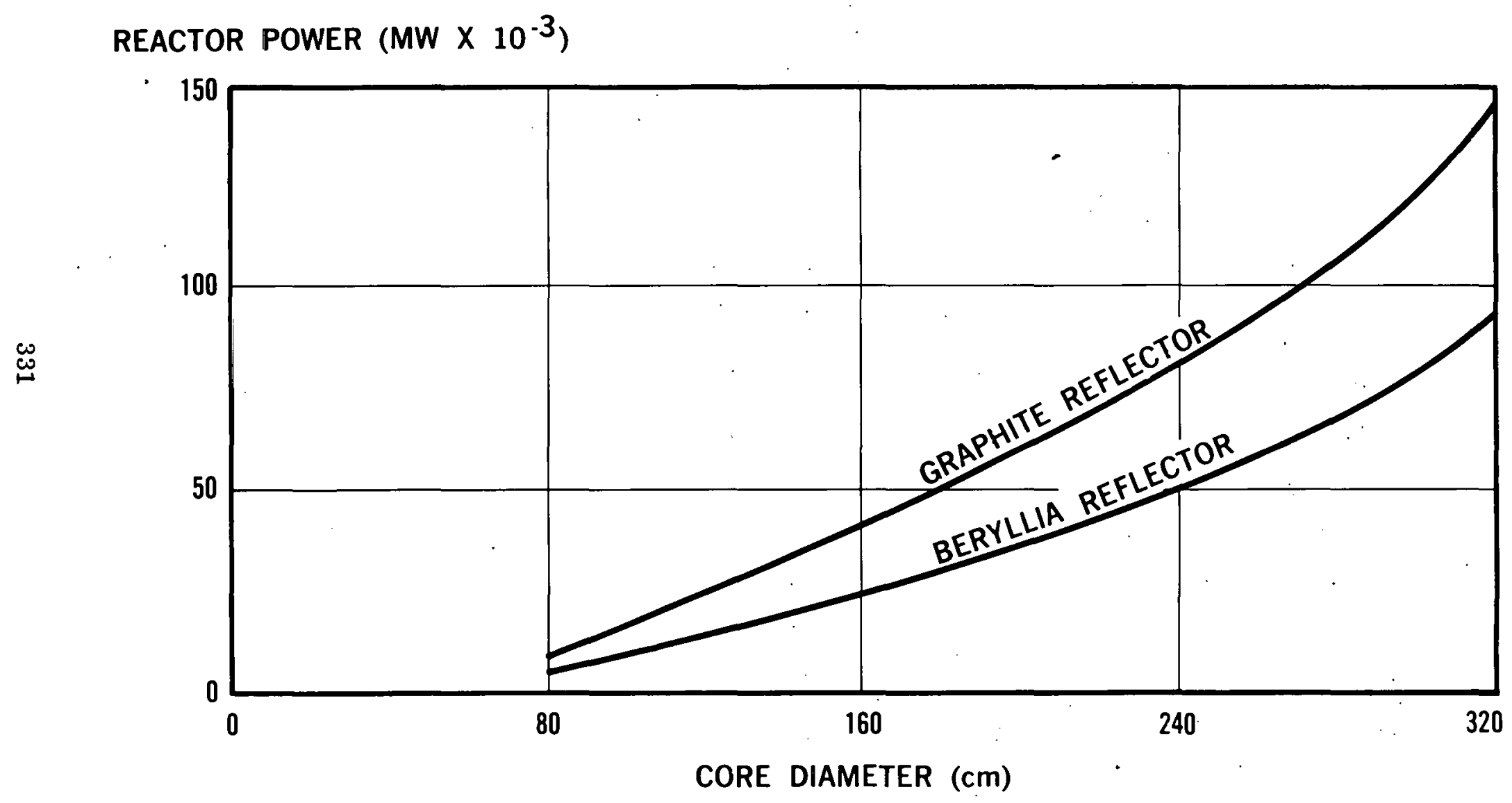

Figure 11 


\section{MAXIMUM 渠 THRUST LEVEL}

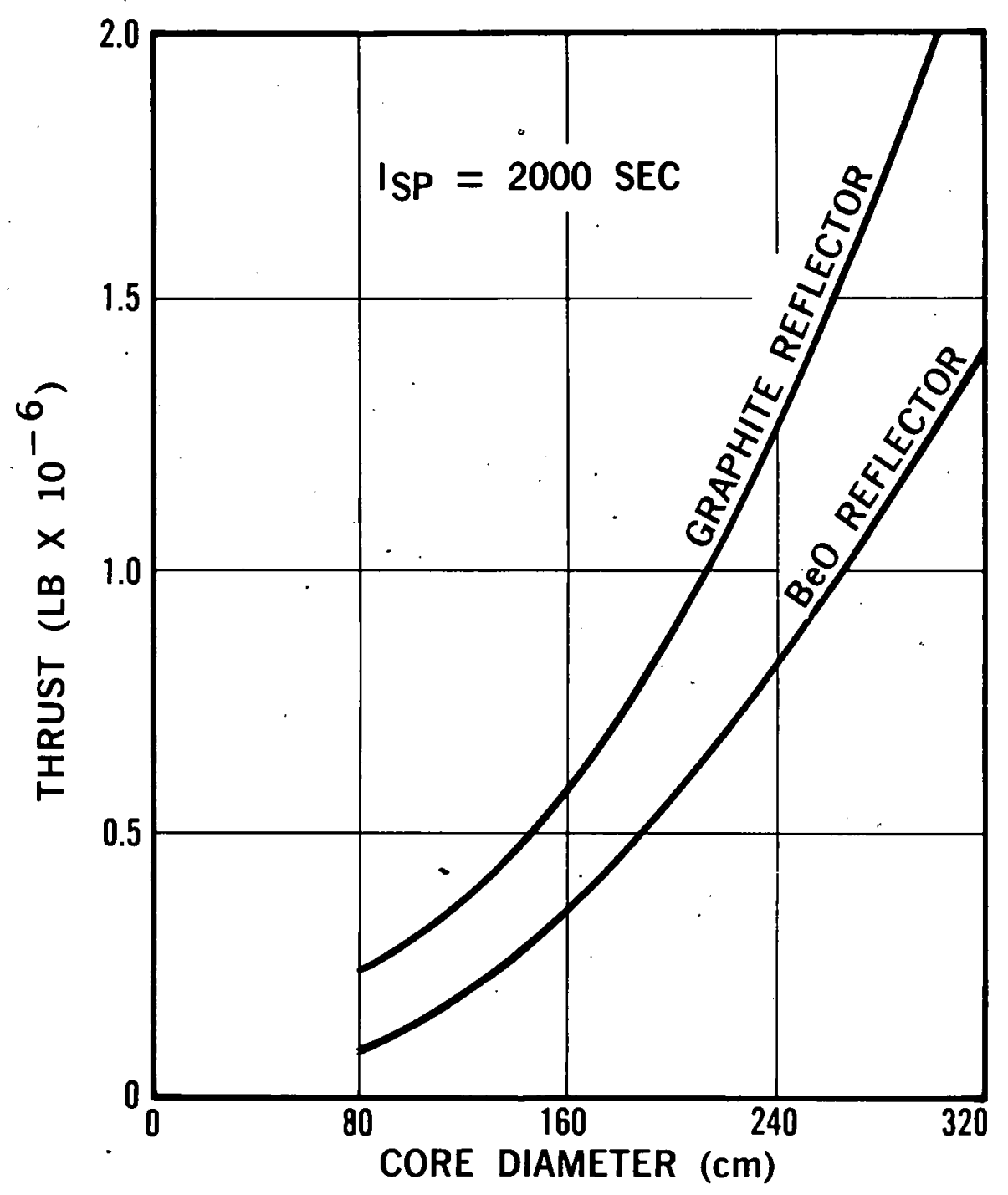

Figure 12 


\section{ENGINE WEIGHTS}

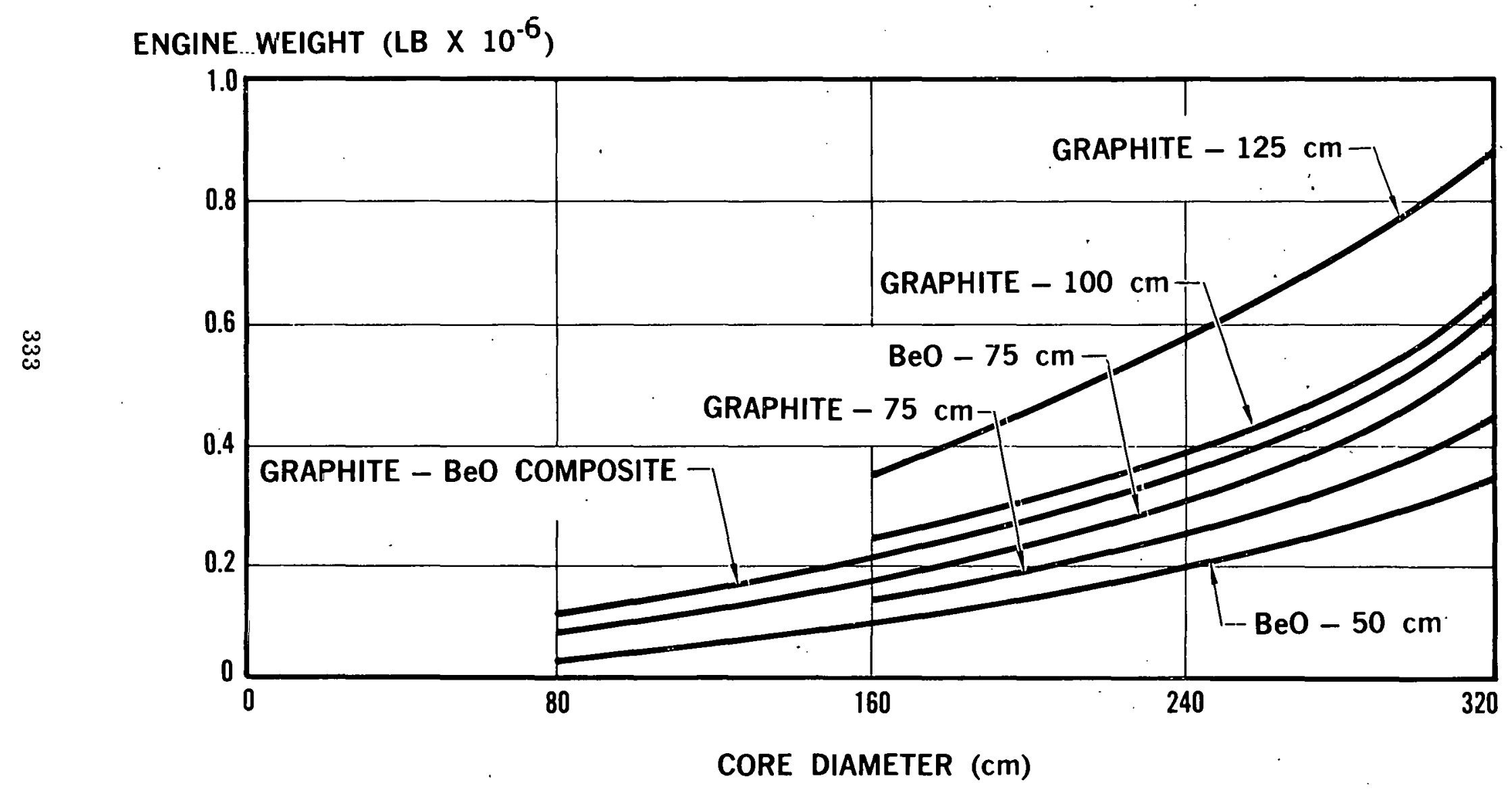

Figure 13 


\section{RATIO OF THRUST TO} ¿ ENGINE WEIGHT

$$
I_{S P}=2000 \mathrm{SEC}
$$

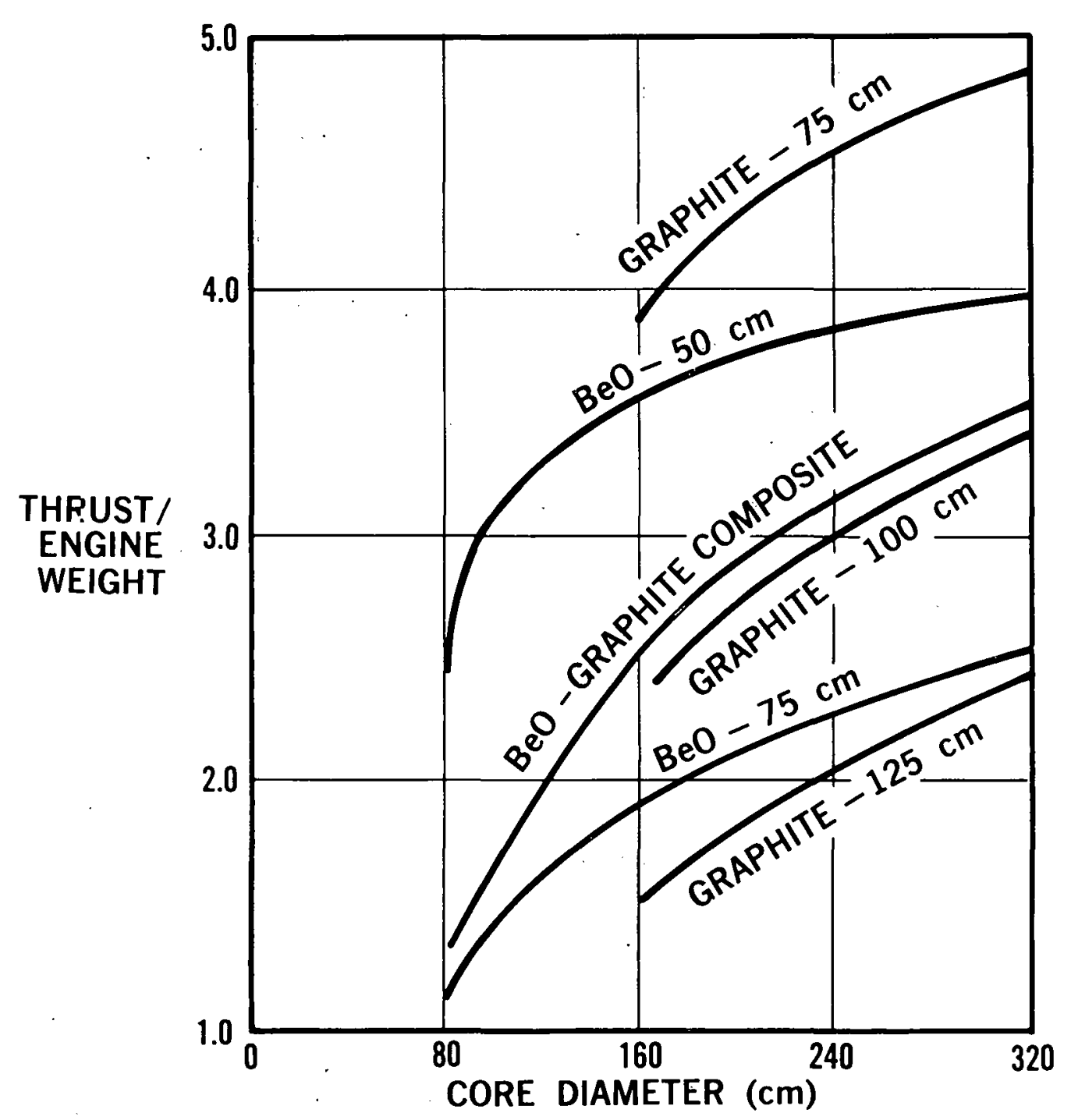

Figure 14 


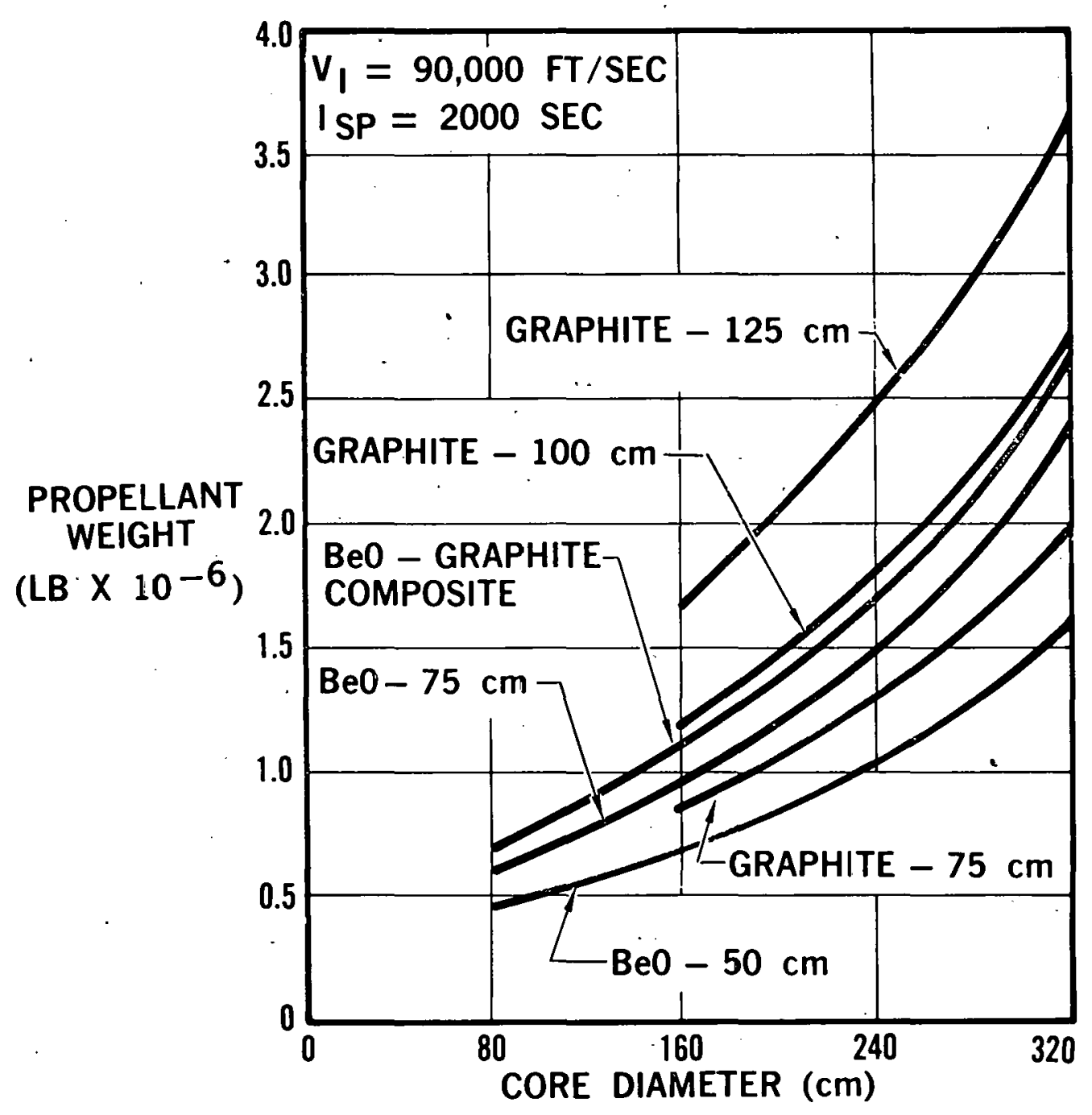


be evaluated for this one payload. Applying the earlier calculations of the reactor power, the enthalpy required to achieve $2000 \mathrm{sec}$, and the amount of propellant required to accomplish the mission, we now can define the operation time of the system. Figure 16 shows this result for an impulsive velocity mission of $90,000 \mathrm{ft} / \mathrm{sec}$. We can then combine this with the fuel content, determined from the nuclear analysis, to define the efficiency needed from the separation scheme to make it interesting. Operation times of at least $2000 \mathrm{sec}$ are required to accomplish this mission with large systems.

Figure 17 shows the thrust-to-takeoff weight with these restrictions. For the graphite-beryllium oxide composite, it is around 0.5 to 0.6 . For none of the systems is it as large as unity. Since this is done parametrically, the fact that it comes out less than unity should not have any particular significance. Only modest improvements in reflector cooling would be required to have a thrust to gross weight ratio exceeding unity.

Figure 18 shows the critical mass for these two-dimensional systems. The nozzle diameter was adjusted for each case to the size of the throat of the nozzle which would yield the thrust shown in Figure.12. The critical masses are then combined with the operating time to show what the fuel retention time has to be for an arbitrary fuel loss criterion, say $10^{-4}$ of the propellant flow. This is shown in Figure 19. Of course, this is an arbitrary criterion. For a different criterion, one can just ratio from these results. The graphite-beryllium oxide composite appears to be a favorable system with respect to fuel loss. This fuel retention time shown is really the reciprocal of the fuel loss time constant i.e., the time it takes to lose one critical fuel loading. This is asymptotic to about $2000 \mathrm{sec}$ for this particular criterion. I think it is interesting to see that it is really flattening out for large systems. We did not carry this any further because we thought that the system weight was becoming excessive. But, it is apparent that no significant further improvement can be expected as the core size is increased. Figure 19 showed the fuel retention time required for a criterion based upon the fraction of fuel-to-propellant in the exhaust. It is more interesting to look at an absolute amount of fuel as a criterion. Again, for normalization purposes, $100 \mathrm{Kg}$ is used as the amount of fuel loss. Figure 20 shows the required fuel retention time to lose only $100 \mathrm{Kg}$. This moves the optimum back toward the smaller size system. While; when the criterion was based on the fraction of fuel in the exhaust, performance improved, although with diminishing returns, as the system became larger. The reason for this is that for a given mission, more hydrogen was expended for the larger system. We expect that the optimum, as shown in Figure 20, which approximates the minimum critical mass case, will be more favorable than any other criterion. Figure 21 shows the fuel loss, in hundreds of kilograms, 


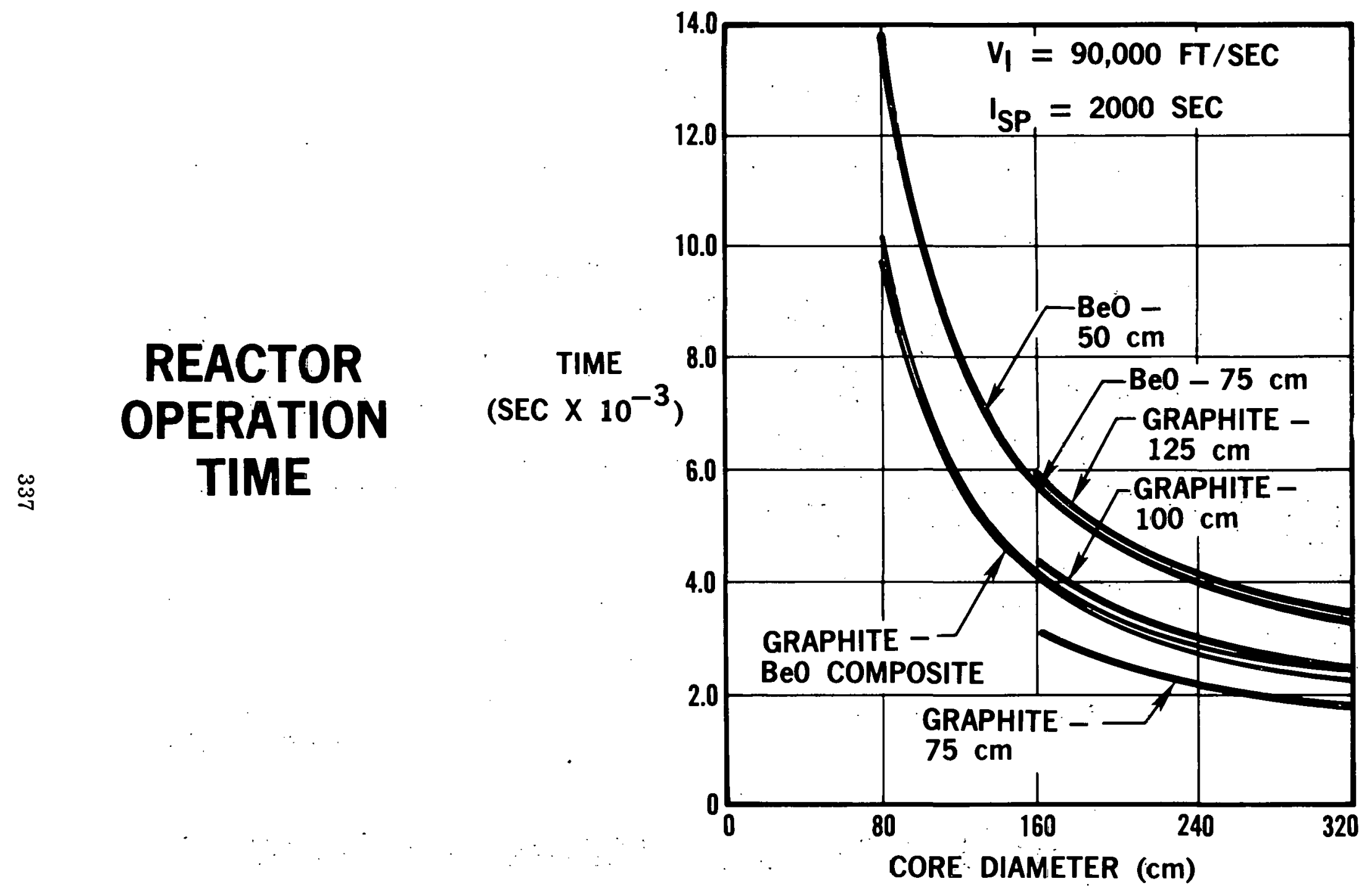

Figure 16 


\section{RATIO OF THRUST TO TAKE OFF WEIGHT}

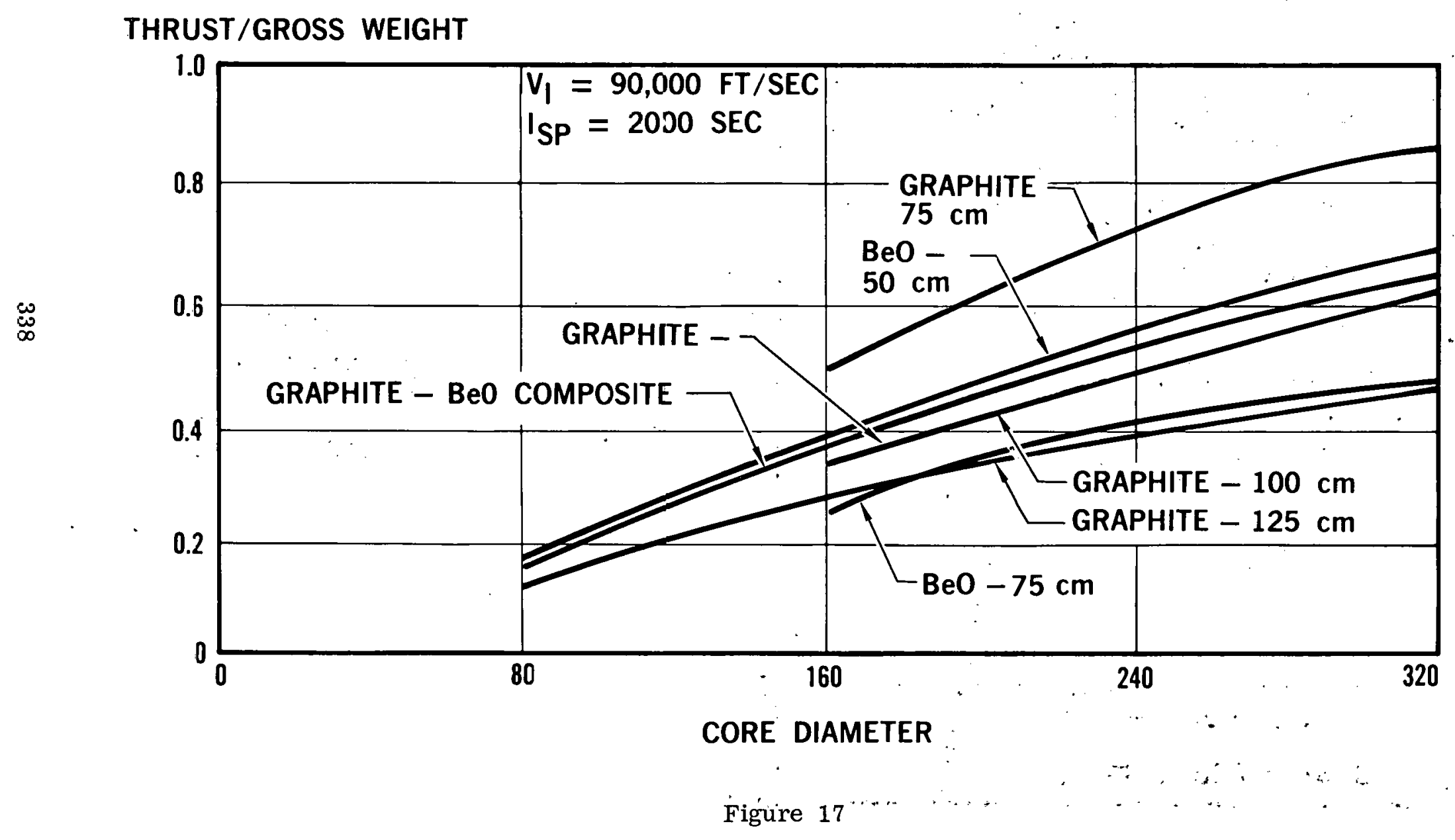



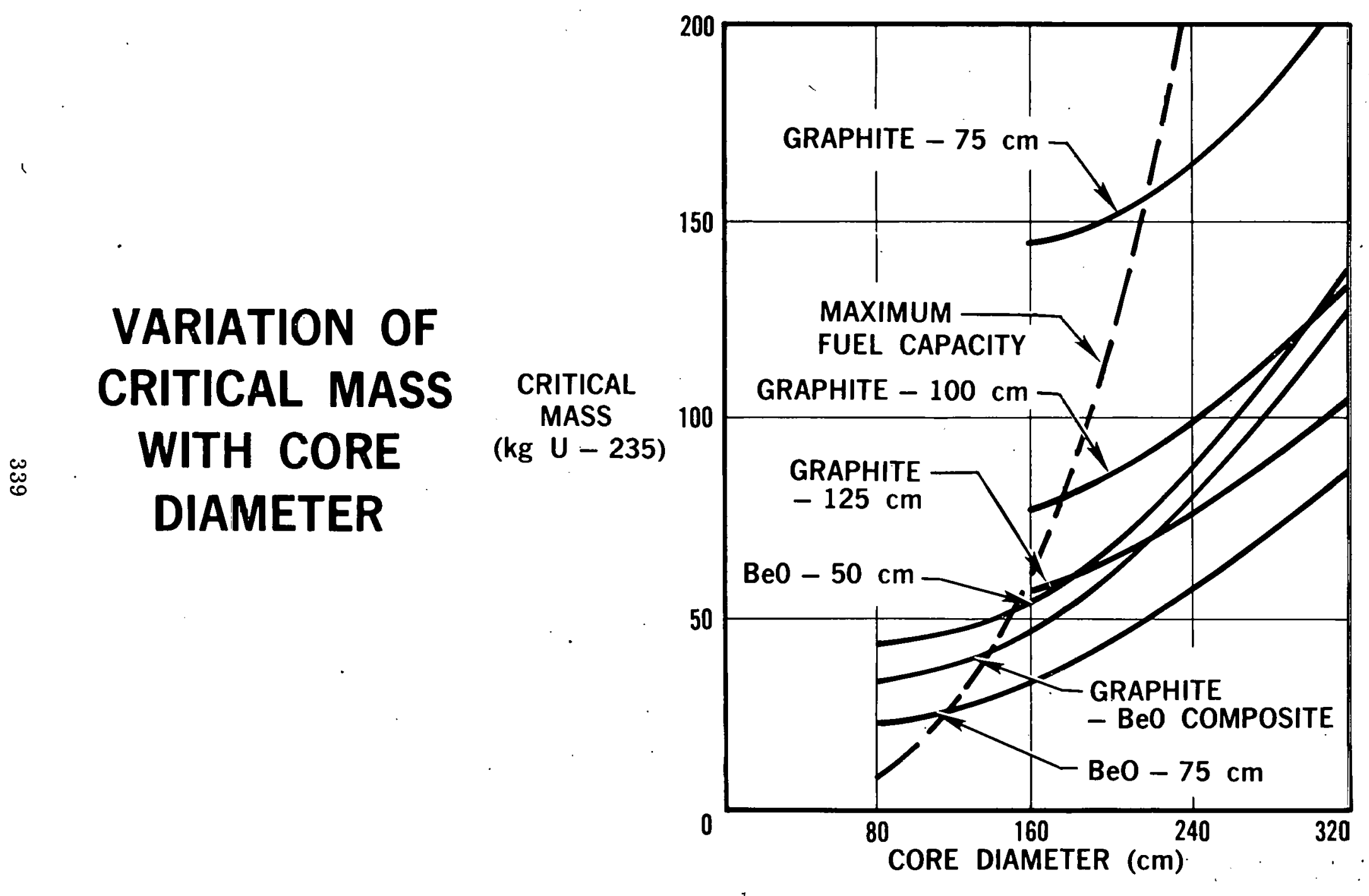

Figure 18 


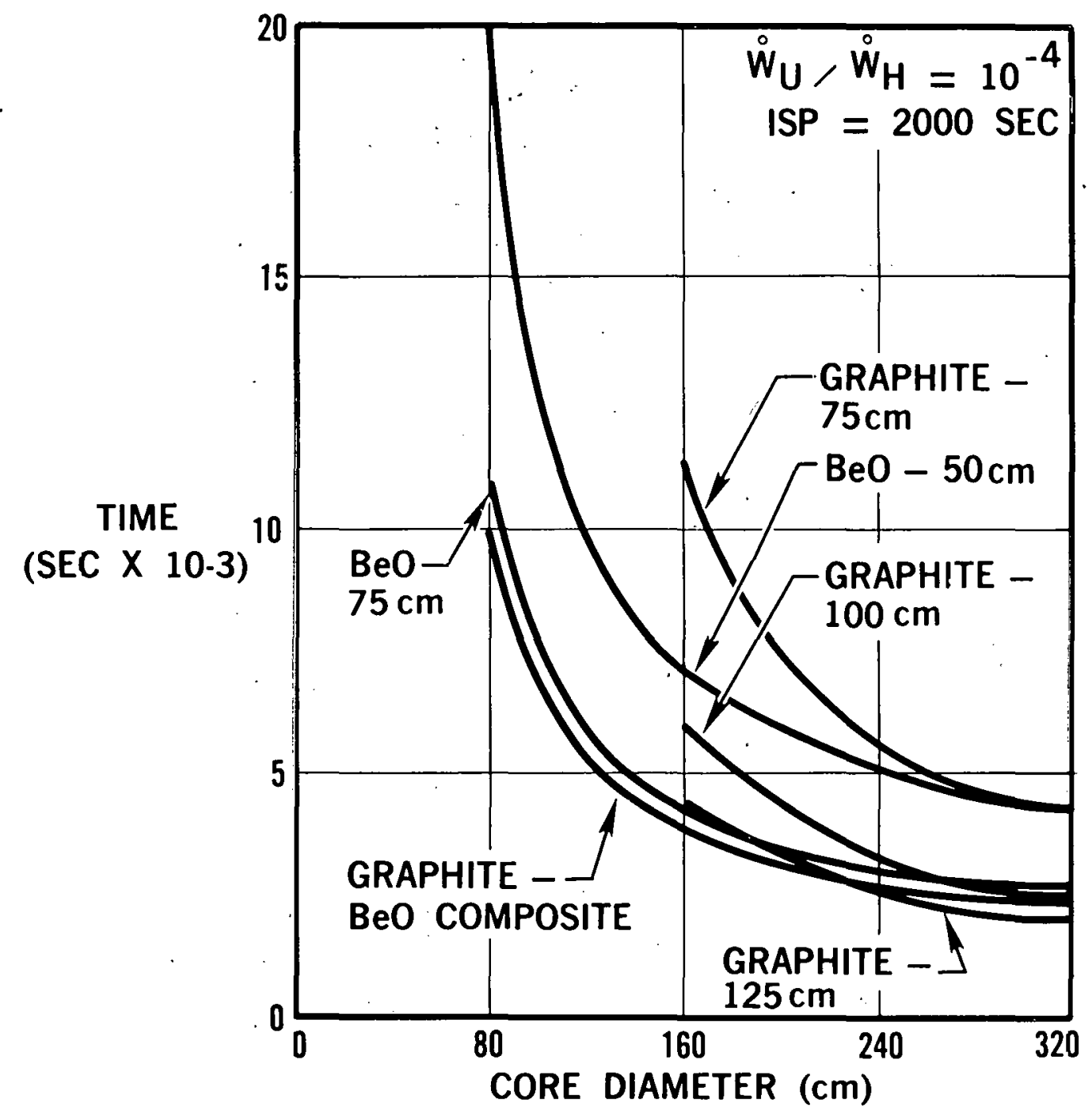

Figure 19 


\section{FUEL RETENTION TIME}

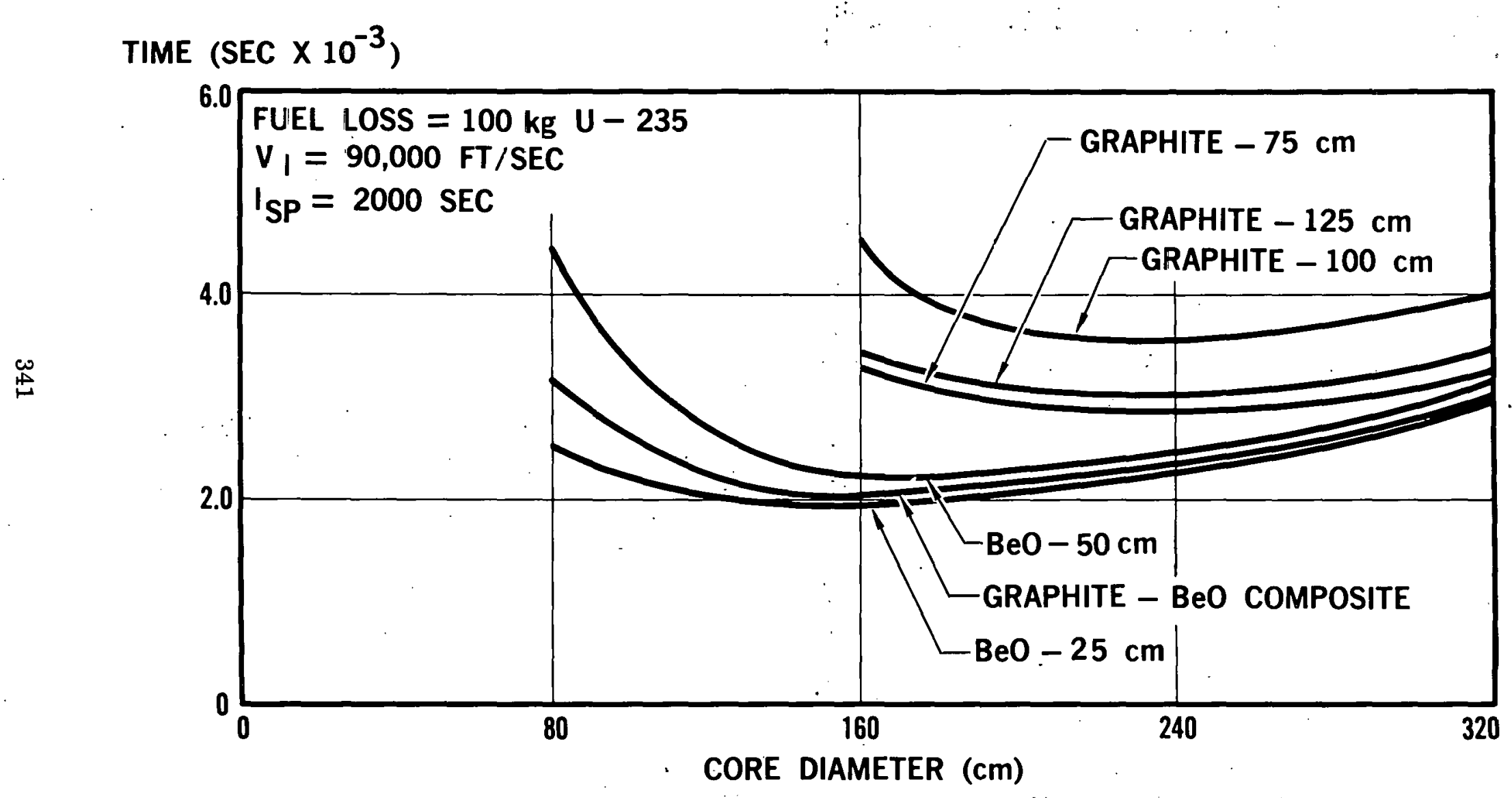

Figure 20 
FUEL LOSS VS RETENTION TIME

$\stackrel{W}{\stackrel{P}{N}}$

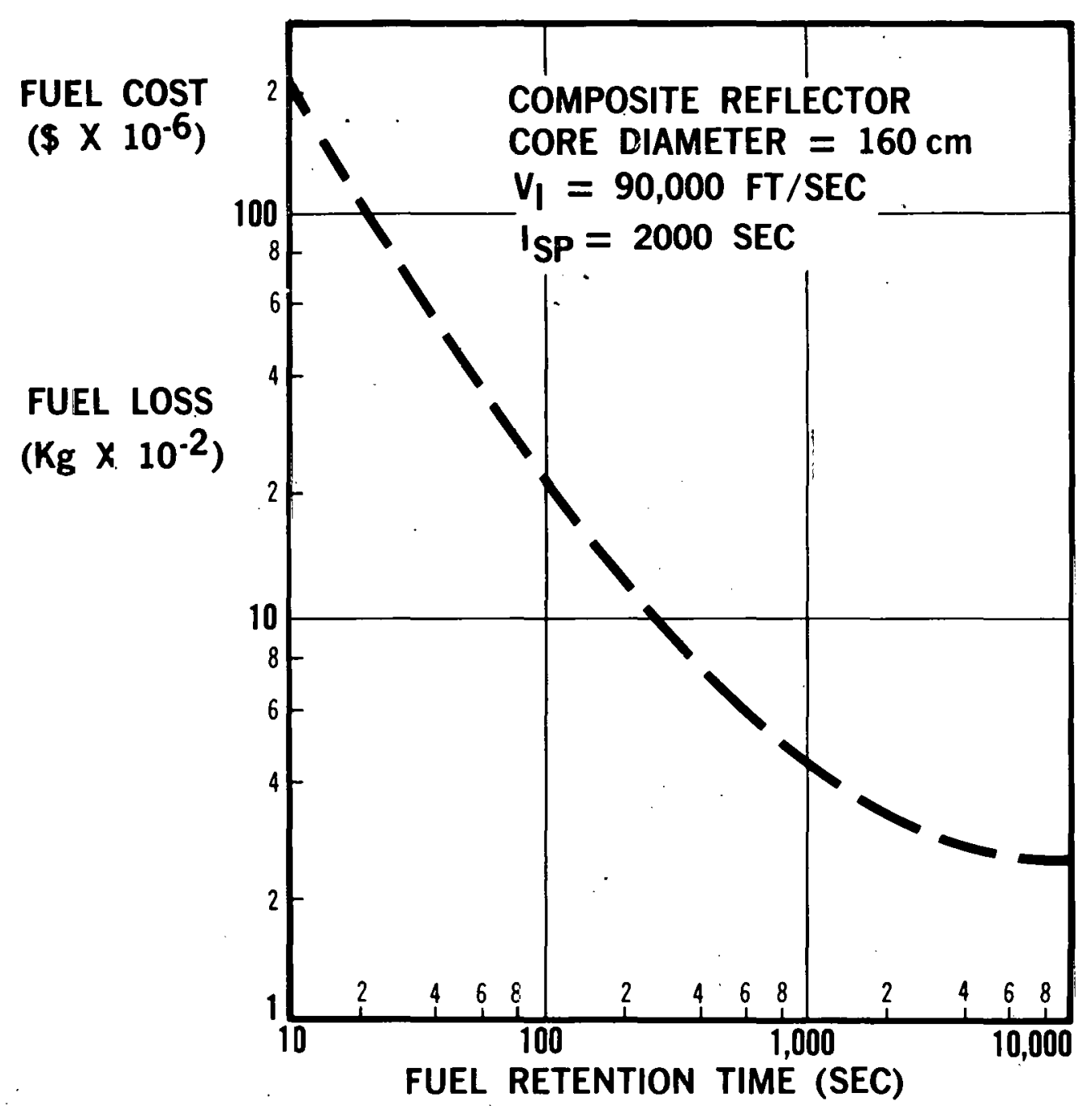

Figure 21 
and fuel cost, in millions of dollars, as a function of the fuel retention time. For fuel retention times around $10 \mathrm{sec}$, this becomes rather large $\$ 200,000,000$ for the fuel alone to accomplish this mission. As stated previously, we think that $\mathrm{U}-235$ is sufficient to evaluate the economics of this system. 


\section{CAVITY REACTOR}

George A. Jarvis

\section{Los Alamos Scientific Laboratory \\ Los Alamos, New Mexico}

\section{MEASUREMENTS WITH URANIUM-GRAPHITE FUEL RODS}

A series of measurements are currently being carried out on the $\mathrm{D}_{2} \mathrm{O}-$ reflected cavity reactor mockup. These measurements are of interest to groups working on gaseous core propulsion reactor concepts, and were initiated at the request of the NASA Lewis Propulsion Laboratory and others. The measurements underway and planned include the following:

1. Fuel Distribution Studies. Measure the effect of decreasing the fuel region diameter within a fixed diameter cavity on critical mass and flux distribution.

2. Struclusal Maleridals. Study the effect of cavity-moderator interface structural materials on critical mass and flux distribution.

3. Nozzle Mockups. Insert voids in the end $\mathrm{D}_{2} \mathrm{O}$ reflector tank to mockup rocket nozzles and measure effect on criticality and flux distribution.

4. Reflector Heating. Study the effect on critical mass and thermal flux distribution when the $\mathrm{D}_{2} \mathrm{O}$ is heated.

5. Composite Reflector. Study the effect on critical máss when a composite reflector is used. Beryllium and graphite cavity liners are being considered.

6. Plutonium vs uranium as fuel.

Figure 1 shows the essential geometry and dimensions of the $\mathrm{D}_{2} \mathrm{O}-$ reflected cavity reactor mockup. Figure 2 shows the fuel element support fixture resting on the lower $\mathrm{D}_{2} \mathrm{O}$ reflector plug tank.

Critical mass determinations have been made for four different fuel 


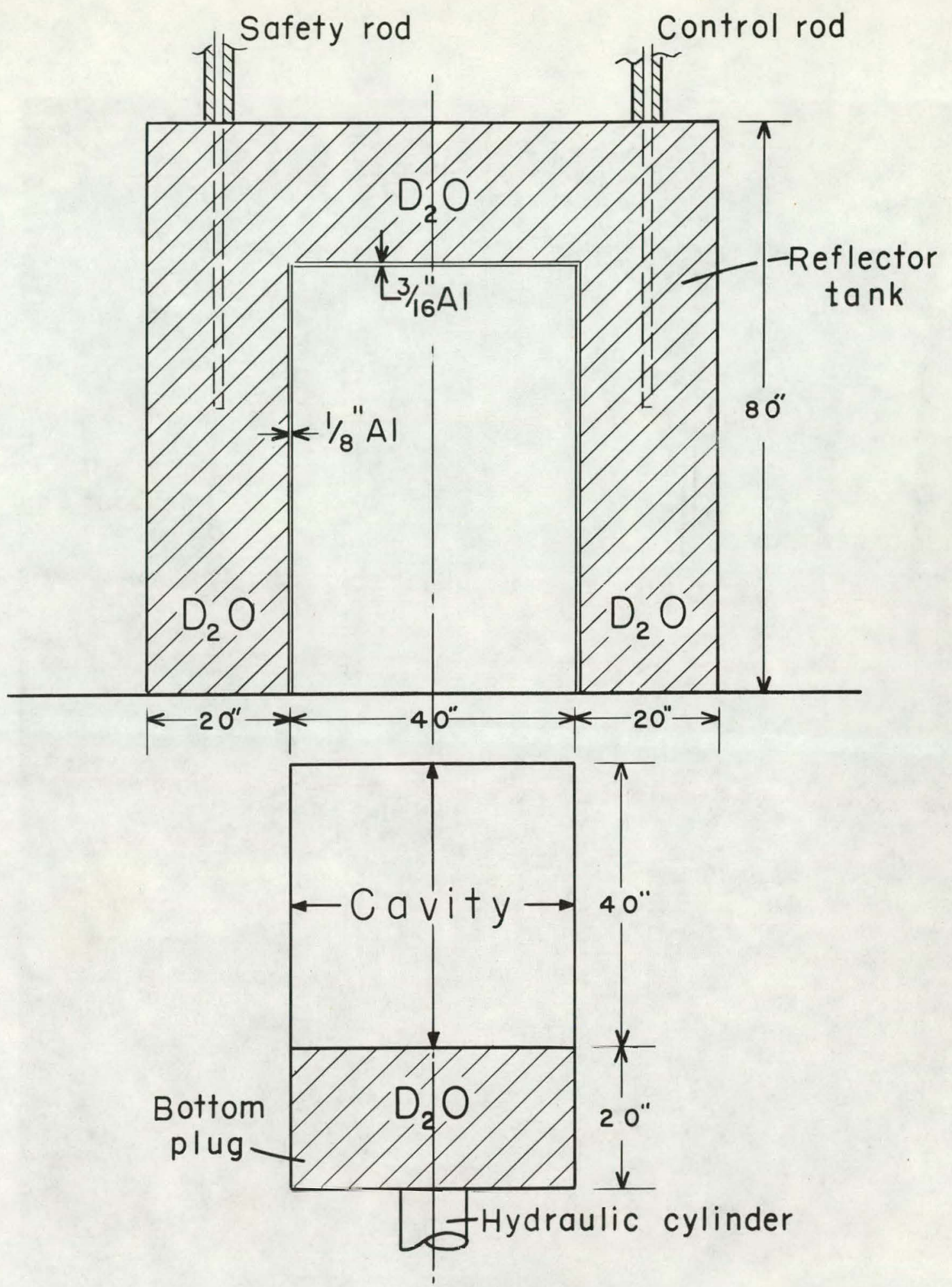

Figure 1. Basic Cavity Assembly Schematic 


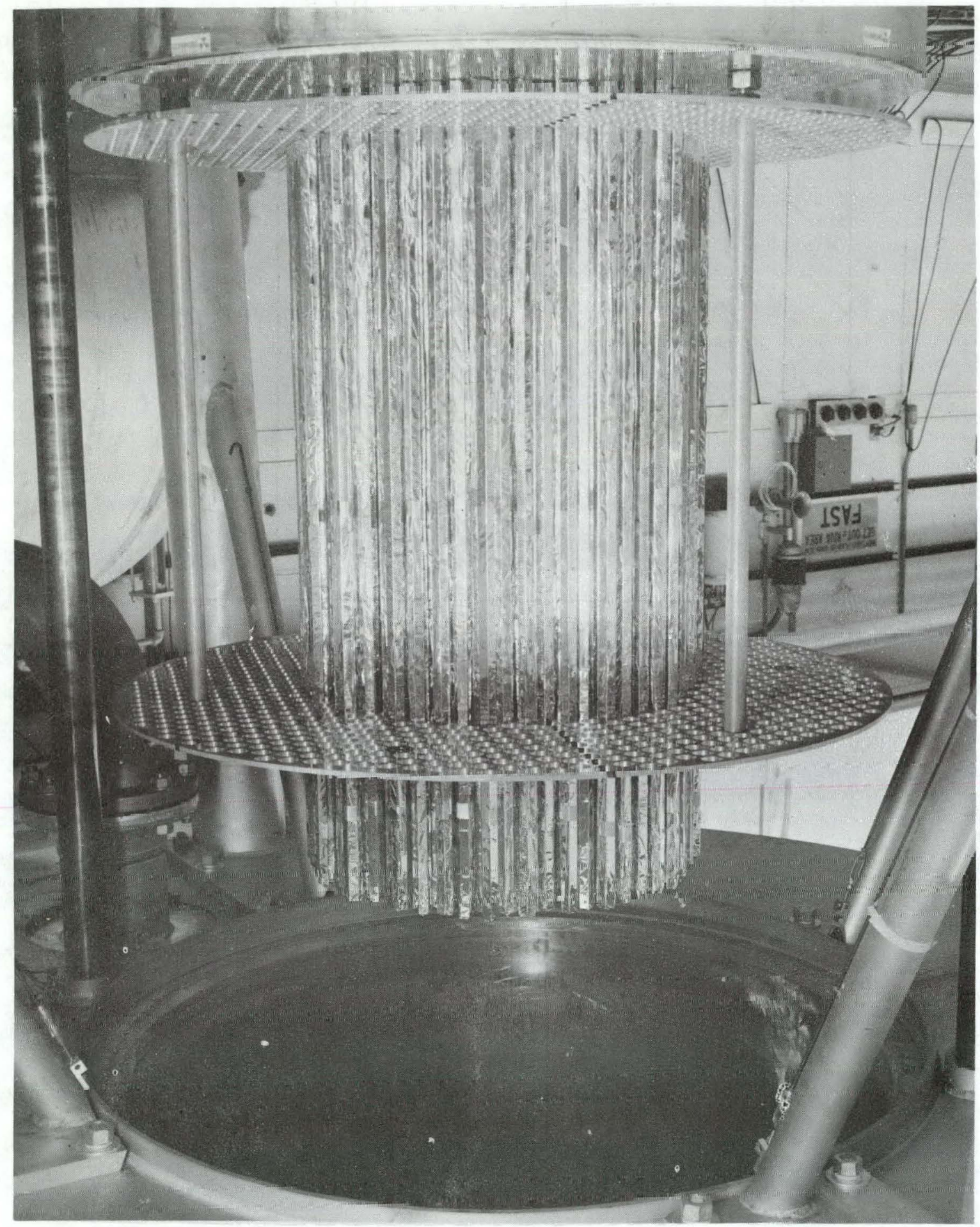

Figure 2. Fuel Element Support Fixture Resting on Reflector Plug Tank 
distributions. Figures 3, 4, 5, and 6 indicate the fuel distribution for each of these cases. Table I summarizes the results obtained in these measurements. Most significant is the rapid rise in the critical mass with decreasing fuel zone radius. Measurements to determine the fuel penalty for the aluminum in the fuel support fixture and cavity-reflector interface walls are underway but incomplete. The results of these determinations will reduce the critical masses shown in Table I. Flux survey measurements are now ready to proceed using autoradiography of irradiated thin uranium foil strips. The $\mathrm{D}_{2} \mathrm{O}$ heating equipment, needed for the temperature coefficient measurement, is ready for installation.

\section{NOZZLE EXPERIMENT}

An experiment was carried out to evaluate the effect of introducing a nozzle in one end of the cavity reactor. For this purpose, several empty cylindrical tanks (ranging in diameter from 4 to 10 inches) were placed in the lower end reflector, and the critical mass for each configuration determined.

Figure 7 shows the fuel penalty determined as a function of orifice diameter.

The fuel was in the form of 3-mil uranium foil lining the surface of the cavity.

\section{$\mathrm{D}_{2} \mathrm{O}$ HEATING EXPERIMENT}

An experiment has been carried out to determine the reactivity change as a function of the reflector $\mathrm{D}_{2} \mathrm{O}$ temperature. For this purpose, a heatexchanger 1oop was installed in the storage tank for the annular reflectur $\mathrm{D}_{2} \mathrm{O}$. A $600,000 \mathrm{BTU}$ gas fired heater was connected to the heat exchanger, permitting an approximate $5^{\circ} \mathrm{C}$ per hour temperature rise in the $\mathrm{D}_{2} \mathrm{O}$. Although there was no attempt to heat the fixed reflector in the end plug tanks, the $\mathrm{D}_{2} \mathrm{O}$ in the upper tank did increase somewhat in temperature due to the proximity of the hot annular reflector.

The $\mathrm{D}_{2} \mathrm{O}$ in the storage tank was heated to about $61^{\circ} \mathrm{C}$, the maximum temperature obtained; pumped into the annular reflector tank; and the delayed critical condition determined. The $\mathrm{D}_{2} \mathrm{O}$ was returned to the storage tank and cooled to $44^{\circ} \mathrm{C}$ by running cold water through the heat exchanger. At this temperature, the delayed critical position of the control rod was again established. This measurement was repeated for two successively 
Table I

\section{Critical conditions (for control rod withdrawn)}

Fuel element distribution ${ }^{a}$

No. 1

Cylindrical shell (see fig. 3)

No. 2

Uniform distribution (see fig. 4)

No. 3

Half density loading in central region (see fig. 5)

No. 4

Maximum density loading in central region

(see fig. 6)
Number of

radius in inctes

fuel elements

92.0

95.8

8.6

36.7

$12.5^{\prime \prime}$

186.9

16.7

71.6

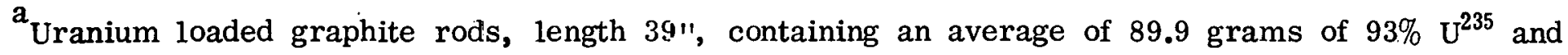
383.3 grams $C$.

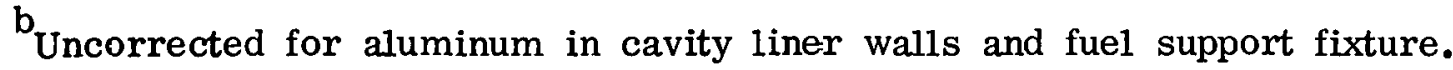




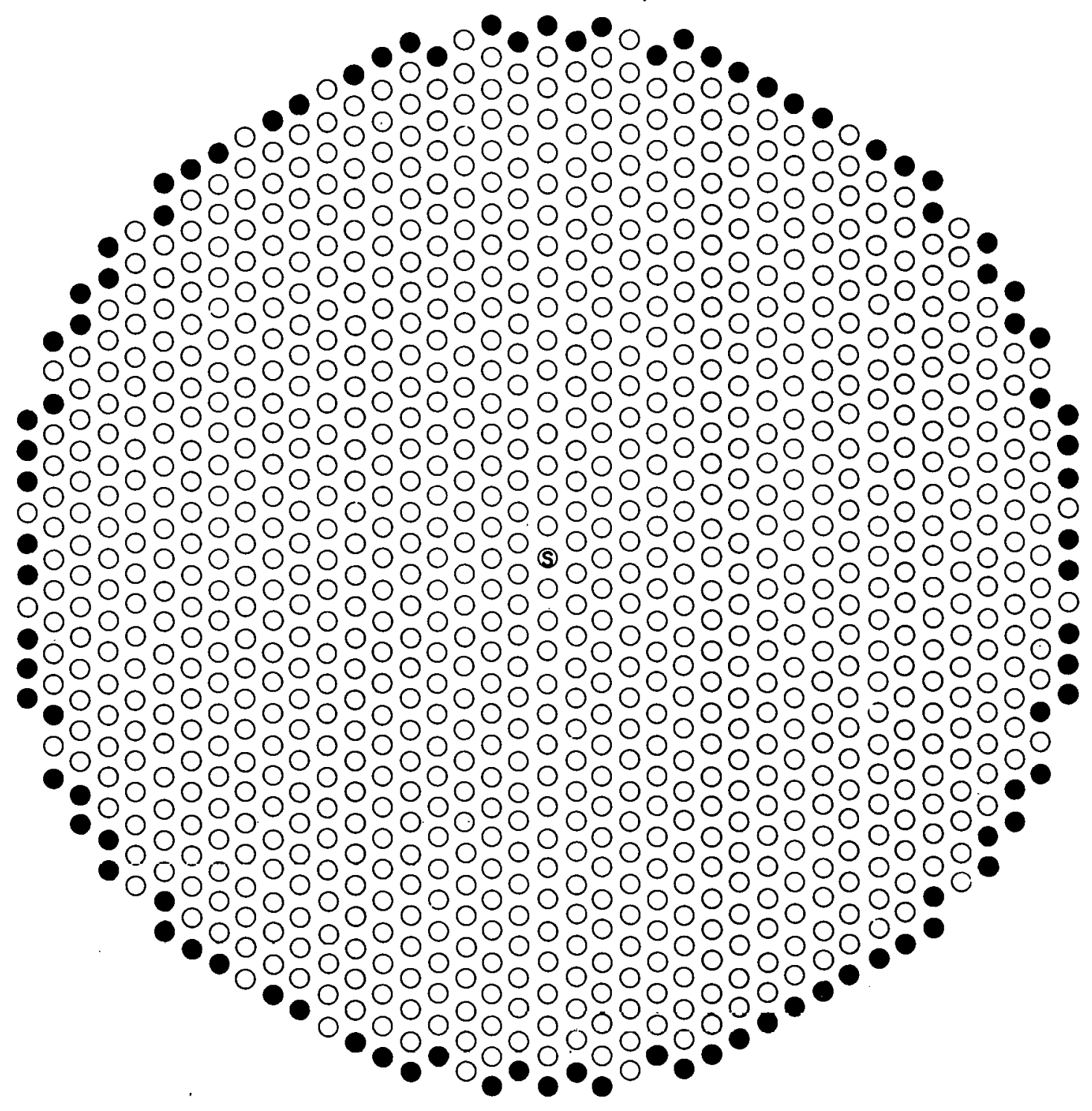

Figure 3. Fuel Element Distribution for Experimental Fuel Loading No. 1, Approximating Cylindrical Shell Loading of 90 Fuel Elements. Cavity Radius $=20 "$, Fuel Zone Radius $=\sim 19 "$, Fuel Element Length $=39 "$, Cavity Length $=40 "$. 


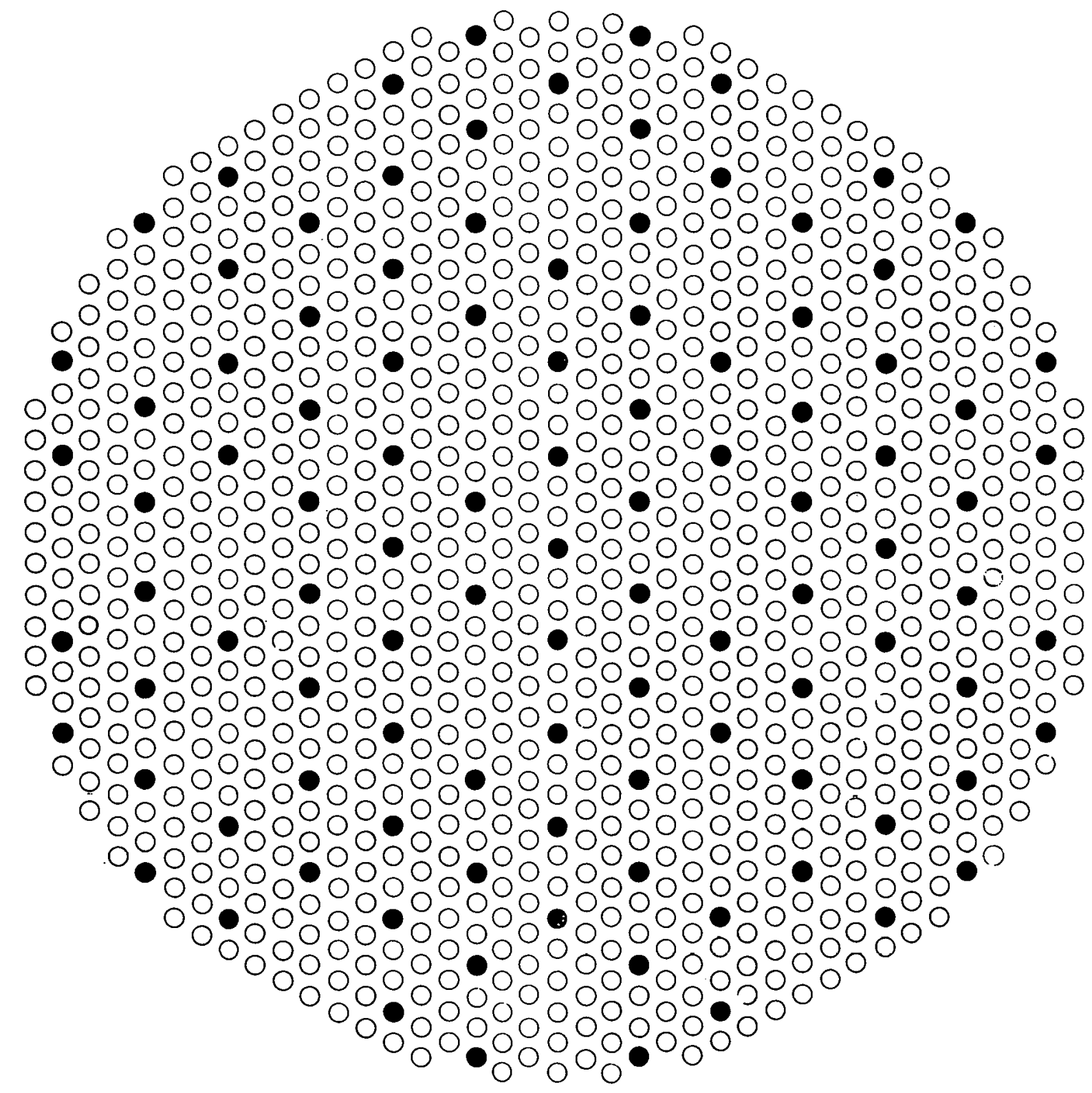

Figure 4. Fuel Element Distribution for Experimental Fuel Loading No. 2, Approximately Uniform Loading of 103 Elements. Fuel Zone Radius $=19 "$. 


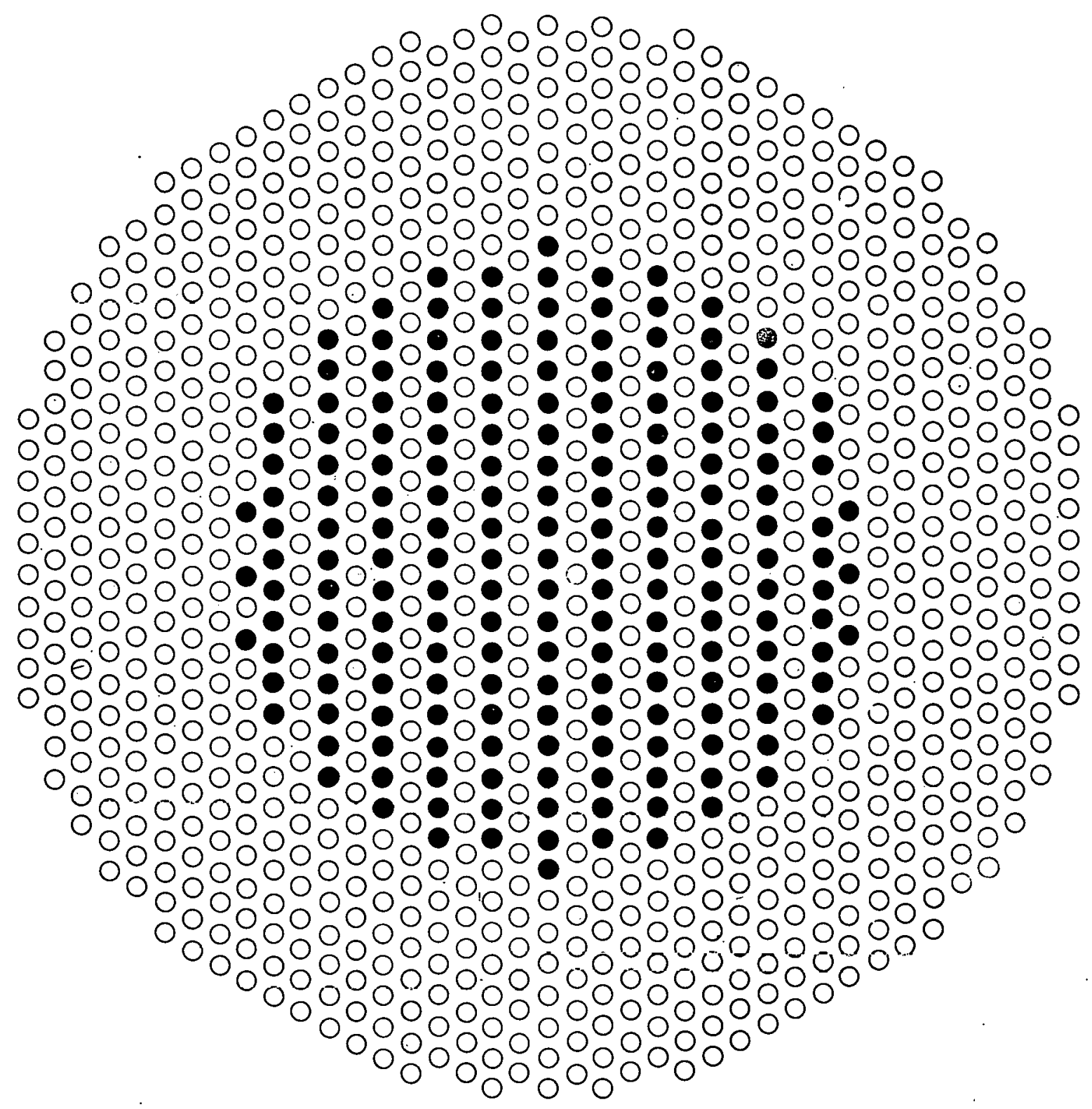

Figure 5. Fuel Element Distribution for Experimental Fuel Loading No. 3, Half Density Loading in Central Zone. Fuel Zone Radius $=\sim 12.5$ ". 


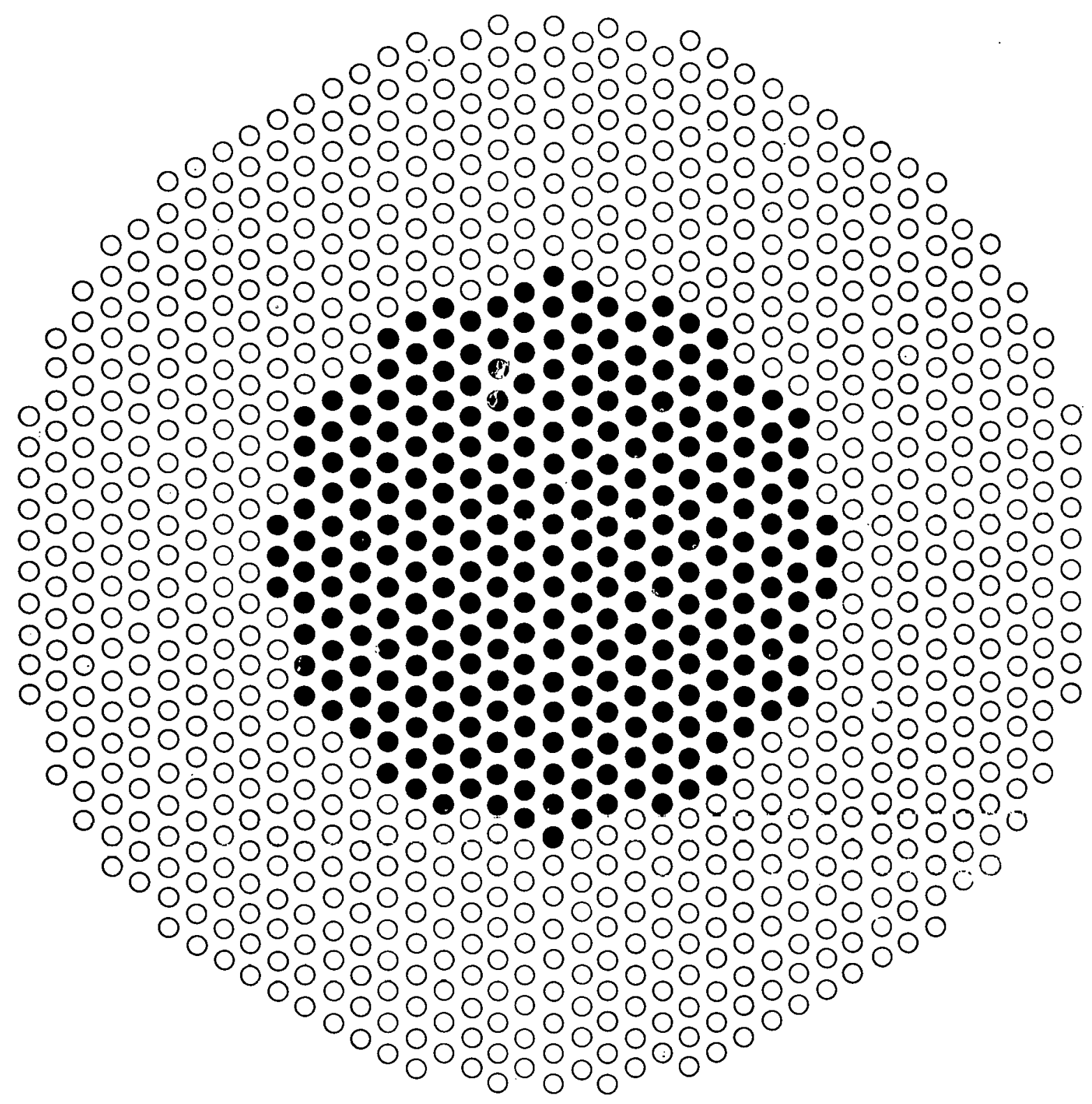

Figure 6. Fuel Element Distribution for Experimental Fuel Loading No. 4, Maximum Density Loading in Central Zone. Fuel Zone Radius = $\sim 11.6 "$. 


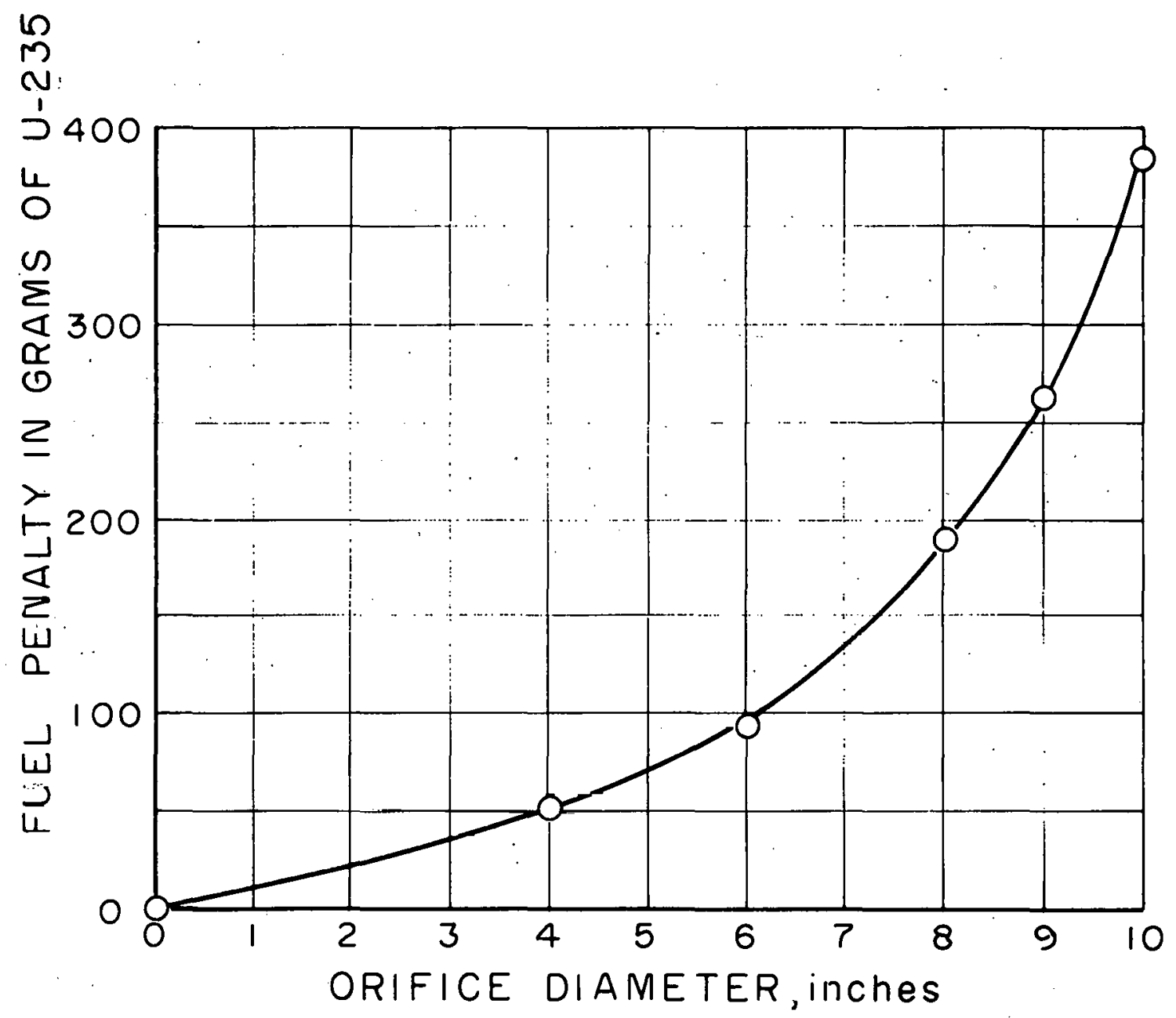

Figure 7. Fuel Configuration - Cavity Lined with 3-mil Uranium Foil, Enriched to $93.1 \% \mathrm{U}-235$. Critical Mass $=5.886 \mathrm{Kg} \mathrm{U}-235$. 
lower temperatures of the $\mathrm{D}_{2} \mathrm{O}$. Figure 8 illustrates the geometry of the $\mathrm{D}_{2} \mathrm{O}$ reflector tanks, gives cavity fuel loading and temperatures of the $\mathrm{D}_{2} \mathrm{O}$ in the reflector tanks for the several criticality determinations, and presents a curve showing the reactivity penalty in grams of $U^{235}$ vs temperature rise in degrees centigrade of the $\mathrm{D}_{2} \mathrm{O}$ in the annular reflector tank.

\section{CRITICAL MASSES OF VARIOUS FUEL ZONES}

A series of critical mass determinations as a function of fuel zone. radius have been made with the $\mathrm{D}_{2} \mathrm{O}$ reflected cavity reactor mockup. Uranium loaded graphite elements were distributed within four different radii, both uniformly and as cylindrical shells.

The fuel loading patterns are shown in Figure 9. Figure 10 gives a summary of the critical mass values as a function of fuel zone radius for the two types of stackings studied. The critical mass rises rapidly as radii are decreased below about two thirds of the cavity radius.

An experiment was performed to determine the fuel penalty resulting from shifting the fuel zone off axis. Stacking type (4-b) having a radius of about 12 inches was used. Measurements were made with the fuel zone shifted out to the position shown in Figure 9, where the fuel became tangent to the cylindrical cavity wall. Figure 11 gives the critical mass as a function of fuel zone displacement.

Flux mapping for ocveral of the stackings has been carried uut, using the autoradiographic technique. Typical results are given in Figures 12, 13, and 14. 


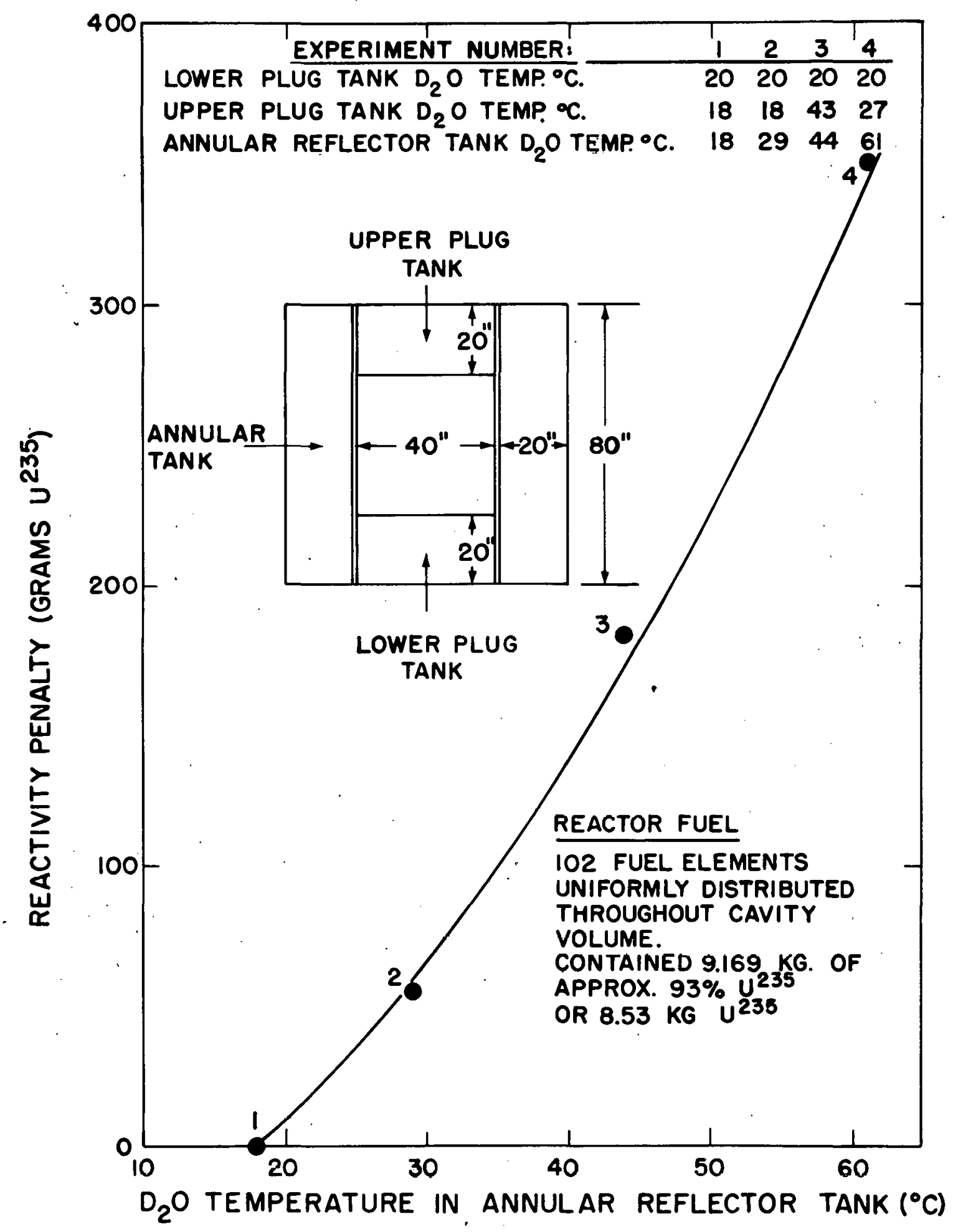

Figure 8. Cavity Reactor $\mathrm{D}_{2} \mathrm{O}$ Reflector Heating Experiment 

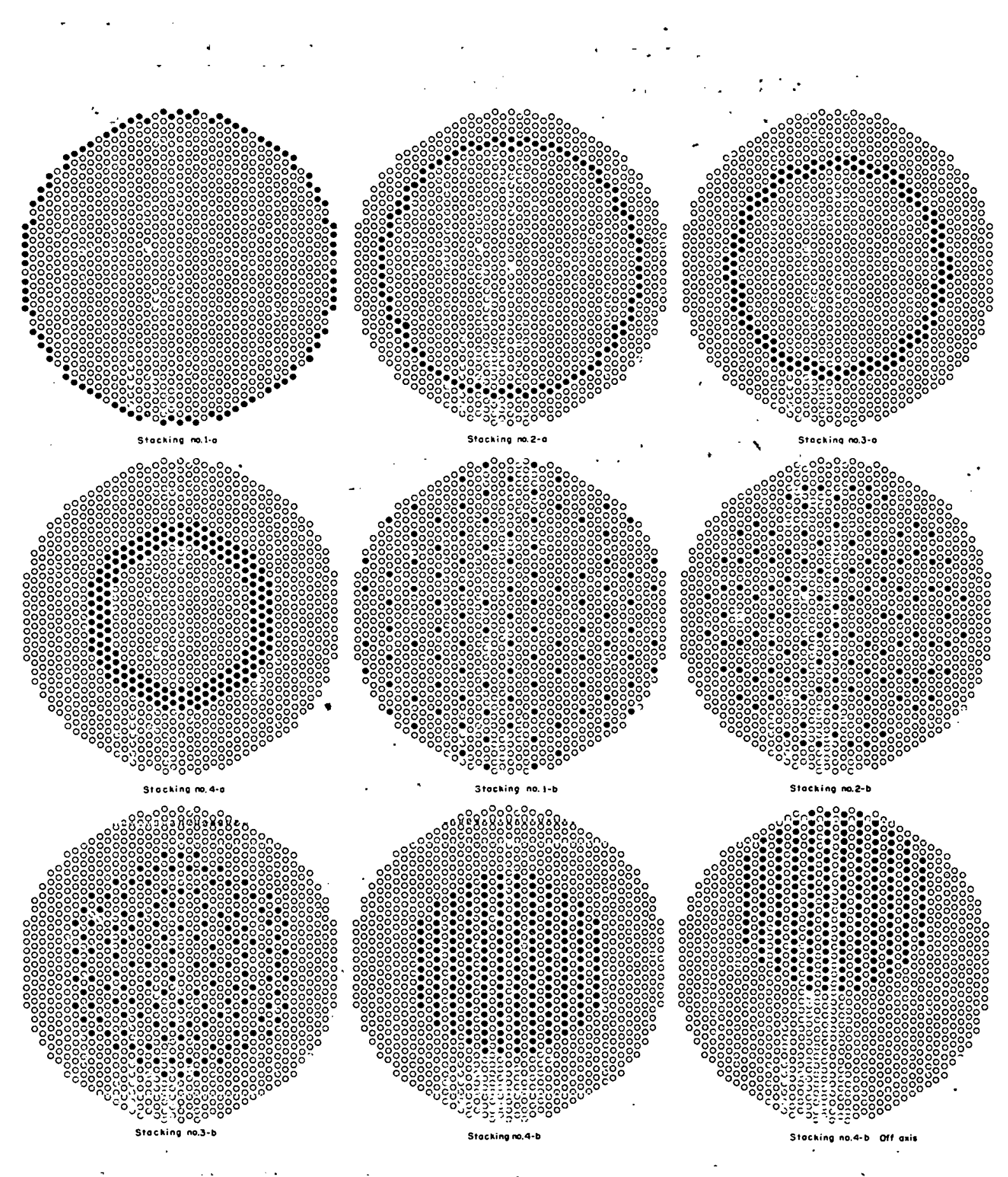

Figure 9. Loading Patterns for Fuel Elements in Cavity Reactor 


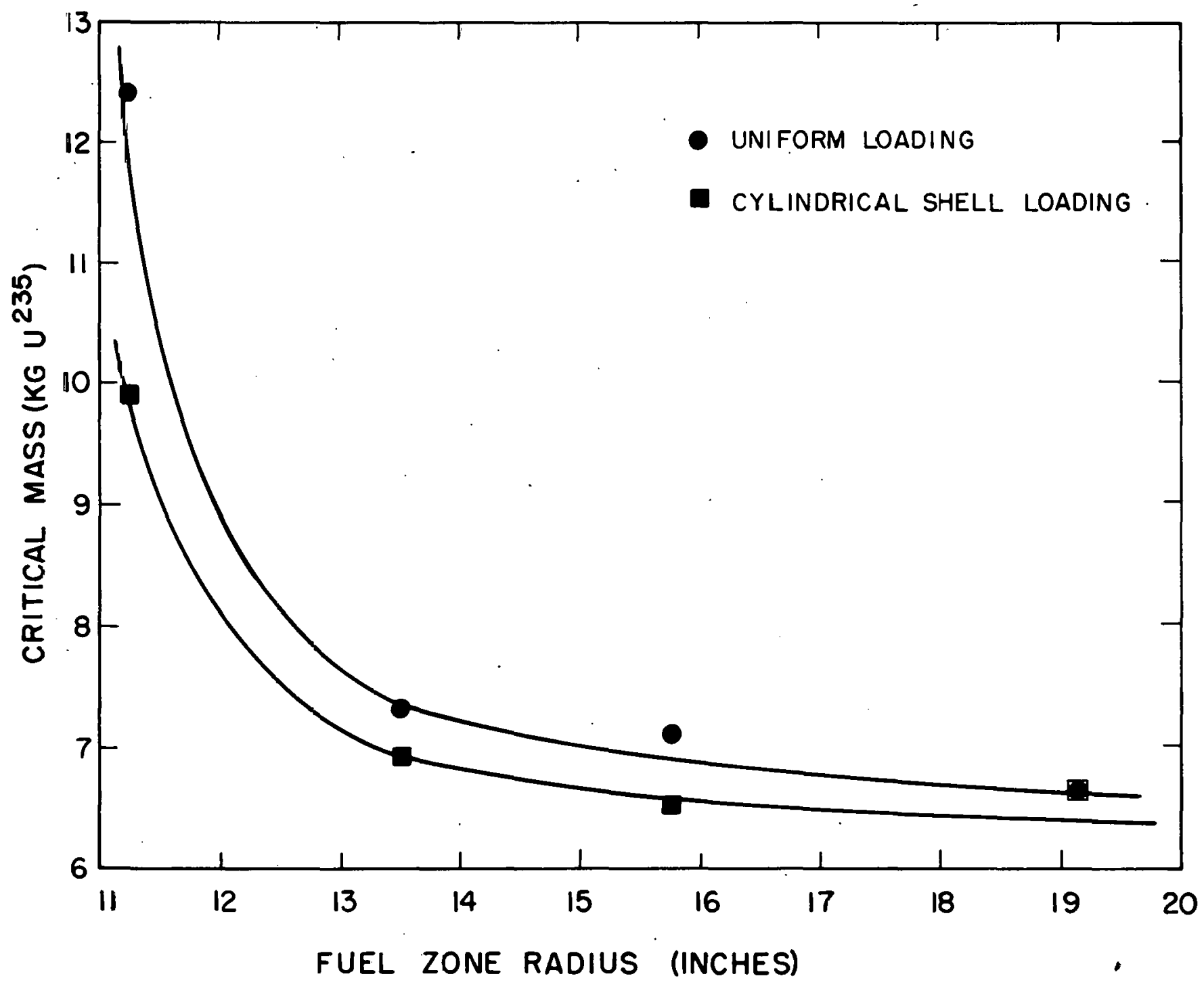

Figure 10. Critical Mass vs Fuel Zone Radius 


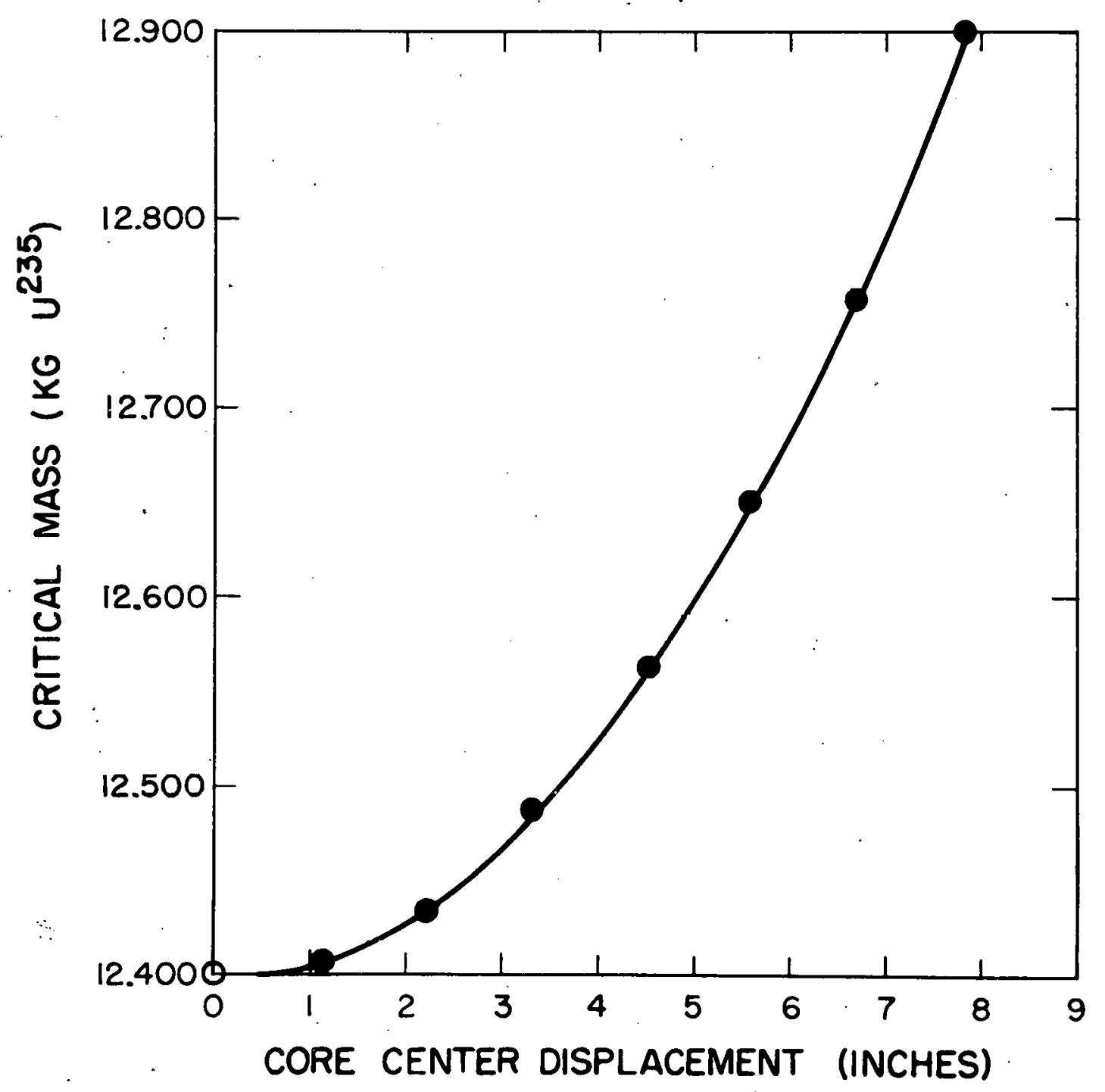

Figure 11. Critical Mass vs Fuel Cylinder Displacement 


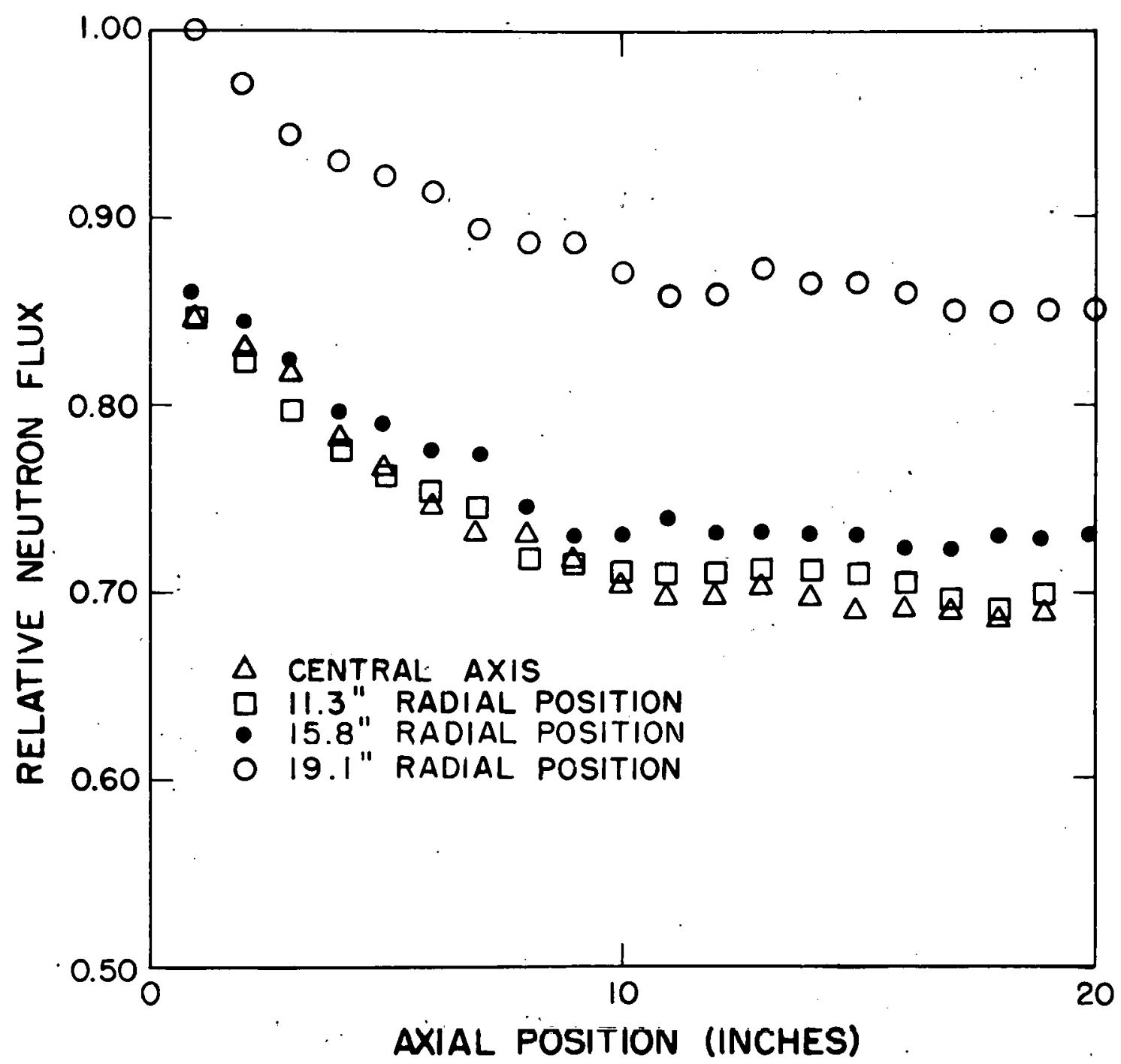

Figure 12. For Stacking No. 1-b, Neutron Flux Intensity along Lines Parallel to Cylindrical Axis of Cavity at Indicated Radial Positions 


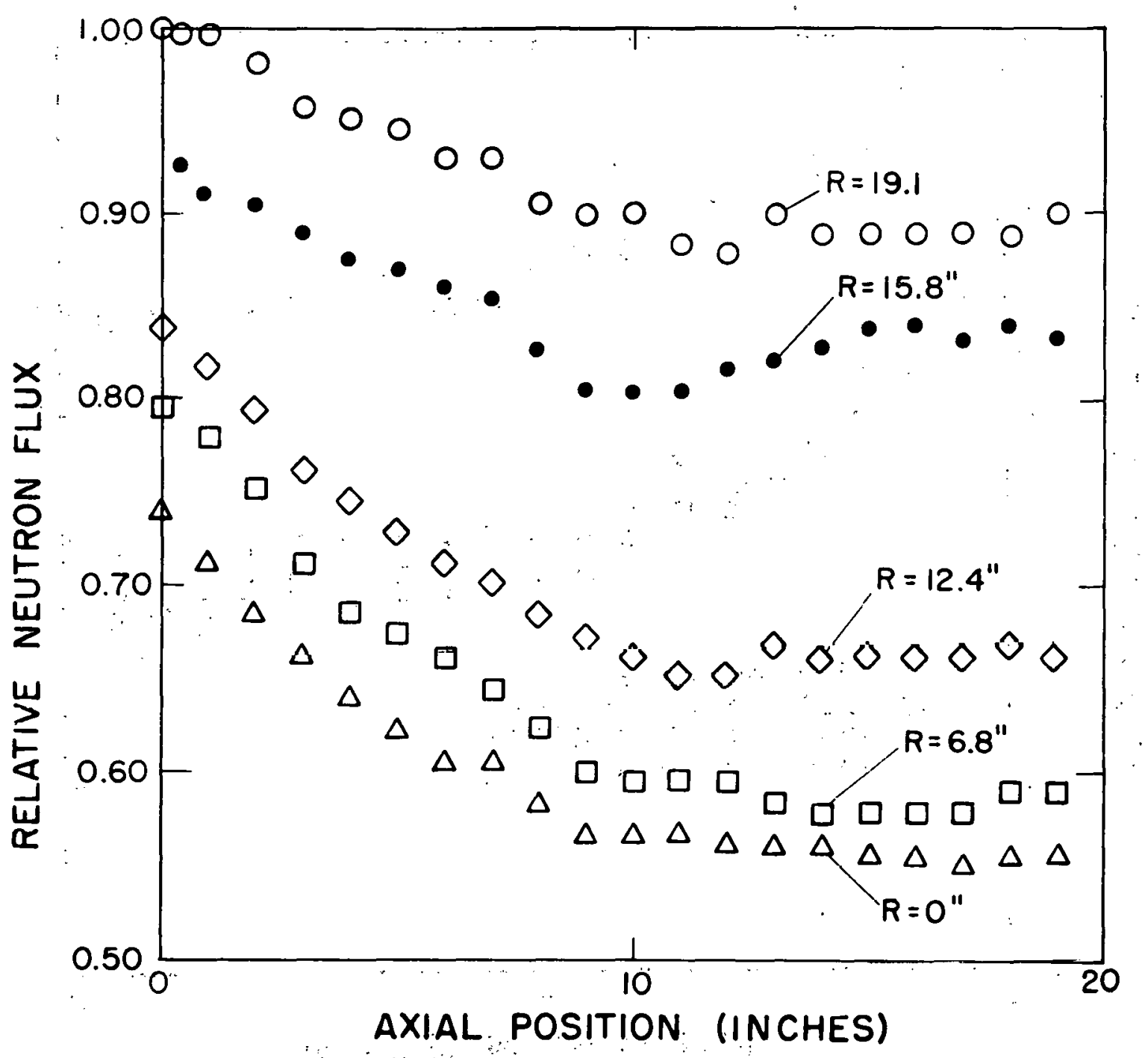

Figure 13. For Stacking No. 2-b, Neutron Flux Intensity along Lines Parallel to Cylindrical Axis of Cavity at Indicated Radial Positions. (*Bump belicved due to flaw in photographic film.) 


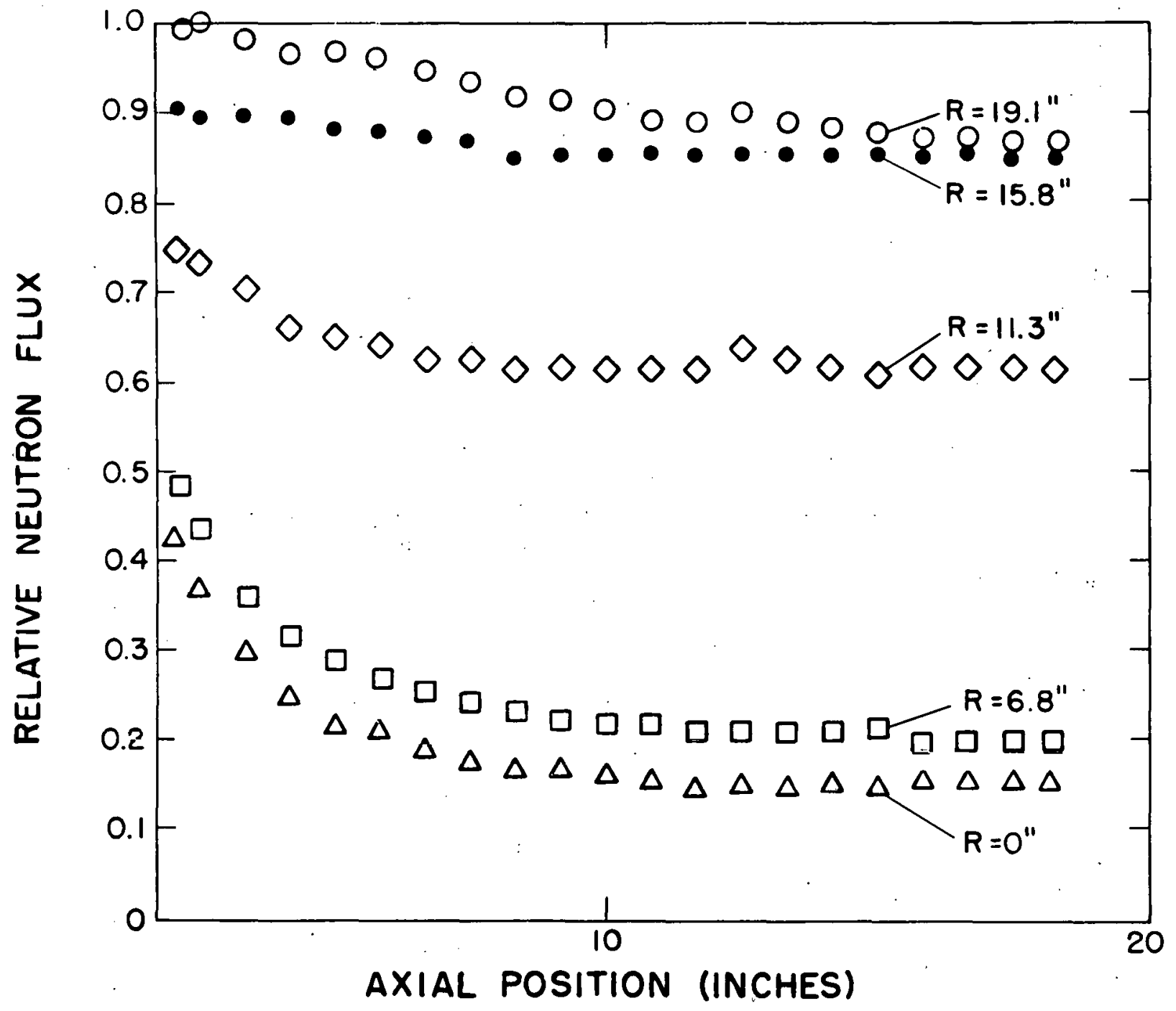

Figure 14. For Stacking No. 4-b, Neutron Flux Intensity along Lines Parallel to Cylindrical Axis of Cavity at Indicated Radial Positions. 


\section{CRITICALITY \\ C. B. Mills \\ Los Alamos, New Mexico}

Los Alamos Scientific Laboratory

The essential elements of reactor neutron transport methods and their use can be most clearly understood by a simple illustration, rather than by a discussion of a highly developed system. In 1950 we had the problem of computing the "muclear" characteristics of a $\mathrm{BeO}$ aircraft reactor fueled by fused fluorides in tubes. The transport equations were, as always, as exact as one wanted; but the constants of the equations, the neutron cross sections, were very poorly known. In fact, a calculation of a simple Be critical experiment gave $k_{\text {eff }} \simeq 0.90$. Rather than adjusting the constants of the "four factor formula" to give "useful" results for the similar BeO reactor prototype experiment, we tried to develop energy dependent cross sections for $\mathrm{Be}$ and $\mathrm{U}^{235}$ that could, in principle, generalize the transport equations. This involved exploration of the $(n, 2 n),(n, \alpha)$, and $\left(n, n^{\prime}\right)$ cross sections that would be consistent with both the neutron age experiment for $\mathrm{Be}$, which required some assumptions for $\sigma(\mathrm{E}, \theta)$, and the critical reactor calculation. In addition, the possibility of errors in the $U^{235}$ cross sections and other elements of the computing system, now a multigroup code, was investigated by studying a second reactor experiment, this time constructed of graphite. The cross-section problem with graphite was much simpler than that with $\mathrm{Be}$, as is the problem with oxygen. As a result of these studies, it was possible to evolve reasonably accurate $\mathrm{Be}, \mathrm{C}, \mathrm{O}$, and $\mathrm{U}^{235}$ cross sections as functions of energy. This in turn served to generalize in a limited sense the transport equations, by that time in the numerical diffusion approximation. The largest implication of the situation at that point was that "exact" transport equation, $\varphi(\mathrm{r}, \mathrm{E})$, completed by reasonably exact cross sections, could then be used to compute the reactor physics of an arbitrarily specified reactor assembly with errors of the order of $1 \%$. The "exact" equations provided the extension to arbitrary geometries, with the restriction that the crosssection constants for the thermal region had still not been studied, so that 
complete generality in flux spectrum variation could not be included with confidence. It is the matter of "confidence" that makes a calculation applicable in design studies, and this has always followed the path from experimental verification and close theory to experimental collaboration and back.

Returning to the aircraft problem, it was clear to the design engineer (A. P. Fraas) that since reactor power, weight, and shielding were directly connected, the use of a moderator for both low critical mass and shielding would result in a significant improvement. The "generalized" computing system, with some thermalization assumptions, showed that this should be possible by simply surrounding the fuel with a reasonably thick moderatorreflector. Experiments verified the computed results, so the first of the cavity reactor concepts was born.

As a by-product of the reactor studies discussed above and of the use of computing methods to uncover new possibilities in the reactor design, the value of detailed studies of theoretical methods in reactor evaluation was shown to be real. As one result, it was made possible in the following years to integrate a wide variety of reactor experiments and detailed experimental neutron cross-section studies into a simple, fast, and flexible numerical computing system. This system is currently of significant help in the study of reactor performance and in particular in the extrapolation of experimental assemblies to high-power reactors.

\section{NEUTRONICS OF THE GASEOUS REACTOR}

The use of a heat absorbing gas, seeded hydrogen for one, to contain very hot gaseous fissionable material removes one basic limit to the attainable temperatures that might be reached with fission energy as the driving heat source. This limit, due to the stress yielding point of solid materials, is presently a basic obstacle to the use of nuclear fission energy for the source of very high gas temperature. Accordingly a gaseous container backed hy solid materials, behind the large temperature difference that can occur in radiation absorbing gases, is conceptually interesting. The area of study shifts from an approach to maximum attainable temperature in solids (that retain the fuel very well) to geometries that minimize critical reactor fuel concentration. This is because the fuel loss rate for gaseous systems can be large and is at least proportional to the fuel concentrations. Once the criticality problem is in hand, the problem area shifts to that of fissionable fuel conservation.

In this note the effects of simple and complex geometries on critical concentration are computed for the most familiar fuel $\mathrm{U}^{235}$, with the reservation 
that $\mathrm{U}^{233}$ and $\mathrm{Pu}^{239}$ will have somewhat lower values of concentration. The effects of different geometries on criticality are then pointed out for the conceptually simplest situation, that of a single large rocket power plant. The same problem areas will be found in the use of nuclear power for generation of very high gas temperature for laboratory use, with the exception that the heat dump problem is smaller. This same gadget can be developed as a small system with low critical mass and volume of hydrogen propellant simply by shifting to smaller radii on the figures. Since criticality is closely connected with rocket reaction design, some space will be spent on design ideas.

\section{Criticality}

Criticality is connected with geometry and with moderating and structural materials. Minima in critical mass are reached with small cavity fuel critical concentrations. This is illustrated by Figures 1 and 2 for the two types of systems. Figure 1 shows the effect of $U^{235}$ critical concentration of (filled) gaseous spherical cavity radius reflected by $\mathrm{Be}, \mathrm{C}$, or $\mathrm{D}_{2} \mathrm{O}$. The same variables are shown (dotted line) for a homogeneous sphere of $\mathrm{U}^{235}$ and $\mathrm{Be}$. Figure 2 shows the critical concentration of $\mathrm{U}^{235}$ in homogeneous spheres of moderator $\mathrm{Be}, \mathrm{C}$, or $\mathrm{D}_{2} \mathrm{O}$. Both systems have critical concentrations of $\mathrm{U}^{235}$ near $10^{18}$ atoms per cc for large $(300 \mathrm{~cm})$ radii.

Neither the gaseous cavity nor the homogeneous reactors described in Figures 1 and 2 approach the "gaseous reactor" condition, since this is defined as a gas contained by a gas. Figure 3 shows the first truly gaseous reactor, if an annulus of hydrogen can be visualized between the "cold" wall (located by the intercept of dotted and solid lines) and the restricted $U^{235}$ gas radius located by any point on the solid line. The hydrogen (or any other heat absorbing gas) is the container. An order of magnitude increase in $\mathrm{U}^{235}$ concentration is required for criticality with; perhaps, $10 \%$ of the area filled with the fuel and $90 \%$ by the "container" gas (presumably hydrogen for a rocket).

The development of a gaseous reactor from a homogeneous geometry (Figure 2) is indicated to be easily feasible using the results of Figure 4. This shows the radius of a fisssionable gas in a cylindrical cell to not affect criticality in first order above area ratios of fuel to cavity of about $1 / 100$. By way of a heterogeneous cylindrical geometry shown in Figure 5 (equivalent to a homogeneous geometry according to Figure 4) we reach a.."gaseous reactor" shown in Figure 6. A geometry such as this was reduced to a section shown in Figure $6 \mathrm{~b}$ from homogeneous reactor dimensions: A twodimensional calculation must agree with the critical $\mathrm{U}^{235}$ concentration 


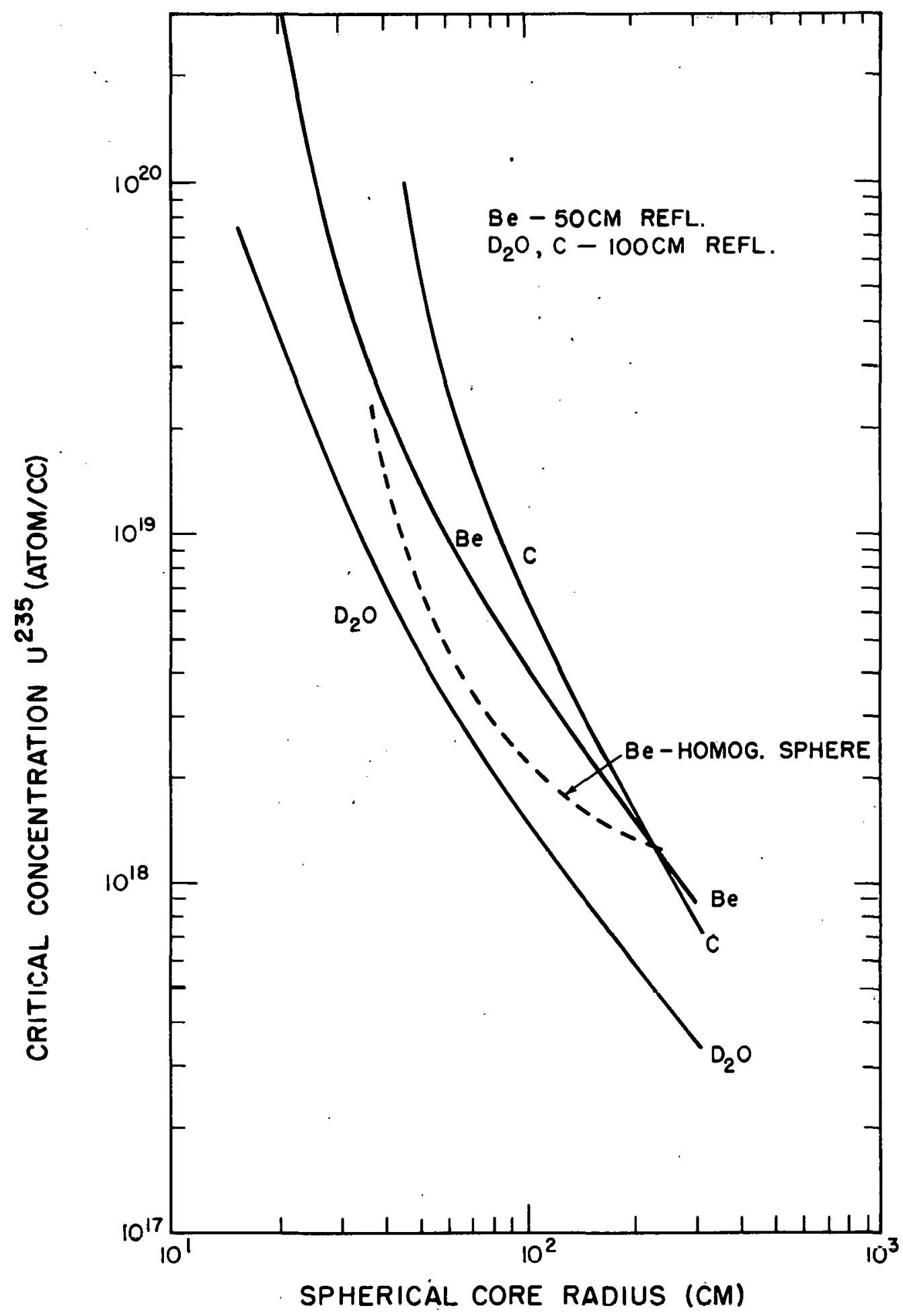

Figure 1. Reflector Moderated Reactors 


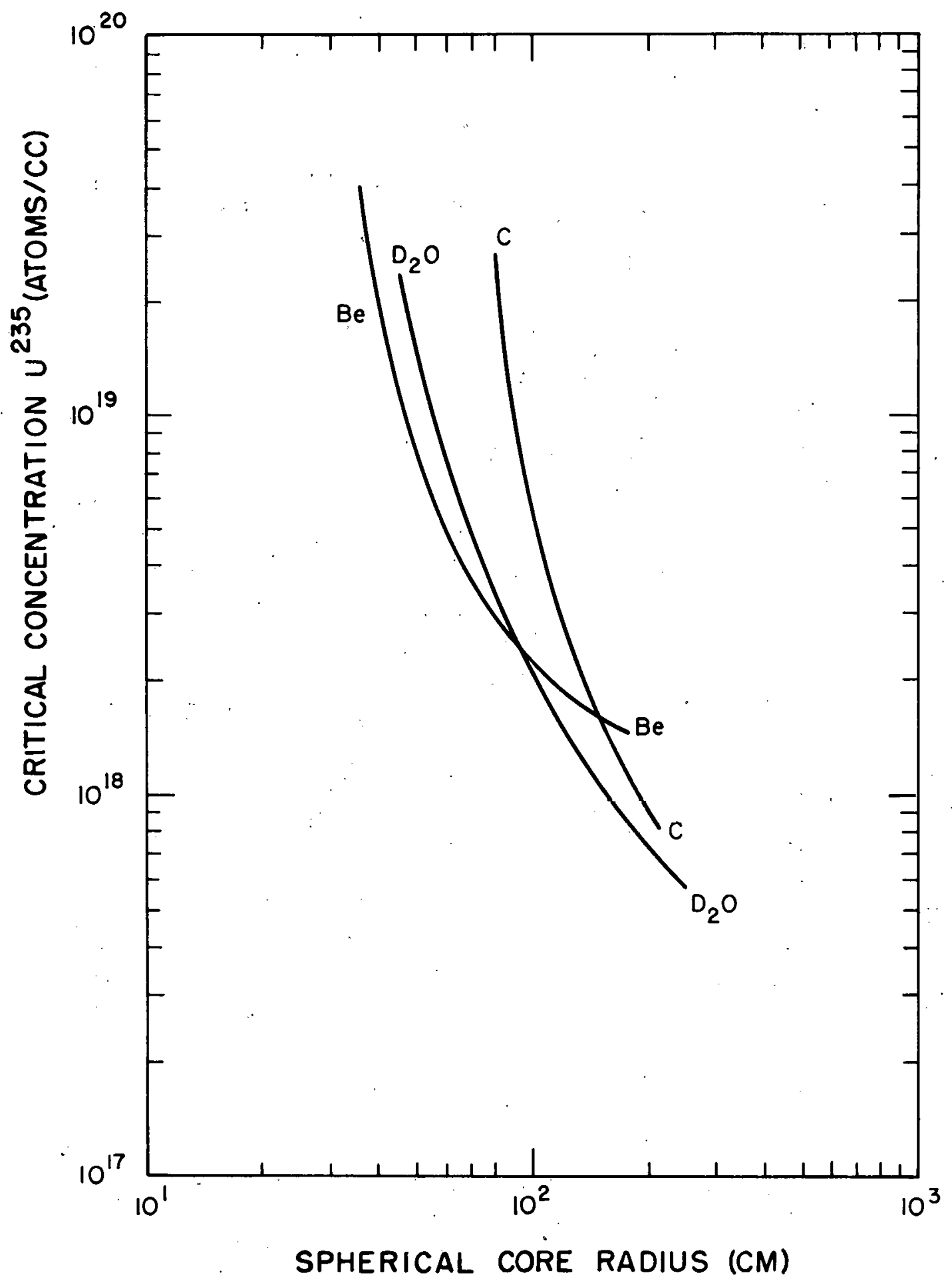

Figure 2. Core Moderated Homogeneous Reactors 


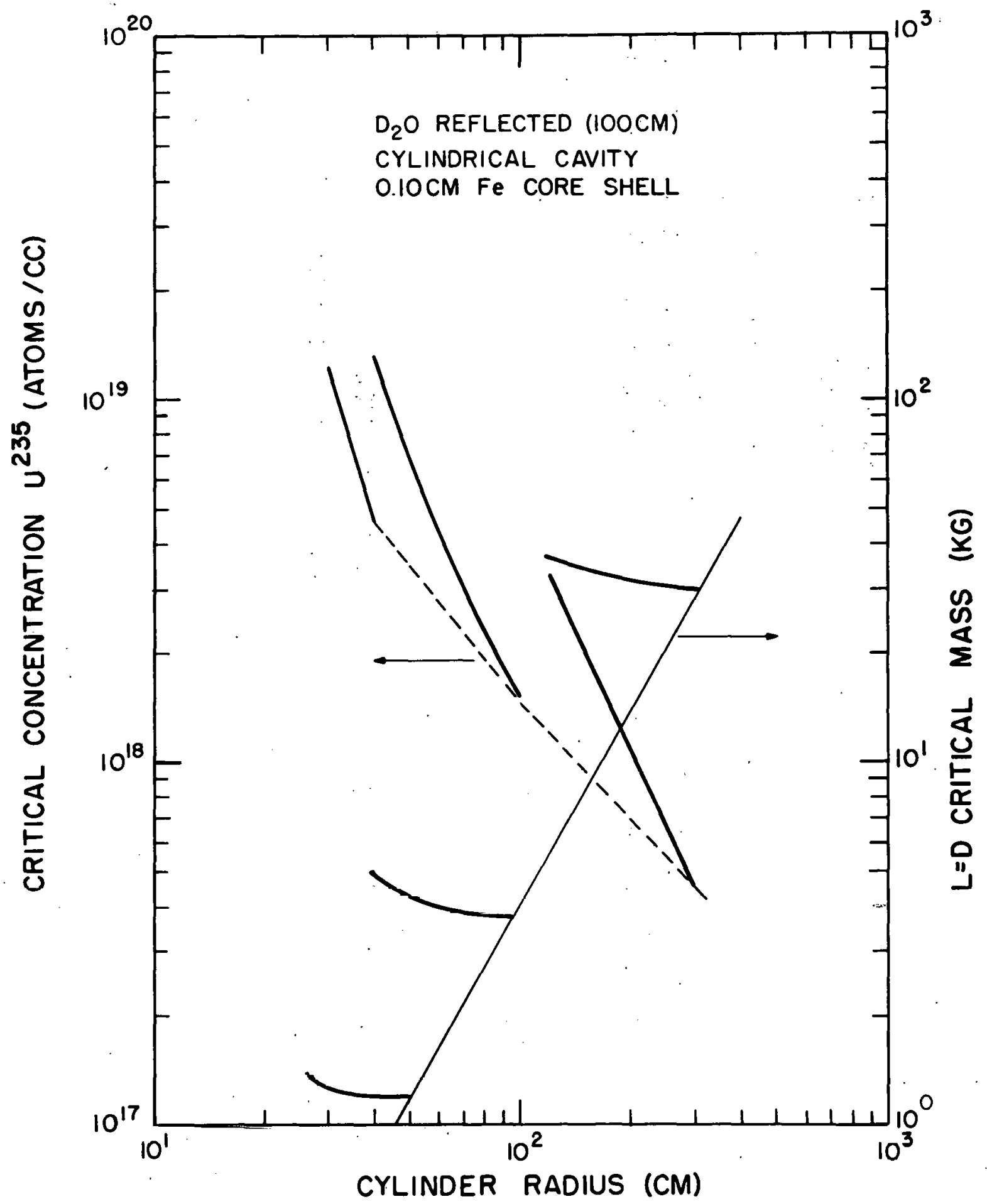

Figure 3. Reflector Moderated Gaseous Reactors 


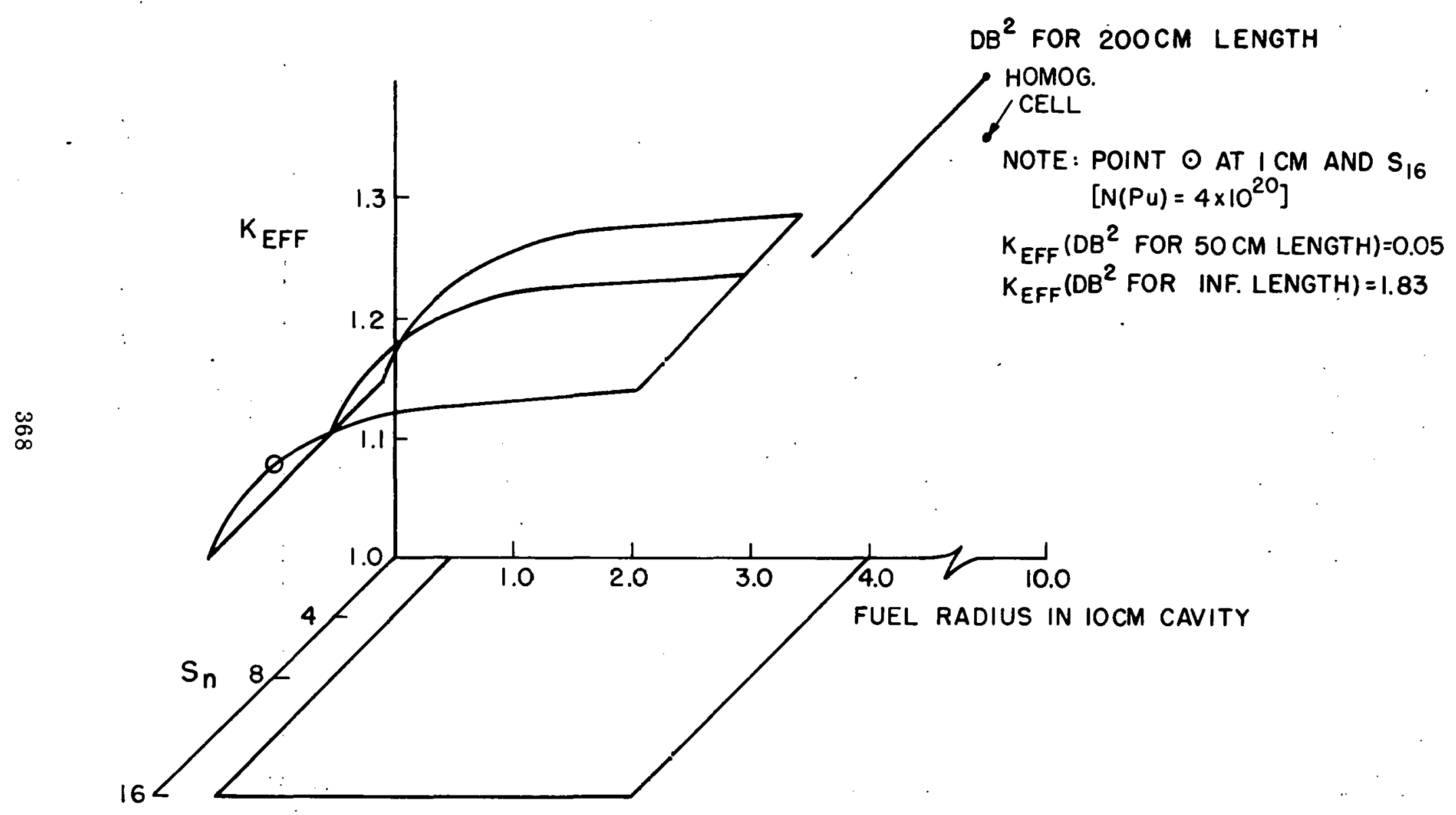

Figure 4. Cylindrical Cell 


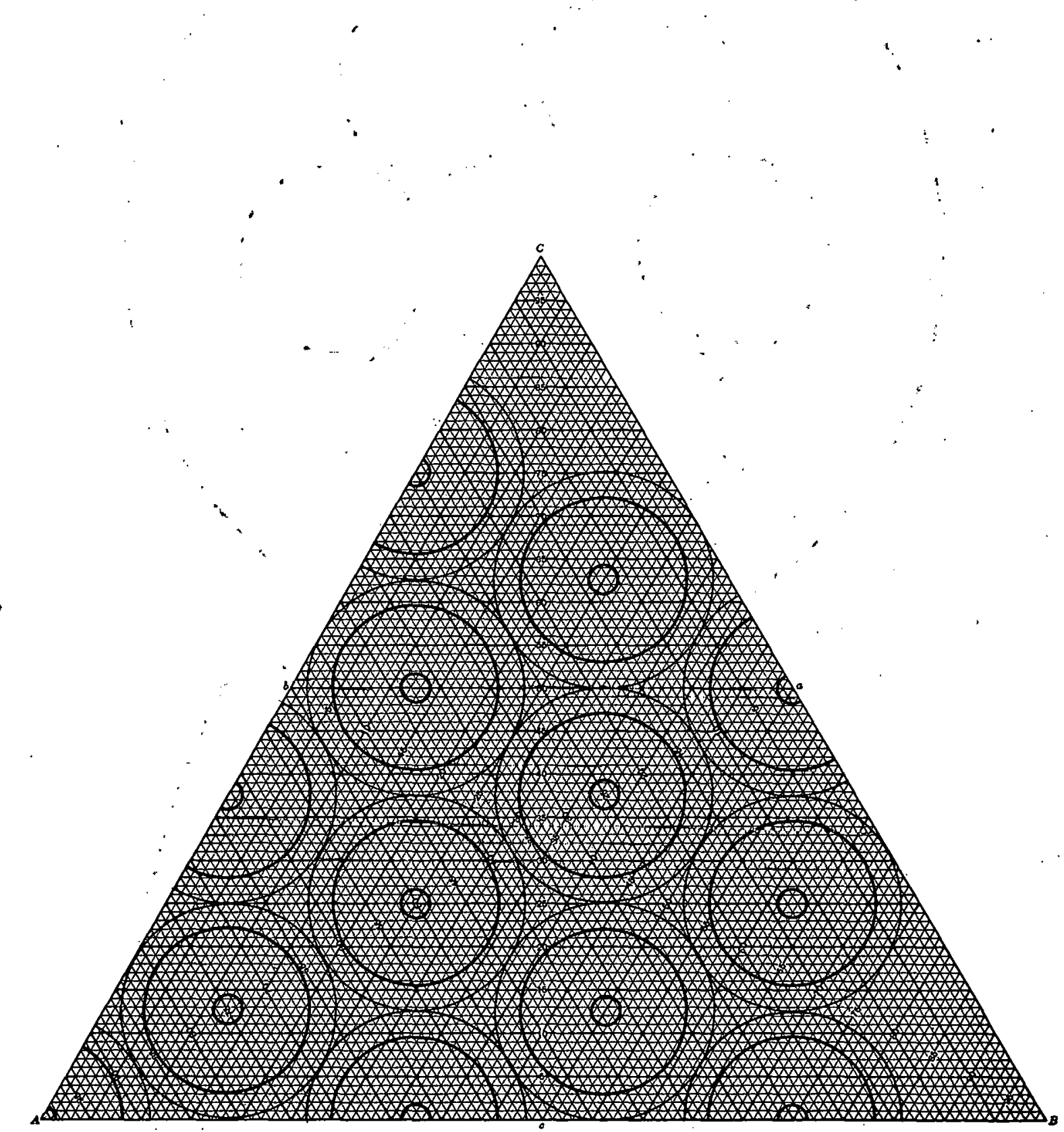

Figure 5. Core Moderated Gaseous Reactor 


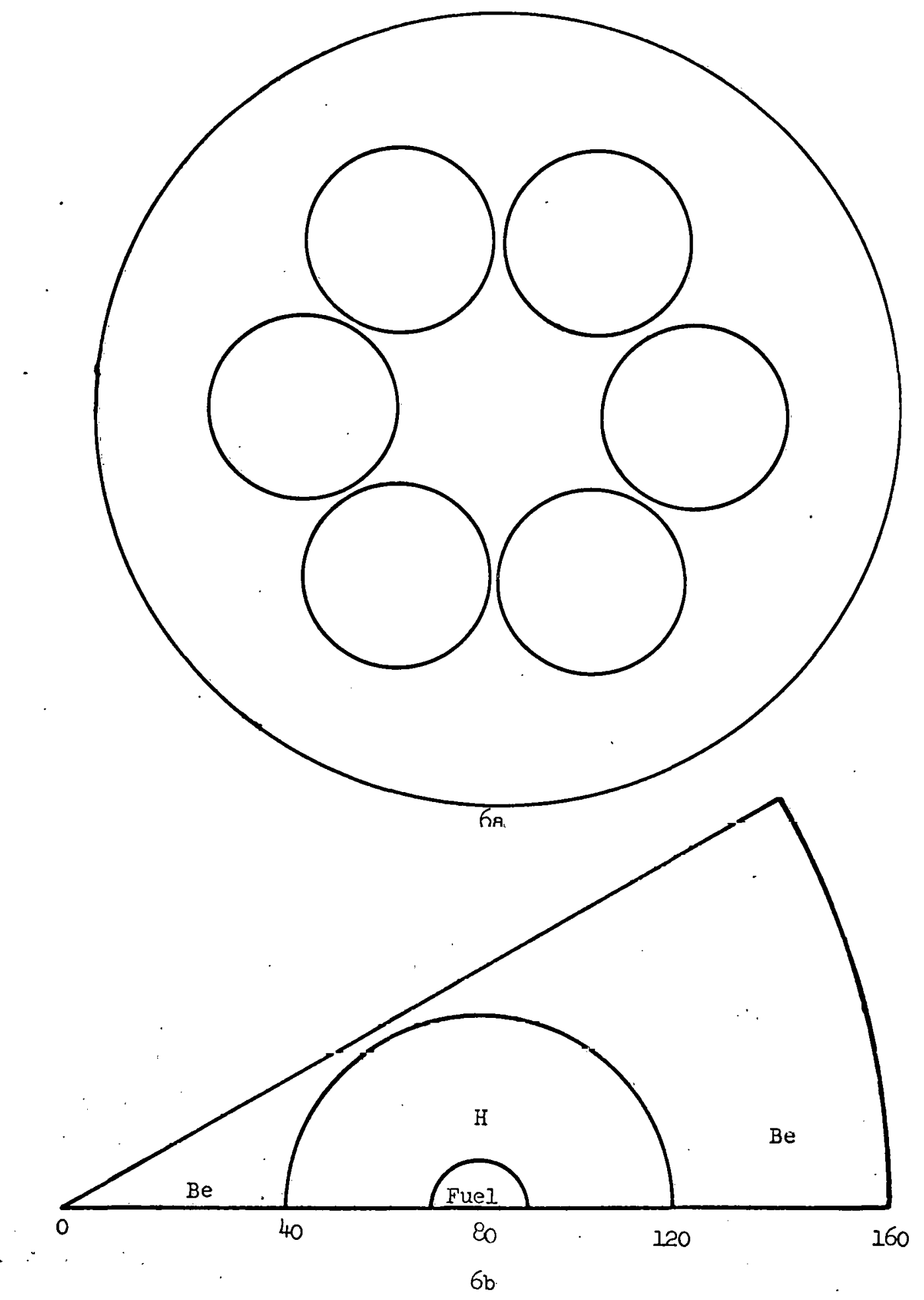

Figure 6. Gaseous Reactor 
obtained by an area ratio factor on fuel concentration from the simple system. This reduction is illustrated below under the design section. A summary of the criticality situation at this point in the discussion is shown in Figure 7. The simple cavity and homogeneous reactors are shown by solid and dotted lines, respectively. A multiple cavity described as a simple homogeneous reactor can suffer from radial streaming effects so two 6cavity "homogeneous" critical points are shown for 40-cm-radius cylindrical cavities in a $160-\mathrm{cm}$-radius cylinder $\left(\mathrm{DB}^{2}\right.$ axial $\left.=10^{-4}\right)$. The simple annular geometry obtained from Figure 6 by using an island (Be) and a reflector (Be) as inner and outer bounds of a "gaseous reactor" is shown by circles (critical mass by dots) as an intermediate position. Critical mass (per $100 \mathrm{~cm}$ length) in each of the six cavities is shown by the diamond. Clearly there is little cost associated with internal details; in fact the cost of removing moderator is small, for a thick reflector.

Summary remarks, as a review of the situation of neutronic design of gaseous reactors, can be made. The results above of a quantitative if not specific nature show that a gaseous reactor can be designed with either the simple or multiple cavity geometry. The selection of the better type cannot be made solely on neutronic arguments, since both have roughly the same critical mass and size. The physical properties of the gaseous reactor connected with radiation transfer, flow stability and gas mixing, and fuel conservation techniques are also fundamental; and a few qualitative arguments will be indicated below.

Heat Balance

The total heat $Q_{T}$ generated by the fissionable fuel is deposited in large part in the fuel from which it is radiated to the surrounding gas. Two to $5 \%$ is deposited in the moderator and structure by neutrons (slowing down) and gammas ( $n, \gamma$, and decay product reactions). A larger energy sink is that due to fuel conservation. The heat of condensation of uranium is $\mathrm{h}=110 \mathrm{k} \mathrm{cal} / \mathrm{mol}=4.75 \mathrm{eV} /$ atom. If all of the fuel were to be separated by condensation the thermal heat sink $(Q=N k T)$ required would in addition be $Q(U) \simeq \frac{N(U)}{N(H)} Q_{T}$ (where $N$ is the number of atoms) if fuel and hydrogen were heated to approximately the same temperature. $Q(U)$ is small only for a small fuel area fraction in the gas flow, if all the fuel $N(U)$ is cooled and condensed for retention. Since there is a relatively small amount of heat available for cooling in a simple hydrogen exhaust cycle - the heat absorbed while heating hydrogen by a solid heat exchanger to its maximum temperature, say $2300^{\circ} \mathrm{K}$ - only about $10 \%$ of the total heat absorbed before 


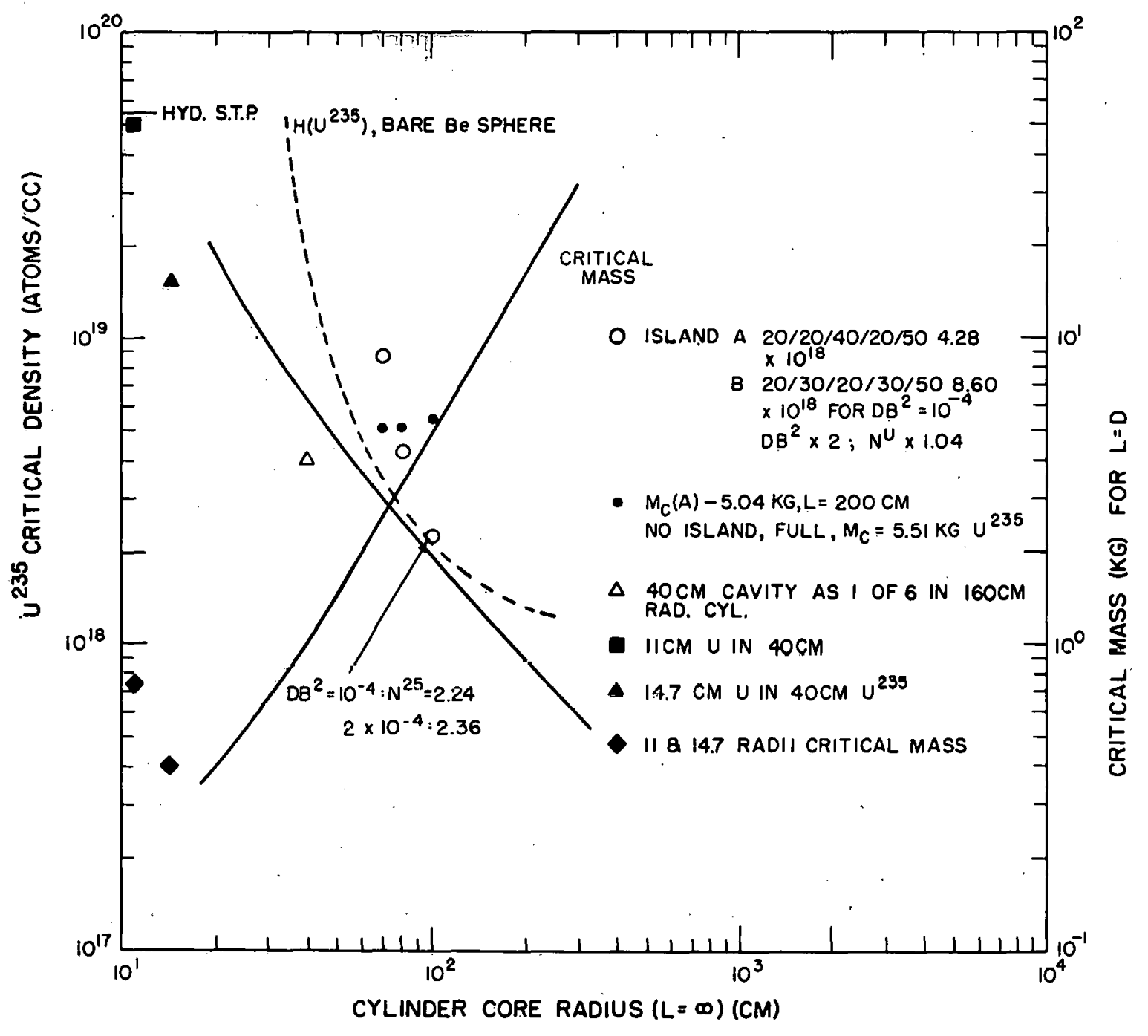

Figure 7. Cylindrical Cavity. Be Refl. $50 \mathrm{~cm}\left(10 \%\right.$ void) $\mathrm{U}^{235}$. 
exhaust is available for auxiliary cooling. In other words, the heat made sink available by initial heating of the "containing" gas, hydrogen, is not large enough, roughly by the amount required to condense the fuel from gas to liquid in its own loop. This adds about $4 \mathrm{eV}$ per fuel atom to the energy $(\mathrm{kT} \simeq 2 \mathrm{eV})$ of the fully heated gas.

The flexibility of gaseous reactor design parameters at this point permits moving out of the heat balance dilemma in three ways. First, areas and temperatures can be moved by significant amounts toward a more favorable design. The insensitivity of the multiple cavity concept to geometry permits these changes with small effect, and we move in that direction. Second, recognition that molecular evaporation requires less heat significantly reduces this problem if $\mathrm{UF}_{6}(\mathrm{~h} \simeq 7 \mathrm{kilocal} / \mathrm{mol}$ or $0.3 \mathrm{eV} / \mathrm{molecule}$ ), for example, can be used; also $\mathrm{H}_{2} \mathrm{O}$ rather than $\mathrm{H}_{2}$ adds $10 \mathrm{kilocal} / \mathrm{mole}$ of heat sink. Third, the coolant gas heated to perhaps $0.2 \mathrm{eV}$ can then be (wastefully) ejected in part.

\section{Gas Density}

The lower limit to gas density in a gaseous reactor is set by the criticality requirement. This is connected strongly to geometry and temperature if the (room temperature) reflectors used for the evaluations above are to be strongly heated. The gas density to be used can be set approximately by noticing that a factor of 100 in temperature (to $2 \mathrm{eV}$ ) causes a factor of 100 in pressure (to perhaps 100 atmospheres) for constant atomic density. This value for hydrogen $\left(5.4 \times 10^{19}\right)$ is shown on Figure 7 for reference. It is clear from Figure 8, showing critical fuel atomic density vs bare reactor weight, that little is gained in going below $2 \times 10^{18}$ atoms/cc $\left(\mathrm{U}^{235}\right)$ for the homogeneous reactor condition. This is seen more clearly by noting that the near-asymptotio (from Figure 4) reactor to fuel area ratio of 35 gives an atomic density ratio $\mathrm{N}(\mathrm{U}) / \mathrm{N}(\mathrm{H})=1$. In particular, this ratio is unity for a 11-cm-radius fuel cylinder in a 40-cm-radius $\mathrm{H}$ filled cavity (this is the gaseous reactor shown in Figure 6) as an assembly of six in a 160-cm-radius Be moderator geometry.

This point, and a perturbation to $14.7 \mathrm{~cm}$, is shown in Figure 7 . We note that an area factor of 35 raises the critical concentration of fuel at $160 \mathrm{~cm}$ reactor radius from $1.5 \times 10^{18}$ to $50 \times 10^{18}$ per cc (that of $\mathrm{H}_{2}$ at $\mathrm{STP}$ ) for relatively small axial neutron escape (length $\simeq 300 \mathrm{~cm}$ ). 


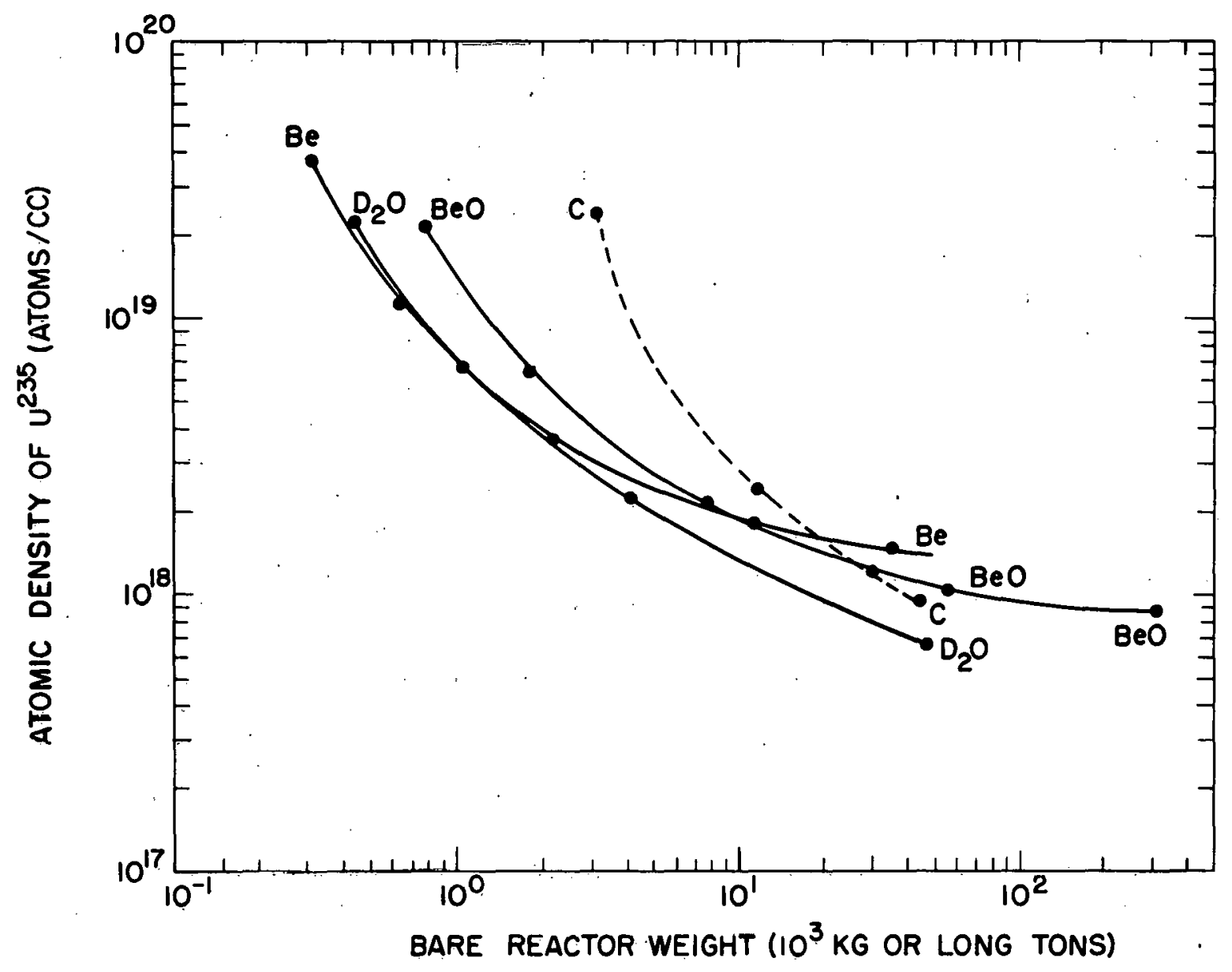

Figure 8. Critical Fuel Atomic Density vs Bare Reactor Weight 
Geometry

Holes in the moderator-reflector are costly in terms of keff. Experimental variation in hole diameter in a 50-cm-thick $\mathrm{D}_{2} \mathrm{O}$ reflector is shown in Figure 9. A computed point is shown for $40-\mathrm{cm}$ diameter. The exhaust port end of a cavity reactor should have a large $\Delta \mathrm{k}$ associated with it, also, although the magnitude would be reduced by the natural displacement of the neutron flux profile toward the colder, inlet end. No evaluation has been made as yet of fast neutron vs thermal neutron streaming effects.

Summary

The general characteristics of gaseous reactors include simplicity and sensitivity to any neutron sink, whether by structural material absorption or streaming through holes. The one-cavity reactor will tolerate relatively small fuel radii. Low fuel atomic densities are consistent with large reactor sizes, with both types having roughly the same values of fuel concentration near $300-\mathrm{cm}$ radius. Neither type is clearly better with regard to reactor neutronics. 


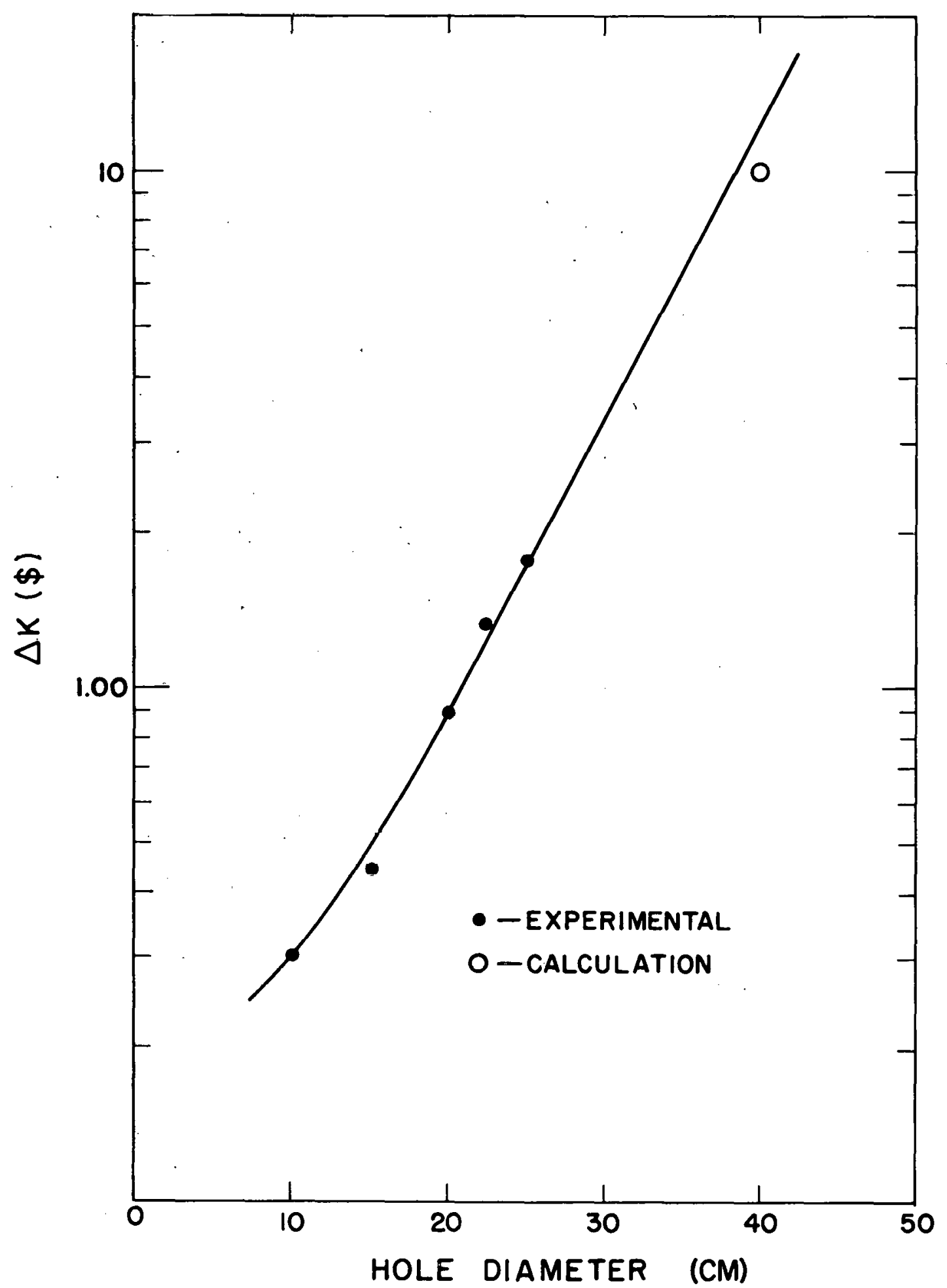

Figure 9. Cost of Hole in One End of Cavity Reactor vs Hole Diameter 